\title{
Access to S-Stereogenic Free Sulfoximines via Bifunctional Phosphonium Salt-catalyzed Desymmetrization of Bisphenols**
}

Siqiang Fang,,$^{\dagger}$ Zanjiao Liu, ${ }^{\dagger}$ Hongkui Zhang, ${ }^{\dagger}$ Jianke Pan, ${ }^{\dagger}$ Yuan Chen, ${ }^{\dagger}$ Xiaoyu Ren, ${ }^{\dagger}$ and Tianli Wang ${ }^{*} \dagger$

$\dagger$ Key Laboratory of Green Chemistry \& Technology of Ministry of Education, College of Chemistry, Sichuan University, 29 Wangjiang Road, Chengdu 610064, P. R. China.

E-mail: wangtl@scu.edu.cn

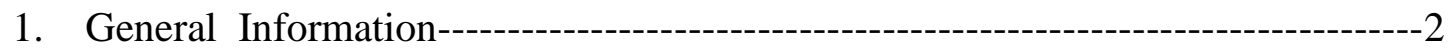

2. Optimization of Reaction Conditions for Desymmetrizing Asymmetric A-T-type

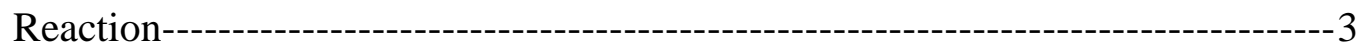

3. Preparation of Phosphonium Salt Catalysts-----------------------------------------7

4. Preparation of Free Sulfoximines 1--------13

5. General Procedure for the Desymmetrization of Free Sulfoximines via A-T-type Reaction-

6. Investigation on the Active Species of the A-T Process and Desymmetrizing NAS-type Reaction Development------------------------------------------------'67

7. Optimization of Reaction Conditions for Desymmetrizing Asymmetric NAS-type Reaction-

8. General Procedure for the Desymmetrization of Free Sulfoximines via NAS-type

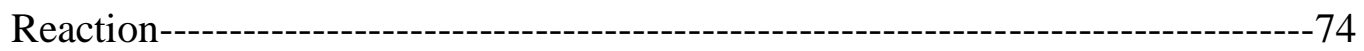

9. Synthetic Applications of the Desymmetrizing Products------------------------125

10. Determination of Absolute Configuration of the Chiral Products--------------136

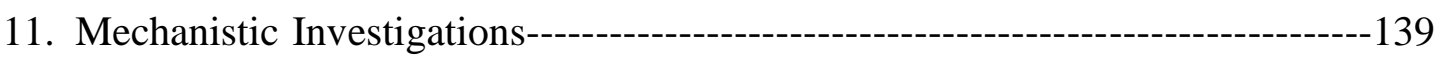

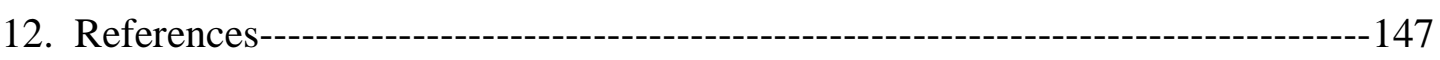

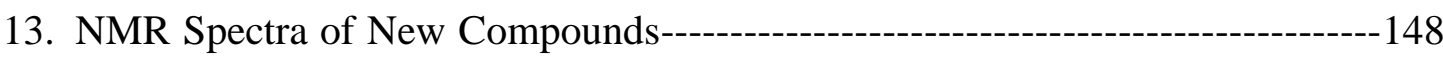




\section{General Information}

All the starting materials were obtained from commercial sources and used without further purification unless otherwise stated. ${ }^{1} \mathrm{H}$ and ${ }^{13} \mathrm{C}$ NMR spectra were recorded at ambient temperature in $\mathrm{CDCl}_{3}$ or DMSO on a Brüker Advance 400 spectrometer. Chemical shifts $(\delta)$ were given in parts per million $(\mathrm{ppm})$, and the residual solvent peak was used as an internal reference $\left(\mathrm{CDCl}_{3}: \delta 7.26 \mathrm{ppm}{ }^{1} \mathrm{H} ; \delta\right.$ $77.16 \mathrm{ppm}{ }^{13} \mathrm{C}$; DMSO-d6: $\left.\delta 2.50 \mathrm{ppm}{ }^{1} \mathrm{H} ; \delta 39.51 \mathrm{ppm}{ }^{13} \mathrm{C}\right)$. Multiplicity was indicated as follows: s (singlet), $\mathrm{d}$ (doublet), $\mathrm{t}$ (triplet), $\mathrm{q}$ (quartet), m (multiplet), dd (doublet of doublet), br s (broad singlet). Coupling constants $(J)$ were reported in Hertz $(\mathrm{Hz})$. High resolution mass spectra were obtained on a Thermo LTQ mass spectrometer. For thin layer chromatography (TLC), Merck pre-coated TLC plates (Merck 60 F254) were used, and compounds were visualized with a UV light at 254 $\mathrm{nm}$. Further visualization was achieved by staining with potassium permanganate followed by heating on a hot plate. Flash chromatographic separations were performed on Merck $60(0.040-0.063 \mathrm{~mm})$ mesh silica gel. Enantiomeric excess was determined by HPLC analysis using chiral column described below in detail. Optical rotations were measured with polarimeter. 


\section{Optimization of Reaction Conditions for Asymmetric A-T-type}

\section{Reaction}

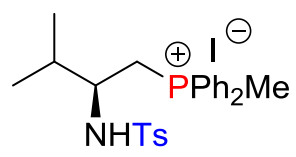

P1<smiles></smiles>

P5<smiles>CC(C)[C@H](N)C[PbH2][PbH2]</smiles>

P2<smiles></smiles>

P3<smiles>CC(C)C(N[C@@H](C[PbH])C(=O)OCc1ccccc1)C(C)C</smiles>

P4

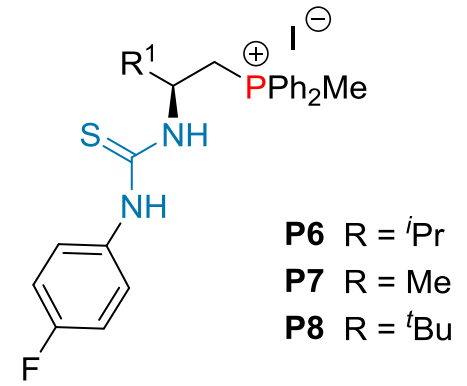<smiles>[R]O[C@H](C)[C@H](C[Pb](C)=O)NC(=S)Nc1ccc(F)cc1</smiles><smiles>CO[13CH2]c1ccccc1</smiles>

Scheme S1. Chiral phosphonium salt catalysts examined in this study. $($ TBDPS $=$ tert-butyldiphenylsilyl, TBS $=$ tert-butyldimethylsilyl $)$

Table S1. Optimization of the catalysts ${ }^{[\mathrm{a}, \mathrm{b}]}$<smiles>N=S(=O)(c1ccccc1O)c1ccccc1O</smiles>

$1 \mathrm{a}$

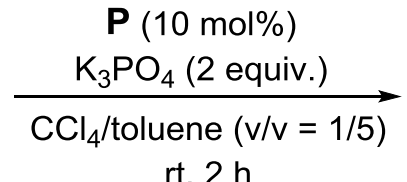
$\mathrm{rt}, 2 \mathrm{~h}$<smiles>N=S(=O)(c1ccccc1O)c1ccccc1O[Po+](O)c1ccccc1</smiles>

$3 a$

$\begin{array}{cccc}\text { entry } & \text { P } & \text { yield }(\%) & \text { ee }(\%) \\ 1 & \text { P1 } & 66 & 0 \\ 2 & \text { P2 } & 52 & 0 \\ 3 & \text { P3 } & 57 & 0\end{array}$




$\begin{array}{lllc}4 & \text { P4 } & 61 & 5 \\ 5 & \text { P5 } & 68 & 8 \\ 6 & \text { P6 } & 54 & 12 \\ 7 & \mathbf{P 7} & 56 & 8 \\ 8 & \mathbf{P 8} & 70 & 16 \\ 9 & \mathbf{P 9} & 75 & 19 \\ 10 & \mathbf{P 1 0} & 78 & 20 \\ 11 & \mathbf{P 1 1} & 86 & 51 \\ 12 & \mathbf{P 1 2} & 72 & 43 \\ 13 & \mathbf{P 1 3} & 79 & 68 \\ 14 & \mathbf{P 1 4} & 85 & 72 \\ 15 & \mathbf{P 1 5} & 82 & 81\end{array}$

[a] Reaction conditions: 1a $(0.1 \mathrm{mmol}), \mathbf{2 a}(0.12 \mathrm{mmol}), \mathrm{K}_{3} \mathrm{PO}_{4}(0.2 \mathrm{mmol})$ and the catalyst $(0.01$ $\mathrm{mmol})$ in $\mathrm{CCl}_{4} /$ toluene $(2.0 \mathrm{~mL}, \mathrm{v} / \mathrm{v}=1 / 5)$ at room temperature for $2 \mathrm{~h}$. [b] All ee values were determined by HPLC analysis on a chiral stationary phase, and all yields were isolated yields.

Table S2. Optimization of the temperatures ${ }^{[a, b]}$<smiles>N=S(=O)(c1ccccc1O)c1ccccc1O</smiles>

$1 \mathrm{a}$

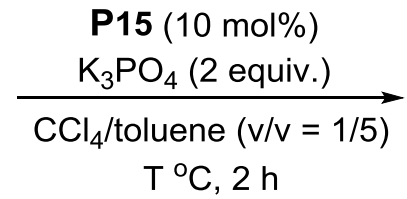
$\mathrm{T}^{\circ} \mathrm{C}, 2 \mathrm{~h}$<smiles>N=S(=O)(c1ccccc1OP)c1ccccc1OP</smiles>

3a

$\begin{array}{cccc}\text { entry } & \mathrm{T} & \text { yield }(\%) & \text { ee }(\%) \\ 1 & 25 & 82 & 81 \\ 2^{[\mathrm{c}]} & 0 & 78 & 96\end{array}$

[a] Reaction conditions: $1 \mathbf{a}(0.1 \mathrm{mmol}), \mathbf{2 a}(0.12 \mathrm{mmol}), \mathrm{K}_{3} \mathrm{PO}_{4}(0.2 \mathrm{mmol})$ and $\mathbf{P 1 5}(0.01 \mathrm{mmol})$ in $\mathrm{CCl}_{4} /$ toluene $(2.0 \mathrm{~mL}$, v/v $=1 / 5)$ at $\mathrm{T}{ }^{\circ} \mathrm{C}$ for $2 \mathrm{~h}$. [b] All ee values were determined by HPLC analysis on a chiral stationary phase, and all yields were isolated yields. [C] $12 \mathrm{~h}$.

Table S3. Optimization of the bases ${ }^{[a, b]}$<smiles></smiles>

$1 \mathrm{a}$

$2 a$

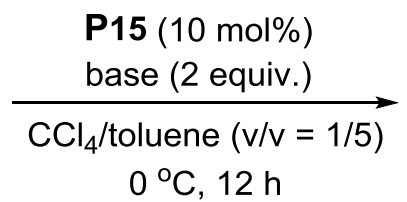

$0^{\circ} \mathrm{C}, 12 \mathrm{~h}$

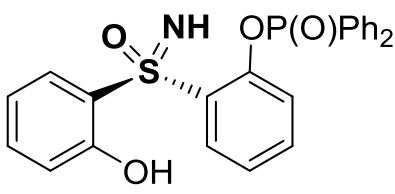

$3 a$

$$
\text { entry }
$$

yield $(\%)$

ee $(\%)$ 


$\begin{array}{cccc}1 & \mathrm{Cs}_{2} \mathrm{CO}_{3} & 87 & 80 \\ 2 & \mathrm{~K}_{2} \mathrm{CO}_{3} & 72 & 74 \\ 3 & \mathrm{KHCO}_{3} & 70 & 35 \\ 4 & \mathrm{~K}_{3} \mathrm{PO}_{4} & 78 & 96 \\ 5 & \mathrm{~K}_{3} \mathrm{PO}_{4} \cdot 3 \mathrm{H}_{2} \mathrm{O} & 70 & 96 \\ 6 & \mathrm{~K}_{3} \mathrm{PO}_{4} \cdot 7 \mathrm{H}_{2} \mathrm{O} & 66 & 55 \\ 7 & \mathrm{~K}_{2} \mathrm{HPO}_{4} & \text { trace } & -- \\ 8 & \mathrm{KOH} & 91 & 65\end{array}$

[a] Reaction conditions: 1a $(0.1 \mathrm{mmol}), \mathbf{2 a}(0.12 \mathrm{mmol})$, base $(0.2 \mathrm{mmol})$ and $\mathbf{P 1 5}(0.01 \mathrm{mmol})$ in $\mathrm{CCl}_{4} /$ toluene $(2.0 \mathrm{~mL}, \mathrm{v} / \mathrm{v}=1 / 5)$ at $0{ }^{\circ} \mathrm{C}$ for $12 \mathrm{~h}$. [b] All ee values were determined by HPLC analysis on a chiral stationary phase, and all yields were isolated yields.

Table S4. Optimization of the solvents ${ }^{[a, b]}$<smiles></smiles>

$1 \mathrm{a}$ entry

1

2

3

4

5

6

7

8

9

10

11

12

$\mathbf{2 a}$

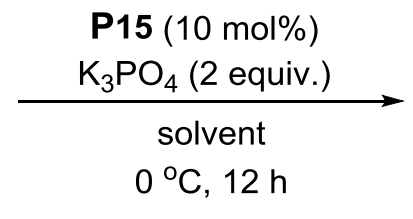

$0^{\circ} \mathrm{C}, 12 \mathrm{~h}$<smiles>NS(=O)(=O)c1ccccc1OP(O)OCc1ccccc1</smiles>

$3 a$ yield (\%) 78 26

10 13 96 84 73 76 73 75

86 97

$$
\mathrm{CCl}_{4} / \text { toluene }(\mathrm{v} / \mathrm{v}=1 / 10)
$$

84

94

$$
\left.\mathrm{CCl}_{4}\right)
$$

toluene (with 1.0 equivent of $\mathrm{CCl}_{4}$ ) 
[a] Reaction conditions: 1a $(0.1 \mathrm{mmol}), \mathbf{2 a}(0.12 \mathrm{mmol}), \mathrm{K}_{3} \mathrm{PO}_{4}(0.2 \mathrm{mmol})$ and $\mathbf{P 1 5}(0.01 \mathrm{mmol})$ in solvent $(2.0 \mathrm{~mL})$ at $0{ }^{\circ} \mathrm{C}$ for $12 \mathrm{~h}$. [b] All ee values were determined by HPLC analysis on a chiral stationary phase, and all yields were isolated yields.

Table S5. Optimization of the equivalent of $\mathbf{2 a}^{[\mathrm{a}, \mathrm{b}]}$<smiles>N=S(=O)(c1ccccc1O)c1ccccc1O</smiles><smiles></smiles>

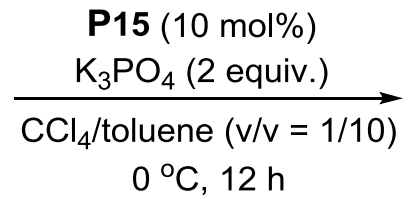

$1 \mathrm{a}$ 2a ( $n$ equiv.)<smiles>NS(=O)(=O)c1ccccc1OPOCOc1ccccc1</smiles>
entry 2a (n equiv.) yield $(\%)$

3a

$\begin{array}{cccc}\text { entry } & \text { 2a (n equiv.) } & \text { yield }(\%) & \text { ee }(\%) \\ 1 & 1.0 & 89 & 77 \\ 2 & 1.1 & 87 & 86 \\ 3 & 1.2 & 86 & 97\end{array}$

[a] Reaction conditions: 1a $(0.1 \mathrm{mmol}), \mathbf{2 a}$ (n equiv.), $\mathrm{K}_{3} \mathrm{PO}_{4}(0.2 \mathrm{mmol})$ and $\mathbf{P 1 5}(0.01 \mathrm{mmol})$ in $\mathrm{CCl}_{4} /$ toluene $(2.0 \mathrm{~mL}, \mathrm{v} / \mathrm{v}=1 / 10)$ at $0{ }^{\circ} \mathrm{C}$ for $12 \mathrm{~h}$. [b] All ee values were determined by HPLC analysis on a chiral stationary phase, and all yields were isolated yields. 


\section{Preparation of Phosphonium Salt Catalysts}

All bifunctional phosphonium salt catalysts P1-15 used in this study were prepared via a $P$-alkylation reaction of our previously reported organophosphines according to the known procedures. ${ }^{[1]}$ Except for P9, P12, ent-P12, P13, P14, P15 and ent-P15 other catalysts are known compounds, and their characterization data were in agreement with those reported in the literature. ${ }^{[2]}$ Unknown compounds P9, P12, ent-P12, P13, P14, P15 and ent-P15 were fully characterized.

\section{$\underline{((2 S, 3 R)-3-((t e r t-b u t y l d i m e t h y l s i l y l) o x y)-2-(3-(4-f l u o r o p h e n y l) t h i o u r e i d o) b u t y l)(~}$}

\section{methyl)diphenylphosphonium iodide (P9)}

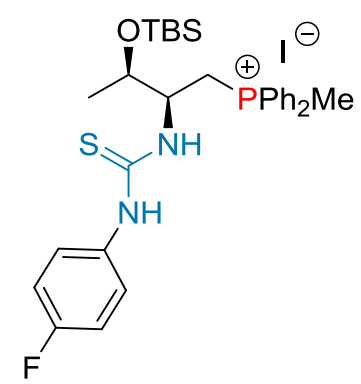

White solid; m.p. $=227-229{ }^{\circ} \mathrm{C} ;{ }^{1} \mathrm{H}$ NMR $\left(400 \mathrm{MHz}, \mathrm{CDCl}_{3}\right) \delta 9.16(\mathrm{~s}, 1 \mathrm{H}), 8.83(\mathrm{~s}$, $1 \mathrm{H}), 7.86-7.52(\mathrm{~m}, 11 \mathrm{H}), 7.45(\mathrm{~d}, J=3.6 \mathrm{~Hz}, 2 \mathrm{H}), 6.92-6.76(\mathrm{~m}, 2 \mathrm{H}), 5.12-4.96(\mathrm{~m}$, $1 \mathrm{H}), 4.12-3.98(\mathrm{~m}, 1 \mathrm{H}), 3.54-3.36(\mathrm{~m}, 1 \mathrm{H}), 3.05(\mathrm{t}, J=14.8 \mathrm{~Hz}, 1 \mathrm{H}), 2.83-2.61(\mathrm{~m}$, 3H), $1.19\left(1.19(\mathrm{~d}, J=5.6 \mathrm{~Hz}, 3 \mathrm{H}), 0.75(\mathrm{~s}, 9 \mathrm{H}),-0.03(\mathrm{~s}, 3 \mathrm{H}),-0.11(\mathrm{~s}, 3 \mathrm{H}),{ }^{13} \mathrm{C}\right.$ NMR $\left(100 \mathrm{MHz}, \mathrm{CDCl}_{3}\right) \delta 180.7,159.6(\mathrm{~d}, J=243.9 \mathrm{~Hz}), 134.8(\mathrm{~d}, J=34.4 \mathrm{~Hz})$, 134.5, 132.2 (d, $J=9.8 \mathrm{~Hz}), 131.9$ (d, $J=10.4 \mathrm{~Hz}), 130.1$ (t, $J=11.6 \mathrm{~Hz}), 128.0$ (d, $J$ $=13.4 \mathrm{~Hz}), 125.4(\mathrm{~d}, J=8.0 \mathrm{~Hz}), 119.5(\mathrm{~d}, J=89.6 \mathrm{~Hz}), 117.5(\mathrm{~d}, J=85.2 \mathrm{~Hz}), 114.7$ $(\mathrm{d}, J=22.4 \mathrm{~Hz}), 68.2(\mathrm{~d}, J=14.8 \mathrm{~Hz}), 52.6,25.6,22.2(\mathrm{~d}, J=52.9 \mathrm{~Hz}), 17.4(\mathrm{~d}, J=$ $45.4 \mathrm{~Hz}), 8.3(\mathrm{~d}, J=53.9 \mathrm{~Hz}),-4.9,-5.1 ;{ }^{31} \mathrm{P} \mathrm{NMR}\left(162 \mathrm{MHz}, \mathrm{CDCl}_{3}\right) \delta 23.55 ;{ }^{19} \mathrm{~F}$ NMR (376 MHz, $\left.\mathrm{CDCl}_{3}\right) \delta-117.25$; HRMS (ESI) $\mathrm{m} / z$ calcd for $\mathrm{C}_{30} \mathrm{H}_{41} \mathrm{FIN}_{2} \mathrm{OPSSi}$ $[\mathrm{M}-\mathrm{I}]^{+}=555.2430$, found $=555.2432$.

(3,5-bis(trifluoromethyl)benzyl)((2S,3R)-3-((tert-butyldiphenylsilyl)oxy)-2-(3-(4-f luorophenyl)thioureido)butyl)diphenylphosphonium bromide (P12) 


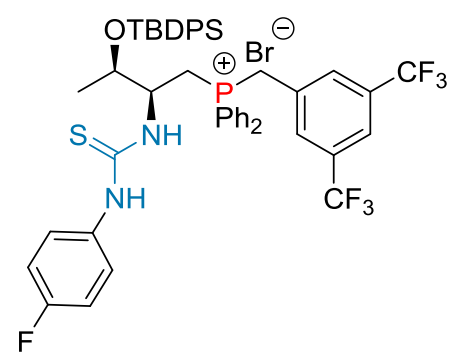

White solid; m.p. $=119-121{ }^{\circ} \mathrm{C} ;{ }^{1} \mathrm{H}$ NMR $\left(400 \mathrm{MHz}, \mathrm{CDCl}_{3}\right) \delta$ 9.25-9.20 (m, 2H), 7.98-7.89 (m, 2H), 7.78-7.71 (m, 2H), 7.69-7.62 (m, 3H), 7.61-7.48 (m, 8H), 7.46-7.37 (m, 4H), 7.36-7.28 (m, 6H), 6.95-6.87 (m, 2H), $5.66(\mathrm{t}, J=15.4 \mathrm{~Hz}, 1 \mathrm{H})$, 5.43-5.26 (m, 1H), $4.50(\mathrm{t}, J=14.6 \mathrm{~Hz}, 1 \mathrm{H}), 4.26-4.08(\mathrm{~m}, 2 \mathrm{H}), 3.07-2.89(\mathrm{~m}, 1 \mathrm{H})$, $1.16(\mathrm{~d}, J=6.2 \mathrm{~Hz}, 3 \mathrm{H}), 0.92(\mathrm{~s}, 9 \mathrm{H}) ;{ }^{13} \mathrm{C} \mathrm{NMR}\left(100 \mathrm{MHz}, \mathrm{CDCl}_{3}\right) \delta 181.1,160.0(\mathrm{~d}$, $J=244.2 \mathrm{~Hz}), 135.8(\mathrm{~d}, J=7.4 \mathrm{~Hz}), 135.5(\mathrm{~d}, J=2.4 \mathrm{~Hz}), 134.9(\mathrm{~d}, J=2.7 \mathrm{~Hz})$, $134.0(\mathrm{~d}, J=9.7 \mathrm{~Hz}), 133.6(\mathrm{~d}, J=9.0 \mathrm{~Hz}), 133.2(\mathrm{~d}, J=16.7 \mathrm{~Hz}), 132.2(\mathrm{qd}, J=$ 33.8, $3.1 \mathrm{~Hz}), 130.9$ (d, $J=8.9 \mathrm{~Hz}), 130.8,130.4$ (dd, $J=12.4,7.6 \mathrm{~Hz}), 130.0$ (d, $J=$ $14.1 \mathrm{~Hz}), 127.8(\mathrm{~d}, J=5.5 \mathrm{~Hz}), 125.8(\mathrm{~d}, J=8.0 \mathrm{~Hz}), 123.9,122.2,121.2,116.3$, $115.5(\mathrm{~d}, J=8.1 \mathrm{~Hz}), 115.0(\mathrm{~d}, J=22.6 \mathrm{~Hz}), 114.6,69.7(\mathrm{~d}, J=12.9 \mathrm{~Hz}), 52.6(\mathrm{~d}, J=$ $4.0 \mathrm{~Hz}), 29.3$ (d, $J=45.4 \mathrm{~Hz}), 27.1,23.0$ (d, $J=52.4 \mathrm{~Hz}), 19.2,17.0 ;{ }^{31} \mathrm{P}$ NMR $(162$ $\left.\mathrm{MHz}, \mathrm{CDCl}_{3}\right) \delta 28.45 ;{ }^{19} \mathrm{~F}$ NMR $\left(376 \mathrm{MHz}, \mathrm{CDCl}_{3}\right) \delta-63.13,-117.40$; HRMS (ESI) $m / z$ calcd for $\mathrm{C}_{48} \mathrm{H}_{47} \mathrm{BrF}_{7} \mathrm{~N}_{2} \mathrm{OPSSi}[\mathrm{M}-\mathrm{Br}]^{+}=891.2805$, found $=891.2781$.

(3,5-bis(trifluoromethyl)benzyl)((2R,3S)-3-((tert-butyldiphenylsilyl)oxy)-2-(3-(4-f luorophenyl)thioureido)butyl)diphenylphosphonium bromide (ent-P12)

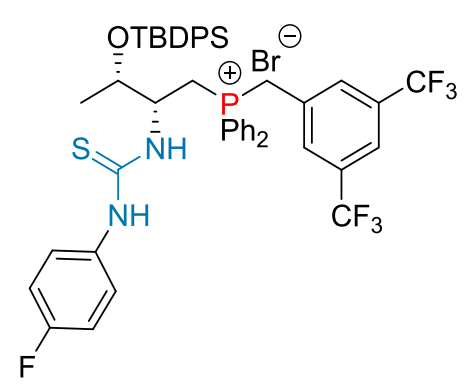

White solid; m.p. $=119-121{ }^{\circ} \mathrm{C} ;{ }^{1} \mathrm{H}$ NMR $\left(400 \mathrm{MHz}, \mathrm{CDCl}_{3}\right) \delta 9.36(\mathrm{~d}, J=8.7 \mathrm{~Hz}$, $1 \mathrm{H}), 9.19(\mathrm{~s}, 1 \mathrm{H}), 7.96-7.87(\mathrm{~m}, 2 \mathrm{H}), 7.78-7.71(\mathrm{~m}, 2 \mathrm{H}), 7.70-7.62(\mathrm{~m}, 3 \mathrm{H})$, 7.60-7.49 (m, 8H), 7.47-7.37 (m, 4H), 7.36-7.27 (m, 6H), 6.96-6.88 (m, 2H), 5.69 (t, $J=15.4 \mathrm{~Hz}, 1 \mathrm{H}), 5.40-5.24(\mathrm{~m}, 1 \mathrm{H}), 4.52(\mathrm{t}, J=14.6 \mathrm{~Hz}, 1 \mathrm{H}), 4.27-4.07(\mathrm{~m}, 2 \mathrm{H})$, 3.01-2.90 (m, 1H), $1.17(\mathrm{~d}, J=6.2 \mathrm{~Hz}, 3 \mathrm{H}), 0.91(\mathrm{~s}, 9 \mathrm{H}) ;{ }^{13} \mathrm{C}$ NMR $(100 \mathrm{MHz}$, 
$\left.\mathrm{CDCl}_{3}\right) \delta 181.2,160.1(\mathrm{~d}, J=244.3 \mathrm{~Hz}), 135.9(\mathrm{~d}, J=1.4 \mathrm{~Hz}), 135.6(\mathrm{~d}, J=3.0 \mathrm{~Hz})$, 135.6, $135.0(\mathrm{~d}, J=3.0 \mathrm{~Hz}), 133.8(\mathrm{dd}, J=38.4,9.3 \mathrm{~Hz}), 133.2(\mathrm{~d}, J=24.9 \mathrm{~Hz})$, $132.2(\mathrm{qd}, J=33.8,3.0 \mathrm{~Hz}), 131.0(\mathrm{~d}, J=9.0 \mathrm{~Hz}), 130.7,130.5(\mathrm{dd}, J=12.0,11.0$ Hz), $130.0(\mathrm{~d}, J=12.0 \mathrm{~Hz}), 127.9(\mathrm{~d}, J=6.0 \mathrm{~Hz}), 125.9$ (d, $J=8.2 \mathrm{~Hz}), 123.9,122.2$ $(\mathrm{d}, J=3.3 \mathrm{~Hz}), 121.2,116.5,115.5(\mathrm{~d}, J=33.6 \mathrm{~Hz}), 115.1(\mathrm{~d}, J=22.6 \mathrm{~Hz}), 114.5$, $69.8(\mathrm{~d}, J=13.0 \mathrm{~Hz}), 52.6(\mathrm{~d}, J=4.0 \mathrm{~Hz}), 28.8(\mathrm{~d}, J=44.8 \mathrm{~Hz}), 27.1,23.6(\mathrm{~d}, J=$ $52.2 \mathrm{~Hz}), 19.2,17.1 ;{ }^{31} \mathrm{P}$ NMR $\left(162 \mathrm{MHz}, \mathrm{CDCl}_{3}\right) \delta 28.62 ;{ }^{19} \mathrm{~F}$ NMR $(376 \mathrm{MHz}$, $\left.\mathrm{CDCl}_{3}\right) \delta-63.13,-117.37$; HRMS (ESI) $m / z$ calcd for $\mathrm{C}_{48} \mathrm{H}_{47} \mathrm{BrF}_{7} \mathrm{~N}_{2} \mathrm{OPSSi}[\mathrm{M}-\mathrm{Br}]^{+}=$ 891.2805 , found $=891.2788$.

((2S,3R)-3-((tert-butyldiphenylsilyl)oxy)-2-(3-(4-fluorophenyl)thioureido)butyl)di phenyl (3,4,5-trimethoxybenzyl)phosphonium bromide (P13)

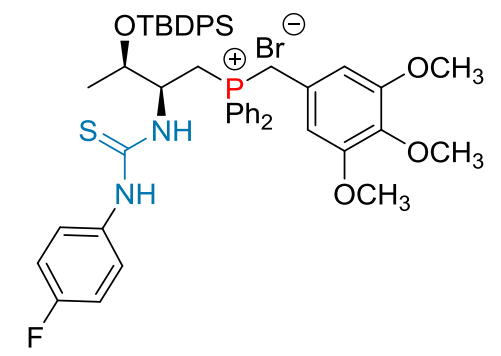

White solid; m.p. $=123-125{ }^{\circ} \mathrm{C} ;{ }^{1} \mathrm{H}$ NMR $\left(400 \mathrm{MHz}, \mathrm{CDCl}_{3}\right) \delta 9.25(\mathrm{~d}, J=8.6 \mathrm{~Hz}$, $1 \mathrm{H}), 9.16(\mathrm{~s}, 1 \mathrm{H}), 7.89-7.80(\mathrm{~m}, 2 \mathrm{H}), 7.73-7.60(\mathrm{~m}, 4 \mathrm{H}), 7.59-7.55(\mathrm{~m}, 2 \mathrm{H})$, 7.53-7.44 (m, 6H), 7.44-7.39 (m, 2H), 7.39-7.34 (m, 2H), 7.33-7.27 (m, 4H), 6.94$6.84(\mathrm{~m}, 2 \mathrm{H}), 6.08(\mathrm{~d}, J=2.5 \mathrm{~Hz}, 2 \mathrm{H}), 5.31-5.19(\mathrm{~m}, 1 \mathrm{H}), 5.13(t, J=14.6 \mathrm{~Hz}, 1 \mathrm{H})$, 4.29-4.13 (m, 2H), 3.95-3.80 (m, 1H), $3.72(\mathrm{~s}, 3 \mathrm{H}), 3.47$ (s, 6H), 2.94 (t, J= 14.4 Hz, $1 \mathrm{H}), 1.13(\mathrm{~d}, J=6.2 \mathrm{~Hz}, 3 \mathrm{H}), 0.93(\mathrm{~s}, 9 \mathrm{H}) ;{ }^{13} \mathrm{C} \mathrm{NMR}\left(100 \mathrm{MHz}, \mathrm{CDCl}_{3}\right) \delta 181.1$, $159.9(\mathrm{~d}, J=244.0 \mathrm{~Hz}), 153.3(\mathrm{~d}, J=3.6 \mathrm{~Hz}), 137.9$ (d, $J=4.6 \mathrm{~Hz}), 135.9,135.0$, $135.0(\mathrm{~d}, J=4.2 \mathrm{~Hz}), 134.8(\mathrm{~d}, J=3.0 \mathrm{~Hz}), 134.0(\mathrm{~d}, J=9.4 \mathrm{~Hz}), 133.7(\mathrm{~d}, J=8.8$ Hz), 133.2 (d, $J=25.6 \mathrm{~Hz}), 130.0$ (d, $J=13.6 \mathrm{~Hz}), 129.9$ (d, $J=10.8 \mathrm{~Hz}), 127.8$ (d, $J$ $=5.6 \mathrm{~Hz}), 125.8(\mathrm{~d}, J=8.2 \mathrm{~Hz}), 122.4(\mathrm{~d}, J=9.4 \mathrm{~Hz}), 117.9,117.0(\mathrm{~d}, J=16.8 \mathrm{~Hz})$, $116.1,114.9(\mathrm{~d}, J=22.6 \mathrm{~Hz}), 107.6(\mathrm{~d}, J=5.8 \mathrm{~Hz}), 69.7$ (d, $J=12.8 \mathrm{~Hz}), 60.9$ (d, $J=$ $2.4 \mathrm{~Hz}), 56.0,52.6$ (d, $J=3.8 \mathrm{~Hz}), 29.7$ (d, $J=44.6 \mathrm{~Hz}), 27.1,22.6(\mathrm{~d}, J=51.8 \mathrm{~Hz})$, 19.2, 17.1; ${ }^{31} \mathrm{P}$ NMR $\left(162 \mathrm{MHz}, \mathrm{CDCl}_{3}\right) \delta 26.02 ;{ }^{19} \mathrm{~F} \mathrm{NMR}\left(376 \mathrm{MHz}, \mathrm{CDCl}_{3}\right) \delta$ 
-117.62; HRMS (ESI) $m / z$ calcd for $\mathrm{C}_{49} \mathrm{H}_{55} \mathrm{BrFN}_{2} \mathrm{O}_{4} \mathrm{PSSi}[\mathrm{M}-\mathrm{Br}]^{+}=845.3374$, found $=$ 845.3371.

$\underline{((2 S, 3 R)-2-(3-(4-(t e r t-b u t y l) p h e n y l) t h i o u r e i d o)-3-((t e r t-b u t y l d i p h e n y l s i l y l) o x y) b u}$ tyl)diphenyl(3,4,5-trimethoxybenzyl)phosphonium bromide (P14)

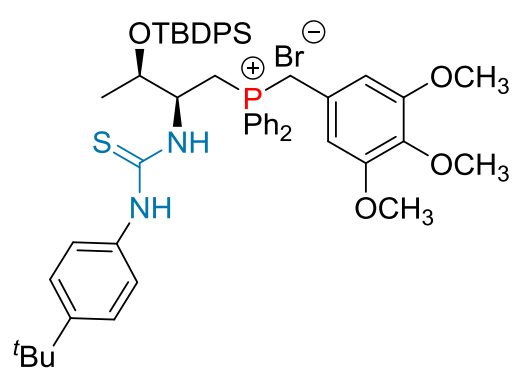

White solid; m.p. $=131-133{ }^{\circ} \mathrm{C} ;{ }^{1} \mathrm{H}$ NMR $\left(400 \mathrm{MHz}, \mathrm{CDCl}_{3}\right) \delta 9.27(\mathrm{~d}, J=7.8 \mathrm{~Hz}$, $1 \mathrm{H}), 8.99(\mathrm{~s}, 1 \mathrm{H}), 7.95-7.86(\mathrm{~m}, 2 \mathrm{H}), 7.75-7.62(\mathrm{~m}, 4 \mathrm{H}), 7.60-7.45(\mathrm{~m}, 8 \mathrm{H})$, 7.43-7.35 (m, 4H), 7.36-7.29 (m, 4H), 7.29-7.21 (m, 2H), 6.09 (d, $J=2.6 \mathrm{~Hz}, 2 \mathrm{H})$, 5.39-5.28 (m, 1H), $5.22(\mathrm{t}, J=14.8 \mathrm{~Hz}, 1 \mathrm{H}), 4.33-4.17(\mathrm{~m}, 2 \mathrm{H}), 4.09-3.97(\mathrm{~m}, 1 \mathrm{H})$, $3.74(\mathrm{~s}, 3 \mathrm{H}), 3.49$ (s, 6H), 2.95 (t, $J=14.2 \mathrm{~Hz}, 1 \mathrm{H}), 1.26$ (s, 9H), 1.14 (d, J=6.2 Hz, 3H), $0.93(\mathrm{~s}, 9 \mathrm{H}) ;{ }^{13} \mathrm{C} \mathrm{NMR}\left(100 \mathrm{MHz}, \mathrm{CDCl}_{3}\right) \delta 180.9,153.4,147.9,138.0(\mathrm{~d}, J=$ $4.6 \mathrm{~Hz}), 136.3,135.9$ (d, $J=1.8 \mathrm{~Hz}), 135.1$ (d, $J=3.0 \mathrm{~Hz}), 134.8$ (d, $J=3.0 \mathrm{~Hz})$, $134.3(\mathrm{~d}, J=9.4 \mathrm{~Hz}), 133.9$ (d, $J=9.0 \mathrm{~Hz}), 133.4(\mathrm{~d}, J=37.5 \mathrm{~Hz}), 130.1$ (d, $J=12.4$ $\mathrm{Hz}), 130.0(\mathrm{~d}, J=10.8 \mathrm{~Hz}), 129.9,127.9$ (d, $J=5.6 \mathrm{~Hz}), 125.3,123.9,122.8$ (d, $J=$ $9.3 \mathrm{~Hz}), 118.3,117.3(\mathrm{~d}, J=34.0 \mathrm{~Hz}), 107.8(\mathrm{~d}, J=5.6 \mathrm{~Hz}), 69.8,61.0(\mathrm{~d}, J=2.4$ $\mathrm{Hz}), 56.1,52.7(\mathrm{~d}, J=4.0 \mathrm{~Hz}), 34.5,31.5,27.2,19.3,17.2 ;{ }^{31} \mathrm{P}$ NMR (162 MHz, $\left.\mathrm{CDCl}_{3}\right) \delta$ 26.64; HRMS (ESI) $m / z$ calcd for $\mathrm{C}_{53} \mathrm{H}_{64} \mathrm{BrN}_{2} \mathrm{O}_{4} \mathrm{PSSi}[\mathrm{M}-\mathrm{Br}]^{+}=883.4094$, found $=883.4084$.

((2S,3R)-2-(3-(4-(tert-butyl)phenyl)thioureido)-3-((tert-butyldiphenylsilyl)oxy)bu

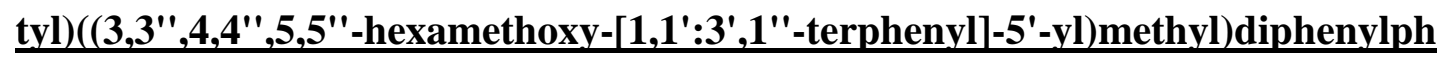
osphonium bromide (P15). 


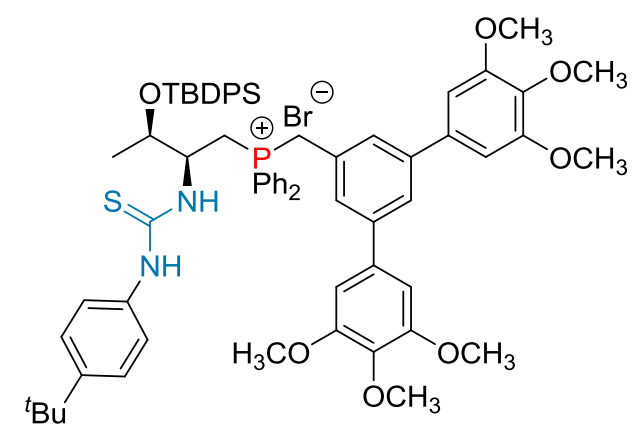

Light yellow solid; m.p. $=140-142{ }^{\circ} \mathrm{C} ;{ }^{1} \mathrm{H}$ NMR $\left(400 \mathrm{MHz}, \mathrm{CDCl}_{3}\right) \delta$ 9.08-8.92 (m, 2H), 8.05-7.95 (m, 2H), 7.76-7.68 (m, 2H), 7.68-7.60 (m, 4H), 7.59-7.47 (m, 6H), 7.47-7.41 (m, 5H), 7.39-7.32 (m, 3H), 7.31-7.25 (m, 2H), 7.22 (dd, J = 8.8, 2.0 Hz, 1H), $7.15(\mathrm{~s}, 2 \mathrm{H}), 6.52(\mathrm{~s}, 4 \mathrm{H}), 5.45-4.26(\mathrm{~m}, 2 \mathrm{H}), 4.42(\mathrm{t}, J=14.4 \mathrm{~Hz}, 1 \mathrm{H})$, 4.31-4.22 (m, 1H), 4.22-4.10 (m, 1H), $3.86(\mathrm{~s}, 6 \mathrm{H}), 3.85(\mathrm{~s}, 12 \mathrm{H}), 3.02(\mathrm{t}, J=14.2 \mathrm{~Hz}$, 1H), $1.23(\mathrm{~s}, 9 \mathrm{H}), 1.13(\mathrm{~d}, J=6.2 \mathrm{~Hz}, 3 \mathrm{H}), 0.90(\mathrm{~s}, 9 \mathrm{H}) ;{ }^{13} \mathrm{C} \mathrm{NMR}\left(100 \mathrm{MHz}, \mathrm{CDCl}_{3}\right)$ $\delta 180.7,153.5,147.7,142.5(\mathrm{~d}, J=3.0 \mathrm{~Hz}), 138.2,136.2,135.7,135.6,135.0(\mathrm{~d}, J=$ $2.5 \mathrm{~Hz}), 134.7(\mathrm{~d}, J=2.7 \mathrm{~Hz}), 134.2(\mathrm{~d}, J=9.6 \mathrm{~Hz}), 133.8(\mathrm{~d}, J=8.9 \mathrm{~Hz}), 133.3(\mathrm{~d}, J$ $=29.8 \mathrm{~Hz}), 132.8,132.0(\mathrm{~d}, J=10.0 \mathrm{~Hz}), 131.8,130.1(\mathrm{~d}, J=12.4 \mathrm{~Hz}), 129.8(\mathrm{~d}, J=$ $11.6 \mathrm{~Hz}), 129.8(\mathrm{~d}, J=12.2 \mathrm{~Hz}), 128.5(\mathrm{~d}, J=12.4 \mathrm{~Hz}), 128.0(\mathrm{~d}, J=5.6 \mathrm{~Hz}), 127.8$ $(\mathrm{d}, J=6.8 \mathrm{~Hz}), 125.6(\mathrm{~d}, J=3.8 \mathrm{~Hz}), 124.4(\mathrm{~d}, J=154.7 \mathrm{~Hz}), 117.4(\mathrm{~d}, J=84.6 \mathrm{~Hz})$, 116.2, 104.4, 69.7 (d, $J=12.6 \mathrm{~Hz}), 61.0,56.4,52.5$ (d, $J=3.4 \mathrm{~Hz}), 34.3,31.3,29.3$ $(\mathrm{d}, J=44.8 \mathrm{~Hz}), 27.1,22.5(\mathrm{~d}, J=52.2 \mathrm{~Hz}), 19.1,17.0 ;{ }^{31} \mathrm{P}$ NMR $\left(162 \mathrm{MHz}, \mathrm{CDCl}_{3}\right)$ $\delta$ 26.85; HRMS (ESI) $m / z$ calcd for $\mathrm{C}_{68} \mathrm{H}_{78} \mathrm{BrN}_{2} \mathrm{O}_{7} \mathrm{PSSi}[\mathrm{M}-\mathrm{Br}]^{+}=1125.5037$, found $=$ 1125.5030.

((2R,3S)-2-(3-(4-(tert-butyl)phenyl)thioureido)-3-((tert-butyldiphenylsilyl)oxy)bu tyl) $\left(\left(3,3^{\prime \prime}, 4,4^{\prime \prime}, 5,5^{\prime \prime}-\right.\right.$ hexamethoxy-[1,1':3',1'-terphenyl $]-5^{\prime}$-yl $)$ methyl $)$ diphenylph osphonium bromide (ent-P15).

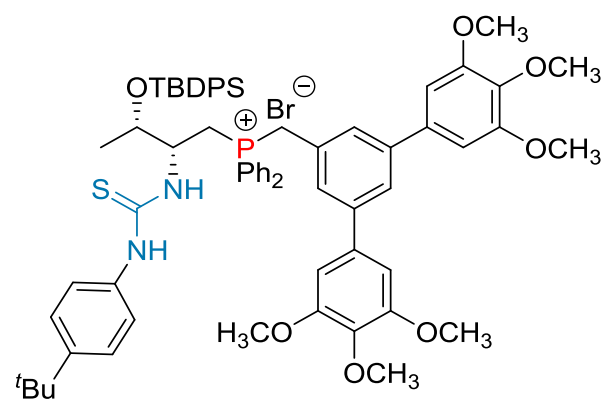


White solid; m.p. $=140-142{ }^{\circ} \mathrm{C} ;{ }^{1} \mathrm{H}$ NMR $\left(400 \mathrm{MHz}, \mathrm{CDCl}_{3}\right) \delta 9.27(\mathrm{~d}, J=7.8 \mathrm{~Hz}$, 1H), 8.99 (s, 1H), 8.04-7.95 (m, 2H), 7.75-7.9 (m, 2H), 7.68-7.63 (m, 4H), 7.57-7.51 (m, 6H), 7.49-7.44 (m, 5H), 7.40-7.35 (m, 3H), 7.32-7.29 (m, 2H), 7.24-7.22 (m, 1H), $7.12(\mathrm{~s}, 2 \mathrm{H}), 6.51(\mathrm{~s}, 4 \mathrm{H}), 5.43(\mathrm{t}, J=15.0 \mathrm{~Hz}, 1 \mathrm{H}), 5.40-5.29(\mathrm{~m}, 1 \mathrm{H}), 4.45(\mathrm{t}, J=$ 14.4 Hz, 1H), 4.34-4.22 (m, 1H), 4.22-4.10 (m, 1H), 3.86 (s, 6H), 3.86 (s, 12H), 3.00 $(\mathrm{t}, J=14.2 \mathrm{~Hz}, 1 \mathrm{H}), 1.24(\mathrm{~s}, 9 \mathrm{H}), 1.14(\mathrm{~d}, J=6.2 \mathrm{~Hz}, 3 \mathrm{H}), 0.91(\mathrm{~s}, 9 \mathrm{H}) ;{ }^{13} \mathrm{C} \mathrm{NMR}$ $\left(100 \mathrm{MHz}, \mathrm{CDCl}_{3}\right) \delta 180.8,153.6,147.8,142.6(\mathrm{~d}, J=3.2 \mathrm{~Hz}), 138.2,136.2,135.7$ (d, $J=17.4 \mathrm{~Hz}), 135.1(\mathrm{~d}, J=2.6 \mathrm{~Hz}), 134.8(\mathrm{~d}, J=2.5 \mathrm{~Hz}), 134.3(\mathrm{~d}, J=9.4 \mathrm{~Hz})$, $133.9(\mathrm{~d}, J=8.8 \mathrm{~Hz}), 133.3(\mathrm{~d}, J=34.8 \mathrm{~Hz}), 133.0,132.1$ (d, $J=9.8 \mathrm{~Hz}), 132.0$ (d, $J$ $=2.8 \mathrm{~Hz}), 130.2(\mathrm{~d}, J=12.4 \mathrm{~Hz}), 129.9(\mathrm{~d}, J=10.8 \mathrm{~Hz}), 129.8(\mathrm{~d}, J=12.2 \mathrm{~Hz})$, $129.8(\mathrm{~d}, J=12.2 \mathrm{~Hz}), 128.6(\mathrm{~d}, J=12.2 \mathrm{~Hz}), 128.1(\mathrm{~d}, J=5.8 \mathrm{~Hz}), 127.8(\mathrm{~d}, J=6.4$ Hz), $125.6(\mathrm{~d}, J=3.6 \mathrm{~Hz}), 124.5(\mathrm{~d}, J=155.2 \mathrm{~Hz}), 117.6(\mathrm{~d}, J=85.2 \mathrm{~Hz}), 116.6(\mathrm{~d}, J$ = 81.9 Hz), 104.4, 69.8 (d, $J=12.8 \mathrm{~Hz}), 61.0,56.5,52.6$ (d, $J=3.7 \mathrm{~Hz}), 34.4,31.4$, $29.0(\mathrm{~d}, J=44.0 \mathrm{~Hz}), 27.1,22.9(\mathrm{~d}, J=51.8 \mathrm{~Hz}), 19.2,17.1 ;{ }^{31} \mathrm{P} \mathrm{NMR}(162 \mathrm{MHz}$, $\mathrm{CDCl}_{3}$ ) $\delta$ 26.98; HRMS (ESI) $m / z$ calcd for $\mathrm{C}_{68} \mathrm{H}_{78} \mathrm{BrN}_{2} \mathrm{O}_{7} \mathrm{PSSi}[\mathrm{M}-\mathrm{Br}]^{+}=1125.5037$, found $=1125.6006$. 


\section{Preparation of Free Sulfoximines 1}

\section{A. General Procedure for Synthesis of 1a}<smiles>Oc1ccccc1I</smiles>

S1

$$
\underset{\mathrm{CH}_{2} \mathrm{Cl}_{2}, \mathrm{rt}, 12 \mathrm{~h}}{\stackrel{\mathrm{MOMCl}}{\mathrm{DIEA}}}
$$<smiles>COc1ccccc1I</smiles>

S2

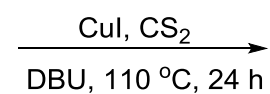

$\stackrel{3 \mathrm{NHCl}}{\mathrm{MeOH}, 80^{\circ} \mathrm{C}, 2 \mathrm{~h}}$ ammonium carbamate $\mathrm{Phl}(\mathrm{OAc})_{2}$ $\mathrm{MeOH}, 3 \mathrm{~h}$, rt

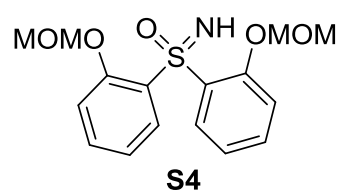<smiles>COc1ccccc1Sc1ccccc1OC</smiles>

S3

$1 \mathrm{a}$

Step 1: To a solution of $\mathbf{S 1}(5.00 \mathrm{~g}, 22.7 \mathrm{mmol})$ and $N, N$-diisopropylethylamine (3.43 $\mathrm{mL}, 45.4 \mathrm{mmol})$ in dry dichloromethane $(10.0 \mathrm{~mL})$ was added chloromethyl methyl ether $(11.3 \mathrm{~mL}, 68.2 \mathrm{mmol})$ at $0{ }^{\circ} \mathrm{C}$ for a $10 \mathrm{~min}$ period, and the reaction mixture was stirred for $12 \mathrm{~h}$ at room temperature. Then, the reaction mixture was diluted with water, and extracted with dichloromethane $(10 \mathrm{~mL} \times 3)$. The organic layer was washed with brine, dried over anhydrous sodium sulfate, filtered, and evaporated in vacuo. The 2-iodo-1-methoxymethoxy-benzene $\mathbf{S 2}$ was used in the next synthetic step without further purification. ${ }^{[3]}$

Step 2: To a $100 \mathrm{~mL}$ round-bottom flask with magnetic stirring bar and reflux condenser were added the crude 2-iodo-1-methoxymethoxy-benzene S2 (5.4 g, 20.3 mmol), copper iodide (387 mg, $2.0 \mathrm{mmol}$ ), carbon disulfide (414 mg, $10.2 \mathrm{mmol}$ ) and 1,8-diazabicyclo(5.4.0)undec-7-ene $(6.1 \mathrm{~mL}, 40.7 \mathrm{mmol})$ purged with nitrogen atmosphere. Then, it was dissolved with dry toluene $(10 \mathrm{~mL})$. The mixture was vigorously stirred at $110{ }^{\circ} \mathrm{C}$ for $24 \mathrm{~h}$, and then it was cooled to room temperature. Water $(5 \mathrm{~mL})$ was added, and the mixture was extracted with ethyl acetate $(5 \mathrm{~mL} \times 3)$. The organic layer was washed with brine, dried over anhydrous sodium sulfate, filtered, and evaporated in vacuo. The bis(2-(methoxymethoxy)phenyl)sulfane $\mathbf{S 3}$ was used in the next synthetic step without further purification. ${ }^{[4]}$ 
Step 3: To a $50 \mathrm{~mL}$ round-bottom flask with magnetic stirring bar were added the crude bis(2-(methoxymethoxy)phenyl)sulfane S3 (3.9 g, $12.7 \mathrm{mmol})$, ammonium carbamate $(2.0 \mathrm{~g}, 25.4 \mathrm{mmol})$ and iodobenzene diacetate $(10.2 \mathrm{~g}, 31.8 \mathrm{mmol})$. The mixture was dissolved in methanol $(15 \mathrm{~mL})$, and then stirred at $\mathrm{rt}$ for $3 \mathrm{~h}$. The solvent was removed in vacuo, and the residue was used in the next synthetic step without further purification. ${ }^{[5]}$

Step 4: To a $50 \mathrm{~mL}$ round-bottom flask with magnetic stirring bar were added the crude S4 (1.8 g, $5.3 \mathrm{mmol})$ and $3 \mathrm{M}$ hydrochloric acid solution (10 mL, 0.5 M). Then, the mixture was dissolved in methanol $(20 \mathrm{~mL})$, and stirred at $80{ }^{\circ} \mathrm{C}$ for $2 \mathrm{~h}$. The solvent was removed in vacuo, diluted with dichloromethane and filtered. The residue was washed with dichloromethane $(10 \mathrm{~mL} \times 3)$ and ethyl acetate $(10 \mathrm{~mL} \times 3)$ to afford the desired product 1a (618 $\mathrm{mg}, 11 \%$ overall yield) as a white solid.

The free N-H sulfoximines $\mathbf{1 b}-\mathbf{u}$ were synthesized by the similar procedure. The unknown compounds $\mathbf{1 b}$-u were fully characterized.

\section{Bis(2-hydroxyphenyl)(imino)- $\lambda^{6}$-sulfanone (1a)}<smiles>N=S(=O)(c1ccccc1O)c1ccccc1O</smiles>

White solid; m.p. $=139-141{ }^{\circ} \mathrm{C} ;{ }^{1} \mathrm{H}$ NMR $\left(400 \mathrm{MHz}, \mathrm{CDCl}_{3}\right) \delta 8.78(\mathrm{~s}, 2 \mathrm{H}), 7.63(\mathrm{dd}$, $J=8.2,1.6 \mathrm{~Hz}, 2 \mathrm{H}), 7.43(\mathrm{ddd}, J=8.6,7.2,1.6 \mathrm{~Hz}, 2 \mathrm{H}), 6.99(\mathrm{dd}, J=8.6,1.2 \mathrm{~Hz}$, $2 \mathrm{H}), 6.91(\mathrm{ddd}, J=8.2,7.2,1.2 \mathrm{~Hz}, 2 \mathrm{H}) ;{ }^{13} \mathrm{C} \mathrm{NMR}\left(100 \mathrm{MHz}, \mathrm{CDCl}_{3}\right) \delta 156.1,136.2$, 128.7, 124.1, 120.5, 119.6; HRMS (ESI) $\mathrm{m} / z$ calcd for $\mathrm{C}_{12} \mathrm{H}_{11} \mathrm{NO}_{3} \mathrm{~S}[\mathrm{M}+\mathrm{H}]^{+}=$ 250.0538, found $=250.0538$.

\section{$\underline{\text { Bis(2-hydroxy-5-methylphenyl)(imino) }-\lambda^{6} \text {-sulfanone (1b) }}$}<smiles>Cc1ccc(O)c(S(=N)(=O)c2cc(C)ccc2O)c1</smiles> 
White solid; $702 \mathrm{mg}, 12 \%$ overall yield; m.p. $=212-214{ }^{\circ} \mathrm{C} ;{ }^{1} \mathrm{H}$ NMR $(400 \mathrm{MHz}$, DMSO) $\delta 7.70(\mathrm{~d}, J=1.8 \mathrm{~Hz}, 2 \mathrm{H}), 7.41(\mathrm{dd}, J=8.4,1.8 \mathrm{~Hz}, 2 \mathrm{H}), 7.00(\mathrm{~d}, J=8.4 \mathrm{~Hz}$, 2H), $2.30(\mathrm{~s}, 6 \mathrm{H}) ;{ }^{13} \mathrm{C}$ NMR (100 MHz, DMSO) $\delta 154.7,137.6,129.2,127.8,120.0$, 117.8, 19.9; HRMS (ESI) $m / z$ calcd for $\mathrm{C}_{14} \mathrm{H}_{15} \mathrm{NO}_{3} \mathrm{~S}[\mathrm{M}+\mathrm{H}]^{+}=278.0851$, found $=$ 278.0848 .

\section{Bis(2-hydroxy-5-ethylphenyl)(imino)- $\lambda^{6}$-sulfanone (1c)}<smiles>CCc1ccc(O)c(S(=N)(=O)c2cc(CC)ccc2O)c1</smiles>

Light yellow solid; $757 \mathrm{mg}, 12 \%$ overall yield; m.p. $=196-198{ }^{\circ} \mathrm{C} ;{ }^{1} \mathrm{H}$ NMR $(400$ MHz, DMSO) $\delta 7.77(\mathrm{~d}, J=2.2 \mathrm{~Hz}, 2 \mathrm{H}), 7.48(\mathrm{dd}, J=8.4,2.2 \mathrm{~Hz}, 2 \mathrm{H}), 7.07(\mathrm{~d}, J=$ $8.4 \mathrm{~Hz}, 2 \mathrm{H}), 2.62(\mathrm{q}, J=7.6 \mathrm{~Hz}, 4 \mathrm{H}), 1.18(\mathrm{t}, J=7.6 \mathrm{~Hz}, 6 \mathrm{H}) ;{ }^{13} \mathrm{C} \mathrm{NMR}(100 \mathrm{MHz}$, DMSO) $\delta 154.9,136.8,134.2,128.4,119.3,117.8,27.1,15.6$; HRMS (ESI) $m / z$ calcd for $\mathrm{C}_{16} \mathrm{H}_{19} \mathrm{NO}_{3} \mathrm{~S}[\mathrm{M}+\mathrm{H}]^{+}=306.1164$, found $=306.1164$.

\section{$\underline{\text { Bis(2-hydroxy-5-isopropylphenyl)(imino)- } \lambda^{6} \text {-sulfanone (1d) }}$}<smiles>CCCc1ccc(O)c(S(=N)(=O)c2cc(C(C)C)ccc2O)c1</smiles>

Light yellow solid; $816 \mathrm{mg}, 13 \%$ overall yield; m.p. $=182-184{ }^{\circ} \mathrm{C} ;{ }^{1} \mathrm{H}$ NMR $(400$ MHz, DMSO) $\delta 7.75(\mathrm{~d}, J=2.2 \mathrm{~Hz}, 2 \mathrm{H}), 7.50(\mathrm{dd}, J=8.6,2.2 \mathrm{~Hz}, 2 \mathrm{H}), 7.02(\mathrm{~d}, J=$ $8.6 \mathrm{~Hz}, 2 \mathrm{H}), 5.75(\mathrm{~s}, 2 \mathrm{H}), 2.97-2.87(\mathrm{~m}, 2 \mathrm{H}), 1.20(\mathrm{~d}, J=6.9 \mathrm{~Hz}, 6 \mathrm{H}),{ }^{13} \mathrm{C}$ NMR $(100$ MHz, DMSO) $\delta 155.3,139.2,135.6,127.4,120.4,118.2,32.9,24.3$; HRMS (ESI) $m / z$ calcd for $\mathrm{C}_{18} \mathrm{H}_{23} \mathrm{NO}_{3} \mathrm{~S}[\mathrm{M}+\mathrm{H}]^{+}=334.1477$, found $=334.1486$.

\section{$\underline{\text { Bis(2-hydroxy-5-(tert-butyl)phenyl)(imino)- } \lambda^{6} \text {-sulfanone (1e) }}$}<smiles>CC(C)(C)c1ccc(O)c(S(=N)(=O)c2cc(C(C)(C)C)ccc2O)c1</smiles> 
White solid; $1.2 \mathrm{~g}, 18 \%$ overall yield; m.p. $=149-151{ }^{\circ} \mathrm{C} ;{ }^{1} \mathrm{H}$ NMR $(400 \mathrm{MHz}$, DMSO) $\delta 7.89(\mathrm{~d}, J=2.4 \mathrm{~Hz}, 2 \mathrm{H}), 7.69(\mathrm{dd}, J=8.7,2.4 \mathrm{~Hz}, 2 \mathrm{H}), 7.07(\mathrm{~d}, J=8.7 \mathrm{~Hz}$, 2H), 1.29 (s, 18H); ${ }^{13} \mathrm{C}$ NMR (100 MHz, DMSO) $\delta 159.8,146.4,139.8,131.2,123.9$, 122.8, 39.4, 36.2; HRMS (ESI) $m / z$ calcd for $\mathrm{C}_{20} \mathrm{H}_{27} \mathrm{NO}_{3} \mathrm{~S}[\mathrm{M}+\mathrm{H}]^{+}=362.1790$, found $=362.1782$.

\section{$\underline{\text { Bis(2-hydroxy-5-benzylphenyl)(imino)- } \lambda^{6} \text {-sulfanone (1f) }}$}<smiles>N=S(=O)(c1cc(Br)ccc1O)c1cc(Br)ccc1O</smiles>

White solid; $1.1 \mathrm{~g}, 16 \%$ overall yield; m.p. $=189-191{ }^{\circ} \mathrm{C} ;{ }^{1} \mathrm{H}$ NMR $(400 \mathrm{MHz}$, $\left.\mathrm{CDCl}_{3}\right) \delta 8.59(\mathrm{~s}, 2 \mathrm{H}), 7.43(\mathrm{~d}, J=2.1 \mathrm{~Hz}, 2 \mathrm{H}), 7.30-7.15(\mathrm{~m}, 8 \mathrm{H}), 7.11-7.02(\mathrm{~m}, 4 \mathrm{H})$, $6.90(\mathrm{~d}, J=8.5 \mathrm{~Hz}, 2 \mathrm{H}), 3.85(\mathrm{~s}, 4 \mathrm{H}) ;{ }^{13} \mathrm{C} \mathrm{NMR}\left(100 \mathrm{MHz}, \mathrm{CDCl}_{3}\right) \delta$ 154.4, 140.2, 136.9, 133.5, 128.9, 128.8, 128.1, 126.5, 123.7, 119.7, 40.8; HRMS (ESI) $m / z$ calcd for $\mathrm{C}_{26} \mathrm{H}_{23} \mathrm{NO}_{3} \mathrm{~S}[\mathrm{M}+\mathrm{H}]^{+}=430.1477$, found $=430.1482$.

\section{$\underline{B i s(4-h y d r o x y}-\left[1,1^{\prime}\right.$-biphenyl]-3-yl)(imino)- $\lambda^{6}$-sulfanone (1g)}<smiles>N=S(=O)(c1cc(-c2ccccc2)ccc1O)c1cc(-c2ccccc2)ccc1O</smiles>

Light yellow solid; $985 \mathrm{mg}, 14 \%$ overall yield; m.p. $=206-208{ }^{\circ} \mathrm{C} ;{ }^{1} \mathrm{H}$ NMR $(400$ MHz, DMSO) $\delta 8.21(\mathrm{~d}, J=2.3 \mathrm{~Hz}, 2 \mathrm{H}), 7.94(\mathrm{dd}, J=8.6,2.3 \mathrm{~Hz}, 2 \mathrm{H}), 7.71-7.65(\mathrm{~m}$, 4H), $7.49(\mathrm{t}, J=7.7 \mathrm{~Hz}, 4 \mathrm{H}), 7.38(\mathrm{t}, J=7.4 \mathrm{~Hz}, 2 \mathrm{H}), 7.20(\mathrm{~d}, J=8.6 \mathrm{~Hz}, 2 \mathrm{H}) ;{ }^{13} \mathrm{C}$ NMR (100 MHz, DMSO) $\delta$ 156.3, 138.3, 135.1, 131.0, 129.2, 127.6, 127.5, 126.3, 121.1, 118.6; HRMS (ESI) $m / z$ calcd for $\mathrm{C}_{24} \mathrm{H}_{19} \mathrm{NO}_{3} \mathrm{~S}[\mathrm{M}+\mathrm{H}]^{+}=402.1164$, found $=$ 402.1162 .

\section{$\underline{\text { Bis(5-chloro-2-hydroxyphenyl)- } \lambda^{6} \text {-sulfanone (1h) }}$}


<smiles>N=S(=O)(c1cc(Cl)ccc1O)c1cc(Cl)ccc1O</smiles>

White solid; $649 \mathrm{mg}, 10 \%$ overall yield; m.p. $=202-204{ }^{\circ} \mathrm{C} ;{ }^{1} \mathrm{H}$ NMR $(400 \mathrm{MHz}$, DMSO) $\delta 12.11(\mathrm{~s}, 1 \mathrm{H}), 7.97(\mathrm{~d}, J=2.7 \mathrm{~Hz}, 1 \mathrm{H}), 7.79(\mathrm{~d}, J=2.7 \mathrm{~Hz}, 1 \mathrm{H}), 7.69(\mathrm{dd}$, $J=8.9,2.7 \mathrm{~Hz}, 1 \mathrm{H}), 7.57(\mathrm{dd}, J=8.9,2.7 \mathrm{~Hz}, 1 \mathrm{H}), 7.20(\mathrm{~d}, J=8.9 \mathrm{~Hz}, 1 \mathrm{H}), 7.05(\mathrm{~d}$, $J=8.9 \mathrm{~Hz}, 1 \mathrm{H}), 5.75(\mathrm{~s}, 2 \mathrm{H}) ;{ }^{13} \mathrm{C} \mathrm{NMR}(100 \mathrm{MHz}, \mathrm{DMSO}) \delta 155.8,155.5,136.9$, 135.7, 130.2, 128.4, 124.3, 122.5, 122.1, 121.4, 119.8; HRMS (ESI) $\mathrm{m} / \mathrm{z}$ calcd for $\mathrm{C}_{12} \mathrm{H}_{9} \mathrm{Cl}_{2} \mathrm{NO}_{3} \mathrm{~S}[\mathrm{M}+\mathrm{H}]^{+}=317.9758$, found $=317.9762$.

\section{$\underline{\text { Bis(5-bromo-2-hydroxyphenyl)- } \lambda^{6} \text {-sulfanone (1i) }}$}<smiles>N=S(=O)(c1cc(Br)ccc1O)c1cc(Br)ccc1O</smiles>

White solid; $614 \mathrm{mg}, 9 \%$ overall yield; m.p. $=196-198{ }^{\circ} \mathrm{C} ;{ }^{1} \mathrm{H}$ NMR $(400 \mathrm{MHz}$, DMSO) $\delta 8.72(\mathrm{~s}, 2 \mathrm{H}), 7.68(7.68(\mathrm{~d}, J=0.6 \mathrm{~Hz}, 1 \mathrm{H})), 7.53(\mathrm{~d}, J=7.4 \mathrm{~Hz}, 2 \mathrm{H}), 6.92$ $(\mathrm{d}, J=8.3 \mathrm{~Hz}, 2 \mathrm{H}) ;{ }^{13} \mathrm{C} \mathrm{NMR}(100 \mathrm{MHz}, \mathrm{DMSO}) \delta 155.2,139.5,130.6,125.0,121.8$, 112.1; HRMS (ESI) $\mathrm{m} / z$ calcd for $\mathrm{C}_{12} \mathrm{H}_{9} \mathrm{Br}_{2} \mathrm{NO}_{3} \mathrm{~S}[\mathrm{M}+\mathrm{H}]^{+}=405.8748$, found $=$ 405.8702 .

\section{Bis(5-cyclohexyl-2-hydroxyphenyl)- $\lambda^{6}$-sulfanone (1j)}<smiles>N=S(=O)(c1cc(C2CCCCC2)ccc1O)c1cc(C2CCCCC2)ccc1O</smiles>

Light yellow solid; $1.5 \mathrm{~g}, 22 \%$ overall yield; m.p. $=136-138{ }^{\circ} \mathrm{C} ;{ }^{1} \mathrm{H}$ NMR $(400 \mathrm{MHz}$, $\left.\mathrm{CDCl}_{3}\right) \delta 8.59(\mathrm{~s}, 2 \mathrm{H}), 7.43(\mathrm{~s}, 2 \mathrm{H}), 7.29(\mathrm{~d}, J=5.1 \mathrm{~Hz}, 2 \mathrm{H}), 6.93(\mathrm{~d}, J=5.1 \mathrm{~Hz}, 2 \mathrm{H})$, 2.50-2.30(m, 2H), 1.85-1.68 (m, 10H), 1.37-1.21 (m, 10H); ${ }^{13} \mathrm{C}$ NMR (100 MHz, 
$\left.\mathrm{CDCl}_{3}\right) \delta 154.0,140.4,134.8,126.1,123.7,119.3,43.4,34.4,34.3,26.8,26.0$ HRMS (ESI) $m / z$ calcd for $\mathrm{C}_{24} \mathrm{H}_{31} \mathrm{NO}_{3} \mathrm{~S}[\mathrm{M}+\mathrm{H}]^{+}=414.2103$, found $=414.2092$.

$\underline{\text { Bis(2-hydroxy-5-(2-phenylpropan-2-yl)phenyl)- } \lambda^{6} \text {-sulfanone (1k) }}$<smiles>CC(C)(c1ccccc1)c1ccc(O)c(S(=N)(=O)c2cc(C(C)(C)c3ccccc3)ccc2O)c1</smiles>

Light yellow solid; $927 \mathrm{mg}, 13 \%$ overall yield; m.p. $=98-100{ }^{\circ} \mathrm{C} ;{ }^{1} \mathrm{H}$ NMR $(400$ $\left.\mathrm{MHz}, \mathrm{CDCl}_{3}\right) \delta 8.12(\mathrm{~s}, 2 \mathrm{H}), 7.43(\mathrm{~d}, J=2.4 \mathrm{~Hz}, 2 \mathrm{H}), 7.15-7.08(\mathrm{~m}, 6 \mathrm{H}), 7.08-6.98$ $(\mathrm{m}, 6 \mathrm{H}), 6.77(\mathrm{~d}, J=8.8 \mathrm{~Hz}, 2 \mathrm{H}), 1.50(\mathrm{~s}, 12 \mathrm{H}) ;{ }^{13} \mathrm{C} \mathrm{NMR}\left(100 \mathrm{MHz}, \mathrm{CDCl}_{3}\right) \delta$ $154.1,149.5,143.0,135.5,128.3,126.6,126.0,125.4,122.8,119.2,42.5,30.6,30.6$; HRMS (ESI) $m / z$ calcd for $\mathrm{C}_{30} \mathrm{H}_{31} \mathrm{NO}_{3} \mathrm{~S}[\mathrm{M}+\mathrm{H}]^{+}=486.2103$, found $=486.2074$.

\section{$\underline{\text { Bis(2-hydroxy-5-tritylphenyl)phenyl) }-\lambda^{6} \text {-sulfanone (11) }}$}<smiles>N=S(=O)(c1ccccc1)c1cc(C(c2ccccc2)(c2ccccc2)c2ccccc2)ccc1O</smiles>

White solid; $498 \mathrm{mg}, 6 \%$ overall yield; m.p. $=206-208{ }^{\circ} \mathrm{C} ;{ }^{1} \mathrm{H}$ NMR $(400 \mathrm{MHz}$, DMSO) $\delta 7.28(\mathrm{~m}, 7 \mathrm{H}), 7.23-7.16(\mathrm{~m}, 5 \mathrm{H}), 7.09-6.96(\mathrm{~m}, 9 \mathrm{H}) ;{ }^{13} \mathrm{C}$ NMR $(100 \mathrm{MHz}$, DMSO) $\delta 155.2,145.9,136.7,130.4,130.2,127.9,127.6,126.2,125.9,117.4,63.6$; HRMS (ESI) $m / z$ calcd for $\mathrm{C}_{50} \mathrm{H}_{39} \mathrm{NO}_{3} \mathrm{~S}[\mathrm{M}+\mathrm{H}]^{+}=734.2729$, found $=734.2797$.

\section{$\underline{\text { Bis(4-(tert-butyl)-2-hydroxyphenyl)- } \lambda^{6} \text {-sulfanone (1m) }}$}<smiles>CC(C)(C)c1ccc(S(=N)(=O)c2ccc(C(C)(C)C)cc2O)c(O)c1</smiles>

Yellow solid; $614 \mathrm{mg}, 9 \%$ overall yield; m.p. $=153-155{ }^{\circ} \mathrm{C} ;{ }^{1} \mathrm{H}$ NMR $(400 \mathrm{MHz}$, DMSO) $\delta 9.86(\mathrm{~s}, 2 \mathrm{H}), 7.58(\mathrm{~d}, J=8.5 \mathrm{~Hz}, 2 \mathrm{H}), 6.96(\mathrm{dd}, J=8.5,1.8 \mathrm{~Hz}, 2 \mathrm{H}), 6.84$ $(\mathrm{d}, J=1.8 \mathrm{~Hz}, 2 \mathrm{H}), 1.22(\mathrm{~s}, 18 \mathrm{H}) ;{ }^{13} \mathrm{C} \mathrm{NMR}(100 \mathrm{MHz}, \mathrm{DMSO}) \delta 158.1,156.4$, 
128.5, 122.7, 116.1, 114.3, 34.7, 30.6; HRMS (ESI) $\mathrm{m} / z$ calcd for $\mathrm{C}_{20} \mathrm{H}_{27} \mathrm{NO}_{3} \mathrm{~S}$ $[\mathrm{M}+\mathrm{H}]^{+}=362.1790$, found $=362.1788$.

\section{$\underline{\text { Bis(4-chloro-2-hydroxyphenyl)- } \lambda^{6} \text {-sulfanone (1n) }}$}<smiles>N=S(=O)(c1ccc(Cl)cc1O)c1ccc(Cl)cc1O</smiles>

Pink solid; $752 \mathrm{mg}, 12 \%$ overall yield; m.p. $=196-198{ }^{\circ} \mathrm{C} ;{ }^{1} \mathrm{H}$ NMR $(400 \mathrm{MHz}$, $\left.\mathrm{CDCl}_{3}\right) \delta 8.80(\mathrm{~s}, 2 \mathrm{H}), 7.53(\mathrm{~d}, J=8.7 \mathrm{~Hz}, 2 \mathrm{H}), 7.02(\mathrm{~d}, J=2.0 \mathrm{~Hz}, 2 \mathrm{H}), 6.91(\mathrm{dd}, J$ $=8.7,2.0 \mathrm{~Hz}, 2 \mathrm{H}) ;{ }^{13} \mathrm{C} \mathrm{NMR}\left(100 \mathrm{MHz}, \mathrm{CDCl}_{3}\right) \delta 156.7,142.4,129.6,122.5,121.3$, 119.7; HRMS (ESI) $\mathrm{m} / z$ calcd for $\mathrm{C}_{12} \mathrm{H}_{9} \mathrm{Cl}_{2} \mathrm{NO}_{3} \mathrm{~S}[\mathrm{M}+\mathrm{H}]^{+}=317.9758$, found $=$ 317.9760.

\section{$\underline{\text { Bis(2-hydroxy-4,5-dimethylphenyl) }-\lambda^{6} \text {-sulfanone (10) }}$}<smiles>Cc1cc(O)c(S(=N)(=O)c2cc(C)c(C)cc2O)cc1C</smiles>

White solid; 1.4 g, $23 \%$ overall yield; m.p. $=180-182{ }^{\circ} \mathrm{C} ;{ }^{1} \mathrm{H}$ NMR $(400 \mathrm{MHz}$, DMSO) $\delta 7.63(\mathrm{~s}, 2 \mathrm{H}), 6.88(\mathrm{~s}, 2 \mathrm{H}), 2.21(\mathrm{~s}, 6 \mathrm{H}), 2.21$ (s, 6H); ${ }^{13} \mathrm{C}$ NMR $(100 \mathrm{MHz}$, DMSO) $\delta 154.7,146.9,129.5,127.0,118.4,117.2$, 19.8, 18.4; HRMS (ESI) $\mathrm{m} / z$ calcd for $\mathrm{C}_{16} \mathrm{H}_{19} \mathrm{NO}_{3} \mathrm{~S}[\mathrm{M}+\mathrm{H}]^{+}=306.1164$, found $=306.1163$.

\section{Bis(4-chloro-2-hydroxy-5-methylphenyl)- $\lambda^{6}$-sulfanone (1p)}<smiles>Cc1cc(S(=N)(=O)c2cc(C)c(Cl)cc2O)c(O)cc1Cl</smiles>

White solid; 1.1 g, $17 \%$ overall yield; m.p. $=134-136{ }^{\circ} \mathrm{C} ;{ }^{1} \mathrm{H}$ NMR $(400 \mathrm{MHz}$, DMSO) $\delta 7.88(\mathrm{~s}, 2 \mathrm{H}), 7.23(\mathrm{~s}, 2 \mathrm{H}), 5.48(\mathrm{~s}, 2 \mathrm{H}), 2.31(\mathrm{~s}, 6 \mathrm{H}) ;{ }^{13} \mathrm{C}$ NMR $(100 \mathrm{MHz}$, DMSO) $\delta 155.5,141.6,131.3,126.2,119.3,117.8,18.7$; HRMS (ESI) $m / z$ calcd for 
$\mathrm{C}_{14} \mathrm{H}_{13} \mathrm{Cl}_{2} \mathrm{NO}_{3} \mathrm{~S}[\mathrm{M}+\mathrm{H}]^{+}=346.0071$, found $=346.0068$.

\section{Bis(4-bromo-2-hydroxy-5-methylphenyl)- $\lambda^{6}$-sulfanone (1q)}<smiles>Cc1cc(S(=N)(=O)c2cc(C)c(Br)cc2O)c(O)cc1Br</smiles>

Light yellow solid; $908 \mathrm{mg}, 13 \%$ overall yield; m.p. $=153-155{ }^{\circ} \mathrm{C} ;{ }^{1} \mathrm{H}$ NMR $(400$

$\left.\mathrm{MHz}, \mathrm{CDCl}_{3}\right) \delta 8.64(\mathrm{~s}, 2 \mathrm{H}), 7.39(\mathrm{~s}, 2 \mathrm{H}), 7.24(\mathrm{~s}, 2 \mathrm{H}), 2.29(\mathrm{~s}, 6 \mathrm{H}) ;{ }^{13} \mathrm{C}$ NMR $(100$ $\left.\mathrm{MHz}, \mathrm{CDCl}_{3}\right) \delta 154.1,133.2,130.7,129.0,123.2,122.9,22.1$; HRMS (ESI) $\mathrm{m} / \mathrm{z}$ calcd for $\mathrm{C}_{14} \mathrm{H}_{13} \mathrm{Br}_{2} \mathrm{NO}_{3} \mathrm{~S}[\mathrm{M}+\mathrm{H}]^{+}=433.9061$, found $=433.9061$.

\section{Bis(2-hydroxy-5-isopropyl-4-methylphenylphenyl)- $\lambda^{6}$-sulfanone (1r)}<smiles>CCCc1cc(S(=N)(=O)c2cc(C(C)C)c(C)cc2O)c(O)cc1C</smiles>

White solid; 1.6 g, 24\% overall yield; m.p. $=214-216{ }^{\circ} \mathrm{C} ;{ }^{1} \mathrm{H}$ NMR $(400 \mathrm{MHz}$, DMSO) $\delta 7.72(\mathrm{~s}, 2 \mathrm{H}), 6.87(\mathrm{~s}, 2 \mathrm{H}), 3.13-3.00(\mathrm{~m}, 2 \mathrm{H}), 2.29(\mathrm{~s}, 6 \mathrm{H}), 1.18(\mathrm{~d}, J=6.8$ $\mathrm{Hz}, 12 \mathrm{H}) ;{ }^{13} \mathrm{C}$ NMR (100 MHz, DMSO) $\delta 154.2,145.5,137.3,125.8,118.8,117.4$, 28.1, 23.1, 19.2; HRMS (ESI) $m / z$ calcd for $\mathrm{C}_{20} \mathrm{H}_{27} \mathrm{NO}_{3} \mathrm{~S}[\mathrm{M}+\mathrm{H}]^{+}=362.1790$, found $=$ 362.1786 .

\section{$\underline{\text { Bis(6-hydroxy-2,3-dihydro-1H-inden-5-yl)- } \lambda^{6} \text {-sulfanone (1s) }}$}

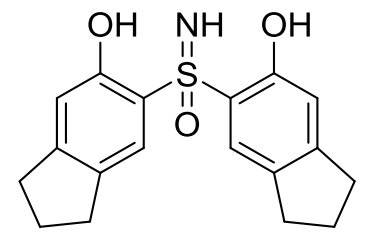

Brown solid; 1.4 g, 22\% overall yield; m.p. $=208-210{ }^{\circ} \mathrm{C} ;{ }^{1} \mathrm{H}$ NMR $(400 \mathrm{MHz}$, $\left.\mathrm{CDCl}_{3}\right) \delta 8.69(\mathrm{~s}, 2 \mathrm{H}), 7.43(\mathrm{~s}, 2 \mathrm{H}), 6.83(\mathrm{~s}, 2 \mathrm{H}), 2.85(\mathrm{t}, J=7.4 \mathrm{~Hz}, 4 \mathrm{H}), 2.78(\mathrm{t}, J=$ $7.4 \mathrm{~Hz}, 4 \mathrm{H}), 2.06-2.00(\mathrm{~m}, 4 \mathrm{H}) ;{ }^{13} \mathrm{C} \mathrm{NMR}\left(100 \mathrm{MHz}, \mathrm{CDCl}_{3}\right) \delta 154.8,154.1,136.5$, 123.3, 121.9, 114.8, 33.4, 31.9, 25.7; HRMS (ESI) $m / z$ calcd for $\mathrm{C}_{20} \mathrm{H}_{27} \mathrm{NO}_{3} \mathrm{~S}[\mathrm{M}+\mathrm{H}]^{+}$ 
$=330.1164$, found $=330.1143$.

$\underline{B i s\left(2^{\prime}-h y d r o x y-\left[1,1^{\prime}-\text { biphenyl }\right]-2-y l\right)-\lambda^{6} \text {-sulfanone (1t) }}$<smiles>O=S(=O)(c1ccccc1-c1ccccc1O)c1ccccc1-c1ccccc1O</smiles>

Gray solid; $872 \mathrm{mg}, 16 \%$ overall yield; m.p. $=203-204{ }^{\circ} \mathrm{C} ;{ }^{1} \mathrm{H}$ NMR $(400 \mathrm{MHz}$, $\left.\mathrm{CDCl}_{3}\right) \delta$ 7.50-7.44 (m, 2H), 7.44-7.38 (m, 1H), 7.22-7.12 (m, 4H), 7.12-7.02 (m, 2H), $6.97(\mathrm{~d}, J=8.0 \mathrm{~Hz}, 2 \mathrm{H}), 6.75-6.63(\mathrm{~m}, 1 \mathrm{H}), 6.55-6.43(\mathrm{~m}, 2 \mathrm{H}), 6.29-6.20(\mathrm{~m}$, 1H), 6.22-6.07 (m, 1H); $\left.{ }^{13} \mathrm{C} \mathrm{NMR} \mathrm{(100} \mathrm{MHz,} \mathrm{CDCl}_{3}\right) \delta$ 153.9, 153.4, 141.1, 137.9, 137.6, 137.0, 133.3, 133.2, 133.1, 132.8, 132.4, 129.9, 129.8, 129.7, 129.7, 129.6, 128.1, 127.9, 127.8, 127.4, 127.1, 121.0, 120.7, 118.9; HRMS (ESI) $\mathrm{m} / z$ calcd for $\mathrm{C}_{24} \mathrm{H}_{19} \mathrm{NO}_{3} \mathrm{~S}[\mathrm{M}+\mathrm{H}]^{+}=402.1164$, found $=402.1164$.

\section{$\underline{\text { Bis(3-hydroxyphenyl) }-\lambda^{6} \text {-sulfanone (1u) }}$}<smiles>NS(=O)(=O)c1cccc(O)c1</smiles>

White solid; $698 \mathrm{mg}, 9 \%$ overall yield; m.p. $=202-204{ }^{\circ} \mathrm{C} ;{ }^{1} \mathrm{H}$ NMR $(400 \mathrm{MHz}$, DMSO) $\delta 10.78(\mathrm{~s}, 1 \mathrm{H}), 7.58-7.43(\mathrm{~m}, 6 \mathrm{H}), 7.27-7.17(\mathrm{~m}, 2 \mathrm{H}), 4.98(\mathrm{~s}, 2 \mathrm{H}) ;{ }^{13} \mathrm{C}$ NMR (100 MHz, DMSO) $\delta 159.0,158.7,137.5,137.2,131.7,131.4,122.6,122.3$, 118.6, 118.2, 114.3, 114.0; HRMS (ESI) $m / z$ calcd for $\mathrm{C}_{24} \mathrm{H}_{19} \mathrm{NO}_{3} \mathrm{~S}[\mathrm{M}+\mathrm{H}]^{+}=$ 250.0538, found $=250.0530$.

\section{B. General Procedure for Synthesis of 1v}<smiles>COc1ccccc1Sc1ccccc1OC</smiles>

S3

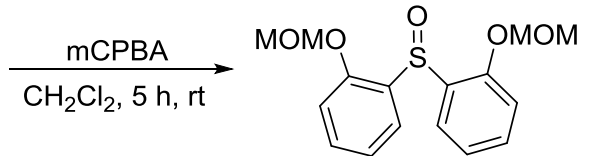

S5

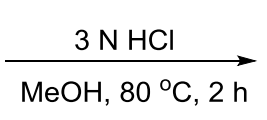

$\mathrm{MeOH}, 80^{\circ} \mathrm{C}, 2 \mathrm{~h}$

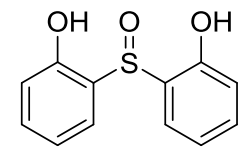

$1 \mathrm{v}$

Step 1: To a $50 \mathrm{~mL}$ round-bottom flask with magnetic stirring bar were added the bis(2-(methoxymethoxy)phenyl)sulfane $\mathbf{S 3}(2.0 \mathrm{~g}, 6.54 \mathrm{mmol})$ and dichloromethane 
$(10 \mathrm{~mL})$ followed by 3 -chloroperbenzoic acid $(1.1 \mathrm{~g}, 6.54 \mathrm{mmol})$ at $0{ }^{\circ} \mathrm{C}$. Then, the mixture was stirred at $\mathrm{rt}$ for $5 \mathrm{~h}$. Until completion, it was washed with aqueous solution of sodium hydroxide $(10 \mathrm{~mL}, 1.0 \mathrm{M})$, aqueous solution of hydrochloric acid $(10 \mathrm{~mL}, 2.0 \mathrm{M})$ and saturated solution of sodium bicarbonate $(20 \mathrm{~mL})$. The organic layer was collected and dried with anhydrous magnesium sulfate. The solvent was removed in vacuo, and the residue was used in the next synthetic step without further purification. $^{[6]}$

Step 2: To a $50 \mathrm{~mL}$ round-bottom flask with magnetic stirring bar were added the crude S5 (1.2 g, $3.73 \mathrm{mmol})$ and $3 \mathrm{M}$ hydrochloric acid solution (5 $\mathrm{mL}, 0.5 \mathrm{M})$. Then, the mixture was dissolved in methanol $(10 \mathrm{~mL})$, and stirred at $80{ }^{\circ} \mathrm{C}$ for $2 \mathrm{~h}$. The solvent was removed in vacuo, diluted with dichloromethane and filtered. The residue was washed with dichloromethane $(5 \mathrm{~mL}$ x 3$)$ and ethyl acetate $(5 \mathrm{~mL} \times 3)$ to afford the desired product $\mathbf{1 v}$ (487 $\mathrm{mg}, 32 \%$ overall yield) as a white solid.

The sulfoxide $\mathbf{1 w}$ was synthesized by the similar procedure. The unknown compounds $1 \mathbf{w}$ was fully characterized.

\section{$\underline{2,2^{\prime}-\text { Sulfinyldiphenol (1v) }}$}<smiles>O=S(c1ccccc1O)c1ccccc1O</smiles>

Light yellow solid; m.p. $=156-158{ }^{\circ} \mathrm{C} ;{ }^{1} \mathrm{H}$ NMR (400 MHz, DMSO) $\delta 10.34$ (s, 2H), 7.46-7.11 (m, 4H), 7.03-6.60 (m, 4H); ${ }^{13} \mathrm{C}$ NMR (100 MHz, DMSO) $\delta 155.2,132.3$, 129.6, 126.1, 119.5, 116.0; HRMS (ESI) $m / z$ calcd for $\mathrm{C}_{24} \mathrm{H}_{19} \mathrm{NO}_{3} \mathrm{~S}[\mathrm{M}+\mathrm{H}]^{+}=$ 250.0538 , found $=250.0426$.

\section{$\underline{2,2 '-S u l f i n y l b i s(4-(t e r t-b u t y l) p h e n o l) ~(1 w) ~}$}<smiles>CC(C)(C)c1ccc(O)c(S(=O)c2cc(C(C)(C)C)ccc2O)c1</smiles> 
White solid; $624 \mathrm{mg}, 38 \%$ overall yield; m.p. $=154-156{ }^{\circ} \mathrm{C} ;{ }^{1} \mathrm{H}$ NMR $(400 \mathrm{MHz}$, DMSO) $\delta 10.11(\mathrm{~s}, 2 \mathrm{H}), 7.38(\mathrm{~d}, J=2.4 \mathrm{~Hz}, 2 \mathrm{H}), 7.33(\mathrm{dd}, J=8.4,2.4 \mathrm{~Hz}, 2 \mathrm{H}), 6.80$ (d, $J=8.4 \mathrm{~Hz}, 2 \mathrm{H}), 1.18(\mathrm{~s}, 18 \mathrm{H}) ;{ }^{13} \mathrm{C} \mathrm{NMR}(100 \mathrm{MHz}, \mathrm{DMSO}) \delta$ 153.0, 141.7, 129.1, 128.8, 122.2, 115.7, 34.0, 31.1; HRMS (ESI) $\mathrm{m} / z$ calcd for $\mathrm{C}_{20} \mathrm{H}_{26} \mathrm{O}_{3} \mathrm{~S}[\mathrm{M}+\mathrm{H}]^{+}$ $=347.1681$, found $=347.1680$. 


\section{General Procedure for the Desymmetrization of Free Sulfoximines}

via A-T-type Reaction

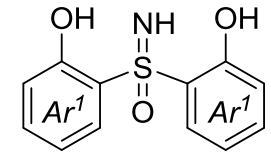

1

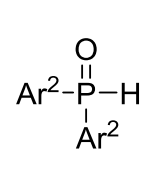

2

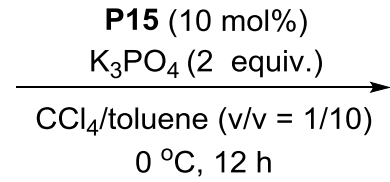

$0{ }^{\circ} \mathrm{C}, 12 \mathrm{~h}$

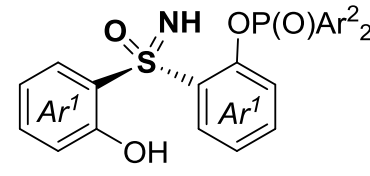

$3 / 4$

Condition A: to a tube $(10 \mathrm{~mL})$ with a magnetic stirring bar were added $\mathbf{1}(0.1 \mathrm{mmol})$, 2 (0.12 mmol), phosphonium salt P15 $(0.01 \mathrm{mmol})$ and potassium phosphate $(0.2$ $\mathrm{mmol})$, followed by the addition of $\mathrm{CCl}_{4} /$ toluene $(2.0 \mathrm{~mL}, \mathrm{v} / \mathrm{v}=1 / 10)$. The reaction mixture was stirred at $0{ }^{\circ} \mathrm{C}$ for $12 \mathrm{~h}$. Until completion, the reaction mixture was transfered to room temperature, and diluted with dichloromethane. Purification by column chromatography on silica gel (petroleum ether/ethyl acetate $=3: 1$ to $1: 1$ ) afforded the prodcut $\mathbf{3 / 4}$.

\section{(S)-2-(2-hydroxyphenylsulfonimidoyl)phenyl diphenylphosphinate (3a)}<smiles>NS(=O)(=O)c1ccccc1OPOCOc1ccccc1</smiles>

White solid; $39 \mathrm{mg}, 86 \%$ yield; m.p. $=146-148{ }^{\circ} \mathrm{C} ;{ }^{1} \mathrm{H}$ NMR $\left(400 \mathrm{MHz}, \mathrm{CDCl}_{3}\right) \delta$ $12.05(\mathrm{~s}, 1 \mathrm{H}), 8.00-7.92(\mathrm{~m}, 2 \mathrm{H}), 7.84-7.77(\mathrm{~m}, 3 \mathrm{H}), 7.66(\mathrm{~d}, J=8.4 \mathrm{~Hz}, 1 \mathrm{H})$, 7.63-7.54 (m, 2H), 7.54-7.47 (m, 2H), 7.47-7.41 (m, 3H), 7.41-7.33 (m, 2H), 7.16-7.10 (m, 1H), $6.81(\mathrm{dd}, J=8.4,1.0 \mathrm{~Hz}, 1 \mathrm{H}), 6.78-6.71(\mathrm{~m}, 1 \mathrm{H}) ;{ }^{13} \mathrm{C}$ NMR $(100$ $\left.\mathrm{MHz}, \mathrm{CDCl}_{3}\right) \delta 157.5,149.2(\mathrm{~d}, J=7.6 \mathrm{~Hz}), 135.3(\mathrm{~d}, J=81.2 \mathrm{~Hz}), 134.2(\mathrm{~d}, J=7.2$ Hz), $133.2(\mathrm{~d}, J=2.8 \mathrm{~Hz}), 133.1(\mathrm{~d}, J=2.8 \mathrm{~Hz}), 132.2(\mathrm{~d}, J=11.0 \mathrm{~Hz}), 130.3(\mathrm{~d}, J=$ $34.6 \mathrm{~Hz}), 129.3(\mathrm{~d}, J=9.2 \mathrm{~Hz}), 129.1,129.0(\mathrm{~d}, J=5.0 \mathrm{~Hz}), 128.8(\mathrm{~d}, J=5.0 \mathrm{~Hz})$, 124.1, $121.5(\mathrm{~d}, J=4.8 \mathrm{~Hz}), 120.0,121.5(\mathrm{~d}, J=4.8 \mathrm{~Hz}) ;{ }^{31} \mathrm{P} \mathrm{NMR}\left(162 \mathrm{MHz}, \mathrm{CDCl}_{3}\right)$ $\delta$ 34.19; HRMS (ESI) $m / z$ calcd for $\mathrm{C}_{24} \mathrm{H}_{20} \mathrm{NO}_{4} \mathrm{PS}[\mathrm{M}+\mathrm{H}]^{+}=450.0929$, found $=$ 450.0929 . 
Optical Rotation: $[\alpha]_{\mathrm{D}}^{25}=76.77\left(c=0.31, \mathrm{CHCl}_{3}\right) .97 \%$ ee (HPLC condition: Chiralpak AD-H column, $n$-Hexane $/ i-\mathrm{PrOH}=70: 30$, flow rate $=1.0 \mathrm{~mL} / \mathrm{min}$, wavelength $=254 \mathrm{~nm}, t_{\mathrm{R}}=24.16$ min for major isomer, $t_{\mathrm{R}}=30.39$ min for minor isomer).

$\mathrm{mV}$

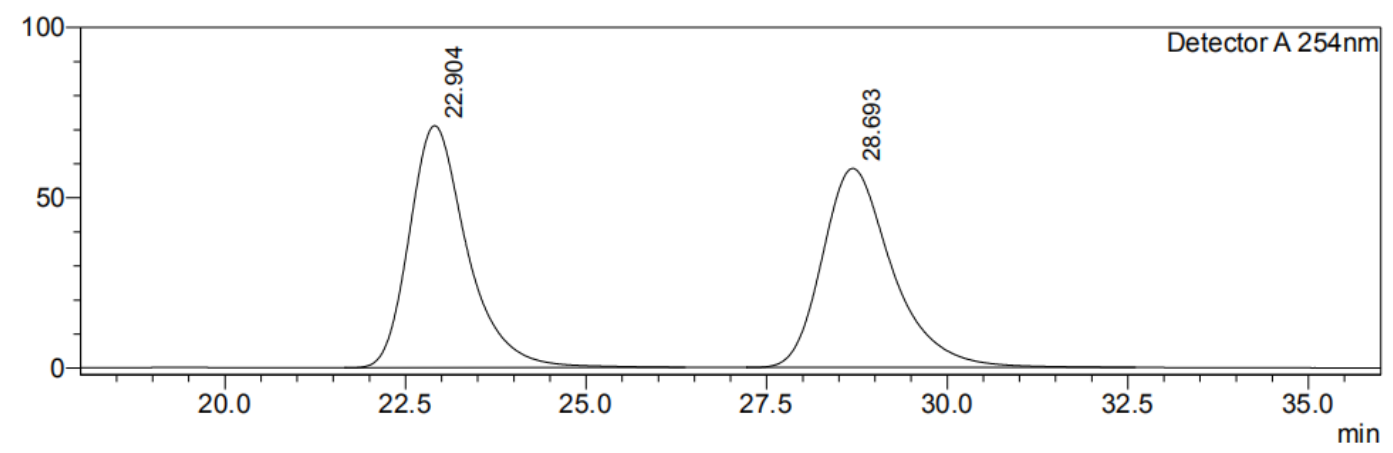

\begin{tabular}{|c|c|c|c|c|c|}
\hline Peak\# & $\begin{array}{l}\text { or A 254nm } \\
\text { Ret. Time }\end{array}$ & Height & Height\% & Area & Area\% \\
\hline 1 & 22.904 & 71046 & 54.874 & 3962141 & 50.048 \\
\hline 2 & 28.693 & 58425 & 45.126 & 3954560 & 49.952 \\
\hline Total & & 129471 & 100.000 & 7916700 & 100.000 \\
\hline
\end{tabular}

$\mathrm{mV}$

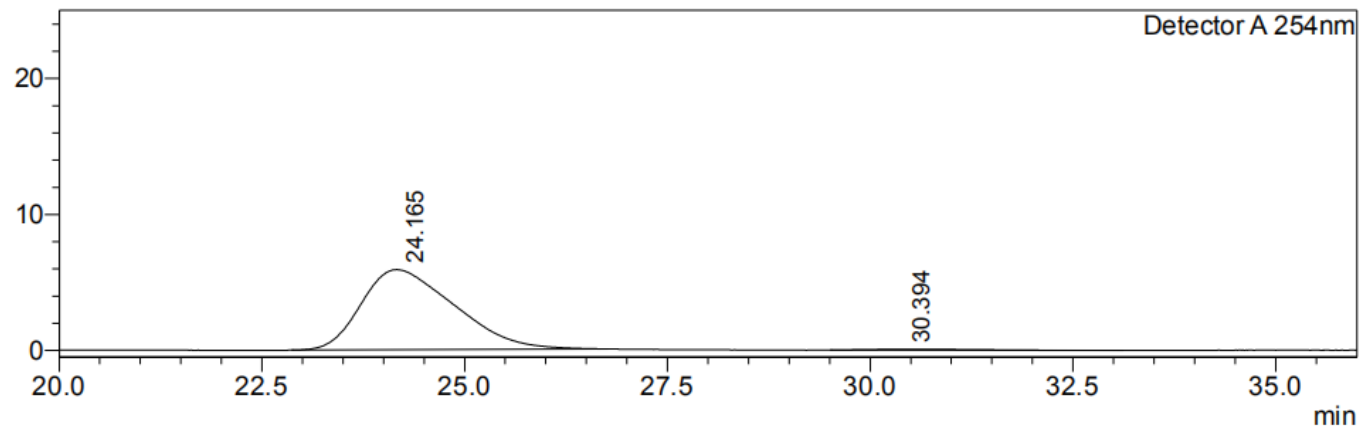

Detector A 254nm

\begin{tabular}{|r|r|r|r|r|r|}
\hline Peak\# & Ret. Time & \multicolumn{1}{c|}{ Height } & Height\% & \multicolumn{1}{c|}{ Area } & \multicolumn{1}{c|}{ Area\% } \\
\hline 1 & 24.165 & 5891 & 98.794 & 470632 & 98.620 \\
\hline 2 & 30.394 & 72 & 1.206 & 6584 & 1.380 \\
\hline Total & & 5963 & 100.000 & 477216 & 100.000 \\
\hline
\end{tabular}

(S)-2-(2-hydroxy-5-methylphenylsulfonimidoyl)-4-methylphenyl

\section{diphenylphosphinate (3b)}

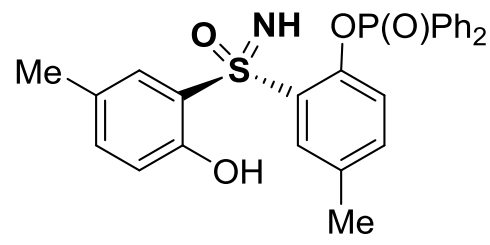

White solid; $40 \mathrm{mg}, 83 \%$ yield; m.p. $=184-186{ }^{\circ} \mathrm{C} ;{ }^{1} \mathrm{H}$ NMR $\left(400 \mathrm{MHz}, \mathrm{CDCl}_{3}\right) \delta$ $11.75(\mathrm{~s}, 1 \mathrm{H}), 8.05-7.96(\mathrm{~m}, 2 \mathrm{H})$, 7.88-7.77 (m, 2H), $7.64(\mathrm{~s}, 1 \mathrm{H})$, 7.61-7.46 (m, 5H), $7.42(\mathrm{dt}, J=7.3,3.7 \mathrm{~Hz}, 2 \mathrm{H}), 7.29$ (d, $J=1.5 \mathrm{~Hz}, 1 \mathrm{H}), 7.18-7.09$ (m, 2H), 6.70 (d, $J$ 
$=8.5 \mathrm{~Hz}, 1 \mathrm{H}), 2.27(\mathrm{~s}, 3 \mathrm{H}), 1.99(\mathrm{~s}, 3 \mathrm{H}) ;{ }^{13} \mathrm{C} \mathrm{NMR}\left(100 \mathrm{MHz}, \mathrm{CDCl}_{3}\right) \delta 155.0,147.0$ (d, $J=7.4 \mathrm{~Hz}), 136.6,134.7$ (d, $J=126.4 \mathrm{~Hz}), 133.6$ (d, $J=7.2 \mathrm{~Hz}), 133.0$ (d, $J=2.9$ Hz), $132.9(\mathrm{~d}, J=2.9 \mathrm{~Hz}), 132.2(\mathrm{~d}, J=1.8 \mathrm{~Hz}), 132.1(\mathrm{~d}, J=1.8 \mathrm{~Hz}), 130.6(\mathrm{~d}, J=$ $25.4 \mathrm{~Hz}), 129.5,129.2(\mathrm{~d}, J=22.6 \mathrm{~Hz}), 128.9$ (d, $J=2.5 \mathrm{~Hz}), 128.8(\mathrm{~d}, J=5.5 \mathrm{~Hz})$, $128.7(\mathrm{~d}, J=9.6 \mathrm{~Hz}), 121.3(\mathrm{~d}, J=4.9 \mathrm{~Hz}), 119.4(\mathrm{~d}, J=86.5 \mathrm{~Hz}), 20.8,20.2 ;{ }^{31} \mathrm{P}$ NMR $\left(162 \mathrm{MHz}, \mathrm{CDCl}_{3}\right) \delta 33.35$; HRMS (ESI) $m / z$ calcd for $\mathrm{C}_{26} \mathrm{H}_{24} \mathrm{NO}_{4} \mathrm{PS}[\mathrm{M}+\mathrm{H}]^{+}=$ 478.1242 , found $=478.1242$.

Optical Rotation: $[\alpha]_{\mathrm{D}}^{25}=96.78\left(c=0.59, \mathrm{CHCl}_{3}\right) .90 \%$ ee (HPLC condition: Chiralpak OD-H column, $n$-Hexane $/ i-\mathrm{PrOH}=80: 20$, flow rate $=1.0 \mathrm{~mL} / \mathrm{min}$, wavelength $=254 \mathrm{~nm}, t_{\mathrm{R}}=10.64$ min for major isomer, $t_{\mathrm{R}}=20.16$ min for minor isomer).

$\mathrm{mV}$

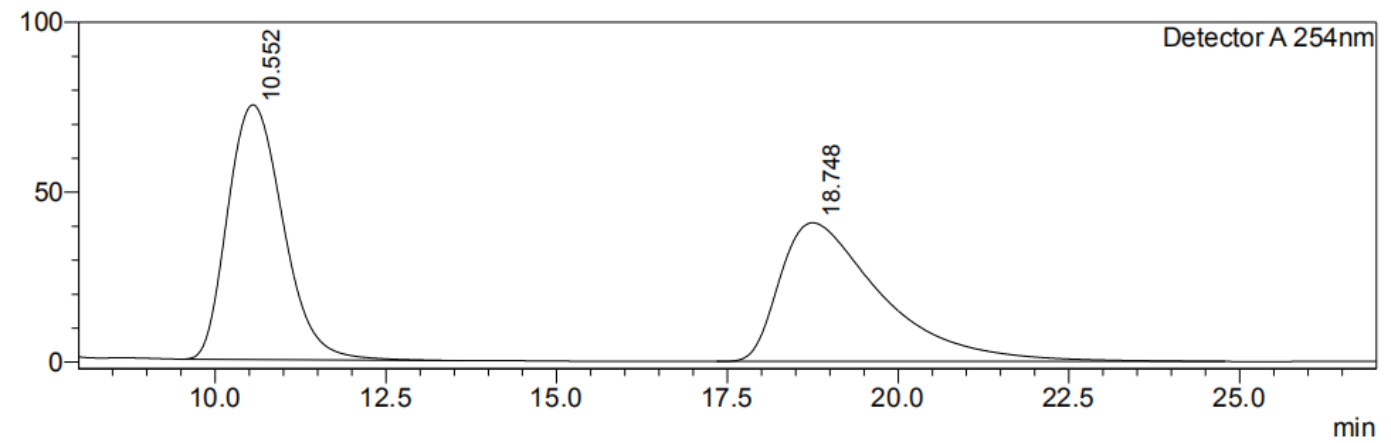

Detector A 254nm

\begin{tabular}{|r|r|r|r|r|r|}
\hline Peak\# & Ret. Time & \multicolumn{1}{c|}{ Height } & Height\% & Area & \multicolumn{1}{c|}{ Area\% } \\
\hline 1 & 10.552 & 74972 & 64.740 & 4236840 & 49.989 \\
\hline 2 & 18.748 & 40832 & 35.260 & 4238749 & 50.011 \\
\hline Total & & 115805 & 100.000 & 8475589 & 100.000 \\
\hline
\end{tabular}

$\mathrm{mV}$

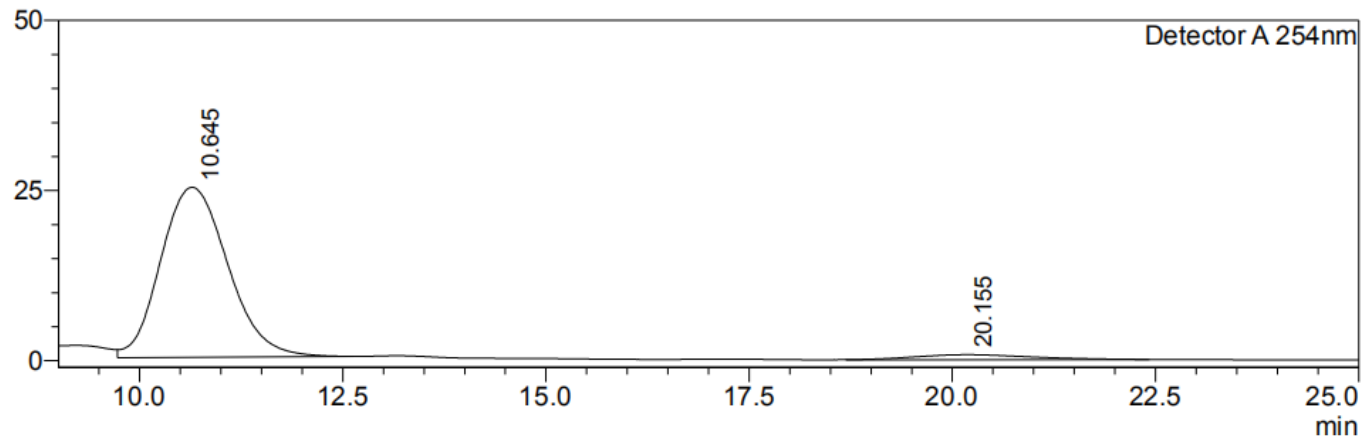

Detector A 254nm

\begin{tabular}{|r|r|r|r|r|r|}
\hline Peak\# & Ret. Time & Height & Height $\%$ & \multicolumn{1}{c|}{ Area } & \multicolumn{1}{c|}{ Area\% } \\
\hline 1 & 10.645 & 24989 & 97.143 & 1438665 & 95.118 \\
\hline 2 & 20.155 & 735 & 2.857 & 73835 & 4.882 \\
\hline Total & & 25723 & 100.000 & 1512499 & 100.000 \\
\hline
\end{tabular}




\section{$\underline{\text { (S)-4-ethyl-2-(5-ethyl-2-hydroxyphenylsulfonimidoyl)phenyl }}$}

\section{diphenylphosphinate (3c)}<smiles>CCOc1ccc(CC)cc1S(=O)(=O)c1cc(CC)ccc1O</smiles>

Light yellow solid; $42 \mathrm{mg}, 84 \%$ yield; m.p. $=174-176{ }^{\circ} \mathrm{C} ;{ }^{1} \mathrm{H}$ NMR $(400 \mathrm{MHz}$, $\left.\mathrm{CDCl}_{3}\right) \delta 11.78(\mathrm{~s}, 1 \mathrm{H}), 8.05(\mathrm{~d}, J=7.3 \mathrm{~Hz}, 1 \mathrm{H}), 8.02(\mathrm{~d}, J=7.3 \mathrm{~Hz}, 1 \mathrm{H}), 7.87(\mathrm{~d}, J$ $=7.4 \mathrm{~Hz}, 1 \mathrm{H}), 7.84(\mathrm{~d}, J=7.4 \mathrm{~Hz}, 1 \mathrm{H}), 7.63(\mathrm{~s}, 1 \mathrm{H}), 7.61-7.47(\mathrm{~m}, 5 \mathrm{H}), 7.42(\mathrm{td}, J=$ 7.4, 3.6 Hz, 2H), 7.35 (s, 1H), $7.21(\mathrm{~d}, J=8.1 \mathrm{~Hz}, 1 \mathrm{H}), 7.16$ (d, $J=7.6 \mathrm{~Hz}, 1 \mathrm{H}), 6.74$ $(\mathrm{d}, J=8.4 \mathrm{~Hz}, 1 \mathrm{H}), 2.58(\mathrm{q}, J=7.6 \mathrm{~Hz}, 2 \mathrm{H}), 2.29$ (q, $J=7.6 \mathrm{~Hz}, 2 \mathrm{H}), 1.15(\mathrm{t}, J=7.6$ $\mathrm{Hz}, 3 \mathrm{H}), 1.00(\mathrm{t}, J=7.6 \mathrm{~Hz}, 3 \mathrm{H}) ;{ }^{13} \mathrm{C} \mathrm{NMR}\left(100 \mathrm{MHz}, \mathrm{CDCl}_{3}\right) \delta 155.2,147.0(\mathrm{~d}, J=$ $7.4 \mathrm{~Hz}), 140.3,135.3,134.8$ (d, $J=132.5 \mathrm{~Hz}), 133.8$ (d, $J=6.8 \mathrm{~Hz}), 133.0$ (d, $J=2.6$ Hz), $132.9(\mathrm{~d}, J=2.6 \mathrm{~Hz}), 132.3(\mathrm{~d}, J=7.8 \mathrm{~Hz}), 132.2(\mathrm{~d}, J=7.7 \mathrm{~Hz}), 130.7$ (d, $J=$ $19.1 \mathrm{~Hz}), 129.3$ (d, $J=16.3 \mathrm{~Hz}), 128.9$ (d, $J=2.7 \mathrm{~Hz}), 128.8$ (d, $J=2.8 \mathrm{~Hz}), 128.4$, 127.7, 121.4 (d, $J=4.7 \mathrm{~Hz}), 119.8,119.1,28.1,27.8,15.6,15.2 ;{ }^{31} \mathrm{P} \mathrm{NMR}(162 \mathrm{MHz}$, $\left.\mathrm{CDCl}_{3}\right) \delta 33.18$; HRMS (ESI) $m / z$ calcd for $\mathrm{C}_{28} \mathrm{H}_{28} \mathrm{NO}_{4} \mathrm{PS}[\mathrm{M}+\mathrm{H}]^{+}=506.1555$, found $=506.1555$.

Optical Rotation: $[\alpha]_{\mathrm{D}}^{25}=116.33\left(c=0.60, \mathrm{CHCl}_{3}\right) .81 \%$ ee (HPLC condition: Chiralpak OD-H column, $n$-Hexane $/ i-\mathrm{PrOH}=90: 10$, flow rate $=1.0 \mathrm{~mL} / \mathrm{min}$, wavelength $=254 \mathrm{~nm}, t_{\mathrm{R}}=14.34$ min for major isomer, $t_{\mathrm{R}}=31.57$ min for minor isomer). 
$\mathrm{mV}$

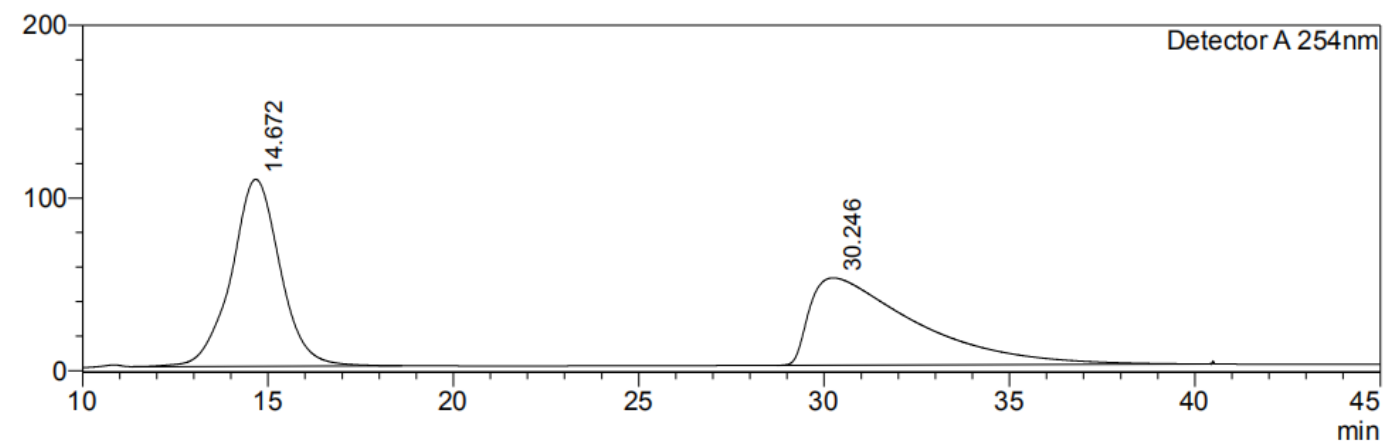

Detector A 254nm

\begin{tabular}{|r|r|r|r|r|r|}
\hline Peak\# & Ret. Time & \multicolumn{1}{c|}{ Height } & Height\% & \multicolumn{1}{c|}{ Area } & \multicolumn{1}{c|}{ Area\% } \\
\hline 1 & 14.672 & 108340 & 68.193 & 9944576 & 49.759 \\
\hline 2 & 30.246 & 50532 & 31.807 & 10040940 & 50.241 \\
\hline Total & & 158872 & 100.000 & 19985515 & 100.000 \\
\hline
\end{tabular}

$\mathrm{mV}$

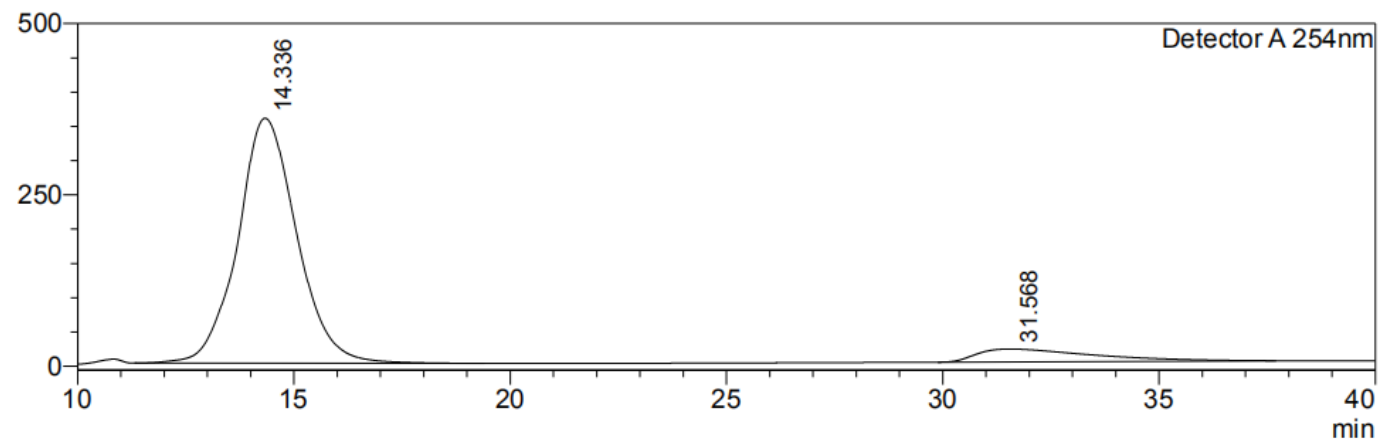

Detector A 254nm

\begin{tabular}{|r|r|r|r|r|r|}
\hline Peak\# & Ret. Time & \multicolumn{1}{c|}{ Height } & \multicolumn{1}{c|}{ Height\% } & \multicolumn{1}{c|}{ Area } & \multicolumn{1}{c|}{ Area\% } \\
\hline 1 & 14.336 & 357551 & 95.004 & 33987118 & 90.714 \\
\hline 2 & 31.568 & 18802 & 4.996 & 3479220 & 9.286 \\
\hline Total & & 376352 & 100.000 & 37466337 & 100.000 \\
\hline
\end{tabular}

\section{(S)-2-(2-hydroxy-5-isopropylphenylsulfonimidoyl)-4-isopropylphenyl}

\section{diphenylphosphinate (3d)}<smiles>CCCOc1ccc(C(C)C)cc1S(=O)(=O)c1cc(C(C)C)ccc1O</smiles>

White powder; $43 \mathrm{mg}, 82 \%$ yield; m.p. $=149-151{ }^{\circ} \mathrm{C} ;{ }^{1} \mathrm{H}$ NMR $\left(400 \mathrm{MHz}, \mathrm{CDCl}_{3}\right) \delta$ $12.07(\mathrm{~s}, 1 \mathrm{H}), 8.09-8.05(\mathrm{~m}, 1 \mathrm{H}), 8.05-8.01(\mathrm{~m}, 1 \mathrm{H}), 7.92-7.88(\mathrm{~m}, 1 \mathrm{H}), 7.88-7.83(\mathrm{~m}$, 1H), 7.61-7.54 (m, 2H), 7.54-7.47 (m, 4H), $7.42(\mathrm{dt}, J=5.6,3.6 \mathrm{~Hz}, 3 \mathrm{H}), 7.27(\mathrm{~d}, J=$ $2.2 \mathrm{~Hz}, 1 \mathrm{H}), 7.18(\mathrm{dd}, J=8.6,2.2 \mathrm{~Hz}, 1 \mathrm{H}), 6.77(\mathrm{~d}, J=8.6 \mathrm{~Hz}, 1 \mathrm{H}), 4.09(\mathrm{~s}, 1 \mathrm{H})$, 2.82 (hept, $J=6.9 \mathrm{~Hz}, 1 \mathrm{H}$ ), 2.53 (hept, $J=6.9 \mathrm{~Hz}, 1 \mathrm{H}$ ), 1.14 (d, $J=6.9 \mathrm{~Hz}, 6 \mathrm{H}$ ), 
$1.04(\mathrm{~d}, J=6.9 \mathrm{~Hz}, 3 \mathrm{H}), 1.01(\mathrm{~d}, J=6.9 \mathrm{~Hz}, 3 \mathrm{H}) ;{ }^{13} \mathrm{C}$ NMR $\left(100 \mathrm{MHz}, \mathrm{CDCl}_{3}\right) \delta$ 155.3, 146.8 (d, $J=7.4 \mathrm{~Hz}), 145.0,140.0,134.1(\mathrm{~d}, J=7.0 \mathrm{~Hz}), 133.2$ (d, $J=134.2$ Hz), $133.0(\mathrm{~d}, J=2.9 \mathrm{~Hz}), 132.8(\mathrm{~d}, J=2.9 \mathrm{~Hz}), 132.3(\mathrm{~d}, J=10.8 \mathrm{~Hz}), 132.1(\mathrm{~d}, J=$ $10.8 \mathrm{~Hz}), 130.8$ (d, $J=8.3 \mathrm{~Hz}), 129.4$ (d, $J=6.3 \mathrm{~Hz}), 128.9$ (d, $J=13.8 \mathrm{~Hz}), 126.9$, 126.5, 121.4, $121.4(\mathrm{~d}, J=4.9 \mathrm{~Hz}), 119.1,33.4,33.1,24.0,23.9,23.8,23.7 ;{ }^{31} \mathrm{P}$ NMR $\left(162 \mathrm{MHz}, \mathrm{CDCl}_{3}\right) \delta 33.05$; HRMS (ESI) $\mathrm{m} / z$ calcd for $\mathrm{C}_{30} \mathrm{H}_{32} \mathrm{NO}_{4} \mathrm{PS}[\mathrm{M}+\mathrm{H}]^{+}=$ 534.1868 , found $=534.1869$.

Optical Rotation: $[\alpha]_{\mathrm{D}}^{25}=57.55\left(c=0.49, \mathrm{CHCl}_{3}\right) .97 \%$ ee (HPLC condition: Chiralpak OD-H column, $n$-Hexane $/ i-\mathrm{PrOH}=90: 10$, flow rate $=1.0 \mathrm{~mL} / \mathrm{min}$, wavelength $=254 \mathrm{~nm}, t_{\mathrm{R}}=10.06$ min for major isomer, $t_{\mathrm{R}}=15.96$ min for minor isomer).

$\mathrm{mV}$

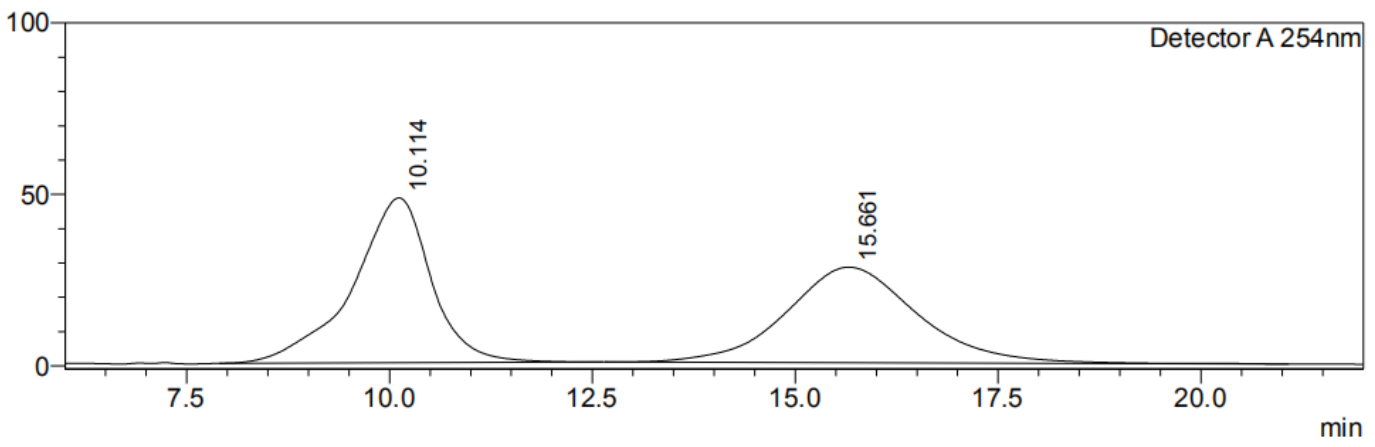

Detector A 254nm
\begin{tabular}{|r|r|r|r|r|r|}
\hline Peak\# & Ret. Time & Height & Height\% & Area & \multicolumn{1}{|c|}{ Area\% } \\
\hline 1 & 10.114 & 48076 & 63.292 & 3226670 & 50.551 \\
\hline 2 & 15.661 & 27883 & 36.708 & 3156387 & 49.449 \\
\hline Total & & 75960 & 100.000 & 6383058 & 100.000 \\
\hline
\end{tabular}

$\mathrm{mV}$

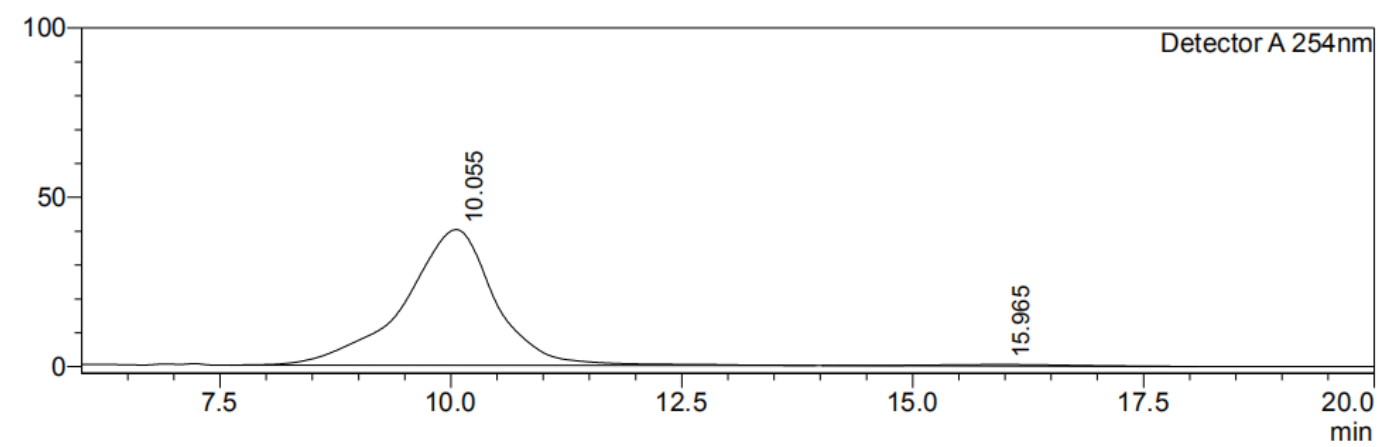

Detector A 254nm

\begin{tabular}{|r|r|r|r|r|r|}
\hline Peak\# & Ret. Time & \multicolumn{1}{c|}{ Height } & \multicolumn{1}{c|}{ Height\% } & \multicolumn{1}{c|}{ Area } & \multicolumn{1}{c|}{ Area\% } \\
\hline 1 & 10.055 & 40068 & 98.911 & 2762555 & 98.367 \\
\hline 2 & 15.965 & 441 & 1.089 & 45851 & 1.633 \\
\hline Total & & 40509 & 100.000 & 2808406 & 100.000 \\
\hline
\end{tabular}




\section{(S)-4-(tert-butyl)-2-(5-(tert-butyl)-2-hydroxyphenylsulfonimidoyl)phenyl}

\section{diphenylphosphinate (3e)}

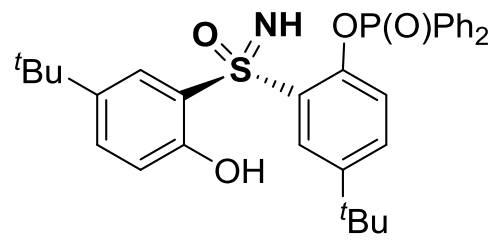

White solid; $49 \mathrm{mg}, 87 \%$ yield; m.p. $=179-181{ }^{\circ} \mathrm{C} ;{ }^{1} \mathrm{H}$ NMR $\left(400 \mathrm{MHz}, \mathrm{CDCl}_{3}\right) \delta$ $8.07(\mathrm{~d}, J=7.2 \mathrm{~Hz}, 1 \mathrm{H}), 8.04(\mathrm{~d}, J=7.2 \mathrm{~Hz}, 1 \mathrm{H}), 7.91(\mathrm{~d}, J=7.3 \mathrm{~Hz}, 1 \mathrm{H}), 7.88(\mathrm{~d}, J$ $=7.3 \mathrm{~Hz}, 1 \mathrm{H}), 7.62(\mathrm{~d}, J=1.4 \mathrm{~Hz}, 1 \mathrm{H}), 7.58(\mathrm{~d}, J=2.4 \mathrm{~Hz}, 1 \mathrm{H}), 7.56-7.40(\mathrm{~m}, 8 \mathrm{H})$, $7.32(\mathrm{dd}, J=8.6,2.1 \mathrm{~Hz}, 1 \mathrm{H}), 6.84(\mathrm{~d}, J=8.6 \mathrm{~Hz}, 1 \mathrm{H}), 1.18(\mathrm{~s}, 9 \mathrm{H}), 1.11(\mathrm{~s}, 9 \mathrm{H}) ;{ }^{13} \mathrm{C}$ NMR $\left(100 \mathrm{MHz}, \mathrm{CDCl}_{3}\right) \delta 155.3,147.5,146.4(\mathrm{~d}, J=7.7 \mathrm{~Hz}), 142.6,133.9(\mathrm{~d}, J=$ $6.9 \mathrm{~Hz}), 133.4,133.0(\mathrm{~d}, J=2.8 \mathrm{~Hz}), 132.9(\mathrm{~d}, J=2.8 \mathrm{~Hz}), 132.3(\mathrm{~d}, J=10.9 \mathrm{~Hz})$, $132.0(\mathrm{~d}, J=10.8 \mathrm{~Hz}), 131.4,130.8(\mathrm{~d}, J=8.2 \mathrm{~Hz}), 129.5$ (d, $J=8.9 \mathrm{~Hz}), 129.0(\mathrm{~d}, J$ $=1.8 \mathrm{~Hz}), 128.8(\mathrm{~d}, J=1.6 \mathrm{~Hz}), 125.6(\mathrm{~d}, J=69.3 \mathrm{~Hz}), 121.2(\mathrm{~d}, J=4.7 \mathrm{~Hz}), 118.8$ $(\mathrm{d}, J=10.3 \mathrm{~Hz}), 34.4(\mathrm{~d}, J=46.3 \mathrm{~Hz}), 31.1(\mathrm{~d}, J=4.2 \mathrm{~Hz}) ;{ }^{31} \mathrm{P}$ NMR $(162 \mathrm{MHz}$, $\left.\mathrm{CDCl}_{3}\right) \delta 33.30$; HRMS (ESI) $\mathrm{m} / z$ calcd for $\mathrm{C}_{32} \mathrm{H}_{36} \mathrm{NO}_{4} \mathrm{PS}[\mathrm{M}+\mathrm{H}]^{+}=562.2181$, found $=562.2181$.

Optical Rotation: $[\alpha]_{\mathrm{D}}^{25}=75.34\left(c=0.50, \mathrm{CHCl}_{3}\right) .98 \%$ ee (HPLC condition: Chiralpak OD-H column, $n-\mathrm{Hexane} / i-\mathrm{PrOH}=90: 10$, flow rate $=1.0 \mathrm{~mL} / \mathrm{min}$, wavelength $=254 \mathrm{~nm}, t_{\mathrm{R}}=9.21 \mathrm{~min}$ for major isomer, $t_{\mathrm{R}}=12.50 \mathrm{~min}$ for minor isomer). 
$\mathrm{mV}$
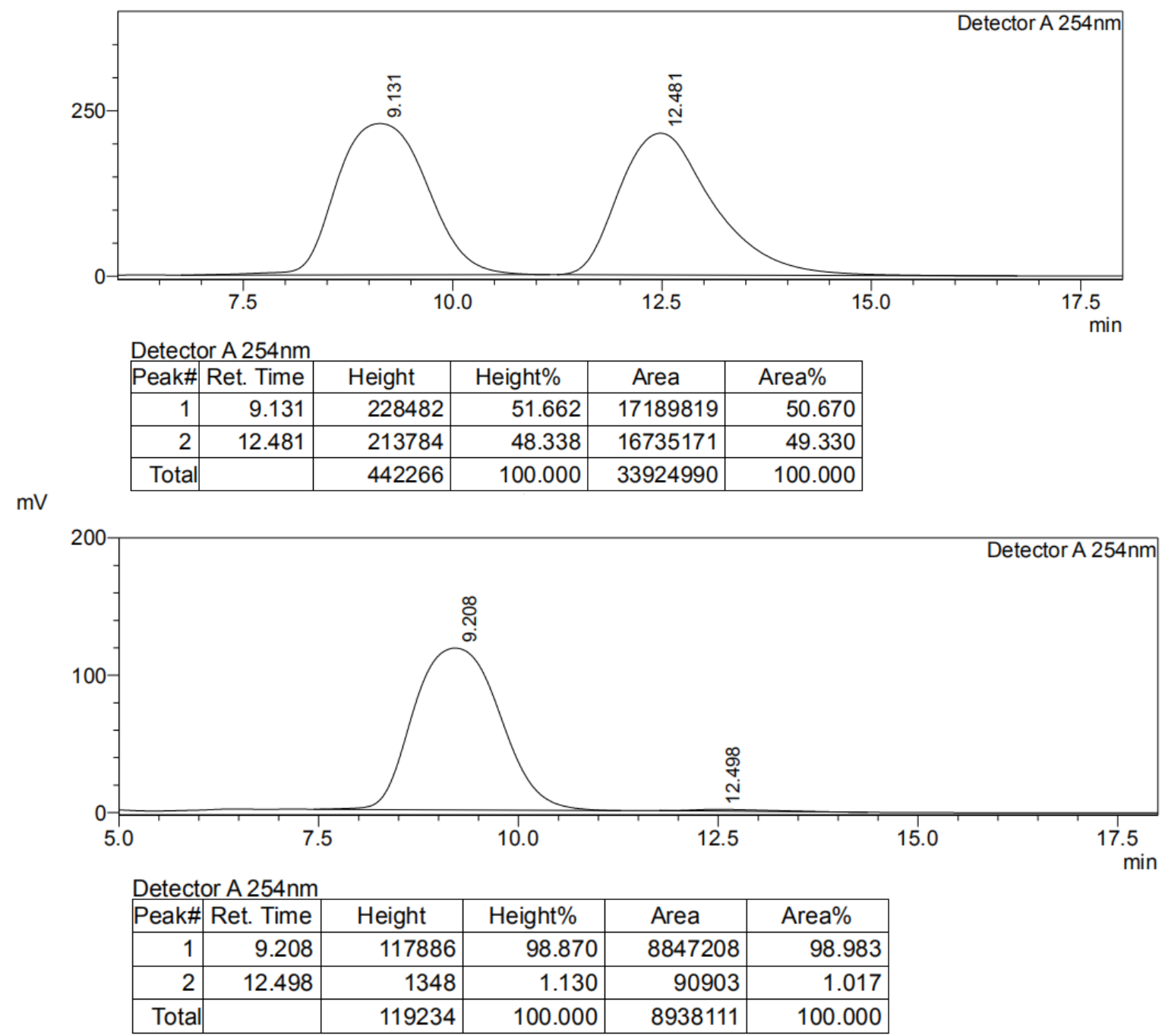

(S)-2-(2-hydroxy-5-(2-phenylpropan-2-yl)phenylsulfonimidoyl)-4-(2-phenylprop an-2-yl)phenyl diphenylphosphinate (3f)<smiles>CC(C)(c1ccccc1)c1ccc(O)c(S(=O)(=O)c2cc(C(C)(C)c3ccccc3)ccc2OCCCCOc2ccccc2)c1</smiles>

Yellow oil; 60 mg, 87\% yield; ${ }^{1} \mathrm{H}$ NMR (400 MHz, $\left.\mathrm{CDCl}_{3}\right) \delta 12.07$ (s, 1H), 8.04 (d, $J$ $=7.3 \mathrm{~Hz}, 1 \mathrm{H}), 8.00(\mathrm{~d}, J=7.3 \mathrm{~Hz}, 1 \mathrm{H}), 7.90(\mathrm{~d}, J=7.4 \mathrm{~Hz}, 1 \mathrm{H}), 7.86(\mathrm{~d}, J=7.4 \mathrm{~Hz}$, 1H), 7.60-7.38 (m, 9H), 7.25-7.18 (m, 4H), 7.18-7.12 (m, 4H), 7.12-7.04 (m, 4H), $6.69(\mathrm{~d}, J=8.8 \mathrm{~Hz}, 1 \mathrm{H}), 4.19(\mathrm{~s}, 1 \mathrm{H}), 1.57(\mathrm{~s}, 6 \mathrm{H}), 1.50(\mathrm{~s}, 3 \mathrm{H}), 1.48(\mathrm{~s}, 3 \mathrm{H}) ;{ }^{13} \mathrm{C}$ NMR $\left(100 \mathrm{MHz}, \mathrm{CDCl}_{3}\right) \delta 155.7,149.4(\mathrm{~d}, J=65.8 \mathrm{~Hz}), 147.2,146.6(\mathrm{~d}, J=7.5 \mathrm{~Hz})$, 
$142.1,135.2,134.2(\mathrm{~d}, J=6.6 \mathrm{~Hz}), 133.1,133.1(\mathrm{~d}, J=2.4 \mathrm{~Hz}), 133.0,132.9(\mathrm{~d}, J=$ $2.5 \mathrm{~Hz}), 132.3(\mathrm{~d}, J=10.9 \mathrm{~Hz}), 131.94(\mathrm{~d}, J=10.8 \mathrm{~Hz}), 130.8$ (d, $J=16.1 \mathrm{~Hz}), 129.4$ $(\mathrm{d}, J=14.3 \mathrm{~Hz}), 128.9(\mathrm{~d}, J=13.8 \mathrm{~Hz}), 128.3(\mathrm{~d}, J=12.7 \mathrm{~Hz}), 126.6(\mathrm{~d}, J=5.8 \mathrm{~Hz})$, $126.5(\mathrm{~d}, J=57.4 \mathrm{~Hz}), 125.9(\mathrm{~d}, J=6.1 \mathrm{~Hz}), 121.2(\mathrm{~d}, J=4.4 \mathrm{~Hz}), 118.8(\mathrm{~d}, J=52.6$ $\mathrm{Hz}), 42.8,42.4,30.7,30.6,30.5 ;{ }^{31} \mathrm{P}$ NMR (162 MHz, $\left.\mathrm{CDCl}_{3}\right) \delta 33.57$; HRMS (ESI) $m / z$ calcd for $\mathrm{C}_{42} \mathrm{H}_{40} \mathrm{NO}_{4} \mathrm{PS}[\mathrm{M}+\mathrm{H}]^{+}=686.2494$, found $=686.2493$.

Optical Rotation: $[\alpha]^{25}=43.47\left(c=1.01, \mathrm{CHCl}_{3}\right) .98 \%$ ee (HPLC condition: Chiralpak OD-H column, $n-\mathrm{Hexane} / i-\mathrm{PrOH}=90: 10$, flow rate $=1.0 \mathrm{~mL} / \mathrm{min}$, wavelength $=254 \mathrm{~nm}, t_{\mathrm{R}}=14.75 \min$ for major isomer, $t_{\mathrm{R}}=25.04 \mathrm{~min}$ for minor isomer).

$\mathrm{mV}$

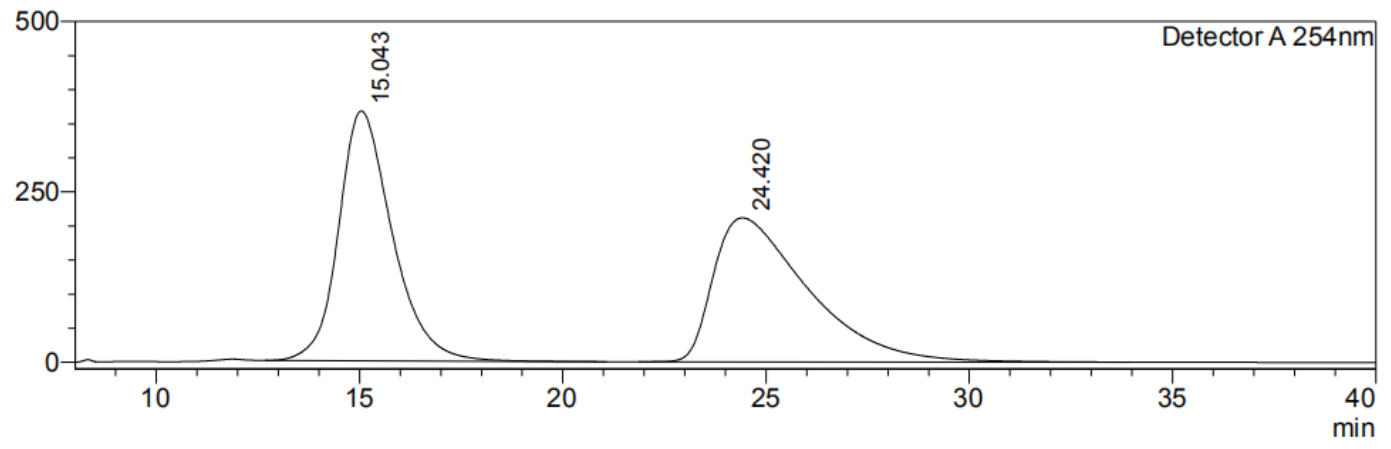

Detector A 254nm

\begin{tabular}{|r|r|r|r|r|r|}
\hline Peak\# & Ret. Time & Height & \multicolumn{1}{c|}{ Height\% } & \multicolumn{1}{c|}{ Area } & \multicolumn{1}{c|}{ Area\% } \\
\hline 1 & 15.043 & 366637 & 63.445 & 33747624 & 49.611 \\
\hline 2 & 24.420 & 211243 & 36.555 & 34277266 & 50.389 \\
\hline Total & & 577880 & 100.000 & 68024890 & 100.000 \\
\hline
\end{tabular}


$\mathrm{mV}$

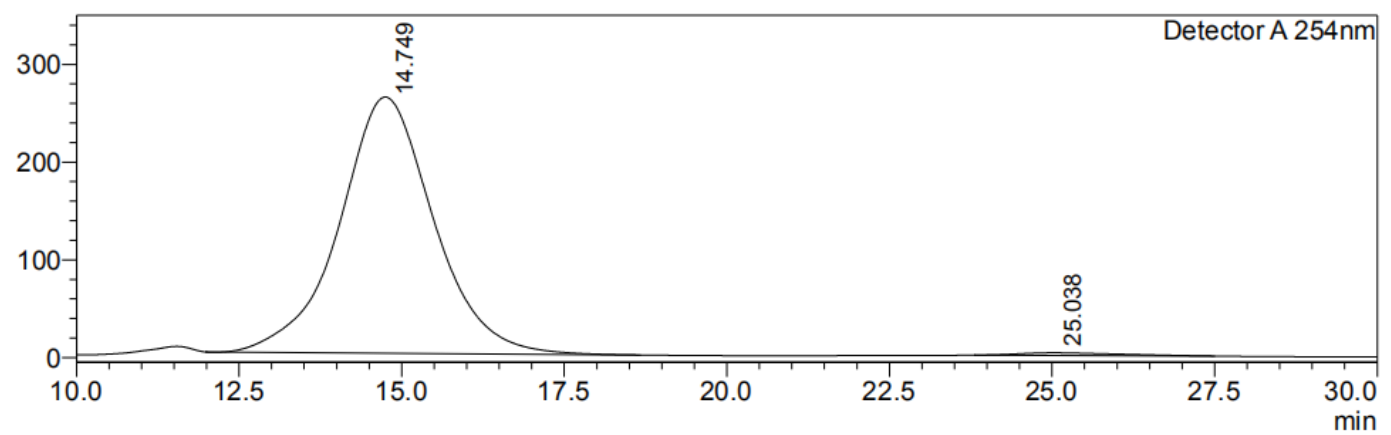

Detector A 254nm

\begin{tabular}{|r|r|r|r|r|r|}
\hline Peak\# & Ret. Time & \multicolumn{1}{c|}{ Height } & Height\% & \multicolumn{1}{c|}{ Area } & \multicolumn{1}{c|}{ Area\% } \\
\hline 1 & 14.749 & 262179 & 98.963 & 26469787 & 98.881 \\
\hline 2 & 25.038 & 2746 & 1.037 & 299646 & 1.119 \\
\hline Total & & 264925 & 100.000 & 26769434 & 100.000 \\
\hline
\end{tabular}

\section{(S)-4-cyclohexyl-2-(5-cyclohexyl-2-hydroxyphenylsulfonimidoyl)phenyl}

\section{diphenylphosphinate (3g)}<smiles>O=C(OCCOCCOc1ccc(C2CCCCC2)cc1)S(=O)(=O)c1cc(C2CCCCC2)ccc1O</smiles>

Light yellow solid; $51 \mathrm{mg}, 83 \%$ yield; m.p. $=183-185{ }^{\circ} \mathrm{C} ;{ }^{1} \mathrm{H}$ NMR $(400 \mathrm{MHz}$, $\left.\mathrm{CDCl}_{3}\right) \delta 8.14-8.09(\mathrm{~m}, 1 \mathrm{H}), 8.08(\mathrm{~d}, J=7.2 \mathrm{~Hz}, 1 \mathrm{H}), 7.90(\mathrm{~d}, J=7.2 \mathrm{~Hz}, 1 \mathrm{H}), 7.87$ (d, $J=7.2 \mathrm{~Hz}, 1 \mathrm{H}), 7.63(\mathrm{~d}, J=1.2 \mathrm{~Hz}, 1 \mathrm{H}), 7.60-7.48(\mathrm{~m}, 5 \mathrm{H}), 7.44-7.36(\mathrm{~m}, 3 \mathrm{H})$, $7.22(\mathrm{dd}, J=8.6,2.0 \mathrm{~Hz}, 1 \mathrm{H}), 7.16(\mathrm{dd}, J=8.6,2.0 \mathrm{~Hz}, 1 \mathrm{H}), 6.75(\mathrm{~d}, J=8.6 \mathrm{~Hz}, 1 \mathrm{H})$, $2.42(\mathrm{dd}, J=15.7,7.0 \mathrm{~Hz}, 1 \mathrm{H}), 2.09(\mathrm{dd}, J=16.2,7.2 \mathrm{~Hz}, 1 \mathrm{H}), 1.86-1.51(\mathrm{~m}, 10 \mathrm{H})$, 1.33-1.05 (m, 10H); ${ }^{13} \mathrm{C}$ NMR (100 MHz, $\left.\mathrm{CDCl}_{3}\right) \delta 155.1,146.8(\mathrm{~d}, J=7.4 \mathrm{~Hz})$, 144.2, 139.3, 133.9 (d, $J=7.1 \mathrm{~Hz}), 133.6(\mathrm{~d}, J=127.8 \mathrm{~Hz}), 133.0(\mathrm{~d}, J=2.8 \mathrm{~Hz})$, $132.8(\mathrm{~d}, \mathrm{~J}=2.8 \mathrm{~Hz}), 132.2(\mathrm{~d}, J=11.0 \mathrm{~Hz}), 132.1(\mathrm{~d}, J=10.8 \mathrm{~Hz}), 130.9(\mathrm{~d}, J=$ $13.0 \mathrm{~Hz}), 129.5(\mathrm{~d}, J=9.2 \mathrm{~Hz}), 128.9(\mathrm{~d}, J=1.8 \mathrm{~Hz}), 128.8(\mathrm{~d}, J=1.6 \mathrm{~Hz}), 127.5$, 126.7, 121.3 (d, $J=4.9 \mathrm{~Hz}), 119.8,118.9,67.2$, 43.6, 43.3, 34.4, 34.3, 34.2, 26.8, 26.7, 26.6, 25.9, 25.8; ${ }^{31} \mathrm{P}$ NMR (162 MHz, $\left.\mathrm{CDCl}_{3}\right) \delta 32.78$; HRMS (ESI) $\mathrm{m} / z$ calcd for $\mathrm{C}_{36} \mathrm{H}_{40} \mathrm{NO}_{4} \mathrm{PS}[\mathrm{M}+\mathrm{H}]^{+}=614.2494$, found $=614.2494$. 
Optical Rotation: $[\alpha]^{25}=32.13\left(c=0.61, \mathrm{CHCl}_{3}\right) .97 \%$ ee (HPLC condition: Chiralpak AD-H column, $n$-Hexane $/ i-\mathrm{PrOH}=60: 40$, flow rate $=1.0 \mathrm{~mL} / \mathrm{min}$, wavelength $=254 \mathrm{~nm}, t_{\mathrm{R}}=33.10$ min for major isomer, $t_{\mathrm{R}}=53.73$ min for minor isomer).

$\mathrm{mV}$

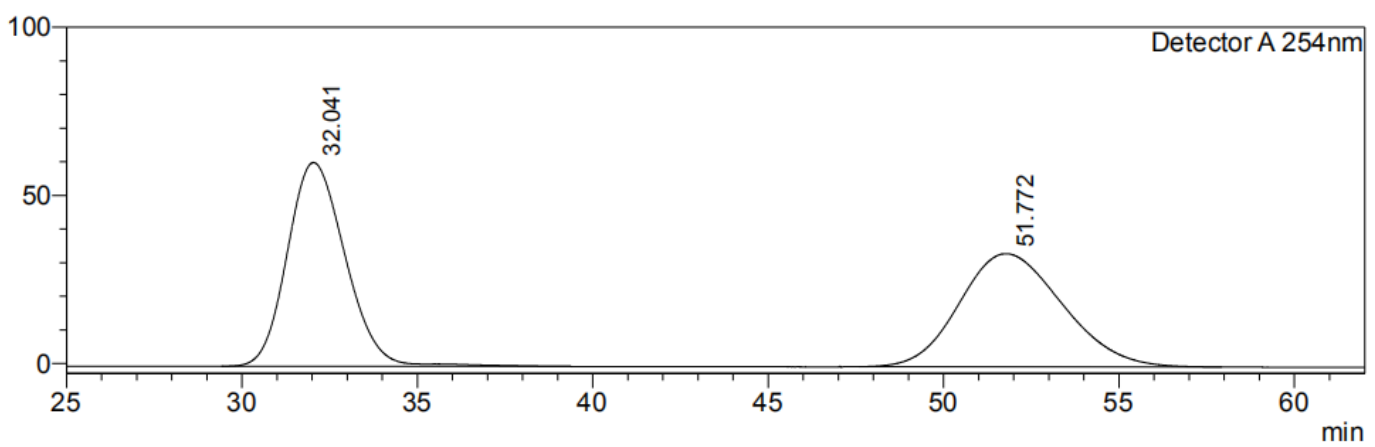

Detector A 254nm

\begin{tabular}{|r|r|r|r|r|r|}
\hline Peak\# & Ret. Time & Height & Height\% & \multicolumn{1}{c|}{ Area } & \multicolumn{1}{c|}{ Area\% } \\
\hline 1 & 32.041 & 60624 & 64.300 & 6979532 & 50.353 \\
\hline 2 & 51.772 & 33659 & 35.700 & 6881609 & 49.647 \\
\hline Total & & 94283 & 100.000 & 13861141 & 100.000 \\
\hline
\end{tabular}

$\mathrm{mV}$

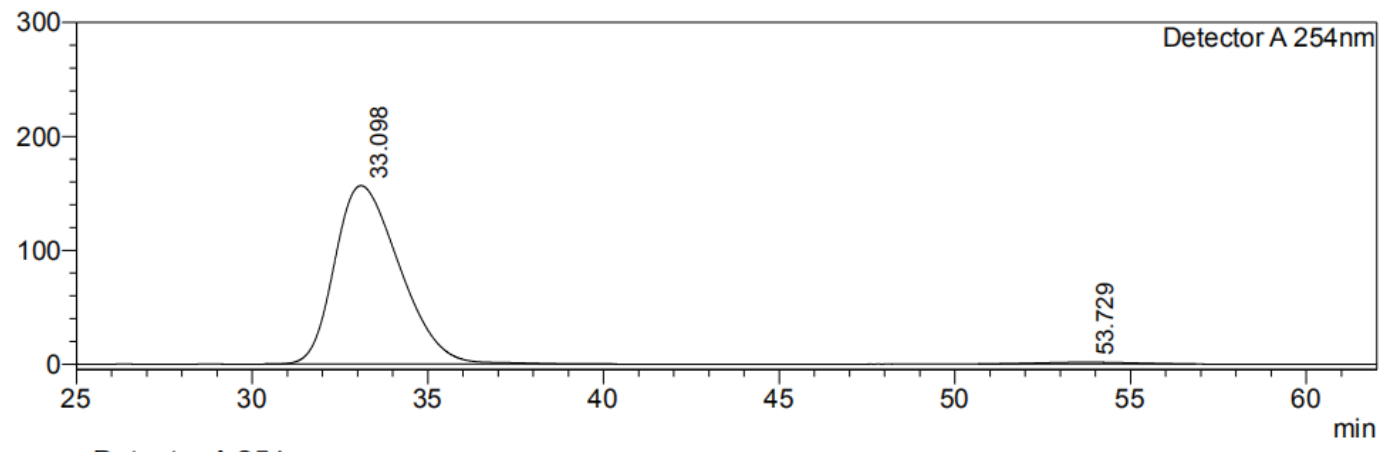

Detector A $254 \mathrm{~nm}$

\begin{tabular}{|r|r|r|r|r|r|}
\hline Peak\# & Ret. Time & Height & \multicolumn{1}{c|}{ Height\% } & \multicolumn{1}{c|}{ Area } & \multicolumn{1}{c|}{ Area\% } \\
\hline 1 & 33.098 & 156765 & 98.996 & 20229415 & 98.529 \\
\hline 2 & 53.729 & 1590 & 1.004 & 302013 & 1.471 \\
\hline Total & & 158355 & 100.000 & 20531427 & 100.000 \\
\hline
\end{tabular}

\section{$\underline{\text { (S)-4-benzyl-2-(5-benzyl-2-hydroxyphenylsulfonimidoyl)phenyl }}$}

\section{diphenylphosphinate (3h)}<smiles>N=S(=O)(c1cc(Br)ccc1O)c1cc(Br)ccc1OP</smiles> 
Light yellow oil; $57 \mathrm{mg}, 90 \%$ yield; ${ }^{1} \mathrm{H}$ NMR (400 MHz, $\left.\mathrm{CDCl}_{3}\right) \delta 11.91(\mathrm{~s}, 1 \mathrm{H})$, $8.06(\mathrm{~d}, J=7.2 \mathrm{~Hz}, 1 \mathrm{H}), 8.02(\mathrm{~d}, J=7.2 \mathrm{~Hz}, 1 \mathrm{H}), 7.89$ (d, $J=7.4 \mathrm{~Hz}, 1 \mathrm{H}), 7.86(\mathrm{~d}, J$ $=7.4 \mathrm{~Hz}, 1 \mathrm{H}), 7.61(\mathrm{~s}, 1 \mathrm{H}), 7.58-7.46(\mathrm{~m}, 5 \mathrm{H}), 7.44-7.38(\mathrm{~m}, 3 \mathrm{H}), 7.27-7.17(\mathrm{~m}, 6 \mathrm{H})$, 7.17-7.11 (m, 2H), 7.07 (d, $J=7.0 \mathrm{~Hz}, 2 \mathrm{H}), 7.00(\mathrm{~d}, J=7.0 \mathrm{~Hz}, 2 \mathrm{H}), 6.73(\mathrm{~d}, J=8.5$ $\mathrm{Hz}, 1 \mathrm{H}), 3.89$ (s, 2H), 3.60 (q, $J=15.3 \mathrm{~Hz}, 2 \mathrm{H}) ;{ }^{13} \mathrm{C} \mathrm{NMR}\left(100 \mathrm{MHz}, \mathrm{CDCl}_{3}\right) \delta$ 155.6, 147.2 (d, $J=7.4 \mathrm{~Hz}), 140.4,139.4,136.9$ (d, $J=110.6 \mathrm{~Hz}), 134.9,134.0$ (d, $J$ $=6.9 \mathrm{~Hz}), 133.0(\mathrm{~d}, J=2.6 \mathrm{~Hz}), 132.9(\mathrm{~d}, J=2.6 \mathrm{~Hz}), 132.2(\mathrm{~d}, J=11.0 \mathrm{~Hz}), 132.0$ $(\mathrm{d}, J=11.0 \mathrm{~Hz}), 130.6(\mathrm{~d}, J=8.8 \mathrm{~Hz}), 129.3,129.2,128.9,128.8,128.6(\mathrm{~d}, J=13.8$ $\mathrm{Hz}), 126.5$ (d, $J=34.7 \mathrm{~Hz}), 121.5(\mathrm{~d}, J=4.8 \mathrm{~Hz}), 119.6,119.4,40.9,40.5 ;{ }^{31} \mathrm{P} \mathrm{NMR}$ $\left(162 \mathrm{MHz}, \mathrm{CDCl}_{3}\right) \delta$ 33.34; HRMS (ESI) $\mathrm{m} / z$ calcd for $\mathrm{C}_{38} \mathrm{H}_{32} \mathrm{NO}_{4} \mathrm{PS}[\mathrm{M}+\mathrm{H}]^{+}=$ 630.1868 , found $=630.1869$.

Optical Rotation: $[\alpha]_{\mathrm{D}}^{25}=59.43\left(c=0.70, \mathrm{CHCl}_{3}\right) .97 \%$ ee (HPLC condition: Chiralpak OD-H column, $n-\mathrm{Hexane} / i-\mathrm{PrOH}=75: 25$, flow rate $=1.0 \mathrm{~mL} / \mathrm{min}$, wavelength $=254 \mathrm{~nm}, t_{\mathrm{R}}=16.41$ min for major isomer, $t_{\mathrm{R}}=31.78$ min for minor isomer).

$\mathrm{mV}$

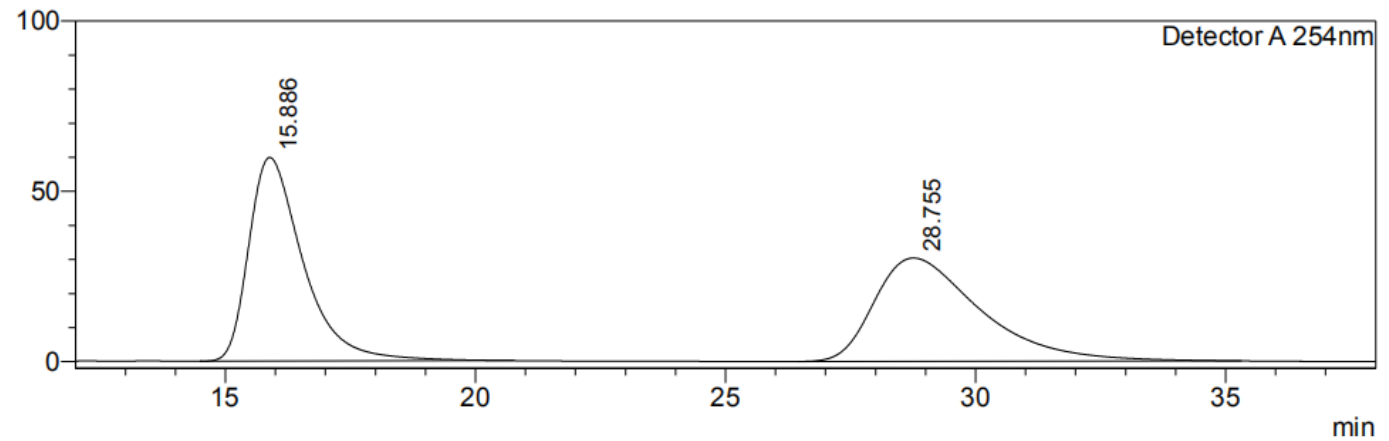

Detector A 254nm
\begin{tabular}{|r|r|r|r|r|r|}
\hline Peak\# & Ret. Time & Height & Height\% & Area & \multicolumn{1}{c|}{ Area\% } \\
\hline 1 & 15.886 & 59796 & 66.328 & 4536635 & 50.291 \\
\hline 2 & 28.755 & 30356 & 33.672 & 4484126 & 49.709 \\
\hline Total & & 90152 & 100.000 & 9020761 & 100.000 \\
\hline
\end{tabular}


$\mathrm{mV}$

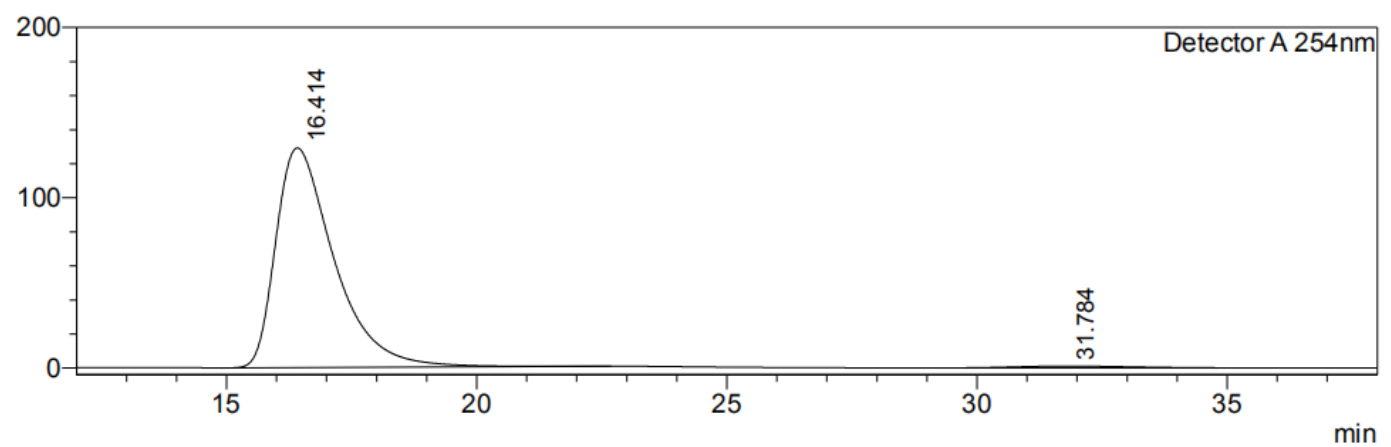

Detector A 254nm

\begin{tabular}{|r|r|r|r|r|r|}
\hline Peak\# & Ret. Time & Height & Height\% & \multicolumn{1}{c|}{ Area } & \multicolumn{1}{c|}{ Area\% } \\
\hline 1 & 16.414 & 128952 & 99.232 & 10467740 & 98.564 \\
\hline 2 & 31.784 & 999 & 0.768 & 152538 & 1.436 \\
\hline Total & & 129951 & 100.000 & 10620277 & 100.000 \\
\hline
\end{tabular}

\section{(S)-3-(4-hydroxy-[1,1'-biphenyl]-3-sulfonimidoyl)-[1,1'-biphenyl]-4-yl}

\section{diphenylphosphinate (3i)}<smiles>NS(=O)(=O)c1cc(-c2ccccc2)ccc1OCCOCCO</smiles>

Light yellow solid; $51 \mathrm{mg}, 84 \%$ yield; m.p. $=209-211{ }^{\circ} \mathrm{C} ;{ }^{1} \mathrm{H}$ NMR $(400 \mathrm{MHz}$, $\left.\mathrm{CDCl}_{3}\right) \delta 12.25(\mathrm{~s}, 1 \mathrm{H}), 8.20(\mathrm{~d}, J=1.2 \mathrm{~Hz}, 1 \mathrm{H}), 8.08-8.03(\mathrm{~m}, 1 \mathrm{H}), 8.01(\mathrm{~d}, J=7.5$ $\mathrm{Hz}, 1 \mathrm{H}), 7.88-7.78(\mathrm{~m}, 3 \mathrm{H}), 7.76(\mathrm{~d}, J=8.6 \mathrm{~Hz}, 1 \mathrm{H}), 7.64(\mathrm{dd}, J=8.6,1.9 \mathrm{~Hz}, 1 \mathrm{H})$, 7.61-7.44 (m, 7H), 7.44-7.32 (m, 5H), 7.32-7.26 (m, 5H), 6.89 (d, J = 8.6 Hz, 1H); ${ }^{13} \mathrm{C}$ NMR $\left(100 \mathrm{MHz}, \mathrm{CDCl}_{3}\right) \delta 156.7,148.4(\mathrm{~d}, J=7.2 \mathrm{~Hz}), 139.0,138.4,137.4$, 134.2, 133.8 (d, $J=109.0 \mathrm{~Hz}), 133.1$ (d, $J=2.4 \mathrm{~Hz}), 133.0(\mathrm{~d}, J=2.4 \mathrm{~Hz}), 132.8$, $132.2(\mathrm{~d}, J=4.3 \mathrm{~Hz}), 132.0(\mathrm{~d}, J=4.5 \mathrm{~Hz}), 130.4,130.2,129.1,129.0,128.9,128.8$ $(\mathrm{d}, J=3.6 \mathrm{~Hz}), 128.0(\mathrm{~d}, J=20.0 \mathrm{~Hz}), 127.2,126.9,126.8,126.5,122.0(\mathrm{~d}, J=4.8$ $\mathrm{Hz}), 120.7,119.8 ;{ }^{31} \mathrm{P}$ NMR $\left(162 \mathrm{MHz}, \mathrm{CDCl}_{3}\right) \delta 33.43$; HRMS (ESI) $\mathrm{m} / z$ calcd for $\mathrm{C}_{36} \mathrm{H}_{28} \mathrm{NO}_{4} \mathrm{PS}[\mathrm{M}+\mathrm{H}]^{+}=602.1555$, found $=602.1544$.

Optical Rotation: $[\alpha]_{\mathrm{D}}^{25}=40.34\left(c=0.59, \mathrm{CHCl}_{3}\right) .97 \%$ ee (HPLC condition: Chiralpak OD-H column, $n-\mathrm{Hexane} / i-\mathrm{PrOH}=75: 25$, flow rate $=1.0 \mathrm{~mL} / \mathrm{min}$, wavelength $=254 \mathrm{~nm}, t_{\mathrm{R}}=11.02$ min for major isomer, $t_{\mathrm{R}}=31.70$ min for minor isomer). 
$\mathrm{mV}$

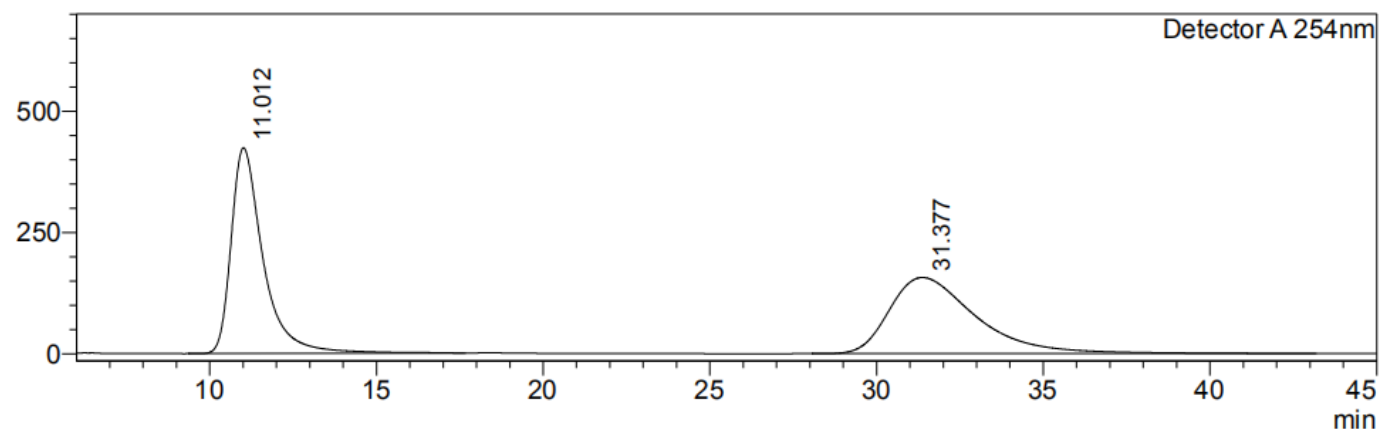

Detector A 254nm

\begin{tabular}{|r|r|r|r|r|r|}
\hline Peak\# & Ret. Time & Height & Height $\%$ & \multicolumn{1}{c|}{ Area } & \multicolumn{1}{c|}{ Area\% } \\
\hline 1 & 11.012 & 423878 & 72.997 & 28396359 & 50.030 \\
\hline 2 & 31.377 & 156803 & 27.003 & 28362706 & 49.970 \\
\hline Total & & 580682 & 100.000 & 56759065 & 100.000 \\
\hline
\end{tabular}

$\mathrm{mV}$

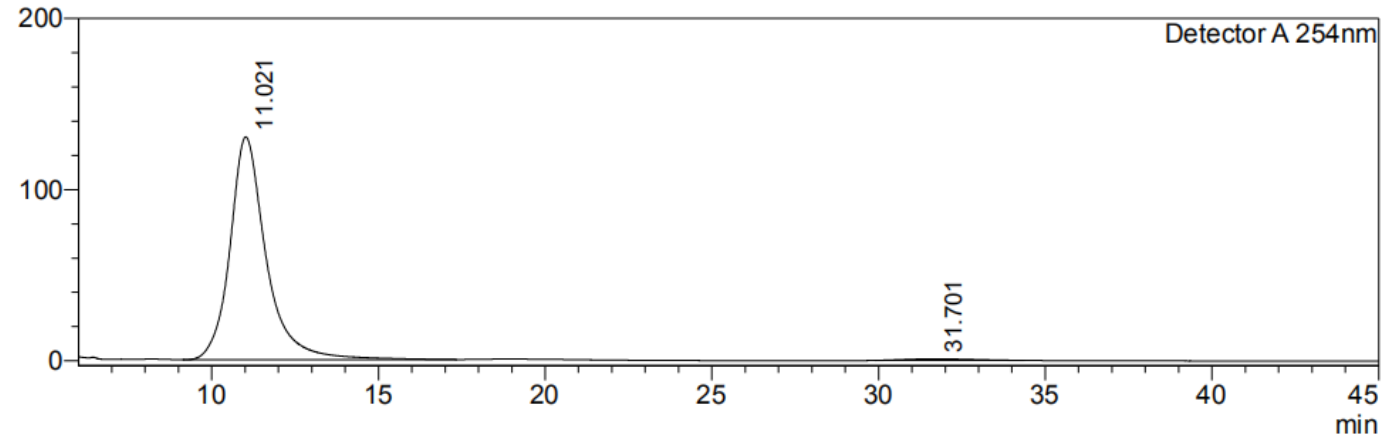

\begin{tabular}{|c|c|c|c|c|c|}
\hline Peak\# & Ret. Time & Height & Height $\%$ & Area & Area $\%$ \\
\hline 1 & 11.021 & 130245 & 99.311 & 9867903 & 98.701 \\
\hline 2 & 31.701 & 903 & 0.689 & 129847 & 1.299 \\
\hline Total & & 131148 & 100.000 & 9997750 & 100.000 \\
\hline
\end{tabular}

(S)-4'-ethyl-3-(4'-ethyl-4-hydroxy-[1,1'-biphenyl]-3-sulfonimidoyl)-[1,1'-bipheny

\section{1]-4-yl diphenylphosphinate (3j)}<smiles>CCc1ccc(-c2ccc(O)c(S(N)(=O)(Oc3ccccc3)c3cc(-c4ccc(CC)cc4)ccc3OCCCCCOc3ccccc3)c2)cc1</smiles>

White powder; $54 \mathrm{mg}, 83 \%$ yield; m.p. $=105-107{ }^{\circ} \mathrm{C} ;{ }^{1} \mathrm{H}$ NMR $\left(400 \mathrm{MHz}, \mathrm{CDCl}_{3}\right) \delta$ $12.33(\mathrm{~s}, 1 \mathrm{H}), 8.14(\mathrm{~d}, J=2.1 \mathrm{~Hz}, 1 \mathrm{H}), 8.04-8.00(\mathrm{~m}, 1 \mathrm{H}), 8.00-7.96(\mathrm{~m}, 1 \mathrm{H})$, 7.86-7.76 (m, 3H), $7.73(\mathrm{~d}, J=8.6 \mathrm{~Hz}, 1 \mathrm{H}), 7.66-7.60(\mathrm{~m}, 1 \mathrm{H}), 7.59-7.53(\mathrm{~m}, 2 \mathrm{H})$, 7.54-7.45 (m, 3H), 7.43-7.36 (m, 4H), 7.26-7.21 (m, 4H), $7.14(\mathrm{~d}, J=8.2 \mathrm{~Hz}, 2 \mathrm{H})$, 
$6.87(\mathrm{~d}, J=8.6 \mathrm{~Hz}, 1 \mathrm{H}), 2.71-2.61(\mathrm{~m}, 4 \mathrm{H}), 1.30-1.21(\mathrm{~m}, 6 \mathrm{H}) ;{ }^{13} \mathrm{C}$ NMR $(100 \mathrm{MHz}$, $\left.\mathrm{CDCl}_{3}\right) \delta 156.6,148.3,148.2,148.1(\mathrm{~d}, J=7.3 \mathrm{~Hz}), 137.4,136.2(\mathrm{~d}, J=71.4 \mathrm{~Hz})$, $134.3(\mathrm{~d}, J=7.1 \mathrm{~Hz}), 133.6(\mathrm{~d}, J=121.6 \mathrm{~Hz}), 133.1(\mathrm{~d}, J=2.8 \mathrm{~Hz}), 132.9(\mathrm{~d}, J=6.9$ $\mathrm{Hz}), 132.2(\mathrm{~d}, J=3.3 \mathrm{~Hz}), 132.1$ (d, $J=3.5 \mathrm{~Hz}), 130.4(\mathrm{~d}, J=32.4 \mathrm{~Hz}), 129.2,129.0$ $(\mathrm{d}, J=5.6 \mathrm{~Hz}), 128.8(\mathrm{~d}, J=5.8 \mathrm{~Hz}), 128.6(\mathrm{~d}, J=16.7 \mathrm{~Hz}), 128.5,127.7,126.7(\mathrm{~d}, J$ = 42.1 Hz), 126.8, 126.6, $122.0(\mathrm{~d}, J=4.9 \mathrm{~Hz}), 120.6,119.8,28.7,28.6,15.7,15.6$; ${ }^{31} \mathrm{P}$ NMR (162 MHz, $\left.\mathrm{CDCl}_{3}\right) \delta$ 34.33; HRMS (ESI) $\mathrm{m} / z$ calcd for $\mathrm{C}_{40} \mathrm{H}_{36} \mathrm{NO}_{4} \mathrm{PS}$ $[\mathrm{M}+\mathrm{H}]^{+}=658.2181$, found $=658.2178$.

Optical Rotation: $[\alpha]_{\mathrm{D}}^{25}=-51.67\left(c=0.60, \mathrm{CHCl}_{3}\right) .95 \%$ ee (HPLC condition: Chiralpak AD-H column, $n-\mathrm{Hexane} / i-\mathrm{PrOH}=70: 30$, flow rate $=1.0 \mathrm{~mL} / \mathrm{min}$, wavelength $=254 \mathrm{~nm}, t_{\mathrm{R}}=49.19 \min$ for minor isomer, $t_{\mathrm{R}}=55.15 \mathrm{~min}$ for major isomer).

$\mathrm{mV}$

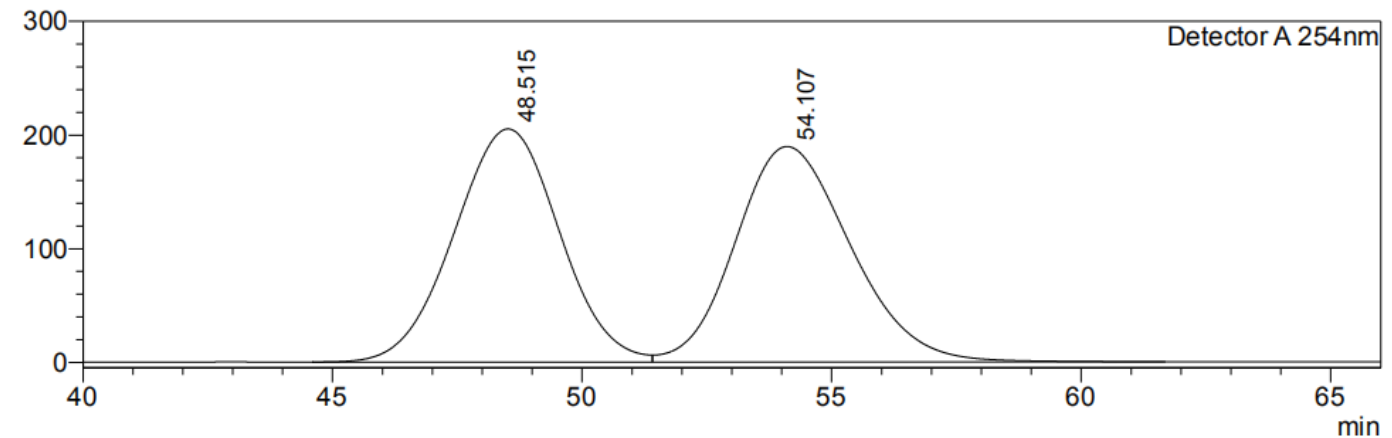

Detector A 254nm

\begin{tabular}{|r|r|r|r|r|r|}
\hline Peak\# & Ret. Time & Height & Height $\%$ & \multicolumn{1}{c|}{ Area } & \multicolumn{1}{c|}{ Area\% } \\
\hline 1 & 48.515 & 205077 & 51.966 & 30150456 & 49.756 \\
\hline 2 & 54.107 & 189562 & 48.034 & 30445849 & 50.244 \\
\hline Total & & 394638 & 100.000 & 60596305 & 100.000 \\
\hline
\end{tabular}

$\mathrm{mV}$

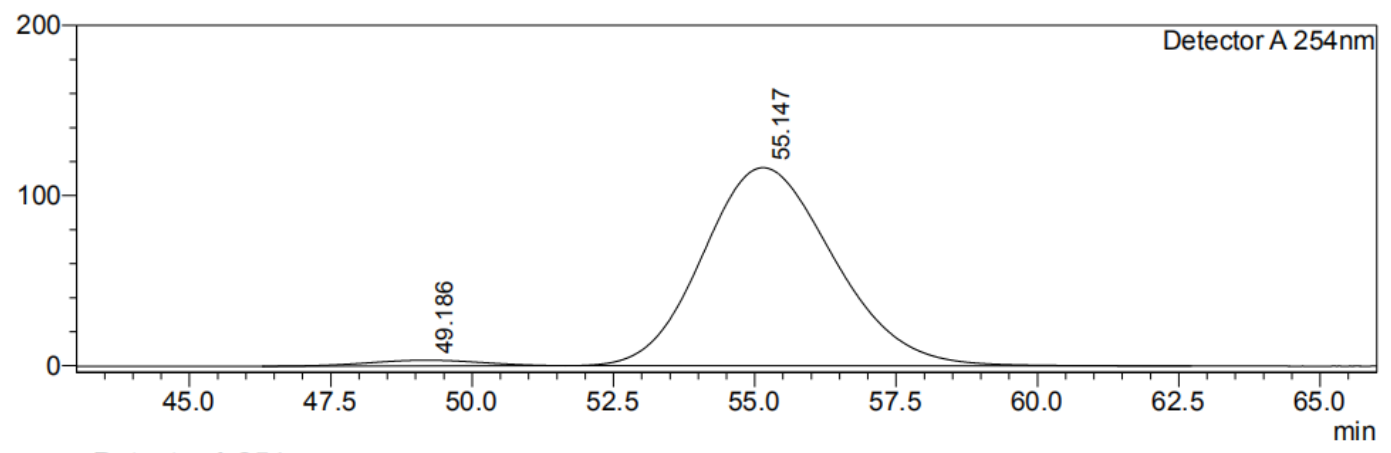

Detector A 254nm

\begin{tabular}{|r|r|r|r|r|r|}
\hline Peak\# & Ret. Time & \multicolumn{1}{c|}{ Height } & \multicolumn{1}{c|}{ Height\% } & \multicolumn{1}{c|}{ Area } & \multicolumn{1}{c|}{ Area\% } \\
\hline 1 & 49.186 & 3369 & 2.812 & 466015 & 2.418 \\
\hline 2 & 55.147 & 116447 & 97.188 & 18809304 & 97.582 \\
\hline Total & & 119817 & 100.000 & 19275319 & 100.000 \\
\hline
\end{tabular}




\section{-5-yl diphenylphosphinate (3k)}

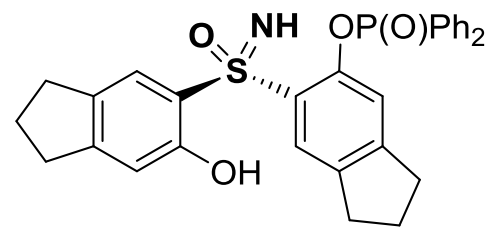

Yellow solid; $45 \mathrm{mg}, 86 \%$ yield; m.p. $=112-114{ }^{\circ} \mathrm{C} ;{ }^{1} \mathrm{H}$ NMR $\left(400 \mathrm{MHz}, \mathrm{CDCl}_{3}\right) \delta$ $11.92(\mathrm{~s}, 1 \mathrm{H}), 8.05-7.97(\mathrm{~m}, 2 \mathrm{H}), 7.92-7.83(\mathrm{~m}, 2 \mathrm{H}), 7.64(\mathrm{~s}, 1 \mathrm{H}), 7.58-7.53(\mathrm{~m}, 2 \mathrm{H})$, 7.52-7.45 (m, 3H), 7.44-7.38 (m, 2H), $7.28(\mathrm{~s}, 1 \mathrm{H}), 6.64(\mathrm{~s}, 1 \mathrm{H}), 2.82-2.74(\mathrm{~m}, 6 \mathrm{H})$, 2.61-2.45 (m, 2H), 2.03-1,93 (m, 4H); $\left.{ }^{13} \mathrm{C} \mathrm{NMR} \mathrm{(100} \mathrm{MHz,} \mathrm{CDCl}_{3}\right) \delta 155.9,152.7(\mathrm{~d}$, $J=100.8 \mathrm{~Hz}), 147.9(\mathrm{~d}, J=7.4 \mathrm{~Hz}), 139.9,135.0,132.9(\mathrm{~d}, J=2.8 \mathrm{~Hz}), 132.8(\mathrm{~d}, J=$ $2.8 \mathrm{~Hz}), 132.2(\mathrm{~d}, J=10.9 \mathrm{~Hz}), 132.0(\mathrm{~d}, J=7.2 \mathrm{~Hz}), 130.7$ (d, $J=23.8 \mathrm{~Hz}), 129.3$ (d, $J=21.9 \mathrm{~Hz}), 128.8(\mathrm{~d}, J=1.3 \mathrm{~Hz}), 128.7(\mathrm{~d}, J=1.4 \mathrm{~Hz}), 124.2(\mathrm{~d}, J=53.5 \mathrm{~Hz})$, 118.1, $117.2(\mathrm{~d}, J=4.9 \mathrm{~Hz}), 114.4,33.4,33.3,32.1,31.6,25.6,25.4 ;{ }^{31} \mathrm{P}$ NMR $(162$ $\left.\mathrm{MHz}, \mathrm{CDCl}_{3}\right) \delta 33.14$; HRMS (ESI) $m / z$ calcd for $\mathrm{C}_{30} \mathrm{H}_{28} \mathrm{NO}_{4} \mathrm{PS}[\mathrm{M}+\mathrm{H}]^{+}=530.1555$, found $=530.1555$.

Optical Rotation: $[\alpha]_{\mathrm{D}}^{25}=21.76\left(c=0.51, \mathrm{CHCl}_{3}\right) .90 \%$ ee (HPLC condition: Chiralpak OD-H column, $n$-Hexane $/ i-\mathrm{PrOH}=80: 20$, flow rate $=1.0 \mathrm{~mL} / \mathrm{min}$, wavelength $=254 \mathrm{~nm}, t_{\mathrm{R}}=13.00$ min for major isomer, $t_{\mathrm{R}}=20.02$ min for minor isomer).

$\mathrm{mV}$

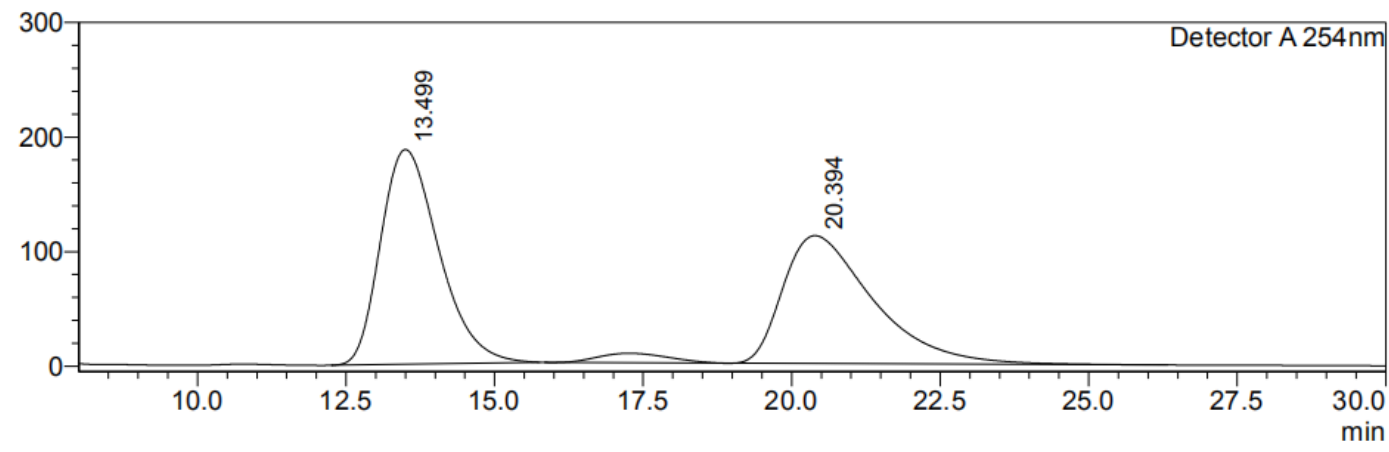

Detector A 254nm
\begin{tabular}{|r|r|r|r|r|r|}
\hline Peak\# & Ret. Time & Height & Height\% & Area & \multicolumn{1}{c|}{ Area $\%$} \\
\hline 1 & 13.499 & 187445 & 62.669 & 12808418 & 50.609 \\
\hline 2 & 20.394 & 111657 & 37.331 & 12500260 & 49.391 \\
\hline Total & & 299102 & 100.000 & 25308678 & 100.000 \\
\hline
\end{tabular}


$\mathrm{mV}$

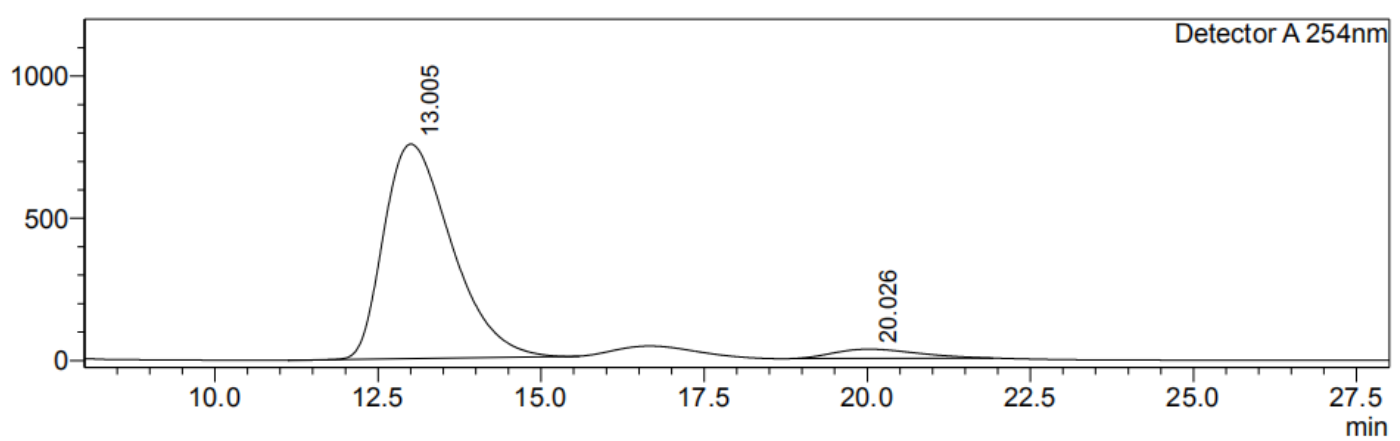

\begin{tabular}{|c|c|c|c|c|c|}
\hline Peak\# & Ret. Time & Height & Height $\%$ & Area & Area\% \\
\hline 1 & 13.005 & 754364 & 95.892 & 54537370 & 95.010 \\
\hline 2 & 20.026 & 32316 & 4.108 & 2864411 & 4.990 \\
\hline Total & & 786681 & 100.000 & 57401781 & 100.000 \\
\hline
\end{tabular}

\section{(S)-2-(2-hydroxy-4,5-dimethylphenylsulfonimidoyl)-4,5-dimethylphenyl}

\section{diphenylphosphinate (31)}<smiles>COc1cc(C)c(OCCCCO)cc1S(=O)(=O)c1cc(C)c(C)cc1O</smiles>

White solid; $43 \mathrm{mg}, 86 \%$ yield; m.p. $=187-189{ }^{\circ} \mathrm{C} ;{ }^{1} \mathrm{H}$ NMR $\left(400 \mathrm{MHz}, \mathrm{CDCl}_{3}\right) \delta$ $11.83(\mathrm{~s}, 1 \mathrm{H}), 8.07-8.03(\mathrm{~m}, 1 \mathrm{H}), 8.03-8.00$ (m, 1H), 7.89-7.85 (m, 1H), 7.85-7.81 (m, 1H), 7.59 (s, 1H), 7.58-7.46 (m, 5H), $7.41(\mathrm{td}, J=7.6,3.8 \mathrm{~Hz}, 2 \mathrm{H}), 7.22(\mathrm{~s}, 1 \mathrm{H}), 6.58$ (s, 1H), 3.83 (s, 1H), 2.17 (s, 3H), 2.16 (s, 3H), 2.12 (s, 3H), 1.86 (s, 3H); ${ }^{13} \mathrm{C}$ NMR $\left(100 \mathrm{MHz}, \mathrm{CDCl}_{3}\right) \delta 154.9,146.9(\mathrm{~d}, J=7.5 \mathrm{~Hz}), 145.5,144.7,132.9(\mathrm{~d}, J=2.9 \mathrm{~Hz})$, $132.8(\mathrm{~d}, J=2.9 \mathrm{~Hz}), 132.7,132.2(\mathrm{~d}, J=11.0 \mathrm{~Hz}), 131.2(\mathrm{~d}, J=7.2 \mathrm{~Hz}), 130.8(\mathrm{~d}, J$ $=26.7 \mathrm{~Hz}), 129.5(\mathrm{~d}, J=23.6 \mathrm{~Hz}), 129.4(\mathrm{~d}, J=103.0 \mathrm{~Hz}), 128.8(\mathrm{~d}, J=1.2 \mathrm{~Hz})$, 128.7 (d, $J=1.1 \mathrm{~Hz}), 127.9,122.3$ (d, $J=4.8 \mathrm{~Hz}), 119.6,117.7,20.2,20.1,19.2$, 18.5; ${ }^{31} \mathrm{P}$ NMR (162 MHz, $\left.\mathrm{CDCl}_{3}\right) \delta 32.96$; HRMS (ESI) $\mathrm{m} / z$ calcd for $\mathrm{C}_{28} \mathrm{H}_{28} \mathrm{NO}_{4} \mathrm{PS}$ $[\mathrm{M}+\mathrm{H}]^{+}=506.1555$, found $=506.1551$.

Optical Rotation: $[\alpha]^{25}=37.50\left(c=0.60, \mathrm{CHCl}_{3}\right) .82 \%$ ee (HPLC condition: Chiralpak OD-H column, $n$-Hexane $/ i-\mathrm{PrOH}=80: 20$, flow rate $=1.0 \mathrm{~mL} / \mathrm{min}$, wavelength $=254 \mathrm{~nm}, t_{\mathrm{R}}=9.68 \mathrm{~min}$ for major isomer, $t_{\mathrm{R}}=18.18 \mathrm{~min}$ for minor isomer). 
$\mathrm{mV}$

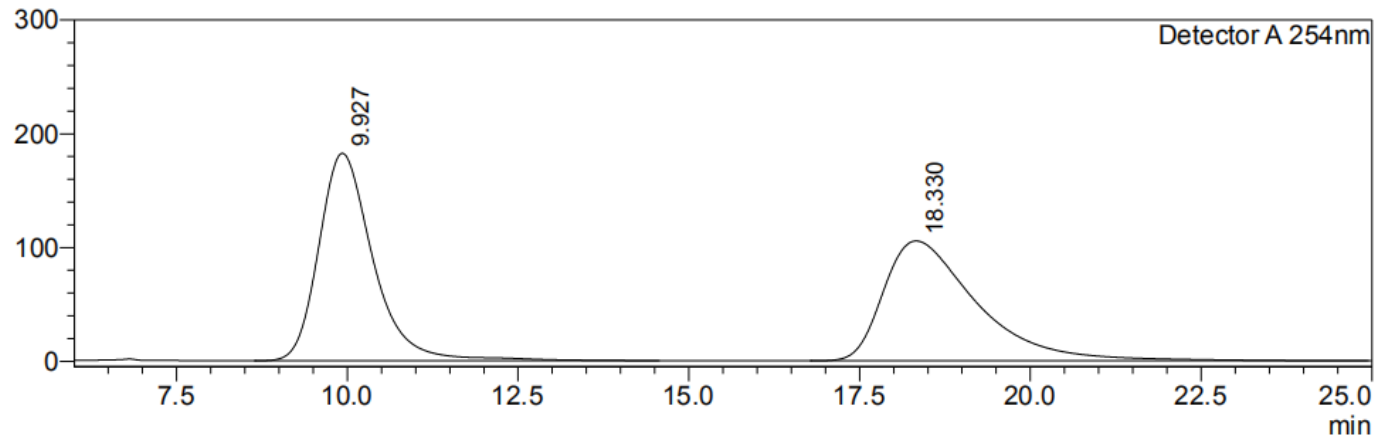

Detector A 254nm
\begin{tabular}{|r|r|r|r|r|r|}
\hline Peak\# & Ret. Time & Height & Height\% & Area & Area $\%$ \\
\hline 1 & 9.927 & 182374 & 63.381 & 10102025 & 50.181 \\
\hline 2 & 18.330 & 105369 & 36.619 & 10029085 & 49.819 \\
\hline Total & & 287742 & 100.000 & 20131110 & 100.000 \\
\hline
\end{tabular}

$\mathrm{mV}$

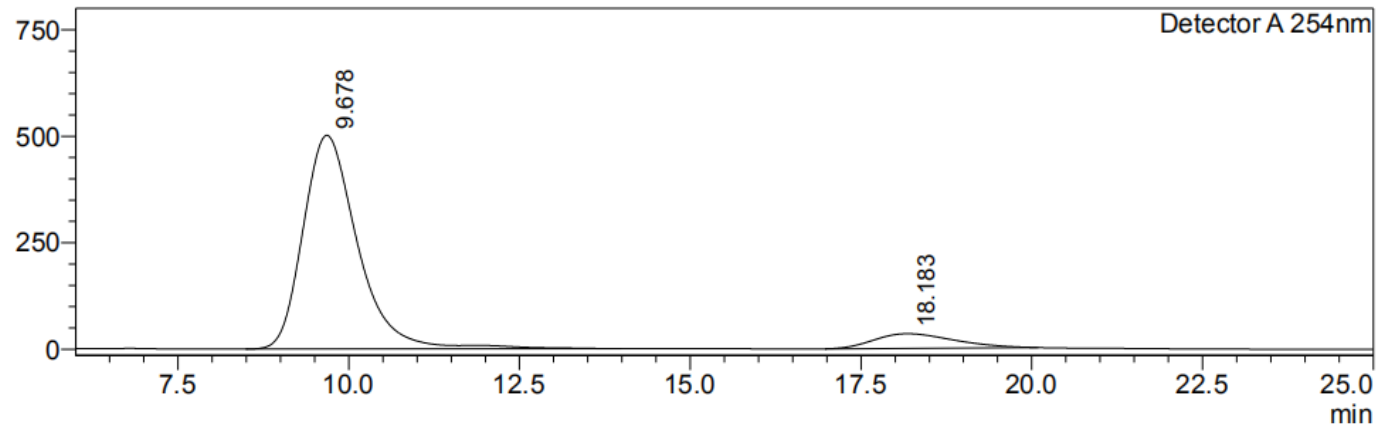

Detector A 254nm

\begin{tabular}{|r|r|r|r|r|r|}
\hline Peak\# & Ret. Time & Height & Height\% & \multicolumn{1}{c|}{ Area } & \multicolumn{1}{c|}{ Area\% } \\
\hline 1 & 9.678 & 501806 & 93.590 & 28025169 & 90.909 \\
\hline 2 & 18.183 & 34367 & 6.410 & 2802470 & 9.091 \\
\hline Total & & 536173 & 100.000 & 30827639 & 100.000 \\
\hline
\end{tabular}

(S)-2-(2-hydroxy-5-isopropyl-4-methylphenylsulfonimidoyl)-4-isopropyl-5-meth ylphenyl diphenylphosphinate $(3 \mathrm{~m})$<smiles>CCCOc1cc(C)c(OP)cc1S(=O)(=O)c1cc(C(C)C)c(C)cc1O</smiles>

Light yellow solid; $45 \mathrm{mg}, 80 \%$ yield; m.p. $=173-175{ }^{\circ} \mathrm{C} ;{ }^{1} \mathrm{H}$ NMR $(400 \mathrm{MHz}$, $\left.\mathrm{CDCl}_{3}\right) \delta 11.22(\mathrm{~s}, 1 \mathrm{H}), 8.11(\mathrm{dd}, J=8.2,1.4 \mathrm{~Hz}, 1 \mathrm{H}), 8.07(\mathrm{dd}, J=8.2,1.4 \mathrm{~Hz}, 1 \mathrm{H})$, 7.92-7.89 (m, 1H), 7.89-7.84 (m, 1H), 7.59-7.53 (m, 1H), 7.50 (dt, $J=6.9,3.9 \mathrm{~Hz}$, 4H), 7.44-7.36 (m, 4H), $6.62(\mathrm{~s}, 1 \mathrm{H}), 3.90(\mathrm{~s}, 1 \mathrm{H}), 3.02-2.94(\mathrm{~m}, 1 \mathrm{H})$, 2.94-2.85 (m, 1H), $2.26(\mathrm{~s}, 3 \mathrm{H}), 2.17(\mathrm{~s}, 3 \mathrm{H}), 1.09(\mathrm{dd}, J=9.2,6.8 \mathrm{~Hz}, 6 \mathrm{H}), 0.98(\mathrm{~d}, J=6.8 \mathrm{~Hz}$, 3H), $0.90(\mathrm{~d}, J=6.8 \mathrm{~Hz}, 3 \mathrm{H}) ;{ }^{13} \mathrm{C} \mathrm{NMR}\left(100 \mathrm{MHz}, \mathrm{CDCl}_{3}\right) \delta 154.7,146.2(\mathrm{~d}, J=7.6$ 
Hz), $143.7(\mathrm{~d}, J=117.6 \mathrm{~Hz}), 142.8,138.7,132.9(\mathrm{~d}, J=2.9 \mathrm{~Hz}), 132.7(\mathrm{~d}, J=2.9$ Hz), 132.4, 132.3 (d, $J=10.9 \mathrm{~Hz}), 132.1$ (d, $J=10.8 \mathrm{~Hz}), 131.0$ (d, $J=6.4 \mathrm{~Hz})$, $129.7(\mathrm{~d}, J=4.1 \mathrm{~Hz}), 128.9(\mathrm{~d}, J=7.7 \mathrm{~Hz}), 128.7(\mathrm{~d}, J=7.6 \mathrm{~Hz}), 125.5,125.0,122.9$ $(\mathrm{d}, J=4.7 \mathrm{~Hz}), 120.1,117.7,29.0,28.6,23.2,23.1,23.00,22.9,19.6,19.4 ;{ }^{31} \mathrm{P}$ NMR $\left(162 \mathrm{MHz}, \mathrm{CDCl}_{3}\right) \delta 32.80$; HRMS (ESI) $\mathrm{m} / z$ calcd for $\mathrm{C}_{32} \mathrm{H}_{36} \mathrm{NO}_{4} \mathrm{PS}[\mathrm{M}+\mathrm{H}]^{+}=$ 562.2181 , found $=562.2181$.

Optical Rotation: $[\alpha]_{\mathrm{D}}^{25}=22.20\left(c=1.00, \mathrm{CHCl}_{3}\right) .92 \%$ ee (HPLC condition: Chiralpak OD-H column, $n-\mathrm{Hexane} / i-\mathrm{PrOH}=90: 10$, flow rate $=0.8 \mathrm{~mL} / \mathrm{min}$, wavelength $=254 \mathrm{~nm}, t_{\mathrm{R}}=10.97$ min for major isomer, $t_{\mathrm{R}}=16.86$ min for minor isomer).

$\mathrm{mV}$

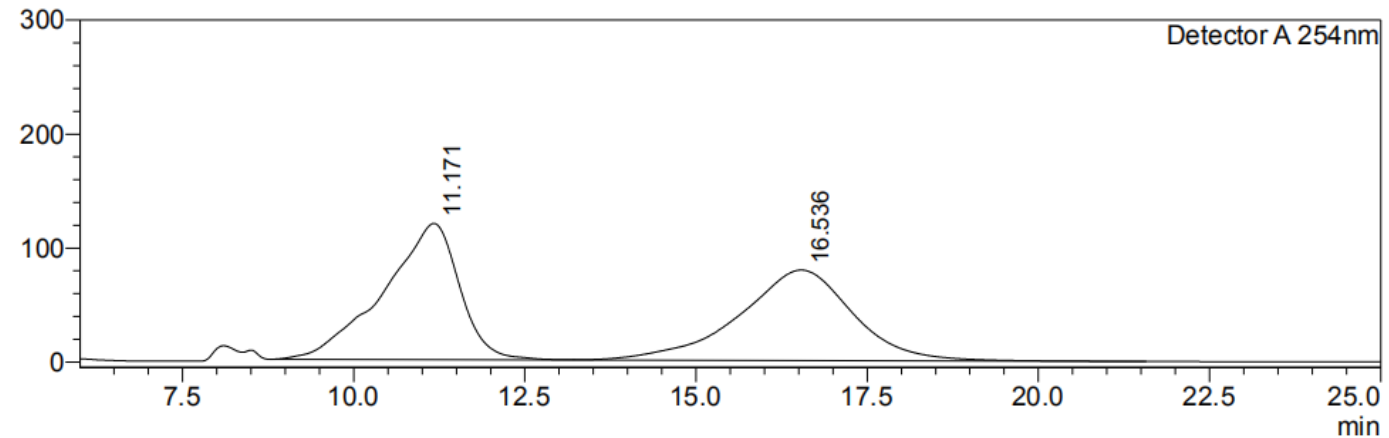

Detector A 254nm

\begin{tabular}{|r|r|r|r|r|r|}
\hline Peak\# & Ret. Time & \multicolumn{1}{c|}{ Height } & Height\% & \multicolumn{1}{c|}{ Area } & \multicolumn{1}{c|}{ Area\% } \\
\hline 1 & 11.171 & 119568 & 60.071 & 9238053 & 50.055 \\
\hline 2 & 16.536 & 79475 & 39.929 & 9217617 & 49.945 \\
\hline Total & & 199042 & 100.000 & 18455671 & 100.000 \\
\hline
\end{tabular}

$\mathrm{mV}$

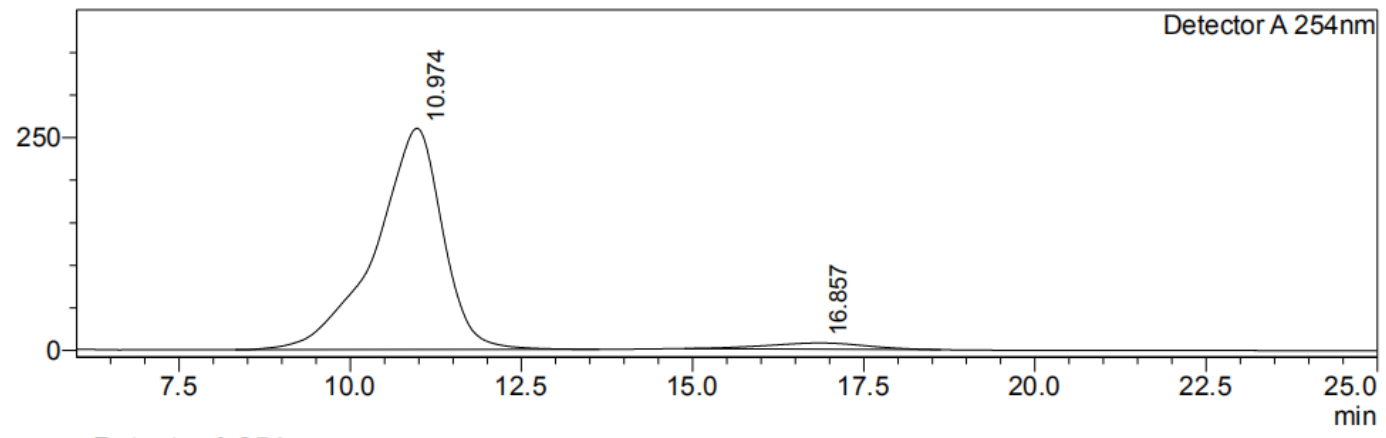

\begin{tabular}{|c|c|c|c|c|c|}
\hline Peak\# & Ret. Time & Height & Height $\%$ & Area & Area $\%$ \\
\hline 1 & 10.974 & 259869 & 97.278 & 17734411 & 96.024 \\
\hline 2 & 16.857 & 7272 & 2.722 & 734299 & 3.976 \\
\hline Total & & 267140 & 100.000 & 18468710 & 100.000 \\
\hline
\end{tabular}

$\underline{(S)-2 '-\left(2 '-h y d r o x y-\left[1,1^{\prime} \text {-biphenyl]-2-sulfonimidoyl)-[1,1'-biphenyl]-2-yl }\right.\right.}$ 


\section{diphenylphosphinate (3n)}

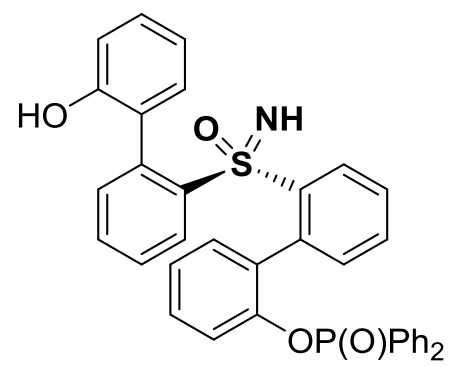

Yellow solid; $53 \mathrm{mg}, 88 \%$ yield; m.p. $=91-93{ }^{\circ} \mathrm{C} ;{ }^{1} \mathrm{H}$ NMR $\left(400 \mathrm{MHz}, \mathrm{CDCl}_{3}\right) \delta$ 7.85-7.68 (m, 2H), 7.63-7.49 (m, 3H), 7.46-7.39 (m, 3H), 7.35-7.26 (m, 4H), 7.23-7.06 (m, 7H), $7.03(\mathrm{~d}, J=7.6 \mathrm{~Hz}, 2 \mathrm{H}), 6.98-6.82(\mathrm{~m}, 1 \mathrm{H}), 6.65-6.44(\mathrm{~m}, 2 \mathrm{H})$, 6.29-6.18 (m, 1H), 6.02-5.77 (m, 1H); $\left.{ }^{13} \mathrm{C} \mathrm{NMR} \mathrm{(100} \mathrm{MHz,} \mathrm{CDCl}_{3}\right) \delta$ 154.3, 149.0, 141.7, 140.1 (d, $J=119.1 \mathrm{~Hz}), 138.1,137.3,137.2,136.3,133.2(\mathrm{~d}, J=17.8 \mathrm{~Hz})$, $132.2(\mathrm{~d}, J=10.3 \mathrm{~Hz}), 131.1(\mathrm{~d}, J=10.8 \mathrm{~Hz}), 129.8(\mathrm{~d}, J=3.1 \mathrm{~Hz}), 129.7,129.3$, $128.7(\mathrm{~d}, J=13.6 \mathrm{~Hz}), 128.4,128.2$ (d, $J=13.7 \mathrm{~Hz}), 127.9$ (d, $J=9.8 \mathrm{~Hz}), 127.8$, 127.4, 123.7, 122.8, 121.2, 121.0, 120.4, 119.8; ${ }^{31} \mathrm{P}$ NMR (162 MHz, $\left.\mathrm{CDCl}_{3}\right) \delta 29.63$; HRMS (ESI) $m / z$ calcd for $\mathrm{C}_{36} \mathrm{H}_{28} \mathrm{NO}_{4} \mathrm{PS}[\mathrm{M}+\mathrm{H}]^{+}=602.1555$, found $=602.1543$.

Optical Rotation: $[\alpha]_{\mathrm{D}}^{25}=4.48\left(c=0.29, \mathrm{CHCl}_{3}\right) .17 \%$ ee (HPLC condition: Chiralpak AD-H column, $n$-Hexane $/ i-\mathrm{PrOH}=60: 40$, flow rate $=0.8 \mathrm{~mL} / \mathrm{min}$, wavelength $=254 \mathrm{~nm}, t_{\mathrm{R}}=21.59 \min$ for major isomer, $t_{\mathrm{R}}=38.43 \mathrm{~min}$ for minor isomer).

$\mathrm{mV}$

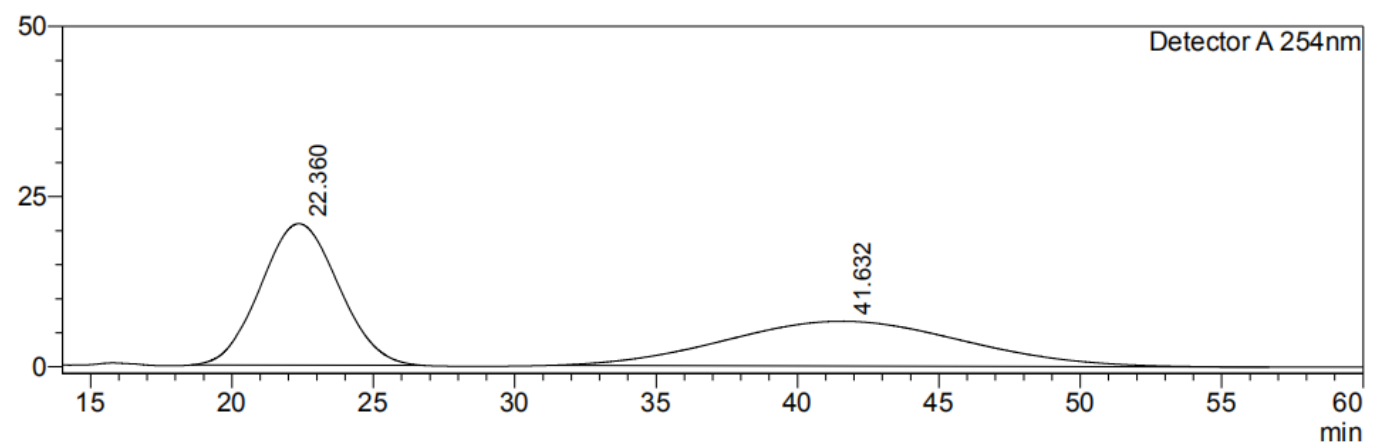

Detector A 254nm

\begin{tabular}{|r|r|r|r|r|r|}
\hline Peak\# & Ret. Time & Height & Height $\%$ & \multicolumn{1}{l|}{ Area } & \multicolumn{1}{c|}{ Area\% } \\
\hline 1 & 22.360 & 20781 & 75.928 & 3988116 & 50.703 \\
\hline 2 & 41.632 & 6588 & 24.072 & 3877501 & 49.297 \\
\hline Total & & 27369 & 100.000 & 7865617 & 100.000 \\
\hline
\end{tabular}


$\mathrm{mV}$

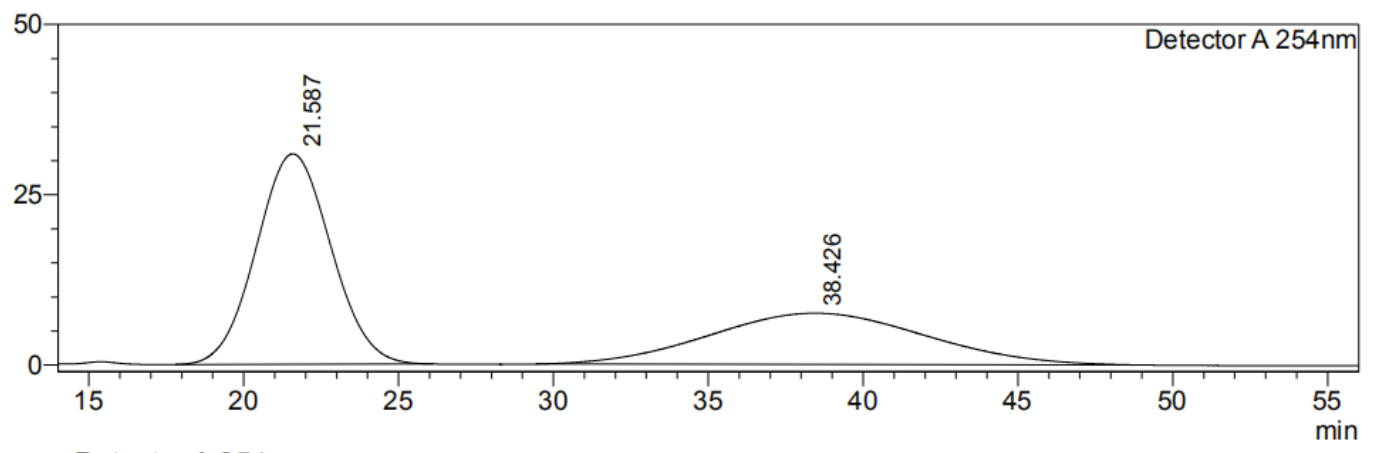

Detector A 254nm

\begin{tabular}{|r|r|r|r|r|r|}
\hline Peak\# & Ret. Time & \multicolumn{1}{c|}{ Height } & Height\% & \multicolumn{1}{c|}{ Area } & \multicolumn{1}{c|}{ Area\% } \\
\hline 1 & 21.587 & 30885 & 80.417 & 5185190 & 58.660 \\
\hline 2 & 38.426 & 7521 & 19.583 & 3654203 & 41.340 \\
\hline Total & & 38406 & 100.000 & 8839393 & 100.000 \\
\hline
\end{tabular}

$\underline{(R)-4-(t e r t-b u t y l)-2-((5-(t e r t-b u t y l)-2-h y d r o x y p h e n y l) s u l f i n y l) p h e n y l ~}$

\section{diphenylphosphinate (3o)}

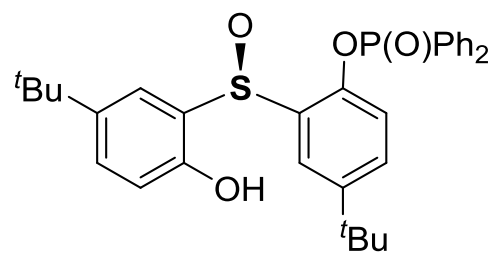

White powder; $47 \mathrm{mg}, 86 \%$ yield; m.p. $=94-96{ }^{\circ} \mathrm{C} ;{ }^{1} \mathrm{H} \mathrm{NMR}\left(400 \mathrm{MHz}, \mathrm{CDCl}_{3}\right) \delta$ $10.04(\mathrm{~s}, 1 \mathrm{H}), 8.04(\mathrm{dd}, J=8.2,1.3 \mathrm{~Hz}, 1 \mathrm{H}), 8.01(\mathrm{dd}, J=8.2,1.3 \mathrm{~Hz}, 1 \mathrm{H}), 7.95$ (dd, $J=8.2,1.3 \mathrm{~Hz}, 1 \mathrm{H}), 7.91(\mathrm{dd}, J=8.2,1.3 \mathrm{~Hz}, 1 \mathrm{H}), 7.79-7.73(\mathrm{~m}, 1 \mathrm{H}), 7.60-7.42(\mathrm{~m}$, 6H), 7.31-7.26 (m, 3H), $7.19(\mathrm{~d}, J=2.4 \mathrm{~Hz}, 1 \mathrm{H}), 6.82(\mathrm{~d}, J=8.7 \mathrm{~Hz}, 1 \mathrm{H}), 1.20$ (s, 9H), $1.02(\mathrm{~s}, 9 \mathrm{H}) ;{ }^{13} \mathrm{C} \mathrm{NMR}\left(100 \mathrm{MHz}, \mathrm{CDCl}_{3}\right) \delta 156.4,148.6,146.1(\mathrm{~d}, J=7.5 \mathrm{~Hz})$, 143.0, 133.3 (d, $J=6.4 \mathrm{~Hz}), 133.1(\mathrm{~d}, J=2.8 \mathrm{~Hz}), 133.0$ (d, $J=2.9 \mathrm{~Hz}), 132.0$ (d, $J=$ $7.0 \mathrm{~Hz}), 131.9(\mathrm{~d}, J=6.7 \mathrm{~Hz}), 130.9(\mathrm{~d}, J=24.7 \mathrm{~Hz}), 130.3,130.2,129.6$ (d, $J=18.0$ Hz), 129.5 (d, $J=18.0 \mathrm{~Hz}), 122.8$ (d, $J=49.8 \mathrm{~Hz}), 122.7,120.0$ (d, $J=4.3 \mathrm{~Hz})$, 118.8, 34.8, 34.1, 31.2, 31.2; ${ }^{31} \mathrm{P}$ NMR (162 MHz, $\left.\mathrm{CDCl}_{3}\right) \delta 32.34$; HRMS (ESI) $\mathrm{m} / \mathrm{z}$ calcd for $\mathrm{C}_{32} \mathrm{H}_{35} \mathrm{O}_{4} \mathrm{PS}[\mathrm{M}+\mathrm{H}]^{+}=547.2072$, found $=547.2076$.

Optical Rotation: $[\alpha]_{\mathrm{D}}^{25}=31.44\left(c=0.90, \mathrm{CHCl}_{3}\right) .77 \%$ ee (HPLC condition: Chiralpak ID-H column, $n$-Hexane $/ i$-PrOH $=70: 30$, flow rate $=1.0 \mathrm{~mL} / \mathrm{min}$, wavelength $=254 \mathrm{~nm}, t_{\mathrm{R}}=9.31 \mathrm{~min}$ for major isomer, $t_{\mathrm{R}}=15.32 \mathrm{~min}$ for minor isomer). 
$\mathrm{mV}$

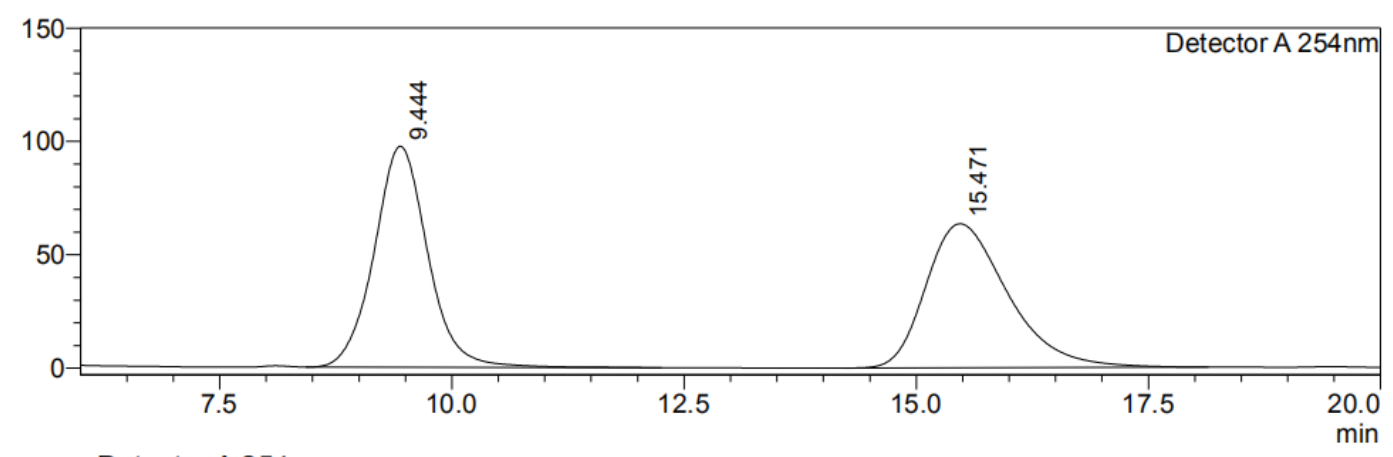

Detector A 254nm

\begin{tabular}{|r|r|r|r|r|r|}
\hline Peak\# & Ret. Time & Height & Height\% & \multicolumn{1}{c|}{ Area } & \multicolumn{1}{c|}{ Area\% } \\
\hline 1 & 9.444 & 97433 & 60.547 & 3917026 & 50.109 \\
\hline 2 & 15.471 & 63488 & 39.453 & 3899959 & 49.891 \\
\hline Total & & 160922 & 100.000 & 7816985 & 100.000 \\
\hline
\end{tabular}

$\mathrm{mV}$

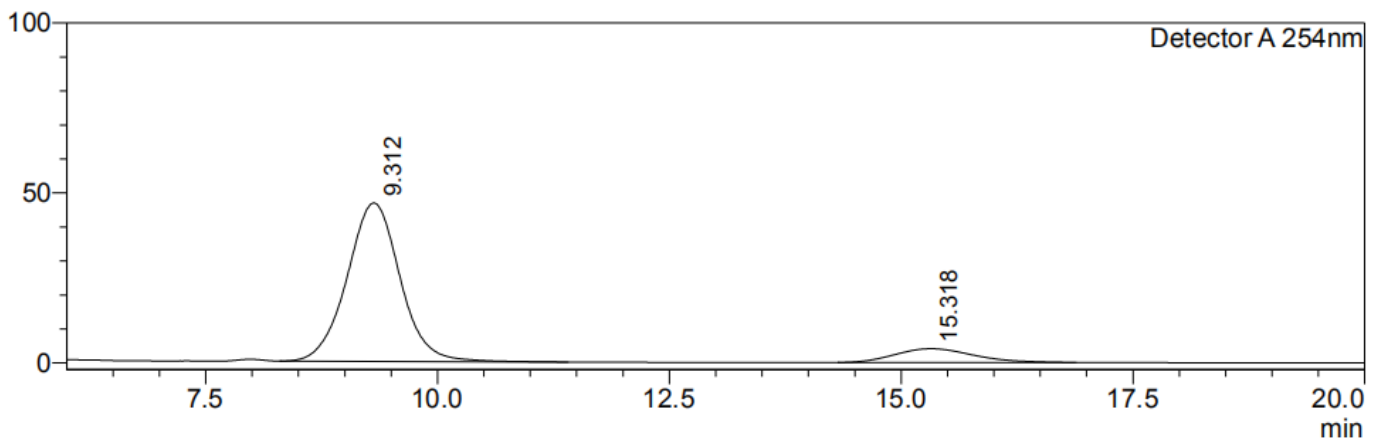

Detector A 254nm
\begin{tabular}{|r|r|r|r|r|r|}
\hline Peak\# & Ret. Time & Height & Height\% & \multicolumn{1}{c|}{ Area } & \multicolumn{1}{c|}{ Area\% } \\
\hline 1 & 9.312 & 46589 & 91.987 & 1858093 & 88.567 \\
\hline 2 & 15.318 & 4059 & 8.013 & 239861 & 11.433 \\
\hline Total & & 50647 & 100.000 & 2097954 & 100.000 \\
\hline
\end{tabular}

\section{$\underline{(S) \text {-2-(2-hydroxyphenylsulfonimidoyl)phenyl di-p-tolylphosphinate (4a) }}$}

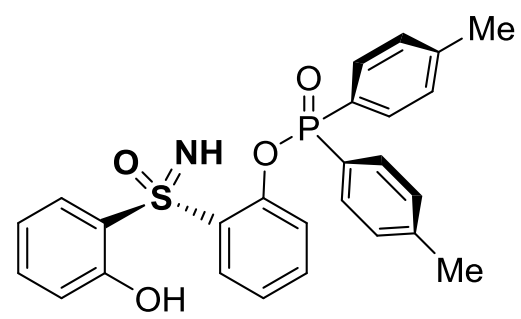

Yellow solid; $36 \mathrm{mg}, 75 \%$ yield; m.p. $=62-64{ }^{\circ} \mathrm{C} ;{ }^{1} \mathrm{H}$ NMR $\left(400 \mathrm{MHz}, \mathrm{CDCl}_{3}\right) \delta$ $12.12(\mathrm{~s}, 1 \mathrm{H}), 7.84(\mathrm{~d}, J=8.2 \mathrm{~Hz}, 1 \mathrm{H}), 7.81(\mathrm{~d}, J=8.2 \mathrm{~Hz}, 1 \mathrm{H}), 7.74(\mathrm{~d}, J=7.8 \mathrm{~Hz}$, 1H), $7.70(\mathrm{~d}, J=8.2 \mathrm{~Hz}, 1 \mathrm{H}), 7.66(\mathrm{t}, J=8.2 \mathrm{~Hz}, 2 \mathrm{H}), 7.47(\mathrm{dd}, J=8.2,1.6 \mathrm{~Hz}, 1 \mathrm{H})$, $7.39(\mathrm{ddd}, J=8.8,7.3,1.6 \mathrm{~Hz}, 1 \mathrm{H}), 7.36-7.31(\mathrm{~m}, 1 \mathrm{H}), 7.29(\mathrm{dd}, J=8.0,3.4 \mathrm{~Hz}, 2 \mathrm{H})$, $7.23(\mathrm{dd}, J=8.0,3.4 \mathrm{~Hz}, 2 \mathrm{H}), 7.11(\mathrm{t}, J=7.7 \mathrm{~Hz}, 1 \mathrm{H}), 6.82(\mathrm{dd}, J=8.4,0.8 \mathrm{~Hz}, 1 \mathrm{H})$, 6.80-6.71 (m, 1H), $4.09(\mathrm{~s}, 1 \mathrm{H}), 2.38(\mathrm{~d}, J=9.7 \mathrm{~Hz}, 6 \mathrm{H}) ;{ }^{13} \mathrm{C}$ NMR (100 MHz, 
$\left.\mathrm{CDCl}_{3}\right) \delta 157.5,149.3(\mathrm{~d}, J=7.4 \mathrm{~Hz}), 143.7(\mathrm{~d}, J=7.1 \mathrm{~Hz}), 135.2(\mathrm{~d}, J=91.2 \mathrm{~Hz})$, $134.3(\mathrm{~d}, J=7.0 \mathrm{~Hz}), 132.2(\mathrm{~d}, J=3.3 \mathrm{~Hz}), 132.1$ (d, $J=3.2 \mathrm{~Hz}), 129.6$ (d, $J=5.0$ Hz), $129.5(\mathrm{~d}, J=5.1 \mathrm{~Hz}), 129.0,127.3(\mathrm{~d}, J=27.7 \mathrm{~Hz}), 126.0(\mathrm{~d}, J=26.9 \mathrm{~Hz}), 123.9$, $121.6(\mathrm{~d}, J=4.9 \mathrm{~Hz}), 120.0,119.3(\mathrm{~d}, J=10.0 \mathrm{~Hz}), 21.8(\mathrm{~d}, J=3.7 \mathrm{~Hz}) ;{ }^{31} \mathrm{P} \mathrm{NMR}$ $\left(162 \mathrm{MHz}, \mathrm{CDCl}_{3}\right) \delta 35.27$; HRMS (ESI) $\mathrm{m} / z$ calcd for $\mathrm{C}_{26} \mathrm{H}_{24} \mathrm{NO}_{4} \mathrm{PS}[\mathrm{M}+\mathrm{H}]^{+}=$ 478.1242 , found $=478.1247$.

Optical Rotation: $[\alpha]_{\mathrm{D}}^{25}=60.00\left(c=0.51, \mathrm{CHCl}_{3}\right) .99 \%$ ee (HPLC condition: Chiralpak OD-H column, $n-\mathrm{Hexane} / i-\mathrm{PrOH}=80: 20$, flow rate $=1.0 \mathrm{~mL} / \mathrm{min}$, wavelength $=254 \mathrm{~nm}, t_{\mathrm{R}}=12.69 \min$ for major isomer, $t_{\mathrm{R}}=15.84 \mathrm{~min}$ for minor isomer).

$\mathrm{mV}$

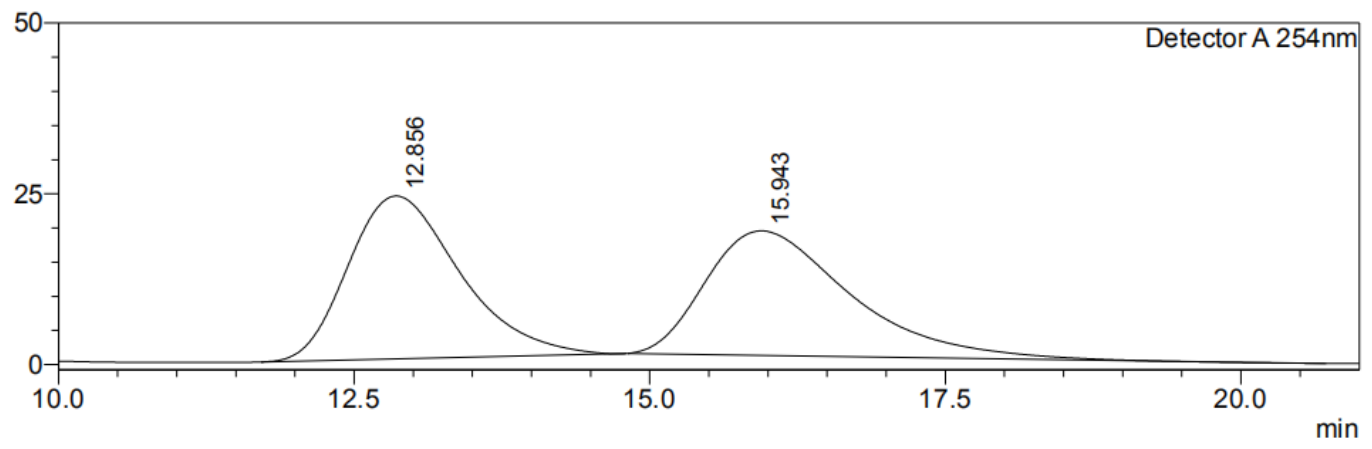

Detector A 254nm

\begin{tabular}{|r|r|r|r|r|r|}
\hline Peak\# & Ret. Time & Height & Height $\%$ & \multicolumn{1}{c|}{ Area } & \multicolumn{1}{c|}{ Area\% } \\
\hline 1 & 12.856 & 23848 & 56.689 & 1566665 & 50.294 \\
\hline 2 & 15.943 & 18220 & 43.311 & 1548349 & 49.706 \\
\hline Total & & 42068 & 100.000 & 3115014 & 100.000 \\
\hline
\end{tabular}

$\mathrm{mV}$

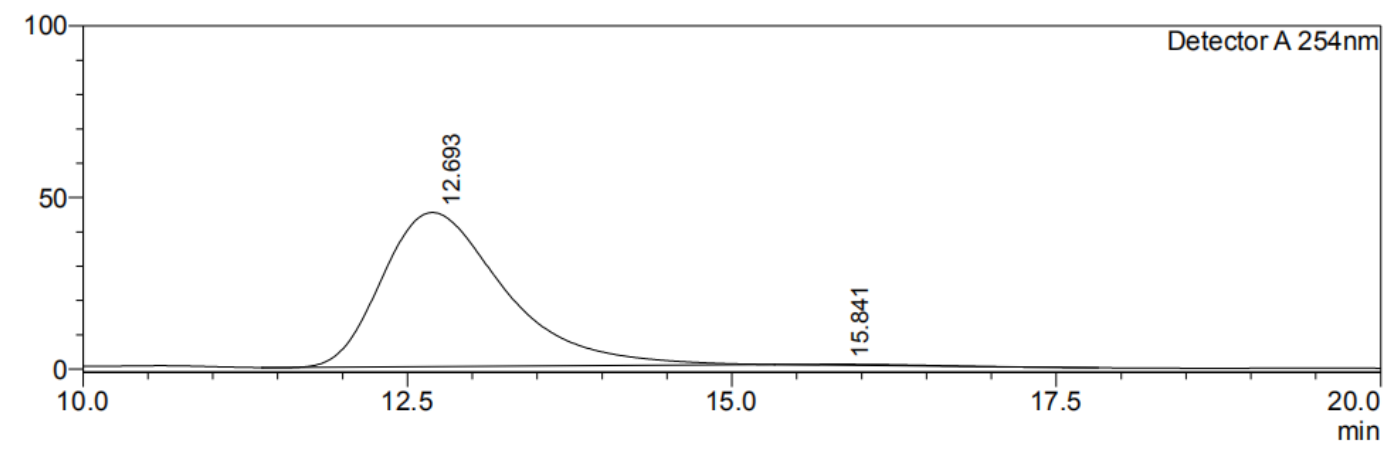

Detector A 254nm

\begin{tabular}{|r|r|r|r|r|r|}
\hline Peak\# & Ret. Time & \multicolumn{1}{c|}{ Height } & Height $\%$ & \multicolumn{1}{c|}{ Area } & \multicolumn{1}{c|}{ Area\% } \\
\hline 1 & 12.693 & 44895 & 99.430 & 2955384 & 99.498 \\
\hline 2 & 15.841 & 257 & 0.570 & 14902 & 0.502 \\
\hline Total & & 45152 & 100.000 & 2970287 & 100.000 \\
\hline
\end{tabular}

$\underline{\text { (S)-2-(2-hydroxyphenylsulfonimidoyl)phenyl bis(4-butylphenyl)phosphinate (4b) }}$ 


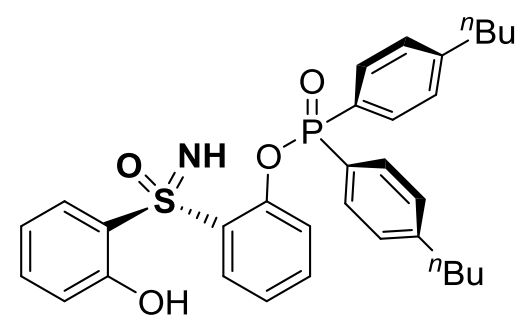

Light yellow oil; $43 \mathrm{mg}, 76 \%$ yield; ${ }^{1} \mathrm{H}$ NMR (400 MHz, $\left.\mathrm{CDCl}_{3}\right) \delta 12.16(\mathrm{~s}, 1 \mathrm{H})$, $7.87(\mathrm{~d}, J=8.2 \mathrm{~Hz}, 1 \mathrm{H}), 7.83(\mathrm{~d}, J=8.2 \mathrm{~Hz}, 1 \mathrm{H}), 7.74(\mathrm{~d}, J=8.2 \mathrm{~Hz}, 2 \mathrm{H}), 7.71(\mathrm{~d}, J$ $=8.2 \mathrm{~Hz}, 1 \mathrm{H}), 7.66(\mathrm{~d}, J=8.4 \mathrm{~Hz}, 1 \mathrm{H}), 7.46(\mathrm{dd}, J=8.2,1.6 \mathrm{~Hz}, 1 \mathrm{H}), 7.41-7.33(\mathrm{~m}$, 2H), 7.33-7.27 (m, 2H), 7.24 (dd, $J=8.2,3.7 \mathrm{~Hz}, 2 \mathrm{H}), 7.15-7.07(\mathrm{~m}, 1 \mathrm{H}), 6.81(\mathrm{dd}, J$ $=8.4,0.9 \mathrm{~Hz}, 1 \mathrm{H}), 6.77-6.68(\mathrm{~m}, 1 \mathrm{H}), 4.00(\mathrm{~s}, 1 \mathrm{H}), 2.72-.53(\mathrm{~m}, 4 \mathrm{H}), 1.65-1.53(\mathrm{~m}$, $4 \mathrm{H}), 1.39-1.30(\mathrm{~m}, 4 \mathrm{H}), 0.91(\mathrm{t}, J=7.3 \mathrm{~Hz}, 6 \mathrm{H}) ;{ }^{13} \mathrm{C} \mathrm{NMR}\left(100 \mathrm{MHz}, \mathrm{CDCl}_{3}\right) \delta$ $157.5,149.4(\mathrm{~d}, J=7.2 \mathrm{~Hz}), 148.6(\mathrm{~d}, J=2.9 \mathrm{~Hz}), 148.5(\mathrm{~d}, J=3.0 \mathrm{~Hz}), 135.2(\mathrm{~d}, J=$ $88.6 \mathrm{~Hz}), 134.3$ (d, $J=7.0 \mathrm{~Hz}), 132.2$ (d, $J=4.0 \mathrm{~Hz}), 132.1$ (d, $J=4.0 \mathrm{~Hz}), 129.4$, $129.0(\mathrm{~d}, J=5.0 \mathrm{~Hz}), 128.9(\mathrm{~d}, J=3.8 \mathrm{~Hz}), 127.6(\mathrm{~d}, J=26.5 \mathrm{~Hz}), 126.2(\mathrm{~d}, J=25.9$ $\mathrm{Hz}), 123.9,121.6(\mathrm{~d}, J=5.0 \mathrm{~Hz}), 120.0,119.2(\mathrm{~d}, J=6.9 \mathrm{~Hz}), 35.9,33.3$ (d, $J=1.7$ $\mathrm{Hz}), 22.4(\mathrm{~d}, J=2.7 \mathrm{~Hz}), 14.0 ;{ }^{31} \mathrm{P}$ NMR $\left(162 \mathrm{MHz}, \mathrm{CDCl}_{3}\right) \delta 35.22$; HRMS (ESI) $m / z$ calcd for $\mathrm{C}_{32} \mathrm{H}_{36} \mathrm{NO}_{4} \mathrm{PS}[\mathrm{M}+\mathrm{H}]^{+}=562.2181$, found $=562.2189$.

Optical Rotation: $[\alpha]^{25}=74.10\left(c=0.61, \mathrm{CHCl}_{3}\right) .96 \%$ ee (HPLC condition: Chiralpak OD-H column, $n-\mathrm{Hexane} / i-\mathrm{PrOH}=90: 10$, flow rate $=1.0 \mathrm{~mL} / \mathrm{min}$, wavelength $=254 \mathrm{~nm}, t_{\mathrm{R}}=15.43$ min for major isomer, $t_{\mathrm{R}}=23.62 \min$ for minor isomer). $\mathrm{mV}$

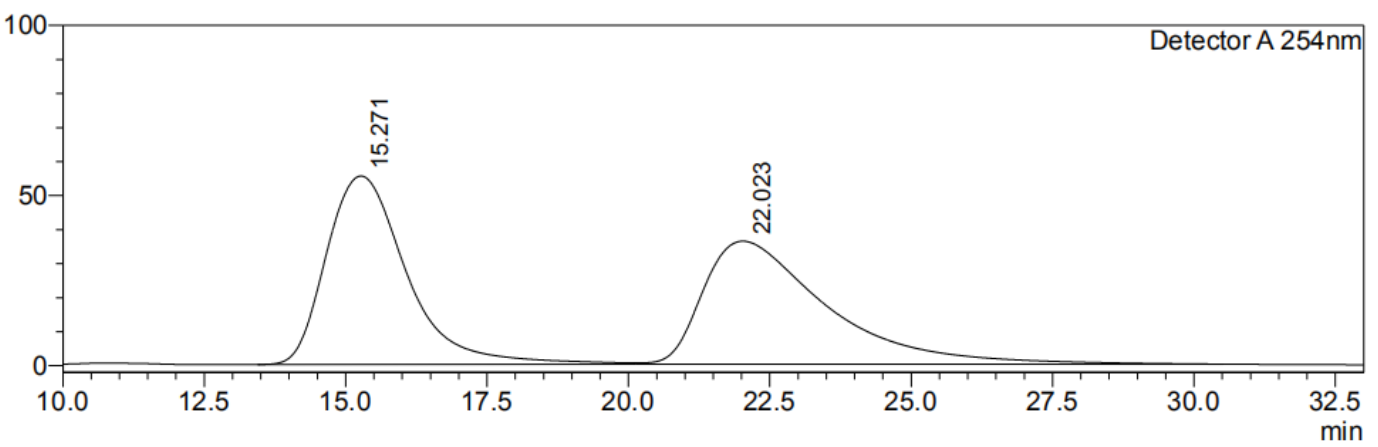

\begin{tabular}{|c|c|c|c|c|c|}
\hline Peak\# & Ret. Time & Height & Height\% & Area & Area\% \\
\hline 1 & 15.271 & 55385 & 60.473 & 5498138 & 49.919 \\
\hline 2 & 22.023 & 36201 & 39.527 & 5515892 & 50.081 \\
\hline Total & & 91587 & 100.000 & 11014030 & 100.000 \\
\hline
\end{tabular}


$\mathrm{mV}$

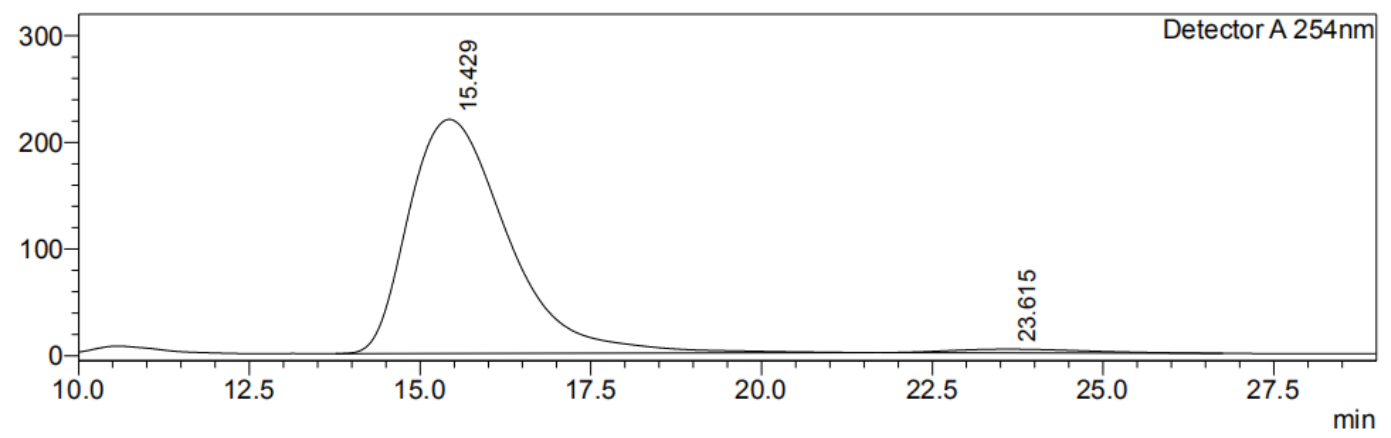

Detector A 254nm
\begin{tabular}{|r|r|r|r|r|r|}
\hline Peak\# & Ret. Time & Height & Height\% & \multicolumn{1}{c|}{ Area } & \multicolumn{1}{c|}{ Area\% } \\
\hline 1 & 15.429 & 219419 & 98.437 & 22176653 & 97.856 \\
\hline 2 & 23.615 & 3484 & 1.563 & 485954 & 2.144 \\
\hline Total & & 222904 & 100.000 & 22662608 & 100.000 \\
\hline
\end{tabular}

\section{(S)-2-(2-hydroxyphenylsulfonimidoyl)phenyl}

\section{bis(4-(tert-butyl)phenyl)phosphinate (4c)}

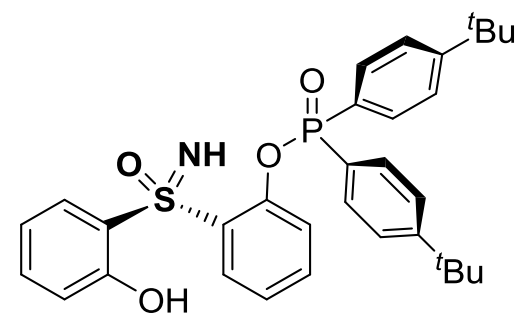

Yellow oil; $48 \mathrm{mg}, 85 \%$ yield; ${ }^{1} \mathrm{H}$ NMR $\left(400 \mathrm{MHz}, \mathrm{CDCl}_{3}\right) \delta 7.90(\mathrm{~d}, J=8.4 \mathrm{~Hz}, 1 \mathrm{H})$, $7.86(\mathrm{~d}, J=8.4 \mathrm{~Hz}, 1 \mathrm{H}), 7.80(\mathrm{~d}, J=8.4 \mathrm{~Hz}, 1 \mathrm{H}), 7.77(\mathrm{~d}, J=8.4 \mathrm{~Hz}, 1 \mathrm{H}), 7.73$ (d, $J$ $=7.8 \mathrm{~Hz}, 1 \mathrm{H}), 7.66(\mathrm{~d}, J=8.4 \mathrm{~Hz}, 1 \mathrm{H}), 7.52(\mathrm{~d}, J=3.5 \mathrm{~Hz}, 1 \mathrm{H}), 7.49(\mathrm{~d}, J=3.5 \mathrm{~Hz}$, 1H), 7.48-7.43 (m, 3H), 7.40-7.32 (m, 2H), $7.10(\mathrm{t}, J=7.8 \mathrm{~Hz}, 1 \mathrm{H}), 6.85(\mathrm{~d}, J=8.4$ $\mathrm{Hz}, 1 \mathrm{H}), 6.72-6.64(\mathrm{~m}, 1 \mathrm{H}), 1.32(\mathrm{~s}, 9 \mathrm{H}), 1.31(\mathrm{~s}, 9 \mathrm{H}) ;{ }^{13} \mathrm{C} \mathrm{NMR}\left(100 \mathrm{MHz}, \mathrm{CDCl}_{3}\right)$ $\delta 157.4,156.6(\mathrm{~d}, J=2.9 \mathrm{~Hz}), 156.5(\mathrm{~d}, J=2.9 \mathrm{~Hz}), 149.4(\mathrm{~d}, J=7.4 \mathrm{~Hz}), 135.4(\mathrm{~d}, J$ $=87.3 \mathrm{~Hz}), 133.7,132.1(\mathrm{~d}, J=8.4 \mathrm{~Hz}), 132.0(\mathrm{~d}, J=8.4 \mathrm{~Hz}), 129.2(\mathrm{~d}, J=37.1 \mathrm{~Hz})$, $127.3(\mathrm{~d}, J=16.2 \mathrm{~Hz}), 126.0(\mathrm{~d}, J=4.2 \mathrm{~Hz}), 125.8(\mathrm{~d}, J=4.2 \mathrm{~Hz}), 123.9,121.5(\mathrm{~d}, J$ $=5.0 \mathrm{~Hz}), 119.8,119.2(\mathrm{~d}, J=8.6 \mathrm{~Hz}), 35.2(\mathrm{~d}, J=3.2 \mathrm{~Hz}), 31.2(\mathrm{~d}, J=1.9 \mathrm{~Hz}) ;{ }^{31} \mathrm{P}$ NMR $\left(162 \mathrm{MHz}, \mathrm{CDCl}_{3}\right) \delta 34.96$; HRMS (ESI) $m / z$ calcd for $\mathrm{C}_{32} \mathrm{H}_{36} \mathrm{NO}_{4} \mathrm{PS}[\mathrm{M}+\mathrm{H}]^{+}=$ 562.2181 , found $=562.2182$.

Optical Rotation: $[\alpha]_{\mathrm{D}}^{25}=36.47\left(c=0.51, \mathrm{CHCl}_{3}\right) .96 \%$ ee (HPLC condition: Chiralpak OD-H column, $n$-Hexane $/ i-\mathrm{PrOH}=90: 10$, flow rate $=0.5 \mathrm{~mL} / \mathrm{min}$, 
wavelength $=254 \mathrm{~nm}, t_{\mathrm{R}}=24.83 \mathrm{~min}$ for minor isomer, $t_{\mathrm{R}}=37.27$ min for major isomer).

$\mathrm{mV}$

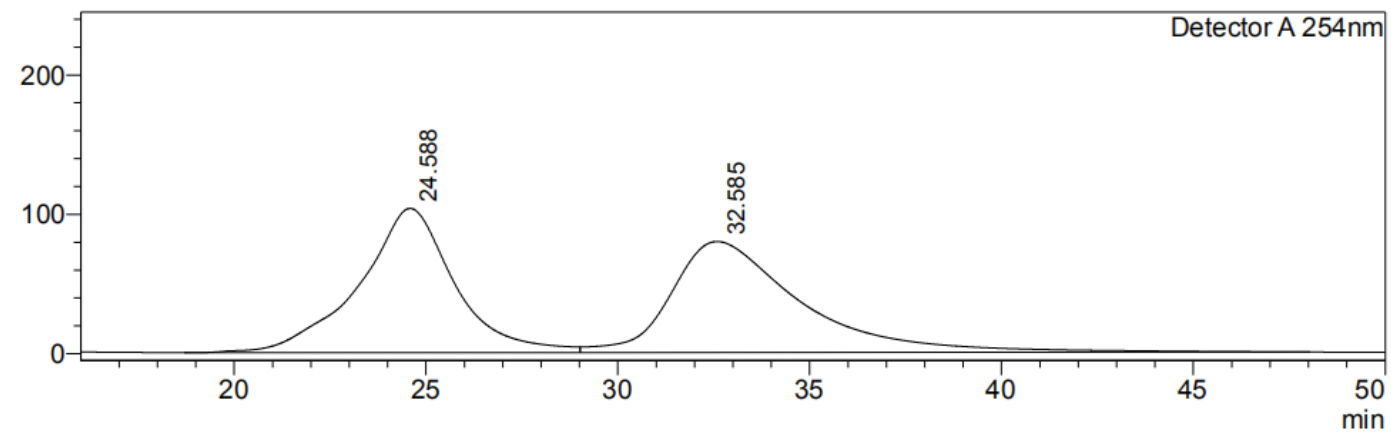

Detector A 254nm

\begin{tabular}{|r|r|r|r|r|r|}
\hline Peak\# & Ret. Time & Height & Height\% & \multicolumn{1}{c|}{ Area } & \multicolumn{1}{c|}{ Area\% } \\
\hline 1 & 24.588 & 103446 & 56.520 & 17923266 & 48.857 \\
\hline 2 & 32.585 & 79580 & 43.480 & 18761976 & 51.143 \\
\hline Total & & 183026 & 100.000 & 36685243 & 100.000 \\
\hline
\end{tabular}

$\mathrm{mV}$

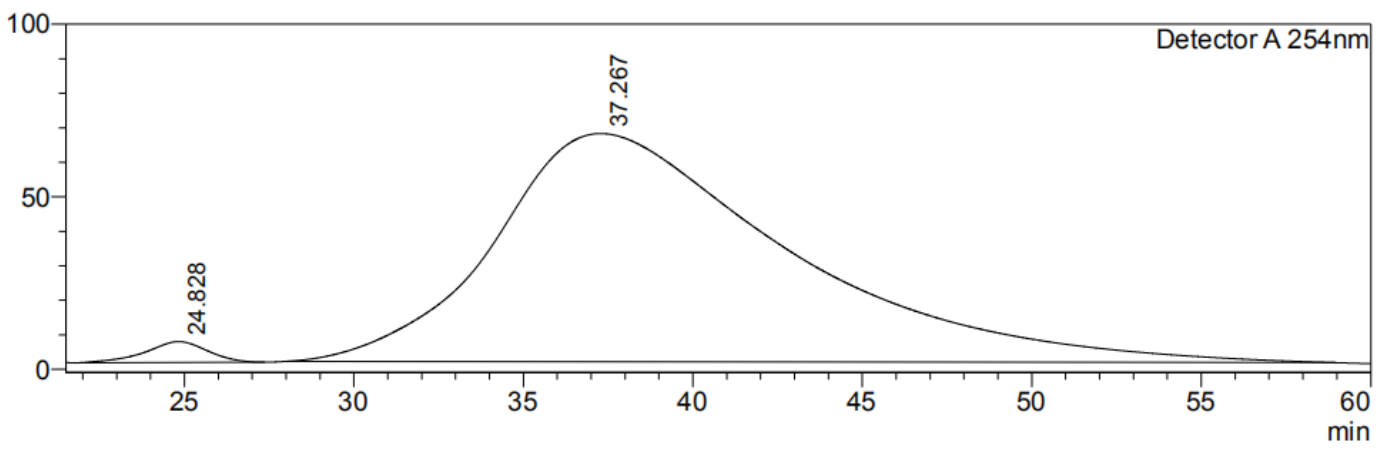

Detector A 254nm

\begin{tabular}{|r|r|r|r|r|r|}
\hline Peak\# & Ret. Time & \multicolumn{1}{c|}{ Height } & Height $\%$ & \multicolumn{1}{c|}{ Area } & \multicolumn{1}{c|}{ Area\% } \\
\hline 1 & 24.828 & 6071 & 8.405 & 777308 & 1.911 \\
\hline 2 & 37.267 & 66159 & 91.595 & 39903169 & 98.089 \\
\hline Total & & 72229 & 100.000 & 40680477 & 100.000 \\
\hline
\end{tabular}

\section{$\underline{\text { (S)-2-(2-hydroxyphenylsulfonimidoyl)phenyl di([1,1'-biphenyl]-4-yl)phosphinate }}$}

$\underline{(4 d)}$<smiles>N[SH](=O)(Oc1ccccc1S(=O)(=O)c1ccc(-c2ccccc2)cc1)c1ccccc1</smiles>

Colorless oil; $50 \mathrm{mg}, 83 \%$ yield; ${ }^{1} \mathrm{H}$ NMR $\left(400 \mathrm{MHz}, \mathrm{CDCl}_{3}\right) \delta 8.06(\mathrm{~d}, J=8.2 \mathrm{~Hz}$,

1H), $8.03(\mathrm{~d}, J=8.2 \mathrm{~Hz}, 1 \mathrm{H}), 7.95(\mathrm{~d}, J=8.2 \mathrm{~Hz}, 1 \mathrm{H}), 7.92(\mathrm{~d}, J=8.2 \mathrm{~Hz}, 1 \mathrm{H}), 7.85$ $(\mathrm{d}, J=7.8 \mathrm{~Hz}, 1 \mathrm{H}), 7.76-7.65(\mathrm{~m}, 5 \mathrm{H}), 7.65-7.56(\mathrm{~m}, 4 \mathrm{H}), 7.47(\mathrm{~m}, 5 \mathrm{H}), 7.44-7.33$ 
(m, 4H), $7.15(\mathrm{t}, J=7.6 \mathrm{~Hz}, 1 \mathrm{H}), 6.92(\mathrm{~d}, J=8.4 \mathrm{~Hz}, 1 \mathrm{H}), 6.73(\mathrm{t}, J=7.6 \mathrm{~Hz}, 1 \mathrm{H})$; ${ }^{13} \mathrm{C}$ NMR $\left(100 \mathrm{MHz}, \mathrm{CDCl}_{3}\right) \delta 157.4,149.3(\mathrm{~d}, J=7.2 \mathrm{~Hz}), 145.9(\mathrm{~d}, J=3.0 \mathrm{~Hz})$, $145.8(\mathrm{~d}, J=3.0 \mathrm{~Hz}), 139.8,135.6(\mathrm{~d}, J=73.3 \mathrm{~Hz}), 132.7$ (d, $J=2.0 \mathrm{~Hz}), 132.6$ (d, $J$ $=1.8 \mathrm{~Hz}), 129.4(\mathrm{~d}, J=29.5 \mathrm{~Hz}), 129.1,128.8(\mathrm{~d}, J=25.4 \mathrm{~Hz}), 128.5,127.7(\mathrm{~d}, J=$ $5.8 \mathrm{~Hz}), 127.5(\mathrm{~d}, J=5.8 \mathrm{~Hz}), 127.4(\mathrm{~d}, J=2.0 \mathrm{~Hz}), 127.3,124.3,121.6(\mathrm{~d}, J=4.9$ $\mathrm{Hz}), 119.7,119.3(\mathrm{~d}, J=11.8 \mathrm{~Hz}) ;{ }^{31} \mathrm{P}$ NMR $\left(162 \mathrm{MHz}, \mathrm{CDCl}_{3}\right) \delta 34.41 ; \mathrm{HRMS}(\mathrm{ESI})$ $m / z$ calcd for $\mathrm{C}_{36} \mathrm{H}_{28} \mathrm{NO}_{4} \mathrm{PS}[\mathrm{M}+\mathrm{H}]^{+}=602.1555$, found $=602.1555$.

Optical Rotation: $[\alpha]^{25}=78.52\left(c=0.27, \mathrm{CHCl}_{3}\right) .90 \%$ ee (HPLC condition: Chiralpak AD-H column, $n$-Hexane $/ i-\mathrm{PrOH}=60: 40$, flow rate $=1.0 \mathrm{~mL} / \mathrm{min}$, wavelength $=254 \mathrm{~nm}, t_{\mathrm{R}}=25.26$ min for major isomer, $t_{\mathrm{R}}=31.96$ min for minor isomer).

$\mathrm{mV}$

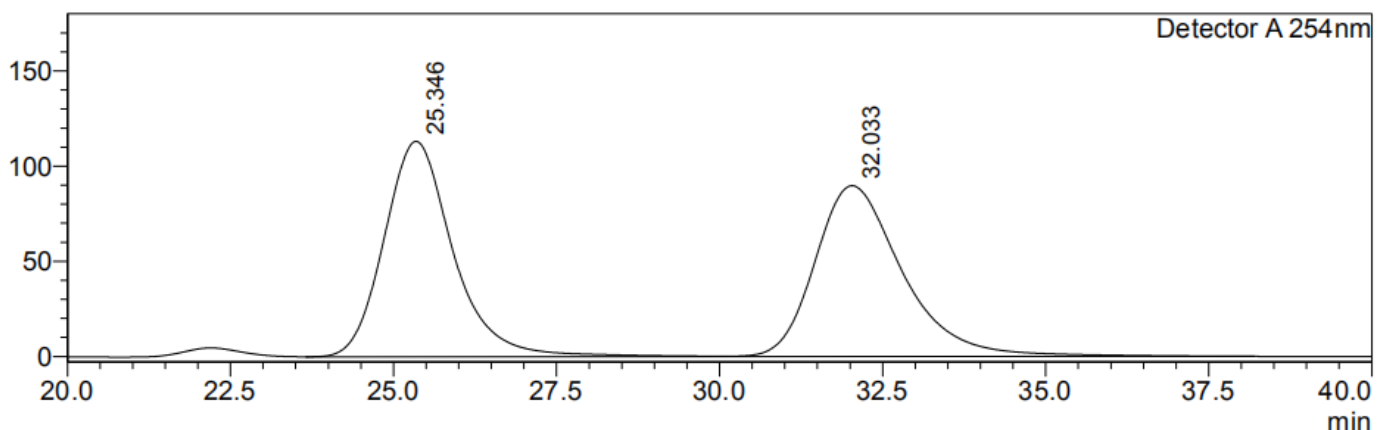

Detector A 254nm

\begin{tabular}{|r|r|r|r|r|r|}
\hline Peak\# & Ret. Time & Height & Height $\%$ & \multicolumn{1}{c|}{ Area } & \multicolumn{1}{c|}{ Area $\%$} \\
\hline 1 & 25.346 & 113176 & 55.756 & 8355503 & 49.832 \\
\hline 2 & 32.033 & 89810 & 44.244 & 8411819 & 50.168 \\
\hline Total & & 202985 & 100.000 & 16767322 & 100.000 \\
\hline
\end{tabular}

$\mathrm{mV}$

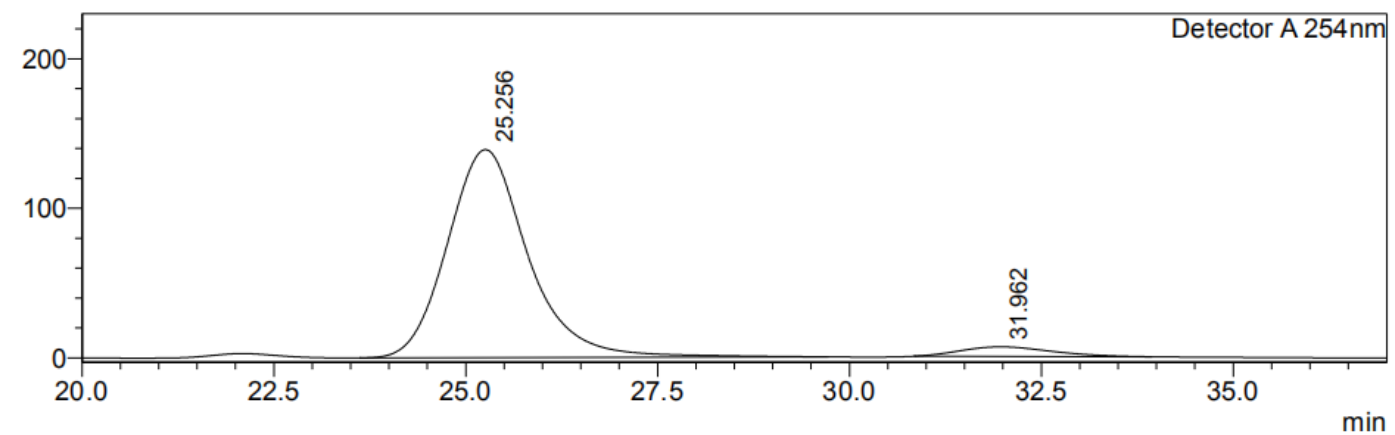

Detector A 254nm

\begin{tabular}{|r|r|r|r|r|r|}
\hline Peak\# & Ret. Time & \multicolumn{1}{c|}{ Height } & Height $\%$ & \multicolumn{1}{c|}{ Area } & \multicolumn{1}{c|}{ Area\% } \\
\hline 1 & 25.256 & 139077 & 95.587 & 10185174 & 95.047 \\
\hline 2 & 31.962 & 6421 & 4.413 & 530791 & 4.953 \\
\hline Total & & 145498 & 100.000 & 10715965 & 100.000 \\
\hline
\end{tabular}


(4e)<smiles>COc1ccc(P(=O)(Oc2ccccc2S(=O)(=O)c2ccccc2O)c2ccc(OC)cc2)cc1</smiles>

Colorless oil; $41 \mathrm{mg}, 80 \%$ yield; ${ }^{1} \mathrm{H}$ NMR $\left(400 \mathrm{MHz}, \mathrm{CDCl}_{3}\right) \delta 7.86(\mathrm{~d}, J=8.8 \mathrm{~Hz}$, $1 \mathrm{H}), 7.83(\mathrm{~d}, J=8.8 \mathrm{~Hz}, 1 \mathrm{H}), 7.72(\mathrm{~m}, 3 \mathrm{H}), 7.62(\mathrm{~d}, J=8.4 \mathrm{~Hz}, 1 \mathrm{H}), 7.46(\mathrm{dd}, J=$ 8.2, $1.6 \mathrm{~Hz}, 1 \mathrm{H}), 7.38(\mathrm{ddd}, J=8.8,7.4,1.6 \mathrm{~Hz}, 1 \mathrm{H}), 7.35-7.28(\mathrm{~m}, 1 \mathrm{H}), 7.09$ (t, $J=$ $7.4 \mathrm{~Hz}, 1 \mathrm{H}), 6.98$ (d, $J=3.0 \mathrm{~Hz}, 1 \mathrm{H}), 6.96(\mathrm{~d}, J=3.0 \mathrm{~Hz}, 1 \mathrm{H}), 6.92(\mathrm{~d}, J=3.0 \mathrm{~Hz}$, 1H), $6.90(\mathrm{~d}, J=3.0 \mathrm{~Hz}, 1 \mathrm{H}), 6.85(\mathrm{dd}, J=8.4,0.8 \mathrm{~Hz}, 1 \mathrm{H}), 6.77-6.70(\mathrm{~m}, 1 \mathrm{H}), 3.81$ $(\mathrm{d}, J=7.4 \mathrm{~Hz}, 6 \mathrm{H}) ;{ }^{13} \mathrm{C} \mathrm{NMR}\left(100 \mathrm{MHz}, \mathrm{CDCl}_{3}\right) \delta 163.3(\mathrm{~d}, J=3.0 \mathrm{~Hz}), 163.2(\mathrm{~d}, J$ $=3.0 \mathrm{~Hz}), 157.4,149.3(\mathrm{~d}, J=7.3 \mathrm{~Hz}), 135.3(\mathrm{~d}, J=90.9 \mathrm{~Hz}), 134.1(\mathrm{~d}, J=3.0 \mathrm{~Hz})$, $134.0(\mathrm{~d}, J=2.9 \mathrm{~Hz}), 129.2(\mathrm{~d}, J=29.9 \mathrm{~Hz}), 123.9,121.8(\mathrm{~d}, J=28.3 \mathrm{~Hz}), 121.6(\mathrm{~d}$, $J=5.0 \mathrm{~Hz}), 120.4(\mathrm{~d}, J=27.5 \mathrm{~Hz}), 119.9,119.2(\mathrm{~d}, J=11.9 \mathrm{~Hz}), 114.4(\mathrm{~d}, J=4.8$ $\mathrm{Hz}), 114.3(\mathrm{~d}, J=5.0 \mathrm{~Hz}), 55.5(\mathrm{~d}, J=2.7 \mathrm{~Hz}) ;{ }^{31} \mathrm{P} \mathrm{NMR}\left(162 \mathrm{MHz}, \mathrm{CDCl}_{3}\right) \delta 35.28$; HRMS (ESI) $m / z$ calcd for $\mathrm{C}_{26} \mathrm{H}_{24} \mathrm{NO}_{6} \mathrm{PS}[\mathrm{M}+\mathrm{H}]^{+}=510.1140$, found $=510.1131$.

Optical Rotation: $[\alpha]^{25}=82.33\left(c=0.30, \mathrm{CHCl}_{3}\right) .97 \%$ ee (HPLC condition: Chiralpak AD-H column, $n$-Hexane $/ i-\mathrm{PrOH}=60: 40$, flow rate $=1.0 \mathrm{~mL} / \mathrm{min}$, wavelength $=254 \mathrm{~nm}, t_{\mathrm{R}}=28.46 \min$ for major isomer, $t_{\mathrm{R}}=33.82 \mathrm{~min}$ for minor isomer).

$\mathrm{mV}$

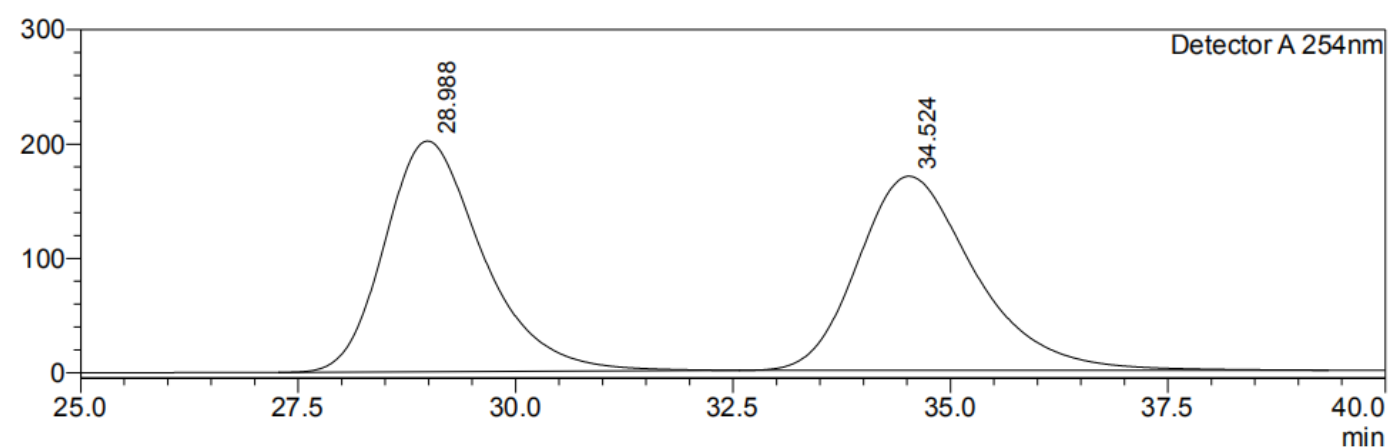

Detector A 254nm
\begin{tabular}{|r|r|r|r|r|r|}
\hline Peak\#\# & Ret. Time & Height & \multicolumn{1}{c|}{ Height\% } & Area & \multicolumn{1}{c|}{ Area\% } \\
\hline 1 & 28.988 & 201768 & 54.302 & 15926198 & 49.976 \\
\hline 2 & 34.524 & 169797 & 45.698 & 15941202 & 50.024 \\
\hline Total & & 371565 & 100.000 & 31867400 & 100.000 \\
\hline
\end{tabular}


$\mathrm{mV}$

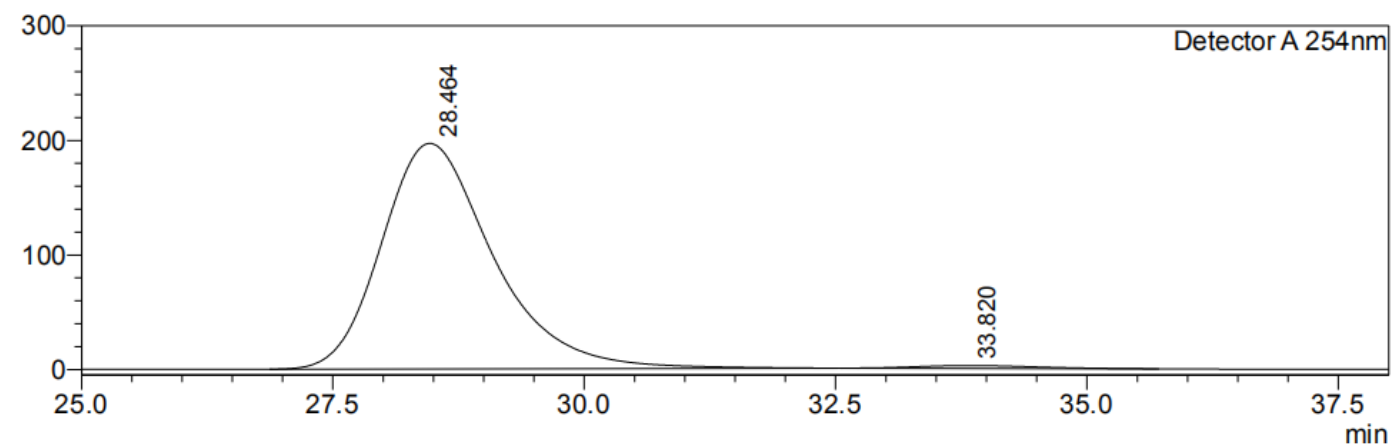

Detector A 254nm
\begin{tabular}{|r|r|r|r|r|r|}
\hline Peak\# & Ret. Time & Height & Height\% & \multicolumn{1}{c|}{ Area } & \multicolumn{1}{c|}{ Area\% } \\
\hline 1 & 28.464 & 196907 & 98.737 & 15244025 & 98.642 \\
\hline 2 & 33.820 & 2518 & 1.263 & 209904 & 1.358 \\
\hline Total & & 199425 & 100.000 & 15453929 & 100.000 \\
\hline
\end{tabular}

\section{(S)-2-(2-hydroxyphenylsulfonimidoyl)phenyl bis(4-ethoxyphenyl)phosphinate}

$\underline{(4 \mathbf{f})}$

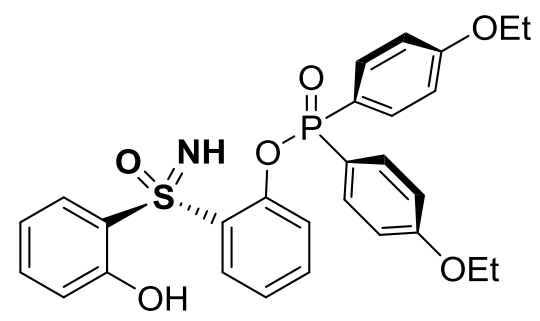

Light yellow solid; $44 \mathrm{mg}, 81 \%$ yield; m.p. $=137-139{ }^{\circ} \mathrm{C} ;{ }^{1} \mathrm{H}$ NMR $(400 \mathrm{MHz}$, $\left.\mathrm{CDCl}_{3}\right) \delta 11.96(\mathrm{~s}, 1 \mathrm{H}), 7.86(\mathrm{~d}, J=8.7 \mathrm{~Hz}, 1 \mathrm{H}), 7.83(\mathrm{~d}, J=8.7 \mathrm{~Hz}, 1 \mathrm{H}), 7.72(\mathrm{~d}, J$ $=8.7 \mathrm{~Hz}, 2 \mathrm{H}), 7.69(\mathrm{~d}, J=8.7 \mathrm{~Hz}, 1 \mathrm{H}), 7.65(\mathrm{~d}, J=8.2 \mathrm{~Hz}, 1 \mathrm{H}), 7.47(\mathrm{dd}, J=8.2$, $1.5 \mathrm{~Hz}, 1 \mathrm{H}), 7.42-7.36(\mathrm{~m}, 1 \mathrm{H}), 7.36-7.30(\mathrm{~m}, 1 \mathrm{H}), 7.10(\mathrm{t}, J=7.6 \mathrm{~Hz}, 1 \mathrm{H}), 6.96$ (dd, $J=8.7,2.8 \mathrm{~Hz}, 2 \mathrm{H}), 6.90(\mathrm{dd}, J=8.7,2.8 \mathrm{~Hz}, 2 \mathrm{H}), 6.83(\mathrm{~d}, J=8.2 \mathrm{~Hz}, 1 \mathrm{H})$, 6.79-6.71 (m, 1H), $4.06(\mathrm{dq}, J=14.2,7.0 \mathrm{~Hz}, 4 \mathrm{H}), 1.41(\mathrm{td}, J=7.0,4.0 \mathrm{~Hz}, 6 \mathrm{H}) ;{ }^{13} \mathrm{C}$ NMR $\left(100 \mathrm{MHz}, \mathrm{CDCl}_{3}\right) \delta 162.7(\mathrm{~d}, J=3.0 \mathrm{~Hz}), 162.6(\mathrm{~d}, J=3.0 \mathrm{~Hz}), 157.5,149.4$ $(\mathrm{d}, J=7.3 \mathrm{~Hz}), 135.2(\mathrm{~d}, J=93.1 \mathrm{~Hz}), 134.2(\mathrm{~d}, J=7.0 \mathrm{~Hz}), 134.1(\mathrm{~d}, J=3.4 \mathrm{~Hz})$, $134.0(\mathrm{~d}, J=3.2 \mathrm{~Hz}), 129.2(\mathrm{~d}, J=53.0 \mathrm{~Hz}), 123.8,121.7$ (d, $J=31.8 \mathrm{~Hz}), 121.6$ (d, $J=4.9 \mathrm{~Hz}), 120.3(\mathrm{~d}, J=30.9 \mathrm{~Hz}), 120.0,119.3(\mathrm{~d}, J=9.9 \mathrm{~Hz}), 114.9(\mathrm{~d}, J=4.8 \mathrm{~Hz})$, $114.7(\mathrm{~d}, J=5.0 \mathrm{~Hz}), 63.8(\mathrm{~d}, J=2.1 \mathrm{~Hz}), 14.8 ;{ }^{31} \mathrm{P} \mathrm{NMR}\left(162 \mathrm{MHz}, \mathrm{CDCl}_{3}\right) \delta 35.43$; HRMS (ESI) $m / z$ calcd for $\mathrm{C}_{28} \mathrm{H}_{28} \mathrm{NO}_{6} \mathrm{PS}[\mathrm{M}+\mathrm{H}]^{+}=538.1453$, found $=538.1459$.

Optical Rotation: $[\alpha]_{\mathrm{D}}^{25}=33.33\left(c=0.66, \mathrm{CHCl}_{3}\right) .97 \%$ ee (HPLC condition: Chiralpak AD-H column, $n$-Hexane $/ i-\mathrm{PrOH}=70: 30$, flow rate $=1.0 \mathrm{~mL} / \mathrm{min}$, 
wavelength $=254 \mathrm{~nm}, t_{\mathrm{R}}=23.01$ min for major isomer, $t_{\mathrm{R}}=28.77$ min for minor isomer).

$\mathrm{mV}$

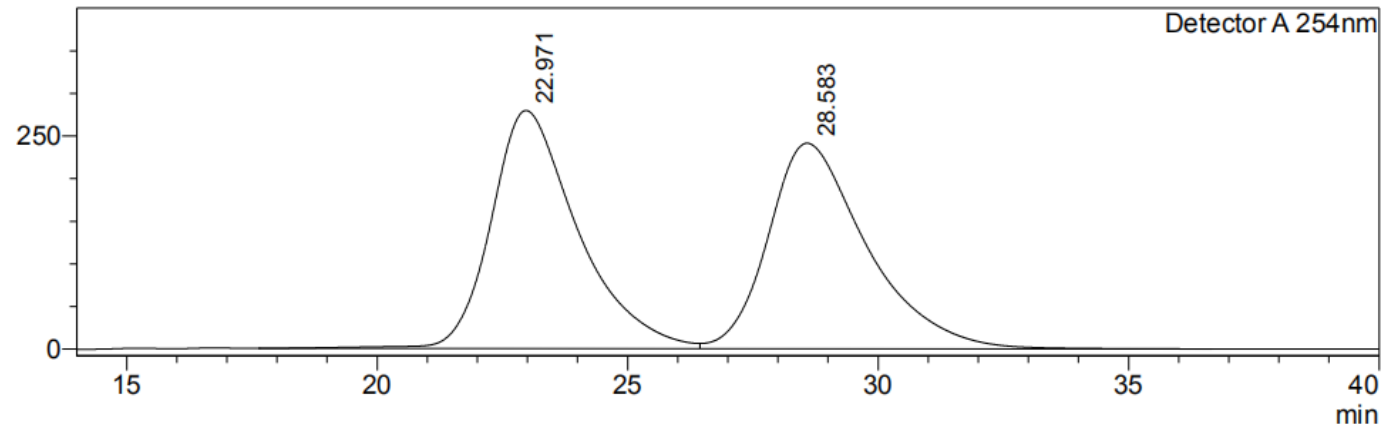

Detector A 254nm

\begin{tabular}{|r|r|r|r|r|r|}
\hline Peak\# & Ret. Time & \multicolumn{1}{c|}{ Height } & \multicolumn{1}{c|}{ Height\% } & \multicolumn{1}{c|}{ Area } & \multicolumn{1}{c|}{ Area\% } \\
\hline 1 & 22.971 & 279226 & 53.670 & 33731825 & 50.464 \\
\hline 2 & 28.583 & 241034 & 46.330 & 33111650 & 49.536 \\
\hline Total & & 520260 & 100.000 & 66843475 & 100.000 \\
\hline
\end{tabular}

$\mathrm{mV}$

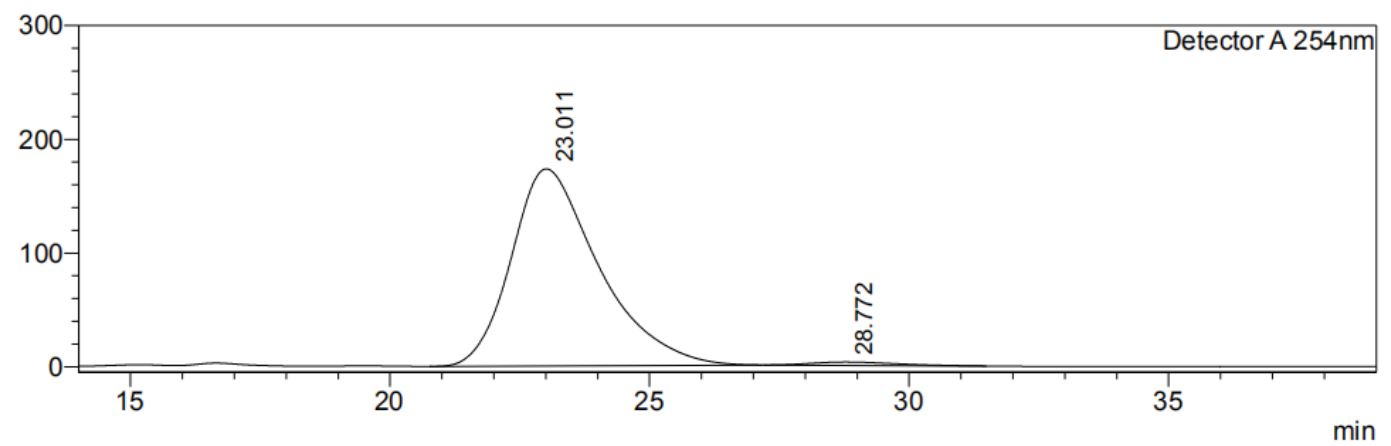

Detector A 254nm

\begin{tabular}{|r|r|r|r|r|r|}
\hline Peak\# & Ret. Time & \multicolumn{1}{c|}{ Height } & \multicolumn{1}{c|}{ Height $\%$} & \multicolumn{1}{c|}{ Area } & \multicolumn{1}{c|}{ Area\% } \\
\hline 1 & 23.011 & 173268 & 98.361 & 20376288 & 98.406 \\
\hline 2 & 28.772 & 2887 & 1.639 & 330139 & 1.594 \\
\hline Total & & 176156 & 100.000 & 20706427 & 100.000 \\
\hline
\end{tabular}

\section{(S)-2-(2-hydroxyphenylsulfonimidoyl)phenyl bis(4-phenoxyphenyl)phosphinate}

$\underline{(4 g)}$<smiles>N[SH](=O)(Oc1ccccc1OP(=O)(c1ccccc1)c1ccc(Oc2ccccc2)cc1)c1ccccc1O</smiles>

White powder; $49 \mathrm{mg}, 78 \%$ yield; m.p. $=81-83{ }^{\circ} \mathrm{C} ;{ }^{1} \mathrm{H}$ NMR $\left(400 \mathrm{MHz}, \mathrm{CDCl}_{3}\right) \delta$ $7.86(\mathrm{~d}, J=8.6 \mathrm{~Hz}, 1 \mathrm{H}), 7.82(\mathrm{~d}, J=8.6 \mathrm{~Hz}, 1 \mathrm{H}), 7.75(\mathrm{~d}, J=8.7 \mathrm{~Hz}, 1 \mathrm{H}), 7.72(\mathrm{~d}, J$ $=8.7 \mathrm{~Hz}, 1 \mathrm{H}), 7.64(\mathrm{~d}, J=8.2 \mathrm{~Hz}, 1 \mathrm{H}), 7.44(\mathrm{dd}, J=8.2,1.4 \mathrm{~Hz}, 1 \mathrm{H}), 7.42-7.36(\mathrm{~m}$, $5 \mathrm{H}), 7.20(\mathrm{t}, J=7.4 \mathrm{~Hz}, 2 \mathrm{H}), 7.15(\mathrm{t}, J=7.6 \mathrm{~Hz}, 1 \mathrm{H}), 7.09-7.03(\mathrm{~m}, 6 \mathrm{H}), 6.99(\mathrm{dd}, J$ 
$=8.7,3.0 \mathrm{~Hz}, 2 \mathrm{H}), 6.93(\mathrm{~d}, J=8.2 \mathrm{~Hz}, 1 \mathrm{H}), 6.74(\mathrm{t}, J=7.6 \mathrm{~Hz}, 1 \mathrm{H}) ;{ }^{13} \mathrm{C} \mathrm{NMR}(100$ $\left.\mathrm{MHz}, \mathrm{CDCl}_{3}\right) \delta 162.1(\mathrm{~d}, J=1.6 \mathrm{~Hz}), 162.0(\mathrm{~d}, J=1.7 \mathrm{~Hz}), 157.4,155.3(\mathrm{~d}, J=14.0$ Hz), $149.4(\mathrm{~d}, J=7.2 \mathrm{~Hz}), 135.7(\mathrm{~d}, J=78.4 \mathrm{~Hz}), 134.3(\mathrm{~d}, J=3.0 \mathrm{~Hz}), 134.2(\mathrm{~d}, J=$ $3.1 \mathrm{~Hz}), 130.2$, 129.6, 129.3, 125.0 (d, $J=5.4 \mathrm{~Hz}), 124.2$, 123.9, 123.4, 122.4, 122.0, $121.5(\mathrm{~d}, J=5.0 \mathrm{~Hz}), 120.4(\mathrm{~d}, J=6.3 \mathrm{~Hz}), 119.4,119.3(\mathrm{~d}, J=8.6 \mathrm{~Hz}), 117.8(\mathrm{~d}, J=$ $4.4 \mathrm{~Hz}), 117.7(\mathrm{~d}, J=4.3 \mathrm{~Hz}) ;{ }^{31} \mathrm{P}$ NMR $\left(162 \mathrm{MHz}, \mathrm{CDCl}_{3}\right) \delta 34.31$; HRMS (ESI) $m / z$ calcd for $\mathrm{C}_{36} \mathrm{H}_{28} \mathrm{NO}_{6} \mathrm{PS}[\mathrm{M}+\mathrm{H}]^{+}=634.1453$, found $=634.1453$.

Optical Rotation: $[\alpha]^{25}=34.00\left(c=0.50, \mathrm{CHCl}_{3}\right) .93 \%$ ee (HPLC condition: Chiralpak AD-H column, $n$-Hexane $/ i-\mathrm{PrOH}=60: 40$, flow rate $=1.0 \mathrm{~mL} / \mathrm{min}$, wavelength $=254 \mathrm{~nm}, t_{\mathrm{R}}=15.64$ min for major isomer, $t_{\mathrm{R}}=23.06$ min for minor isomer).

$\mathrm{mV}$

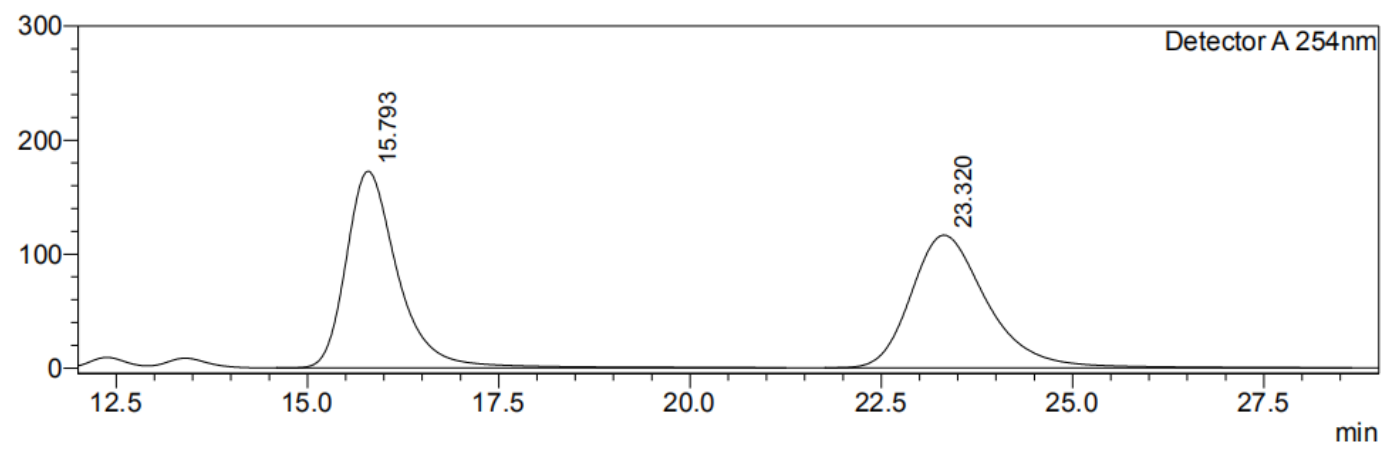

Detector A 254nm

\begin{tabular}{|r|r|r|r|r|r|}
\hline Peak\# & Ret. Time & Height & \multicolumn{1}{c|}{ Height\% } & \multicolumn{1}{c|}{ Area } & \multicolumn{1}{c|}{ Area\% } \\
\hline 1 & 15.793 & 172567 & 59.722 & 8141050 & 50.199 \\
\hline 2 & 23.320 & 116385 & 40.278 & 8076651 & 49.801 \\
\hline Total & & 288952 & 100.000 & 16217701 & 100.000 \\
\hline
\end{tabular}

$\mathrm{mV}$

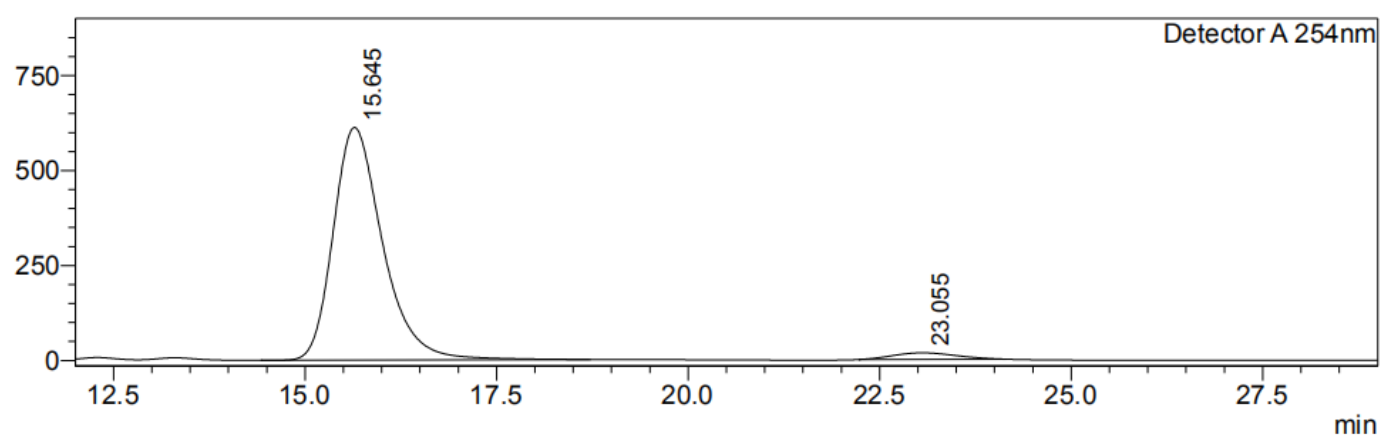

Detector A 254nm

\begin{tabular}{|r|r|r|r|r|r|}
\hline Peak\# & Ret. Time & Height & Height\% & \multicolumn{1}{c|}{ Area } & \multicolumn{1}{c|}{ Area\% } \\
\hline 1 & 15.645 & 612338 & 97.261 & 27530648 & 96.545 \\
\hline 2 & 23.055 & 17246 & 2.739 & 985128 & 3.455 \\
\hline Total & & 629584 & 100.000 & 28515776 & 100.000 \\
\hline
\end{tabular}




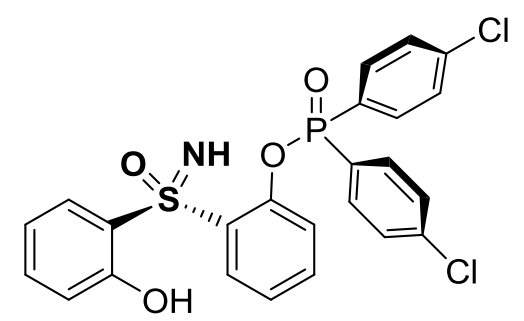

White solid; $42 \mathrm{mg}, 81 \%$ yield; m.p. $=72-73{ }^{\circ} \mathrm{C} ;{ }^{1} \mathrm{H}$ NMR $\left(400 \mathrm{MHz}, \mathrm{CDCl}_{3}\right) \delta$ $12.16(\mathrm{~s}, 1 \mathrm{H}), 7.95-7.91(\mathrm{~m}, 1 \mathrm{H}), 7.91-7.87(\mathrm{~m}, 1 \mathrm{H}), 7.80(\mathrm{dd}, J=6.6,1.1 \mathrm{~Hz}, 1 \mathrm{H})$, 7.77-7.73 (m, 1H), 7.73-7.69 (m, 1H), $7.66(\mathrm{~d}, J=8.4 \mathrm{~Hz}, 1 \mathrm{H}), 7.51-7.49(\mathrm{~m}, 1 \mathrm{H})$, 7.49-7,47 (m, 1H), 7.47-7.36 (m, 5H), 7.22-7.14 (m, 1H), $6.84(\mathrm{dd}, J=8.4,1.0 \mathrm{~Hz}$, $1 \mathrm{H}), 6.79(\mathrm{ddd}, J=8.4,7.3,1.0 \mathrm{~Hz}, 1 \mathrm{H}), 4.02(\mathrm{~s}, 1 \mathrm{H}) ;{ }^{13} \mathrm{C} \mathrm{NMR}\left(100 \mathrm{MHz}, \mathrm{CDCl}_{3}\right)$ $\delta 157.5,148.8(\mathrm{~d}, J=7.2 \mathrm{~Hz}), 140.2(\mathrm{~d}, J=3.8 \mathrm{~Hz}), 140.1(\mathrm{~d}, J=3.8 \mathrm{~Hz}), 135.4(\mathrm{~d}, J$ $=81.2 \mathrm{~Hz}), 134.3(\mathrm{~d}, J=7.2 \mathrm{~Hz}), 133.6(\mathrm{~d}, J=1.2 \mathrm{~Hz}), 133.5(\mathrm{~d}, J=0.9 \mathrm{~Hz}), 129.5$ $(\mathrm{d}, J=3.6 \mathrm{~Hz}), 129.4,129.3(\mathrm{~d}, J=3.8 \mathrm{~Hz}), 129.1,128.5(\mathrm{~d}, J=31.1 \mathrm{~Hz}), 127.1(\mathrm{~d}, J$ $=30.3 \mathrm{~Hz}), 124.6,121.4(\mathrm{~d}, J=4.9 \mathrm{~Hz}), 119.9,119.5(\mathrm{~d}, J=10.0 \mathrm{~Hz}) ;{ }^{31} \mathrm{P}$ NMR $(162$ $\left.\mathrm{MHz}, \mathrm{CDCl}_{3}\right) \delta$ 31.92; HRMS (ESI) $\mathrm{m} / \mathrm{z}$ calcd for $\mathrm{C}_{24} \mathrm{H}_{18} \mathrm{C}_{12} \mathrm{NO}_{4} \mathrm{PS}[\mathrm{M}+\mathrm{H}]^{+}=$ 518.0149 , found $=518.0151$.

Optical Rotation: $[\alpha]_{\mathrm{D}}^{25}=15.80\left(c=0.50, \mathrm{CHCl}_{3}\right) .70 \%$ ee (HPLC condition: Chiralpak AD-H column, $n$-Hexane $/ i-\mathrm{PrOH}=60: 40$, flow rate $=1.0 \mathrm{~mL} / \mathrm{min}$, wavelength $=254 \mathrm{~nm}, t_{\mathrm{R}}=15.84 \mathrm{~min}$ for minor isomer, $t_{\mathrm{R}}=20.06 \mathrm{~min}$ for major isomer).

$\mathrm{mV}$

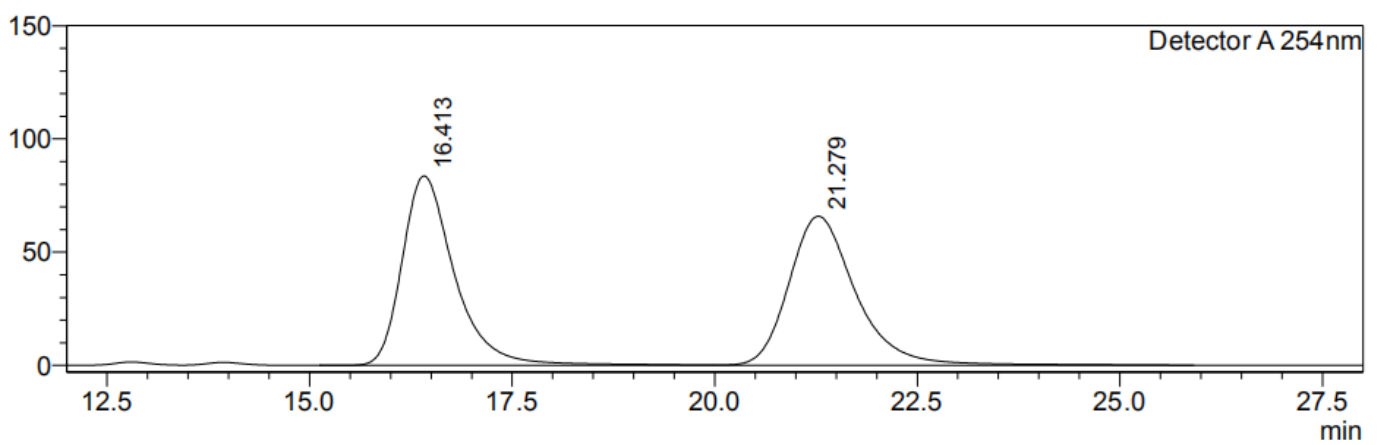

\begin{tabular}{|c|c|c|c|c|c|}
\hline \multicolumn{5}{|c|}{ Lectol $A 254$ IIII } & Area\% \\
\hline 1 & 16.413 & 83679 & 55.967 & 3787083 & 49.969 \\
\hline 2 & 21.279 & 65836 & 44.033 & 3791830 & 50.031 \\
\hline Total & & 149516 & 100.000 & 7578913 & 100.000 \\
\hline
\end{tabular}


$\mathrm{mV}$

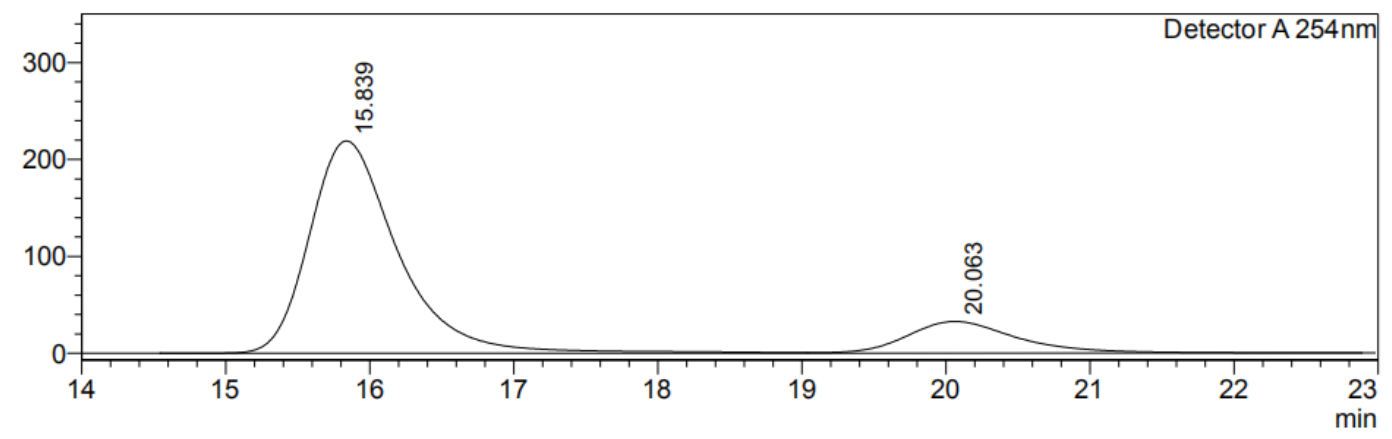

Detector A 254nm
\begin{tabular}{|r|r|r|r|r|r|}
\hline Peak\# & Ret. Time & Height & Height\% & \multicolumn{1}{c|}{ Area } & Area\% \\
\hline 1 & 15.839 & 219046 & 87.051 & 9395923 & 84.344 \\
\hline 2 & 20.063 & 32585 & 12.949 & 1744052 & 15.656 \\
\hline Total & & 251631 & 100.000 & 11139975 & 100.000 \\
\hline
\end{tabular}

(S)-2-(2-hydroxyphenylsulfonimidoyl)phenyl bis(3-methoxyphenyl)phosphinate

$\underline{(4 \mathbf{i})}$<smiles>COc1cccc(P(=O)(Oc2ccccc2S(=N)(=O)c2ccccc2O)c2cccc(OC)c2)c1</smiles>

Colorless oil; 49 mg, 84\% yield; ${ }^{1} \mathrm{H}$ NMR (400 MHz, $\left.\mathrm{CDCl}_{3}\right) \delta$ 7.68-7.52 (m, 6H), 7.45-7.32 (m, 5H), 7.14-7.06 (m, 3H), $6.84(\mathrm{~d}, J=8.4 \mathrm{~Hz}, 1 \mathrm{H}), 6.77(\mathrm{t}, J=7.6 \mathrm{~Hz}$, $1 \mathrm{H}), 3.83(\mathrm{~d}, J=1.8 \mathrm{~Hz}, 6 \mathrm{H}) ;{ }^{13} \mathrm{C} \mathrm{NMR}\left(100 \mathrm{MHz}, \mathrm{CDCl}_{3}\right) \delta 159.9(\mathrm{~d}, J=4.5$ Hz),159.8 (d, $J=4.6 \mathrm{~Hz}), 157.5,149.0(\mathrm{~d}, J=7.4 \mathrm{~Hz}), 136.1,135.3(\mathrm{~d}, J=126.0 \mathrm{~Hz})$, 134.8, $131.6(\mathrm{~d}, J=5.6 \mathrm{~Hz}), 130.2(\mathrm{~d}, J=6.0 \mathrm{~Hz}), 130.2(\mathrm{~d}, J=16.6 \mathrm{~Hz}), 129.0(\mathrm{~d}, J$ $=86.9 \mathrm{~Hz}), 124.6(\mathrm{~d}, J=10.9 \mathrm{~Hz}), 124.4(\mathrm{~d}, J=10.8 \mathrm{~Hz}), 121.5(\mathrm{~d}, J=5.0 \mathrm{~Hz})$, $119.9(\mathrm{~d}, J=6.9 \mathrm{~Hz}), 119.6,119.3(\mathrm{~d}, J=19.6 \mathrm{~Hz}), 116.4(\mathrm{~d}, J=12.3 \mathrm{~Hz}), 116.2(\mathrm{~d}$, $J=12.1 \mathrm{~Hz}), 55.8(\mathrm{~d}, J=7.4 \mathrm{~Hz}) ;{ }^{31} \mathrm{P}$ NMR $\left(162 \mathrm{MHz}, \mathrm{CDCl}_{3}\right) \delta 33.80 ;$ HRMS (ESI) $m / z$ calcd for $\mathrm{C}_{26} \mathrm{H}_{24} \mathrm{NO}_{6} \mathrm{PS}[\mathrm{M}+\mathrm{H}]^{+}=510.1140$, found $=510.1132$.

Optical Rotation: $[\alpha]_{\mathrm{D}}^{25}=55.00\left(c=0.20, \mathrm{CHCl}_{3}\right) .80 \%$ ee (HPLC condition: Chiralpak AD-H column, $n$-Hexane $/ i-\mathrm{PrOH}=70: 30$, flow rate $=1.0 \mathrm{~mL} / \mathrm{min}$, wavelength $=254 \mathrm{~nm}, t_{\mathrm{R}}=17.74$ min for major isomer, $t_{\mathrm{R}}=24.79 \min$ for minor isomer). 
$\mathrm{mV}$

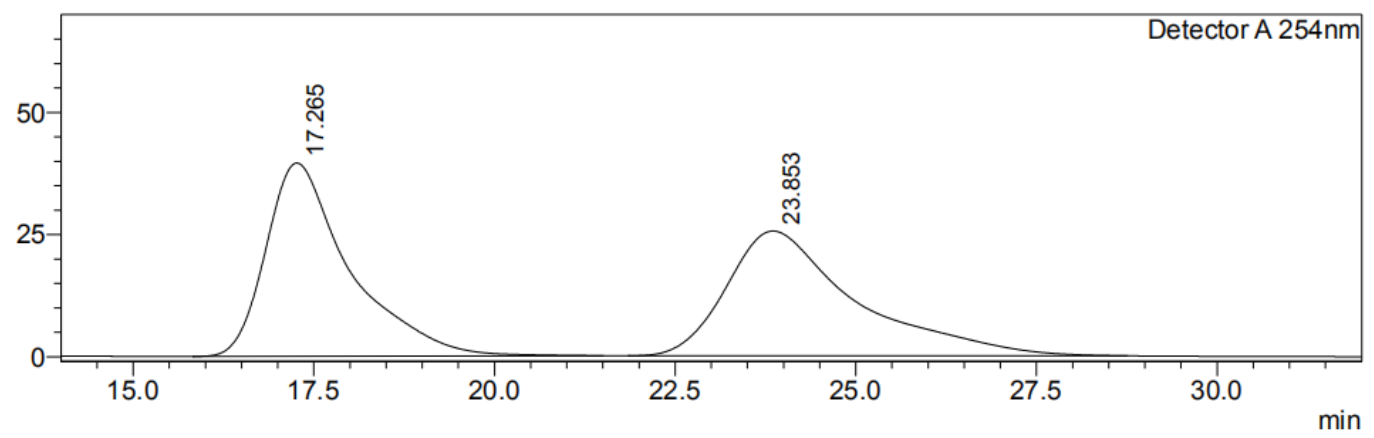

Detector A $254 \mathrm{~nm}$
\begin{tabular}{|r|r|r|r|r|r|}
\hline Peak\# & Ret. Time & Height & Height\% & Area & \multicolumn{1}{|c|}{ Area\% } \\
\hline 1 & 17.265 & 39546 & 60.762 & 3178768 & 50.123 \\
\hline 2 & 23.853 & 25538 & 39.238 & 3163169 & 49.877 \\
\hline Total & & 65084 & 100.000 & 6341937 & 100.000 \\
\hline
\end{tabular}

$\mathrm{mV}$

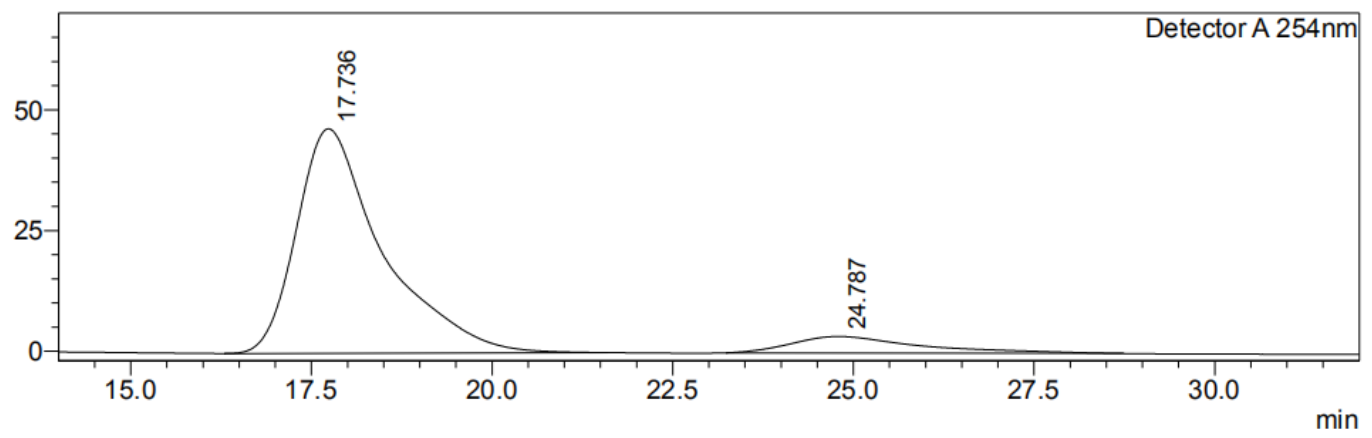

Detector A 254nm

\begin{tabular}{|r|r|r|r|r|r|}
\hline Peak\# & Ret. Time & Height & Height\% & \multicolumn{1}{c|}{ Area } & \multicolumn{1}{c|}{ Area\% } \\
\hline 1 & 17.736 & 46456 & 93.150 & 3840983 & 90.139 \\
\hline 2 & 24.787 & 3416 & 6.850 & 420218 & 9.861 \\
\hline Total & & 49872 & 100.000 & 4261202 & 100.000 \\
\hline
\end{tabular}

\section{$\underline{(S)-2-(2-h y d r o x y p h e n y l s u l f o n i m i d o y l})$ phenyl bis(3,5-dimethylphenyl)phosphinate}

$\underline{(4 \mathbf{j})}$<smiles>Cc1cc(C)cc(P(=O)(Oc2ccccc2S(=N)(=O)c2ccccc2O)c2cc(C)cc(C)c2)c1</smiles>

White solid; $38 \mathrm{mg}, 75 \%$ yield; m.p. $=192-194{ }^{\circ} \mathrm{C} ;{ }^{1} \mathrm{H}$ NMR $\left(400 \mathrm{MHz}, \mathrm{CDCl}_{3}\right) \delta$ $12.11(\mathrm{~s}, 1 \mathrm{H}), 7.75(\mathrm{~d}, J=7.6 \mathrm{~Hz}, 1 \mathrm{H}), 7.60-7.52(\mathrm{~m}, 3 \mathrm{H}), 7.48(\mathrm{dd}, J=8.2,1.6 \mathrm{~Hz}$, 1H), 7.46-7.43 (m, 2H), 7.41-7.31 (m, 2H), $7.20(\mathrm{~s}, 1 \mathrm{H}), 7.17(\mathrm{~s}, 1 \mathrm{H})$, 7.15-7.07 (m, 1H), 6.80 (dd, $J=8.4,0.9 \mathrm{~Hz}, 1 \mathrm{H}), 6.78-6.73(\mathrm{~m}, 1 \mathrm{H}), 4.19$ (s, 1H), 2.33 (d, $J=11.8$ $\mathrm{Hz}, 12 \mathrm{H}) ;{ }^{13} \mathrm{C} \mathrm{NMR}\left(100 \mathrm{MHz}, \mathrm{CDCl}_{3}\right) \delta 157.6,149.2(\mathrm{~d}, J=7.6 \mathrm{~Hz}), 138.6(\mathrm{~d}, J=$ 
$9.2 \mathrm{~Hz}), 138.5(\mathrm{~d}, J=9.3 \mathrm{~Hz}), 135.1(\mathrm{~d}, J=85.0 \mathrm{~Hz}), 134.8(\mathrm{~d}, J=2.8 \mathrm{~Hz}), 134.4(\mathrm{~d}$, $J=6.9 \mathrm{~Hz}), 130.3(\mathrm{~d}, J=8.2 \mathrm{~Hz}), 129.7,129.6,129.4(\mathrm{~d}, J=12.0 \mathrm{~Hz}), 129.0,128.9$ $(\mathrm{d}, J=8.0 \mathrm{~Hz}), 123.9,121.7(\mathrm{~d}, J=5.0 \mathrm{~Hz}), 120.1,119.2(\mathrm{~d}, J=11.3 \mathrm{~Hz}), 21.4 ;{ }^{31} \mathrm{P}$ NMR (162 MHz, $\left.\mathrm{CDCl}_{3}\right) \delta 35.71$; HRMS (ESI) $m / z$ calcd for $\mathrm{C}_{28} \mathrm{H}_{28} \mathrm{NO}_{4} \mathrm{PS}[\mathrm{M}+\mathrm{H}]^{+}=$ 506.1555 , found $=506.1545$.

Optical Rotation: $[\alpha]_{\mathrm{D}}^{25}=41.89\left(c=0.53, \mathrm{CHCl}_{3}\right) .96 \%$ ee (HPLC condition: Chiralpak AD-H column, $n$-Hexane $/ i-\mathrm{PrOH}=80: 20$, flow rate $=0.6 \mathrm{~mL} / \mathrm{min}$, wavelength $=254 \mathrm{~nm}, t_{\mathrm{R}}=17.55 \mathrm{~min}$ for minor isomer, $t_{\mathrm{R}}=20.52 \min$ for major isomer).

$\mathrm{mV}$

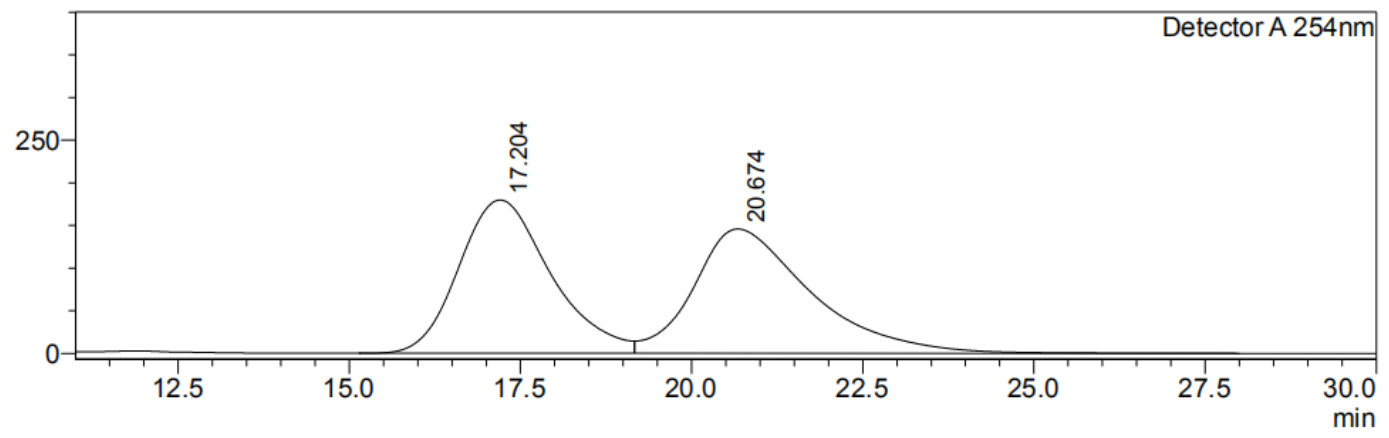

Detector A $254 \mathrm{~nm}$
\begin{tabular}{|r|r|r|r|r|r|}
\hline Peak\# & Ret. Time & Height & Height $\%$ & Area & \multicolumn{1}{c|}{ Area\% } \\
\hline 1 & 17.204 & 179624 & 55.227 & 16788560 & 49.181 \\
\hline 2 & 20.674 & 145624 & 44.773 & 17347842 & 50.819 \\
\hline Total & & 325247 & 100.000 & 34136402 & 100.000 \\
\hline
\end{tabular}

$\mathrm{mV}$

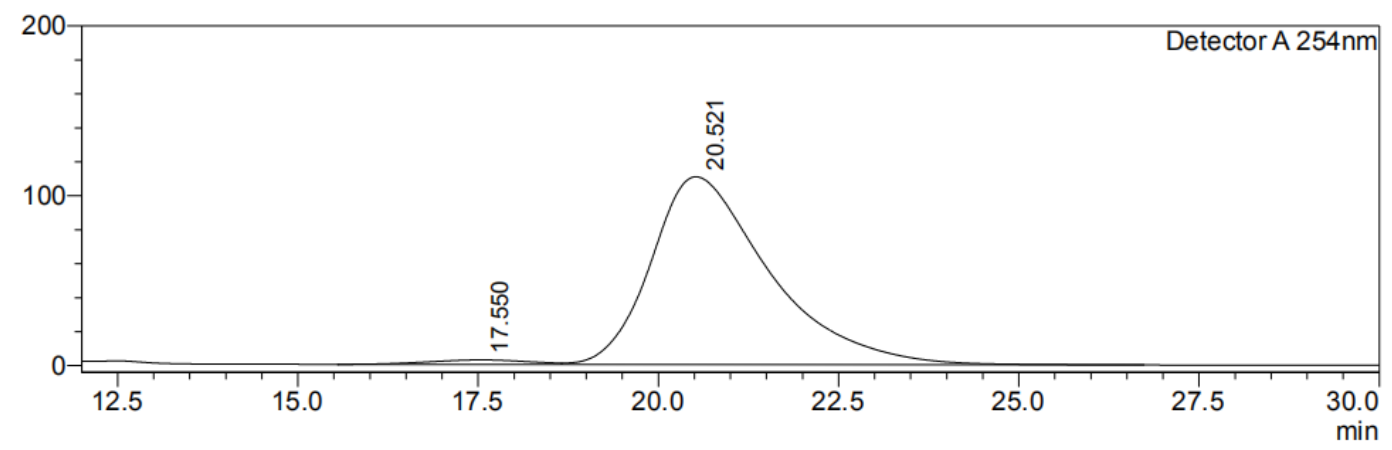

Detector A 254nm

\begin{tabular}{|r|r|r|r|r|r|}
\hline Peak\# & Ret. Time & \multicolumn{1}{c|}{ Height } & \multicolumn{1}{c|}{ Height\% } & \multicolumn{1}{c|}{ Area } & \multicolumn{1}{c|}{ Area\% } \\
\hline 1 & 17.550 & 2711 & 2.393 & 261788 & 2.024 \\
\hline 2 & 20.521 & 110610 & 97.607 & 12673043 & 97.976 \\
\hline Total & & 113321 & 100.000 & 12934832 & 100.000 \\
\hline
\end{tabular}

\section{(S)-2-(2-hydroxyphenylsulfonimidoyl)phenyl bis(3,5-dimethylphenyl)phosphinate}

$\underline{(4 \mathbf{k})}$ 


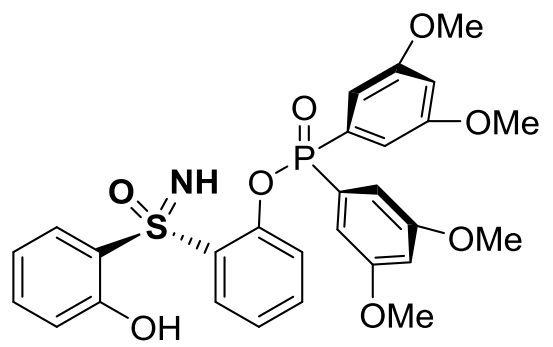

Colorless oil; $44 \mathrm{mg}, 77 \%$ yield; ${ }^{1} \mathrm{H}$ NMR (400 MHz, $\left.\mathrm{CDCl}_{3}\right) \delta$ 7.66-7.54 (m, 3H), 7.45-7.40 (m, 1H), 7.39-7.34 (m, 1H), $7.18(\mathrm{dd}, J=6.6,2.2 \mathrm{~Hz}, 2 \mathrm{H}), 7.14(\mathrm{dd}, J=$ 6.6, 2.2 Hz, 2H), $7.10(\mathrm{t}, J=7.4 \mathrm{~Hz}, 1 \mathrm{H}), 6.96(\mathrm{~d}, J=8.3 \mathrm{~Hz}, 1 \mathrm{H}), 6.77(\mathrm{t}, J=7.6 \mathrm{~Hz}$, $1 \mathrm{H}), 6.63(\mathrm{t}, J=2.2 \mathrm{~Hz}, 1 \mathrm{H}), 6.61(\mathrm{t}, J=2.2 \mathrm{~Hz}, 1 \mathrm{H}), 3.80(\mathrm{~d}, J=1.2 \mathrm{~Hz}, 12 \mathrm{H}) ;{ }^{13} \mathrm{C}$ NMR $\left(100 \mathrm{MHz}, \mathrm{CDCl}_{3}\right) \delta 161.3(\mathrm{~d}, J=4.4 \mathrm{~Hz}), 161.1(\mathrm{~d}, J=4.4 \mathrm{~Hz}), 157.3,149.1$ $(\mathrm{d}, J=8.2 \mathrm{~Hz}), 135.7(\mathrm{~d}, J=143.5 \mathrm{~Hz}), 133.6,132.0,130.6,129.5,128.7,124.4$, $121.4(\mathrm{~d}, J=4.8 \mathrm{~Hz}), 119.5,119.2,118.9,109.6(\mathrm{~d}, J=7.4 \mathrm{~Hz}), 109.4(\mathrm{~d}, J=7.3 \mathrm{~Hz})$, $106.1(\mathrm{~d}, J=2.6 \mathrm{~Hz}), 106.0(\mathrm{~d}, J=2.3 \mathrm{~Hz}), 55.9(\mathrm{~d}, J=8.5 \mathrm{~Hz}) ;{ }^{31} \mathrm{P}$ NMR $(162 \mathrm{MHz}$, $\left.\mathrm{CDCl}_{3}\right) \delta 34.01$; HRMS (ESI) $\mathrm{m} / z$ calcd for $\mathrm{C}_{28} \mathrm{H}_{28} \mathrm{NO}_{4} \mathrm{PS}[\mathrm{M}+\mathrm{H}]^{+}=570.1351$, found $=570.1350$.

Optical Rotation: $[\alpha]_{\mathrm{D}}^{25}=23.08\left(c=0.59, \mathrm{CHCl}_{3}\right) .95 \%$ ee (HPLC condition: Chiralpak IE column, $n$-Hexane $/ i-\mathrm{PrOH}=60: 40$, flow rate $=1.0 \mathrm{~mL} / \mathrm{min}$, wavelength $=254 \mathrm{~nm}, t_{\mathrm{R}}=32.56 \mathrm{~min}$ for major isomer, $t_{\mathrm{R}}=45.97 \mathrm{~min}$ for minor isomer). $\mathrm{mV}$

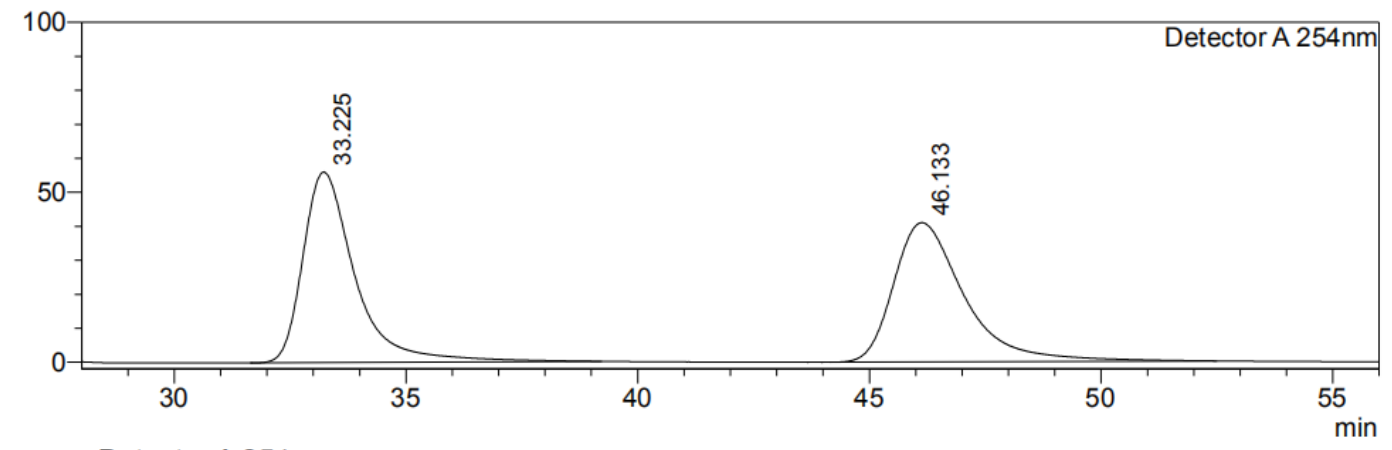

Detector A 254nm
\begin{tabular}{|r|r|r|r|r|r|}
\hline Peak\# & Ret. Time & Height & Height\% & Area & \multicolumn{1}{c|}{ Area\% } \\
\hline 1 & 33.225 & 56075 & 57.768 & 4393914 & 50.236 \\
\hline 2 & 46.133 & 40995 & 42.232 & 4352613 & 49.764 \\
\hline Total & & 97069 & 100.000 & 8746527 & 100.000 \\
\hline
\end{tabular}


$\mathrm{mV}$

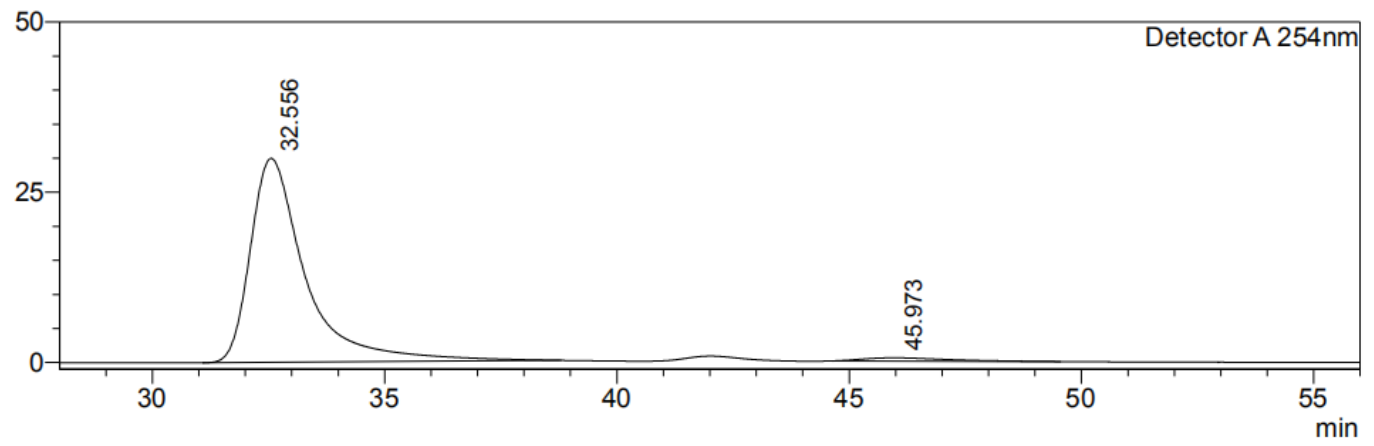

\begin{tabular}{|c|c|c|c|c|c|}
\hline Peak\# & Ret. Time & Height & Height\% & Area & Area\% \\
\hline 1 & 32.556 & 29966 & 98.404 & 2480482 & 97.574 \\
\hline 2 & 45.973 & 486 & 1.596 & 61678 & 2.426 \\
\hline Total & & 30452 & 100.000 & 2542161 & 100.000 \\
\hline
\end{tabular}

\section{$\underline{\text { (S)-2-(2-hydroxyphenylsulfonimidoyl)phenyl di-o-tolylphosphinate (4I) }}$}

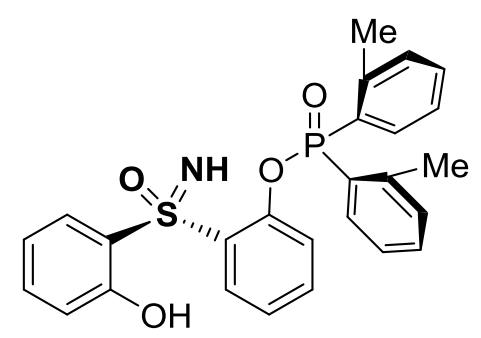

Colorless oil; $44 \mathrm{mg}, 92 \%$ yield; ${ }^{1} \mathrm{H}$ NMR $\left(400 \mathrm{MHz}, \mathrm{CDCl}_{3}\right) \delta 7.93(\mathrm{~d}, J=8.0 \mathrm{~Hz}$, 1H), 7.53-7.40 (m, 4H), 7.40-7.34 (m, 3H), 7.33-7.27 (m, 3H), 7.25-7.13 (m, 3H), $6.76(\mathrm{~d}, J=8.2 \mathrm{~Hz}, 1 \mathrm{H}), 6.70(\mathrm{t}, J=7.6 \mathrm{~Hz}, 1 \mathrm{H}), 2.46(\mathrm{~d}, J=33.8 \mathrm{~Hz}, 6 \mathrm{H}) ;{ }^{13} \mathrm{C} \mathrm{NMR}$ $\left(100 \mathrm{MHz}, \mathrm{CDCl}_{3}\right) \delta 157.7,149.4(\mathrm{~d}, J=7.4 \mathrm{~Hz}), 143.0(\mathrm{~d}, J=11.5 \mathrm{~Hz}), 142.8(\mathrm{~d}, J$ = $10.7 \mathrm{~Hz}), 135.5(\mathrm{~d}, J=71.6 \mathrm{~Hz}), 133.2(\mathrm{~d}, J=12.0 \mathrm{~Hz}), 133.1(\mathrm{~d}, J=1.5 \mathrm{~Hz})$, $133.0(\mathrm{~d}, J=2.0 \mathrm{~Hz}), 132.1(\mathrm{~d}, J=1.3 \mathrm{~Hz}), 131.9(\mathrm{~d}, J=1.6 \mathrm{~Hz}), 129.4(\mathrm{~d}, J=46.3$ Hz), $128.8(\mathrm{~d}, J=41.2 \mathrm{~Hz}), 127.5(\mathrm{~d}, J=40.8 \mathrm{~Hz}), 125.9$ (d, $J=1.7 \mathrm{~Hz}), 125.8(\mathrm{~d}, J$ $=2.3 \mathrm{~Hz}), 124.1,121.7(\mathrm{~d}, J=4.6 \mathrm{~Hz}), 119.6,119.2(\mathrm{~d}, J=2.4 \mathrm{~Hz}), 21.5(\mathrm{~d}, J=4.6$ $\mathrm{Hz}) ;{ }^{31} \mathrm{P}$ NMR $\left(162 \mathrm{MHz}, \mathrm{CDCl}_{3}\right) \delta 33.37$; HRMS (ESI) $\mathrm{m} / z$ calcd for $\mathrm{C}_{26} \mathrm{H}_{24} \mathrm{NO}_{4} \mathrm{PS}$ $[\mathrm{M}+\mathrm{H}]^{+}=478.1242$, found $=478.1246$.

Optical Rotation: $[\alpha]_{\mathrm{D}}^{25}=43.33\left(c=0.50, \mathrm{CHCl}_{3}\right) .81 \%$ ee (HPLC condition: Chiralpak AD-H column, $n$-Hexane $/ i-\mathrm{PrOH}=90: 10$, flow rate $=1.0 \mathrm{~mL} / \mathrm{min}$, wavelength $=254 \mathrm{~nm}, t_{\mathrm{R}}=36.38 \mathrm{~min}$ for minor isomer, $t_{\mathrm{R}}=48.41 \mathrm{~min}$ for major isomer). 
$\mathrm{mV}$

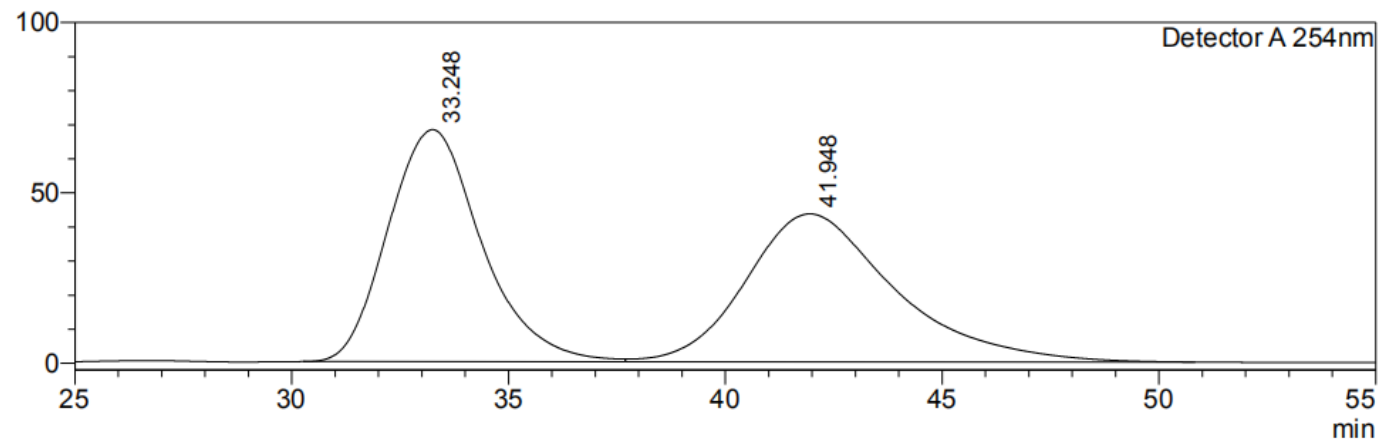

Detector A $254 \mathrm{~nm}$

\begin{tabular}{|r|r|r|r|r|r|}
\hline Peak\# & Ret. Time & Height & Height\% & Area & \multicolumn{1}{c|}{ Area\% } \\
\hline 1 & 33.248 & 68083 & 61.055 & 10248557 & 49.659 \\
\hline 2 & 41.948 & 43428 & 38.945 & 10389417 & 50.341 \\
\hline Total & & 111511 & 100.000 & 20637974 & 100.000 \\
\hline
\end{tabular}

$\mathrm{mV}$

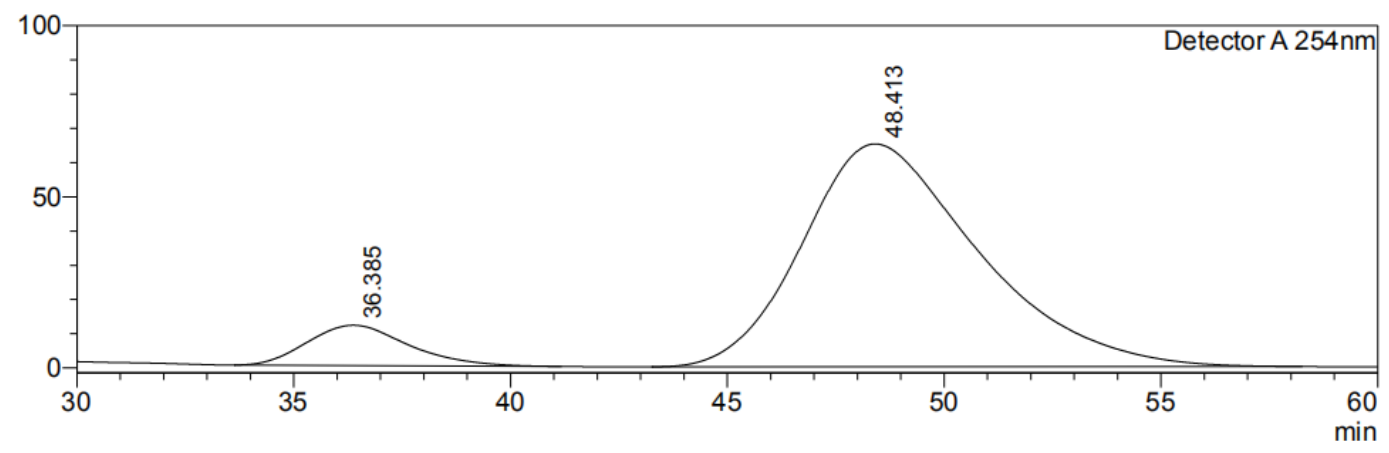

Detector A 254nm

\begin{tabular}{|r|r|r|r|r|r|}
\hline Peak\# & Ret. Time & \multicolumn{1}{c|}{ Height } & Height\% & \multicolumn{1}{|c|}{ Area } & \multicolumn{1}{c|}{ Area\% } \\
\hline 1 & 36.385 & 11796 & 15.338 & 1907666 & 9.403 \\
\hline 2 & 48.413 & 65110 & 84.662 & 18380801 & 90.597 \\
\hline Total & & 76906 & 100.000 & 20288467 & 100.000 \\
\hline
\end{tabular}

(S)-2-(2-hydroxyphenylsulfonimidoyl)phenyl di(naphthalen-2-yl)phosphinate

$\underline{(4 m)}$

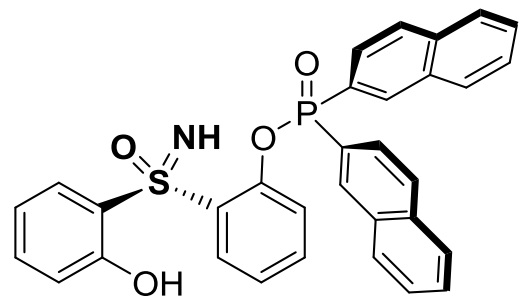

Light yellow solid; $47 \mathrm{mg}, 86 \%$ yield; m.p. $=93-95^{\circ} \mathrm{C} ;{ }^{1} \mathrm{H}$ NMR $\left(400 \mathrm{MHz}, \mathrm{CDCl}_{3}\right) \delta$ 12.09 (s, 1H), 8.62 (d, $J=15.0 \mathrm{~Hz}, 1 \mathrm{H}), 8.56$ (d, $J=15.0 \mathrm{~Hz}, 1 \mathrm{H}), 8.08-8.00$ (m, 1H), 7.98-7.91 (m, 3H), 7.91-7.82 (m, 4H), $7.80(\mathrm{dd}, J=6.0,4.8 \mathrm{~Hz}, 1 \mathrm{H}), 7.74(\mathrm{~d}, J=8.4$ $\mathrm{Hz}, 1 \mathrm{H}), 7.65-7.54(\mathrm{~m}, 4 \mathrm{H}), 7.52(\mathrm{dd}, J=8.2,1.6 \mathrm{~Hz}, 1 \mathrm{H}), 7.34(\mathrm{qd}, J=8.4,1.6 \mathrm{~Hz}$, 2H), 7.15-7.08 (m, 1H), 6.77-6.64 (m, 2H), 4.33 (s, 1H); $\left.{ }^{13} \mathrm{C} \mathrm{NMR} \mathrm{(100} \mathrm{MHz,} \mathrm{CDCl}_{3}\right)$ 
$\delta 157.5,149.2(\mathrm{~d}, J=7.4 \mathrm{~Hz}), 135.4(\mathrm{~d}, J=2.6 \mathrm{~Hz}), 135.3(\mathrm{~d}, J=81.8 \mathrm{~Hz}), 135.2(\mathrm{~d}$, $J=2.5 \mathrm{~Hz}), 134.8(\mathrm{~d}, J=1.3 \mathrm{~Hz}), 134.7(\mathrm{~d}, J=0.8 \mathrm{~Hz}), 134.4(\mathrm{~d}, J=7.0 \mathrm{~Hz}), 132.6$ $(\mathrm{d}, J=2.2 \mathrm{~Hz}), 132.4(\mathrm{~d}, J=2.4 \mathrm{~Hz}), 129.4,129.3,129.2,129.0(\mathrm{~d}, J=3.0 \mathrm{~Hz})$, 128.9, $128.0(\mathrm{~d}, J=6.2 \mathrm{~Hz}), 127.6(\mathrm{~d}, J=18.4 \mathrm{~Hz}), 127.2(\mathrm{~d}, J=3.6 \mathrm{~Hz}), 126.5(\mathrm{~d}, J$ $=8.2 \mathrm{~Hz}), 126.4(\mathrm{~d}, J=8.2 \mathrm{~Hz}), 126.2(\mathrm{~d}, J=17.6 \mathrm{~Hz}), 124.2,121.6(\mathrm{~d}, J=5.0 \mathrm{~Hz})$, 120.1, $119.3(\mathrm{~d}, J=11.8 \mathrm{~Hz}) ;{ }^{31} \mathrm{P}$ NMR $\left(162 \mathrm{MHz}, \mathrm{CDCl}_{3}\right) \delta 34.49 ; \mathrm{HRMS}(\mathrm{ESI}) \mathrm{m} / \mathrm{z}$ calcd for $\mathrm{C}_{32} \mathrm{H}_{24} \mathrm{NO}_{4} \mathrm{PS}[\mathrm{M}+\mathrm{H}]^{+}=550.1242$, found $=550.1242$.

Optical Rotation: $[\alpha]_{\mathrm{D}}^{25}=74.00\left(c=0.20, \mathrm{CHCl}_{3}\right) .96 \%$ ee (HPLC condition: Chiralpak OD-H column, $n$-Hexane $/ i-\mathrm{PrOH}=80: 20$, flow rate $=1.0 \mathrm{~mL} / \mathrm{min}$, wavelength $=254 \mathrm{~nm}, t_{\mathrm{R}}=15.15$ min for major isomer, $t_{\mathrm{R}}=19.33$ min for minor isomer).

$\mathrm{mV}$

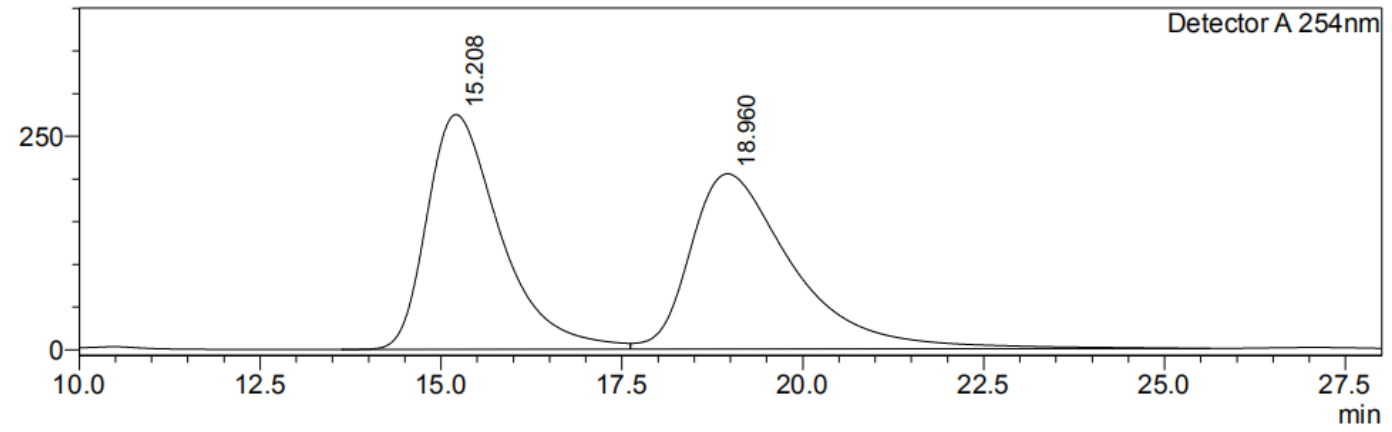

Detector A 254nm

\begin{tabular}{|r|r|r|r|r|r|}
\hline Peak\# & Ret. Time & \multicolumn{1}{c|}{ Height } & Height $\%$ & \multicolumn{1}{c|}{ Area } & \multicolumn{1}{c|}{ Area\% } \\
\hline 1 & 15.208 & 274817 & 57.259 & 19407658 & 48.900 \\
\hline 2 & 18.960 & 205137 & 42.741 & 20280743 & 51.100 \\
\hline Total & & 479954 & 100.000 & 39688401 & 100.000 \\
\hline
\end{tabular}

$\mathrm{mV}$

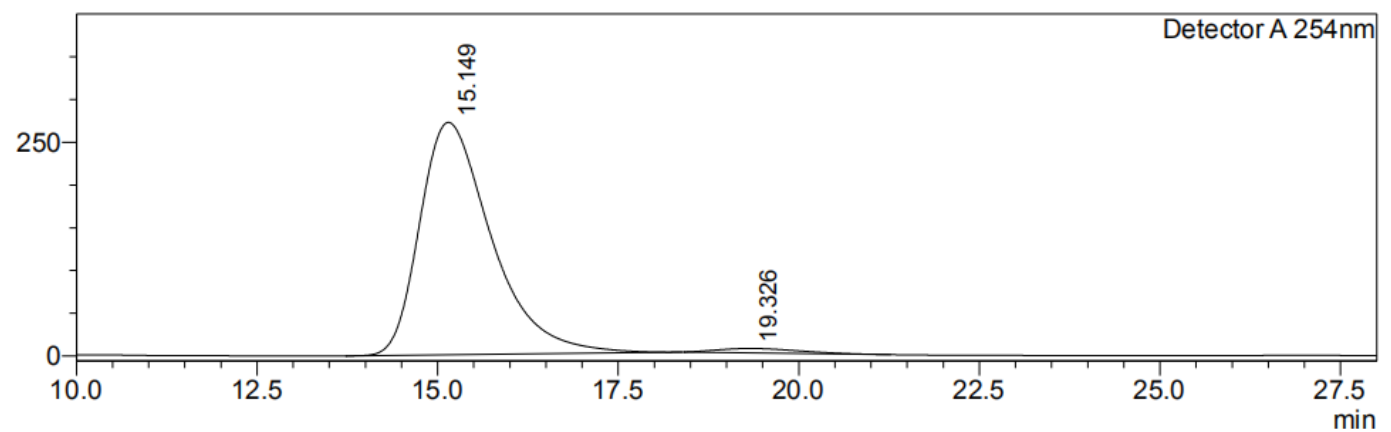

Detector A 254nm

\begin{tabular}{|r|r|r|r|r|r|}
\hline Peak\# & Ret. Time & Height & Height $\%$ & \multicolumn{1}{c|}{ Area } & \multicolumn{1}{c|}{ Area\% } \\
\hline 1 & 15.149 & 271754 & 98.149 & 18781192 & 97.872 \\
\hline 2 & 19.326 & 5126 & 1.851 & 408305 & 2.128 \\
\hline Total & & 276880 & 100.000 & 19189497 & 100.000 \\
\hline
\end{tabular}

$\underline{(S)-2-(2-h y d r o x y p h e n y l s u l f o n i m i d o y l) p h e n y l ~ b i s(b e n z o[b] \text { thiophen-5-yl)phosphin }}$ 


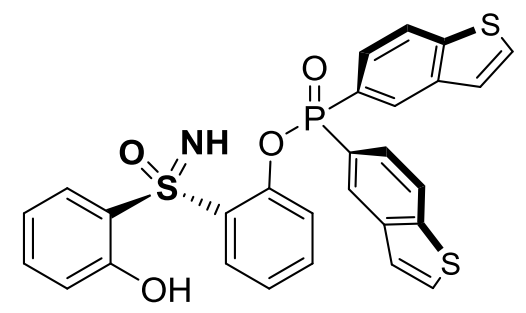

Colorless oil; 46 mg, 81\% yield; ${ }^{1} \mathrm{H}$ NMR (400 MHz, $\left.\mathrm{CDCl}_{3}\right) \delta 12.22$ (s, 1H), 8.49 (d, $J=14.0 \mathrm{~Hz}, 1 \mathrm{H}), 8.38(\mathrm{~d}, J=14.0 \mathrm{~Hz}, 1 \mathrm{H}), 8.00(\mathrm{dd}, J=8.4,3.2 \mathrm{~Hz}, 1 \mathrm{H}), 7.97-7.90$ (m, 2H), $7.80(\mathrm{~d}, J=7.8 \mathrm{~Hz}, 1 \mathrm{H}), 7.78-7.70(\mathrm{~m}, 2 \mathrm{H}), 7.54(\mathrm{t}, J=5.2 \mathrm{~Hz}, 2 \mathrm{H}), 7.47$ $(\mathrm{dd}, J=8.2,1.6 \mathrm{~Hz}, 1 \mathrm{H}), 7.43(\mathrm{~d}, J=5.6 \mathrm{~Hz}, 1 \mathrm{H}), 7.41(\mathrm{~d}, J=5.6 \mathrm{~Hz}, 1 \mathrm{H}), 7.38-7.32$ $(\mathrm{m}, 2 \mathrm{H}), 7.12(\mathrm{t}, J=7.8 \mathrm{~Hz}, 1 \mathrm{H}), 6.77-6.65(\mathrm{~m}, 2 \mathrm{H}), 4.22(\mathrm{~s}, 1 \mathrm{H}) ;{ }^{13} \mathrm{C}$ NMR $(100$ $\left.\mathrm{MHz}, \mathrm{CDCl}_{3}\right) \delta 157.5,149.3(\mathrm{~d}, J=7.4 \mathrm{~Hz}), 144.4(\mathrm{~d}, J=3.0 \mathrm{~Hz}), 144.3(\mathrm{~d}, J=3.0$ $\mathrm{Hz}), 139.5$ (d, $J=3.2 \mathrm{~Hz}), 139.3(\mathrm{~d}, J=3.3 \mathrm{~Hz}), 135.2(\mathrm{~d}, J=69.5 \mathrm{~Hz}), 134.2$ (d, $J=$ $7.2 \mathrm{~Hz}), 129.2(\mathrm{~d}, J=4.2 \mathrm{~Hz}), 128.6(\mathrm{~d}, J=8.2 \mathrm{~Hz}), 128.4(\mathrm{~d}, J=7.9 \mathrm{~Hz}), 128.2(\mathrm{~d}, J$ $=5.8 \mathrm{~Hz}), 126.3(\mathrm{~d}, J=20.6 \mathrm{~Hz}), 126.2(\mathrm{~d}, J=4.8 \mathrm{~Hz}), 124.9(\mathrm{~d}, J=21.4 \mathrm{~Hz}), 124.5$, 124.1, 123.3 (d, $J=3.4 \mathrm{~Hz}), 123.2$ (d, $J=3.4 \mathrm{~Hz}), 121.7$, 121.6, 120.1, 119.4, 119.3; ${ }^{31} \mathrm{P}$ NMR $\left(162 \mathrm{MHz}, \mathrm{CDCl}_{3}\right) \delta 35.89$; HRMS (ESI) $\mathrm{m} / z$ calcd for $\mathrm{C}_{28} \mathrm{H}_{20} \mathrm{NO}_{4} \mathrm{PS}_{3}$ $[\mathrm{M}+\mathrm{H}]^{+}=562.0370$, found $=562.0371$.

Optical Rotation: $[\alpha]_{\mathrm{D}}^{25}=51.43\left(c=0.49, \mathrm{CHCl}_{3}\right) .97 \%$ ee (HPLC condition: Chiralpak AD-H column, $n$-Hexane $/ i-\mathrm{PrOH}=60: 40$, flow rate $=1.0 \mathrm{~mL} / \mathrm{min}$, wavelength $=254 \mathrm{~nm}, t_{\mathrm{R}}=53.46 \min$ for minor isomer, $t_{\mathrm{R}}=70.20 \mathrm{~min}$ for major isomer). 


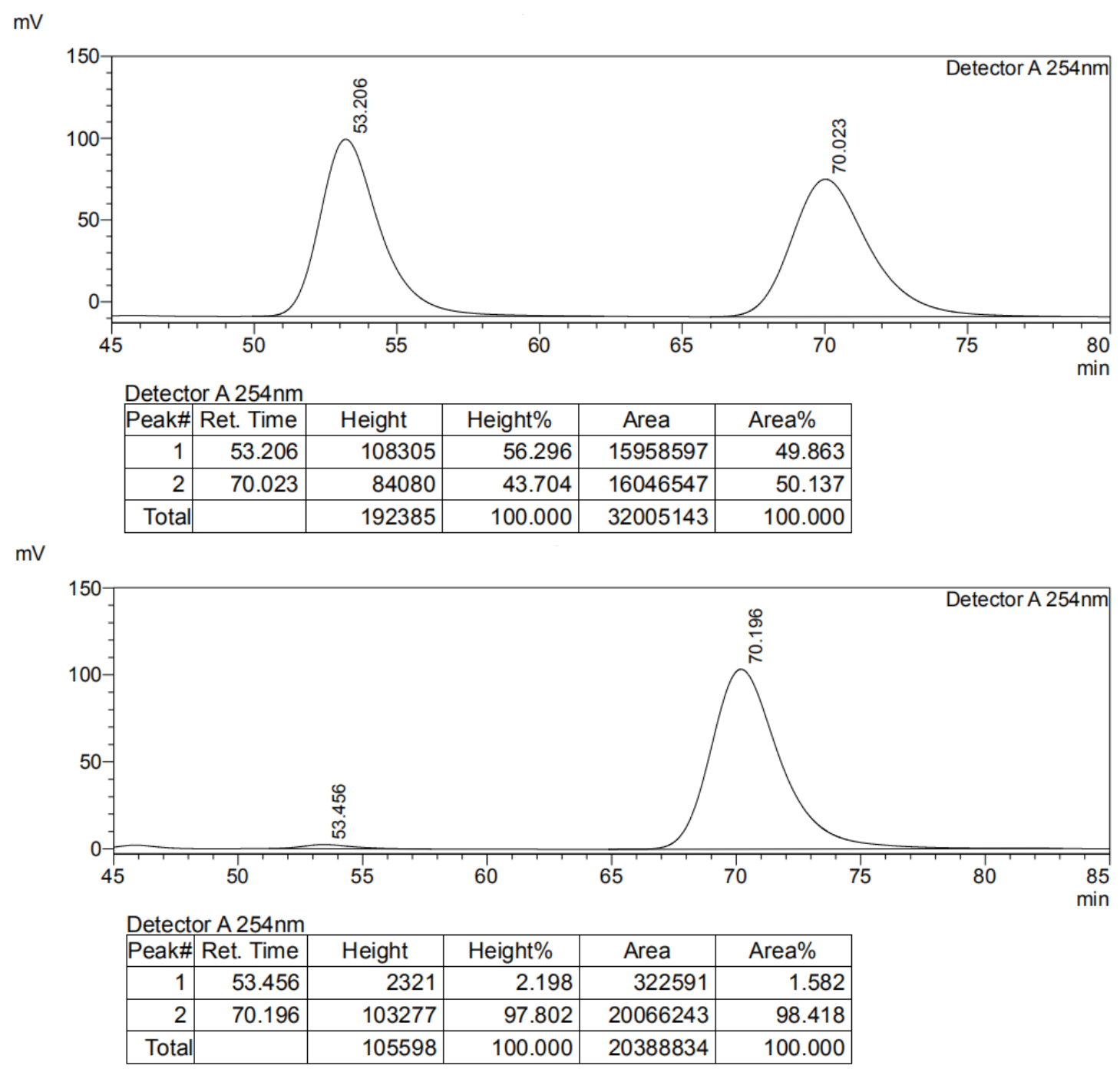

(S)-2-(2-hydroxyphenylsulfonimidoyl)phenyl [1,1'-biphenyl]-4-yl(phenyl)phosphi -nate (40)

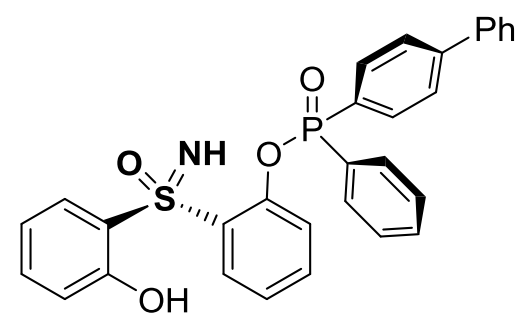

Colorless oil; $43 \mathrm{mg}, 83 \%$ yield; ${ }^{1} \mathrm{H}$ NMR (400 MHz, $\left.\mathrm{CDCl}_{3}\right) \delta 12.04(\mathrm{~s}, 1 \mathrm{H})$, 8.09-7.96 (m, 2H), 7.93-7.84 (m, 2H), 7.83-7.75 (m, 1H), 7.75-7.68 (m, 2H), 7.68-7.63 (m, 1H), 7.63-7.56 (m, 3H), 7.55-7.51 (m, 1H), 7.50-7.43 (m, 4H), 7.42-7.33 (m, 3H), 7.18-7.09 (m, 1H), 6.85-6.79 (m, 1H), $6.75(\mathrm{t}, J=7.8 \mathrm{~Hz}, 1 \mathrm{H})$; ${ }^{13} \mathrm{C} \mathrm{NMR}\left(100 \mathrm{MHz}, \mathrm{CDCl}_{3}\right) \delta 157.5,149.2(\mathrm{~d}, J=3.2 \mathrm{~Hz}), 145.8(\mathrm{~d}, J=3.0 \mathrm{~Hz})$, 
139.8, $135.4(\mathrm{~d}, J=80.0), 134.3(\mathrm{~d}, J=5.0 \mathrm{~Hz}), 134.2(\mathrm{~d}, J=5.1 \mathrm{~Hz}), 133.1(\mathrm{~d}, J=$ $2.8 \mathrm{~Hz}), 132.7(\mathrm{~d}, J=11.4 \mathrm{~Hz}), 132.1(\mathrm{~d}, J=10.7 \mathrm{~Hz}), 130.5(\mathrm{~d}, J=26.6 \mathrm{~Hz}), 129.3$ $(\mathrm{d}, J=1.6 \mathrm{~Hz}), 129.2,129.1(\mathrm{~d}, J=15.8 \mathrm{~Hz}), 128.9(\mathrm{~d}, J=9.2 \mathrm{~Hz}), 128.6(\mathrm{~d}, J=35.1$ Hz), $127.6(\mathrm{~d}, J=4.6 \mathrm{~Hz}), 127.4(\mathrm{~d}, J=4.8 \mathrm{~Hz}), 127.3$ (d, $J=2.4 \mathrm{~Hz}), 124.2,121.7$, $121.6(\mathrm{~d}, J=5.0 \mathrm{~Hz}), 120.1(\mathrm{~d}, J=6.2 \mathrm{~Hz}), 119.3(\mathrm{~d}, J=8.5 \mathrm{~Hz}) ;{ }^{31} \mathrm{P}$ NMR $(162$ $\mathrm{MHz}, \mathrm{CDCl}_{3}$ ) $\delta 34.25,34.17$; HRMS (ESI) $\mathrm{m} / z$ calcd for $\mathrm{C}_{30} \mathrm{H}_{24} \mathrm{NO}_{4} \mathrm{PS}[\mathrm{M}+\mathrm{H}]^{+}=$ 526.1242 , found $=526.1242$.

Optical Rotation: $[\alpha]_{\mathrm{D}}^{25}=37.43\left(c=0.70, \mathrm{CHCl}_{3}\right) .90 \% / 82 \%$ ee $(\mathrm{HPLC}$ condition: Chiralpak AD-H column, $n$-Hexane $/ i$-PrOH $=60: 40$, flow rate $=1.0$ $\mathrm{mL} / \mathrm{min}$, wavelength $=254 \mathrm{~nm}, t_{\mathrm{R} 1}=18.40 \mathrm{~min}$ for minor isomer, $t_{\mathrm{R} 1}=23.08 \mathrm{~min}$ for major isomer; $t_{\mathrm{R} 2}=27.22 \mathrm{~min}$ for major isomer, $t_{\mathrm{R} 2}=35.81 \mathrm{~min}$ for minor isomer). $\mathrm{mV}$

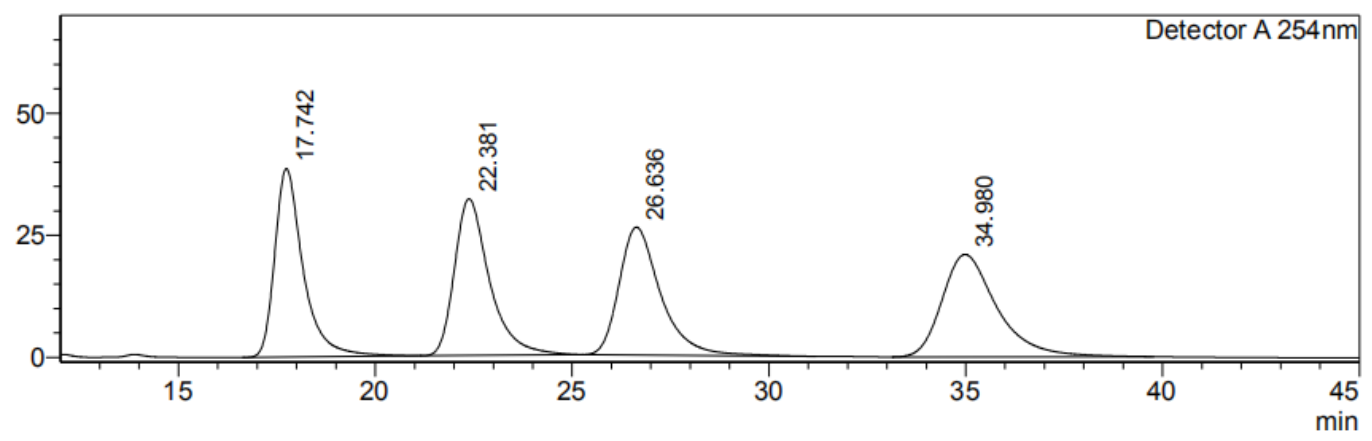

Detector A 254nm
\begin{tabular}{|r|r|r|r|r|r|}
\hline Peak\# & Ret. Time & Height & Height\% & Area & \multicolumn{1}{c|}{ Area\% } \\
\hline 1 & 17.742 & 38579 & 32.754 & 1942730 & 24.633 \\
\hline 2 & 22.381 & 32070 & 27.228 & 1985482 & 25.175 \\
\hline 3 & 26.636 & 26163 & 22.213 & 1922532 & 24.377 \\
\hline 4 & 34.980 & 20971 & 17.805 & 2035927 & 25.815 \\
\hline Total & & 117784 & 100.000 & 7886671 & 100.000 \\
\hline
\end{tabular}


$\mathrm{mV}$

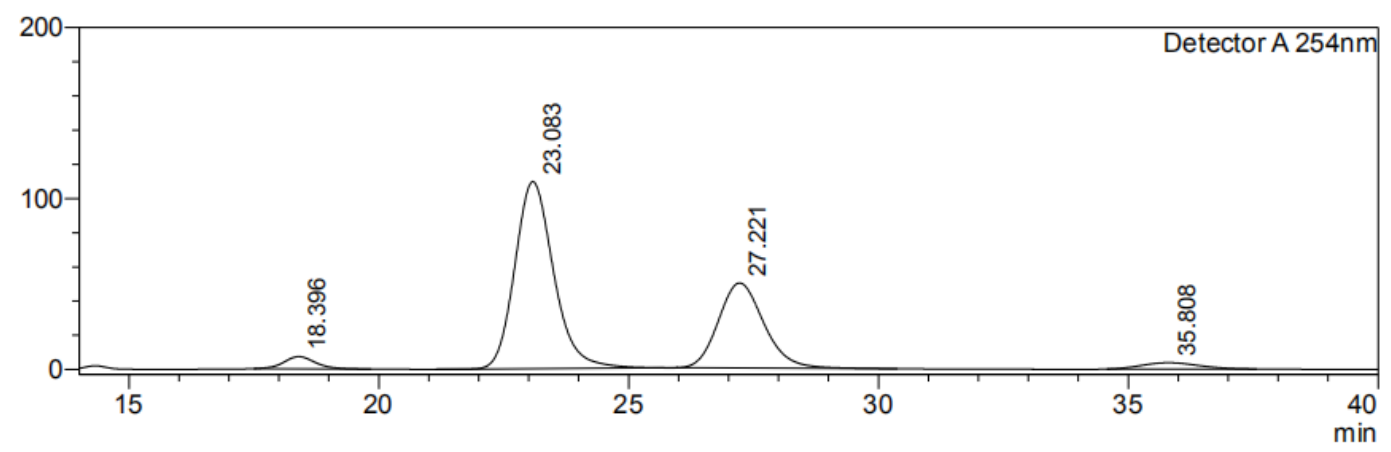

Detector A 254nm

\begin{tabular}{|r|r|r|r|r|r|}
\hline Peak\# & Ret. Time & \multicolumn{1}{c|}{ Height } & Height\% & \multicolumn{1}{c|}{ Area } & \multicolumn{1}{c|}{ Area $\%$} \\
\hline 1 & 18.396 & 7085 & 4.168 & 311639 & 3.216 \\
\hline 2 & 23.083 & 109450 & 64.382 & 5928714 & 61.176 \\
\hline 3 & 27.221 & 49779 & 29.282 & 3160300 & 32.610 \\
\hline 4 & 35.808 & 3686 & 2.168 & 290606 & 2.999 \\
\hline Total & & 170001 & 100.000 & 9691260 & 100.000 \\
\hline
\end{tabular}




\section{Investigation on the Active Species of the A-T Process and Desymmetrizing NAS-type Reaction Development}

Table S6. Transformation of diphenylphosphoine oxide 2a catalyzed by phosphonium salt P15

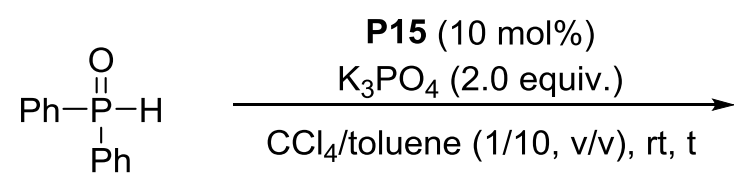

$2 a$<smiles>O=P(Cl)(c1ccccc1)c1ccccc1</smiles>

A<smiles>O=P(OP(=O)(c1ccccc1)c1ccccc1)(c1ccccc1)c1ccccc1</smiles>

B

\begin{tabular}{cccc}
$\mathrm{t}(\min )$ & $\mathbf{m o l}(\%)$ & $\mathbf{B}$ \\
\hline 0 & 100 & $\mathbf{A}$ & 0 \\
10 & 90 & 0 & 3 \\
20 & 83 & 7 & 12 \\
30 & 56 & 15 & 18 \\
40 & 50 & 26 & 20 \\
50 & 42 & 30 & 27 \\
60 & 34 & 31 & 32 \\
70 & 19 & 34 & 39 \\
120 & 12 & 42 & 50 \\
180 & 0 & 38 & 72 \\
260 & 0 & 28 & 100
\end{tabular}

To a tube with a magnetic stirring bar were added 2a (20.2 mg, $0.1 \mathrm{mmol}), \mathbf{P 1 5}$ (12.0 $\mathrm{mg}, 10 \mathrm{~mol} \%$ ) and potassium phosphate $(42.4 \mathrm{mg}, 0.2 \mathrm{mmol}$ ), followed by the addition of $\mathrm{CCl}_{4} /$ toluene $(2.0 \mathrm{~mL}, \mathrm{v} / \mathrm{v}=1 / 10)$. The reaction mixture was stirred at $\mathrm{rt}$ for $260 \mathrm{~min}$ and a ${ }^{31} \mathrm{P}$ NMR spectrum was recorded after a period of time. The phosphoryl chloride $\mathbf{A}$ and phosphoric anhydride $\mathbf{B}$ were detected by the ${ }^{31} \mathrm{P}$ NMR spectrum of the crude mixture. 


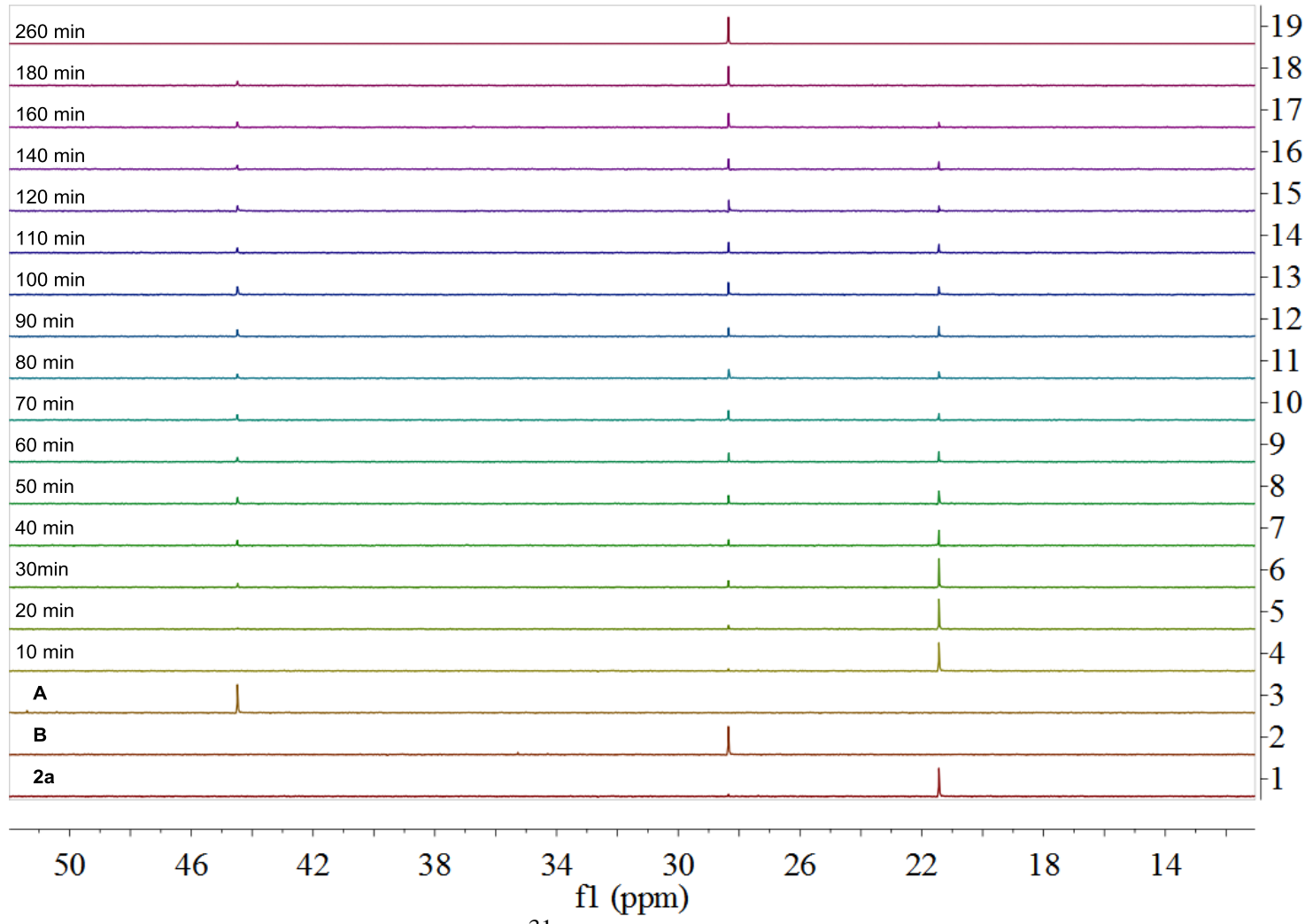

Figure S1. Real-time ${ }^{31} \mathrm{P}$ NMR for the transformation of $\mathbf{2 a}$

Table S7. Transformation of diphenylphosphoine oxide 2a under the P15-involving desymmetrizing conditions

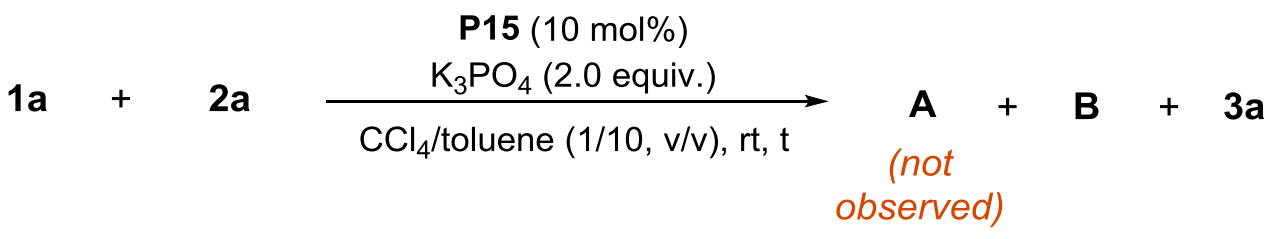

\begin{tabular}{cccc} 
T (min) & \multicolumn{3}{c}{$\operatorname{mol}(\%)$} \\
0 & $\mathbf{2 a}$ & $\mathbf{B}$ & $\mathbf{3 a}$ \\
10 & 100 & 0 & 0 \\
20 & 40 & 16 & 31 \\
30 & 18 & 19 & 45 \\
40 & 2 & 26 & 59 \\
50 & 0 & 16 & 73 \\
60 & 0 & 11 & 79 \\
& 0 & 0 & 83
\end{tabular}

To a tube with a magnetic stirring bar were added $1 \mathbf{a}(24.9 \mathrm{mg}, 0.1 \mathrm{mmol}), \mathbf{2 a}$ 
(24.2 mg, $0.12 \mathrm{mmol}$ ), P15 (12.0 mg, $10 \mathrm{~mol} \%$ ) and potassium phosphate (42.4 mg, $0.2 \mathrm{mmol})$, followed by the addition of $\mathrm{CCl}_{4} /$ toluene $(2.0 \mathrm{~mL}, \mathrm{v} / \mathrm{v}=1 / 10)$. The reaction mixture was stirred at $\mathrm{rt}$ for $60 \mathrm{~min}$ and $\mathrm{a}{ }^{31} \mathrm{P}$ NMR spectrum was recorded after a period of time. Only phosphoric anhydride $\mathbf{B}$ was detected by the ${ }^{31} \mathrm{P}$ NMR spectrum of the crude mixture.

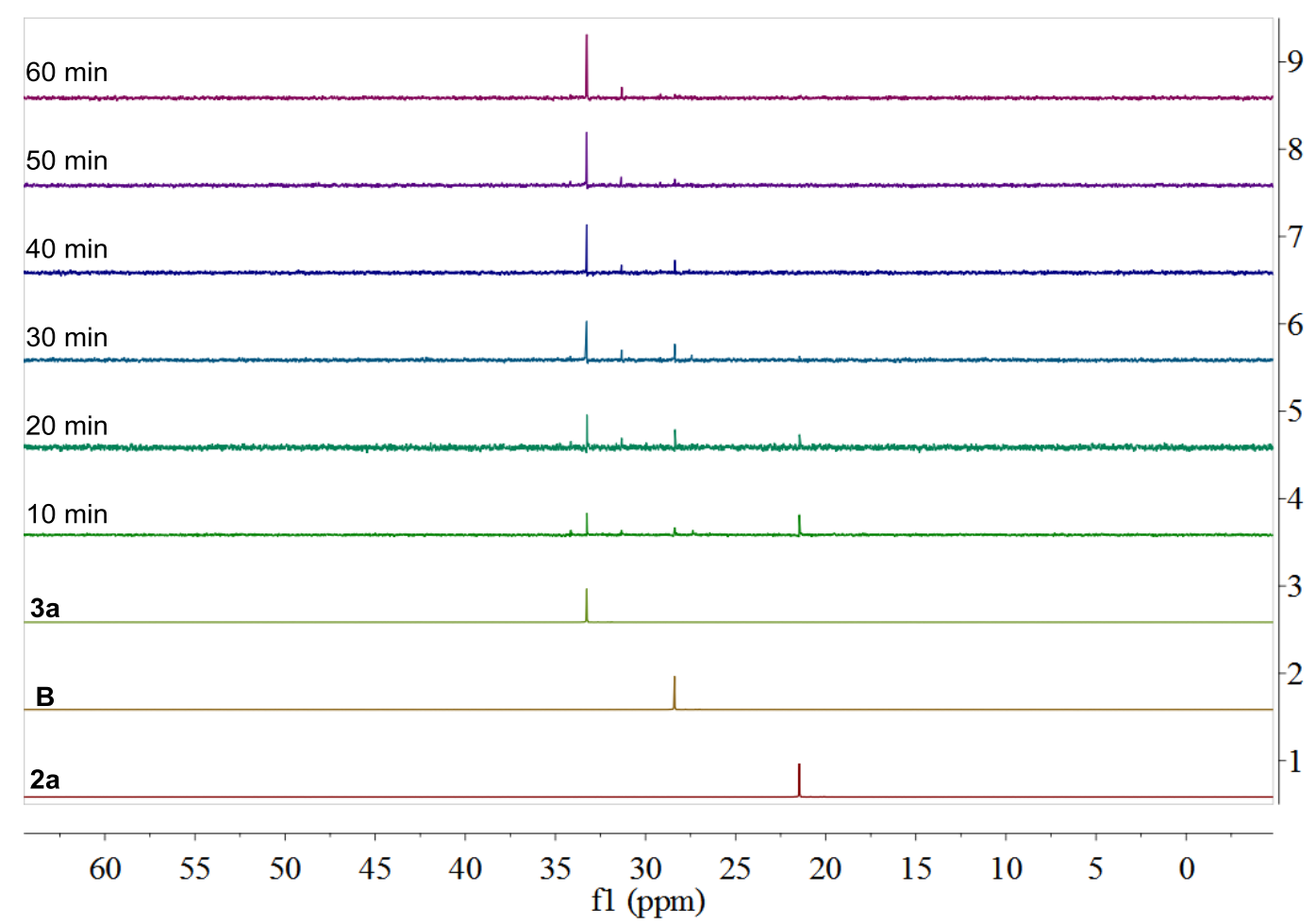

Figure S2. Real-time ${ }^{31} \mathrm{P}$ NMR in the P15-catalyzed desymmetrizing process

Table S8. Reaction of 1a with A or B catalyzed by phosphonium salt P15

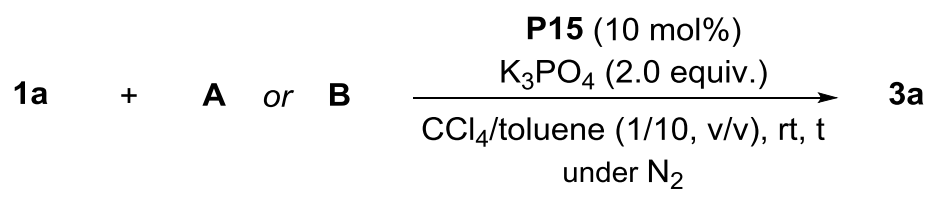

\begin{tabular}{ccccc}
$\mathrm{T}(\min )$ & \multicolumn{3}{c}{$\operatorname{mol}(\%)$} \\
& $\mathbf{1 a}^{a}$ & $\mathbf{3 a}^{a}$ & $\mathbf{1 a}^{b}$ & $\mathbf{3 a}^{b}$ \\
0 & 100 & 0 & 100 & 0 \\
5 & 59 & 38 & & \\
10 & 31 & 62 & 82 & 16 \\
20 & 9 & 82 & 73 & 23 \\
30 & 0 & 80 & 68 & 32
\end{tabular}


${ }^{a}$ Condition A: 1a $(0.1 \mathrm{mmol}), \mathbf{A}(0.12 \mathrm{mmol}), \mathrm{K}_{3} \mathrm{PO}_{4}(0.2 \mathrm{mmol})$ and $\mathbf{P 1 5}(10 \mathrm{~mol} \%)$ in $\mathrm{CCl}_{4} /$ toluene $(2 \mathrm{~mL}, \mathrm{v} / \mathrm{v}=1: 10)$ at $\mathrm{rt}$ for $\mathrm{t}$ min under $\mathrm{N}_{2} \cdot{ }^{b} \mathbf{B}(0.12 \mathrm{mmol})$ instead of $\mathbf{A}$. Yields were determined by HPLC and ${ }^{13} \mathrm{P}$ NMR.

Scheme S2. Control experiments for investigation of the active species in the desymmetrizing asymmetric A-T-type reaction<smiles>[R]c1ccc(O)c(S(=N)(=O)c2cc([R])ccc2O)c1</smiles>

1a, $\mathrm{R}=\mathrm{H}$ $\mathbf{1 d}^{b}, \mathrm{R}={ }^{i} \mathrm{Pr}$ $1 \mathrm{e}^{b}, \mathrm{R}={ }^{t} \mathrm{Bu}$
$2 a$

A

B

$2 a$

A

B $2 \mathbf{a}$

A

B<smiles>[R]c1ccc(NS(=O)(=O)c2cc([R])ccc2O)c(OP)c1</smiles>

3a, $86 \%, 97 \%$ ee 3a, $76 \%$, 98\% ee 3a, $72 \%, 69 \%$ ee

3d, $82 \%, 97 \%$ ee 3d, $79 \%$, 98\% ee 3d, $67 \%$, $18 \%$ ee

3e, $87 \%$, 98\% ee

3e. $82 \%, 97 \%$ ee

3e, $75 \%, 40 \%$ ee

${ }^{a}$ Condition A: 1 (0.1 mmol), 2a/A/B (0.12 mmol), $\mathrm{K}_{3} \mathrm{PO}_{4}(0.2 \mathrm{mmol})$ and $\mathbf{P 1 5}(10 \mathrm{~mol} \%)$ in $\mathrm{CCl}_{4} /$ toluene $(2 \mathrm{~mL}, \mathrm{v} / \mathrm{v}=1: 10)$ at $0{ }^{\circ} \mathrm{C}$ for $12 \mathrm{~h}$ under $\mathrm{N}_{2} \cdot{ }^{b} \mathrm{Cs}_{2} \mathrm{CO}_{3}$ (2 equiv.). 


\section{Optimization of Reaction Conditions for Desymmetrizing Asymmetric NAS-type Reaction}

Table S9. Screening different electrophiles ${ }^{[a, b]}$<smiles>N=S(=O)(c1ccccc1O)c1ccccc1O</smiles>

1a

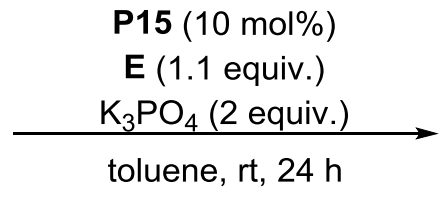

toluene, rt, $24 \mathrm{~h}$<smiles>NS(=O)(=O)c1ccccc1OCCO</smiles>

$3 / 6$

$\begin{array}{ccc}\text { entry } & \mathbf{E} & \text { Yield/ee }(\%) \text { of product } \\ 1 & \mathrm{ClP}(\mathrm{O}) \mathrm{Ph}_{2} & 86 \%, 78 \% \text { ee }(\mathbf{3 a}) \\ \mathbf{2} & \mathbf{T s C l} & \mathbf{8 4 \%}, \mathbf{9 1 \%} \text { ee }(\mathbf{6 a}) \\ 3 & \mathrm{PhCOCl} & 88 \%, 2 \% \text { ee }(\mathbf{6 a - 1}) \\ 4 & \mathrm{TBSCl} & 92 \%, 7 \% \text { ee }(\mathbf{6 a - 2}) \\ 5 & \mathrm{FmocCl} & 85 \%, 43 \%(\mathbf{6 a - 3}) \\ 6 & \mathrm{AcCl} & 69 \%, 7 \%(6 \mathbf{6}-\mathbf{4})\end{array}$

[a] Reaction conditions: $1 \mathbf{a}(0.1 \mathrm{mmol}), \mathbf{E}(0.11 \mathrm{mmol}), \mathrm{K}_{3} \mathrm{PO}_{4}(0.2 \mathrm{mmol})$ and $\mathbf{P 1 5}(0.01 \mathrm{mmol})$ in toluene $(2.0 \mathrm{~mL})$ at room temperature for $24 \mathrm{~h}$. [b] All ee values were determined by HPLC analysis on a chiral stationary phase, and yields were determined by in situ ${ }^{1} \mathrm{H}-\mathrm{NMR}$.

Table S10. Screening the phosphonium salt catalysts for NAS-type reaction ${ }^{[a, b]}$<smiles>N=S(=O)(c1ccccc1O)c1ccccc1O</smiles>

$1 \mathrm{a}$ entry<smiles>Cc1ccc(S(=O)(=O)Cl)cc1</smiles>

$5 a$

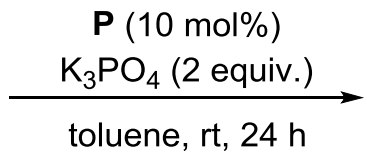

toluene, rt, $24 \mathrm{~h}$<smiles></smiles>

$6 a$

$$
\text { ee }(\%)
$$

1

2

3

4

5

6
P1

P2

P3

P4

P5

P6
54

41

49

53

62

57 


$\begin{array}{llll}7 & \mathbf{P 7} & 60 & 18 \\ 8 & \mathbf{P 8} & 43 & 55 \\ 9 & \mathbf{P 9} & 58 & 61 \\ 10 & \mathbf{P 1 0} & 60 & 78 \\ 11 & \mathbf{P 1 1} & 75 & 93 \\ 12 & \mathbf{P 1 2} & 81 & 98 \\ 13 & \mathbf{P 1 3} & 66 & 82 \\ 14 & \mathbf{P 1 4} & 72 & 79 \\ 15 & \mathbf{P 1 5} & 84 & 91\end{array}$

[a] Reaction conditions: $\mathbf{1 a}(0.1 \mathrm{mmol}), \mathbf{5 a}(0.11 \mathrm{mmol}), \mathrm{K}_{3} \mathrm{PO}_{4}(0.2 \mathrm{mmol})$ and the catalyst $(0.01$ $\mathrm{mmol})$ in toluene $(2.0 \mathrm{~mL})$ at room temperature for $24 \mathrm{~h}$. [b] All ee values were determined by HPLC analysis on a chiral stationary phase, and yields were determined by in situ ${ }^{1} \mathrm{H}-\mathrm{NMR}$.

Table S11. Screening of the bases for NAS-type reaction ${ }^{[a, b]}$<smiles>N=S(=O)(c1ccccc1O)c1ccccc1O</smiles><smiles>Cc1ccc(S(=O)(=O)Cl)cc1</smiles>

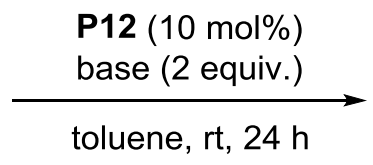<smiles>NS(=O)(=O)c1ccccc1[O-]</smiles>

1a 5a

$\begin{array}{cccc}\text { entry } & \text { base } & \text { yield }(\%) & \text { ee }(\%) \\ 1 & \mathrm{Cs}_{2} \mathrm{CO}_{3} & 84 & 96 \\ 2 & \mathrm{~K}_{2} \mathrm{CO}_{3} & 5 & 65 \\ 3 & \mathrm{Na}_{2} \mathrm{CO}_{3} & \text { trace } & -- \\ 4 & \mathrm{~K}_{3} \mathrm{PO}_{4} & 81 & 98 \\ 5 & \mathrm{~K}_{3} \mathrm{PO}_{4} \cdot 7 \mathrm{H}_{2} \mathrm{O} & 64 & 97 \\ 6 & \mathrm{KHCO}_{3} & 16 & 65 \\ 7 & \mathrm{KOAc}_{8} & 8 & 53 \\ 8 & \mathrm{~K}_{2} \mathrm{HPO}_{4} & 67 & 87 \\ 9 & \mathrm{KOH}^{2} & 86 & 45\end{array}$

[a] Reaction conditions: $\mathbf{1 a}(0.1 \mathrm{mmol}), \mathbf{5 a}(0.11 \mathrm{mmol})$, base $(0.2 \mathrm{mmol})$ and $\mathbf{P 1 2}(0.01 \mathrm{mmol})$ in toluene $(2.0 \mathrm{~mL})$ at room temperature for $24 \mathrm{~h}$. [b] All ee values were determined by HPLC analysis on a chiral stationary phase, and yields were determined by in situ ${ }^{1} \mathrm{H}-\mathrm{NMR}$. 
Table S12. Screening the solvents for NAS-type reaction ${ }^{[\mathrm{a}, \mathrm{b}]}$<smiles>N=S(=O)(c1ccccc1Cl)c1ccccc1Cl</smiles>

$1 \mathrm{a}$<smiles>Cc1ccc(S(=O)(=O)Cl)cc1</smiles>

$5 a$

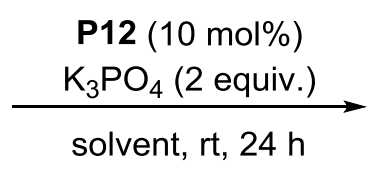

solvent, rt, $24 \mathrm{~h}$<smiles>NS(=O)(=O)c1ccccc1[O-]</smiles>

6a

\begin{tabular}{|c|c|c|c|}
\hline entry & Solvent & yield (\%) & ee $(\%)$ \\
\hline 1 & toluene & 81 & 98 \\
\hline 2 & xylene & 78 & 98 \\
\hline 3 & mesitylene & 63 & 98 \\
\hline 4 & $\mathrm{Et}_{2} \mathrm{O}$ & 87 & 91 \\
\hline 5 & $\mathrm{PE}_{30-60}$ & 54 & 57 \\
\hline 6 & $\mathrm{PE}_{60-90}$ & 35 & 55 \\
\hline 7 & hexane & 32 & 40 \\
\hline 8 & cyclohexane & 46 & 87 \\
\hline 9 & $\mathrm{CH}_{2} \mathrm{Cl}_{2}$ & 88 & 46 \\
\hline 10 & $\mathrm{CHCl}_{3}$ & 85 & 75 \\
\hline
\end{tabular}

[b] Reaction conditions: 1a $(0.1 \mathrm{mmol}), \mathbf{5 a}(0.11 \mathrm{mmol}), \mathrm{K}_{3} \mathrm{PO}_{4}(0.2 \mathrm{mmol})$ and $\mathbf{P 1 2}(0.01 \mathrm{mmol})$ in solvent $(2.0 \mathrm{~mL})$ at room temperature for $24 \mathrm{~h}$. [b] All ee values were determined by HPLC analysis on a chiral stationary phase, and yields were determined by in situ ${ }^{1} \mathrm{H}-\mathrm{NMR}$.

Table S13. Screening the catalyst loading of $\mathbf{P 1 2}{ }^{[a, b]}$<smiles>Cc1ccc(S(=O)(=O)Cl)cc1</smiles>
$1 \mathrm{a}$

\section{entry}

1

2

3

$\mathrm{n}$

20

10
$5 a$

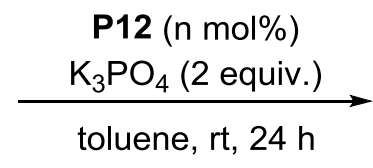

$6 a$

yield (\%)

ee $(\%)$

78

98

81

98

87

93

[a] Reaction conditions: $\mathbf{1 a}(0.1 \mathrm{mmol}), \mathbf{5 a}(0.11 \mathrm{mmol}), \mathrm{K}_{3} \mathrm{PO}_{4}(0.2 \mathrm{mmol})$ and $\mathbf{P 1 2}(\mathrm{n} \mathrm{mol} \%)$ in toluene $(2.0 \mathrm{~mL})$ at room temperature for $24 \mathrm{~h}$. [b] All ee values were determined by HPLC analysis on a chiral stationary phase, and yields were determined by in situ ${ }^{1} \mathrm{H}-\mathrm{NMR}$. 
Table S14. Screening the temperatures for NAS-type reaction ${ }^{[a, b]}$<smiles>Cc1ccc(S(=O)(=O)Cl)cc1</smiles>

$1 \mathrm{a}$

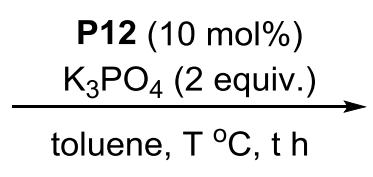

$5 a$<smiles>NS(=O)(=O)c1ccccc1[N+](=O)[O-]</smiles>

$6 a$

$\begin{array}{ccccc}\text { entry } & \mathrm{T}\left(0^{\circ} \mathrm{C}\right) & \mathrm{t}(\mathrm{h}) & \text { yield }(\%) & \text { ee }(\%) \\ 1 & 25 & 24 & 81 & 98 \\ 2 & 0 & 37 & 83 & 99.5 \\ 3 & -10 & 62 & 83 & 99.8\end{array}$

[a] Reaction conditions: $\mathbf{1 a}(0.1 \mathrm{mmol}), \mathbf{5 a}(0.11 \mathrm{mmol}), \mathrm{K}_{3} \mathrm{PO}_{4}(0.2 \mathrm{mmol})$ and $\mathbf{P 1 2}(0.01 \mathrm{mmol})$ in toluene $(2.0 \mathrm{~mL})$ at $\mathrm{T}{ }^{\circ} \mathrm{C}$ for $\mathrm{th}$. [b] All ee values were determined by HPLC analysis on a chiral stationary phase, and yields were determined by in situ ${ }^{1} \mathrm{H}-\mathrm{NMR}$.

\section{General Procedure for the Desymmetrization of Free Sulfoximines} via NAS-type Reaction

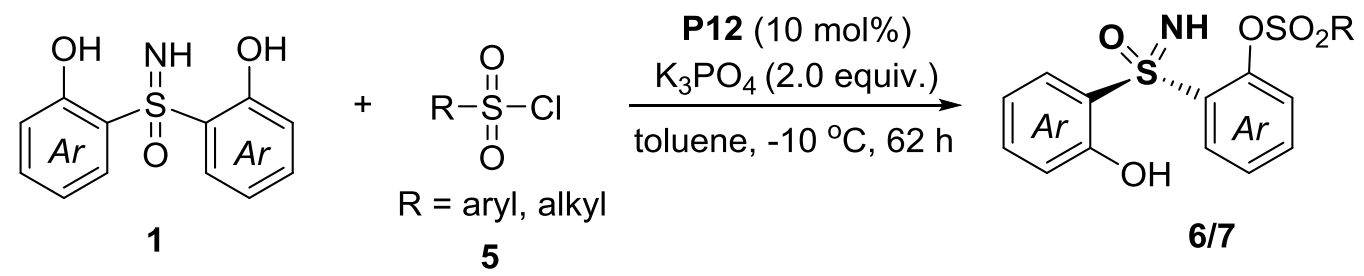

Condition B: to a tube $(10 \mathrm{~mL})$ with a magnetic stirring bar were added $\mathbf{1}(0.1 \mathrm{mmol})$, $5(0.11 \mathrm{mmol})$, phosphonium salt $\mathbf{P 1 2}(0.01 \mathrm{mmol})$ and potassium phosphate $(0.2$ $\mathrm{mmol})$, followed by the addition of toluene $(2.0 \mathrm{~mL})$. The reaction mixture was stirred at $-10{ }^{\circ} \mathrm{C}$ for $62 \mathrm{~h}$. Until completion, the reaction mixture was transfered to room temperature, and diluted with dichloromethane. Purification by column chromatography on silica gel (petroleum ether/ethyl acetate $=5: 1$ to $3: 1$ ) afforded the prodcut 6/7.

\section{$\underline{(S)-2-(2-h y d r o x y p h e n y l s u l f o n i m i d o y l) p h e n y l ~ 4-m e t h y l b e n z e n e s u l f o n a t e ~(6 a) ~}$}




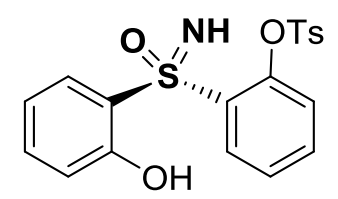

Light yellow solid; $33 \mathrm{mg}, 83 \%$ yield; m.p. $=89-91{ }^{\circ} \mathrm{C} ;{ }^{1} \mathrm{H} \mathrm{NMR}\left(400 \mathrm{MHz}, \mathrm{CDCl}_{3}\right)$ $\delta 12.21$ (s, 1H), $7.98(\mathrm{dd}, J=7.8,1.6 \mathrm{~Hz}, 1 \mathrm{H}), 7.85(\mathrm{~d}, J=8.4 \mathrm{~Hz}, 2 \mathrm{H}), 7.56-7.48$ (m, 2H), 7.44-7.34 (m, 5H), $6.89(\mathrm{dd}, J=8.4,1.0 \mathrm{~Hz}, 1 \mathrm{H}), 6.85-6.77(\mathrm{~m}, 1 \mathrm{H}), 3.58(\mathrm{~s}$, 1H), $2.48(\mathrm{~s}, 3 \mathrm{H}) ;{ }^{13} \mathrm{C}$ NMR $\left(100 \mathrm{MHz}, \mathrm{CDCl}_{3}\right) \delta 157.7,147.0,146.3,136.8,135.8$, $134.7,132.7,130.1,130.0,129.5,128.9,126.7,122.8,120.0,119.4,119.3,22.0$; HRMS (ESI) $m / z$ calcd for $\mathrm{C}_{19} \mathrm{H}_{17} \mathrm{NO}_{5} \mathrm{~S}_{2}[\mathrm{M}+\mathrm{H}]^{+}=404.0626$, found $=404.0620$.

Optical Rotation: $[\alpha]_{\mathrm{D}}^{25}=50.50\left(c=0.20, \mathrm{CHCl}_{3}\right) .>99 \%$ ee (HPLC condition: Chiralpak AD-H column, $n$-Hexane $/ i-\mathrm{PrOH}=70: 30$, flow rate $=1.0 \mathrm{~mL} / \mathrm{min}$, wavelength $=254 \mathrm{~nm}, t_{\mathrm{R}}=21.20$ min for minor isomer, $t_{\mathrm{R}}=26.63$ min for major isomer).

$\mathrm{mV}$

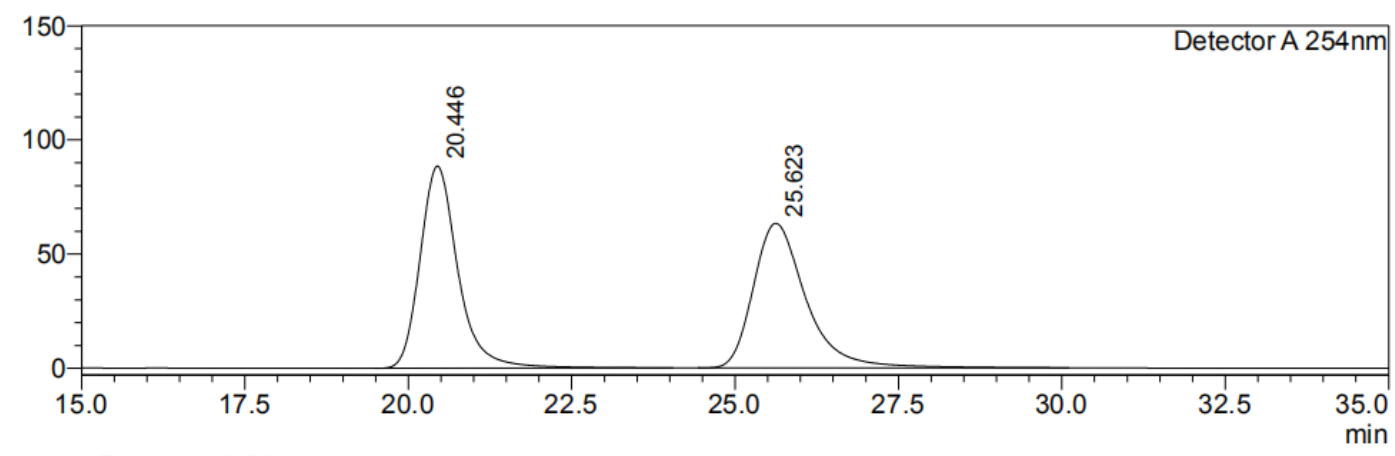

\begin{tabular}{|c|c|c|c|c|c|}
\hline \multicolumn{6}{|c|}{ Detector A 254nm } \\
\hline Peak\# & Ret. Time & Height & Height\% & Area & Area\% \\
\hline 1 & 20.446 & 88505 & 58.307 & 3575309 & 50.150 \\
\hline 2 & 25.623 & 63286 & 41.693 & 3553855 & 49.850 \\
\hline Total & & 151792 & 100.000 & 7129164 & 100.000 \\
\hline
\end{tabular}




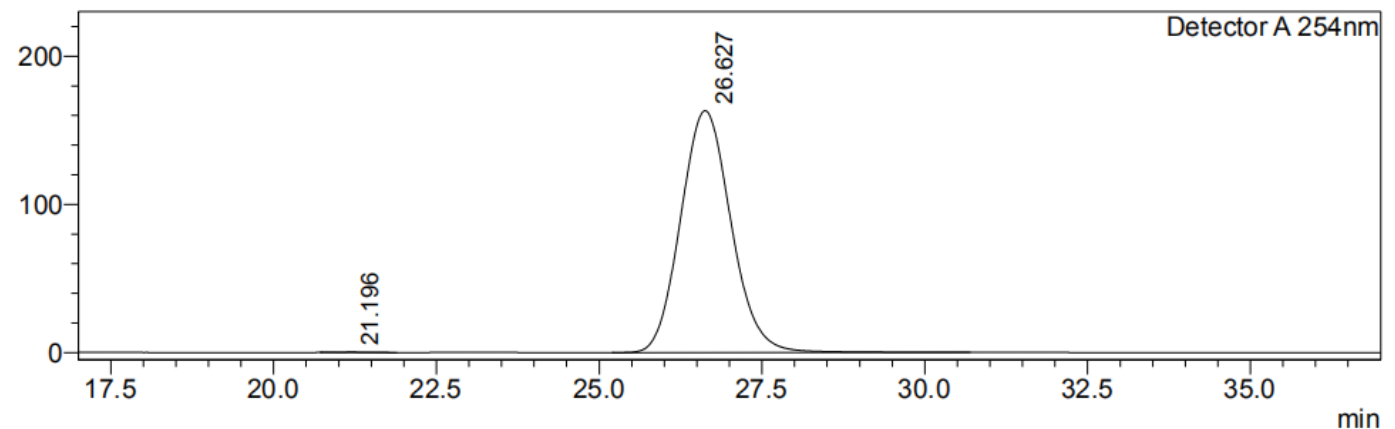

Detector A 254nm
\begin{tabular}{|r|r|r|r|r|r|}
\hline Peak\# & Ret. Time & \multicolumn{1}{|c|}{ Height } & Height\% & \multicolumn{1}{c|}{ Area } & \multicolumn{1}{c|}{ Area\% } \\
\hline 1 & 21.196 & 256 & 0.157 & 8747 & 0.099 \\
\hline 2 & 26.627 & 163169 & 99.843 & 8811642 & 99.901 \\
\hline Total & & 163425 & 100.000 & 8820389 & 100.000 \\
\hline
\end{tabular}

\section{(S)-2-(2-hydroxy-5-methylphenylsulfonimidoyl)-4-methylphenyl}

\section{4-methylbenzenesulfonate $(6 \mathrm{~b})$}<smiles>Cc1ccc(O)c(S(=O)(=O)c2cc(C)ccc2OC(F)(F)F)c1</smiles>

Light yellow solid; $32 \mathrm{mg}, 73 \%$ yield; m.p. $=147-149{ }^{\circ} \mathrm{C} ;{ }^{1} \mathrm{H}$ NMR $(400 \mathrm{MHz}$, $\left.\mathrm{CDCl}_{3}\right) 7.85(\mathrm{~s}, 1 \mathrm{H}), 7.83(\mathrm{~s}, 1 \mathrm{H}), 7.79(\mathrm{~d}, J=1.8 \mathrm{~Hz}, 1 \mathrm{H}), 7.35(\mathrm{dd}, J=8.4,2.2 \mathrm{~Hz}$, 3H), $7.30(\mathrm{dd}, J=8.4,1.8 \mathrm{~Hz}, 1 \mathrm{H}), 7.23-7.14(\mathrm{~m}, 2 \mathrm{H}), 6.81(\mathrm{~d}, J=8.4 \mathrm{~Hz}, 1 \mathrm{H}), 2.47$ (s, 3H), 2.38 (s, 3H), 2.15 (s, 3H); ${ }^{13} \mathrm{C}$ NMR (100 MHz, $\left.\mathrm{CDCl}_{3}\right) \delta 155.4,146.1,144.7$, 137.2 , 136.9, 136.2, 135.1, 132.7, 130.2, 130.0, 128.9, 128.8, 128.7, 122.6, 119.5, 119.1, 21.9, 21.1, 20.4; HRMS (ESI) $m / z$ calcd for $\mathrm{C}_{21} \mathrm{H}_{21} \mathrm{NO}_{5} \mathrm{~S}_{2}[\mathrm{M}+\mathrm{H}]^{+}=432.0939$, found $=432.0927$.

Optical Rotation: $[\alpha]_{\mathrm{D}}^{25}=64.32\left(c=0.81, \mathrm{CHCl}_{3}\right) .90 \%$ ee (HPLC condition: Chiralpak AD-H column, $n-\mathrm{Hexane} / i-\mathrm{PrOH}=75: 25$, flow rate $=1.0 \mathrm{~mL} / \mathrm{min}$, wavelength $=254 \mathrm{~nm}, t_{\mathrm{R}}=14.34 \mathrm{~min}$ for major isomer, $t_{\mathrm{R}}=18.27 \mathrm{~min}$ for minor isomer). 
$\mathrm{mV}$

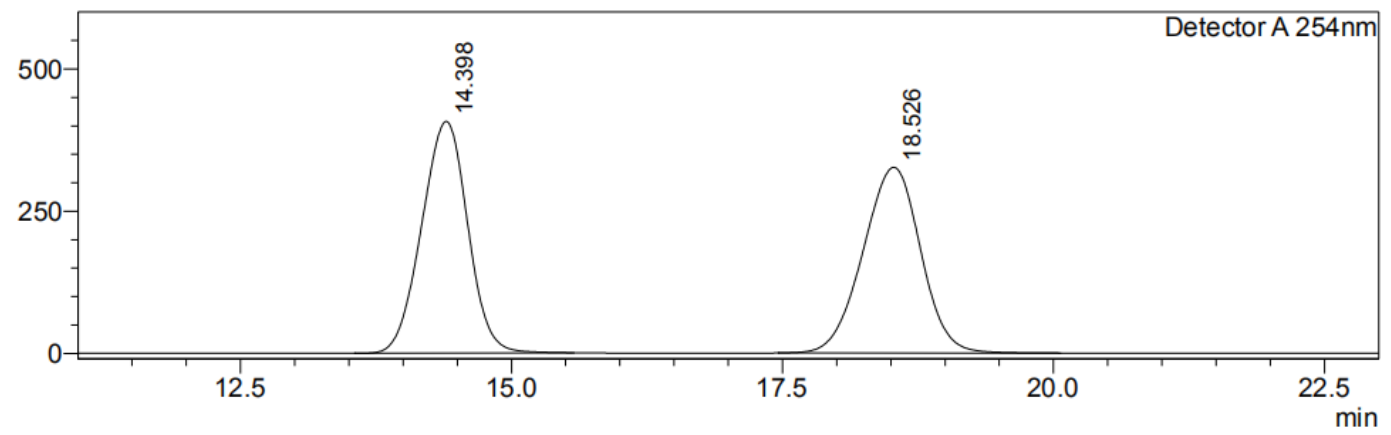

Detector A 254nm
\begin{tabular}{|r|r|r|r|r|r|}
\hline Peak\# & Ret. Time & Height & Height\% & Area & \multicolumn{1}{|c|}{ Area\% } \\
\hline 1 & 14.398 & 407392 & 55.510 & 12056378 & 49.937 \\
\hline 2 & 18.526 & 326514 & 44.490 & 12086897 & 50.063 \\
\hline Total & & 733905 & 100.000 & 24143275 & 100.000 \\
\hline
\end{tabular}

$\mathrm{mV}$

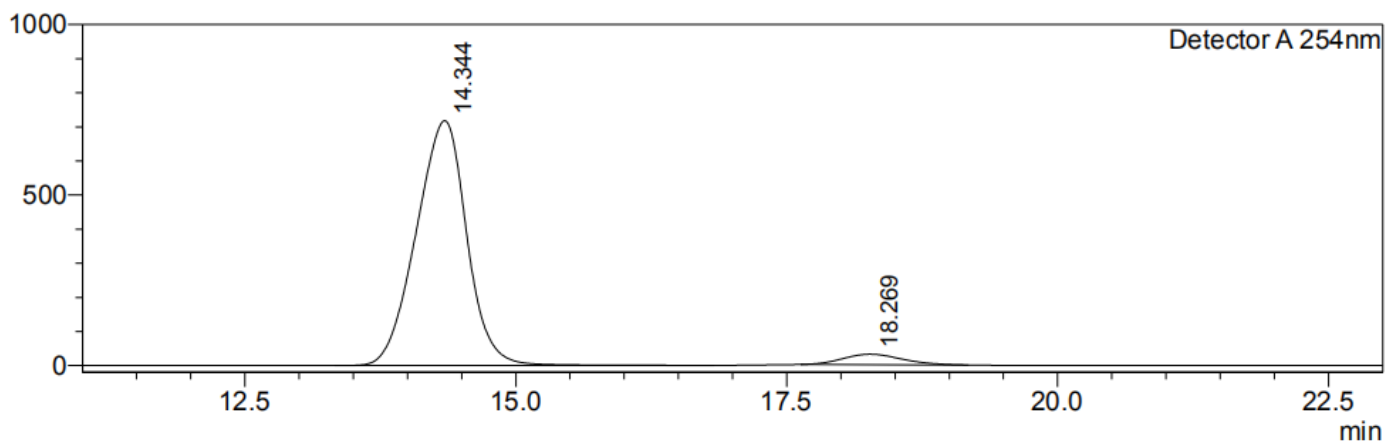

Detector A $254 \mathrm{~nm}$

\begin{tabular}{|r|r|r|r|r|r|}
\hline Peak\# & Ret. Time & \multicolumn{1}{c|}{ Height } & Height\% & \multicolumn{1}{c|}{ Area } & \multicolumn{1}{|c|}{ Area\% } \\
\hline 1 & 14.344 & 717587 & 95.889 & 23291125 & 95.222 \\
\hline 2 & 18.269 & 30763 & 4.111 & 1168770 & 4.778 \\
\hline Total & & 748349 & 100.000 & 24459894 & 100.000 \\
\hline
\end{tabular}

\section{(S)-4-ethyl-2-(5-ethyl-2-hydroxyphenylsulfonimidoyl)phenyl}

\section{4-methylbenzenesulfonate (6c)}<smiles>CCc1ccc(O)c(S(=O)(=O)c2cc(CC)ccc2O)c1</smiles>

White solid; $38 \mathrm{mg}, 84 \%$ yield; m.p. $=79-81{ }^{\circ} \mathrm{C} ;{ }^{1} \mathrm{H}$ NMR $\left(400 \mathrm{MHz}, \mathrm{CDCl}_{3}\right) \delta 7.87$ $(\mathrm{s}, 1 \mathrm{H}), 7.85(\mathrm{~d}, J=1.6 \mathrm{~Hz}, 1 \mathrm{H}), 7.82(\mathrm{~d}, J=2.0 \mathrm{~Hz}, 1 \mathrm{H}), 7.42-7.30(\mathrm{~m}, 4 \mathrm{H}), 7.27(\mathrm{~d}$, $J=2.2 \mathrm{~Hz}, 1 \mathrm{H}), 7.23(\mathrm{dd}, J=8.5,2.2 \mathrm{~Hz}, 1 \mathrm{H}), 6.86(\mathrm{~d}, J=8.5 \mathrm{~Hz}, 1 \mathrm{H}), 2.66(\mathrm{q}, J=$ $7.6 \mathrm{~Hz}, 2 \mathrm{H}), 2.50-2.34(\mathrm{~m}, 5 \mathrm{H}), 1.22(\mathrm{t}, J=7.6 \mathrm{~Hz}, 3 \mathrm{H}), 1.10(\mathrm{t}, J=7.6 \mathrm{~Hz}, 3 \mathrm{H}) ;{ }^{13} \mathrm{C}$ NMR $\left(100 \mathrm{MHz}, \mathrm{CDCl}_{3}\right) \delta 155.4,146.2,144.7,143.3,135.9,135.7,135.3,134.1$, 
$132.7,130.0,129.2,128.9,127.8,122.6,119.3,119.1,28.3,27.8,21.9,15.5,15.2$

HRMS (ESI) $m / z$ calcd for $\mathrm{C}_{23} \mathrm{H}_{25} \mathrm{NO}_{5} \mathrm{~S}_{2}[\mathrm{M}+\mathrm{H}]^{+}=460.1252$, found $=460.1228$.

Optical Rotation: $[\alpha]_{\mathrm{D}}^{25}=54.20\left(c=0.50, \mathrm{CHCl}_{3}\right) .93 \%$ ee (HPLC condition: Chiralpak AD-H column, $n-\mathrm{Hexane} / i-\mathrm{PrOH}=70: 30$, flow rate $=1.0 \mathrm{~mL} / \mathrm{min}$, wavelength $=254 \mathrm{~nm}, t_{\mathrm{R}}=13.45 \min$ for major isomer, $t_{\mathrm{R}}=18.77$ min for minor isomer).

$\mathrm{mV}$

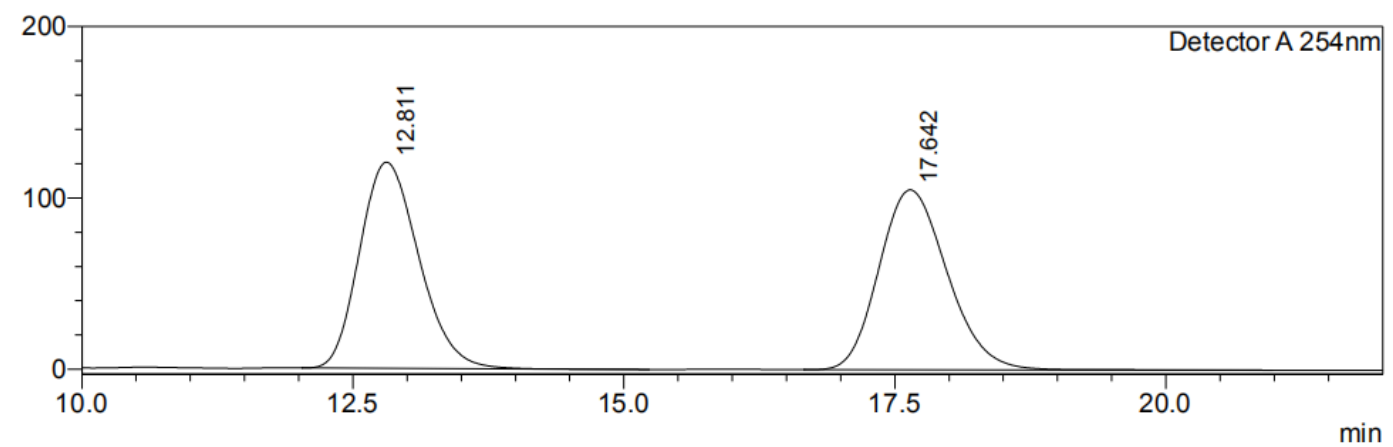

Detector A 254nm

\begin{tabular}{|r|r|r|r|r|r|}
\hline Peak\# & Ret. Time & \multicolumn{1}{c|}{ Height } & Height\% & \multicolumn{1}{c|}{ Area } & \multicolumn{1}{c|}{ Area\% } \\
\hline 1 & 12.811 & 120306 & 53.394 & 4584136 & 49.862 \\
\hline 2 & 17.642 & 105011 & 46.606 & 4609579 & 50.138 \\
\hline Total & & 225318 & 100.000 & 9193715 & 100.000 \\
\hline
\end{tabular}

$\mathrm{mV}$

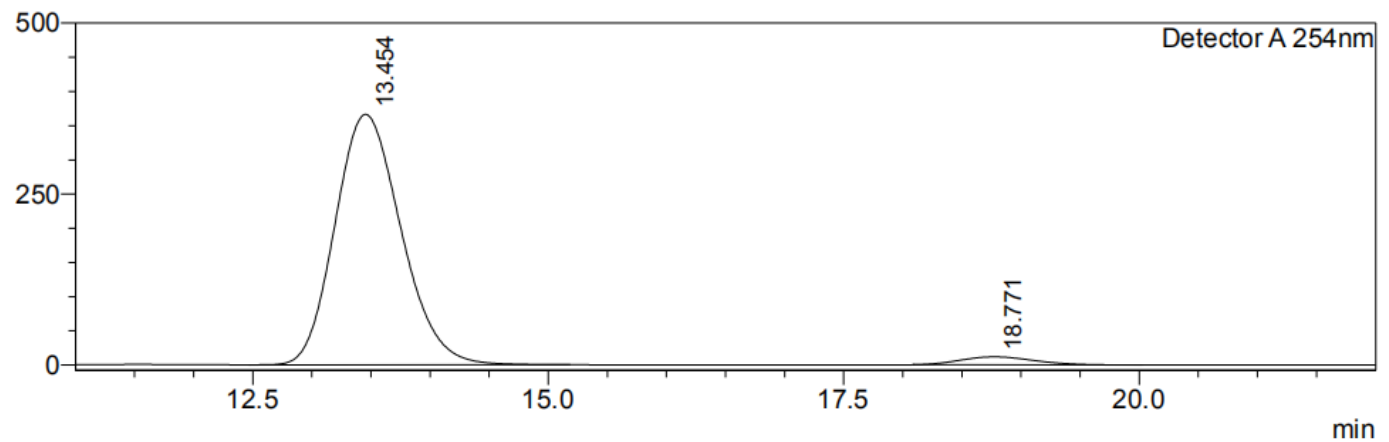

Detector A 254nm
\begin{tabular}{|r|r|r|r|r|r|}
\hline Peak\# & Ret. Time & Height & Height $\%$ & \multicolumn{1}{c|}{ Area } & \multicolumn{1}{c|}{ Area $\%$} \\
\hline 1 & 13.454 & 366210 & 96.891 & 14227750 & 96.523 \\
\hline 2 & 18.771 & 11752 & 3.109 & 512550 & 3.477 \\
\hline Total & & 377962 & 100.000 & 14740301 & 100.000 \\
\hline
\end{tabular}

$\underline{(S)-2-(2-h y d r o x y-5-i s o p r o p y l p h e n y l s u l f o n i m i d o y l)-4-i s o p r o p y l p h e n y l ~}$

\section{4-methylbenzenesulfonate (6d)}<smiles>CCCc1ccc(O)c(S(=O)(=O)c2cc(C(C)C)ccc2O)c1</smiles> 
White powder; $38 \mathrm{mg}, 79 \%$ yield; m.p. $=62-64{ }^{\circ} \mathrm{C} ;{ }^{1} \mathrm{H}$ NMR $\left(400 \mathrm{MHz}, \mathrm{CDCl}_{3}\right) \delta$ 7.93-7.91 (m, 1H), $7.90(\mathrm{~d}, J=1.8 \mathrm{~Hz}, 1 \mathrm{H}), 7.76(\mathrm{~d}, J=1.8 \mathrm{~Hz}, 1 \mathrm{H}), 7.40-7.33(\mathrm{~m}$, 4H), $7.32(\mathrm{~d}, J=2.4 \mathrm{~Hz}, 1 \mathrm{H}), 7.29-7.26(\mathrm{~m}, 1 \mathrm{H}), 6.83$ (d, $J=8.6 \mathrm{~Hz}, 1 \mathrm{H}), 2.94$ (hept, $J=6.9 \mathrm{~Hz}, 1 \mathrm{H}), 2.71$ (hept, $J=6.9 \mathrm{~Hz}, 1 \mathrm{H}), 2.48(\mathrm{~s}, 3 \mathrm{H}), 1.22(\mathrm{~d}, J=6.9 \mathrm{~Hz}, 6 \mathrm{H})$, $1.10(\mathrm{dd}, J=9.1,6.9 \mathrm{~Hz}, 6 \mathrm{H}) ;{ }^{13} \mathrm{C}$ NMR $\left(100 \mathrm{MHz}, \mathrm{CDCl}_{3}\right) \delta 155.5,148.0,146.1$, $144.5,140.0,136.7,134.3,132.9,132.4,130.0,129.0,127.7,126.6,122.9,119.5$, $119.1,33.8,33.2,23.9,23.9,23.8,22.0$; HRMS (ESI) $\mathrm{m} / z$ calcd for $\mathrm{C}_{25} \mathrm{H}_{29} \mathrm{NO}_{5} \mathrm{~S}_{2}$ $[\mathrm{M}+\mathrm{H}]^{+}=488.1565$, found $=488.1542$.

Optical Rotation: $[\alpha]_{\mathrm{D}}^{25}=57.47\left(c=0.40, \mathrm{CHCl}_{3}\right) .>99 \%$ ee (HPLC condition: Chiralpak AD-H column, $n-\mathrm{Hexane} / i-\mathrm{PrOH}=70: 30$, flow rate $=1.0 \mathrm{~mL} / \mathrm{min}$, wavelength $=254 \mathrm{~nm}, t_{\mathrm{R}}=10.34 \mathrm{~min}$ for major isomer, $t_{\mathrm{R}}=12.58 \mathrm{~min}$ for minor isomer).

$\mathrm{mV}$

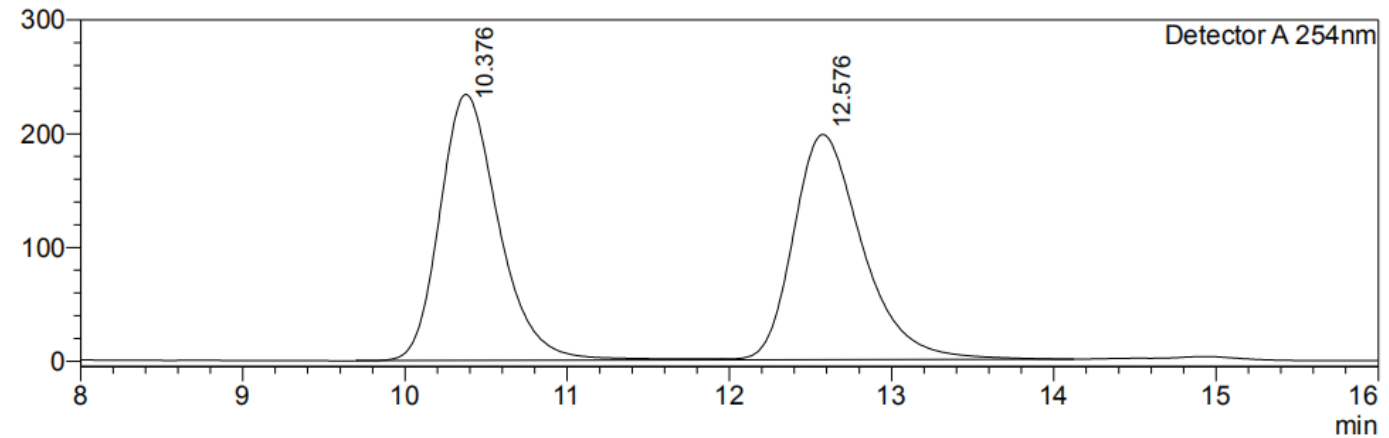

Detector A 254nm

\begin{tabular}{|r|r|r|r|r|r|}
\hline Peak\# & Ret. Time & Height & Height\% & \multicolumn{1}{c|}{ Area } & \multicolumn{1}{c|}{ Area\% } \\
\hline 1 & 10.376 & 234071 & 54.162 & 5946491 & 50.346 \\
\hline 2 & 12.576 & 198096 & 45.838 & 5864649 & 49.654 \\
\hline Total & & 432167 & 100.000 & 11811139 & 100.000 \\
\hline
\end{tabular}

$\mathrm{mV}$

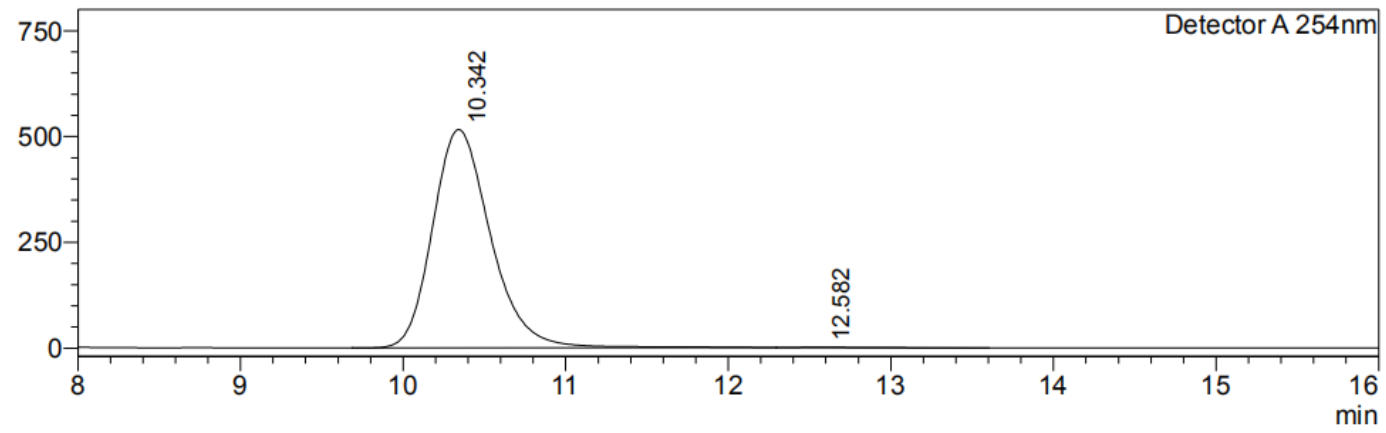

Detector A 254nm

\begin{tabular}{|r|r|r|r|r|r|}
\hline Peak\# & Ret. Time & \multicolumn{1}{|c|}{ Height } & Height $\%$ & \multicolumn{1}{c|}{ Area } & \multicolumn{1}{c|}{ Area\% } \\
\hline 1 & 10.342 & 515925 & 99.866 & 12814296 & 99.866 \\
\hline 2 & 12.582 & 693 & 0.134 & 17187 & 0.134 \\
\hline Total & & 516618 & 100.000 & 12831482 & 100.000 \\
\hline
\end{tabular}




\section{4-methylbenzenesulfonate (6e)}<smiles>CC(C)(C)c1ccc(O)c(S(=O)(=O)c2cc(C(C)(C)C)ccc2[Se-])c1</smiles>

White powder; $42 \mathrm{mg}, 82 \%$ yield; m.p. $=68-70{ }^{\circ} \mathrm{C} ;{ }^{1} \mathrm{H} \mathrm{NMR}\left(400 \mathrm{MHz}, \mathrm{CDCl}_{3}\right) \delta$ $12.00(\mathrm{~s}, 1 \mathrm{H}), 7.94(\mathrm{~s}, 1 \mathrm{H}), 7.92(\mathrm{~s}, 1 \mathrm{H}), 7.86(\mathrm{~d}, J=2.5 \mathrm{~Hz}, 1 \mathrm{H}), 7.52-7.47(\mathrm{~m}, 2 \mathrm{H})$, $7.43(\mathrm{dd}, J=8.8,2.5 \mathrm{~Hz}, 1 \mathrm{H}), 7.39$ (s, 1H), 7.37 (s, 1H), 7.35 (d, $J=8.6 \mathrm{~Hz}, 1 \mathrm{H})$, $6.85(\mathrm{~d}, J=8.8 \mathrm{~Hz}, 1 \mathrm{H}), 3.84(\mathrm{~s}, 1 \mathrm{H}), 2.48(\mathrm{~s}, 3 \mathrm{H}), 1.28(\mathrm{~s}, 9 \mathrm{H}), 1.17(\mathrm{~s}, 9 \mathrm{H}) ;{ }^{13} \mathrm{C}$ NMR (100 MHz, $\left.\mathrm{CDCl}_{3}\right) \delta 155.2,150.5,146.1,144.2,142.5,136.7,133.4,132.9$, 131.2, 130.1, 129.0, 126.8, 125.5, 122.8, 119.1, 118.8, 35.0, 34.3, 31.2, 22.0; HRMS (ESI) $\mathrm{m} / z$ calcd for $\mathrm{C}_{27} \mathrm{H}_{33} \mathrm{NO}_{5} \mathrm{~S}_{2}[\mathrm{M}+\mathrm{H}]^{+}=516.1878$, found $=516.1835$.

Optical Rotation: $[\alpha]^{25}=40.78\left(c=0.51, \mathrm{CHCl}_{3}\right) .98 \%$ ee (HPLC condition: Chiralpak IE column, $n-\mathrm{Hexane} / i-\mathrm{PrOH}=70: 30$, flow rate $=1.0 \mathrm{~mL} / \mathrm{min}$, wavelength $=254 \mathrm{~nm}, t_{\mathrm{R}}=16.50 \mathrm{~min}$ for major isomer, $t_{\mathrm{R}}=18.07 \mathrm{~min}$ for minor isomer). $\mathrm{mV}$

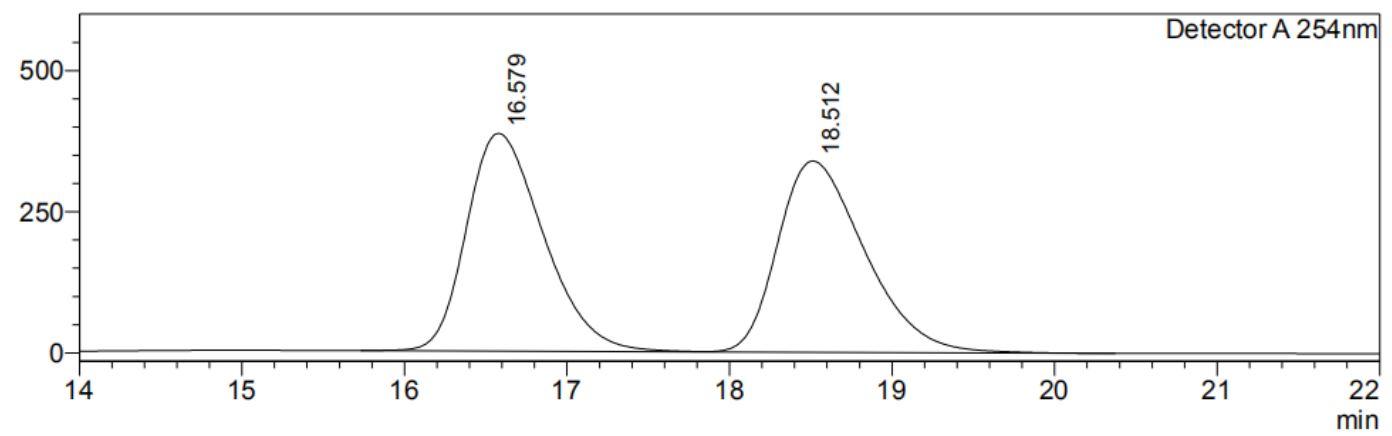

Detector A 254nm
\begin{tabular}{|r|r|r|r|r|r|}
\hline Peak\# & Ret. Time & Height & Height\% & Area & \multicolumn{1}{c|}{ Area\% } \\
\hline 1 & 16.579 & 385408 & 53.237 & 12703149 & 50.207 \\
\hline 2 & 18.512 & 338541 & 46.763 & 12598170 & 49.793 \\
\hline Total & & 723949 & 100.000 & 25301318 & 100.000 \\
\hline
\end{tabular}


$\mathrm{mV}$

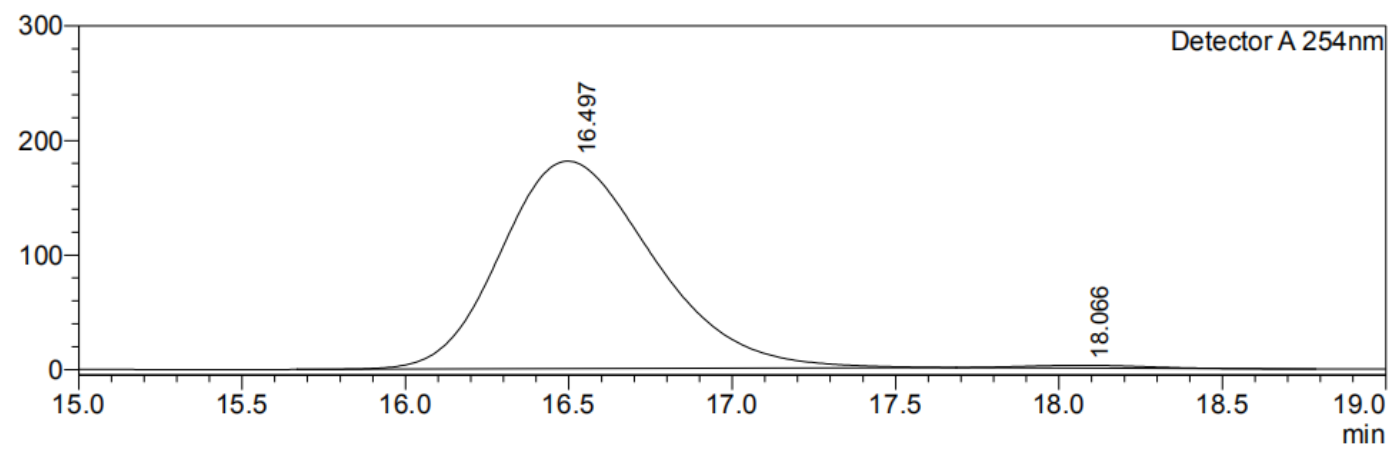

Detector A 254nm

\begin{tabular}{|r|r|r|r|r|r|}
\hline Peak\# & Ret. Time & \multicolumn{1}{c|}{ Height } & \multicolumn{1}{c|}{ Height\% } & \multicolumn{1}{c|}{ Area } & \multicolumn{1}{c|}{ Area\% } \\
\hline 1 & 16.497 & 181279 & 98.700 & 5900460 & 99.001 \\
\hline 2 & 18.066 & 2388 & 1.300 & 59550 & 0.999 \\
\hline Total & & 183668 & 100.000 & 5960010 & 100.000 \\
\hline
\end{tabular}

\section{(S)-4-benzyl-2-(5-benzyl-2-hydroxyphenylsulfonimidoyl)phenyl}

\section{4-methylbenzenesulfonate (6f)}<smiles>COc1ccc(Br)cc1NS(=O)(=O)c1cc(Br)ccc1O</smiles>

White powder; $47 \mathrm{mg}, 80 \%$ yield; m.p. $=65-67{ }^{\circ} \mathrm{C} ;{ }^{1} \mathrm{H} \mathrm{NMR}\left(400 \mathrm{MHz}, \mathrm{CDCl}_{3}\right) \delta$ $11.97(\mathrm{~s}, 1 \mathrm{H}), 7.86(\mathrm{~d}, J=8.4 \mathrm{~Hz}, 2 \mathrm{H}), 7.76(\mathrm{~d}, J=2.0 \mathrm{~Hz}, 1 \mathrm{H}), 7.39-7.34(\mathrm{~m}, 3 \mathrm{H})$, 7.33-7.27 (m, 3H), 7.27-7.21 (m, 4H), 7.20-7.14 (m, 2H), 7.12 (d, J = 7.0 Hz, 2H), $7.03(\mathrm{~d}, J=7.0 \mathrm{~Hz}, 2 \mathrm{H}), 6.79(\mathrm{~d}, J=8.4 \mathrm{~Hz}, 1 \mathrm{H}), 3.98(\mathrm{~s}, 2 \mathrm{H}), 3.76(\mathrm{~s}, 2 \mathrm{H}), 2.45(\mathrm{~s}$, $3 \mathrm{H}) ;{ }^{13} \mathrm{C} \mathrm{NMR}\left(100 \mathrm{MHz}, \mathrm{CDCl}_{3}\right) \delta 155.9,146.2,145.0,140.4,139.3,136.6,134.8$, $132.7,132.2$, 130.0, 129.9, 129.1, 129.0, 129.0, 128.9, 128.8, 128.6, 126.9, 126.4, 122.9, 119.6, 119.4, 41.2, 40.7, 21.9; HRMS (ESI) $\mathrm{m} / \mathrm{z}$ calcd for $\mathrm{C}_{33} \mathrm{H}_{29} \mathrm{NO}_{5} \mathrm{~S}_{2}$ $[\mathrm{M}+\mathrm{H}]^{+}=584.1565$, found $=584.1563$.

Optical Rotation: $[\alpha]_{\mathrm{D}}^{25}=73.00\left(c=0.50, \mathrm{CHCl}_{3}\right) .94 \%$ ee (HPLC condition: Chiralpak AD-H column, $n$-Hexane $/ i-\mathrm{PrOH}=70: 30$, flow rate $=1.0 \mathrm{~mL} / \mathrm{min}$, wavelength $=254 \mathrm{~nm}, t_{\mathrm{R}}=29.77$ min for minor isomer, $t_{\mathrm{R}}=39.92 \min$ for major isomer). 
$\mathrm{mV}$

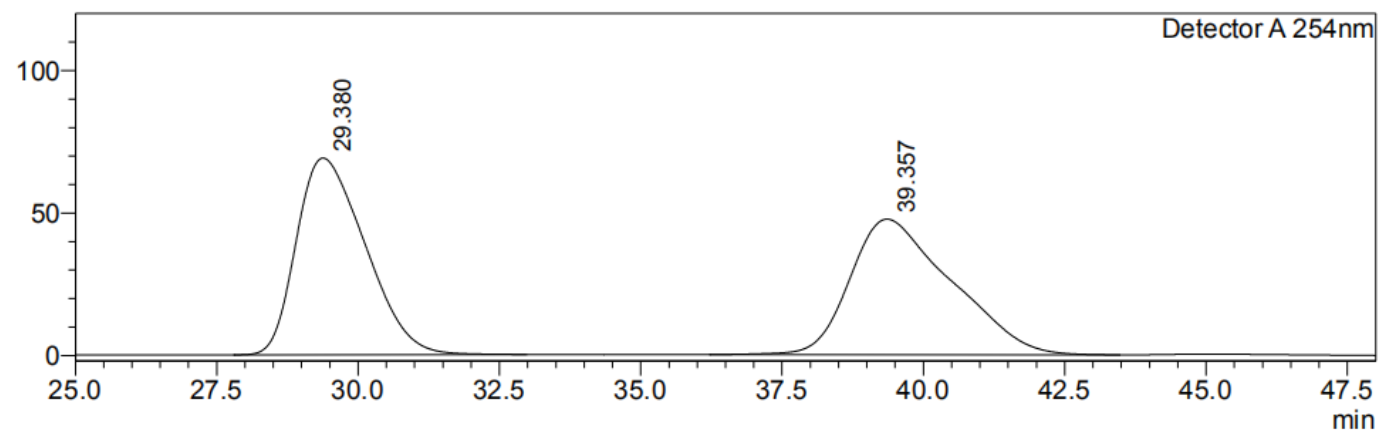

Detector A 254nm

\begin{tabular}{|r|r|r|r|r|r|}
\hline Peak\# & Ret. Time & Height & Height\% & \multicolumn{1}{c|}{ Area } & \multicolumn{1}{c|}{ Area\% } \\
\hline 1 & 29.380 & 69025 & 59.196 & 5848410 & 49.630 \\
\hline 2 & 39.357 & 47579 & 40.804 & 5935709 & 50.370 \\
\hline Total & & 116604 & 100.000 & 11784119 & 100.000 \\
\hline
\end{tabular}

$\mathrm{mV}$

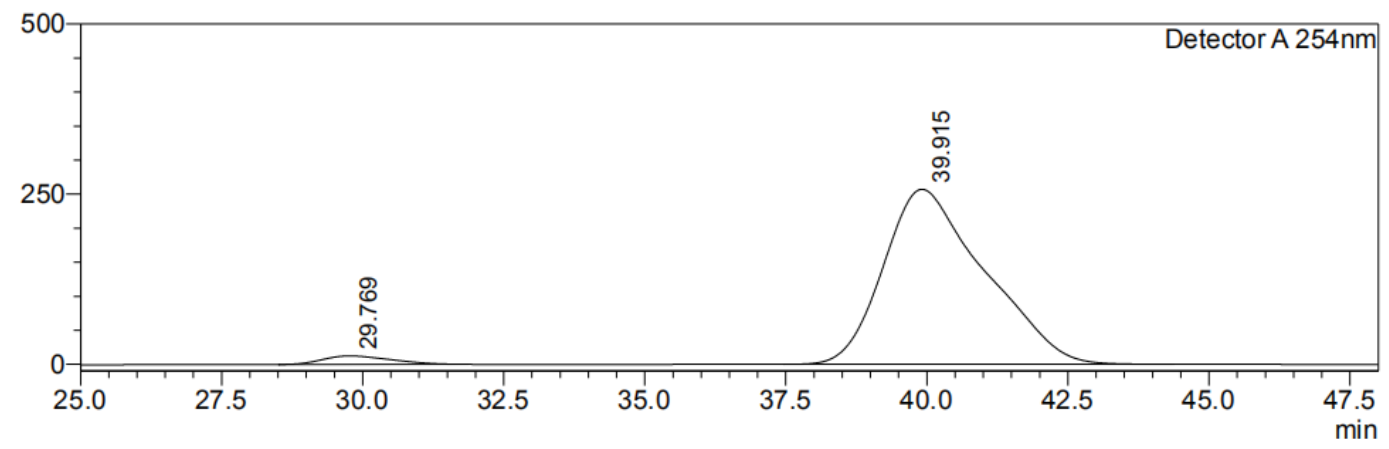

Detector A 254nm

\begin{tabular}{|r|r|r|r|r|r|}
\hline Peak\# & Ret. Time & \multicolumn{1}{c|}{ Height } & \multicolumn{1}{c|}{ Height $\%$} & \multicolumn{1}{c|}{ Area } & \multicolumn{1}{c|}{ Area\% } \\
\hline 1 & 29.769 & 12549 & 4.653 & 1052922 & 3.180 \\
\hline 2 & 39.915 & 257170 & 95.347 & 32056365 & 96.820 \\
\hline Total & & 269719 & 100.000 & 33109287 & 100.000 \\
\hline
\end{tabular}

\section{(S)-3-(4-hydroxy-[1,1'-biphenyl]-3-sulfonimidoyl)-[1,1'-biphenyl]-4-yl}

\section{4-methylbenzenesulfonate $(6 \mathrm{~g})$}<smiles>O=S(=O)(c1cc(-c2ccccc2)ccc1[O-])c1cc(-c2ccccc2)ccc1O</smiles>

White powder; $42 \mathrm{mg}, 76 \%$ yield; m.p. $=123-125{ }^{\circ} \mathrm{C} ;{ }^{1} \mathrm{H}$ NMR $\left(400 \mathrm{MHz}, \mathrm{CDCl}_{3}\right) \delta$ $12.32(\mathrm{~s}, 1 \mathrm{H}), 8.28(\mathrm{~d}, J=2.4 \mathrm{~Hz}, 1 \mathrm{H}), 7.88(\mathrm{~d}, J=8.4 \mathrm{~Hz}, 2 \mathrm{H}), 7.73(\mathrm{dd}, J=8.6,2.4$ Hz, 1H), 7.69 (d, $J=2.4 \mathrm{~Hz}, 1 \mathrm{H}), 7.65(\mathrm{dd}, J=8.6,2.4 \mathrm{~Hz}, 1 \mathrm{H}), 7.59-7.53$ (m, 3H), 7.49-7.45 (m, 2H), 7.43 -7.39 (m, 3H), 7.38-7.34 (m, 4H), 7.31-7.26 (m, 1H), $7.00(\mathrm{~d}$, $J=8.6 \mathrm{~Hz}, 1 \mathrm{H}), 3.85(\mathrm{~s}, 1 \mathrm{H}), 2.47(\mathrm{~s}, 3 \mathrm{H}) ;{ }^{13} \mathrm{C} \mathrm{NMR}\left(100 \mathrm{MHz}, \mathrm{CDCl}_{3}\right) \delta$ 157.1, $146.3,146.1,140.1,139.3,138.2,136.9,134.6,133.0,132.8,132.6,130.1,129.3$, 
129.0, 128.6, 128.4, 127.4, 127.4, 127.2, 126.7, 123.2, 120.4, 119.9, 22.0; HRMS (ESI) $m / z$ calcd for $\mathrm{C}_{31} \mathrm{H}_{25} \mathrm{NO}_{5} \mathrm{~S}_{2}[\mathrm{M}+\mathrm{H}]^{+}=556.1252$, found $=556.1250$.

Optical Rotation: $[\alpha]_{\mathrm{D}}^{25}=64.67\left(c=0.30, \mathrm{CHCl}_{3}\right) .98 \%$ ee (HPLC condition: Chiralpak OD-H column, $n-\mathrm{Hexane} / i-\mathrm{PrOH}=70: 30$, flow rate $=1.0 \mathrm{~mL} / \mathrm{min}$, wavelength $=254 \mathrm{~nm}, t_{\mathrm{R}}=17.03 \mathrm{~min}$ for major isomer, $t_{\mathrm{R}}=36.12 \mathrm{~min}$ for minor isomer).

$\mathrm{mV}$

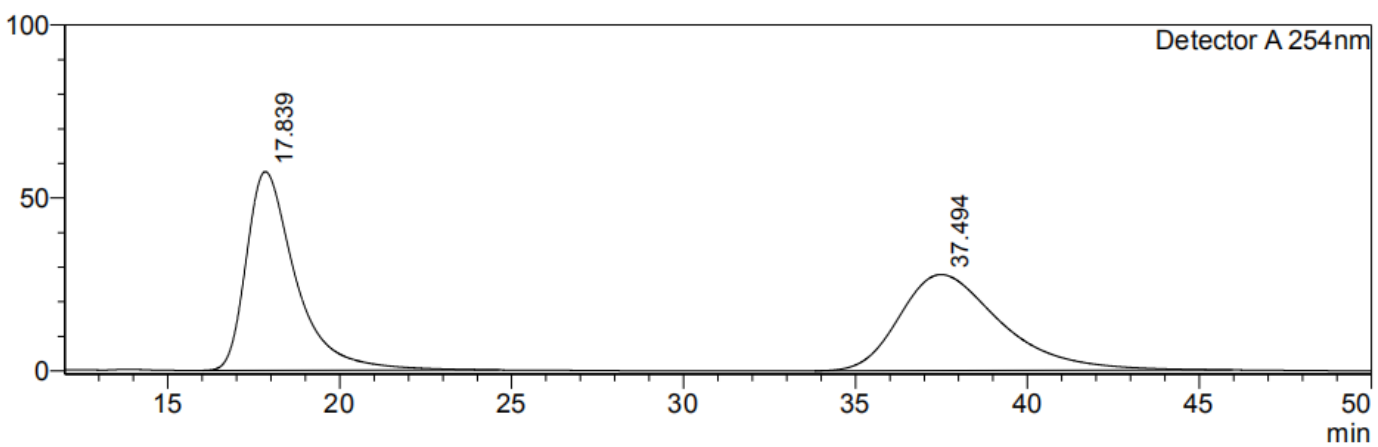

Detector A 254nm

\begin{tabular}{|r|r|r|r|r|r|}
\hline Peak\# & Ret. Time & \multicolumn{1}{c|}{ Height } & Height\% & \multicolumn{1}{c|}{ Area } & \multicolumn{1}{c|}{ Area\% } \\
\hline 1 & 17.839 & 57458 & 67.420 & 5746821 & 50.147 \\
\hline 2 & 37.494 & 27766 & 32.580 & 5713243 & 49.853 \\
\hline Total & & 85224 & 100.000 & 11460064 & 100.000 \\
\hline
\end{tabular}

$\mathrm{mV}$

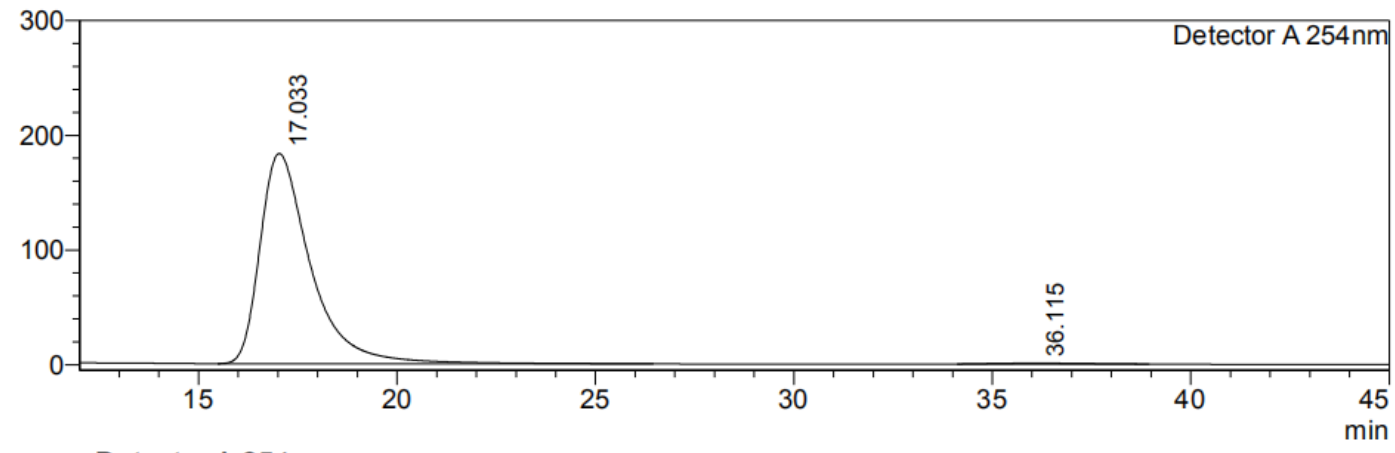

Detector A 254nm
\begin{tabular}{|r|r|r|r|r|r|}
\hline Peak\# & Ret. Time & Height & Height\% & \multicolumn{1}{c|}{ Area } & \multicolumn{1}{c|}{ Area $\%$} \\
\hline 1 & 17.033 & 183223 & 99.572 & 16285594 & 99.242 \\
\hline 2 & 36.115 & 788 & 0.428 & 124409 & 0.758 \\
\hline Total & & 184010 & 100.000 & 16410004 & 100.000 \\
\hline
\end{tabular}

(S)-4-chloro-2-(5-chloro-2-hydroxyphenylsulfonimidoyl)phenyl

\section{4-methylbenzenesulfonate $(6 \mathrm{~h})$}<smiles>O=S(=O)(Nc1ccc(Cl)cc1O)c1cc(Cl)ccc1O</smiles> 
White powder; $36 \mathrm{mg}, 77 \%$ yield; m.p. $=123-125{ }^{\circ} \mathrm{C} ;{ }^{1} \mathrm{H}$ NMR $\left(400 \mathrm{MHz}, \mathrm{CDCl}_{3}\right) \delta$ $11.98(\mathrm{~s}, 1 \mathrm{H}), 8.03(\mathrm{~d}, J=2.6 \mathrm{~Hz}, 1 \mathrm{H}), 7.84(\mathrm{~s}, 1 \mathrm{H}), 7.81(\mathrm{~s}, 1 \mathrm{H}), 7.52(\mathrm{dd}, J=8.8$, $2.6 \mathrm{~Hz}, 1 \mathrm{H}), 7.44(\mathrm{~d}, J=8.8 \mathrm{~Hz}, 1 \mathrm{H}), 7.39$ (d, $J=8.2 \mathrm{~Hz}, 2 \mathrm{H}), 7.36-7.29(\mathrm{~m}, 2 \mathrm{H})$, 6.89-6.81 (m, 1H), 3.85 (s, 1H), $2.49(\mathrm{~s}, 3 \mathrm{H}) ;{ }^{13} \mathrm{C} \mathrm{NMR}\left(100 \mathrm{MHz}, \mathrm{CDCl}_{3}\right) \delta 156.4$, $146.7,145.6,137.5,136.2,134.9,132.6,132.2,130.2,130.0,128.9,128.4,124.2$, 124.1, 121.1, 120.5, 22.0; HRMS (ESI) $\mathrm{m} / \mathrm{z}$ calcd for $\mathrm{C}_{19} \mathrm{H}_{15} \mathrm{Cl}_{2} \mathrm{NO}_{5} \mathrm{~S}_{2}[\mathrm{M}+\mathrm{H}]^{+}=$ 471.9847, found $=471.9845$.

Optical Rotation: $[\alpha]_{\mathrm{D}}^{25}=53.68\left(c=0.70, \mathrm{CHCl}_{3}\right) .91 \%$ ee (HPLC condition: Chiralpak AD-H column, $n$-Hexane $/ i-\mathrm{PrOH}=70: 30$, flow rate $=1.0 \mathrm{~mL} / \mathrm{min}$, wavelength $=254 \mathrm{~nm}, t_{\mathrm{R}}=11.71 \mathrm{~min}$ for major isomer, $t_{\mathrm{R}}=17.94$ min for minor isomer).

$\mathrm{mV}$

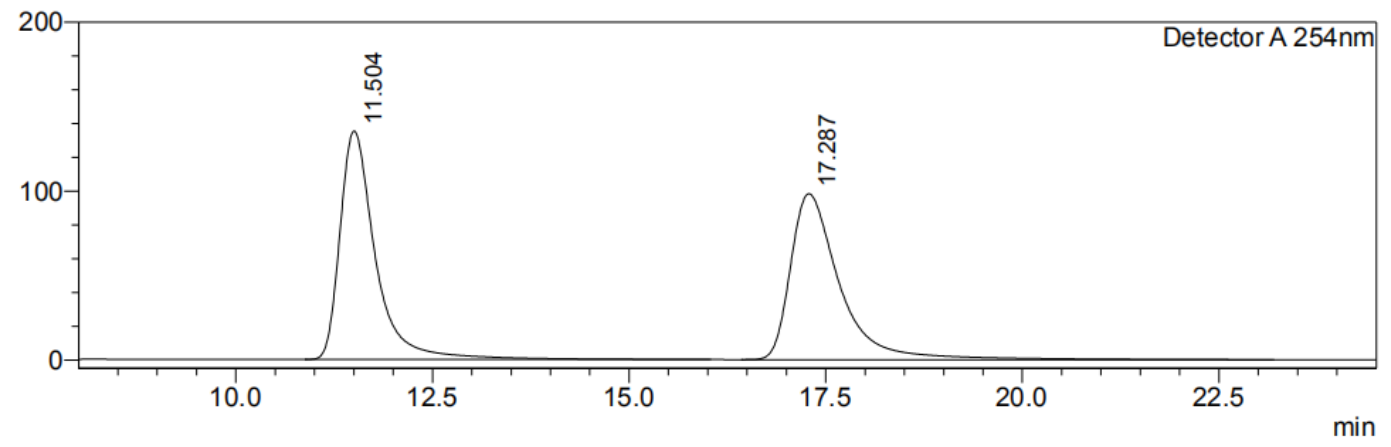

Detector A 254nm

\begin{tabular}{|r|r|r|r|r|r|}
\hline Peak\# & Ret. Time & \multicolumn{1}{c|}{ Height } & Height\% & Area & \multicolumn{1}{c|}{ Area\% } \\
\hline 1 & 11.504 & 135326 & 57.930 & 4274279 & 49.793 \\
\hline 2 & 17.287 & 98276 & 42.070 & 4309815 & 50.207 \\
\hline Total & & 233602 & 100.000 & 8584094 & 100.000 \\
\hline
\end{tabular}

$\mathrm{mV}$

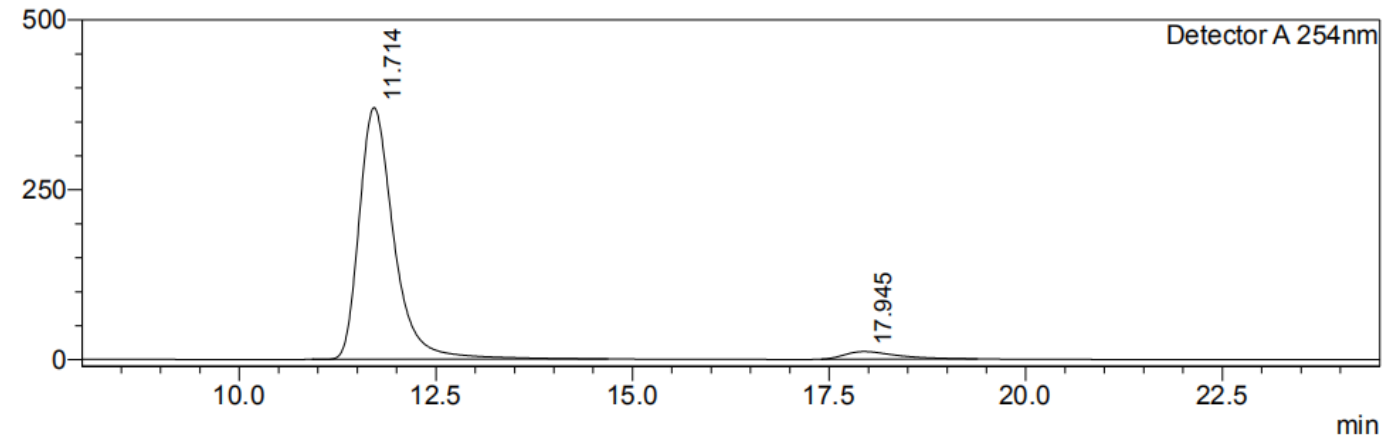

Detector A 254nm
\begin{tabular}{|r|r|r|r|r|r|}
\hline Peak\# & Ret. Time & Height & Height\% & \multicolumn{1}{c|}{ Area } & Area\% \\
\hline 1 & 11.714 & 370490 & 97.092 & 11364717 & 95.732 \\
\hline 2 & 17.945 & 11095 & 2.908 & 506719 & 4.268 \\
\hline Total & & 381585 & 100.000 & 11871436 & 100.000 \\
\hline
\end{tabular}

\section{(S)-4-bromo-2-(5-bromo-2-hydroxyphenylsulfonimidoyl)phenyl}




\section{$\underline{\text { 4-methylbenzenesulfonate (6i) }}$}<smiles>O=S(=O)(Nc1cc(Br)ccc1O)c1cc(Br)ccc1O</smiles>

White powder; $48 \mathrm{mg}, 86 \%$ yield; m.p. $=64-66{ }^{\circ} \mathrm{C} ;{ }^{1} \mathrm{H}$ NMR $\left(400 \mathrm{MHz}, \mathrm{CDCl}_{3}\right) \delta$ $8.18(\mathrm{~d}, J=2.5 \mathrm{~Hz}, 1 \mathrm{H}), 7.82(\mathrm{~s}, 1 \mathrm{H}), 7.80(\mathrm{~s}, 1 \mathrm{H}), 7.66(\mathrm{dd}, J=8.8,2.5 \mathrm{~Hz}, 1 \mathrm{H})$, 7.49-7.43 (m, 2H), 7.41-7.33 (m, 3H), $6.80(\mathrm{~d}, J=9.4 \mathrm{~Hz}, 1 \mathrm{H}), 2.48(\mathrm{~s}, 3 \mathrm{H}) ;{ }^{13} \mathrm{C}$ NMR $\left(100 \mathrm{MHz}, \mathrm{CDCl}_{3}\right) \delta 156.8,146.7,146.2,139.0,137.9,137.6,132.7,132.2$, 131.2, 130.2, 128.8, 124.2, 121.5, 121.0, 119.9, 110.7, 22.0; HRMS (ESI) $\mathrm{m} / z$ calcd for $\mathrm{C}_{19} \mathrm{H}_{15} \mathrm{Br}_{2} \mathrm{NO}_{5} \mathrm{~S}_{2}[\mathrm{M}+\mathrm{H}]^{+}=559.8836$, found $=559.8836$.

Optical Rotation: $[\alpha]_{\mathrm{D}}^{25}=68.90\left(c=0.31, \mathrm{CHCl}_{3}\right) .98 \%$ ee (HPLC condition: Chiralpak AD-H column, $n-\mathrm{Hexane} / i-\mathrm{PrOH}=60: 40$, flow rate $=1.0 \mathrm{~mL} / \mathrm{min}$, wavelength $=254 \mathrm{~nm}, t_{\mathrm{R}}=10.13$ min for major isomer, $t_{\mathrm{R}}=16.13$ min for minor isomer).

$\mathrm{mV}$

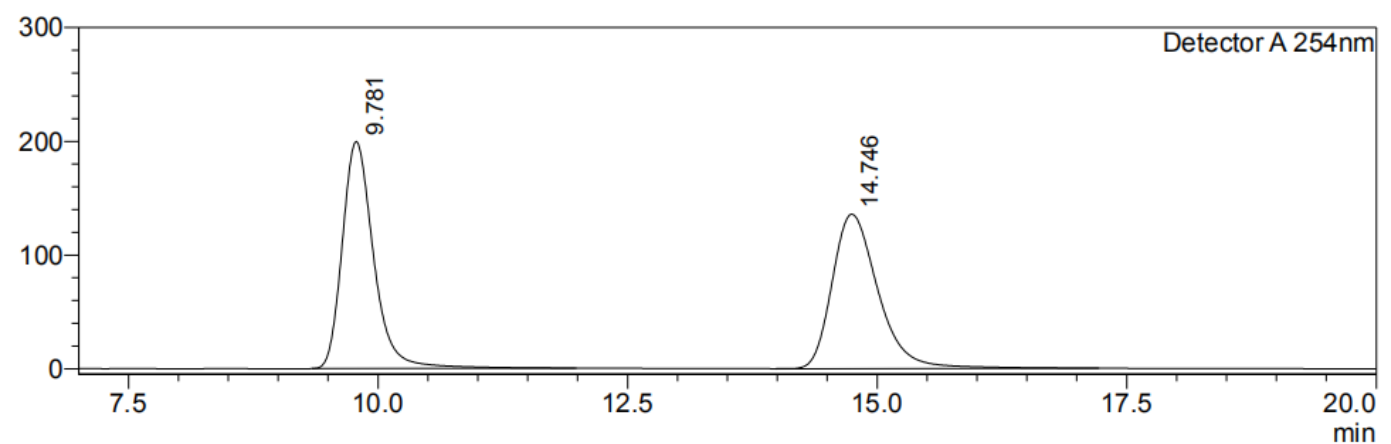

Detector A 254nm

\begin{tabular}{|r|r|r|r|r|r|}
\hline Peak\# & Ret. Time & \multicolumn{1}{c|}{ Height } & Height $\%$ & \multicolumn{1}{c|}{ Area } & \multicolumn{1}{c|}{ Area\% } \\
\hline 1 & 9.781 & 199627 & 59.515 & 4379501 & 50.156 \\
\hline 2 & 14.746 & 135796 & 40.485 & 4352172 & 49.844 \\
\hline Total & & 335424 & 100.000 & 8731673 & 100.000 \\
\hline
\end{tabular}


$\mathrm{mV}$

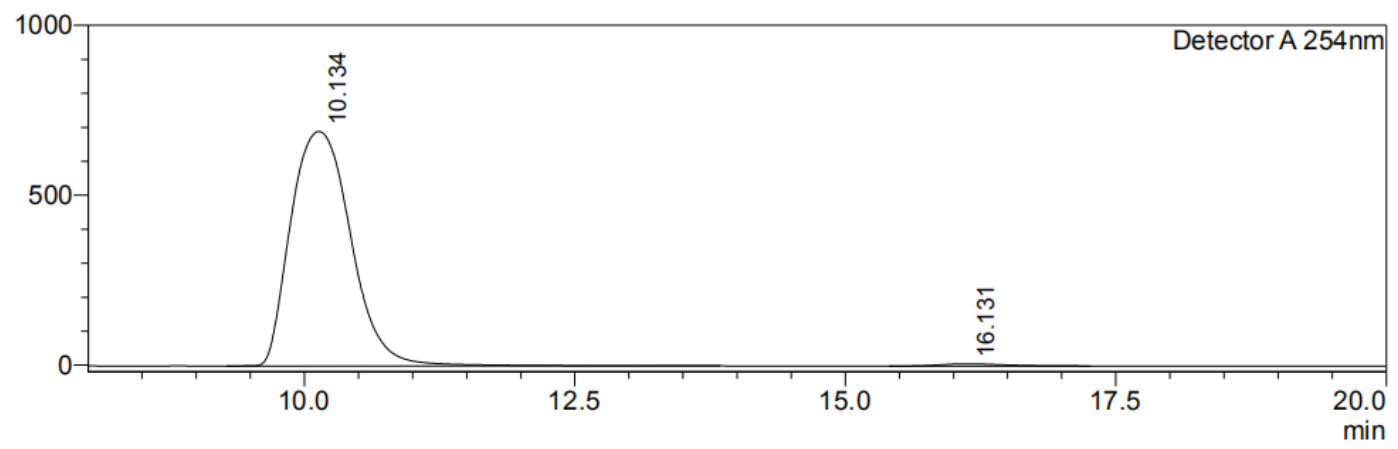

Detector A 254nm

\begin{tabular}{|r|r|r|r|r|r|}
\hline Peak\# & Ret. Time & \multicolumn{1}{c|}{ Height } & Height\% & \multicolumn{1}{c|}{ Area } & \multicolumn{1}{c|}{ Area\% } \\
\hline 1 & 10.134 & 690031 & 99.063 & 26749324 & 99.018 \\
\hline 2 & 16.131 & 6526 & 0.937 & 265307 & 0.982 \\
\hline Total & & 696557 & 100.000 & 27014631 & 100.000 \\
\hline
\end{tabular}

\section{(S)-4-cyclohexyl-2-(5-cyclohexyl-2-hydroxyphenylsulfonimidoyl)phenyl}

\section{4-methylbenzenesulfonate (6j)}<smiles>NS(=O)(=O)c1cc(C2CCCCC2)ccc1O</smiles>

Light yellow oil; 43 mg, 76\% yield; ${ }^{1} \mathrm{H}$ NMR (400 MHz, $\left.\mathrm{CDCl}_{3}\right) \delta 7.92$ (s, 1H), 7.90 (s, 1H), $7.76(\mathrm{~d}, J=1.8 \mathrm{~Hz}, 1 \mathrm{H}), 7.42-7.32(\mathrm{~m}, 4 \mathrm{H}), 7.29$ (d, $J=2.0 \mathrm{~Hz}, 1 \mathrm{H}), 7.24$ $(\mathrm{dd}, J=8.6,2.0 \mathrm{~Hz}, 1 \mathrm{H}), 6.81(\mathrm{~d}, J=8.6 \mathrm{~Hz}, 1 \mathrm{H}), 2.59-2.51(\mathrm{~m}, 1 \mathrm{H}), 2.47(\mathrm{~s}, 3 \mathrm{H})$, 2.35-2.18 (m, 1H), 1.88-1.67 (m, 10H), 1.39-1.17 (m, 10H); ${ }^{13} \mathrm{C}$ NMR (100 MHz, $\left.\mathrm{CDCl}_{3}\right) \delta 155.4,147.1,146.1,144.4,139.2,136.5,134.6,132.9,132.8,130.0,128.9$, $128.1,126.8,122.8,119.6,119.0,43.9,43.4,34.4,34.3,26.8,26.7,26.0,25.9,22.0$ HRMS (ESI) $m / z$ calcd for $\mathrm{C}_{31} \mathrm{H}_{37} \mathrm{NO}_{5} \mathrm{~S}_{2}[\mathrm{M}+\mathrm{H}]^{+}=568.2191$, found $=568.2161$.

Optical Rotation: $[\alpha]_{\mathrm{D}}^{25}=22.18\left(c=0.60, \mathrm{CHCl}_{3}\right) .>99 \%$ ee (HPLC condition: Chiralpak IG column, $n$-Hexane $/ i-\mathrm{PrOH}=70: 30$, flow rate $=1.0 \mathrm{~mL} / \mathrm{min}$, wavelength $=254 \mathrm{~nm}, t_{\mathrm{R}}=26.14 \min$ for major isomer, $t_{\mathrm{R}}=31.55 \mathrm{~min}$ for minor isomer). 
$\mathrm{mV}$

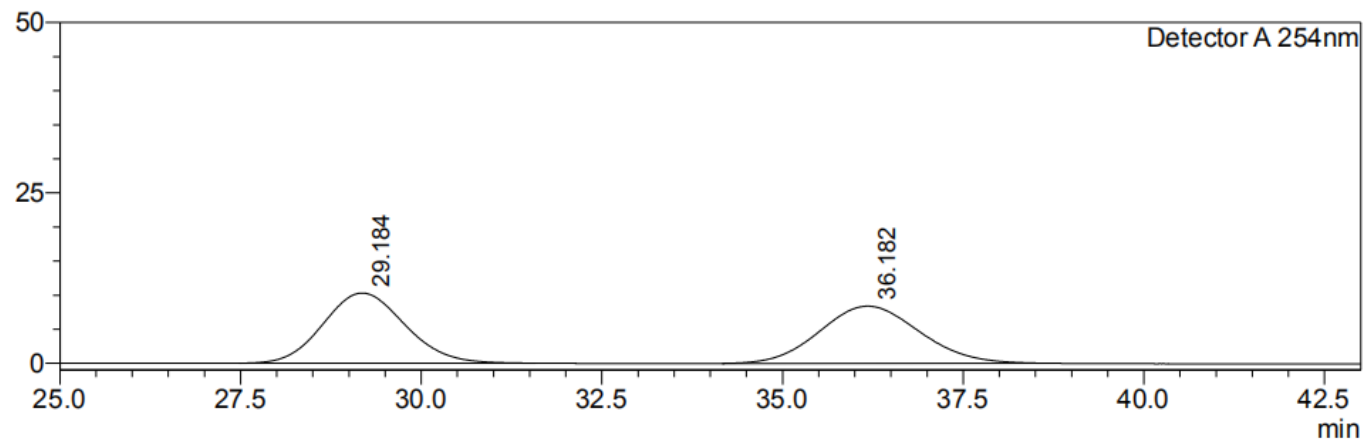

Detector A 254nm

\begin{tabular}{|r|r|r|r|r|r|}
\hline Peak\# & Ret. Time & Height & Height\% & \multicolumn{1}{c|}{ Area } & \multicolumn{1}{c|}{ Area\% } \\
\hline 1 & 29.184 & 10295 & 55.043 & 812315 & 49.919 \\
\hline 2 & 36.182 & 8408 & 44.957 & 814966 & 50.081 \\
\hline Total & & 18703 & 100.000 & 1627281 & 100.000 \\
\hline
\end{tabular}

$\mathrm{mV}$

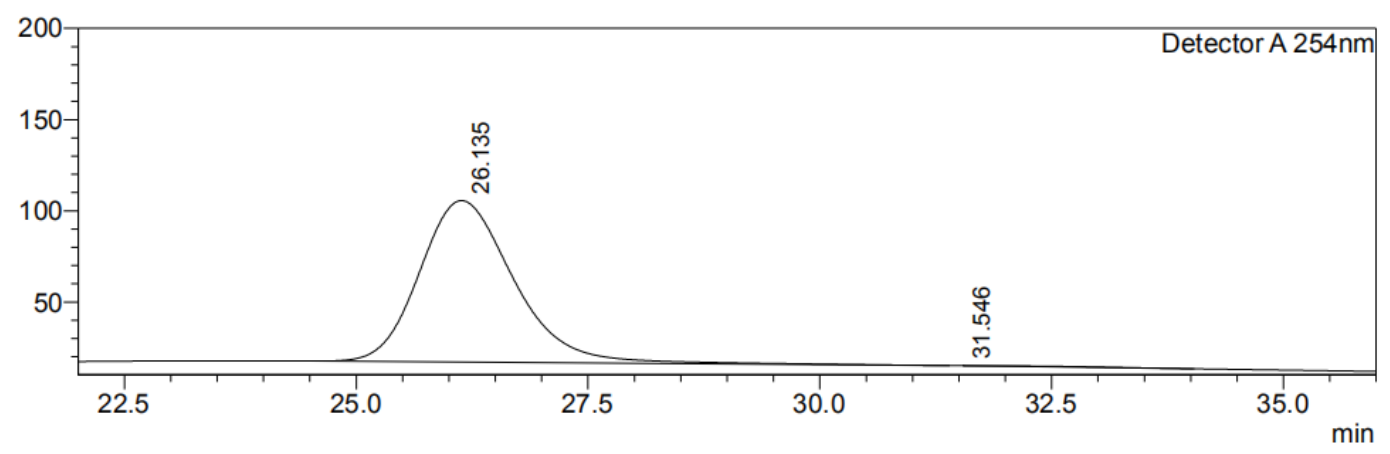

Detector A 254nm

\begin{tabular}{|r|r|r|r|r|r|}
\hline Peak\# & Ret. Time & \multicolumn{1}{c|}{ Height } & \multicolumn{1}{c|}{ Height\% } & \multicolumn{1}{c|}{ Area } & \multicolumn{1}{c|}{ Area\% } \\
\hline 1 & 26.135 & 88582 & 99.997 & 6303700 & 99.572 \\
\hline 2 & 31.546 & 3 & 0.003 & 27074 & 0.428 \\
\hline Total & & 88584 & 100.000 & 6330774 & 100.000 \\
\hline
\end{tabular}

(S)-2-(2-hydroxy-5-(2-phenylpropan-2-yl)phenylsulfonimidoyl)-4-(2-phenylpropa

\section{$\underline{\text { n-2-yl)phenyl 4-methylbenzenesulfonate (6k) }}$}<smiles>CC(C)(c1ccccc1)c1ccc(O)c(S(=O)(=O)c2cc(C(C)(C)c3ccccc3)ccc2O)c1</smiles>

White solid; $47 \mathrm{mg}, 74 \%$ yield; m.p. $=99-101{ }^{\circ} \mathrm{C} ;{ }^{1} \mathrm{H}$ NMR $\left(400 \mathrm{MHz}, \mathrm{CDCl}_{3}\right) \delta 7.90$ (s, 1H), $7.88(\mathrm{~s}, 1 \mathrm{H}), 7.78(\mathrm{~s}, 1 \mathrm{H}), 7.43(\mathrm{~d}, J=2.4 \mathrm{~Hz}, 1 \mathrm{H}), 7.37(\mathrm{~d}, J=8.4 \mathrm{~Hz}, 2 \mathrm{H})$, $7.33(\mathrm{~s}, 2 \mathrm{H}), 7.27(\mathrm{~d}, J=7.2 \mathrm{~Hz}, 2 \mathrm{H}), 7.23-7.16(\mathrm{~m}, 3 \mathrm{H}), 7.16-7.12(\mathrm{~m}, 3 \mathrm{H}), 7.10(\mathrm{dd}$, $J=7.6,6.2 \mathrm{~Hz}, 3 \mathrm{H}), 6.77(\mathrm{~d}, J=8.8 \mathrm{~Hz}, 1 \mathrm{H}), 2.47(\mathrm{~s}, 3 \mathrm{H}), 1.65(\mathrm{~s}, 3 \mathrm{H}), 1.64(\mathrm{~s}, 3 \mathrm{H})$, 1.54 (s, 3H), 1.53 (s, 3H); ${ }^{13} \mathrm{C}$ NMR (100 MHz, $\left.\mathrm{CDCl}_{3}\right) \delta 155.5,150.1,149.8,148.8$, $146.2,144.4,142.0,136.1,135.4,133.1,132.8,130.1,128.8,128.5,128.2,127.8$, 
$126.7,126.6,126.4,126.1,125.9,122.6,119.0,118.8,43.1,42.4,30.7,30.6,30.5$, 21.9; HRMS (ESI) $\mathrm{m} / z$ calcd for $\mathrm{C}_{37} \mathrm{H}_{37} \mathrm{NO}_{5} \mathrm{~S}_{2}[\mathrm{M}+\mathrm{H}]^{+}=640.2191$, found $=640.2156$.

Optical Rotation: $[\alpha]^{25}=31.50\left(c=0.90, \mathrm{CHCl}_{3}\right) .93 \%$ ee (HPLC condition: Chiralpak IE column, $n-\mathrm{Hexane} / i-\mathrm{PrOH}=70: 30$, flow rate $=1.0 \mathrm{~mL} / \mathrm{min}$, wavelength $=254 \mathrm{~nm}, t_{\mathrm{R}}=19.90 \min$ for major isomer, $t_{\mathrm{R}}=22.12 \mathrm{~min}$ for minor isomer).

$\mathrm{mV}$

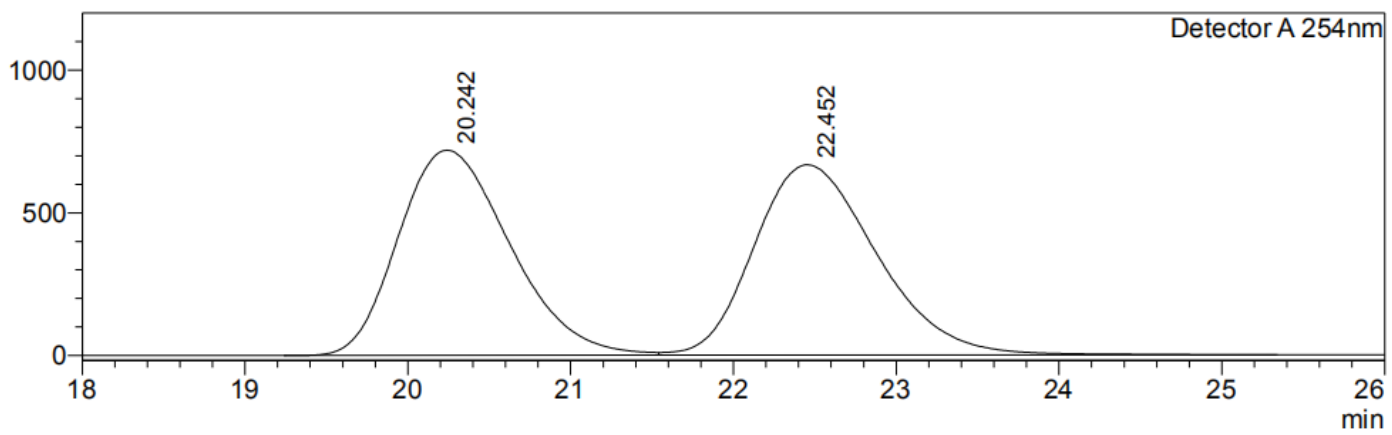

\begin{tabular}{|c|c|c|c|c|c|}
\hline Peak\# & Ret. Time & Height & Height $\%$ & Area & Area $\%$ \\
\hline 1 & 20.242 & 719175 & 51.880 & 34307616 & 49.521 \\
\hline 2 & 22.452 & 667052 & 48.120 & 34971435 & 50.479 \\
\hline Total & & 1386227 & 100.000 & 69279050 & 100.000 \\
\hline
\end{tabular}

$\mathrm{mV}$

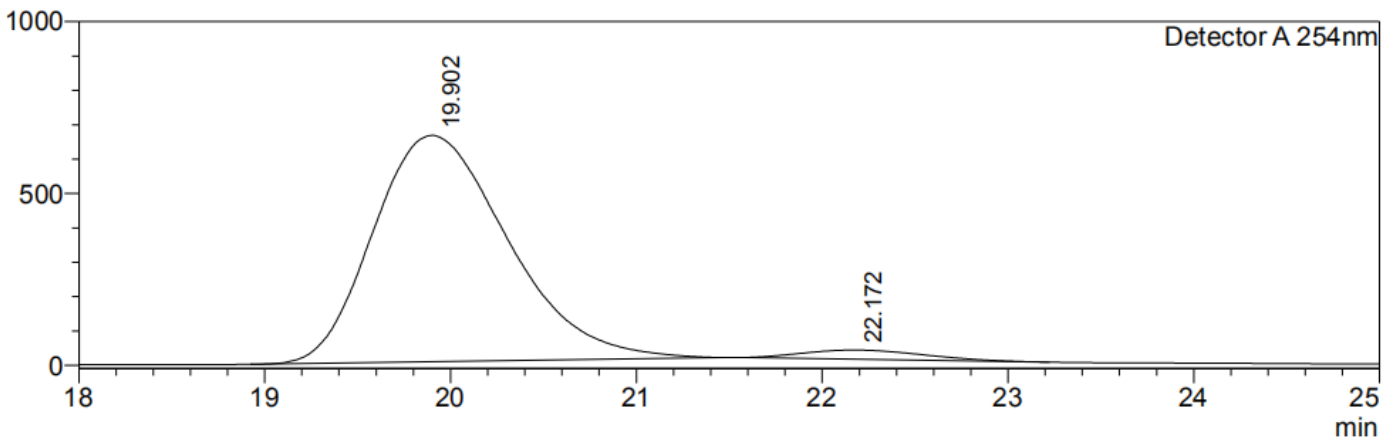

Detector A 254nm

\begin{tabular}{|r|r|r|r|r|r|}
\hline Peak\# & Ret. Time & Height & \multicolumn{1}{c|}{ Height $\%$} & \multicolumn{1}{c|}{ Area } & Area\% \\
\hline 1 & 19.902 & 658689 & 96.160 & 33225626 & 96.572 \\
\hline 2 & 22.172 & 26303 & 3.840 & 1179543 & 3.428 \\
\hline Total & & 684992 & 100.000 & 34405169 & 100.000 \\
\hline
\end{tabular}

(S)-2-(2-hydroxy-5-tritylphenylsulfonimidoyl)-4-tritylphenyl

\section{4-methylbenzenesulfonate (61)}<smiles>NS(=O)(=O)c1cc(C(c2ccccc2)(c2ccccc2)c2ccccc2)ccc1[N+](=O)[O-]</smiles> 
White powder; $69 \mathrm{mg}, 78 \%$ yield; m.p. $=88-90{ }^{\circ} \mathrm{C} ;{ }^{1} \mathrm{H}$ NMR $\left(400 \mathrm{MHz}, \mathrm{CDCl}_{3}\right) \delta$ $7.81(\mathrm{~s}, 1 \mathrm{H}), 7.79(\mathrm{~s}, 1 \mathrm{H}), 7.69$ (d, $J=2.4 \mathrm{~Hz}, 1 \mathrm{H}), 7.44$ (d, $J=8.8 \mathrm{~Hz}, 1 \mathrm{H}), 7.38$ (dd, $J=8.8,2.4 \mathrm{~Hz}, 1 \mathrm{H}), 7.32(\mathrm{~d}, J=8.1 \mathrm{~Hz}, 2 \mathrm{H}), 7.24-7.17(\mathrm{~m}, 11 \mathrm{H}), 7.16-7.09(\mathrm{~m}, 9 \mathrm{H})$, 7.07-6.97 (m, 12H), $6.68(\mathrm{~d}, J=8.8 \mathrm{~Hz}, 1 \mathrm{H}), 2.46(\mathrm{~s}, 3 \mathrm{H}),{ }^{13} \mathrm{C}$ NMR $(100 \mathrm{MHz}$, $\left.\mathrm{CDCl}_{3}\right) \delta 155.8,146.3,146.2,146.0,145.6,144.4,138.6,138.2,137.4,134.8,132.6$ $131.2,130.9,130.8,130.6,130.0,128.8,128.2,128.0,127.8,126.6,126.2,121.7$, 118.5, 64.7, 64.2, 22.0; HRMS (ESI) $m / z$ calcd for $\mathrm{C}_{57} \mathrm{H}_{45} \mathrm{NO}_{5} \mathrm{~S}_{2}[\mathrm{M}+\mathrm{H}]^{+}=888.2817$, found $=888.2769$.

Optical Rotation: $[\alpha]_{\mathrm{D}}^{25}=29.80\left(c=0.50, \mathrm{CHCl}_{3}\right) .92 \%$ ee (HPLC condition: Chiralpak IG column, $n$-Hexane $/ i-\mathrm{PrOH}=75: 25$, flow rate $=1.0 \mathrm{~mL} / \mathrm{min}$, wavelength $=254 \mathrm{~nm}, t_{\mathrm{R}}=11.76 \mathrm{~min}$ for minor isomer, $t_{\mathrm{R}}=18.98 \mathrm{~min}$ for major isomer).

$\mathrm{mV}$

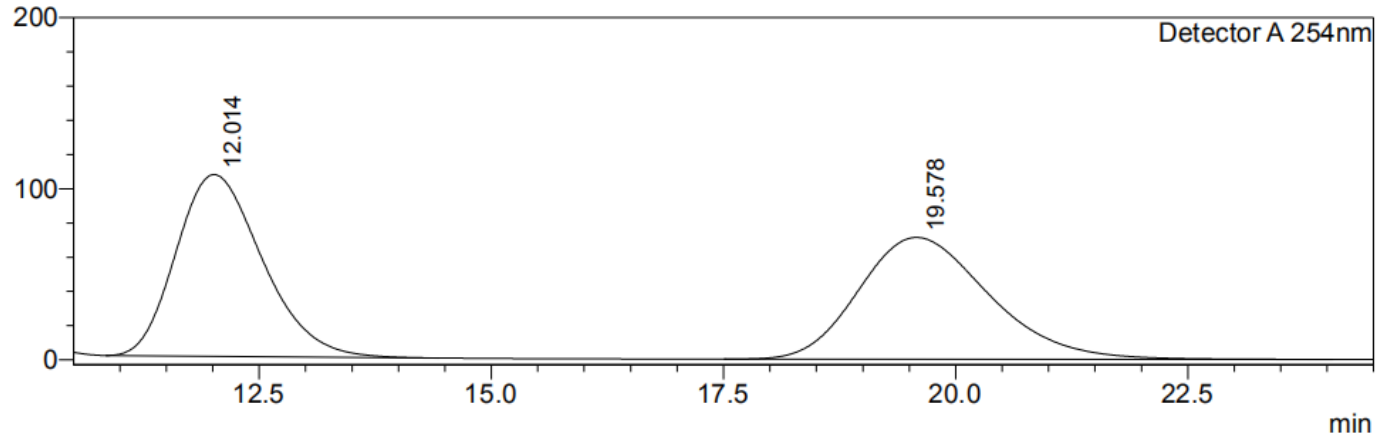

Detector A 254nm
\begin{tabular}{|r|r|r|r|r|r|}
\hline Peak\# & Ret. Time & Height & Height\% & \multicolumn{1}{c|}{ Area } & \multicolumn{1}{|c|}{ Area\% } \\
\hline 1 & 12.014 & 106248 & 59.874 & 6878139 & 49.633 \\
\hline 2 & 19.578 & 71203 & 40.126 & 6979763 & 50.367 \\
\hline Total & & 177452 & 100.000 & 13857902 & 100.000 \\
\hline
\end{tabular}

$\mathrm{mV}$

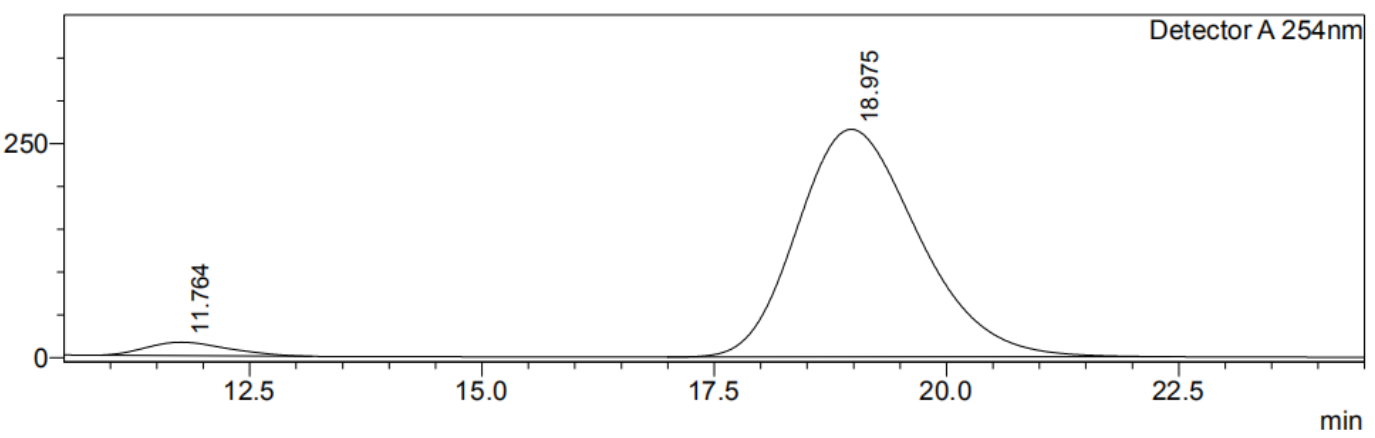

Detector A 254nm
\begin{tabular}{|r|r|r|r|r|r|}
\hline Peak\# & Ret. Time & Height & Height $\%$ & \multicolumn{1}{c|}{ Area } & \multicolumn{1}{|c|}{ Area $\%$} \\
\hline 1 & 11.764 & 15617 & 5.553 & 953308 & 3.794 \\
\hline 2 & 18.975 & 265632 & 94.447 & 24171771 & 96.206 \\
\hline Total & & 281250 & 100.000 & 25125079 & 100.000 \\
\hline
\end{tabular}




\section{4-methylbenzenesulfonate $(6 \mathrm{~m})$}<smiles>CC(C)(C)c1ccc(S(=O)(=O)c2ccc(C(C)(C)C)cc2O)c([O+])c1</smiles>

Colorless oil; $42 \mathrm{mg}, 81 \%$ yield; ${ }^{1} \mathrm{H}$ NMR (400 MHz, $\left.\mathrm{CDCl}_{3}\right) \delta 12.19$ (s, 1H), 7.89-7.82 (m, 3H), $7.38(\mathrm{t}, J=8.6 \mathrm{~Hz}, 3 \mathrm{H}), 7.34-7.30(\mathrm{~m}, 2 \mathrm{H}), 6.92(\mathrm{~d}, J=2.0 \mathrm{~Hz}$, $1 \mathrm{H}), 6.85(\mathrm{dd}, J=8.6,2.0 \mathrm{~Hz}, 1 \mathrm{H}), 3.87(\mathrm{~s}, 1 \mathrm{H}), 2.48(\mathrm{~s}, 3 \mathrm{H}), 1.26(\mathrm{~s}, 9 \mathrm{H}), 1.24$ (s, $9 \mathrm{H}) ;{ }^{13} \mathrm{C} \mathrm{NMR}\left(100 \mathrm{MHz}, \mathrm{CDCl}_{3}\right) \delta 160.0,159.0,157.3,146.7,146.1,134.1,132.9$, 130.0, 129.8, 129.1, 129.0, 123.8, 120.2, 117.4, 117.2, 116.1, 35.4, 35.3, 31.0, 30.9, 21.9; HRMS (ESI) $m / z$ calcd for $\mathrm{C}_{27} \mathrm{H}_{33} \mathrm{NO}_{5} \mathrm{~S}_{2}[\mathrm{M}+\mathrm{H}]^{+}=516.1878$, found $=516.1838$.

Optical Rotation: $[\alpha]_{\mathrm{D}}^{25}=123.00\left(c=0.50, \mathrm{CHCl}_{3}\right) .>99 \%$ ee (HPLC condition: Chiralpak AD-H column, $n-\mathrm{Hexane} / i-\mathrm{PrOH}=75: 25$, flow rate $=1.0 \mathrm{~mL} / \mathrm{min}$, wavelength $=254 \mathrm{~nm}, t_{\mathrm{R}}=10.00$ min for major isomer, $t_{\mathrm{R}}=14.43$ min for minor isomer).

$\mathrm{mV}$

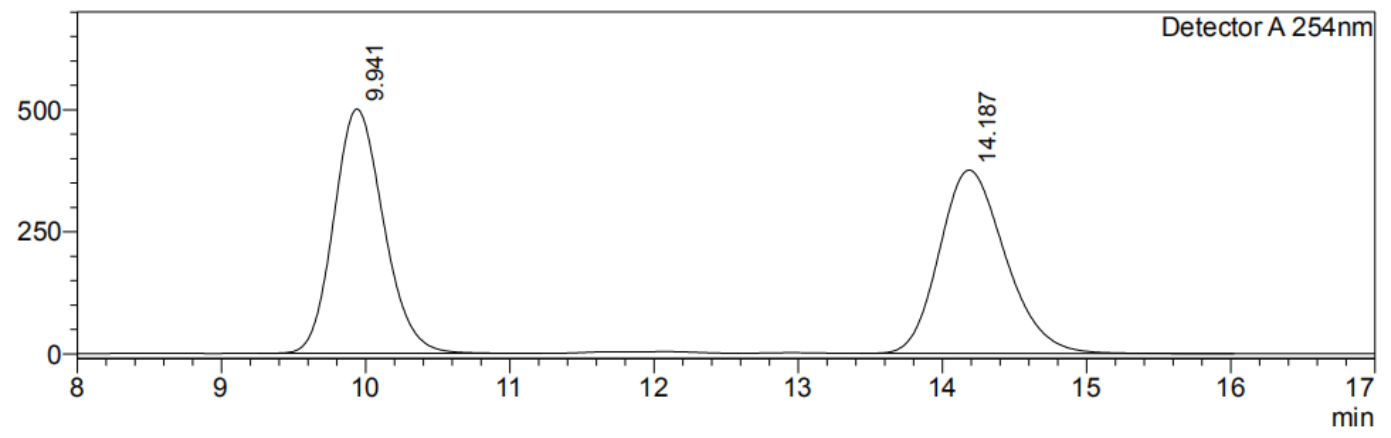

Detector A 254nm

\begin{tabular}{|c|c|c|c|c|c|}
\hline Peak\# & Ret. Time & Height & Height $\%$ & Area & Area $\%$ \\
\hline 1 & 9.941 & 500356 & 57.130 & 12011147 & 50.106 \\
\hline 2 & 14.187 & 375463 & 42.870 & 11960474 & 49.894 \\
\hline Total & & 875820 & 100.000 & 23971620 & 100.000 \\
\hline
\end{tabular}


$\mathrm{mV}$

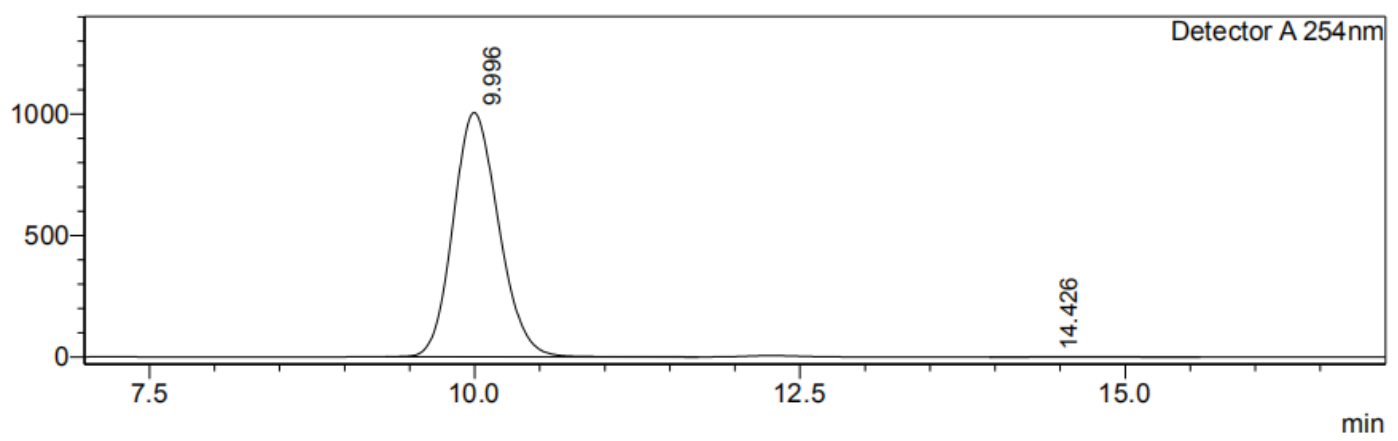

Detector A $254 \mathrm{~nm}$

\begin{tabular}{|r|r|r|r|r|r|}
\hline Peak\# & Ret. Time & Height & Height $\%$ & \multicolumn{1}{c|}{ Area } & \multicolumn{1}{c|}{ Area\% } \\
\hline 1 & 9.996 & 1003896 & 99.924 & 24453359 & 99.814 \\
\hline 2 & 14.426 & 765 & 0.076 & 45576 & 0.186 \\
\hline Total & & 1004661 & 100.000 & 24498935 & 100.000 \\
\hline
\end{tabular}

\section{(S)-5-chloro-2-(4-chloro-2-hydroxyphenylsulfonimidoyl)phenyl}

\section{4-methylbenzenesulfonate (6n)}<smiles>NS(=O)(=O)c1ccc(Cl)cc1[N+](=O)[O-]</smiles>

Yellow oil; $39 \mathrm{mg}, 83 \%$ yield; ${ }^{1} \mathrm{H}$ NMR (400 MHz, $\mathrm{CDCl}_{3}$ ) $\delta 12.27$ (s, 1H), 7.98 (d, $J$ $=8.6 \mathrm{~Hz}, 1 \mathrm{H}), 7.84(\mathrm{~s}, 1 \mathrm{H}), 7.82(\mathrm{~s}, 1 \mathrm{H}), 7.53(\mathrm{~d}, J=2.0 \mathrm{~Hz}, 1 \mathrm{H}), 7.40(\mathrm{~d}, J=8.2 \mathrm{~Hz}$, 2H), $7.35(\mathrm{dd}, J=8.6,2.0 \mathrm{~Hz}, 1 \mathrm{H}), 7.27(\mathrm{~d}, J=8.7 \mathrm{~Hz}, 1 \mathrm{H}), 6.85(\mathrm{~d}, J=2.0 \mathrm{~Hz}, 1 \mathrm{H})$, $6.76(\mathrm{dd}, J=8.7,2.0 \mathrm{~Hz}, 1 \mathrm{H}), 3.96(\mathrm{~s}, 1 \mathrm{H}), 2.50(\mathrm{~s}, 3 \mathrm{H}) ;{ }^{13} \mathrm{C} \mathrm{NMR}\left(100 \mathrm{MHz}, \mathrm{CDCl}_{3}\right)$ $\delta 160.0,159.0,157.3,146.7,146.1,134.1,132.9,130.0,129.8,129.1,129.0,123.8$, 120.2, 117.4, 117.2, 116.1, 35.4, 35.3, 31.0, 30.9, 21.9; HRMS (ESI) $\mathrm{m} / \mathrm{z}$ calcd for $\mathrm{C}_{19} \mathrm{H}_{15} \mathrm{Cl}_{2} \mathrm{NO}_{5} \mathrm{~S}_{2}[\mathrm{M}+\mathrm{H}]^{+}=471.9847$, found $=471.9848$.

Optical Rotation: $[\alpha]_{\mathrm{D}}^{25}=80.00\left(c=0.51, \mathrm{CHCl}_{3}\right) .90 \%$ ee (HPLC condition: Chiralpak IE column, $n$-Hexane $/ i-\mathrm{PrOH}=80: 20$, flow rate $=1.0 \mathrm{~mL} / \mathrm{min}$, wavelength $=254 \mathrm{~nm}, t_{\mathrm{R}}=23.10 \mathrm{~min}$ for minor isomer, $t_{\mathrm{R}}=26.80 \mathrm{~min}$ for major isomer). 
$\mathrm{mV}$

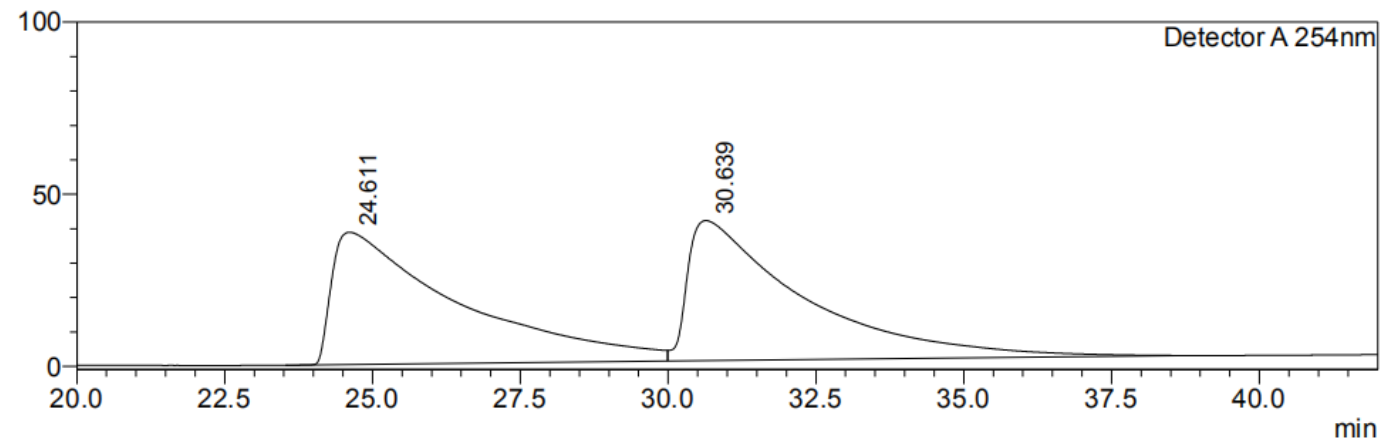

Detector A 254nm
\begin{tabular}{|r|r|r|r|r|r|}
\hline Peak\# & Ret. Time & Height & Height\% & \multicolumn{1}{c|}{ Area } & \multicolumn{1}{c|}{ Area\% } \\
\hline 1 & 24.611 & 38453 & 48.579 & 5679160 & 50.877 \\
\hline 2 & 30.639 & 40702 & 51.421 & 5483293 & 49.123 \\
\hline Total & & 79155 & 100.000 & 11162453 & 100.000 \\
\hline
\end{tabular}

$\mathrm{mV}$

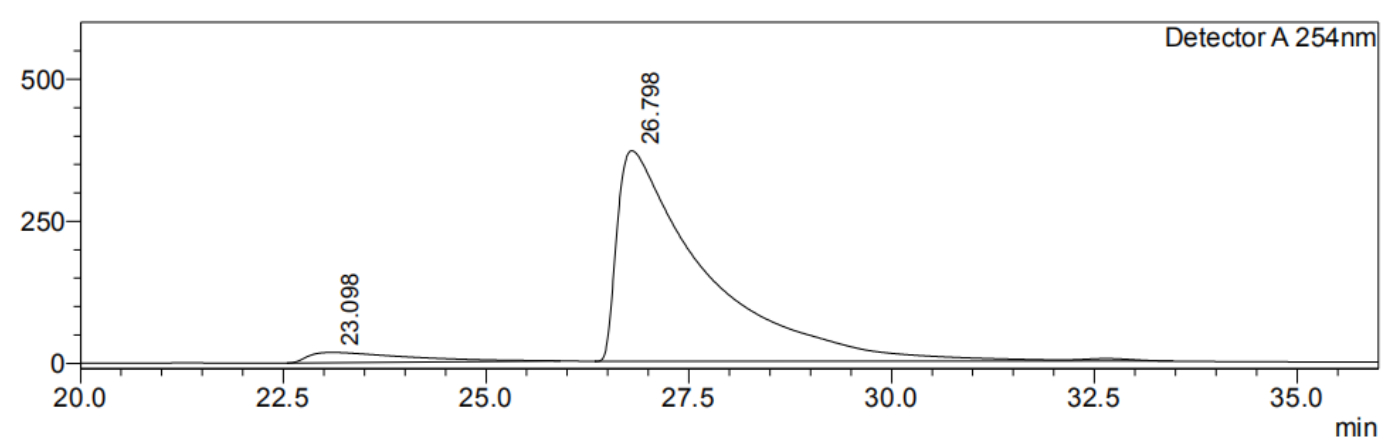

Detector A 254nm
\begin{tabular}{|r|r|r|r|r|r|}
\hline Peak\# & Ret. Time & Height & Height\% & \multicolumn{1}{|c|}{ Area } & \multicolumn{1}{c|}{ Area\% } \\
\hline 1 & 23.098 & 18101 & 4.655 & 1528374 & 5.134 \\
\hline 2 & 26.798 & 370782 & 95.345 & 28242621 & 94.866 \\
\hline Total & & 388883 & 100.000 & 29770994 & 100.000 \\
\hline
\end{tabular}

(S)-2-(2-hydroxy-4,5-dimethylphenylsulfonimidoyl)-4,5-dimethylphenyl

\section{4-methylbenzenesulfonate (6o)}<smiles>Cc1cc(O)c(S(=O)(=O)c2cc(C)c(C)cc2O)cc1C</smiles>

White powder; $37 \mathrm{mg}, 80 \%$ yield; m.p. $=72-74{ }^{\circ} \mathrm{C} ;{ }^{1} \mathrm{H}$ NMR $\left(400 \mathrm{MHz}, \mathrm{CDCl}_{3}\right) \delta$ $7.87(\mathrm{~s}, 1 \mathrm{H}), 7.85(\mathrm{~s}, 1 \mathrm{H}), 7.76(\mathrm{~s}, 1 \mathrm{H}), 7.36(\mathrm{~d}, J=8.2 \mathrm{~Hz}, 2 \mathrm{H}), 7.27$ (s, 1H), 7.12 (s, 1H), $6.72(\mathrm{~s}, 1 \mathrm{H}), 2.47(\mathrm{~s}, 3 \mathrm{H}), 2.26(\mathrm{~s}, 3 \mathrm{H}), 2.25$ (s, 3H), $2.18(\mathrm{~s}, 3 \mathrm{H}), 2.03(\mathrm{~s}, 3 \mathrm{H})$;

${ }^{13} \mathrm{C}$ NMR $\left(100 \mathrm{MHz}, \mathrm{CDCl}_{3}\right) \delta$ 155.2, 146.2, 146.1, 144.8, 144.5, 135.8, 133.0, 132.7, $130.7,129.9,129.1,129.0,128.0,123.8,119.7,116.9,21.9,20.3,20.2,19.4,18.8$ HRMS (ESI) $m / z$ calcd for $\mathrm{C}_{23} \mathrm{H}_{25} \mathrm{NO}_{5} \mathrm{~S}_{2}[\mathrm{M}+\mathrm{H}]^{+}=460.1252$, found $=460.1231$. 
Optical Rotation: $[\alpha]_{\mathrm{D}}^{25}=56.67\left(c=0.51, \mathrm{CHCl}_{3}\right) .90 \%$ ee (HPLC condition: Chiralpak ID column, $n$-Hexane $/ i-\mathrm{PrOH}=80: 20$, flow rate $=1.0 \mathrm{~mL} / \mathrm{min}$, wavelength $=254 \mathrm{~nm}, t_{\mathrm{R}}=12.64$ min for major isomer, $t_{\mathrm{R}}=17.56$ min for minor isomer).

$\mathrm{mv}$

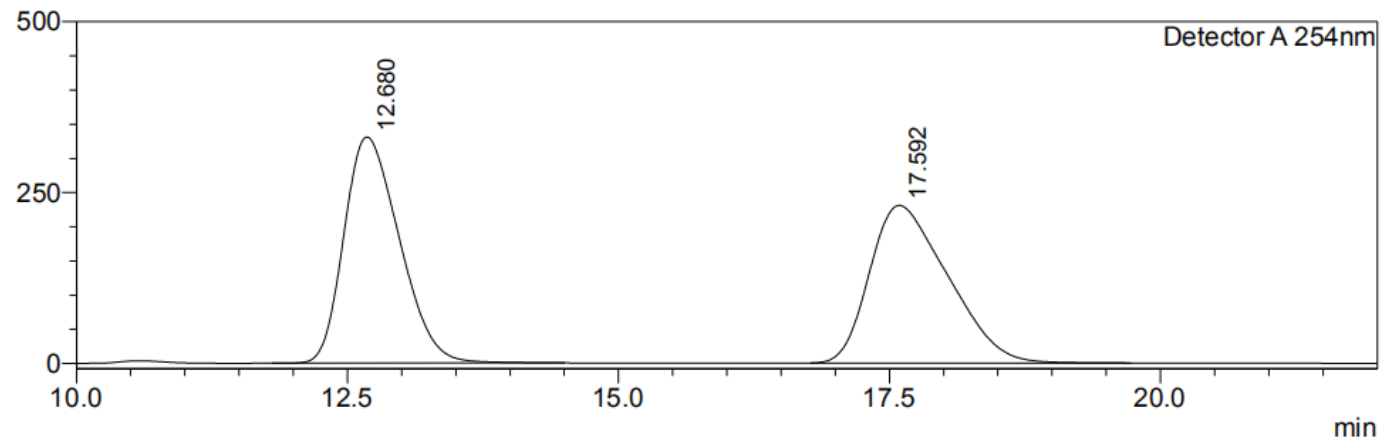

Detector A 254nm

\begin{tabular}{|r|r|r|r|r|r|}
\hline Peak\# & Ret. Time & Height & Height\% & \multicolumn{1}{c|}{ Area } & \multicolumn{1}{c|}{ Area\% } \\
\hline 1 & 12.680 & 330692 & 58.924 & 11767370 & 50.477 \\
\hline 2 & 17.592 & 230524 & 41.076 & 11545134 & 49.523 \\
\hline Total & & 561215 & 100.000 & 23312504 & 100.000 \\
\hline
\end{tabular}

$\mathrm{mV}$

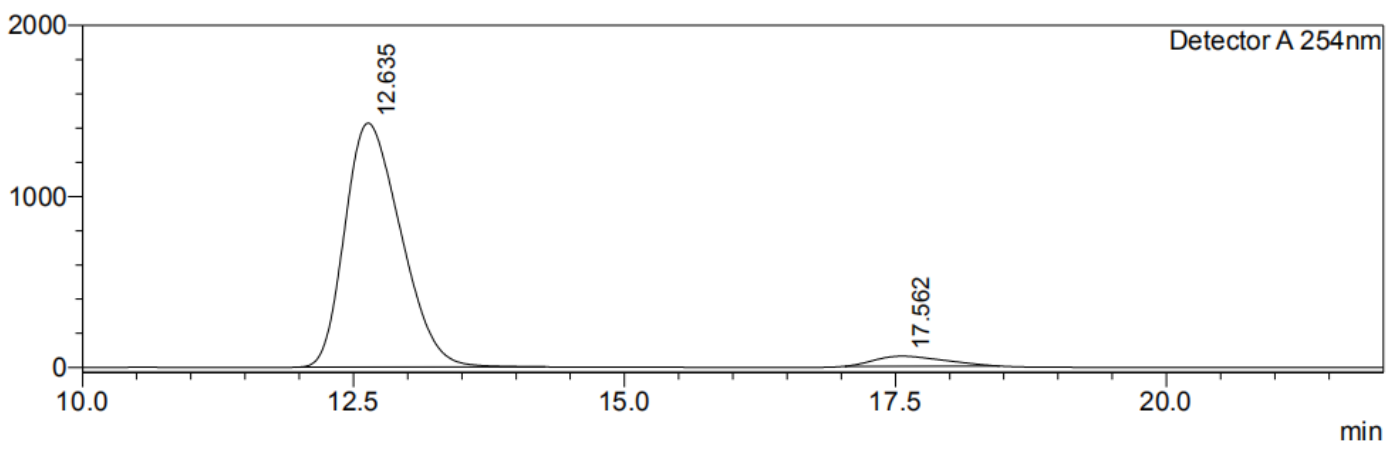

Detector A 254nm

\begin{tabular}{|r|r|r|r|r|r|}
\hline Peak\# & Ret. Time & \multicolumn{1}{c|}{ Height } & Height\% & \multicolumn{1}{c|}{ Area } & \multicolumn{1}{c|}{ Area\% } \\
\hline 1 & 12.635 & 1427148 & 96.061 & 51352503 & 95.172 \\
\hline 2 & 17.562 & 58523 & 3.939 & 2605229 & 4.828 \\
\hline Total & & 1485671 & 100.000 & 53957732 & 100.000 \\
\hline
\end{tabular}

(S)-5-chloro-2-(4-chloro-2-hydroxy-5-methylphenylsulfonimidoyl)-4-methylphen

\section{yl 4-methylbenzenesulfonate (6p)}<smiles>Cc1cc(O)c(S(=O)(=O)c2cc(C)c(Cl)cc2O)cc1O</smiles>

Light yellow solid; $41 \mathrm{mg}, 82 \%$ yield; m.p. $=104-105{ }^{\circ} \mathrm{C} ;{ }^{1} \mathrm{H}$ NMR $(400 \mathrm{MHz}$, $\left.\mathrm{CDCl}_{3}\right) \delta 7.91(\mathrm{~s}, 1 \mathrm{H}), 7.85(\mathrm{~s}, 1 \mathrm{H}), 7.83(\mathrm{~s}, 1 \mathrm{H}), 7.51(\mathrm{~s}, 1 \mathrm{H}), 7.39(\mathrm{~d}, J=8.2 \mathrm{~Hz}$, 
2H), $7.21(\mathrm{~s}, 1 \mathrm{H}), 6.89(\mathrm{~s}, 1 \mathrm{H}), 2.49(\mathrm{~s}, 3 \mathrm{H}), 2.40(\mathrm{~s}, 3 \mathrm{H}), 2.16(\mathrm{~s}, 3 \mathrm{H}),{ }^{13} \mathrm{C}$ NMR $(100$ $\left.\mathrm{MHz}, \mathrm{CDCl}_{3}\right) \delta 156.0,146.6,144.9,142.1,140.6,135.5,134.3,132.2,131.5,130.3$, $130.1,128.9,127.5,123.4,119.5,118.2,22.0,19.9,19.1$; HRMS (ESI) $\mathrm{m} / z$ calcd for $\mathrm{C}_{21} \mathrm{H}_{19} \mathrm{Cl}_{2} \mathrm{NO}_{5} \mathrm{~S}_{2}[\mathrm{M}+\mathrm{H}]^{+}=500.0160$, found $=500.0154$.

Optical Rotation: $[\alpha]_{\mathrm{D}}^{25}=57.90\left(c=0.50, \mathrm{CHCl}_{3}\right) .99 \%$ ee (HPLC condition: Chiralpak IE column, $n-\mathrm{Hexane} / i-\mathrm{PrOH}=70: 30$, flow rate $=1.0 \mathrm{~mL} / \mathrm{min}$, wavelength $=254 \mathrm{~nm}, t_{\mathrm{R}}=16.24 \mathrm{~min}$ for minor isomer, $t_{\mathrm{R}}=19.75 \mathrm{~min}$ for major isomer).

$\mathrm{mV}$

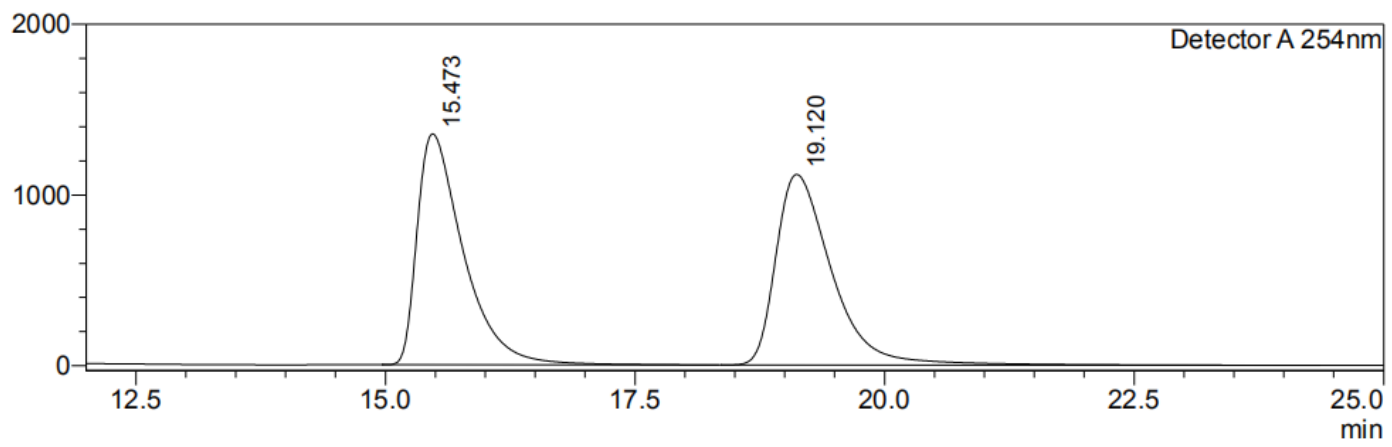

Detector A $254 \mathrm{~nm}$
\begin{tabular}{|r|r|r|r|r|r|}
\hline Peak\# & Ret. Time & Height & Height $\%$ & Area & \multicolumn{1}{c|}{ Area\% } \\
\hline 1 & 15.473 & 1353651 & 54.791 & 43630581 & 49.916 \\
\hline 2 & 19.120 & 1116916 & 45.209 & 43776554 & 50.084 \\
\hline Total & & 2470567 & 100.000 & 87407135 & 100.000 \\
\hline
\end{tabular}

$\mathrm{mV}$

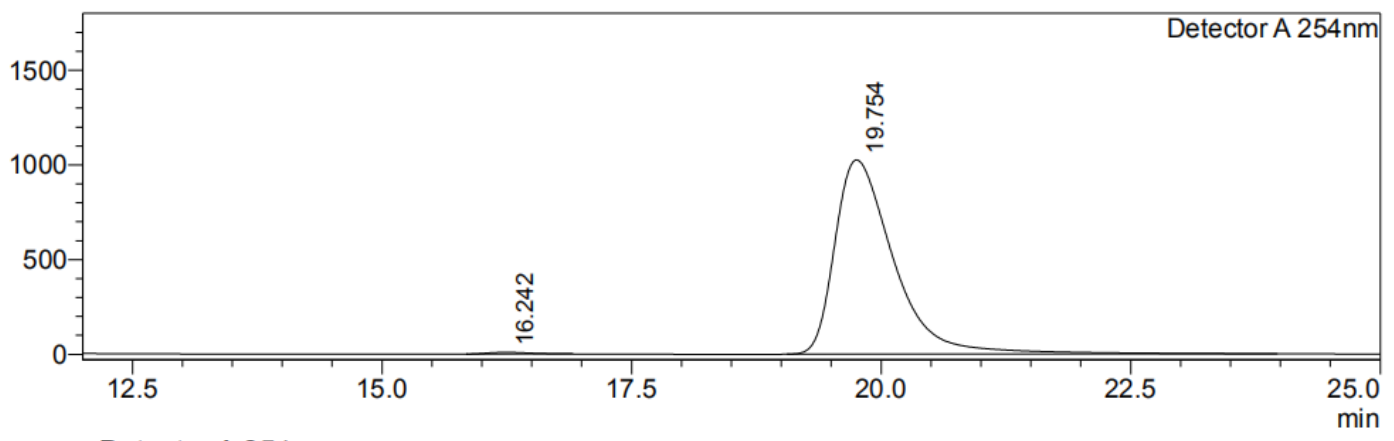

Detector A 254nm
\begin{tabular}{|r|r|r|r|r|r|}
\hline Peak\# & Ret. Time & \multicolumn{1}{l|}{ Height } & Height $\%$ & \multicolumn{1}{c|}{ Area } & \multicolumn{1}{c|}{ Area\% } \\
\hline 1 & 16.242 & 9013 & 0.871 & 264782 & 0.623 \\
\hline 2 & 19.754 & 1025497 & 99.129 & 42243790 & 99.377 \\
\hline Total & & 1034509 & 100.000 & 42508572 & 100.000 \\
\hline
\end{tabular}

(S)-5-bromo-2-(4-bromo-2-hydroxy-5-methylphenylsulfonimidoyl)-4-methylphen yl 4-methylbenzenesulfonate (6q) 
<smiles>Cc1cc(S(=O)(=O)c2cc(C)c(Br)cc2O)c(O)cc1Br</smiles>

Colorless oil; 45 mg, 76\% yield; ${ }^{1} \mathrm{H}$ NMR $\left(400 \mathrm{MHz}, \mathrm{CDCl}_{3}\right) \delta 7.90(\mathrm{~s}, 1 \mathrm{H}), 7.84(\mathrm{~s}$, 1H), $7.82(\mathrm{~s}, 1 \mathrm{H}), 7.67(\mathrm{~s}, 1 \mathrm{H}), 7.39(\mathrm{~d}, J=8.2 \mathrm{~Hz}, 2 \mathrm{H}), 7.20(\mathrm{~s}, 1 \mathrm{H}), 7.09$ (s, 1H), $2.50(\mathrm{~s}, 3 \mathrm{H}), 2.43(\mathrm{~s}, 3 \mathrm{H}), 2.19(\mathrm{~s}, 3 \mathrm{H}),{ }^{13} \mathrm{C} \mathrm{NMR}\left(100 \mathrm{MHz}, \mathrm{CDCl}_{3}\right) \delta$ 155.7, 146.6, 144.6, 137.4, 134.9, 132.8, 132.2, 131.1, 131.0, 130.1, 129.8, 129.3, 128.9, 126.6, 122.9, 118.8, 22.7, 22.0, 21.9; HRMS (ESI) $\mathrm{m} / z$ calcd for $\mathrm{C}_{21} \mathrm{H}_{19} \mathrm{Br}_{2} \mathrm{NO}_{5} \mathrm{~S}_{2}[\mathrm{M}+\mathrm{H}]^{+}=$ 587.9149 , found $=587.9124$.

Optical Rotation: $[\alpha]_{\mathrm{D}}^{25}=60.82\left(c=0.49, \mathrm{CHCl}_{3}\right) .99 \%$ ee (HPLC condition: Chiralpak IE column, $n-\mathrm{Hexane} / i-\mathrm{PrOH}=70: 30$, flow rate $=1.0 \mathrm{~mL} / \mathrm{min}$, wavelength $=254 \mathrm{~nm}, t_{\mathrm{R}}=19.66 \mathrm{~min}$ for minor isomer, $t_{\mathrm{R}}=24.29 \mathrm{~min}$ for major isomer). $\mathrm{mv}$

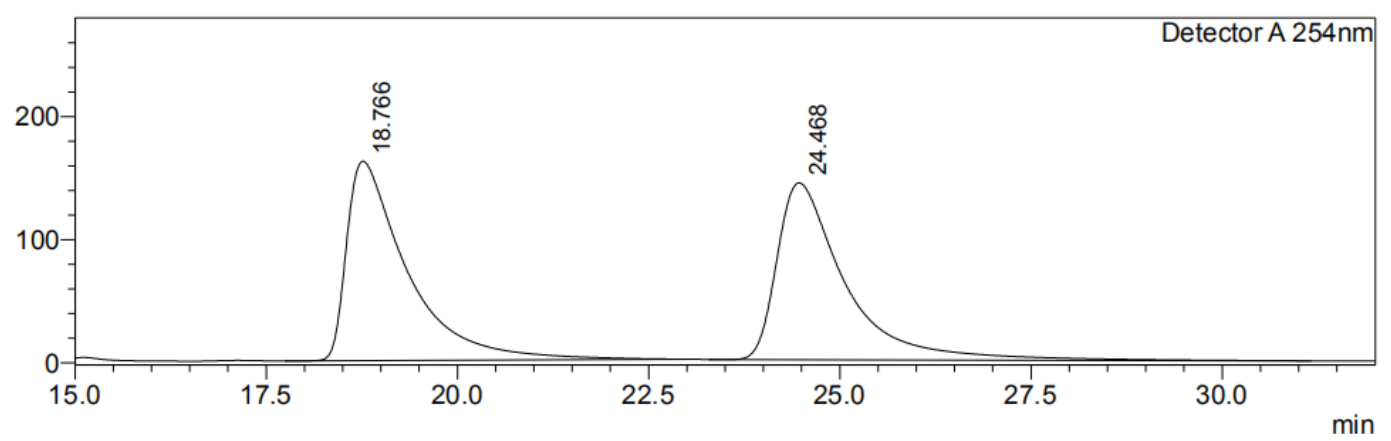

Detector A 254nm

\begin{tabular}{|r|r|r|r|r|r|}
\hline Peak\# & Ret. Time & Height & \multicolumn{1}{c|}{ Height\% } & \multicolumn{1}{c|}{ Area } & \multicolumn{1}{c|}{ Area\% } \\
\hline 1 & 18.766 & 162084 & 53.007 & 9007818 & 50.192 \\
\hline 2 & 24.468 & 143696 & 46.993 & 8939001 & 49.808 \\
\hline Total & & 305779 & 100.000 & 17946820 & 100.000 \\
\hline
\end{tabular}

$\mathrm{mV}$

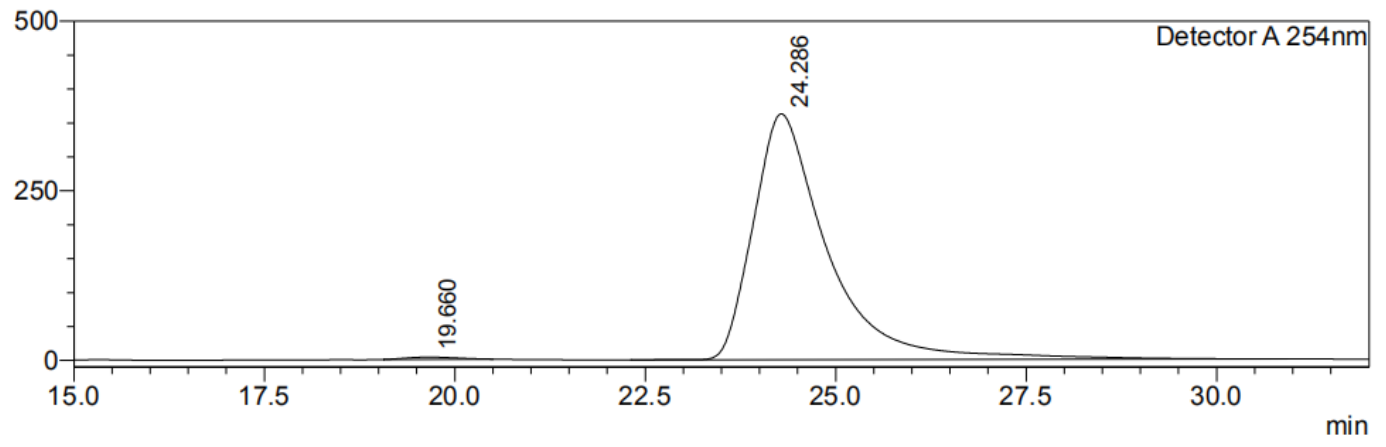

\begin{tabular}{|c|c|c|c|c|c|}
\hline \multirow{2}{*}{\multicolumn{2}{|c|}{$\begin{array}{l}\text { Detector A 254nm } \\
\text { Peak\# Ret. Time }\end{array}$}} & \multirow[b]{2}{*}{ Height } & \multirow[b]{2}{*}{ Height\% } & \multirow[b]{2}{*}{ Area } & \multirow[b]{2}{*}{ Area\% } \\
\hline & & & & & \\
\hline 1 & 19.660 & 3234 & 0.885 & 148512 & 0.599 \\
\hline 2 & 24.286 & 362335 & 99.115 & 24645488 & 99.401 \\
\hline Total & & 365569 & 100.000 & 24794000 & 100.000 \\
\hline
\end{tabular}


<smiles>Cc1cc(O)c(S(=O)(=O)c2cc(C(C)C)c(C)cc2O)cc1C(C)C</smiles>

Colorless oil; 44 mg, 85\% yield; ${ }^{1} \mathrm{H}$ NMR (400 MHz, $\left.\mathrm{CDCl}_{3}\right) \delta 7.93$ (s, 1H), 7.90 (s, 1H), $7.76(\mathrm{~s}, 1 \mathrm{H}), 7.38$ (d, J = 8.2 Hz, 2H), 7.33 (s, 1H), $7.22(\mathrm{~s}, 1 \mathrm{H}), 6.71(\mathrm{~s}, 1 \mathrm{H})$, $3.08(\mathrm{dq}, J=13.6,6.8 \mathrm{~Hz}, 1 \mathrm{H}), 2.92(\mathrm{dq}, J=13.2,6.6 \mathrm{~Hz}, 1 \mathrm{H}), 2.48(\mathrm{~s}, 3 \mathrm{H}), 2.32(\mathrm{~s}$, $3 \mathrm{H}), 2.26(\mathrm{~s}, 3 \mathrm{H}), 1.19(\mathrm{~d}, J=5.5 \mathrm{~Hz}, 3 \mathrm{H}), 1.17(\mathrm{~d}, J=5.5 \mathrm{~Hz}, 3 \mathrm{H}), 1.07(\mathrm{~d}, J=6.8$ $\mathrm{Hz}, 3 \mathrm{H}), 1.00(\mathrm{~d}, J=6.8 \mathrm{~Hz}, 3 \mathrm{H}) ;{ }^{13} \mathrm{C} \mathrm{NMR}\left(100 \mathrm{MHz}, \mathrm{CDCl}_{3}\right) \delta 154.6,146.2,146.1$, $144.9,143.7,143.2,138.8,133.8,132.8,130.0,129.0,126.6,125.2,124.6,120.1$, 117.5, 29.3, 28.7, 23.2, 23.2, 23.1, 22.9, 21.9, 19.6, 19.5; HRMS (ESI) $\mathrm{m} / z$ calcd for $\mathrm{C}_{27} \mathrm{H}_{33} \mathrm{NO}_{5} \mathrm{~S}_{2}[\mathrm{M}+\mathrm{H}]^{+}=516.1878$, found $=516.1839$.

Optical Rotation: $[\alpha]_{\mathrm{D}}^{25}=32.80\left(c=0.50, \mathrm{CHCl}_{3}\right) .94 \%$ ee (HPLC condition: Chiralpak AD-H column, $n-\mathrm{Hexane} / \mathrm{i}-\mathrm{PrOH}=90: 10$, flow rate $=1.0 \mathrm{~mL} / \mathrm{min}$, wavelength $=254 \mathrm{~nm}, t_{\mathrm{R}}=13.31$ min for major isomer, $t_{\mathrm{R}}=18.73 \min$ for minor isomer).

$\mathrm{mV}$

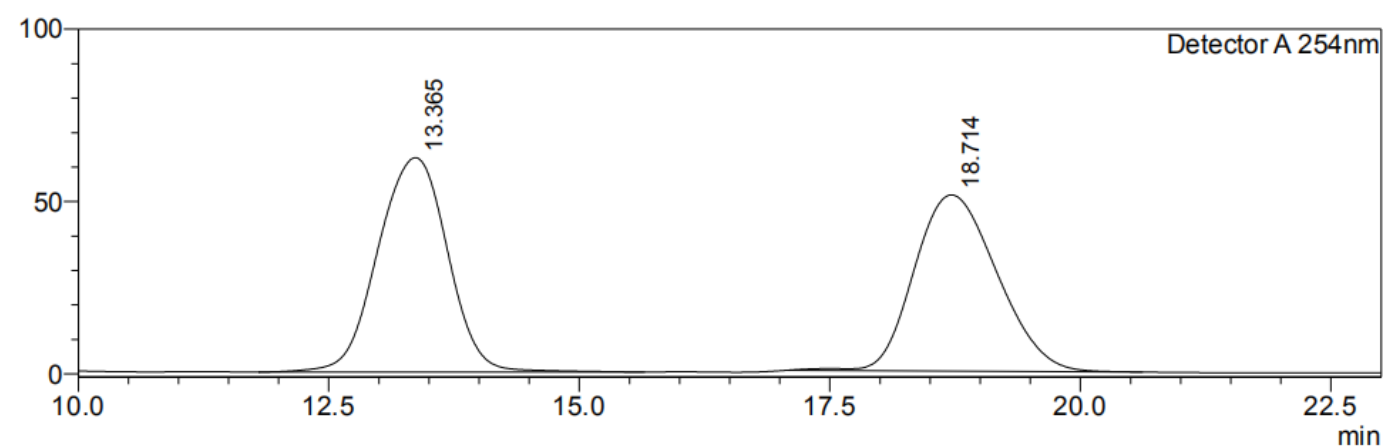

Detector A 254nm

\begin{tabular}{|r|r|r|r|r|r|}
\hline Peak\# & Ret. Time & \multicolumn{1}{c|}{ Height } & \multicolumn{1}{c|}{ Height\% } & \multicolumn{1}{c|}{ Area } & \multicolumn{1}{c|}{ Area\% } \\
\hline 1 & 13.365 & 62197 & 54.887 & 3015597 & 50.643 \\
\hline 2 & 18.714 & 51122 & 45.113 & 2938988 & 49.357 \\
\hline Total & & 113319 & 100.000 & 5954584 & 100.000 \\
\hline
\end{tabular}




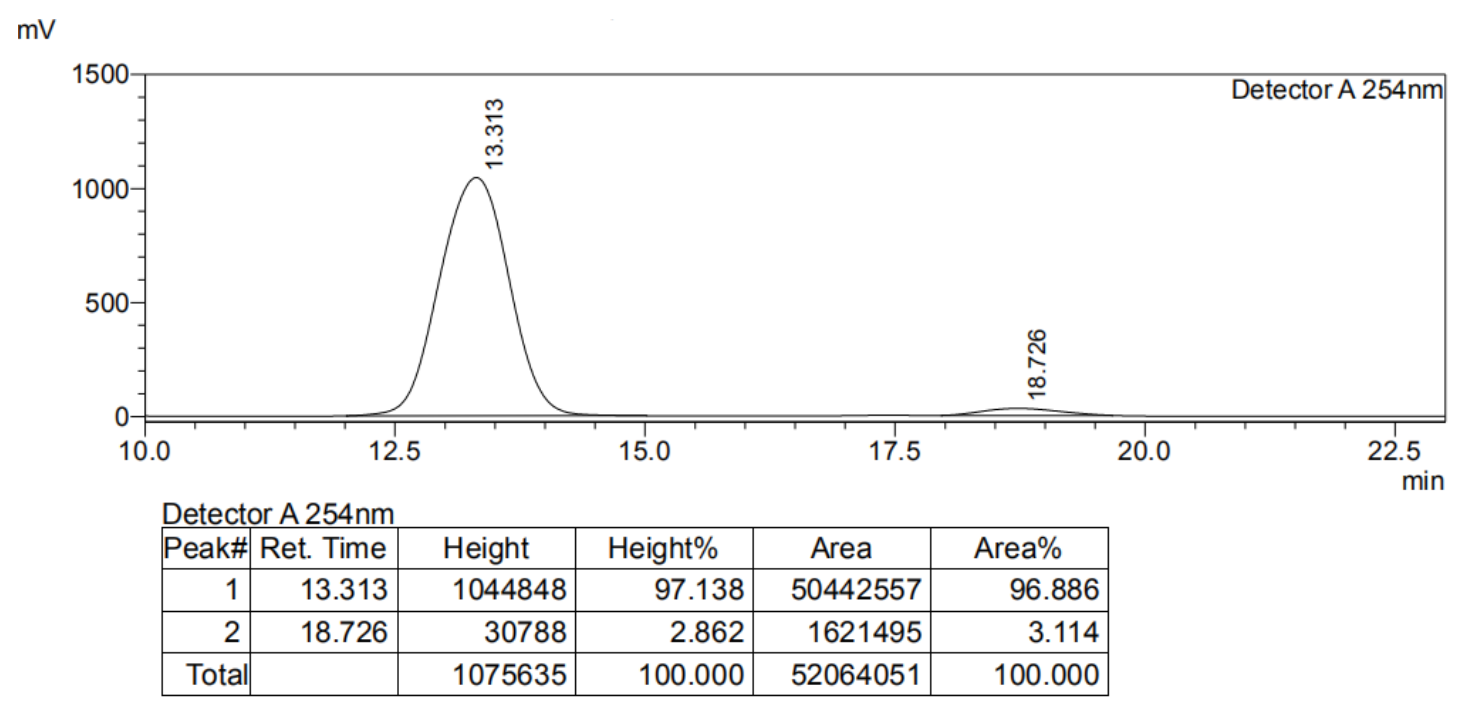

(S)-6-(6-hydroxy-2,3-dihydro-1H-indene-5-sulfonimidoyl)-2,3-dihydro-1H-inden-

\section{5-yl 4-methylbenzenesulfonate (6s)}

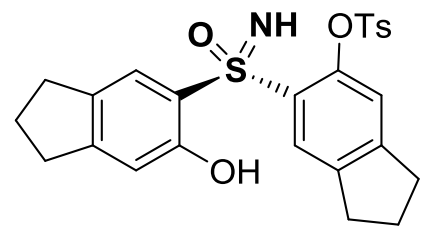

White powder; $42 \mathrm{mg}, 88 \%$ yield; m.p. $=141-143{ }^{\circ} \mathrm{C} ;{ }^{1} \mathrm{H}$ NMR $\left(400 \mathrm{MHz}, \mathrm{CDCl}_{3}\right) \delta$ $7.87(\mathrm{~d}, J=8.4 \mathrm{~Hz}, 2 \mathrm{H}), 7.82(\mathrm{~s}, 1 \mathrm{H}), 7.40-7.34(\mathrm{~m}, 3 \mathrm{H}), 7.19(\mathrm{~s}, 1 \mathrm{H}), 6.79$ (s, 1H), 2.98-2.80 (m, 6H), 2.69 (td, $J=7.4,3.2 \mathrm{~Hz}, 2 \mathrm{H}), 2.48$ (s, 3H), 2.11 (p, $J=7.4 \mathrm{~Hz}$, 2H), $2.00(\mathrm{p}, J=7.4 \mathrm{~Hz}, 2 \mathrm{H}) ;{ }^{13} \mathrm{C} \mathrm{NMR}\left(100 \mathrm{MHz} \mathrm{CDCl}_{3}\right) \delta$ 156.2, 154.0, 152.4, $146.1,145.5,143.0,135.3,132.7,129.9,129.0,125.4,124.1,118.7,117.5,114.5$, 33.4, 32.4, 31.8, 25.6, 25.6, 22.0; HRMS (ESI) $m / z$ calcd for $\mathrm{C}_{25} \mathrm{H}_{25} \mathrm{NO}_{5} \mathrm{~S}_{2}[\mathrm{M}+\mathrm{H}]^{+}=$ 484.1252, found $=484.1227$.

Optical Rotation: $[\alpha]^{25}=50.43\left(c=0.49, \mathrm{CHCl}_{3}\right) .98 \%$ ee (HPLC condition: Chiralpak AD-H column, $n$-Hexane $/ i-\mathrm{PrOH}=70: 30$, flow rate $=1.0 \mathrm{~mL} / \mathrm{min}$, wavelength $=254 \mathrm{~nm}, t_{\mathrm{R}}=11.36$ min for major isomer, $t_{\mathrm{R}}=16.32 \min$ for minor isomer). 
$\mathrm{mV}$

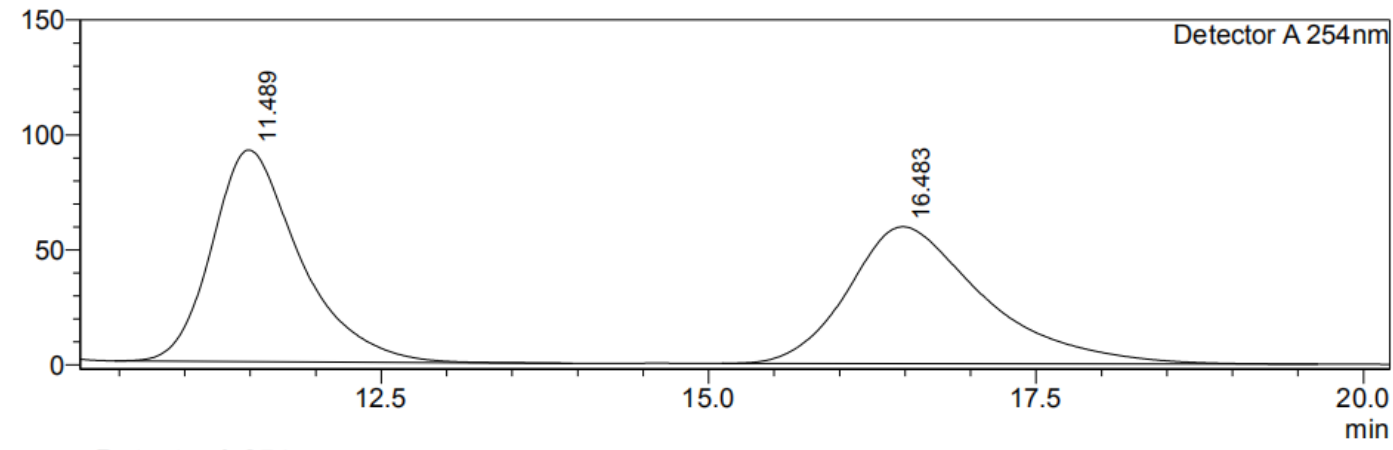

Detector A 254nm
\begin{tabular}{|r|r|r|r|r|r|}
\hline Peak\# & Ret. Time & Height & Height $\%$ & Area & \multicolumn{1}{c|}{ Area\% } \\
\hline 1 & 11.489 & 92064 & 60.723 & 4320554 & 50.154 \\
\hline 2 & 16.483 & 59548 & 39.277 & 4294068 & 49.846 \\
\hline Total & & 151612 & 100.000 & 8614622 & 100.000 \\
\hline
\end{tabular}

$\mathrm{mV}$

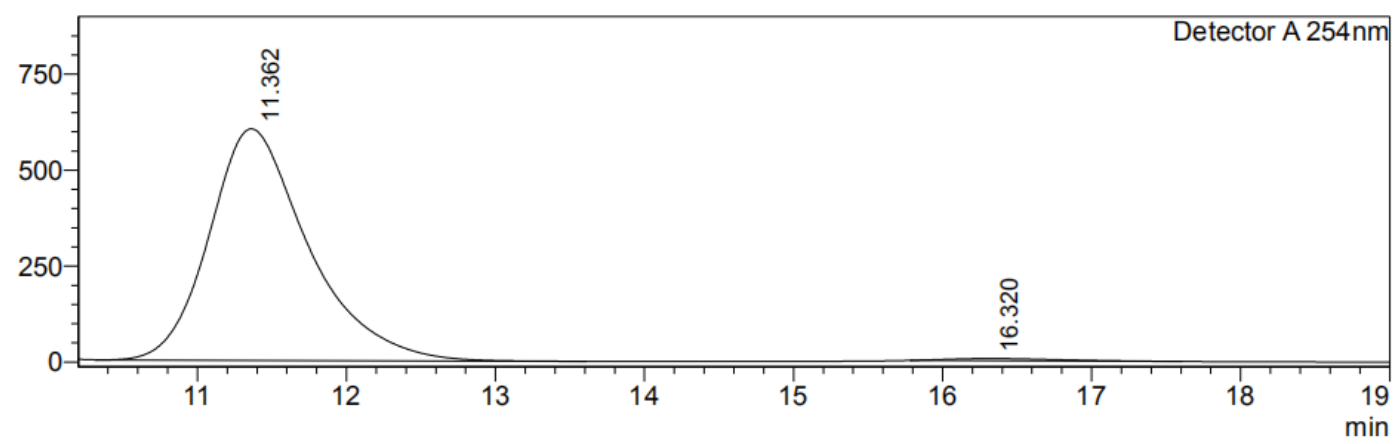

Detector A 254nm
\begin{tabular}{|r|r|r|r|r|r|}
\hline Peak\# & Ret. Time & Height & Height\% & \multicolumn{1}{c|}{ Area } & \multicolumn{1}{c|}{ Area\% } \\
\hline 1 & 11.362 & 603431 & 99.074 & 27949487 & 98.961 \\
\hline 2 & 16.320 & 5639 & 0.926 & 293409 & 1.039 \\
\hline Total & & 609070 & 100.000 & 28242896 & 100.000 \\
\hline
\end{tabular}

(S)-3-(3-hydroxyphenylsulfonimidoyl)phenyl 4-methylbenzenesulfonate (6t)<smiles>NS(=O)(=O)c1cccc([Se])c1</smiles>

Colorless oil; $34 \mathrm{mg}, 86 \%$ yield; ${ }^{1} \mathrm{H}$ NMR (400 MHz, $\left.\mathrm{CDCl}_{3}\right) \delta 7.90(\mathrm{~d}, J=8.0 \mathrm{~Hz}$, $1 \mathrm{H}), 7.63(\mathrm{dd}, J=5.2,3.2 \mathrm{~Hz}, 3 \mathrm{H}), 7.56(\mathrm{~s}, 1 \mathrm{H}), 7.46-7.39(\mathrm{~m}, 2 \mathrm{H}), 7.35-7.26(\mathrm{~m}$, $3 \mathrm{H}), 7.21(\mathrm{dd}, J=8.2,1.6 \mathrm{~Hz}, 1 \mathrm{H}), 7.00(\mathrm{dd}, J=8.2,1.6 \mathrm{~Hz}, 1 \mathrm{H}), 2.44(\mathrm{~s}, 3 \mathrm{H}) ;{ }^{13} \mathrm{C}$ NMR $\left(100 \mathrm{MHz}, \mathrm{CDCl}_{3}\right) \delta 157.4,149.9,146.2,144.8,142.8,131.8,130.8,130.7$, 130.1, 128.6, 127.1, 126.6, 122.2, 121.1, 119.6, 114.8, 21.9; HRMS (ESI) $m / z$ calcd for $\mathrm{C}_{19} \mathrm{H}_{17} \mathrm{NO}_{5} \mathrm{~S}_{2}[\mathrm{M}+\mathrm{H}]^{+}=404.0626$, found $=404.0616$. 
Optical Rotation: $[\alpha]^{25}=7.98\left(c=0.60, \mathrm{CHCl}_{3}\right) .6 \%$ ee (HPLC condition: Chiralpak AD-H column, $n$-Hexane $/ i-\mathrm{PrOH}=70: 30$, flow rate $=1.0 \mathrm{~mL} / \mathrm{min}$, wavelength $=254 \mathrm{~nm}, t_{\mathrm{R}}=15.44$ min for minor isomer, $t_{\mathrm{R}}=17.58 \mathrm{~min}$ for major isomer).

$\mathrm{mV}$

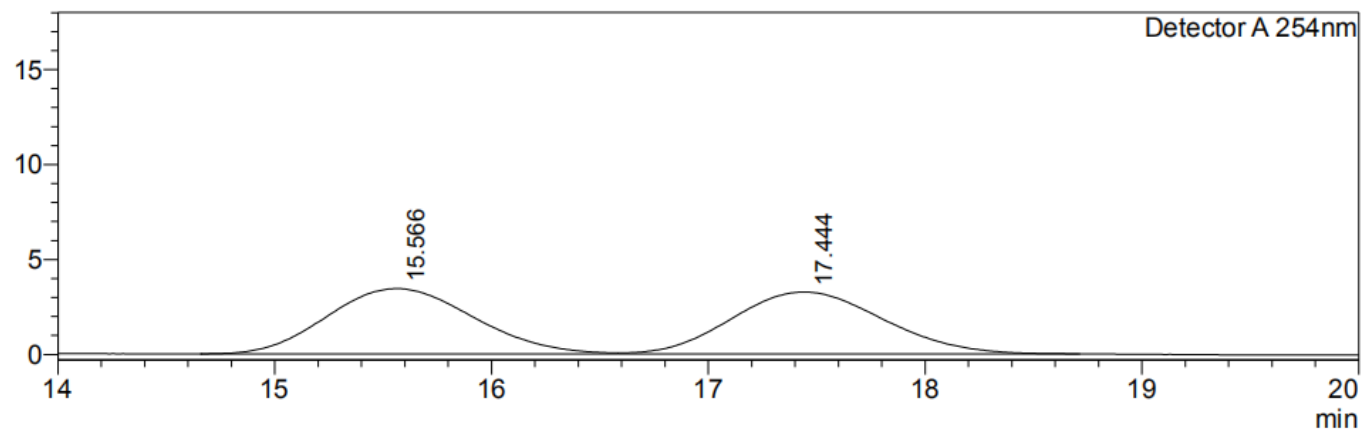

Detector A 254nm

\begin{tabular}{|r|r|r|r|r|r|}
\hline Peak\# & Ret. Time & \multicolumn{1}{c|}{ Height } & Height\% & Area & \multicolumn{1}{c|}{ Area\% } \\
\hline 1 & 15.566 & 3435 & 51.383 & 161249 & 50.500 \\
\hline 2 & 17.444 & 3250 & 48.617 & 158056 & 49.500 \\
\hline Total & & 6685 & 100.000 & 319305 & 100.000 \\
\hline
\end{tabular}

$\mathrm{mV}$

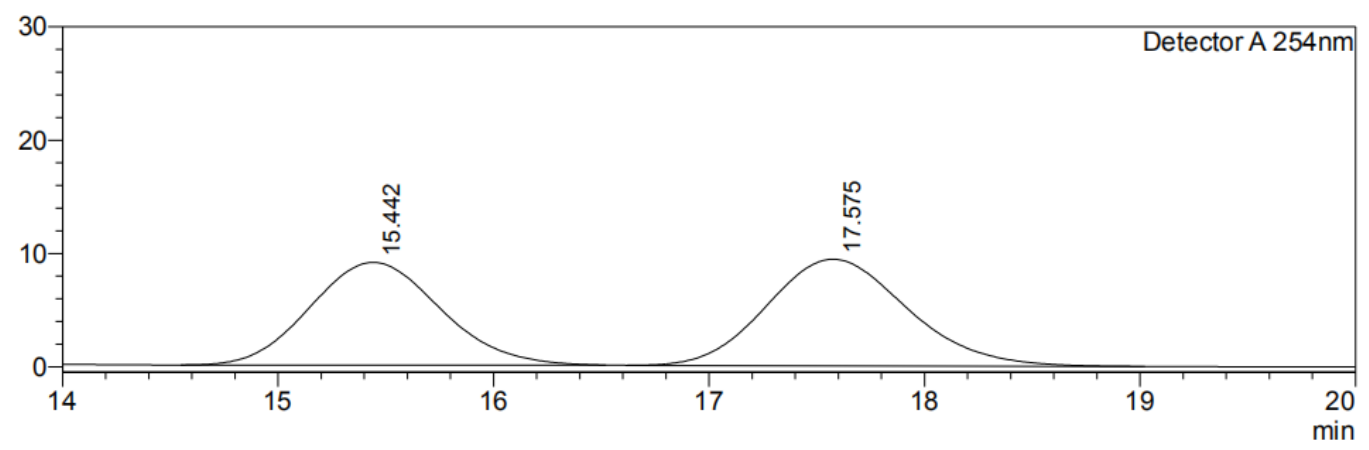

Detector A 254nm

\begin{tabular}{|r|r|r|r|r|r|}
\hline Peak\# & Ret. Time & \multicolumn{1}{c|}{ Height } & Height\% & \multicolumn{1}{|c|}{ Area } & \multicolumn{1}{c|}{ Area\% } \\
\hline 1 & 15.442 & 9056 & 49.063 & 381122 & 47.138 \\
\hline 2 & 17.575 & 9402 & 50.937 & 427399 & 52.862 \\
\hline Total & & 18457 & 100.000 & 808521 & 100.000 \\
\hline
\end{tabular}

$\underline{(R)-2-((2-h y d r o x y p h e n y l) s u l f i n y l) p h e n y l ~ 4-m e t h y l b e n z e n e s u l f o n a t e ~(6 u) ~}$<smiles>O=S(c1ccccc1[O-])c1ccccc1O</smiles>

Colorless oil; $32 \mathrm{mg}, 83 \%$ yield; ${ }^{1} \mathrm{H}$ NMR (400 MHz, $\left.\mathrm{CDCl}_{3}\right) \delta 10.04(\mathrm{~s}, 1 \mathrm{H})$, 7.98-7.90 (m, 1H), $7.82(\mathrm{~s}, 1 \mathrm{H}), 7.79(\mathrm{~s}, 1 \mathrm{H}), 7.49-7.42(\mathrm{~m}, 2 \mathrm{H}), 7.38$ (d, J = 8.2 Hz, 2H), 7.32-7.26 (m, 3H), 6.89-6.84 (m, 1H), $6.82(\mathrm{dd}, J=8.8,1.0 \mathrm{~Hz}, 1 \mathrm{H}), 2.48(\mathrm{~s}$, $3 \mathrm{H}),{ }^{13} \mathrm{C} \mathrm{NMR}\left(100 \mathrm{MHz}, \mathrm{CDCl}_{3}\right) \delta 159.5,146.6,146.0,136.7,133.4,133.0,132.1$, 
130.3, 128.8, 128.0, 126.9, 125.7, 122.7, 121.9, 120.1, 119.7, 22.0; HRMS (ESI) $\mathrm{m} / \mathrm{z}$ calcd for $\mathrm{C}_{19} \mathrm{H}_{16} \mathrm{O}_{5} \mathrm{~S}_{2}[\mathrm{M}+\mathrm{H}]^{+}=389.0517$, found $=389.0513$.

Optical Rotation: $[\alpha]_{\mathrm{D}}^{25}=111.61\left(c=0.72, \mathrm{CHCl}_{3}\right) .91 \%$ ee (HPLC condition: Chiralpak ID column, $n$-Hexane $/ i-\mathrm{PrOH}=80: 20$, flow rate $=1.0 \mathrm{~mL} / \mathrm{min}$, wavelength $=254 \mathrm{~nm}, t_{\mathrm{R}}=25.70 \min$ for minor isomer, $t_{\mathrm{R}}=31.87$ min for major isomer).

$\mathrm{mV}$

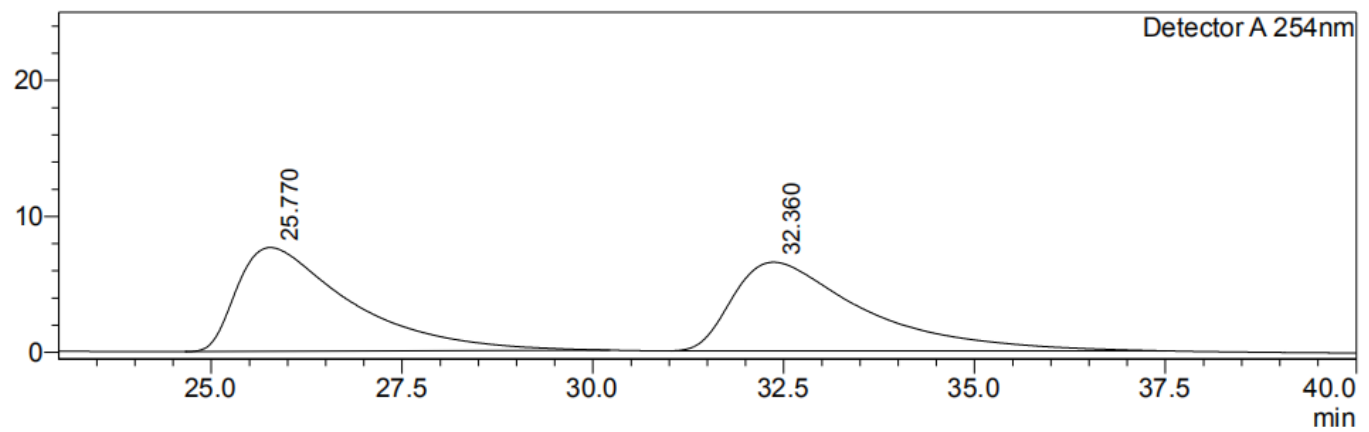

Detector A 254nm

\begin{tabular}{|r|r|r|r|r|r|}
\hline Peak\# & Ret. Time & Height & \multicolumn{1}{c|}{ Height\% } & \multicolumn{1}{c|}{ Area } & \multicolumn{1}{c|}{ Area\% } \\
\hline 1 & 25.770 & 7635 & 53.949 & 787453 & 50.265 \\
\hline 2 & 32.360 & 6517 & 46.051 & 779156 & 49.735 \\
\hline Total & & 14152 & 100.000 & 1566609 & 100.000 \\
\hline
\end{tabular}

$\mathrm{mV}$

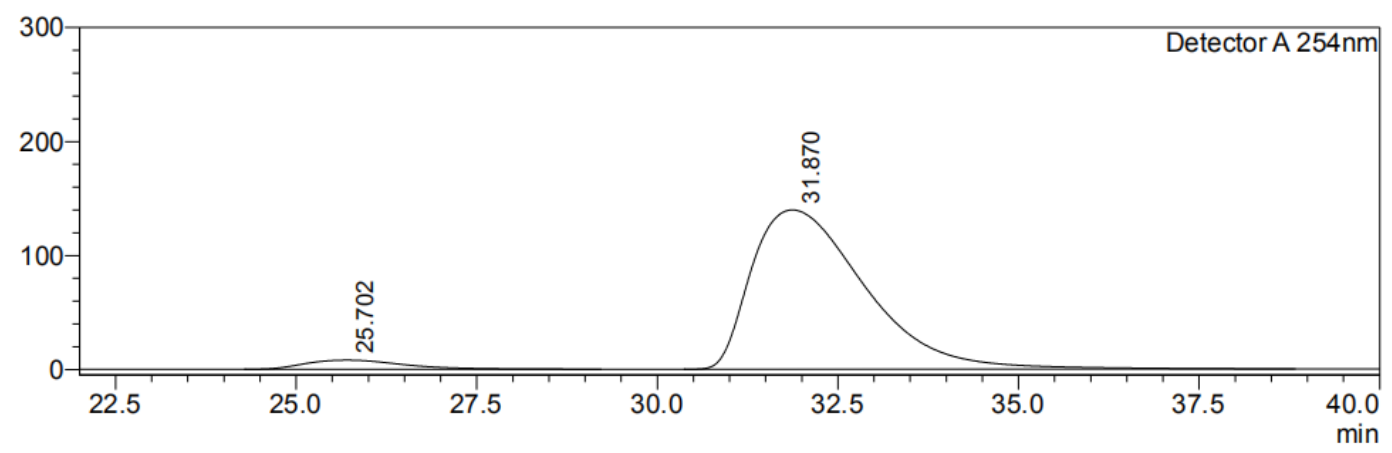

Detector A 254nm

\begin{tabular}{|r|r|r|r|r|r|}
\hline Peak\# & Ret. Time & Height & \multicolumn{1}{c|}{ Height\% } & \multicolumn{1}{c|}{ Area } & \multicolumn{1}{|c|}{ Area\% } \\
\hline 1 & 25.702 & 7993 & 5.410 & 759541 & 4.727 \\
\hline 2 & 31.870 & 139758 & 94.590 & 15307532 & 95.273 \\
\hline Total & & 147751 & 100.000 & 16067073 & 100.000 \\
\hline
\end{tabular}

\section{$\underline{(S)-2-(2-h y d r o x y p h e n y l s u l f o n i m i d o y l) p h e n y l ~ b e n z e n e s u l f o n a t e ~(7 a) ~}$}<smiles>NS(=O)(=O)c1ccccc1OS(=O)(=O)c1ccccc1</smiles> 
White solid; $31 \mathrm{mg}, 80 \%$ yield; m.p. $=106-108{ }^{\circ} \mathrm{C} ;{ }^{1} \mathrm{H}$ NMR $\left(400 \mathrm{MHz}, \mathrm{CDCl}_{3}\right) \delta$ $12.22(\mathrm{~s}, 1 \mathrm{H})$, 8.03-7.94 (m, 3H), 7.78-7.71 (m, 1H), 7.63-7.57 (m, 2H), 7.57-7.48 (m, 2H), 7.42-7.34 (m, 3H), 6.90-6.84 (m, 1H), $6.80(\mathrm{td}, J=7.6,1.2 \mathrm{~Hz}, 1 \mathrm{H}), 3.88(\mathrm{~s}$, $1 \mathrm{H}) ;{ }^{13} \mathrm{C} \mathrm{NMR}\left(100 \mathrm{MHz}, \mathrm{CDCl}_{3}\right) \delta 157.7,146.8,136.8,135.8,135.6,135.0,134.7$, 130.0, 129.5, 129.4, 128.8, 126.8, 122.8, 120.0, 119.4, 119.3; HRMS (ESI) $m / z$ calcd for $\mathrm{C}_{18} \mathrm{H}_{15} \mathrm{NO}_{5} \mathrm{~S}_{2}[\mathrm{M}+\mathrm{H}]^{+}=390.0470$, found $=390.0452$.

Optical Rotation: $[\alpha]_{\mathrm{D}}^{25}=70.00\left(c=0.20, \mathrm{CHCl}_{3}\right) .98 \%$ ee (HPLC condition: Chiralpak AD-H column, $n-\mathrm{Hexane} / i-\mathrm{PrOH}=70: 30$, flow rate $=1.0 \mathrm{~mL} / \mathrm{min}$, wavelength $=254 \mathrm{~nm}, t_{\mathrm{R}}=18.09 \min$ for minor isomer, $t_{\mathrm{R}}=24.34 \mathrm{~min}$ for major isomer).

$\mathrm{mV}$

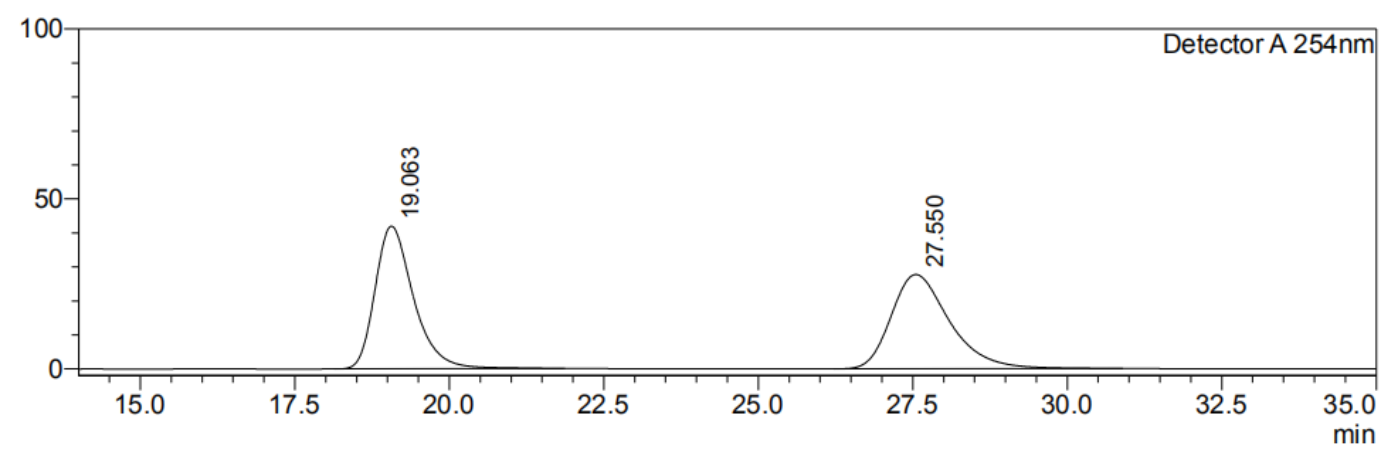

Detector A 254nm

\begin{tabular}{|r|r|r|r|r|r|}
\hline Peak\# & Ret. Time & Height & Height\% & Area & \multicolumn{1}{c|}{ Area\% } \\
\hline 1 & 19.063 & 41983 & 60.221 & 1821058 & 49.923 \\
\hline 2 & 27.550 & 27732 & 39.779 & 1826683 & 50.077 \\
\hline Total & & 69714 & 100.000 & 3647741 & 100.000 \\
\hline
\end{tabular}

$\mathrm{mV}$

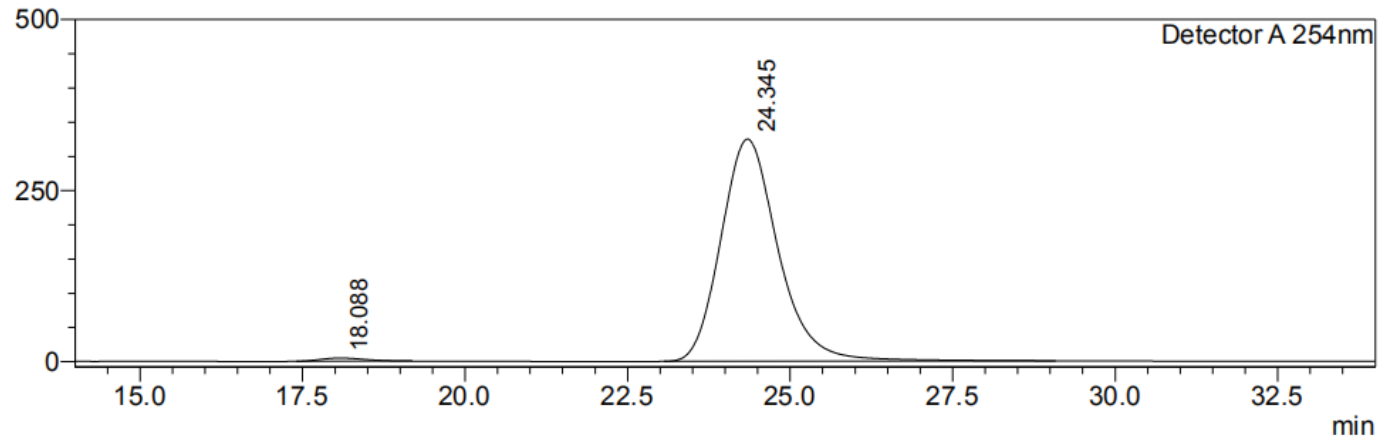

Detector A 254nm
\begin{tabular}{|r|r|r|r|r|r|}
\hline Peak\# & Ret. Time & Height & Height\% & \multicolumn{1}{|c|}{ Area } & \multicolumn{1}{c|}{ Area\% } \\
\hline 1 & 18.088 & 4653 & 1.413 & 221466 & 1.100 \\
\hline 2 & 24.345 & 324717 & 98.587 & 19911516 & 98.900 \\
\hline Total & & 329371 & 100.000 & 20132982 & 100.000 \\
\hline
\end{tabular}

(S)-2-(2-hydroxyphenylsulfonimidoyl)phenyl 4-(tert-butyl)benzenesulfonate (7b) 
<smiles>CC(C)(C)c1ccc(S(=O)(=O)Oc2ccccc2S(=O)(=O)c2ccccc2O)cc1</smiles>

Colorless oil; $37 \mathrm{mg}, 84 \%$ yield; ${ }^{1} \mathrm{H}$ NMR $\left(400 \mathrm{MHz}, \mathrm{CDCl}_{3}\right) \delta 7.98$ (dd, $J=8.0,1.4$ $\mathrm{Hz}, 1 \mathrm{H}), 7.93-7.87$ (m, 2H), 7.61-7.57 (m, 2H), 7.56-7.50 (m, 2H), 7.37 (ddd, $J=8.6$, 8.0, $4.8 \mathrm{~Hz}, 3 \mathrm{H}), 6.86$ (dd, $J=7.6,1.4 \mathrm{~Hz}, 1 \mathrm{H}), 6.79$ (td, $J=7.6,1.2 \mathrm{~Hz}, 1 \mathrm{H}), 1.38$ (s, $9 \mathrm{H}) ;{ }^{13} \mathrm{C} \mathrm{NMR}\left(100 \mathrm{MHz}, \mathrm{CDCl}_{3}\right) \delta 159.2,157.8,146.9,136.8,135.8,134.7,132.6$, 129.9, 129.4, 128.7, 126.7, 126.5, 122.9, 120.0, 119.4, 119.3, 35.6, 31.2; HRMS (ESI) $m / z$ calcd for $\mathrm{C}_{22} \mathrm{H}_{23} \mathrm{NO}_{5} \mathrm{~S}_{2}[\mathrm{M}+\mathrm{H}]^{+}=446.1096$, found $=446.1096$.

Optical Rotation: $[\alpha]_{\mathrm{D}}^{25}=59.50\left(c=0.20, \mathrm{CHCl}_{3}\right) .90 \%$ ee (HPLC condition: Chiralpak ID column, $n$-Hexane $/ i-\mathrm{PrOH}=70: 30$, flow rate $=1.0 \mathrm{~mL} / \mathrm{min}$, wavelength $=254 \mathrm{~nm}, t_{\mathrm{R}}=17.06 \mathrm{~min}$ for minor isomer, $t_{\mathrm{R}}=26.37 \mathrm{~min}$ for major isomer).

$\mathrm{mV}$

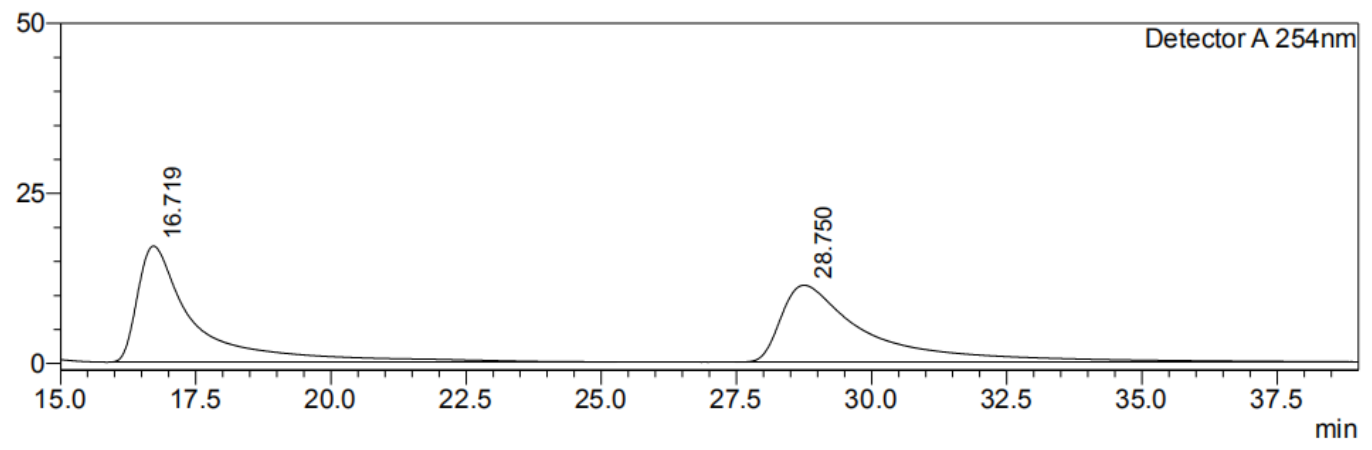

Detector A 254nm

\begin{tabular}{|r|r|r|r|r|r|}
\hline Peak\# & Ret. Time & Height & Height\% & Area & Area\% \\
\hline 1 & 16.719 & 17060 & 60.147 & 1263443 & 49.995 \\
\hline 2 & 28.750 & 11304 & 39.853 & 1263709 & 50.005 \\
\hline Total & & 28365 & 100.000 & 2527153 & 100.000 \\
\hline
\end{tabular}


$\mathrm{mV}$

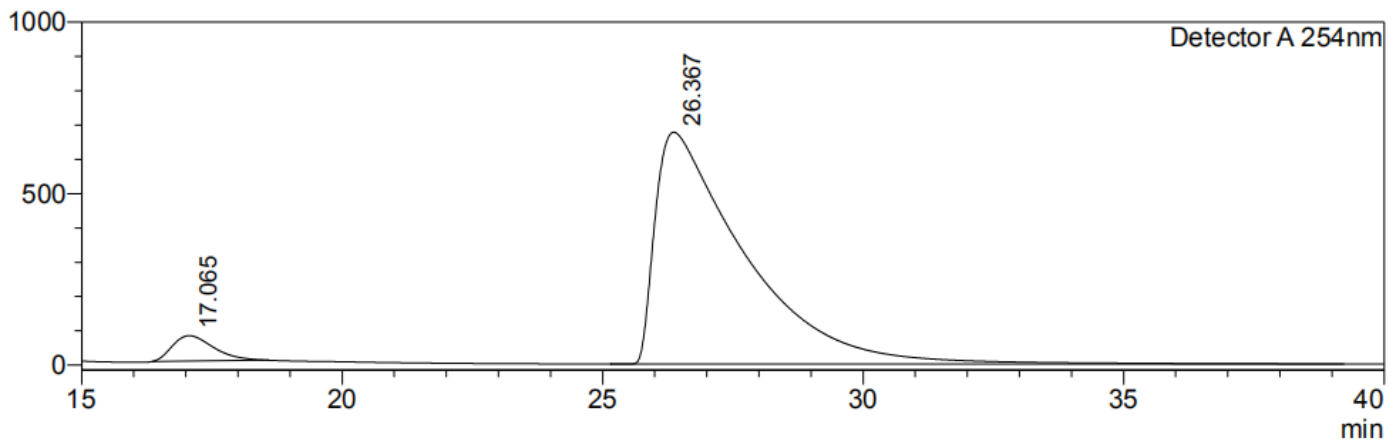

Detector A 254nm

\begin{tabular}{|r|r|r|r|r|r|}
\hline Peak\# & Ret. Time & \multicolumn{1}{c|}{ Height } & \multicolumn{1}{c|}{ Height\% } & \multicolumn{1}{c|}{ Area } & \multicolumn{1}{c|}{ Area\% } \\
\hline 1 & 17.065 & 74023 & 9.873 & 4067545 & 4.781 \\
\hline 2 & 26.367 & 675738 & 90.127 & 81005097 & 95.219 \\
\hline Total & & 749761 & 100.000 & 85072643 & 100.000 \\
\hline
\end{tabular}

$\underline{(S) \text {-2-(2-hydroxyphenylsulfonimidoyl)phenyl 4-methoxybenzenesulfonate (7c) }}$<smiles>COc1ccc(S(=O)(=O)Oc2ccccc2S(=O)(=O)c2ccccc2O)cc1</smiles>

Colorless oil; $32 \mathrm{mg}, 77 \%$ yield; ${ }^{1} \mathrm{H}$ NMR (400 MHz, $\left.\mathrm{CDCl}_{3}\right) \delta 8.00-7.95(\mathrm{~m}, 1 \mathrm{H})$, 7.94-7.86 (m, 2H), 7.57-7.50 (m, 2H), 7.41 (ddd, $J=8.6,7.8,3.8 \mathrm{~Hz}, 2 \mathrm{H}), 7.37-7.32$ (m, 1H), 7.05-7.00 (m, 2H), 6.89 (dd, $J=8.4,1.2 \mathrm{~Hz}, 1 \mathrm{H}), 6.81(\mathrm{ddd}, J=8.4,7.4,1.2$ $\mathrm{Hz}, 1 \mathrm{H}), 3.91(\mathrm{~s}, 3 \mathrm{H}) ;{ }^{13} \mathrm{C} \mathrm{NMR}\left(100 \mathrm{MHz}, \mathrm{CDCl}_{3}\right) \delta 164.8,157.7,147.0,136.7$, $135.8,134.7,131.3,129.9,129.5,126.8,126.7,122.8,120.0,119.4,119.3,114.6$, 56.0; HRMS (ESI) $m / z$ calcd for $\mathrm{C}_{22} \mathrm{H}_{23} \mathrm{NO}_{5} \mathrm{~S}_{2}[\mathrm{M}+\mathrm{H}]^{+}=420.0575$, found $=420.0575$.

Optical Rotation: $[\alpha]_{D}^{25}=42.50\left(c=0.20, \mathrm{CHCl}_{3}\right) .98 \%$ ee (HPLC condition: Chiralpak AD-H column, $n$-Hexane $/ i-\mathrm{PrOH}=70: 30$, flow rate $=0.8 \mathrm{~mL} / \mathrm{min}$, wavelength $=254 \mathrm{~nm}, t_{\mathrm{R}}=31.35$ min for major isomer, $t_{\mathrm{R}}=36.15 \min$ for minor isomer). 
$\mathrm{mV}$

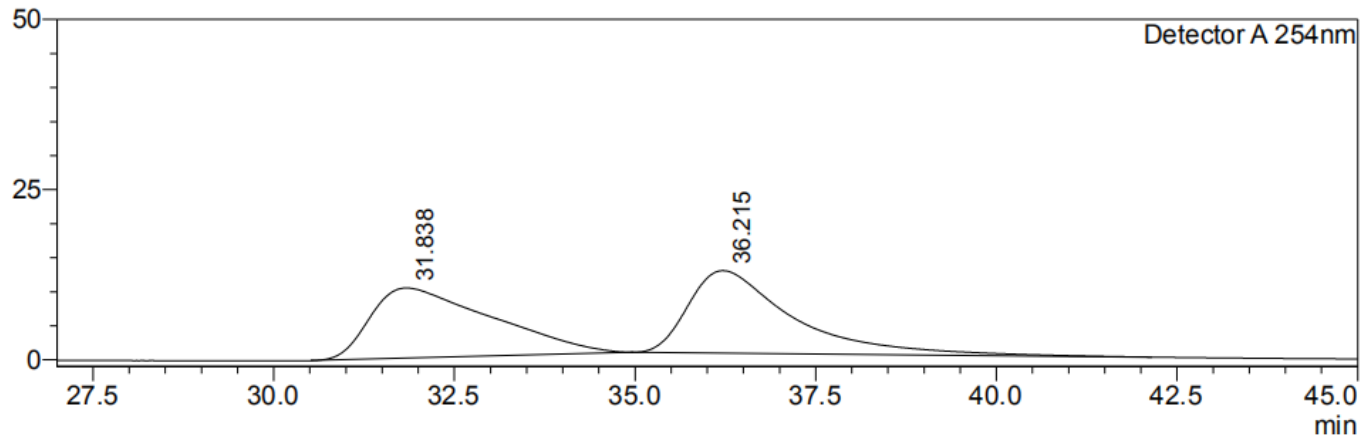

\begin{tabular}{|c|c|c|c|c|c|}
\hline Peak\# & Ret. Time & Height & Height\% & Area & Area\% \\
\hline 1 & 31.838 & 10308 & 45.958 & 1201344 & 49.639 \\
\hline 2 & 36.215 & 12121 & 54.042 & 1218821 & 50.361 \\
\hline Total & & 22429 & 100.000 & 2420165 & 100.000 \\
\hline
\end{tabular}

$\mathrm{mV}$

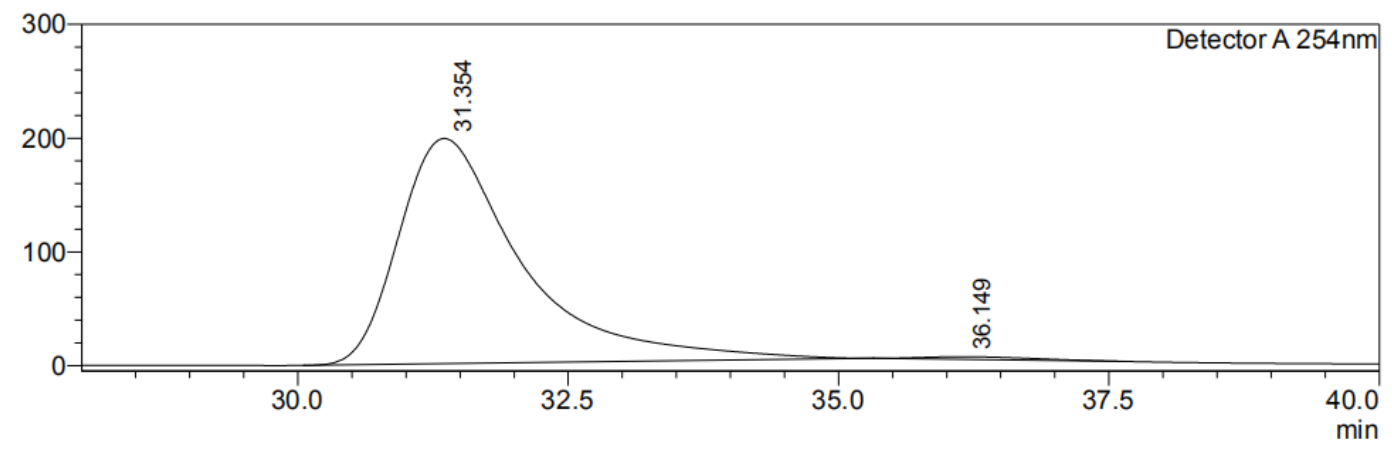

Detector A 254nm
\begin{tabular}{|r|r|r|r|r|r|}
\hline Peak\# & Ret. Time & Height & Height\% & \multicolumn{1}{c|}{ Area } & \multicolumn{1}{|c|}{ Area\% } \\
\hline 1 & 31.354 & 198120 & 98.784 & 15721078 & 98.839 \\
\hline 2 & 36.149 & 2438 & 1.216 & 184599 & 1.161 \\
\hline Total & & 200559 & 100.000 & 15905676 & 100.000 \\
\hline
\end{tabular}

(S)-2-(2-hydroxyphenylsulfonimidoyl)phenyl [1,1'-biphenyl]-4-sulfonate (7d)<smiles>NS(=O)(=O)c1ccccc1OS(=O)(=O)c1ccc(-c2ccccc2)cc1</smiles>

White solid; $37 \mathrm{mg}, 80 \%$ yield; m.p. $=137-139{ }^{\circ} \mathrm{C} ;{ }^{1} \mathrm{H}$ NMR $\left(400 \mathrm{MHz}, \mathrm{CDCl}_{3}\right) \delta$ $12.27(\mathrm{~s}, 1 \mathrm{H}), 8.07-7.94(\mathrm{~m}, 3 \mathrm{H})$, 7.83-7.74 (m, 2H), 7.67-7.61 (m, 2H), 7.59-7.53 (m, 2H), 7.54-7.48 (m, 2H), 7.48-7.41 (m, 2H), 7.41-7.34 (m, 2H), $6.88(\mathrm{dd}, J=8.4,1.0$ $\mathrm{Hz}, 1 \mathrm{H}), 6.81$ (ddd, $J=8.4,7.4,1.0 \mathrm{~Hz}, 1 \mathrm{H}), 3.88(\mathrm{~s}, 1 \mathrm{H}) ;{ }^{13} \mathrm{C}$ NMR $(100 \mathrm{MHz}$, $\left.\mathrm{CDCl}_{3}\right) \delta 157.7,147.9,146.9,138.9,136.8,135.8,134.7,134.0,130.0,129.4,129.3$, 129.1, 128.0, 127.6, 126.8, 122.8, 120.0, 119.5, 119.4; HRMS (ESI) $m / z$ calcd for $\mathrm{C}_{24} \mathrm{H}_{19} \mathrm{NO}_{5} \mathrm{~S}_{2}[\mathrm{M}+\mathrm{H}]^{+}=466.0783$, found $=466.0786$. 
Optical Rotation: $[\alpha]_{\mathrm{D}}^{25}=65.24\left(c=0.21, \mathrm{CHCl}_{3}\right) .>99 \%$ ee (HPLC condition: Chiralpak AD-H column, $n$-Hexane $/ i-\mathrm{PrOH}=70: 30$, flow rate $=1.0 \mathrm{~mL} / \mathrm{min}$, wavelength $=254 \mathrm{~nm}, t_{\mathrm{R}}=28.04$ min for major isomer, $t_{\mathrm{R}}=30.72 \mathrm{~min}$ for minor isomer).

$\mathrm{mV}$

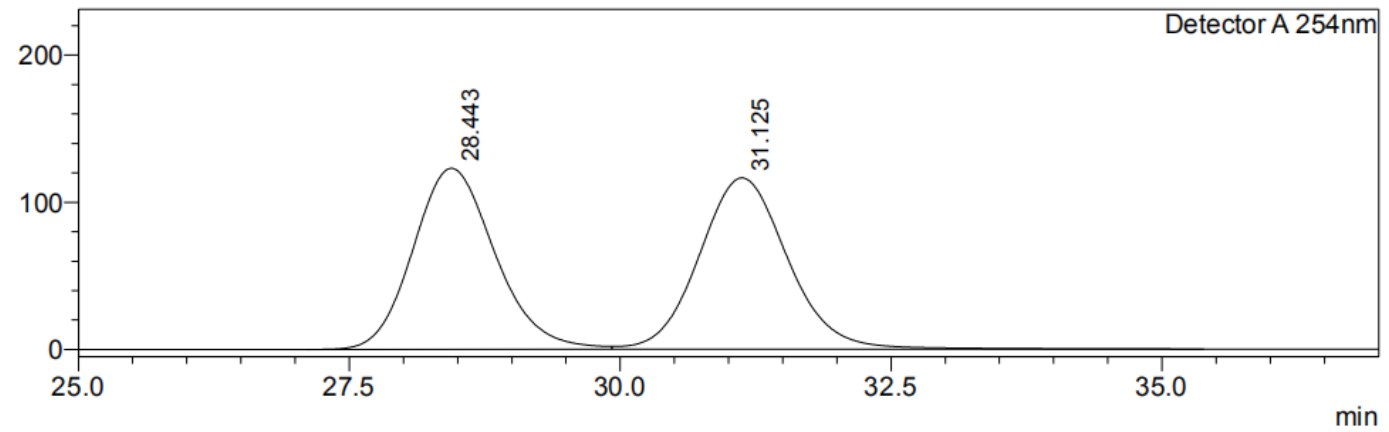

Detector A 254nm

\begin{tabular}{|r|r|r|r|r|r|}
\hline Peak\# & Ret. Time & Height & Height\% & \multicolumn{1}{c|}{ Area } & \multicolumn{1}{|c|}{ Area\% } \\
\hline 1 & 28.443 & 123034 & 51.386 & 6487553 & 49.366 \\
\hline 2 & 31.125 & 116396 & 48.614 & 6654173 & 50.634 \\
\hline Total & & 239430 & 100.000 & 13141726 & 100.000 \\
\hline
\end{tabular}

$\mathrm{mV}$

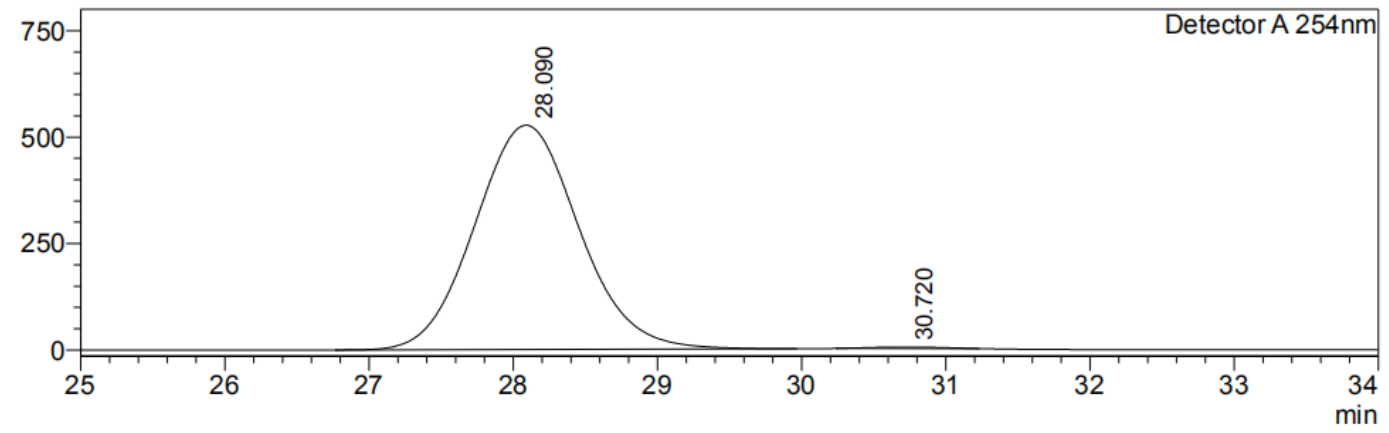

Detector A 254nm

\begin{tabular}{|r|r|r|r|r|r|}
\hline Peak\# & Ret. Time & \multicolumn{1}{c|}{ Height } & \multicolumn{1}{c|}{ Height\% } & \multicolumn{1}{c|}{ Area } & \multicolumn{1}{c|}{ Area\% } \\
\hline 1 & 28.090 & 527022 & 99.388 & 26582417 & 99.573 \\
\hline 2 & 30.720 & 3245 & 0.612 & 114075 & 0.427 \\
\hline Total & & 530267 & 100.000 & 26696492 & 100.000 \\
\hline
\end{tabular}

(S)-2-(2-hydroxyphenylsulfonimidoyl)phenyl 4-fluorobenzenesulfonate (7e)<smiles>NS(=O)(=O)c1ccccc1OS(=O)(=O)c1ccc(F)cc1</smiles>

Light yellow solid; $35 \mathrm{mg}, 85 \%$ yield; m.p. $=100-102{ }^{\circ} \mathrm{C} ;{ }^{1} \mathrm{H}$ NMR $(400 \mathrm{MHz}$, $\left.\mathrm{CDCl}_{3}\right) \delta 12.17(\mathrm{~s}, 1 \mathrm{H})$, 8.07-7.95 (m, 3H), 7.60-7.52 (m, 2H), 7.43-7.34 (m, 3H), 7.30-7.23 (m, 2H), 6.87-6.84 (m, 1H), 6.84-6.78 (m, 1H), $3.88(\mathrm{~s}, 1 \mathrm{H}) ;{ }^{13} \mathrm{C}$ NMR 
$\left(100 \mathrm{MHz}, \mathrm{CDCl}_{3}\right) \delta 167.9,165.3,157.6,146.6,136.8,135.9,134.8,131.9(\mathrm{~d}, J=$ $9.8 \mathrm{~Hz}), 131.5(\mathrm{~d}, J=3.4 \mathrm{~Hz}), 130.1,129.3,127.0,122.7,120.0,119.4(\mathrm{~d}, J=20.8$ $\mathrm{Hz}), 116.8(\mathrm{~d}, J=22.8 \mathrm{~Hz}) ;{ }^{19} \mathrm{~F}$ NMR $\left(376 \mathrm{MHz}, \mathrm{CDCl}_{3}\right) \delta-100.80 ; \mathrm{HRMS}(\mathrm{ESI}) \mathrm{m} / \mathrm{z}$ calcd for $\mathrm{C}_{18} \mathrm{H}_{14} \mathrm{FNO}_{5} \mathrm{~S}_{2}[\mathrm{M}+\mathrm{H}]^{+}=408.0375$, found $=408.0378$.

Optical Rotation: $[\alpha]_{\mathrm{D}}^{25}=58.45\left(c=0.51, \mathrm{CHCl}_{3}\right) .97 \%$ ee (HPLC condition: Chiralpak IG column, $n$-Hexane $/ i-\mathrm{PrOH}=60: 40$, flow rate $=1.0 \mathrm{~mL} / \mathrm{min}$, wavelength $=254 \mathrm{~nm}, t_{\mathrm{R}}=16.75 \mathrm{~min}$ for minor isomer, $t_{\mathrm{R}}=57.10 \mathrm{~min}$ for major isomer).

$\mathrm{mV}$

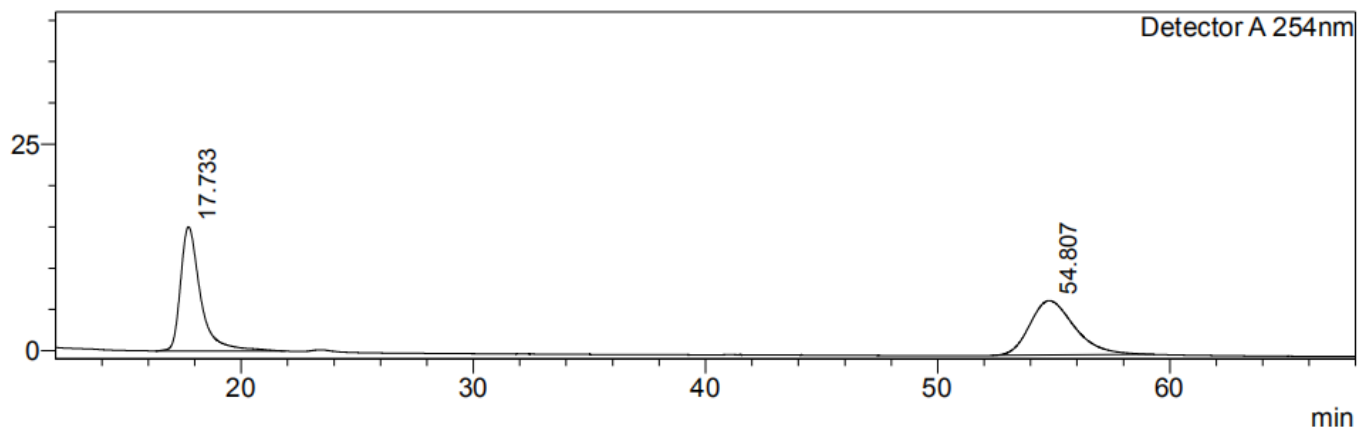

Detector A 254nm

\begin{tabular}{|r|r|r|r|r|r|}
\hline Peak\# & Ret. Time & Height & Height\% & \multicolumn{1}{c|}{ Area } & \multicolumn{1}{c|}{ Area\% } \\
\hline 1 & 17.733 & 15046 & 69.564 & 909739 & 50.657 \\
\hline 2 & 54.807 & 6583 & 30.436 & 886139 & 49.343 \\
\hline Total & & 21630 & 100.000 & 1795878 & 100.000 \\
\hline
\end{tabular}

$\mathrm{mV}$

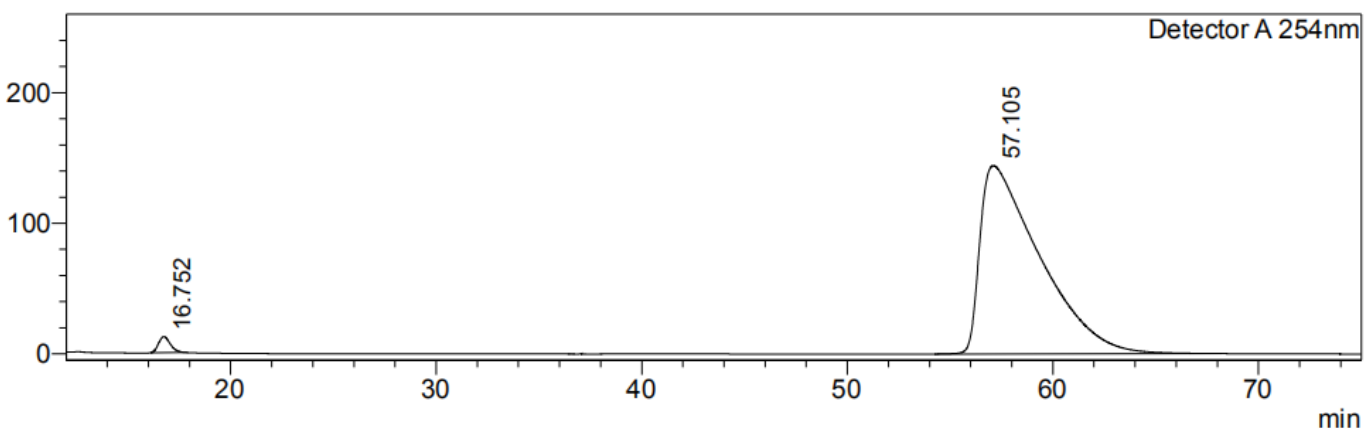

Detector A 254nm

\begin{tabular}{|r|r|r|r|r|r|}
\hline Peak\# & Ret. Time & Height & \multicolumn{1}{c|}{ Height\% } & \multicolumn{1}{c|}{ Area } & \multicolumn{1}{c|}{ Area\% } \\
\hline 1 & 16.752 & 12235 & 7.821 & 486066 & 1.639 \\
\hline 2 & 57.105 & 144199 & 92.179 & 29172836 & 98.361 \\
\hline Total & & 156434 & 100.000 & 29658902 & 100.000 \\
\hline
\end{tabular}

\section{$\underline{(S)-2-(2-h y d r o x y p h e n y l s u l f o n i m i d o y l) p h e n y l ~ 4-c h l o r o b e n z e n e s u l f o n a t e ~(7 f) ~}$}




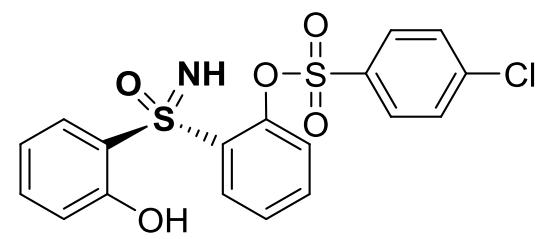

Light yellow oil; $34 \mathrm{mg}, 81 \%$ yield; ${ }^{1} \mathrm{H}$ NMR (400 MHz, $\left.\mathrm{CDCl}_{3}\right) \delta 12.20$ (s, 1H), $8.01(\mathrm{dd}, J=8.0,1.6 \mathrm{~Hz}, 1 \mathrm{H}), 7.95-7.87(\mathrm{~m}, 2 \mathrm{H}), 7.60-7.49(\mathrm{~m}, 4 \mathrm{H}), 7.44-7.35(\mathrm{~m}$, $3 \mathrm{H}), 6.87(\mathrm{dd}, J=7.6,1.4 \mathrm{~Hz}, 1 \mathrm{H}), 6.82(\mathrm{td}, J=7.6,1.4 \mathrm{~Hz}, 1 \mathrm{H}), 3.88(\mathrm{~s}, 1 \mathrm{H}) ;{ }^{13} \mathrm{C}$ NMR $\left(100 \mathrm{MHz}, \mathrm{CDCl}_{3}\right) \delta 157.6,146.6141 .9,136.8,135.9,134.8,133.9,130.3$, 130.1, 129.8, 129.3, 127.0, 122.7, 120.0, 119.5, 119.3; HRMS (ESI) $\mathrm{m} / z$ calcd for $\mathrm{C}_{18} \mathrm{H}_{14} \mathrm{ClNO}_{5} \mathrm{~S}_{2}[\mathrm{M}+\mathrm{H}]^{+}=424.0080$, found $=424.0083$.

Optical Rotation: $[\alpha]_{\mathrm{D}}^{25}=72.90\left(c=0.31, \mathrm{CHCl}_{3}\right) .94 \%$ ee (HPLC condition: Chiralpak ID column, $n$-Hexane $/ i-\mathrm{PrOH}=60: 40$, flow rate $=1.0 \mathrm{~mL} / \mathrm{min}$, wavelength $=254 \mathrm{~nm}, t_{\mathrm{R}}=14.64 \min$ for minor isomer, $t_{\mathrm{R}}=39.32 \min$ for major isomer).

$\mathrm{mV}$

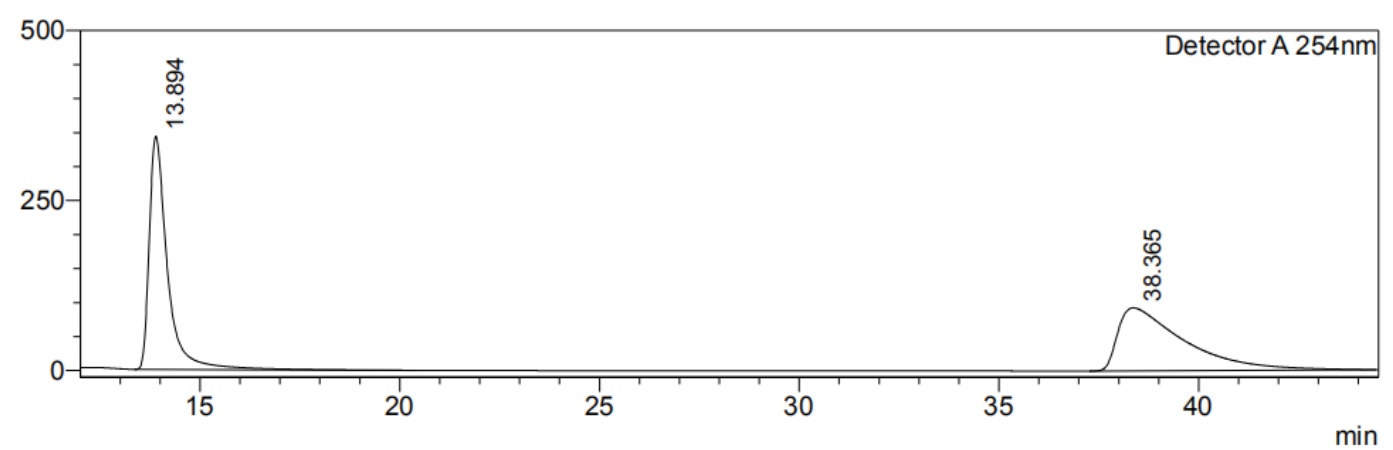

\begin{tabular}{|c|c|c|c|c|c|}
\hline Peak\# & Ret. Time & Height & Height $\%$ & Area & Area\% \\
\hline 1 & 13.894 & 342816 & 78.713 & 10918219 & 50.557 \\
\hline 2 & 38.365 & 92712 & 21.287 & 10677600 & 49.443 \\
\hline Tota & & 435529 & 100.000 & 21595819 & 100.000 \\
\hline
\end{tabular}


$\mathrm{mV}$

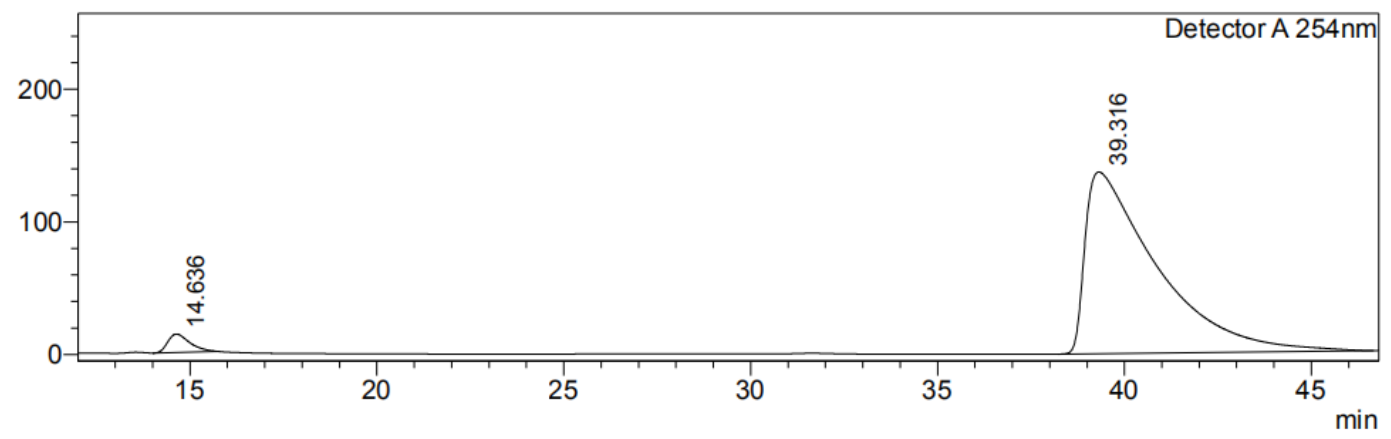

Detector A $254 \mathrm{~nm}$
\begin{tabular}{|r|r|r|r|r|r|}
\hline Peak\# & Ret. Time & Height & Height $\%$ & \multicolumn{1}{c|}{ Area } & \multicolumn{1}{|c|}{ Area $\%$} \\
\hline 1 & 14.636 & 13898 & 9.214 & 574874 & 3.046 \\
\hline 2 & 39.316 & 136937 & 90.786 & 18300572 & 96.954 \\
\hline Total & & 150835 & 100.000 & 18875446 & 100.000 \\
\hline
\end{tabular}

(S)-2-(2-hydroxyphenylsulfonimidoyl)phenyl 4-bromobenzenesulfonate $(7 \mathrm{~g})$<smiles>NS(=O)(=O)c1ccccc1OS(=O)(=O)c1ccc(Br)cc1</smiles>

Colorless oil; $38 \mathrm{mg}, 82 \%$ yield; ${ }^{1} \mathrm{H}$ NMR (400 MHz, $\mathrm{CDCl}_{3}$ ) $\delta 12.23$ (s, 1H), 8.01 $(\mathrm{dd}, J=8.0,1.6 \mathrm{~Hz}, 1 \mathrm{H}), 7.86-7.80(\mathrm{~m}, 2 \mathrm{H}), 7.75-7.70(\mathrm{~m}, 2 \mathrm{H}), 7.60-7.49(\mathrm{~m}, 2 \mathrm{H})$, 7.43-7.35 (m, 3H), $6.87(\mathrm{dd}, J=7.6,1.6 \mathrm{~Hz}, 1 \mathrm{H}), 6.85-6.78(\mathrm{~m}, 1 \mathrm{H}), 3.88(\mathrm{~s}, 1 \mathrm{H}) ;{ }^{13} \mathrm{C}$ NMR $\left(100 \mathrm{MHz}, \mathrm{CDCl}_{3}\right) \delta 157.6,146.6,136.8,135.9,134.8,134.5,132.8,130.5$, 130.3, 130.1, 129.3, 127.1, 122.7, 120.0, 119.5, 119.3; HRMS (ESI) $\mathrm{m} / z$ calcd for $\mathrm{C}_{18} \mathrm{H}_{14} \mathrm{BrNO}_{5} \mathrm{~S}_{2}[\mathrm{M}+\mathrm{H}]^{+}=467.9575$, found $=467.9579$.

Optical Rotation: $[\alpha]_{\mathrm{D}}^{25}=60.98\left(c=0.41, \mathrm{CHCl}_{3}\right) .94 \%$ ee (HPLC condition: Chiralpak AD-H column, $n-\mathrm{Hexane} / \mathrm{i}-\mathrm{PrOH}=70: 30$, flow rate $=1.0 \mathrm{~mL} / \mathrm{min}$, wavelength $=254 \mathrm{~nm}, t_{\mathrm{R}}=21.41 \mathrm{~min}$ for minor isomer, $t_{\mathrm{R}}=31.05$ min for major isomer). 
$\mathrm{mV}$

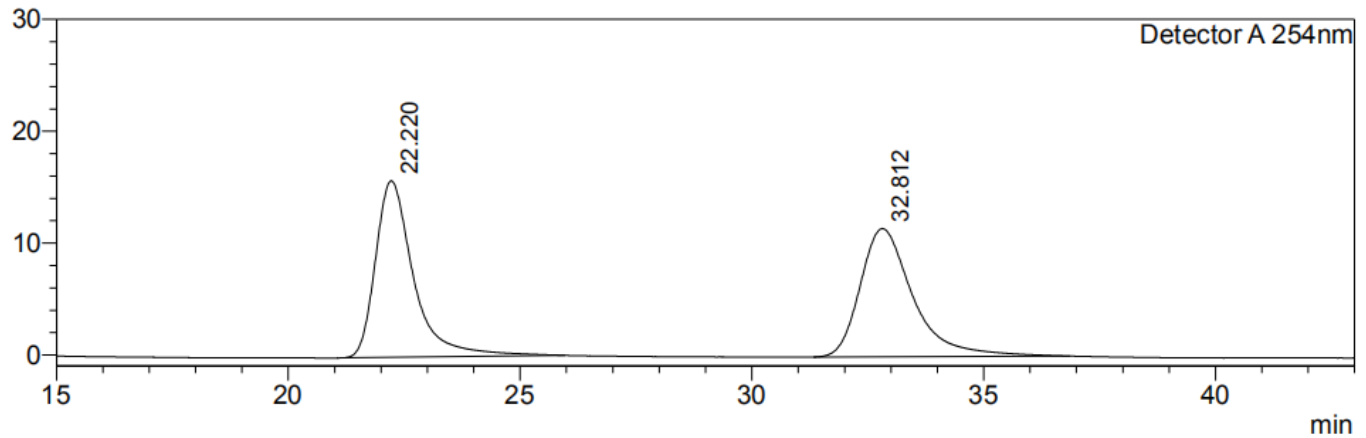

\begin{tabular}{|c|c|c|c|c|c|}
\hline Peak\# & Ret. Time & Height & Height\% & Area & Area\% \\
\hline 1 & 22.220 & 15784 & 57.943 & 904976 & 49.940 \\
\hline 2 & 32.812 & 11456 & 42.057 & 907137 & 50.060 \\
\hline Total & & 27240 & 100.000 & 1812113 & 100.000 \\
\hline
\end{tabular}

$\mathrm{mV}$

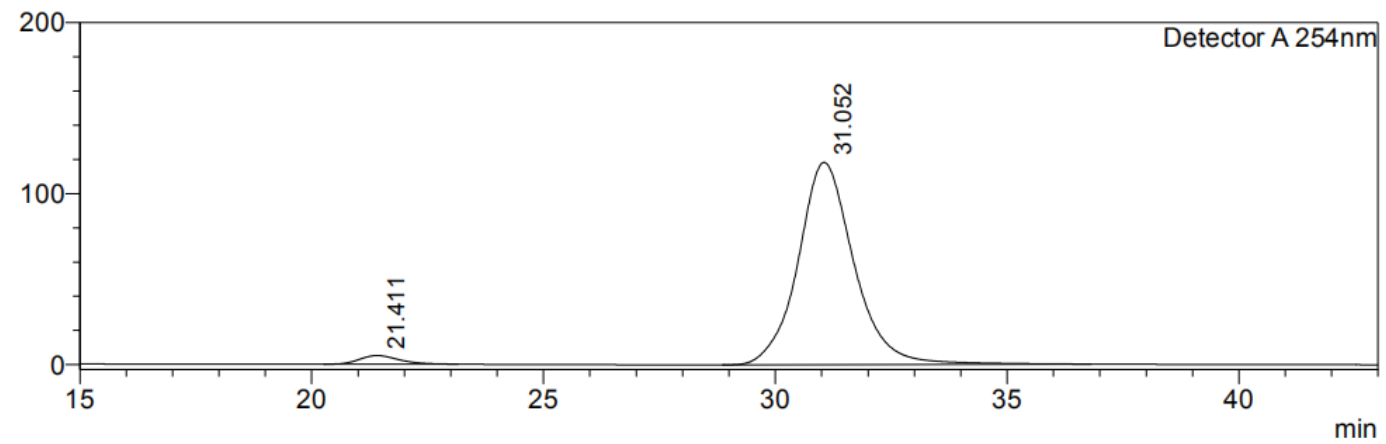

Detector A 254nm

\begin{tabular}{|r|r|r|r|r|r|}
\hline Peak\# & Ret. Time & \multicolumn{1}{c|}{ Height } & \multicolumn{1}{c|}{ Height $\%$} & \multicolumn{1}{c|}{ Area } & \multicolumn{1}{c|}{ Area\% } \\
\hline 1 & 21.411 & 5005 & 4.058 & 295010 & 2.880 \\
\hline 2 & 31.052 & 118328 & 95.942 & 9947497 & 97.120 \\
\hline Total & & 123334 & 100.000 & 10242508 & 100.000 \\
\hline
\end{tabular}

$\underline{(S)-2-(2-h y d r o x y p h e n y l s u l f o n i m i d o y l) p h e n y l ~ 3-m e t h y l b e n z e n e s u l f o n a t e ~(7 h) ~}$<smiles>Cc1cccc(S(=O)(=O)Oc2ccccc2S(=N)(=O)c2ccccc2O)c1</smiles>

White solid; $33 \mathrm{mg}, 81 \%$ yield; m.p. $=173-175{ }^{\circ} \mathrm{C} ;{ }^{1} \mathrm{H}$ NMR $\left(400 \mathrm{MHz}, \mathrm{CDCl}_{3}\right) \delta$ $12.23(\mathrm{~s}, 1 \mathrm{H}), 8.01(\mathrm{dd}, J=8.0,1.6 \mathrm{~Hz}, 1 \mathrm{H}), 7.80(\mathrm{~s}, 1 \mathrm{H}), 7.77(\mathrm{~d}, J=7.8 \mathrm{~Hz}, 1 \mathrm{H})$, 7.59-7.50 (m, 2H), 7.51-7.44 (m, 2H), 7.43-7.34 (m, 3H), $6.87(\mathrm{~d}, J=8.2 \mathrm{~Hz}, 1 \mathrm{H})$, $6.80(\mathrm{t}, J=7.8 \mathrm{~Hz}, 1 \mathrm{H}), 3.91(\mathrm{~s}, 1 \mathrm{H}), 2.47(\mathrm{~s}, 3 \mathrm{H}) ;{ }^{13} \mathrm{C} \mathrm{NMR}\left(100 \mathrm{MHz}, \mathrm{CDCl}_{3}\right) \delta$ $157.7,146.9,139.9,136.8,135.8,135.5,134.7,130.0,129.4,129.4,129.3,129.1$, $126.8,125.9,122.8,120.0,119.4,119.3,21.5$; HRMS (ESI) $\mathrm{m} / z$ calcd for $\mathrm{C}_{19} \mathrm{H}_{17} \mathrm{NO}_{5} \mathrm{~S}_{2}[\mathrm{M}+\mathrm{H}]^{+}=404.0626$, found $=404.0606$. 
Optical Rotation: $[\alpha]_{\mathrm{D}}^{25}=59.75\left(c=0.40, \mathrm{CHCl}_{3}\right) .>99 \%$ ee (HPLC condition: Chiralpak ID column, $n$-Hexane $/ i-\mathrm{PrOH}=60: 40$, flow rate $=1.0 \mathrm{~mL} / \mathrm{min}$, wavelength $=254 \mathrm{~nm}, t_{\mathrm{R}}=20.98$ min for minor isomer, $t_{\mathrm{R}}=25.08$ min for major isomer).

$\mathrm{mV}$

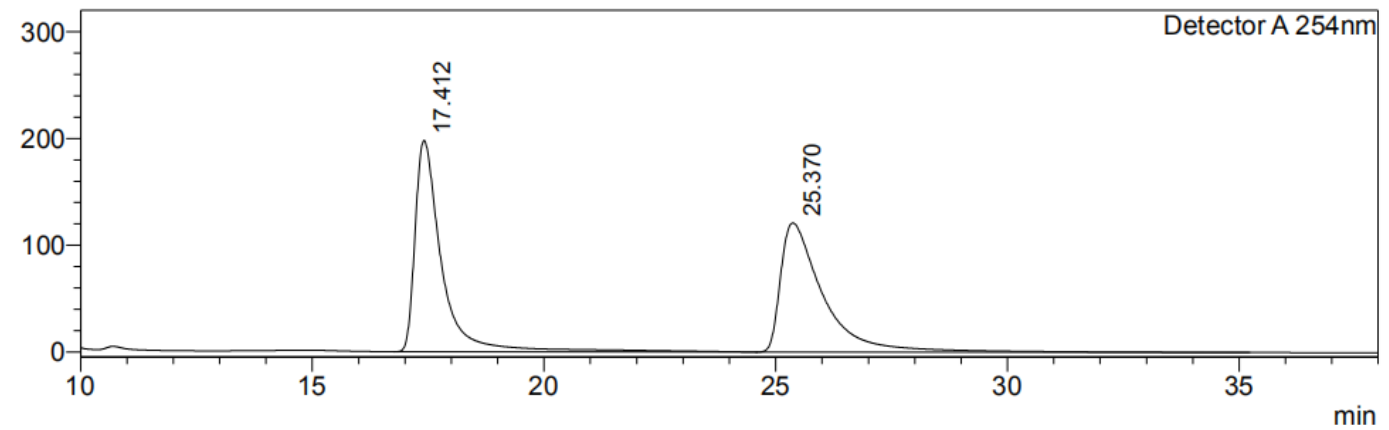

Detector A 254nm

\begin{tabular}{|r|r|r|r|r|r|}
\hline Peak\# & Ret. Time & Height & \multicolumn{1}{c|}{ Height\% } & \multicolumn{1}{c|}{ Area } & \multicolumn{1}{|c|}{ Area\% } \\
\hline 1 & 17.412 & 198098 & 62.075 & 8075848 & 50.767 \\
\hline 2 & 25.370 & 121030 & 37.925 & 7831822 & 49.233 \\
\hline Total & & 319128 & 100.000 & 15907671 & 100.000 \\
\hline
\end{tabular}

$\mathrm{mV}$

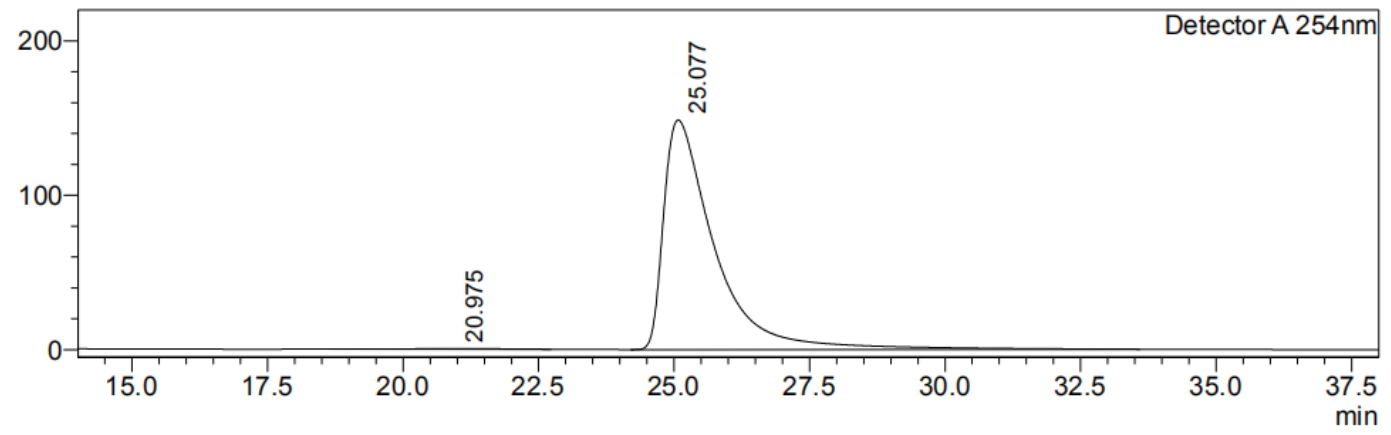

Detector A 254nm

\begin{tabular}{|r|r|r|r|r|r|}
\hline Peak\# & Ret. Time & \multicolumn{1}{|c|}{ Height } & Height\% & \multicolumn{1}{c|}{ Area } & \multicolumn{1}{|c|}{ Area\% } \\
\hline 1 & 20.975 & 372 & 0.249 & 46187 & 0.471 \\
\hline 2 & 25.077 & 148711 & 99.751 & 9758708 & 99.529 \\
\hline Total & & 149083 & 100.000 & 9804895 & 100.000 \\
\hline
\end{tabular}

(S)-2-(2-hydroxyphenylsulfonimidoyl)phenyl 3-fluorobenzenesulfonate (7i)<smiles>NS(=O)(=O)c1ccccc1OS(=O)(=O)c1cccc(F)c1</smiles>

Colorless oil, $34 \mathrm{mg}, 84 \%$ yield; ${ }^{1} \mathrm{H}$ NMR $\left(400 \mathrm{MHz}, \mathrm{CDCl}_{3}\right) \delta 12.20(\mathrm{~s}, 1 \mathrm{H}), 8.04$ $(\mathrm{dd}, J=8.0,1.6 \mathrm{~Hz}, 1 \mathrm{H}), 7.80(\mathrm{ddd}, J=8.0,1.6,1.0 \mathrm{~Hz}, 1 \mathrm{H}), 7.68-7.63(\mathrm{~m}, 1 \mathrm{H})$, 7.62-7.54 (m, 2H), $7.51(\mathrm{dd}, J=8.4,1.2 \mathrm{~Hz}, 1 \mathrm{H}), 7.48-7.35(\mathrm{~m}, 4 \mathrm{H}), 6.88-6.84(\mathrm{~m}$, 
1H), 6.84-6.78 (m, 1H), $3.85(\mathrm{~s}, 1 \mathrm{H}) ;{ }^{13} \mathrm{C} \mathrm{NMR}\left(100 \mathrm{MHz}, \mathrm{CDCl}_{3}\right) \delta$ 163.6, 161.1, 157.7, 146.6, 137.4 (d, $J=7.6 \mathrm{~Hz}), 136.7,135.9,134.9,131.3$ (d, $J=7.8 \mathrm{~Hz}), 130.2$, 129.3, 127.0, $124.7(\mathrm{~d}, J=3.6 \mathrm{~Hz}), 122.6,122.4(\mathrm{~d}, J=21.2 \mathrm{~Hz}), 120.0,119.4(\mathrm{~d}, J=$ 18.7 Hz), $116.2(\mathrm{~d}, J=25.2 \mathrm{~Hz}) ;{ }^{19} \mathrm{~F} \mathrm{NMR}\left(376 \mathrm{MHz} \mathrm{CDCl}_{3}\right) \delta-108.45 ; \mathrm{HRMS}(\mathrm{ESI})$ $m / z$ calcd for $\mathrm{C}_{19} \mathrm{H}_{17} \mathrm{NO}_{5} \mathrm{~S}_{2}[\mathrm{M}+\mathrm{H}]^{+}=408.0375$, found $=408.0360$.

Optical Rotation: $[\alpha]_{\mathrm{D}}^{25}=34.21\left(c=0.39, \mathrm{CHCl}_{3}\right) .>99 \%$ ee (HPLC condition: Chiralpak IG column, $n$-Hexane $/ i$-PrOH $=60: 40$, flow rate $=1.0 \mathrm{~mL} / \mathrm{min}$, wavelength $=254 \mathrm{~nm}, t_{\mathrm{R}}=16.19 \min$ for minor isomer, $t_{\mathrm{R}}=21.93$ min for major isomer).

$\mathrm{mV}$

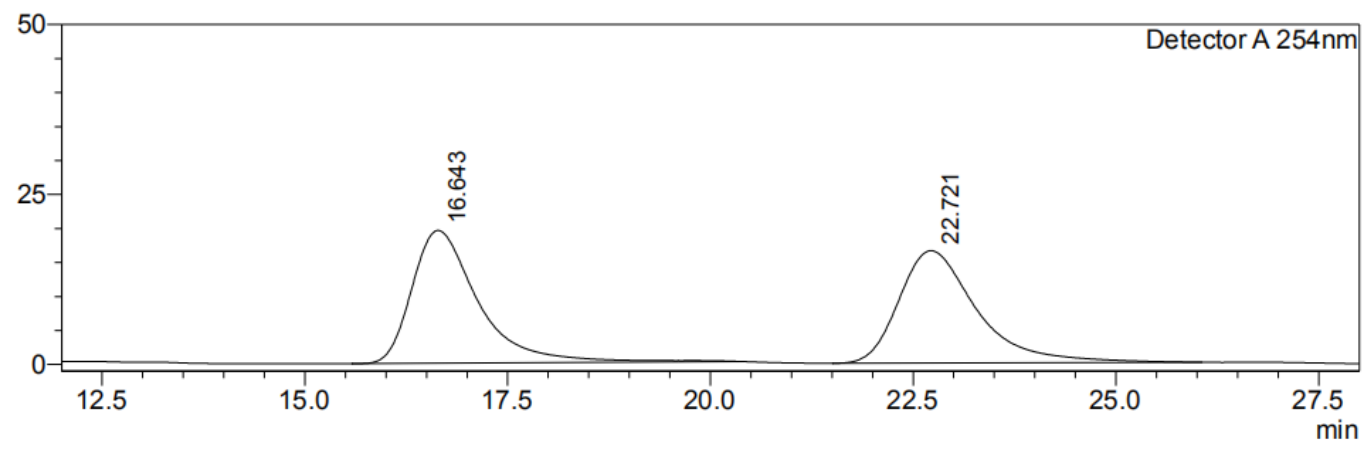

Detector A 254nm
\begin{tabular}{|r|r|r|r|r|r|}
\hline Peak\# & Ret. Time & Height & Height\% & Area & Area\% \\
\hline 1 & 16.643 & 19523 & 54.150 & 1116658 & 50.250 \\
\hline 2 & 22.721 & 16530 & 45.850 & 1105532 & 49.750 \\
\hline Total & & 36053 & 100.000 & 2222190 & 100.000 \\
\hline
\end{tabular}

$\mathrm{mV}$

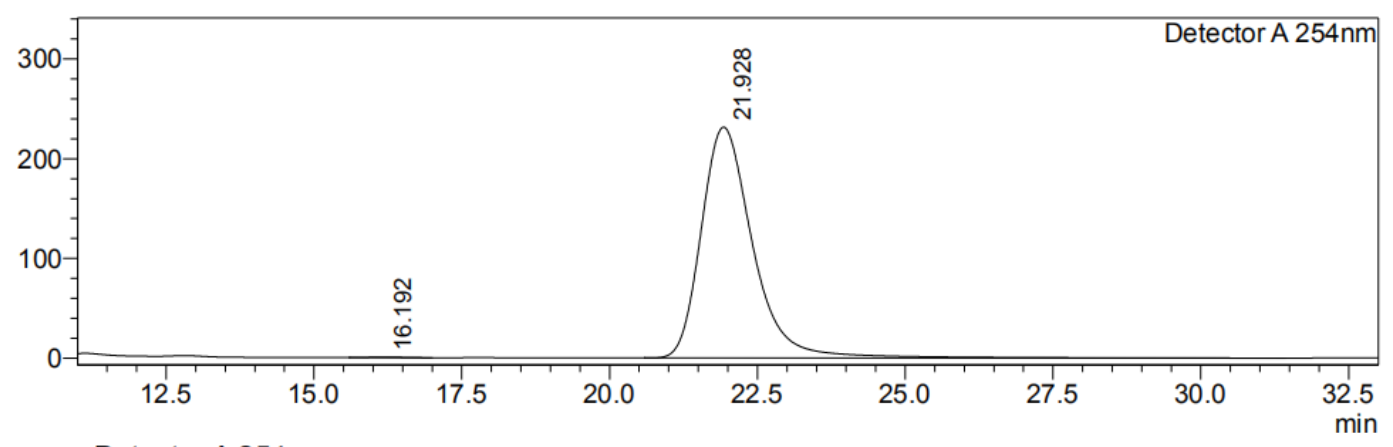

Detector A 254nm
\begin{tabular}{|r|r|r|r|r|r|}
\hline Peak\# & Ret. Time & Height & Height\% & \multicolumn{1}{c|}{ Area } & \multicolumn{1}{|c|}{ Area\% } \\
\hline 1 & 16.192 & 581 & 0.250 & 24140 & 0.171 \\
\hline 2 & 21.928 & 231450 & 99.750 & 14098607 & 99.829 \\
\hline Total & & 232031 & 100.000 & 14122747 & 100.000 \\
\hline
\end{tabular}

\section{(S)-2-(2-hydroxyphenylsulfonimidoyl)phenyl 3-cyanobenzenesulfonate (7j)}




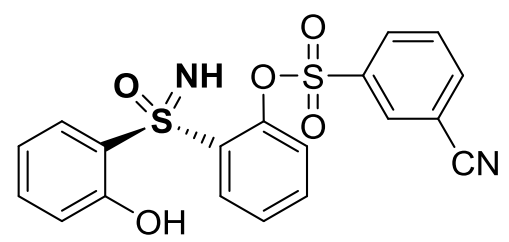

Yellow solid; $35 \mathrm{mg}$, 83\% yield; m.p. $=173-175{ }^{\circ} \mathrm{C} ;{ }^{1} \mathrm{H}$ NMR $\left(400 \mathrm{MHz}, \mathrm{CDCl}_{3}\right) \delta$ 12.11 (s, $1 \mathrm{H}), 8.24$ (ddd, $J=8.0,1.8,1.2 \mathrm{~Hz}, 1 \mathrm{H}), 8.20(\mathrm{t}, J=1.5 \mathrm{~Hz}, 1 \mathrm{H}$ ), 8.06 (dd, $J=8.0,1.2 \mathrm{~Hz}, 1 \mathrm{H}), 8.03-7.97(\mathrm{~m}, 1 \mathrm{H}), 7.76(\mathrm{t}, J=8.0 \mathrm{~Hz}, 1 \mathrm{H}), 7.64-7.56(\mathrm{~m}, 2 \mathrm{H})$, 7.48-7.37 (m, 2H), 7.37-7.32 (m, 1H), 6.85-6.79 (m, 2H), $3.87(\mathrm{~s}, 1 \mathrm{H}) ;{ }^{13} \mathrm{C}$ NMR $\left(100 \mathrm{MHz}, \mathrm{CDCl}_{3}\right) \delta 157.5,146.3,138.0,137.2,136.5,136.0,135.0,132.7,132.3$, 130.5, 130.3, 129.2, 127.3, 122.6, 119.9, 119.6, 119.3, 116.8, 114.2; HRMS (ESI) $\mathrm{m} / \mathrm{z}$ calcd for $\mathrm{C}_{19} \mathrm{H}_{14} \mathrm{~N}_{2} \mathrm{O}_{5} \mathrm{~S}_{2}[\mathrm{M}+\mathrm{H}]^{+}=415.0422$, found $=415.0406$.

Optical Rotation: $[\alpha]^{25}=58.80\left(c=0.50, \mathrm{CHCl}_{3}\right) .96 \%$ ee (HPLC condition: Chiralpak AD-H column, $n$-Hexane $/ i-\mathrm{PrOH}=60: 40$, flow rate $=1.0 \mathrm{~mL} / \mathrm{min}$, wavelength $=254 \mathrm{~nm}, t_{\mathrm{R}}=16.19$ min for major isomer, $t_{\mathrm{R}}=21.93 \mathrm{~min}$ for minor isomer).

$\mathrm{mV}$

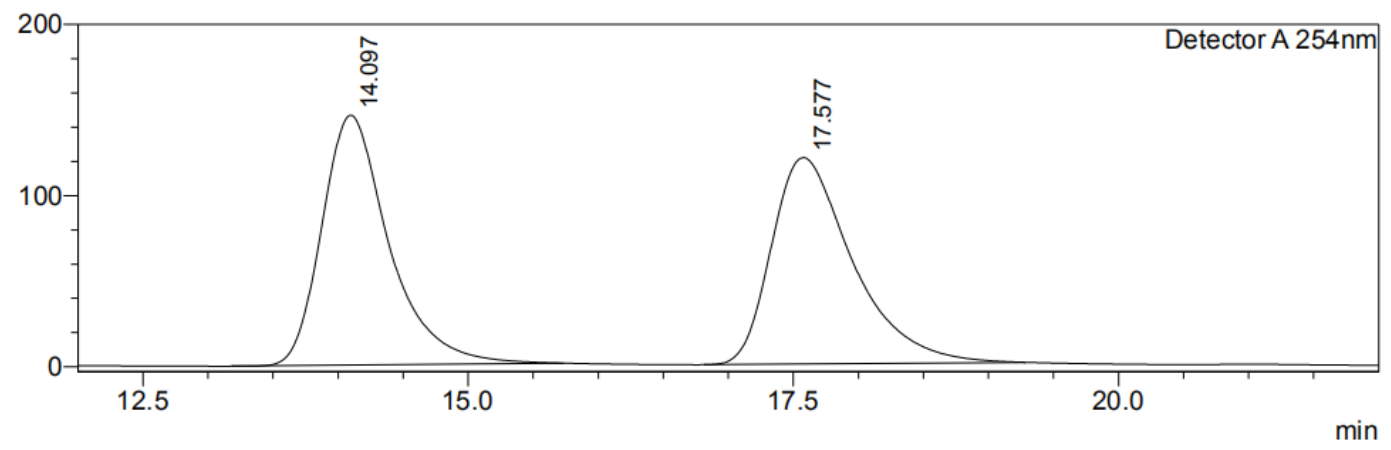

Detector A 254nm

\begin{tabular}{|r|r|r|r|r|r|}
\hline Peak\# & Ret. Time & \multicolumn{1}{c|}{ Height } & Height\% & \multicolumn{1}{c|}{ Area } & \multicolumn{1}{c|}{ Area\% } \\
\hline 1 & 14.097 & 145998 & 54.769 & 5253770 & 50.097 \\
\hline 2 & 17.577 & 120571 & 45.231 & 5233365 & 49.903 \\
\hline Total & & 266569 & 100.000 & 10487135 & 100.000 \\
\hline
\end{tabular}


$\mathrm{mV}$

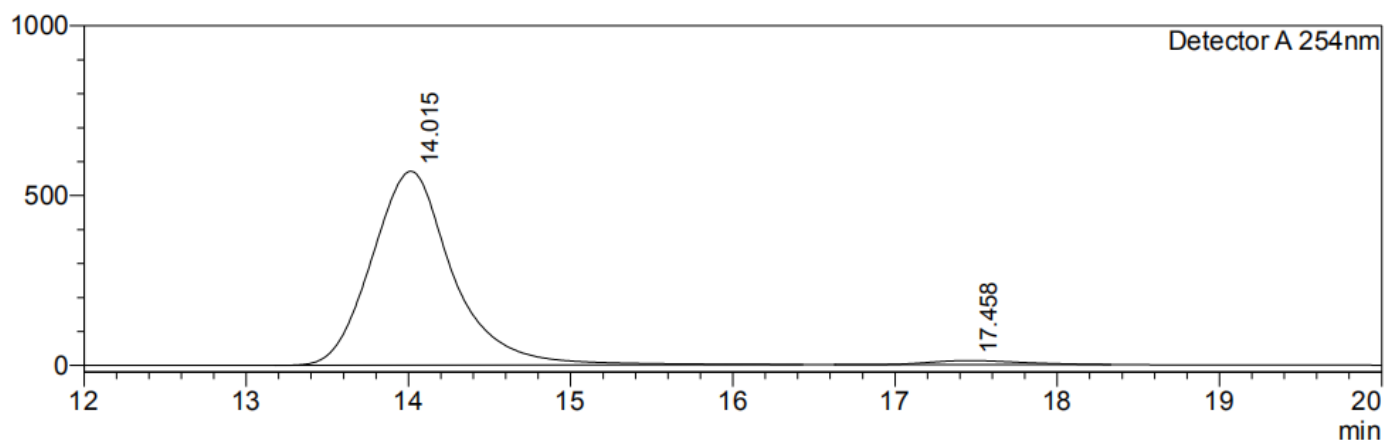

Detector A $254 \mathrm{~nm}$
\begin{tabular}{|r|r|r|r|r|r|}
\hline Peak\#\# & Ret. Time & \multicolumn{1}{c|}{ Height } & \multicolumn{1}{c|}{ Height\% } & \multicolumn{1}{c|}{ Area } & \multicolumn{1}{c|}{ Area\% } \\
\hline 1 & 14.015 & 570752 & 97.968 & 19843156 & 97.757 \\
\hline 2 & 17.458 & 11837 & 2.032 & 455208 & 2.243 \\
\hline Total & & 582589 & 100.000 & 20298364 & 100.000 \\
\hline
\end{tabular}

(S)-2-(2-hydroxyphenylsulfonimidoyl)phenyl 2-methylbenzenesulfonate (7k)<smiles>Cc1ccccc1S(=O)(=O)Oc1ccccc1S(=O)(=O)c1ccccc1O</smiles>

White solid; $35 \mathrm{mg}, 83 \%$ yield; m.p. $=122-124{ }^{\circ} \mathrm{C} ;{ }^{1} \mathrm{H}$ NMR $\left(400 \mathrm{MHz}, \mathrm{CDCl}_{3}\right) \delta$ $12.14(\mathrm{~s}, 1 \mathrm{H}), 8.08(\mathrm{dd}, J=8.0,1.8 \mathrm{~Hz}, 1 \mathrm{H}), 7.97(\mathrm{dd}, J=8.0,1.2 \mathrm{~Hz}, 1 \mathrm{H}), 7.63(\mathrm{td}$, $J=7.6,1.2 \mathrm{~Hz}, 1 \mathrm{H}), 7.55-7.49(\mathrm{~m}, 1 \mathrm{H}), 7.44-7.35(\mathrm{~m}, 5 \mathrm{H}), 7.30(\mathrm{dd}, J=8.2,1.2 \mathrm{~Hz}$ 1H), 6.81-6.73 (m, 2H), $3.90(\mathrm{~s}, 1 \mathrm{H}), 2.62(\mathrm{~s}, 3 \mathrm{H}) ;{ }^{13} \mathrm{C} \mathrm{NMR}\left(100 \mathrm{MHz}, \mathrm{CDCl}_{3}\right) \delta$ $157.7,147.1,139.4,136.8,135.6,135.2$, 134.9, 134.8, 133.0, 130.2, 129.8, 129.3, 128.9, 126.7, 126.5, 122.6, 120.1, 119.3, 20.5; HRMS (ESI) $\mathrm{m} / z$ calcd for $\mathrm{C}_{19} \mathrm{H}_{17} \mathrm{NO}_{5} \mathrm{~S}_{2}[\mathrm{M}+\mathrm{H}]^{+}=404.0626$, found $=404.0615$.

Optical Rotation: $[\alpha]_{\mathrm{D}}^{25}=50.50\left(c=0.20, \mathrm{CHCl}_{3}\right) .>99 \%$ ee (HPLC condition: Chiralpak IG column, $n$-Hexane $/ i-\mathrm{PrOH}=60: 40$, flow rate $=1.0 \mathrm{~mL} / \mathrm{min}$, wavelength $=254 \mathrm{~nm}, t_{\mathrm{R}}=17.84 \min$ for minor isomer, $t_{\mathrm{R}}=21.72 \mathrm{~min}$ for major isomer). 
$\mathrm{mV}$

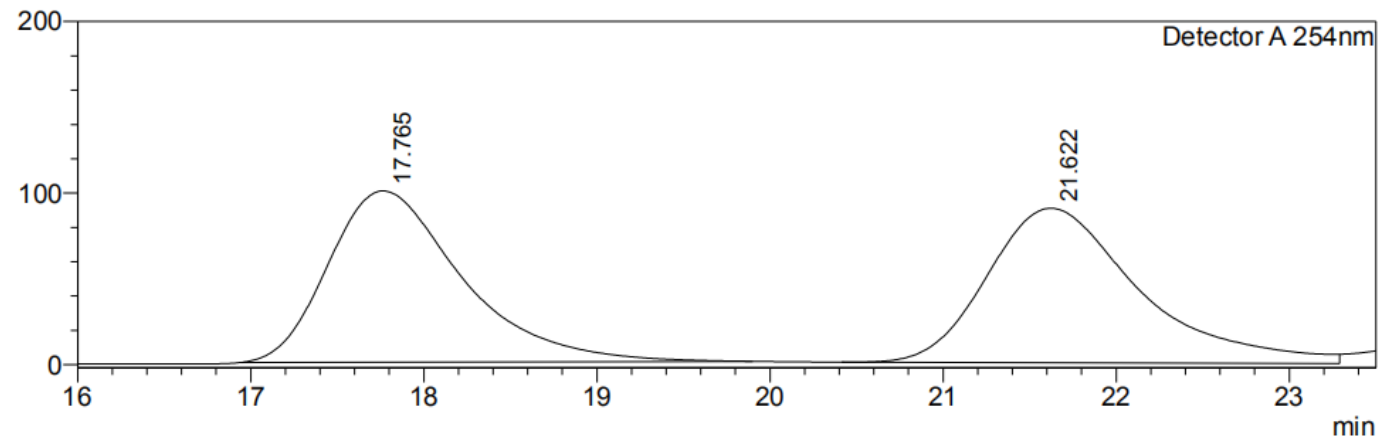

Detector A 254nm

\begin{tabular}{|r|r|r|r|r|r|}
\hline Peak\# & Ret. Time & \multicolumn{1}{c|}{ Height } & Height\% & \multicolumn{1}{c|}{ Area } & \multicolumn{1}{c|}{ Area\% } \\
\hline 1 & 17.765 & 99811 & 52.575 & 5354639 & 49.396 \\
\hline 2 & 21.622 & 90035 & 47.425 & 5485634 & 50.604 \\
\hline Total & & 189846 & 100.000 & 10840273 & 100.000 \\
\hline
\end{tabular}

$\mathrm{mV}$

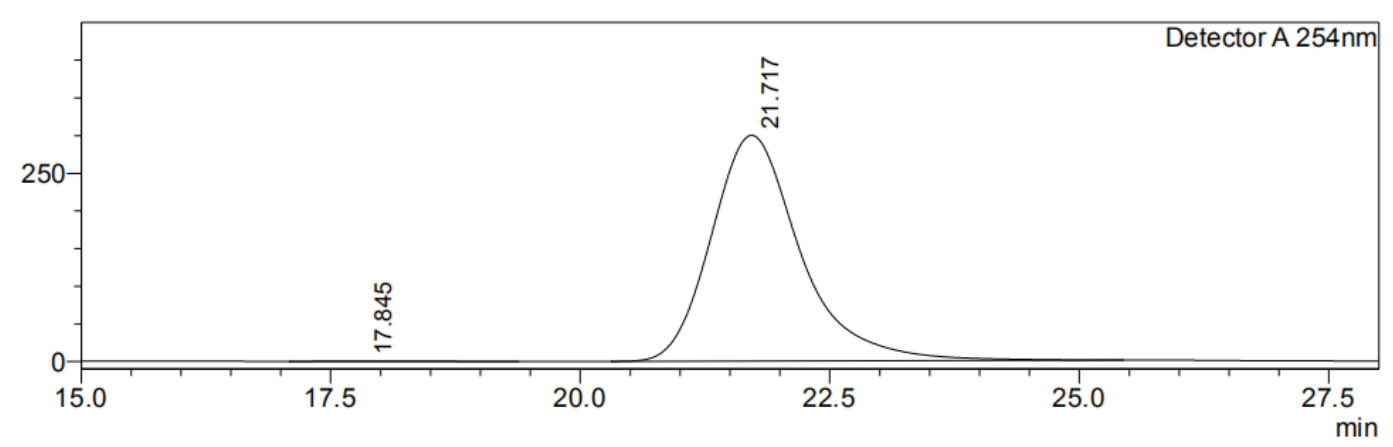

Detector A $254 \mathrm{~nm}$

\begin{tabular}{|r|r|r|r|r|r|}
\hline Peak\# & Ret. Time & \multicolumn{1}{c|}{ Height } & Height\% & \multicolumn{1}{c|}{ Area } & \multicolumn{1}{|c|}{ Area\% } \\
\hline 1 & 17.845 & 324 & 0.108 & 19231 & 0.103 \\
\hline 2 & 21.717 & 299664 & 99.892 & 18658772 & 99.897 \\
\hline Total & & 299988 & 100.000 & 18678003 & 100.000 \\
\hline
\end{tabular}

(S)-2-(2-hydroxyphenylsulfonimidoyl)phenyl 2-fluorobenzenesulfonate (7l)<smiles>NS(=O)(=O)c1ccccc1OS(=O)(=O)c1ccccc1F</smiles>

Yellow oil; $32 \mathrm{mg}, 77 \%$ yield; ${ }^{1} \mathrm{H}$ NMR (400 MHz, $\left.\mathrm{CDCl}_{3}\right) \delta 12.17$ (s, 1H), 8.08-8.02 (m, 1H), 7.99-7.93 (m, 1H), $7.76(\mathrm{ddd}, J=8.4,5.0,1.6 \mathrm{~Hz}, 1 \mathrm{H}), 7.62-7.54(\mathrm{~m}, 2 \mathrm{H})$, 7.44-7.33 (m, 4H), $7.30(\mathrm{t}, J=9.0 \mathrm{~Hz}, 1 \mathrm{H}), 6.81(\mathrm{t}, J=7.8 \mathrm{~Hz}, 1 \mathrm{H}), 6.77(\mathrm{~d}, J=8.4$ $\mathrm{Hz}, 1 \mathrm{H}), 3.88(\mathrm{~s}, 1 \mathrm{H}) ;{ }^{13} \mathrm{C} \mathrm{NMR}\left(100 \mathrm{MHz}, \mathrm{CDCl}_{3}\right) \delta 161.2,158.6,157.7,146.7$, $137.4(\mathrm{~d}, J=8.8 \mathrm{~Hz}), 136.7,135.8,134.8,131.1,130.1,129.3,127.0,124.7(\mathrm{~d}, J=$ $4.0 \mathrm{~Hz}), 124.3(\mathrm{~d}, J=13.6 \mathrm{~Hz}), 123.1,119.8,119.3(\mathrm{~d}, J=24.0 \mathrm{~Hz}), 117.8(\mathrm{~d}, J=$ 
$20.8 \mathrm{~Hz}$ ); ${ }^{19} \mathrm{~F}$ NMR $\left(376 \mathrm{MHz}, \mathrm{CDCl}_{3}\right) \delta$-106.21; HRMS (ESI) $\mathrm{m} / z$ calcd for $\mathrm{C}_{18} \mathrm{H}_{14} \mathrm{FNO}_{5} \mathrm{~S}_{2}[\mathrm{M}+\mathrm{H}]^{+}=408.0375$, found $=408.0375$.

Optical Rotation: $[\alpha]^{25}=63.45\left(c=0.39, \mathrm{CHCl}_{3}\right) .97 \%$ ee (HPLC condition: Chiralpak AD-H column, $n-\mathrm{Hexane} / i-\mathrm{PrOH}=70: 30$, flow rate $=1.0 \mathrm{~mL} / \mathrm{min}$, wavelength $=254 \mathrm{~nm}, t_{\mathrm{R}}=16.61 \mathrm{~min}$ for minor isomer, $t_{\mathrm{R}}=18.46 \min$ for major isomer).

$\mathrm{mV}$

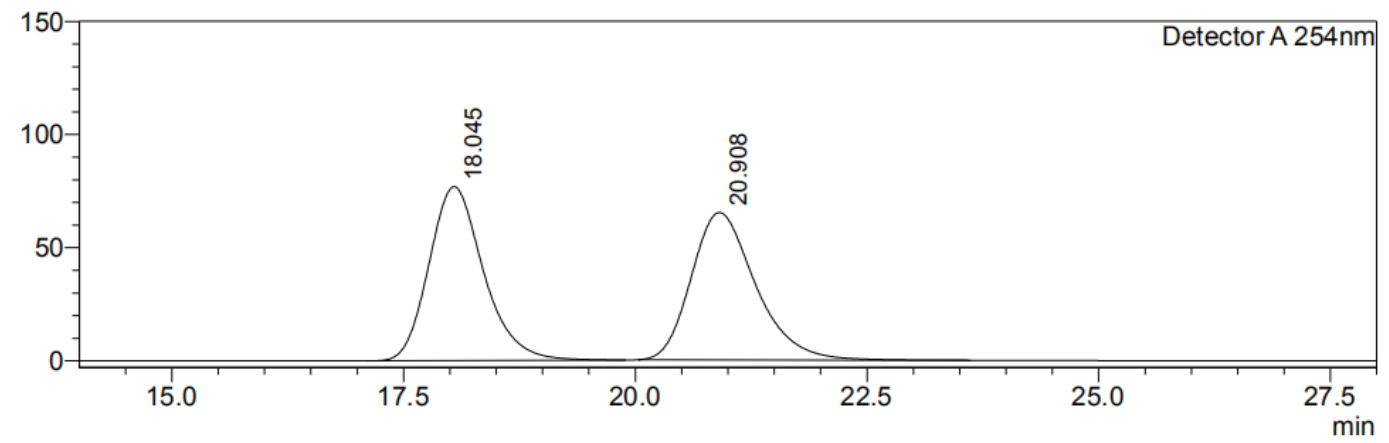

Detector A 254nm

\begin{tabular}{|r|r|r|r|r|r|}
\hline Peak\# & Ret. Time & \multicolumn{1}{c|}{ Height } & Height\% & \multicolumn{1}{c|}{ Area } & \multicolumn{1}{c|}{ Area\% } \\
\hline 1 & 18.045 & 76845 & 54.143 & 3150539 & 50.055 \\
\hline 2 & 20.908 & 65086 & 45.857 & 3143676 & 49.945 \\
\hline Total & & 141931 & 100.000 & 6294216 & 100.000 \\
\hline
\end{tabular}

$\mathrm{mV}$

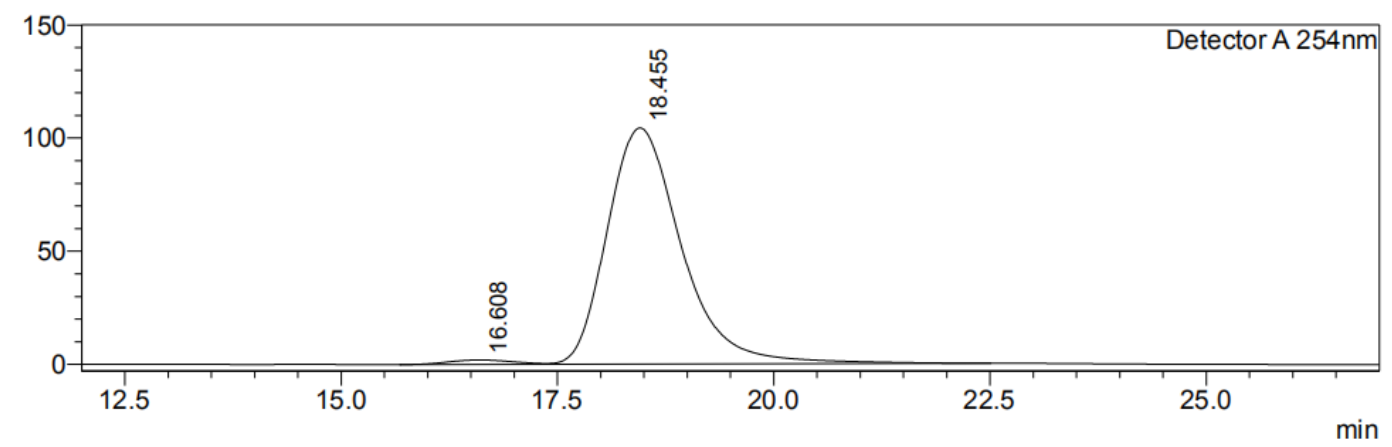

Detector A 254nm

\begin{tabular}{|r|r|r|r|r|r|}
\hline Peak\# & Ret. Time & \multicolumn{1}{c|}{ Height } & \multicolumn{1}{c|}{ Height\% } & \multicolumn{1}{c|}{ Area } & \multicolumn{1}{c|}{ Area\% } \\
\hline 1 & 16.608 & 1981 & 1.863 & 103463 & 1.628 \\
\hline 2 & 18.455 & 104369 & 98.137 & 6252425 & 98.372 \\
\hline Total & & 106351 & 100.000 & 6355888 & 100.000 \\
\hline
\end{tabular}

(S)-2-(2-hydroxyphenylsulfonimidoyl)phenyl 2-chlorobenzenesulfonate (7m)<smiles>NS(=O)(=O)c1ccccc1OS(=O)(=O)c1ccccc1Cl</smiles> 
White solid; $31 \mathrm{mg}, 74 \%$ yield; m.p. $=103-105{ }^{\circ} \mathrm{C} ;{ }^{1} \mathrm{H}$ NMR $\left(400 \mathrm{MHz}, \mathrm{CDCl}_{3}\right) \delta$ $12.20(\mathrm{~s}, 1 \mathrm{H}), 8.09$ (ddd, $J=8.0,5.2,1.6 \mathrm{~Hz}, 2 \mathrm{H}), 7.65(\mathrm{dtd}, J=9.4,8.0,1.6 \mathrm{~Hz}, 2 \mathrm{H})$, 7.58-7.53 (m, 1H), 7.53-7.48 (m, 1H), $7.46(\mathrm{dd}, J=8.4,1.0 \mathrm{~Hz}, 1 \mathrm{H}), 7.43-7.32(\mathrm{~m}$, $3 \mathrm{H}), 6.83-6.76(\mathrm{~m}, 1 \mathrm{H}), 6.74(\mathrm{dd}, J=8.4,1.0 \mathrm{~Hz}, 1 \mathrm{H}), 3.96(\mathrm{~s}, 1 \mathrm{H}) ;{ }^{13} \mathrm{C}$ NMR $(100$ $\left.\mathrm{MHz}, \mathrm{CDCl}_{3}\right) \delta 157.7,146.8,136.8,135.8,135.7,134.8,134.6,133.8,132.4,131.7$, 130.1, 129.3, 127.3, 126.9, 122.9, 119.8, 119.4, 119.2; HRMS (ESI) $\mathrm{m} / \mathrm{z}$ calcd for $\mathrm{C}_{18} \mathrm{H}_{14} \mathrm{ClNO}_{5} \mathrm{~S}_{2}[\mathrm{M}+\mathrm{H}]^{+}=424.0080$, found $=424.0080$.

Optical Rotation: $[\alpha]_{\mathrm{D}}^{25}=57.67\left(c=0.50, \mathrm{CHCl}_{3}\right) .>99 \%$ ee (HPLC condition: Chiralpak ID column, $n$-Hexane $/ i-\mathrm{PrOH}=70: 30$, flow rate $=1.0 \mathrm{~mL} / \mathrm{min}$, wavelength $=254 \mathrm{~nm}, t_{\mathrm{R}}=27.30 \mathrm{~min}$ for minor isomer, $t_{\mathrm{R}}=46.02$ min for major isomer).

$\mathrm{mV}$

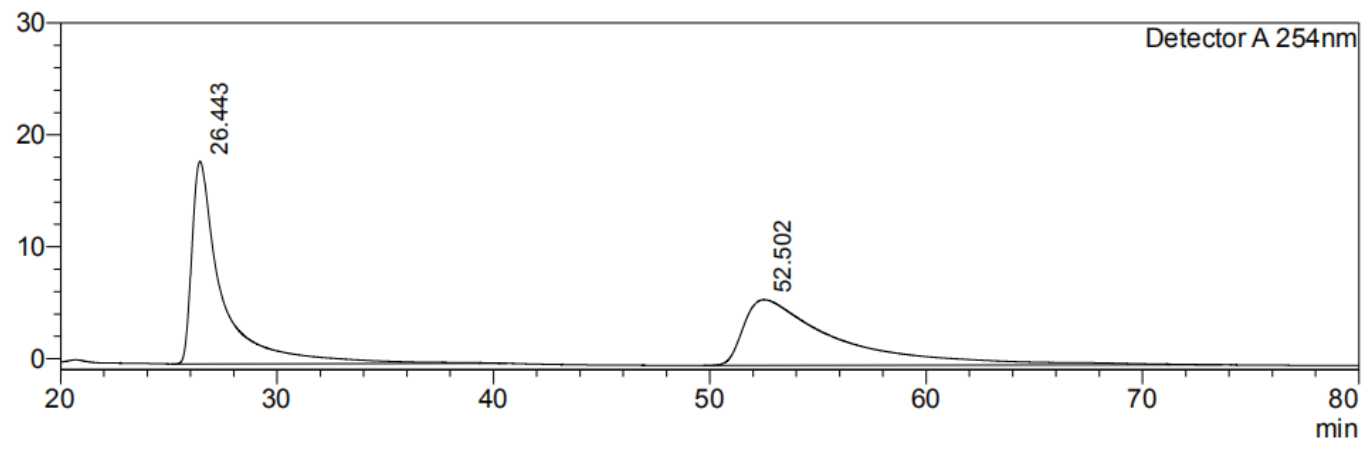

Detector A $254 \mathrm{~nm}$

\begin{tabular}{|r|r|r|r|r|r|}
\hline Peak\# & Ret. Time & \multicolumn{1}{c|}{ Height } & Height\% & \multicolumn{1}{l|}{ Area } & Area\% \\
\hline 1 & 26.443 & 18102 & 75.497 & 1761454 & 50.023 \\
\hline 2 & 52.502 & 5875 & 24.503 & 1759856 & 49.977 \\
\hline Total & & 23977 & 100.000 & 3521311 & 100.000 \\
\hline
\end{tabular}

$\mathrm{mV}$

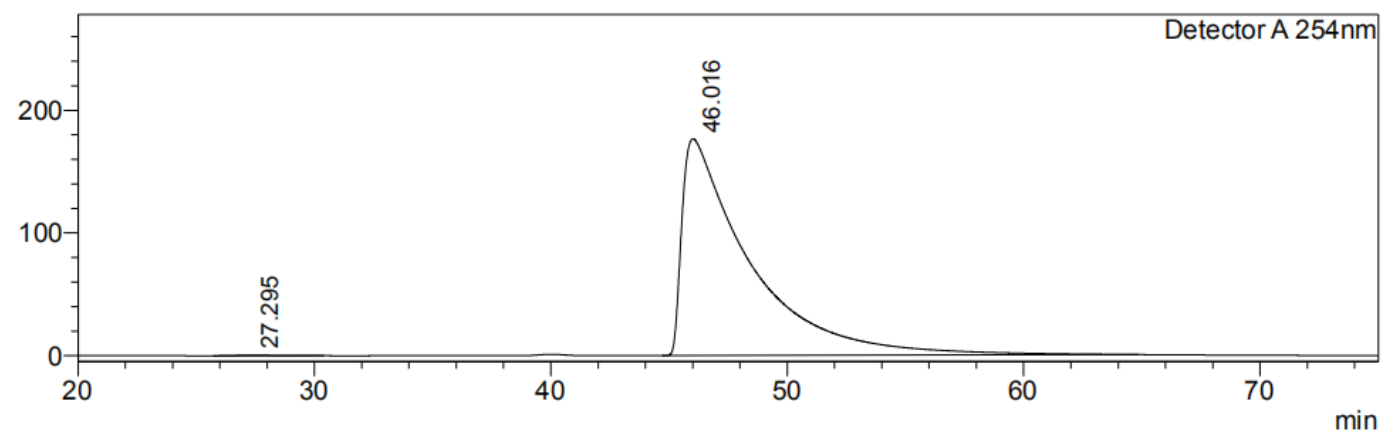

Detector A 254nm

\begin{tabular}{|r|r|r|r|r|r|}
\hline Peak\# & Ret. Time & \multicolumn{1}{|c|}{ Height } & \multicolumn{1}{c|}{ Height\% } & \multicolumn{1}{c|}{ Area } & \multicolumn{1}{c|}{ Area\% } \\
\hline 1 & 27.295 & 455 & 0.257 & 54383 & 0.154 \\
\hline 2 & 46.016 & 176749 & 99.743 & 35209112 & 99.846 \\
\hline Total & & 177204 & 100.000 & 35263495 & 100.000
\end{tabular}

(S)-2-(2-hydroxyphenylsulfonimidoyl)phenyl 2-bromobenzenesulfonate (7n) 


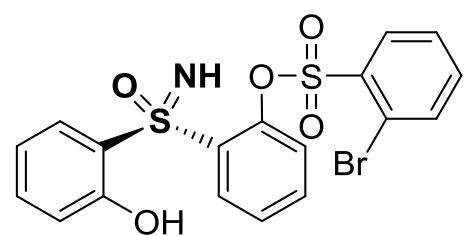

White solid; $36 \mathrm{mg}, 76 \%$ yield; m.p. $=107-109{ }^{\circ} \mathrm{C} ;{ }^{1} \mathrm{H}$ NMR $\left(400 \mathrm{MHz}, \mathrm{CDCl}_{3}\right) \delta$ $12.14(\mathrm{~s}, 1 \mathrm{H}), 8.15-8.11(\mathrm{~m}, 1 \mathrm{H}), 8.09(\mathrm{dd}, J=8.0,1.8 \mathrm{~Hz}, 1 \mathrm{H}), 7.86-7.80(\mathrm{~m}, 1 \mathrm{H})$, 7.61-7.52 (m, 3H), $7.46(\mathrm{dd}, J=8.4,1.2 \mathrm{~Hz}, 1 \mathrm{H}), 7.42(\mathrm{dd}, J=7.8,1.0 \mathrm{~Hz}, 1 \mathrm{H}), 7.39$ $(\mathrm{dd}, J=3.0,1.4 \mathrm{~Hz}, 1 \mathrm{H}), 7.38-7.32(\mathrm{~m}, 1 \mathrm{H}), 6.80(\mathrm{ddd}, J=8.4,7.4,1.2 \mathrm{~Hz}, 1 \mathrm{H}), 6.73$ $(\mathrm{dd}, J=8.4,1.0 \mathrm{~Hz}, 1 \mathrm{H}), 3.99(\mathrm{~s}, 1 \mathrm{H}) ;{ }^{13} \mathrm{C} \mathrm{NMR}\left(100 \mathrm{MHz}, \mathrm{CDCl}_{3}\right) \delta$ 157.7, 146.8, $136.8,136.5,135.9,135.7,135.6,134.8,132.0,130.2,129.3,127.9,126.9,123.0$, 121.6, 119.9, 119.4, 119.3; HRMS (ESI) $m / z$ calcd for $\mathrm{C}_{18} \mathrm{H}_{14} \mathrm{BrNO}_{5} \mathrm{~S}_{2}[\mathrm{M}+\mathrm{H}]^{+}=$ 467.9575 , found $=467.9559$.

Optical Rotation: $[\alpha]_{\mathrm{D}}^{25}=45.68\left(c=0.50, \mathrm{CHCl}_{3}\right) .>99 \%$ ee (HPLC condition: Chiralpak ID column, $n$-Hexane $/ i-\mathrm{PrOH}=70: 30$, flow rate $=1.0 \mathrm{~mL} / \mathrm{min}$, wavelength $=254 \mathrm{~nm}, t_{\mathrm{R}}=29.38 \mathrm{~min}$ for minor isomer, $t_{\mathrm{R}}=46.01 \mathrm{~min}$ for major isomer).

$\mathrm{mV}$

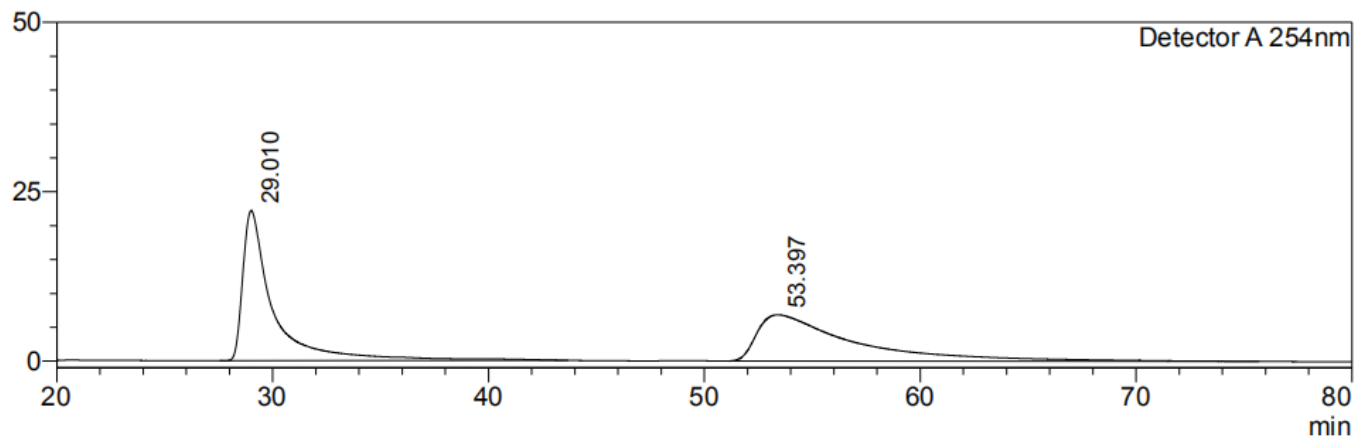

Detector A 254nm

\begin{tabular}{|r|r|r|r|r|r|}
\hline Peak\# & Ret. Time & Height & Height\% & Area & \multicolumn{1}{c|}{ Area\% } \\
\hline 1 & 29.010 & 22133 & 76.446 & 2182541 & 50.774 \\
\hline 2 & 53.397 & 6820 & 23.554 & 2115980 & 49.226 \\
\hline Total & & 28953 & 100.000 & 4298521 & 100.000 \\
\hline
\end{tabular}




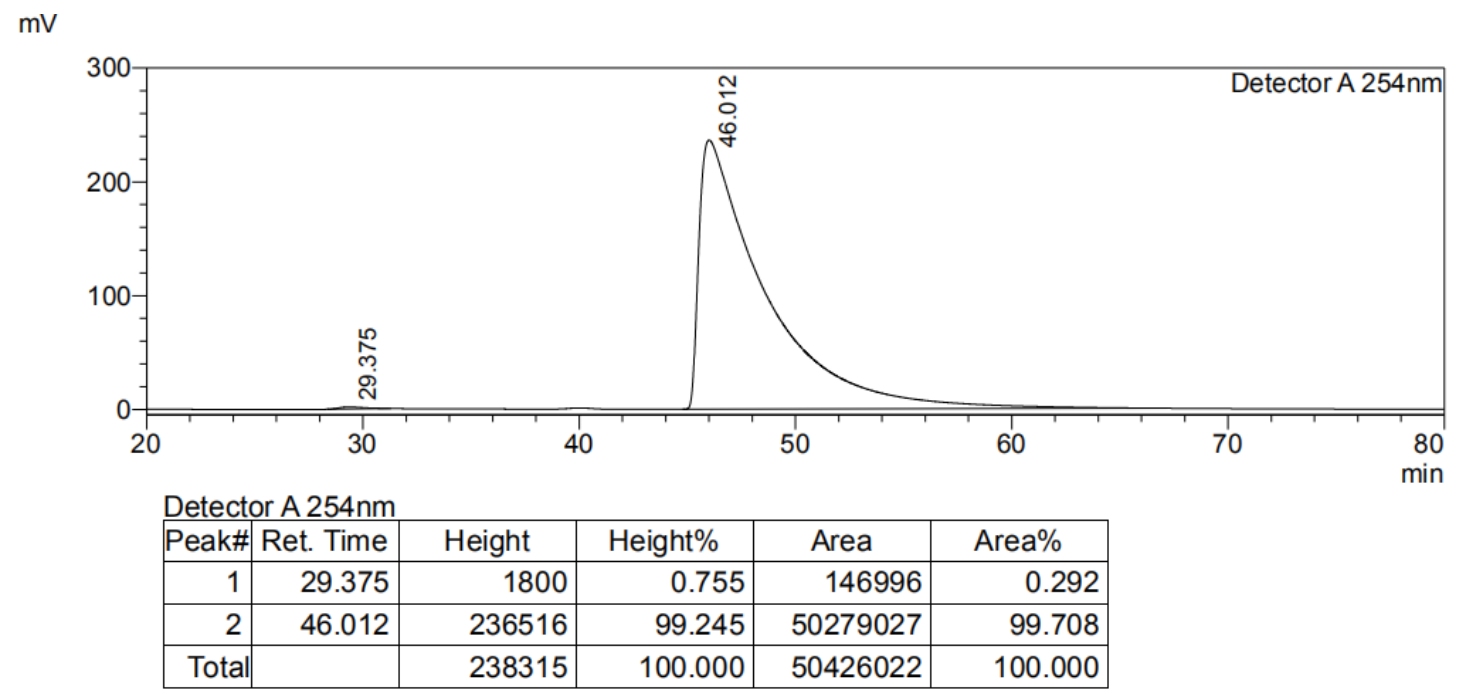

(S)-2-(2-hydroxyphenylsulfonimidoyl)phenyl 2,5-dimethylbenzenesulfonate (7o)<smiles>Cc1ccc(C)c(S(=O)(=O)Oc2ccccc2S(=O)(=O)c2ccccc2O)c1</smiles>

White solid; $33 \mathrm{mg}, 80 \%$ yield; m.p. $=95-97{ }^{\circ} \mathrm{C} ;{ }^{1} \mathrm{H} \mathrm{NMR}\left(400 \mathrm{MHz}, \mathrm{CDCl}_{3}\right) \delta 12.20$ (s, 1H), $8.07(\mathrm{dd}, J=8.0,1.8 \mathrm{~Hz}, 1 \mathrm{H}), 7.76(\mathrm{~s}, 1 \mathrm{H}), 7.52(\mathrm{ddd}, J=8.2,7.6,1.8 \mathrm{~Hz}$, $1 \mathrm{H}), 7.42(\mathrm{dd}, J=8.0,1.2 \mathrm{~Hz}, 1 \mathrm{H}), 7.40-7.32(\mathrm{~m}, 3 \mathrm{H}), 7.29(\mathrm{dd}, J=8.2,1.2 \mathrm{~Hz}, 2 \mathrm{H})$, 6.82-6.73 (m, 2H), $3.97(\mathrm{~s}, 1 \mathrm{H}), 2.56(\mathrm{~s}, 3 \mathrm{H}), 2.41(\mathrm{~s}, 3 \mathrm{H}) ;{ }^{13} \mathrm{C}$ NMR (100 MHz, $\left.\mathrm{CDCl}_{3}\right) \delta 157.8,147.1,136.8,136.6,136.1,135.6,135.5,134.8,134.8,132.9,130.1$, 130.0, 129.3, 126.6, 122.6, 120.1, 119.3, 21.0, 20.0; HRMS (ESI) $\mathrm{m} / z$ calcd for $\mathrm{C}_{20} \mathrm{H}_{19} \mathrm{NO}_{5} \mathrm{~S}_{2}[\mathrm{M}+\mathrm{H}]^{+}=418.0783$, found $=418.0766$.

Optical Rotation: $[\alpha]_{\mathrm{D}}^{25}=92.86\left(c=0.70, \mathrm{CHCl}_{3}\right) .99 \%$ ee (HPLC condition: Chiralpak AD-H column, $n-\mathrm{Hexane} / i-\mathrm{PrOH}=80: 20$, flow rate $=0.8 \mathrm{~mL} / \mathrm{min}$, wavelength $=254 \mathrm{~nm}, t_{\mathrm{R}}=19.92 \min$ for major isomer, $t_{\mathrm{R}}=21.28 \min$ for minor isomer). 
$\mathrm{mV}$

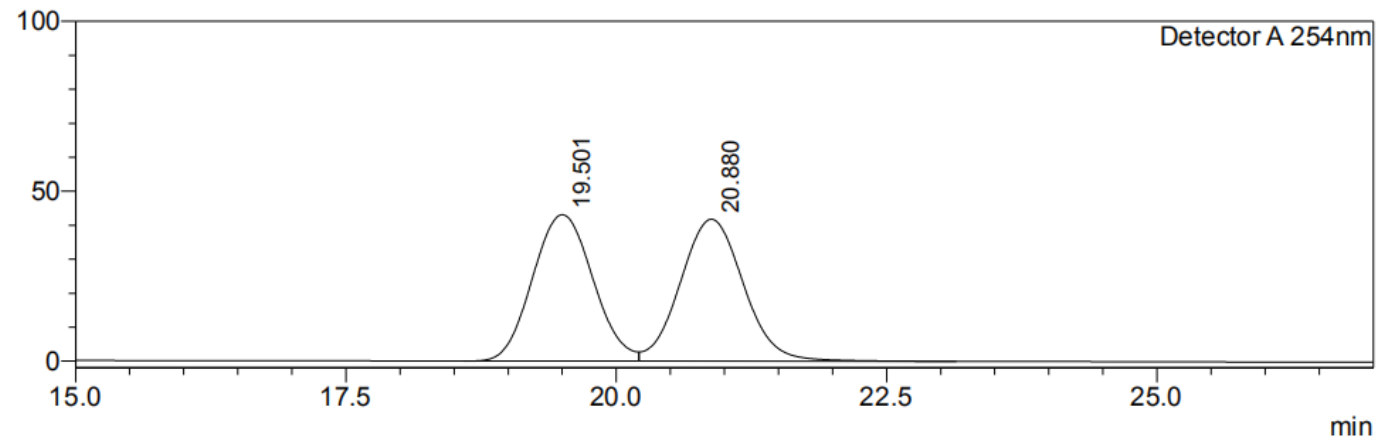

Detector A 254nm

\begin{tabular}{|r|r|r|r|r|r|}
\hline Peak\# & Ret. Time & Height & Height\% & \multicolumn{1}{c|}{ Area } & \multicolumn{1}{c|}{ Area $\%$} \\
\hline 1 & 19.501 & 43103 & 50.780 & 1673396 & 49.591 \\
\hline 2 & 20.880 & 41780 & 49.220 & 1700988 & 50.409 \\
\hline Total & & 84883 & 100.000 & 3374385 & 100.000 \\
\hline
\end{tabular}

$\mathrm{mV}$

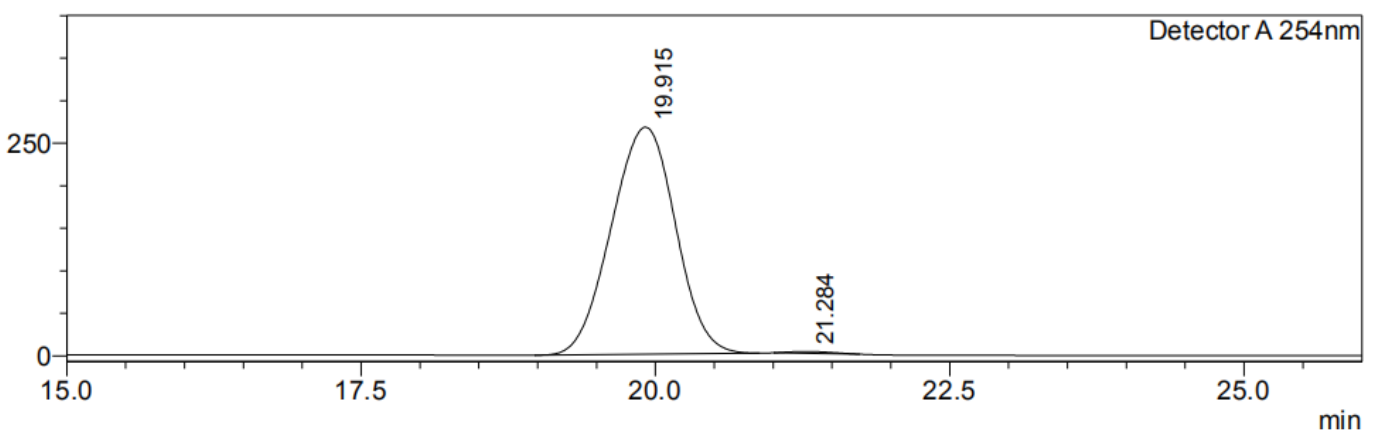

Detector A 254nm

\begin{tabular}{|r|r|r|r|r|r|}
\hline Peak\# & Ret. Time & \multicolumn{1}{c|}{ Height } & Height\% & \multicolumn{1}{c|}{ Area } & \multicolumn{1}{c|}{ Area\% } \\
\hline 1 & 19.915 & 266889 & 99.235 & 10135195 & 99.482 \\
\hline 2 & 21.284 & 2056 & 0.765 & 52760 & 0.518 \\
\hline Total & & 268945 & 100.000 & 10187955 & 100.000 \\
\hline
\end{tabular}

(S)-2-(2-hydroxyphenylsulfonimidoyl)phenyl 3,4-dimethylbenzenesulfonate (7p)<smiles>Cc1ccc(S(=O)(=O)Oc2ccccc2S(N)(=O)=O)cc1C</smiles>

White solid; $34 \mathrm{mg}, 82 \%$ yield; m.p. $=113-115{ }^{\circ} \mathrm{C} ;{ }^{1} \mathrm{H}$ NMR $\left(400 \mathrm{MHz}, \mathrm{CDCl}_{3}\right) \delta$ $7.98(\mathrm{dd}, J=8.0,1.6 \mathrm{~Hz}, 1 \mathrm{H}), 7.74(\mathrm{~s}, 1 \mathrm{H}), 7.68(\mathrm{dd}, J=8.0,2.0 \mathrm{~Hz}, 1 \mathrm{H}), 7.56-7.50$ (m, 1H), $7.46(\mathrm{dd}, J=8.4,1.2 \mathrm{~Hz}, 1 \mathrm{H}), 7.41$ (ddd, $J=8.4,5.6,1.6 \mathrm{~Hz}, 2 \mathrm{H}), 7.38-7.34$ (m, 1H), 7.34-7.29 (m, 1H), 6.89 (dd, $J=8.4,1.0 \mathrm{~Hz}, 1 \mathrm{H}), 6.84-6.72(\mathrm{~m}, 1 \mathrm{H}), 2.37$ (s, 3H), 2.35 (s, 3H); ${ }^{13} \mathrm{C}$ NMR (100 MHz, $\left.\mathrm{CDCl}_{3}\right) \delta 157.8,147.0,145.0,138.4,136.8$, $135.8,134.6,132.8,130.5,129.9,129.6,129.5,126.7,126.3,122.8,120.1,119.4$, 
119.3, 20.4, 19.9; HRMS (ESI) $m / z$ calcd for $\mathrm{C}_{20} \mathrm{H}_{19} \mathrm{NO}_{5} \mathrm{~S}_{2}[\mathrm{M}+\mathrm{H}]^{+}=418.0783$, found $=418.0760$.

Optical Rotation: $[\alpha]_{\mathrm{D}}^{25}=60.00\left(c=0.66, \mathrm{CHCl}_{3}\right) .87 \%$ ee (HPLC condition: Chiralpak IG column, $n$-Hexane $/ i-\mathrm{PrOH}=70: 30$, flow rate $=1.0 \mathrm{~mL} / \mathrm{min}$, wavelength $=254 \mathrm{~nm}, t_{\mathrm{R}}=29.19 \min$ for minor isomer, $t_{\mathrm{R}}=39.50 \mathrm{~min}$ for major isomer).

$\mathrm{mV}$

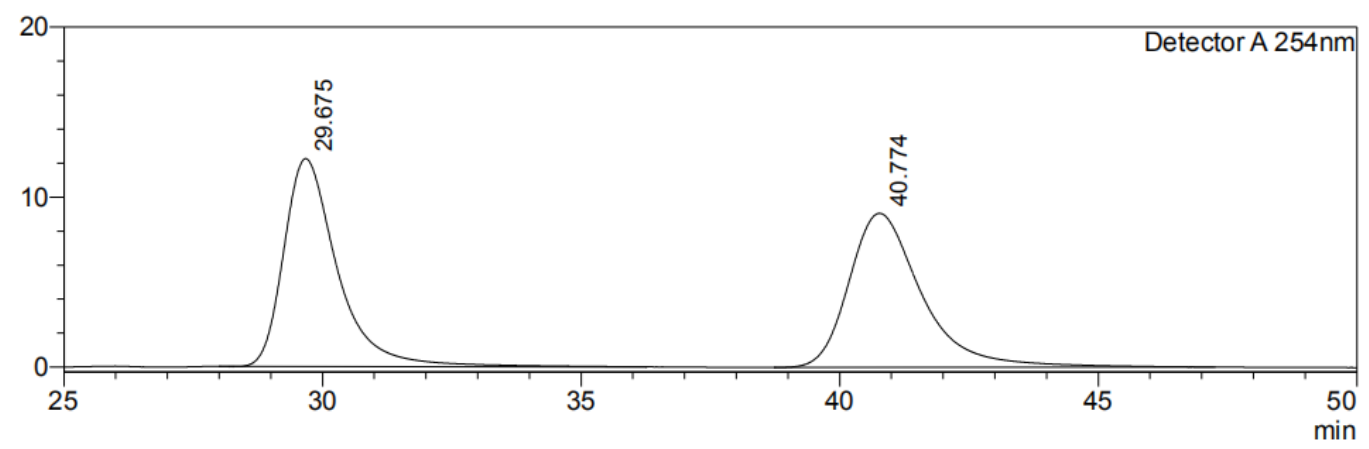

Detector A 254nm

\begin{tabular}{|r|r|r|r|r|r|}
\hline Peak\# & Ret. Time & \multicolumn{1}{c|}{ Height } & Height\% & \multicolumn{1}{c|}{ Area } & \multicolumn{1}{c|}{ Area\% } \\
\hline 1 & 29.675 & 12228 & 57.433 & 885914 & 49.893 \\
\hline 2 & 40.774 & 9063 & 42.567 & 889730 & 50.107 \\
\hline Total & & 21291 & 100.000 & 1775643 & 100.000 \\
\hline
\end{tabular}

$\mathrm{mV}$

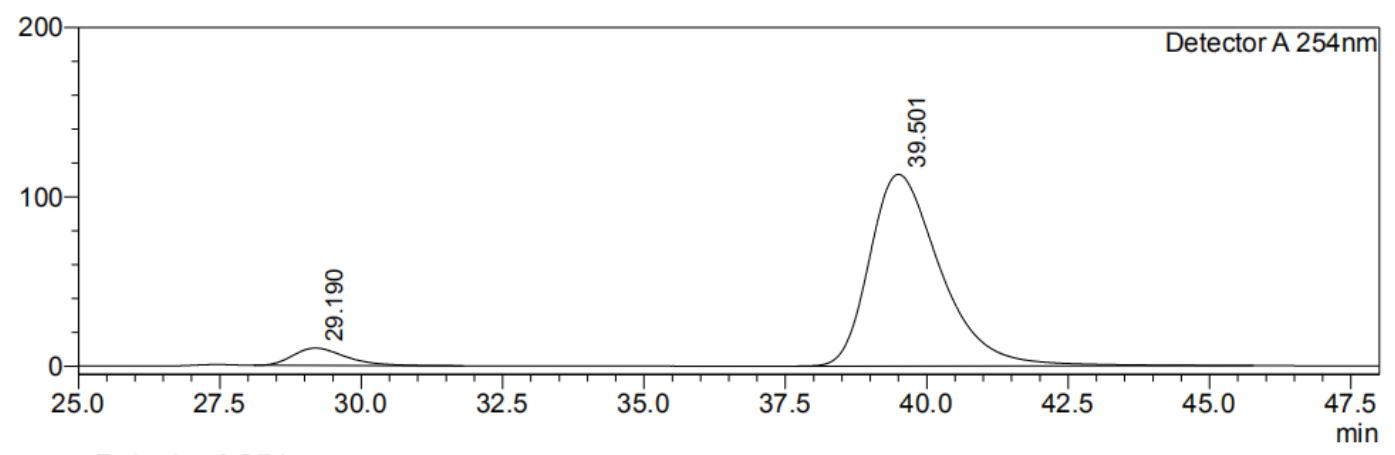

Detector A 254nm

\begin{tabular}{|r|r|r|r|r|r|}
\hline Peak\# & Ret. Time & \multicolumn{1}{c|}{ Height } & \multicolumn{1}{c|}{ Height\% } & \multicolumn{1}{c|}{ Area } & \multicolumn{1}{c|}{ Area\% } \\
\hline 1 & 29.190 & 10283 & 8.324 & 669375 & 6.337 \\
\hline 2 & 39.501 & 113251 & 91.676 & 9893136 & 93.663 \\
\hline Total & & 123535 & 100.000 & 10562511 & 100.000 \\
\hline
\end{tabular}

(S)-2-(2-hydroxyphenylsulfonimidoyl)phenyl naphthalene-1-sulfonate (7q)<smiles>NS(=O)(=O)c1ccccc1OS(=O)(=O)c1cccc2ccccc12</smiles> 
Light yellow solid; $34 \mathrm{mg}, 76 \%$ yield; m.p. $=138-140{ }^{\circ} \mathrm{C} ;{ }^{1} \mathrm{H}$ NMR $(400 \mathrm{MHz}$, $\left.\mathrm{CDCl}_{3}\right) \delta 12.16(\mathrm{~s}, 1 \mathrm{H}), 8.85-8.55(\mathrm{~m}, 1 \mathrm{H}), 8.33(\mathrm{~d}, J=7.4 \mathrm{~Hz}, 1 \mathrm{H}), 8.27(\mathrm{~d}, J=8.2$ $\mathrm{Hz}, 1 \mathrm{H}), 8.11(\mathrm{dd}, J=8.0,1.4 \mathrm{~Hz}, 1 \mathrm{H}), 8.10-7.97(\mathrm{~m}, 1 \mathrm{H}), 7.81-7.55(\mathrm{~m}, 3 \mathrm{H})$, 7.56-7.46 (m, 1H), 7.40 (t, $J=7.6 \mathrm{~Hz}, 1 \mathrm{H}), 7.33$ (dd, $J=8.2,1.4 \mathrm{~Hz}, 1 \mathrm{H}), 7.31-7.20$ $(\mathrm{m}, 2 \mathrm{H}), 6.70(\mathrm{t}, J=7.6 \mathrm{~Hz}, 1 \mathrm{H}), 6.60(\mathrm{~d}, J=8.4 \mathrm{~Hz}, 1 \mathrm{H}), 3.95(\mathrm{~s}, 1 \mathrm{H}) ;{ }^{13} \mathrm{C} \mathrm{NMR}$ $\left(100 \mathrm{MHz}, \mathrm{CDCl}_{3}\right) \delta 157.6,147.3,137.0,136.4,135.5,134.8,134.4,132.2,130.5$, 130.2 , 129.2, 129.1, 129.0, 128.8, 127.6, 126.8, 125.0, 124.2, 122.6, 120.0, 119.2, 119.1; HRMS (ESI) $\mathrm{m} / z$ calcd for $\mathrm{C}_{22} \mathrm{H}_{17} \mathrm{NO}_{5} \mathrm{~S}_{2}[\mathrm{M}+\mathrm{H}]^{+}=440.0626$, found $=$ 440.0626 .

Optical Rotation: $[\alpha]_{\mathrm{D}}^{25}=75.19\left(c=0.52, \mathrm{CHCl}_{3}\right) .98 \%$ ee (HPLC condition: Chiralpak AD-H column, $n-\mathrm{Hexane} / i-\mathrm{PrOH}=70: 30$, flow rate $=1.0 \mathrm{~mL} / \mathrm{min}$, wavelength $=254 \mathrm{~nm}, t_{\mathrm{R}}=18.15 \min$ for minor isomer, $t_{\mathrm{R}}=20.94 \min$ for major isomer).

$\mathrm{mV}$

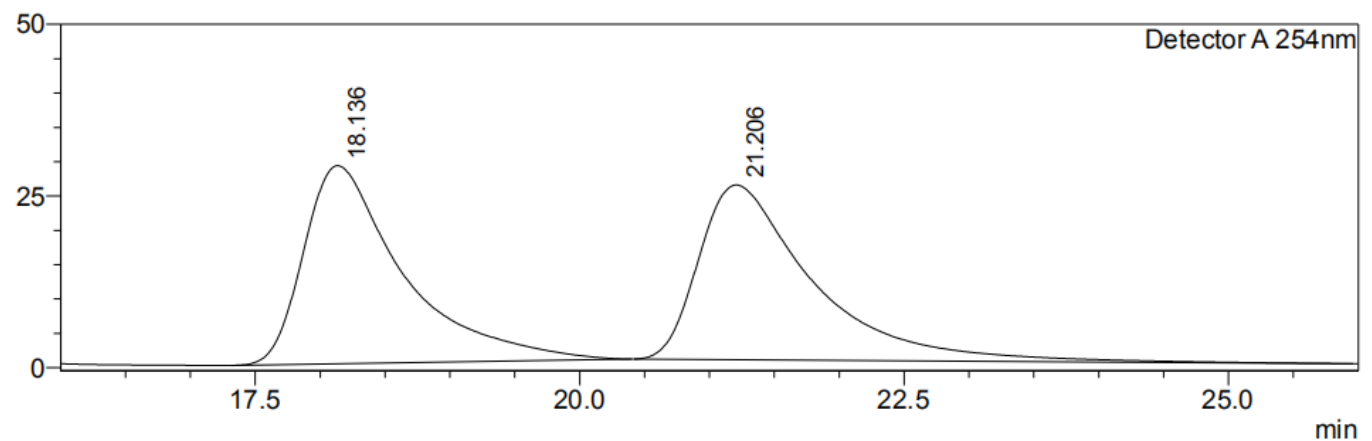

Detector A $254 \mathrm{~nm}$

\begin{tabular}{|r|r|r|r|r|r|}
\hline Peak\# & Ret. Time & Height & Height\% & Area & \multicolumn{1}{|c|}{ Area\% } \\
\hline 1 & 18.136 & 28864 & 53.132 & 1539968 & 49.662 \\
\hline 2 & 21.206 & 25461 & 46.868 & 1560949 & 50.338 \\
\hline Total & & 54326 & 100.000 & 3100918 & 100.000 \\
\hline
\end{tabular}


$\mathrm{mV}$

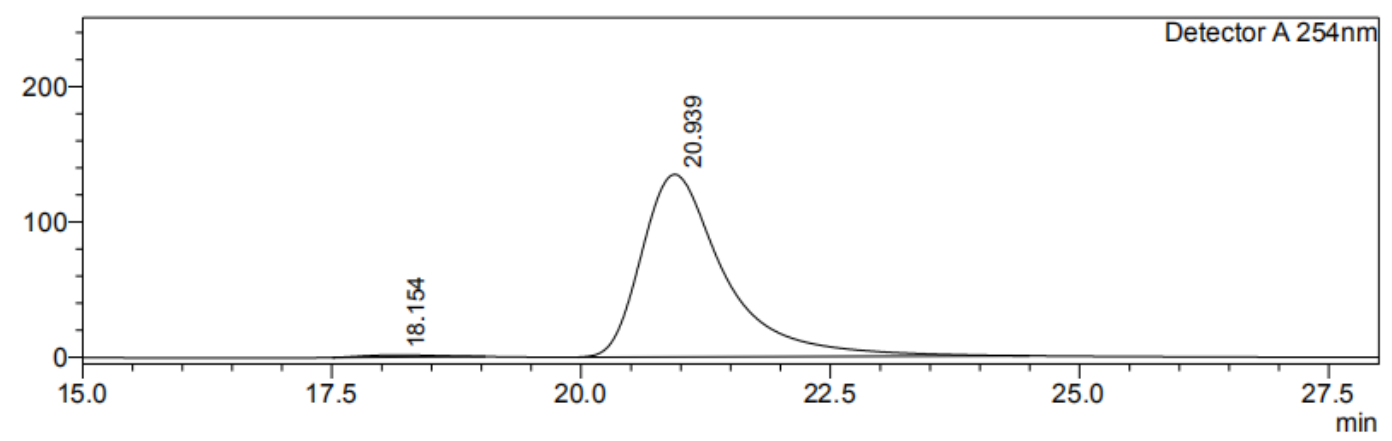

Detector A 254nm
\begin{tabular}{|r|r|r|r|r|r|}
\hline Peak\# & Ret. Time & Height & Height\% & Area & \multicolumn{1}{c|}{ Area\% } \\
\hline 1 & 18.154 & 1584 & 1.162 & 77724 & 0.981 \\
\hline 2 & 20.939 & 134807 & 98.838 & 7847104 & 99.019 \\
\hline Total & & 136391 & 100.000 & 7924828 & 100.000 \\
\hline
\end{tabular}

(S)-2-(2-hydroxyphenylsulfonimidoyl)phenyl naphthalene-2-sulfonate (7r)<smiles>NS(=O)(=O)c1ccccc1OS(=O)(=O)c1ccc2ccccc2c1</smiles>

White solid; $37 \mathrm{mg}, 84 \%$ yield; m.p. $=99-101{ }^{\circ} \mathrm{C} ;{ }^{1} \mathrm{H}$ NMR $\left(400 \mathrm{MHz}, \mathrm{CDCl}_{3}\right) \delta$ $12.21(\mathrm{~s}, 1 \mathrm{H}), 8.56(\mathrm{~d}, J=1.6 \mathrm{~Hz}, 1 \mathrm{H}), 8.05-7.99(\mathrm{~m}, 3 \mathrm{H}), 7.97$ (d, $J=8.2 \mathrm{~Hz}, 1 \mathrm{H})$, $7.93(\mathrm{dd}, J=8.6,1.9 \mathrm{~Hz}, 1 \mathrm{H}), 7.76-7.70(\mathrm{~m}, 1 \mathrm{H}), 7.70-7.64(\mathrm{~m}, 1 \mathrm{H}), 7.56-7.48(\mathrm{~m}$, 2H), $7.40(\mathrm{dd}, J=8.2,1.6 \mathrm{~Hz}, 1 \mathrm{H}), 7.39-7.33(\mathrm{~m}, 2 \mathrm{H}), 6.82-6.74(\mathrm{~m}, 2 \mathrm{H}), 3.88(\mathrm{~s}$, $1 \mathrm{H}) ;{ }^{13} \mathrm{C} \mathrm{NMR}\left(100 \mathrm{MHz}, \mathrm{CDCl}_{3}\right) \delta 157.7,147.0,136.9,135.9,135.8,134.7,132.6$, 132.0, 131.0, 130.2, 130.1, 129.8, 129.8, 129.4, 128.2, 128.2, 126.8, 123.1, 122.7, 120.1, 119.4, 119.3; HRMS (ESI) $m / z$ calcd for $\mathrm{C}_{22} \mathrm{H}_{17} \mathrm{NO}_{5} \mathrm{~S}_{2}[\mathrm{M}+\mathrm{H}]^{+}=440.0626$, found $=440.0624$.

Optical Rotation: $[\alpha]_{\mathrm{D}}^{25}=75.19\left(c=0.52, \mathrm{CHCl}_{3}\right) .98 \%$ ee (HPLC condition: Chiralpak AD-H column, $n-\mathrm{Hexane} / i-\mathrm{PrOH}=70: 30$, flow rate $=1.0 \mathrm{~mL} / \mathrm{min}$, wavelength $=254 \mathrm{~nm}, t_{\mathrm{R}}=18.15 \min$ for minor isomer, $t_{\mathrm{R}}=20.94 \min$ for major isomer). 
$\mathrm{mV}$

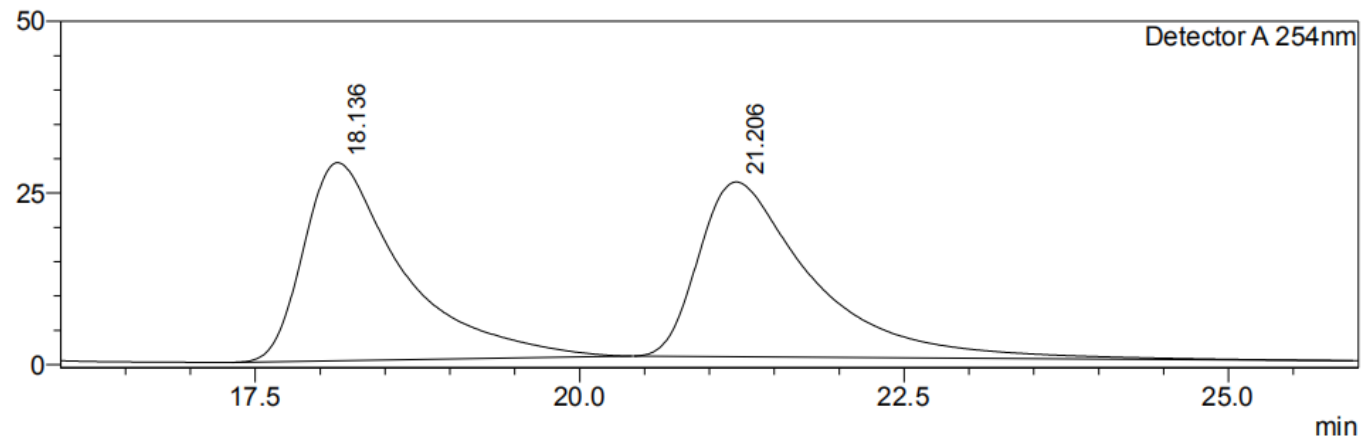

Detector A $254 \mathrm{~nm}$

\begin{tabular}{|r|r|r|r|r|r|}
\hline Peak\# & Ret. Time & Height & Height $\%$ & \multicolumn{1}{c|}{ Area } & \multicolumn{1}{c|}{ Area\% } \\
\hline 1 & 18.136 & 28864 & 53.132 & 1539968 & 49.662 \\
\hline 2 & 21.206 & 25461 & 46.868 & 1560949 & 50.338 \\
\hline Total & & 54326 & 100.000 & 3100918 & 100.000 \\
\hline
\end{tabular}

$\mathrm{mV}$

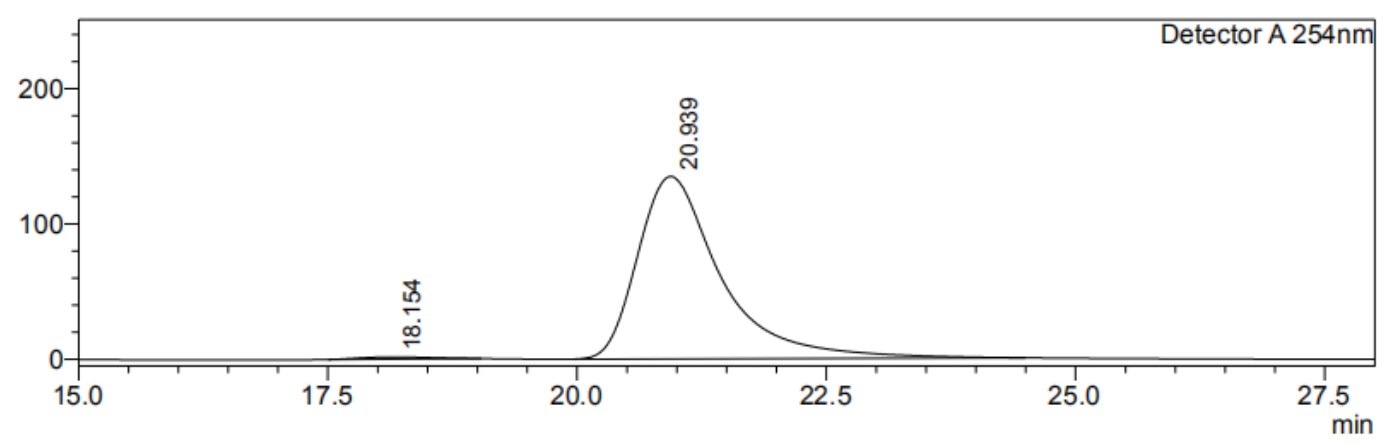

Detector A 254nm

\begin{tabular}{|r|r|r|r|r|r|}
\hline Peak\# & Ret. Time & \multicolumn{1}{c|}{ Height } & Height\% & \multicolumn{1}{c|}{ Area } & \multicolumn{1}{c|}{ Area\% } \\
\hline 1 & 18.154 & 1584 & 1.162 & 77724 & 0.981 \\
\hline 2 & 20.939 & 134807 & 98.838 & 7847104 & 99.019 \\
\hline Total & & 136391 & 100.000 & 7924828 & 100.000 \\
\hline
\end{tabular}

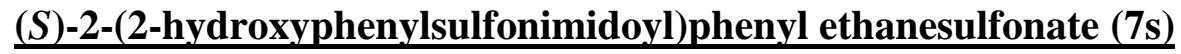<smiles>CCS(=O)(=O)Oc1ccccc1S(=O)(=O)c1ccccc1O</smiles>

Colorless oil; $28 \mathrm{mg}, 81 \%$ yield; ${ }^{1} \mathrm{H}$ NMR (400 MHz, $\left.\mathrm{CDCl}_{3}\right) \delta 12.88$ (s, 1H), 8.08 $(\mathrm{dd}, J=8.0,1.7 \mathrm{~Hz}, 1 \mathrm{H}), 7.66(\mathrm{dd}, J=8.4,1.2 \mathrm{~Hz}, 1 \mathrm{H}), 7.63-7.57(\mathrm{~m}, 1 \mathrm{H}), 7.47-7.38$ (m, 3H), $6.98(\mathrm{dd}, J=8.8,1.2 \mathrm{~Hz}, 1 \mathrm{H}), 6.91-6.85(\mathrm{~m}, 1 \mathrm{H}), 4.12(\mathrm{~s}, 1 \mathrm{H}), 3.61-3.42(\mathrm{~m}$, 2H), $1.50(\mathrm{t}, J=7.4 \mathrm{~Hz}, 3 \mathrm{H}) ;{ }^{13} \mathrm{C} \mathrm{NMR}\left(100 \mathrm{MHz}, \mathrm{CDCl}_{3}\right) \delta 157.7,146.2,136.3$, 136.0, 135.0, 130.2, 129.4, 126.8, 123.5, 120.1, 119.9, 119.3, 47.2, 8.2; HRMS (ESI) $m / z$ calcd for $\mathrm{C}_{14} \mathrm{H}_{15} \mathrm{NO}_{5} \mathrm{~S}_{2}[\mathrm{M}+\mathrm{H}]^{+}=342.0470$, found $=342.0455$. 
Optical Rotation: $[\alpha]_{\mathrm{D}}^{25}=74.00\left(c=0.10, \mathrm{CHCl}_{3}\right) .92 \%$ ee (HPLC condition: Chiralpak IE column, $n$-Hexane $/ i-\mathrm{PrOH}=70: 30$, flow rate $=1.0 \mathrm{~mL} / \mathrm{min}$, wavelength $=254 \mathrm{~nm}, t_{\mathrm{R}}=16.90 \mathrm{~min}$ for minor isomer, $t_{\mathrm{R}}=28.04 \mathrm{~min}$ for major isomer).

$\mathrm{mV}$

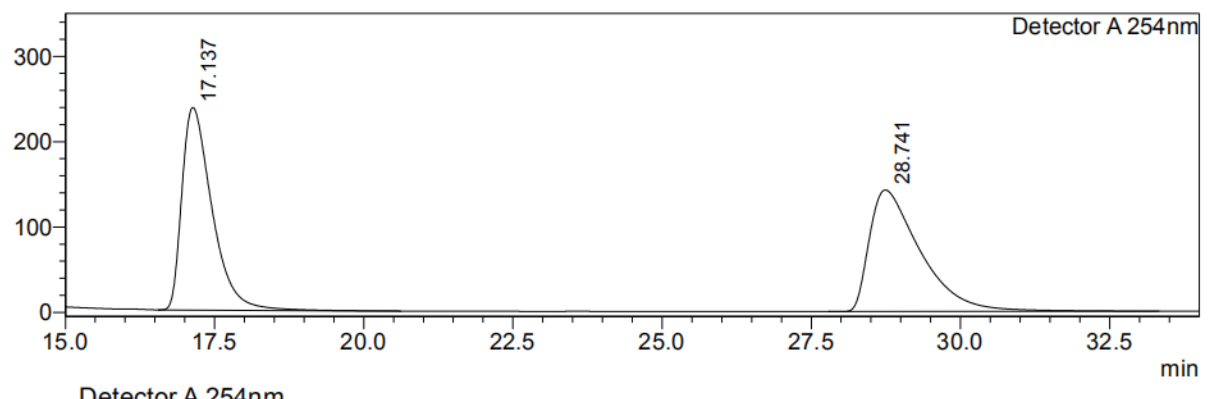

Detector A 254nm

\begin{tabular}{|r|r|r|r|r|r|}
\hline Peak\# & Ret. Time & \multicolumn{1}{c|}{ Height } & Height $\%$ & \multicolumn{1}{c|}{ Area } & Area\% \\
\hline 1 & 17.137 & 237398 & 62.488 & 8355605 & 49.520 \\
\hline 2 & 28.741 & 142514 & 37.512 & 8517638 & 50.480 \\
\hline Total & & 379912 & 100.000 & 16873243 & 100.000 \\
\hline
\end{tabular}

$\mathrm{mV}$

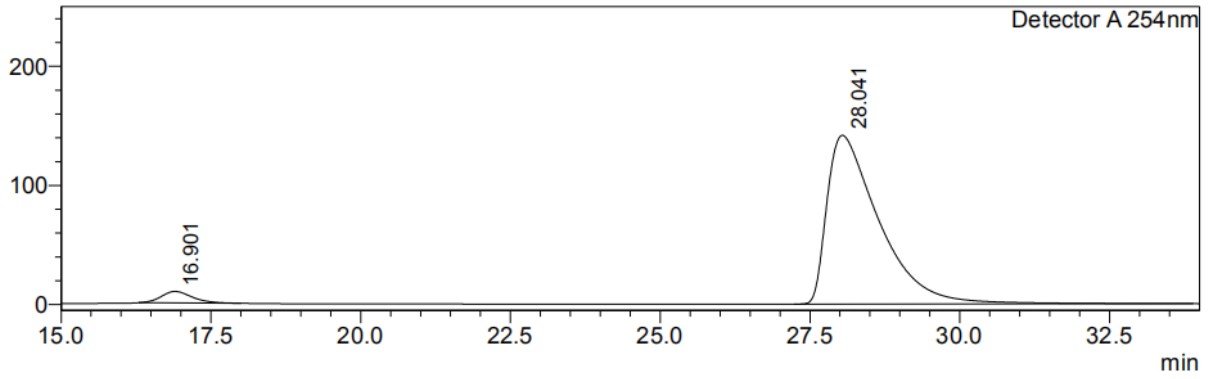

Detector A 254nm
\begin{tabular}{|r|r|r|r|r|r|}
\hline Peak\# & Ret. Time & Height & Height $\%$ & \multicolumn{1}{c|}{ Area } & \multicolumn{1}{c|}{ Area\% } \\
\hline 1 & 16.901 & 9620 & 6.360 & 346025 & 3.864 \\
\hline 2 & 28.041 & 141651 & 93.640 & 8609095 & 96.136 \\
\hline Total & & 151271 & 100.000 & 8955120 & 100.000 \\
\hline
\end{tabular}




\section{Synthetic Applications of the Desymmetrizing Products}

\section{A. Divergent Synthesis of Both Stereoisomers of 3a/6a}<smiles>N=S(=O)(c1ccccc1O)c1ccccc1O</smiles>

$1 \mathrm{a}$

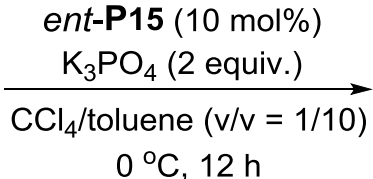

$0{ }^{\circ} \mathrm{C}, 12 \mathrm{~h}$<smiles>N=S(=O)(c1ccccc1OP)c1ccccc1OP</smiles>

$(R)-3 a$

To a tube $(10 \mathrm{~mL})$ with a magnetic stirring bar were added $\mathbf{1 a}(24.9 \mathrm{mg}, 0.1 \mathrm{mmol})$, 2a (24.2, $0.12 \mathrm{mmol})$, phosphonium salt ent-P15 (12.0 $\mathrm{mg}, 0.01 \mathrm{mmol})$ and potassium phosphate $(42.4 \mathrm{mg}, 0.2 \mathrm{mmol})$, followed by the addition of $\mathrm{CCl}_{4} /$ toluene $(2.0 \mathrm{~mL}, \mathrm{v} / \mathrm{v}=1 / 10)$. The reaction mixture was stirred at $0{ }^{\circ} \mathrm{C}$ for $12 \mathrm{~h}$. Until completion, the reaction mixture was transfered to room temperature, and diluted with dichloromethane. Purification by column chromatography on silica gel (petroleum ether/ethyl acetate $=3: 1$ to $1: 1)$ afforded the prodcut $(R)-3 \mathbf{a}(83 \%$ yield, 94\% ee). The enantiomeric excess was determined by HPLC (Chiralpak AD-H column, $n$-Hexane $/ i-\mathrm{PrOH}=70: 30$, flow rate $=1.0 \mathrm{~mL} / \mathrm{min}$, wavelength $=254 \mathrm{~nm}, t_{\mathrm{R}}$ $=23.13$ (minor), $t_{\mathrm{R}}=29.03$ (major)).

$\mathrm{mV}$

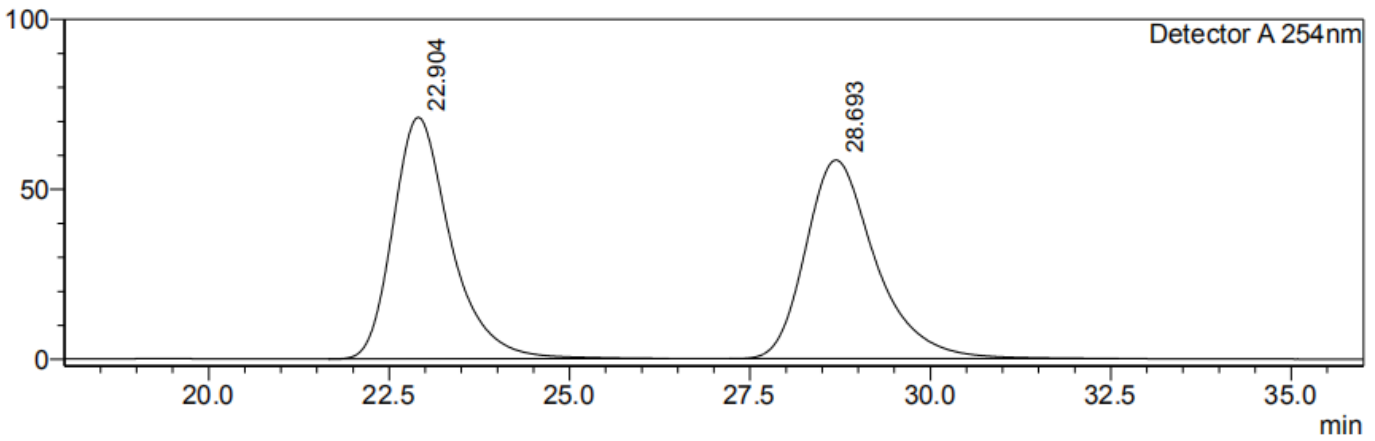

\begin{tabular}{|c|c|c|c|c|c|}
\hline Peak\# & Ret. Time & Height & Height $\%$ & Area & Area\% \\
\hline 1 & 22.904 & 71046 & 54.874 & 3962141 & 50.048 \\
\hline 2 & 28.693 & 58425 & 45.126 & 3954560 & 49.952 \\
\hline Total & & 129471 & 100.000 & 7916700 & 100.000 \\
\hline
\end{tabular}


$\mathrm{mV}$

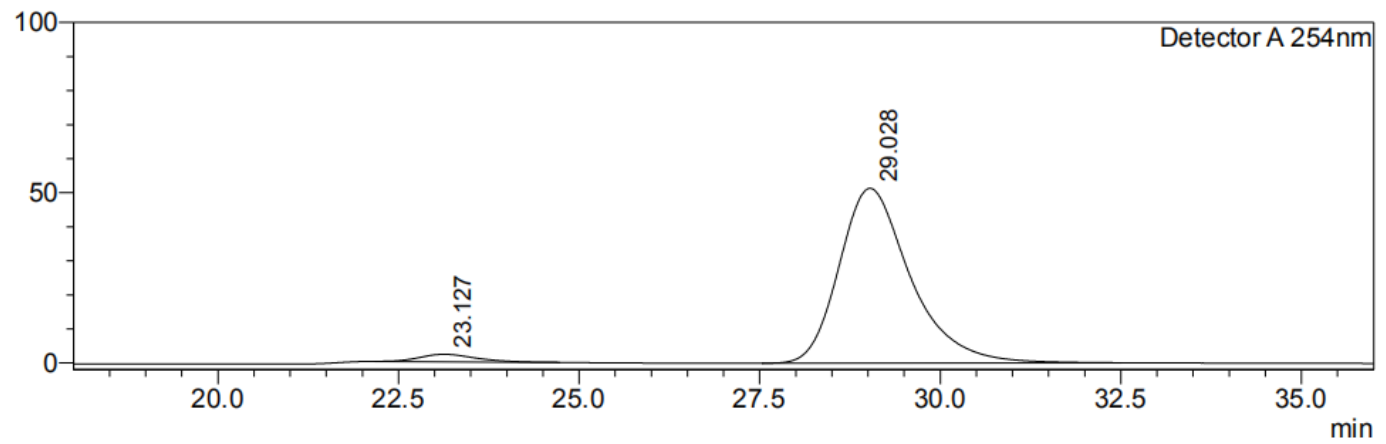

Detector A 254nm

\begin{tabular}{|r|r|r|r|r|r|}
\hline Peak\# & Ret. Time & \multicolumn{1}{c|}{ Height } & \multicolumn{1}{c|}{ Height\% } & \multicolumn{1}{c|}{ Area } & \multicolumn{1}{c|}{ Area\% } \\
\hline 1 & 23.127 & 2185 & 4.084 & 117105 & 3.210 \\
\hline 2 & 29.028 & 51329 & 95.916 & 3531509 & 96.790 \\
\hline Total & & 53514 & 100.000 & 3648613 & 100.000 \\
\hline
\end{tabular}<smiles>N=S(=O)(c1ccccc1O)c1ccccc1O</smiles>

$1 \mathrm{a}$<smiles>Cc1ccc(S(=O)(=O)Cl)cc1</smiles>

$5 a$

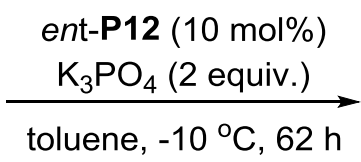

toluene, $-10^{\circ} \mathrm{C}, 62 \mathrm{~h}$<smiles>NS(=O)(=O)c1ccccc1[O-]</smiles>

$(R)-6 \mathbf{a}$

To a tube $(10 \mathrm{~mL})$ with a magnetic stirring bar were added $\mathbf{1 a}(24.9 \mathrm{mg}, 0.1 \mathrm{mmol})$, 5a (21.0 mg, $0.11 \mathrm{mmol})$, phosphonium salt ent-P12 $(9.7 \mathrm{mg}, 0.01 \mathrm{mmol})$ and potassium phosphate $(42.4 \mathrm{mg}, 0.2 \mathrm{mmol})$, followed by the addition of toluene (2.0 $\mathrm{mL})$. The reaction mixture was stirred at $-10{ }^{\circ} \mathrm{C}$ for $62 \mathrm{~h}$. Until completion, the reaction mixture was transfered to room temperature, and diluted with dichloromethane. Purification by column chromatography on silica gel (petroleum ether/ethyl acetate $=5: 1$ to $3: 1)$ afforded the prodcut $(R)-6 \mathbf{a}(80 \%$ yield, $>99 \%$ ee $)$. The enantiomeric excess was determined by HPLC (Chiralpak AD-H column, $n-\mathrm{Hexane} / \mathrm{i}-\mathrm{PrOH}=70: 30$, flow rate $=1.0 \mathrm{~mL} / \mathrm{min}$, wavelength $=254 \mathrm{~nm}, t_{\mathrm{R}}=18.90$ (major), $t_{\mathrm{R}}=21.68$ (minor)). 
$\mathrm{mV}$

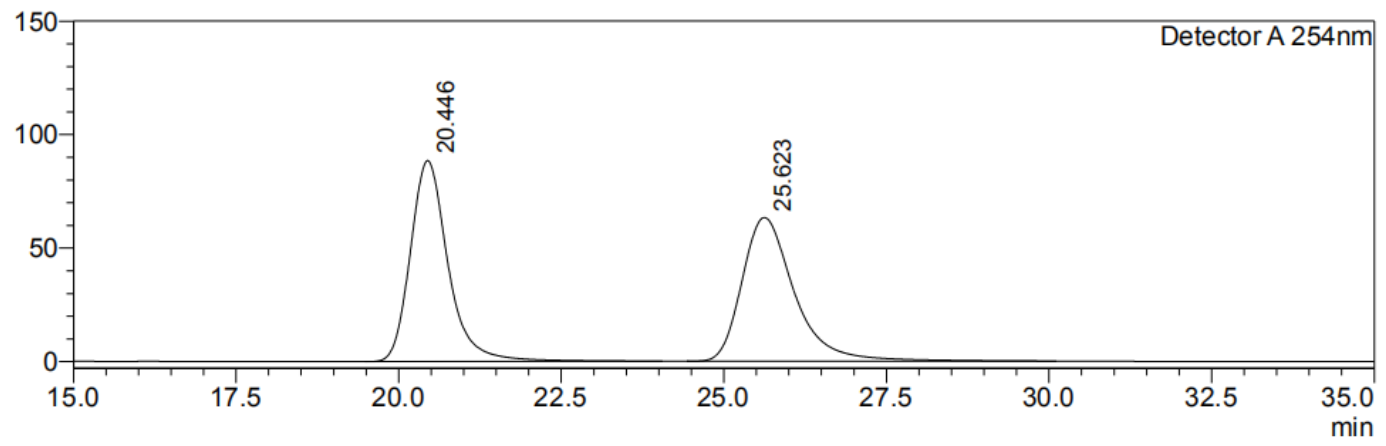

Detector A 254nm

\begin{tabular}{|r|r|r|r|r|r|}
\hline Peak\# & Ret. Time & \multicolumn{1}{c|}{ Height } & Height\% & Area & \multicolumn{1}{c|}{ Area\% } \\
\hline 1 & 20.446 & 88505 & 58.307 & 3575309 & 50.150 \\
\hline 2 & 25.623 & 63286 & 41.693 & 3553855 & 49.850 \\
\hline Total & & 151792 & 100.000 & 7129164 & 100.000 \\
\hline
\end{tabular}

$\mathrm{mV}$

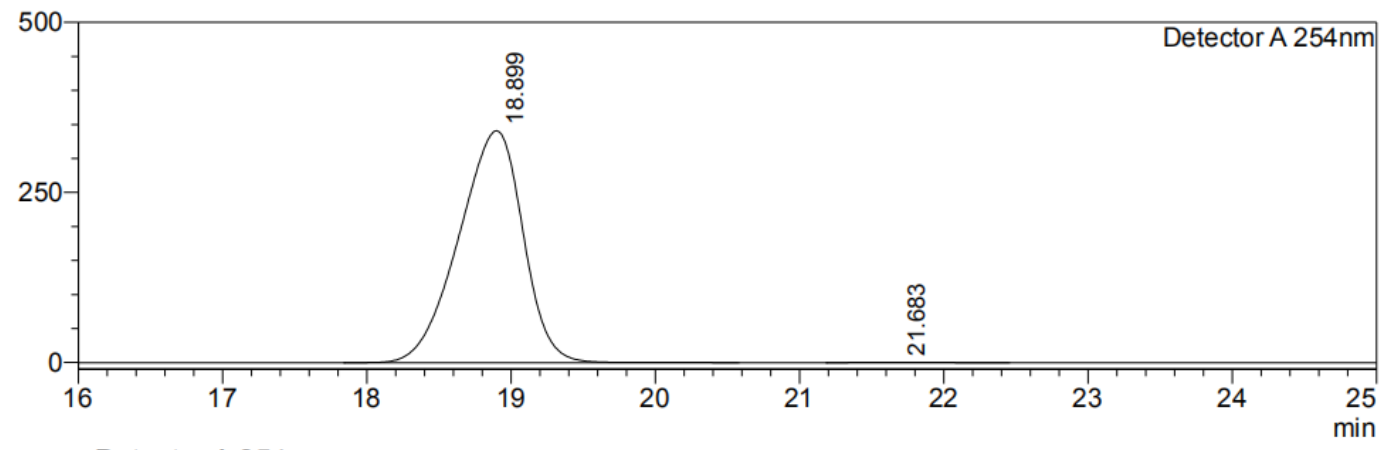

Detector A 254nm

\begin{tabular}{|r|r|r|r|r|r|}
\hline Peak\# & Ret. Time & \multicolumn{1}{c|}{ Height } & Height\% & \multicolumn{1}{c|}{ Area } & \multicolumn{1}{c|}{ Area\% } \\
\hline 1 & 18.899 & 340919 & 99.911 & 10553890 & 99.908 \\
\hline 2 & 21.683 & 304 & 0.089 & 9764 & 0.092 \\
\hline Total & & 341223 & 100.000 & 10563654 & 100.000 \\
\hline
\end{tabular}

\section{B. Scaled-up preparation}<smiles>N=S(=O)(c1ccccc1O)c1ccccc1O</smiles>

$1 \mathrm{a}$<smiles>Cc1ccc(S(=O)(=O)Cl)cc1</smiles>

$5 a$

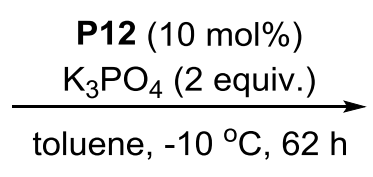

toluene, $-10^{\circ} \mathrm{C}, 62 \mathrm{~h}$<smiles>N[SH](=O)(c1ccccc1[O-])c1ccccc1O</smiles>

$6 a$

To a flame-dried round bottle flask with a magnetic stirring bar were added $\mathbf{1 a}(1.0 \mathrm{~g}$, $4.0 \mathrm{mmol}), 5 \mathbf{a}(845 \mathrm{mg}, 4.4 \mathrm{mmol}), \mathbf{P 1 2}(391 \mathrm{mg}, 0.4 \mathrm{mmol})$ and $\mathrm{K}_{3} \mathrm{PO}_{4}(1.7 \mathrm{~g}, 8.0$ $\mathrm{mmol})$, followed by the addition of toluene $(40 \mathrm{~mL})$. The reaction mixture was stirred at $-10{ }^{\circ} \mathrm{C}$ for $62 \mathrm{~h}$. Until completion, $1 \mathrm{M}$ hydrochloric acid solution (3 mL) was added. Then, the mixture was extracted with dichloromethane $(10 \mathrm{~mL}$ x 3$)$ and water $(10 \mathrm{~mL})$. The solvent was removed under reduced pressure, and the residue was 
purified by column chromatography on silica gel (petroleum ether/ethyl acetate $=5: 1$ to $3: 1)$ to afford the prodcut $\mathbf{6 a}(1.32 \mathrm{~g}, 82 \%$ yield, $98 \%$ ee $)$ as the light yellow solid.

\section{Derivatization of the chiral products}<smiles>NS(=O)(=O)c1ccccc1Cl</smiles>

$6 a, 98 \%$ ee

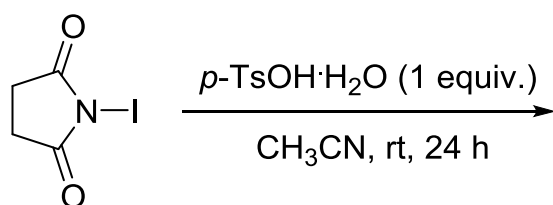

$\mathrm{O}$<smiles>O=S(=O)(Nc1ccccc1[N+](F)(F)F)c1cc(I)cc(I)c1O</smiles>

$8,98 \%$ ee

Path A: to the mixture of compound 6a $(40.3 \mathrm{mg}, 0.1 \mathrm{mmol})$ and $p$-toluenesulfonic acid monohydrate $(38.0 \mathrm{mg}, 0.2 \mathrm{mmol})$ was added $N$-iodosuccinimide $(45.0 \mathrm{mg}, 0.2$ mmol) in acetonitrile $(1 \mathrm{~mL})$. Then, the reaction mixture was stirred at $\mathrm{rt}$ for $24 \mathrm{~h}$. After the completion of the reaction which was indicated by MS, the reaction mixture was purified through column chromatography on silica gel (petroleum ether/ethyl acetate $=5: 1$ to $3: 1)$ to afford the prodcut $\mathbf{8}(62 \mathrm{mg}, 92 \%$ yield $)$ as the yellow oil.

\section{(S)-2-(2-hydroxy-3,5-diiodophenylsulfonimidoyl)phenyl}

\section{4-methylbenzenesulfonate (8)}<smiles>NS(=O)(=O)c1ccccc1[N+](=O)[O-]</smiles>

${ }^{1} \mathrm{H}$ NMR (400 MHz, $\left.\mathrm{CDCl}_{3}\right) \delta 8.10(\mathrm{~d}, J=2.0 \mathrm{~Hz}, 1 \mathrm{H}), 8.05(\mathrm{~d}, J=8.4 \mathrm{~Hz}, 1 \mathrm{H}), 7.87$ $(\mathrm{d}, J=8.2 \mathrm{~Hz}, 2 \mathrm{H}), 7.68-7.53(\mathrm{~m}, 3 \mathrm{H}), 7.46-7.37(\mathrm{~m}, 3 \mathrm{H}), 2.50(\mathrm{~s}, 3 \mathrm{H}) ;{ }^{13} \mathrm{C} \mathrm{NMR}$ $\left(100 \mathrm{MHz}, \mathrm{CDCl}_{3}\right) \delta 156.1,152.3,147.0,146.5,137.3,135.6,135.3,132.4,130.4$, 128.8, 126.9, 123.1, 121.9, 89.0, 79.9, 22.1; HRMS (ESI) $\mathrm{m} / \mathrm{z}$ calcd for $\mathrm{C}_{19} \mathrm{H}_{15} \mathrm{I}_{2} \mathrm{NO}_{5} \mathrm{~S}_{2}[\mathrm{M}+\mathrm{H}]^{+}=655.8559$, found $=655.8555$.

Optical Rotation: $[\alpha]_{\mathrm{D}}^{25}=30.13\left(c=0.75, \mathrm{CHCl}_{3}\right) .98 \%$ ee (HPLC condition: Chiralpak AD-H column, $n-\mathrm{Hexane} / i-\mathrm{PrOH}=70: 30$, flow rate $=1.0 \mathrm{~mL} / \mathrm{min}$, 
wavelength $=254 \mathrm{~nm}, t_{\mathrm{R}}=10.23$ min for major isomer, $t_{\mathrm{R}}=16.33$ min for minor isomer).

$\mathrm{mV}$

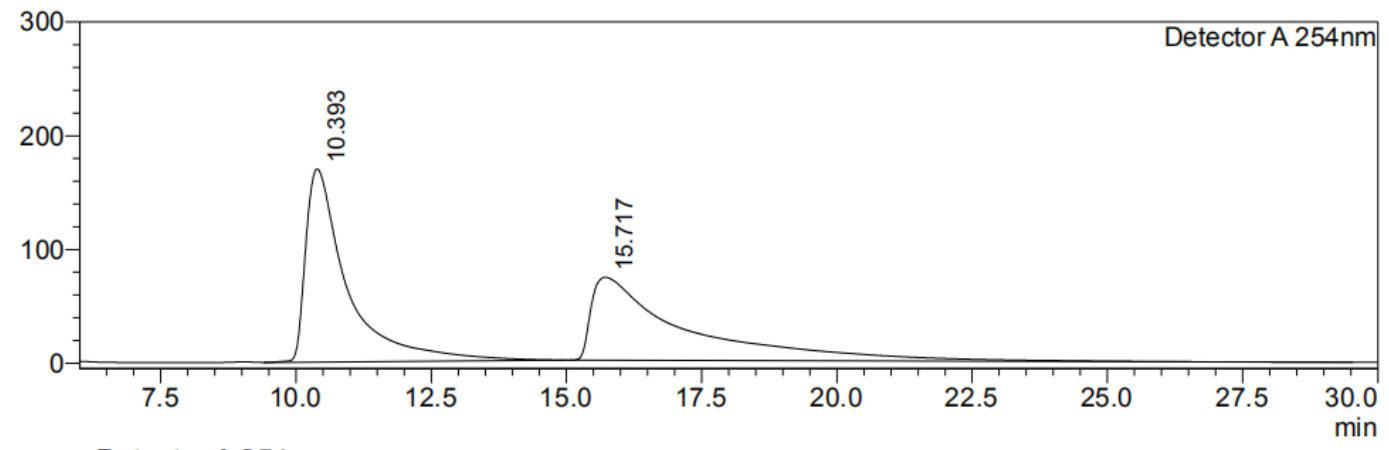

Detector A 254nm

\begin{tabular}{|r|r|r|r|r|r|}
\hline Peak\# & Ret. Time & \multicolumn{1}{c|}{ Height } & Height\% & \multicolumn{1}{c|}{ Area } & \multicolumn{1}{c|}{ Area\% } \\
\hline 1 & 10.393 & 169816 & 69.970 & 9087803 & 50.830 \\
\hline 2 & 15.717 & 72882 & 30.030 & 8791125 & 49.170 \\
\hline Total & & 242698 & 100.000 & 17878928 & 100.000 \\
\hline
\end{tabular}

$\mathrm{mV}$

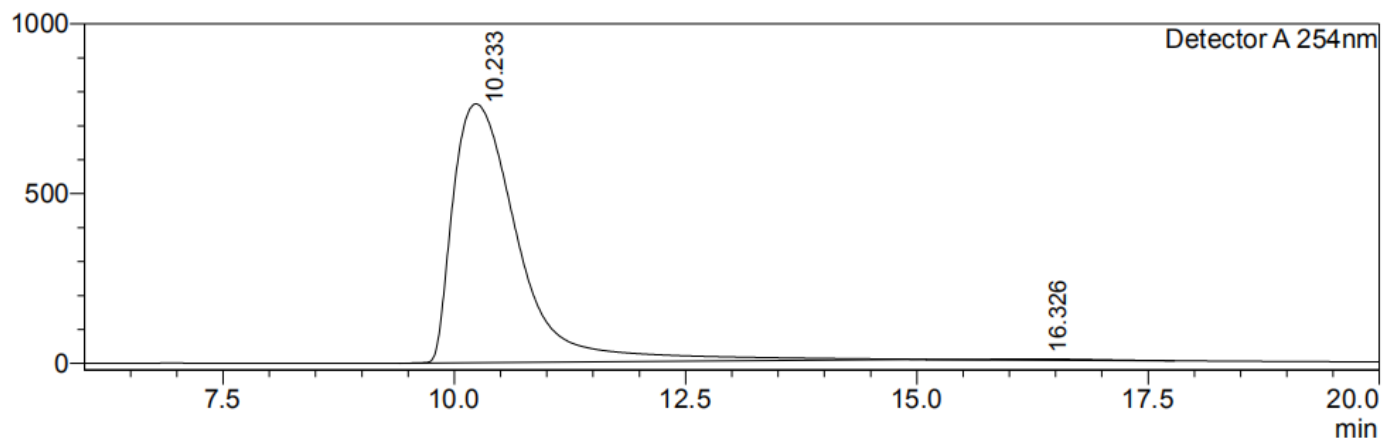

Detector A 254nm

\begin{tabular}{|r|r|r|r|r|r|}
\hline Peak\# & Ret. Time & \multicolumn{1}{c|}{ Height } & \multicolumn{1}{c|}{ Height\% } & \multicolumn{1}{c|}{ Area } & \multicolumn{1}{c|}{ Area\% } \\
\hline 1 & 10.233 & 762414 & 99.528 & 37757282 & 99.078 \\
\hline 2 & 16.326 & 3615 & 0.472 & 351374 & 0.922 \\
\hline Total & & 766029 & 100.000 & 38108656 & 100.000 \\
\hline
\end{tabular}<smiles>NS(=O)(=O)c1ccccc1[N+](=O)[O-]</smiles>

$6 a, 98 \%$ ee

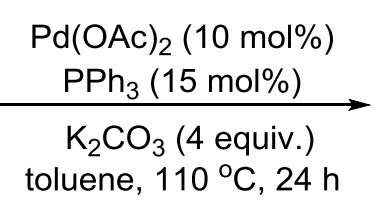

toluene, $110^{\circ} \mathrm{C}, 24 \mathrm{~h}$<smiles>O=S(=O)(c1ccccc1[O-])c1ccccc1Oc1ccccc1</smiles>

$9,92 \%$ ee

Path B: to the mixture of compound $\mathbf{6 a}(40.3 \mathrm{mg}, 0.1 \mathrm{mmol})$, potassium carbonate (55 mg, $0.4 \mathrm{mmol})$, bromobenzene $(21 \mu \mathrm{L}, 0.2 \mathrm{mmol})$ and triphenylphosphine $(3.9$ $\mathrm{mg}, 0.015 \mathrm{mmol})$ was added $\mathrm{Pd}(\mathrm{OAc})_{2}(2.2 \mathrm{mg}, 0.01 \mathrm{mmol})$ in toluene $(1 \mathrm{~mL})$. Then, 
the reaction mixture was stirred at $110{ }^{\circ} \mathrm{C}$ for $24 \mathrm{~h}$. After the completion of the reaction which was indicated by TLC, the reaction mixture was purified through column chromatography on silica gel (petroleum ether/ethyl acetate $=5: 1$ ) to afford the prodcut 9 (29 mg, 60\% yield) as the colorless oil.

\section{(S)-2-(2-hydroxy- $N$-phenylphenylsulfonimidoyl)phenyl}

\section{4-methylbenzenesulfonate (9)}<smiles></smiles>

${ }^{1} \mathrm{H}$ NMR (400 MHz, $\mathrm{CDCl}_{3}$ ) $\delta 12.72(\mathrm{~s}, 1 \mathrm{H}), 8.16(\mathrm{dd}, J=8.0,1.8 \mathrm{~Hz}, 1 \mathrm{H}), 7.73-7.66$ $(\mathrm{m}, 2 \mathrm{H}), 7.59(\mathrm{dd}, J=8.4,1.0 \mathrm{~Hz}, 1 \mathrm{H}), 7.53-7.47(\mathrm{~m}, 1 \mathrm{H}), 7.42(\mathrm{ddd}, J=4.8,4.2,1.4$ $\mathrm{Hz}, 2 \mathrm{H}), 7.33-7.27$ (m, 3H), 7.18-7.10 (m, 2H), 7.02-6.93 (m, 3H), 6.91 (d, $J=8.2$ $\mathrm{Hz}, 1 \mathrm{H}), 6.88-6.80(\mathrm{~m}, 1 \mathrm{H}), 2.46(\mathrm{~s}, 3 \mathrm{H}) ;{ }^{13} \mathrm{C} \mathrm{NMR}\left(100 \mathrm{MHz}, \mathrm{CDCl}_{3}\right) \delta$ 157.7, 147.9, 146.1, 142.0, 135.6, 135.2, 132.6, 132.1, 130.9, 130.0, 129.2, 128.8, 128.7, 126.0, 123.6, 123.3, 120.9, 120.1, 119.5, 22.0; HRMS (ESI) $\mathrm{m} / z$ calcd for $\mathrm{C}_{25} \mathrm{H}_{21} \mathrm{NO}_{5} \mathrm{~S}_{2}[\mathrm{M}+\mathrm{H}]^{+}=480.0939$, found $=480.0936$.

Optical Rotation: $[\alpha]_{\mathrm{D}}^{25}=43.77\left(c=0.61, \mathrm{CHCl}_{3}\right) .92 \%$ ee (HPLC condition: Chiralpak AD-H column, $n-\mathrm{Hexane} / i-\mathrm{PrOH}=70: 30$, flow rate $=1.0 \mathrm{~mL} / \mathrm{min}$, wavelength $=254 \mathrm{~nm}, t_{\mathrm{R}}=15.12 \mathrm{~min}$ for major isomer, $t_{\mathrm{R}}=20.87 \mathrm{~min}$ for minor isomer). 
$\mathrm{mV}$

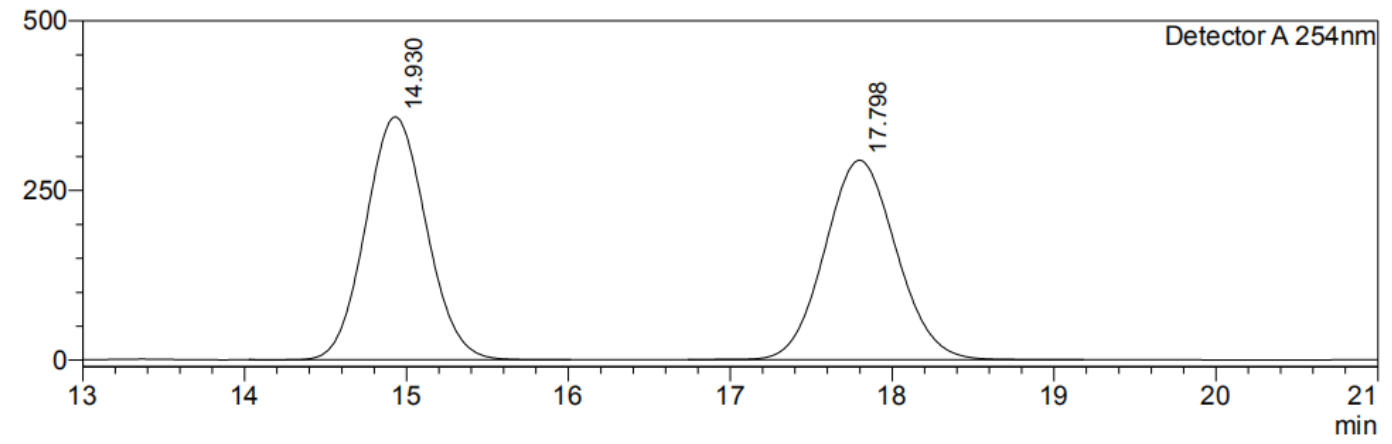

Detector A 254nm

\begin{tabular}{|r|r|r|r|r|r|}
\hline Peak\# & Ret. Time & Height & \multicolumn{1}{|c|}{ Height\% } & \multicolumn{1}{|c|}{ Area } & \multicolumn{1}{c|}{ Area\% } \\
\hline 1 & 14.930 & 358001 & 54.911 & 9343112 & 50.815 \\
\hline 2 & 17.798 & 293960 & 45.089 & 9043346 & 49.185 \\
\hline Total & & 651961 & 100.000 & 18386458 & 100.000 \\
\hline
\end{tabular}

$\mathrm{mV}$

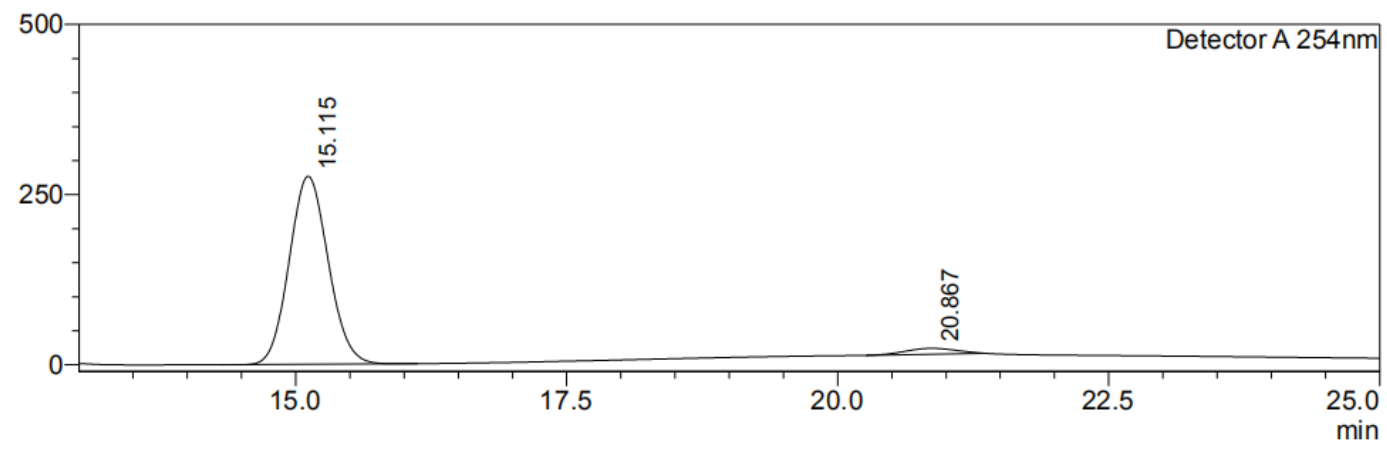

Detector A 254nm
\begin{tabular}{|r|r|r|r|r|r|}
\hline Peak\# & Ret. Time & Height & Height $\%$ & \multicolumn{1}{c|}{ Area } & \multicolumn{1}{|c|}{ Area $\%$} \\
\hline 1 & 15.115 & 276231 & 96.990 & 6927046 & 96.157 \\
\hline 2 & 20.867 & 8572 & 3.010 & 276828 & 3.843 \\
\hline Total & & 284803 & 100.000 & 7203874 & 100.000 \\
\hline
\end{tabular}

\section{$\underline{(S)-2-((2-h y d r o x y p h e n y l) \text { sulfinyl }) \text { phenyl 4-methylbenzenesulfonate }((S)-6 u)}$}<smiles></smiles>

$6 a, 98 \%$ ee

\section{${ }^{t} \mathrm{BuONO}$ (1.1 equiv.) $\mathrm{CHCl}_{3}, \mathrm{rt}, 3 \mathrm{~h}$}<smiles>COc1ccccc1S(=O)c1ccccc1[O-]</smiles>

(S)-6u, 98\% ee

Path C: Chiral 6a $(40.3 \mathrm{mg}, 0.1 \mathrm{mmol})$ was dissolved in chloroform $(1.0 \mathrm{~mL})$ under air and ${ }^{t} \mathrm{BuONO}(13 \mu \mathrm{L}, 0.11 \mathrm{mmol})$ was added. The mixture was stirred at $\mathrm{rt}$ for $3 \mathrm{~h}$. After concentration, the reaction mixture was purified through column chromatography on silica gel (petroleum ether/ethyl acetate $=3: 1$ ) to afford the prodcut (S)-6u (33 mg, 84\% yield) as the yellow solid. 
${ }^{1} \mathrm{H}$ NMR $\left(400 \mathrm{MHz}, \mathrm{CDCl}_{3}\right) \delta 10.03(\mathrm{~s}, 1 \mathrm{H}), 7.99-7.91(\mathrm{~m}, 1 \mathrm{H}), 7.80(\mathrm{~d}, J=8.4 \mathrm{~Hz}$, 2H), 7.51-7.42 (m, 2H), $7.38(\mathrm{~d}, J=8.0 \mathrm{~Hz}, 2 \mathrm{H}), 7.32-7.27(\mathrm{~m}, 3 \mathrm{H}), 6.86(\mathrm{td}, J=7.8$, $1.2 \mathrm{~Hz}, 1 \mathrm{H}), 6.82(\mathrm{dd}, J=8.8,1.2 \mathrm{~Hz}, 1 \mathrm{H}), 2.48(\mathrm{~s}, 3 \mathrm{H}) ;{ }^{13} \mathrm{C} \mathrm{NMR}\left(100 \mathrm{MHz}, \mathrm{CDCl}_{3}\right)$ $\delta 159.5,146.6,145.9,136.6,133.5,133.0,132.1,130.3,128.8,128.1,126.9,125.7$, 122.8, 121.8, 120.1, 119.8, 22.0; HRMS (ESI) $\mathrm{m} / z$ calcd for $\mathrm{C}_{25} \mathrm{H}_{21} \mathrm{NO}_{5} \mathrm{~S}_{2}[\mathrm{M}+\mathrm{H}]^{+}=$ 389.0517 , found $=389.0510$.

Optical Rotation: $[\alpha]^{25}=-64.24\left(c=0.20, \mathrm{CHCl}_{3}\right) .98 \%$ ee (HPLC condition: Chiralpak ID column, $n$-Hexane $/ i-\mathrm{PrOH}=80: 20$, flow rate $=1.0 \mathrm{~mL} / \mathrm{min}$, wavelength $=254 \mathrm{~nm}, t_{\mathrm{R}}=23.32 \min$ for major isomer, $t_{\mathrm{R}}=31.43 \mathrm{~min}$ for minor isomer).

$\mathrm{mV}$

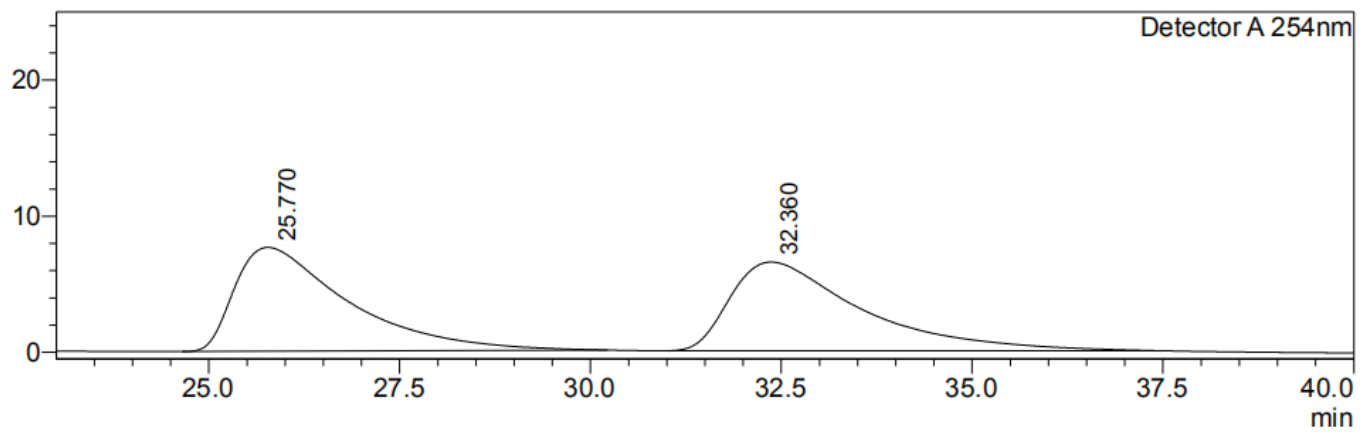

Detector A $254 \mathrm{~nm}$

\begin{tabular}{|r|r|r|r|r|r|}
\hline Peak\# & Ret. Time & Height & Height\% & \multicolumn{1}{c|}{ Area } & \multicolumn{1}{c|}{ Area\% } \\
\hline 1 & 25.770 & 7635 & 53.949 & 787453 & 50.265 \\
\hline 2 & 32.360 & 6517 & 46.051 & 779156 & 49.735 \\
\hline Total & & 14152 & 100.000 & 1566609 & 100.000 \\
\hline
\end{tabular}

$\mathrm{mV}$

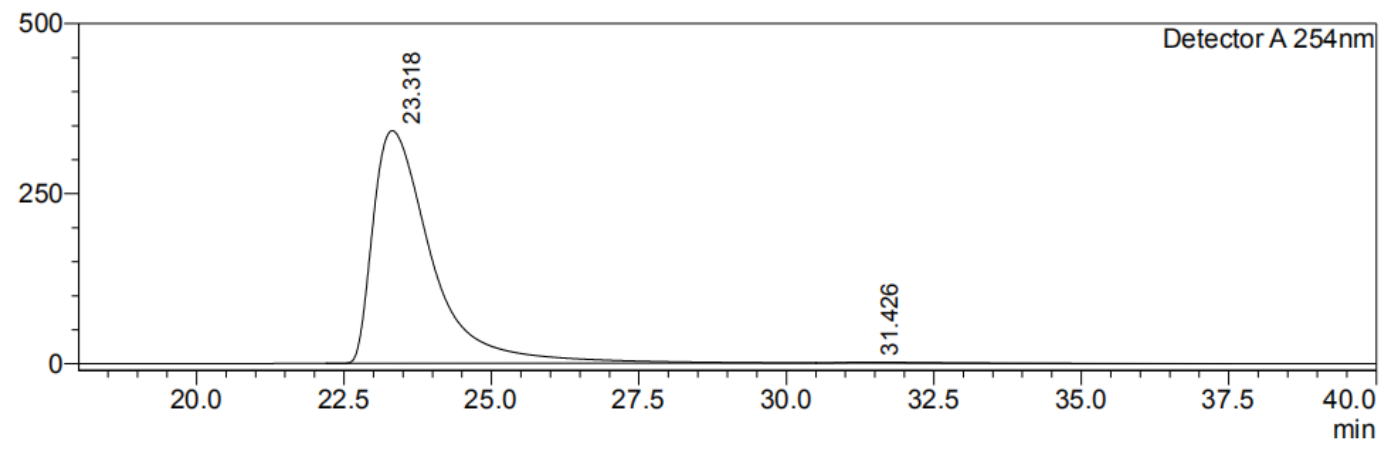

Detector A 254nm
\begin{tabular}{|r|r|r|r|r|r|}
\hline Peak\# & Ret. Time & Height & Height\% & \multicolumn{1}{c|}{ Area } & \multicolumn{1}{|c|}{ Area\% } \\
\hline 1 & 23.318 & 341968 & 99.592 & 23745655 & 99.373 \\
\hline 2 & 31.426 & 1400 & 0.408 & 149925 & 0.627 \\
\hline Total & & 343367 & 100.000 & 23895580 & 100.000 \\
\hline
\end{tabular}




\section{4-methylbenzenesulfonate $(10)$}<smiles>NS(=O)(=O)c1ccccc1[O+]</smiles>

$6 a, 98 \%$ ee<smiles>O=P(Br)(Br)c1ccccc1</smiles>

S6

6

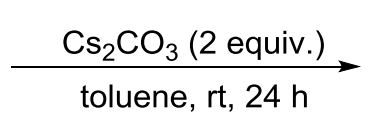<smiles>CC(C)(C)P(=O)(Oc1ccccc1)Oc1ccccc1S(=O)(=O)c1ccccc1[O-]</smiles>

10, $2: 1 d r$ $98 \% / 94 \%$ ee

Path D: to the mixture of compound 6a $(26.0 \mathrm{mg}, 0.1 \mathrm{mmol})$ and S6 (52.0 mg, 0.2 mmol) was added cesium carbonate $(65.2 \mathrm{mg}, 0.2 \mathrm{mmol})$ in toluene $(1 \mathrm{~mL})$. Then, the reaction mixture was stirred at $\mathrm{rt}$ for $24 \mathrm{~h}$. After the completion of the reaction which was indicated by TLC, the reaction mixture was purified through column chromatography on silica gel (petroleum ether/ethyl acetate $=3: 1$ to $1: 1$ ) to afford the prodcut 10 (47 $\mathrm{mg}, 81 \%$ yield) as the colorless oil.

${ }^{1} \mathrm{H}$ NMR $\left(400 \mathrm{MHz}, \mathrm{CDCl}_{3}\right) \delta 8.21(\mathrm{dd}, J=8.0,1.6 \mathrm{~Hz}, 1 \mathrm{H}), 8.03-7.96(\mathrm{~m}, 1 \mathrm{H})$, 7.87-7.72 (m, 4H), $7.62(\mathrm{ddd}, J=8.4,4.2,3.2 \mathrm{~Hz}, 1 \mathrm{H}), 7.55(\mathrm{qd}, J=4.2,2.2 \mathrm{~Hz}, 1 \mathrm{H})$, 7.52-7.39 (m, 2H), 7.38-7.31 (m, 4H), 7.29-7.19 (m, 2H), 6.95-6.80 (m, 1H), $2.48(\mathrm{~s}$, $3 \mathrm{H}), 1.01(\mathrm{~d}, J=16.7 \mathrm{~Hz}, 9 \mathrm{H}) ;{ }^{13} \mathrm{C} \mathrm{NMR}\left(100 \mathrm{MHz}, \mathrm{CDCl}_{3}\right) \delta 150.8,146.2,135.7$, $134.9,134.3,133.9,133.8,132.7,132.5,132.2,131.3,130.0,129.3,129.0,128.6$, 128.5, 126.4, 123.0 (d, $J=12.2 \mathrm{~Hz}), 122.0$ (d, $J=35.6 \mathrm{~Hz}), 120.8$ (d, $J=5.3 \mathrm{~Hz})$, $33.4(\mathrm{~d}, J=99.4 \mathrm{~Hz}), 23.9(\mathrm{~d}, J=7.6 \mathrm{~Hz}), 21.9 ;{ }^{31} \mathrm{P} \mathrm{NMR}\left(162 \mathrm{MHz}, \mathrm{CDCl}_{3}\right) \delta 54.93$, 54.65; HRMS (ESI) $m / z$ calcd for $\mathrm{C}_{29} \mathrm{H}_{30} \mathrm{NO}_{6} \mathrm{PS}_{2}[\mathrm{M}+\mathrm{H}]^{+}=584.1330$, found $=$ 584.1333 .

Optical Rotation: $[\alpha]_{D}^{25}=-132.00\left(c=0.59, \mathrm{CHCl}_{3}\right) .98 \% / 94 \%$ ee (HPLC condition: Chiralpak IA column, $n-\mathrm{Hexane} / i-\mathrm{PrOH}=60: 40$, flow rate $=1.0 \mathrm{~mL} / \mathrm{min}$, wavelength $=254 \mathrm{~nm}, t_{\mathrm{R} 1}=13.49 \mathrm{~min}$ for minor isomer, $t_{\mathrm{R} 1}=16.72 \mathrm{~min}$ for major isomer, $t_{\mathrm{R} 2}=15.16 \mathrm{~min}$ for major isomer, $t_{\mathrm{R} 2}=44.87 \mathrm{~min}$ for minor isomer). 
$\mathrm{mV}$

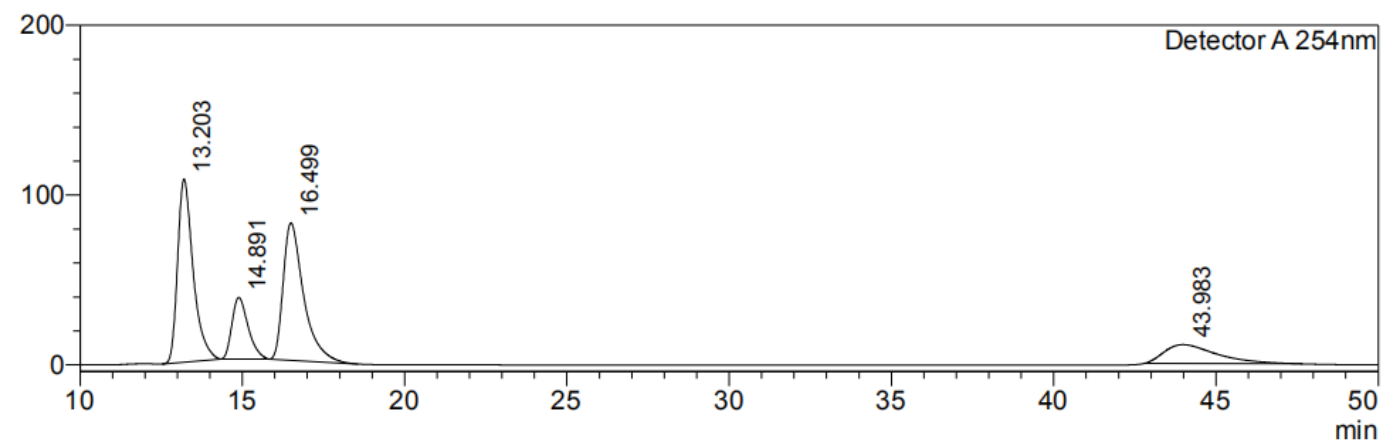

\begin{tabular}{|c|c|c|c|c|c|}
\hline \\
\hline Peak\# & Ret. Time & Height & Height\% & Area & Area\% \\
\hline 1 & 13.203 & 107829 & 45.679 & 3592993 & 37.234 \\
\hline 2 & 14.891 & 36286 & 15.372 & 1257358 & 13.030 \\
\hline 3 & 16.499 & 80908 & 34.274 & 3550682 & 36.796 \\
\hline 4 & 43.983 & 11037 & 4.675 & 1248702 & 12.940 \\
\hline Total & & 236060 & 100.000 & 9649735 & 100.000 \\
\hline
\end{tabular}

$\mathrm{mV}$

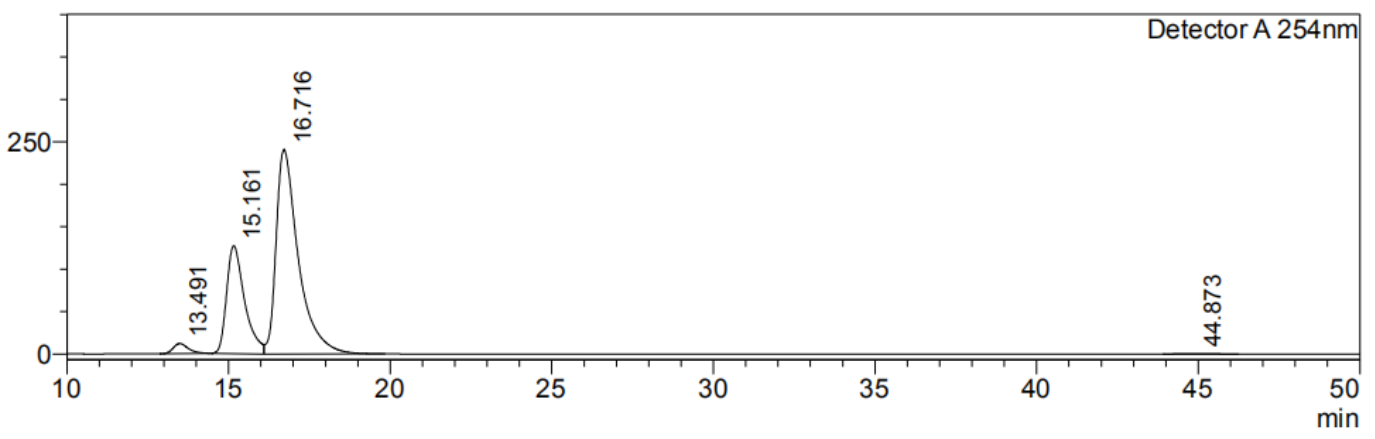

Detector A 254nm

\begin{tabular}{|r|r|r|r|r|r|}
\hline Peak\# & Ret. Time & Height & Height\% & \multicolumn{1}{c|}{ Area } & \multicolumn{1}{c|}{ Area\% } \\
\hline 1 & 13.491 & 11907 & 3.131 & 385581 & 2.281 \\
\hline 2 & 15.161 & 127383 & 33.491 & 4928986 & 29.159 \\
\hline 3 & 16.716 & 240739 & 63.293 & 11564396 & 68.413 \\
\hline 4 & 44.873 & 325 & 0.086 & 24886 & 0.147 \\
\hline Total & & 380354 & 100.000 & 16903849 & 100.000 \\
\hline
\end{tabular}

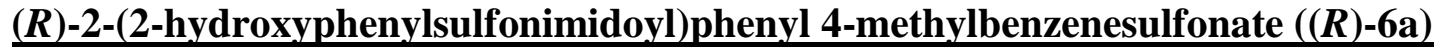<smiles>NS(=O)(=O)c1ccccc1OCCOCCO</smiles>

3a, $97 \%$ ee

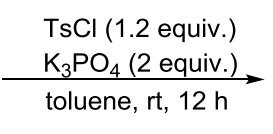

oluene, it, $12 \mathrm{~h}$

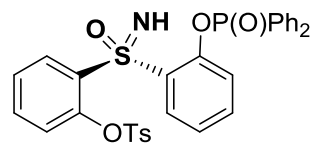

S7

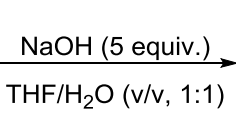
$\mathrm{rt}, 0.5 \mathrm{~h}$

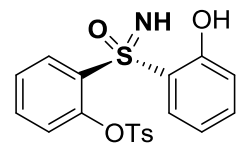

$(R)-6 a, 97 \%$ ee

Path E: to the mixture of compound 3a $(44.95 \mathrm{mg}, 0.1 \mathrm{mmol})$ and $\mathbf{5 a}(21.0 \mathrm{mg}, 0.1$ mmol) was added potassium phosphate $(42.4 \mathrm{mg}, 0.2 \mathrm{mmol})$ in toluene $(1 \mathrm{~mL})$. Then, the reaction mixture was stirred at $\mathrm{rt}$ for $12 \mathrm{~h}$. After the completion of the reaction which was indicated by TLC, the solvent was removed in vacuo, and the residue was 
used in the next synthetic step without further purification. Then, S7 was dissolved in $\mathrm{THF} / \mathrm{H}_{2} \mathrm{O}(2.0 \mathrm{~mL}, \mathrm{v} / \mathrm{v}, 1: 1)$ and sodium hydroxide $(20 \mathrm{mg}, 0.5 \mathrm{mmol})$ was added. The mixture was stirred at $\mathrm{rt}$ for $0.5 \mathrm{~h}$. Then, $1 \mathrm{M} \mathrm{HCl}(1.0 \mathrm{~mL})$ was added, the organic layer was separated and the aqueous layer was extracted with ethyl acetate (3 x $5 \mathrm{~mL}$ ). The organic layer was dried over anhydrous sodium sulfate and concentrated under reduced pressure. The crude mixture was purified through column chromatography on silica gel (petroleum ether/ethyl acetate $=5: 1$ ) to afford the prodcut $(R)-6 \mathbf{a}(36 \mathrm{mg}, 89 \%$ overall yield) as the yellow solid.

Optical Rotation: $[\alpha]_{\mathrm{D}}^{25}=-56.80\left(c=0.50, \mathrm{CHCl}_{3}\right) .97 \%$ ee (HPLC condition: Chiralpak AD-H column, $n$-Hexane $/ i-\mathrm{PrOH}=70: 30$, flow rate $=1.0 \mathrm{~mL} / \mathrm{min}$, wavelength $=254 \mathrm{~nm}, t_{\mathrm{R}}=19.00$ min for major isomer, $t_{\mathrm{R}}=23.18$ min for minor isomer).

$\mathrm{mV}$

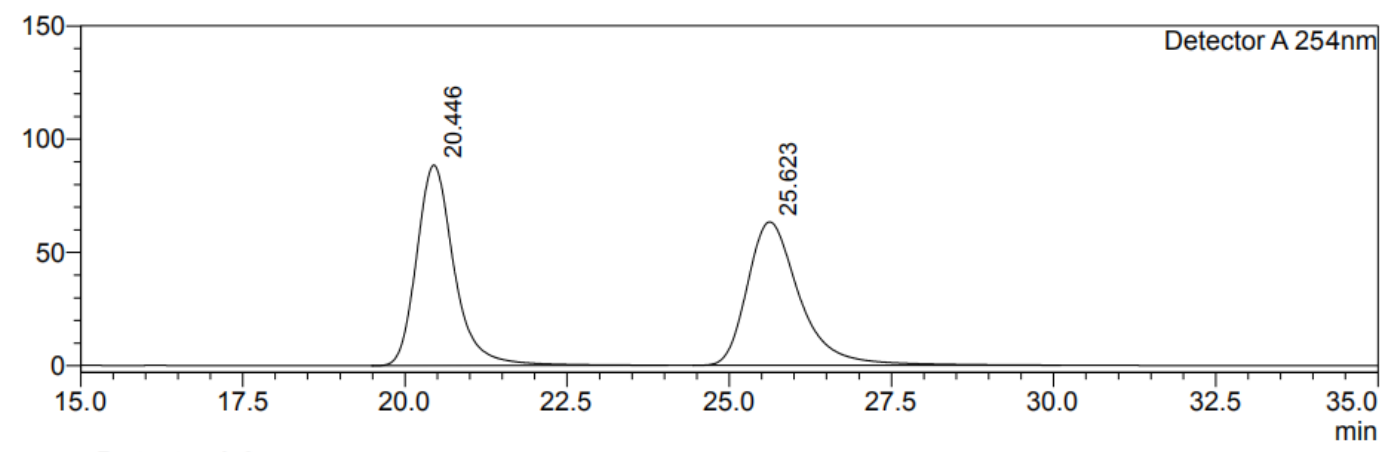

\begin{tabular}{|c|c|c|c|c|c|}
\hline Peak\# & Ret. Time & Height & Height $\%$ & Area & Area $\%$ \\
\hline 1 & 20.446 & 88505 & 58.307 & 3575309 & 50.150 \\
\hline 2 & 25.623 & 63286 & 41.693 & 3553855 & 49.850 \\
\hline Total & & 151792 & 100.000 & 7129164 & 100.000 \\
\hline
\end{tabular}




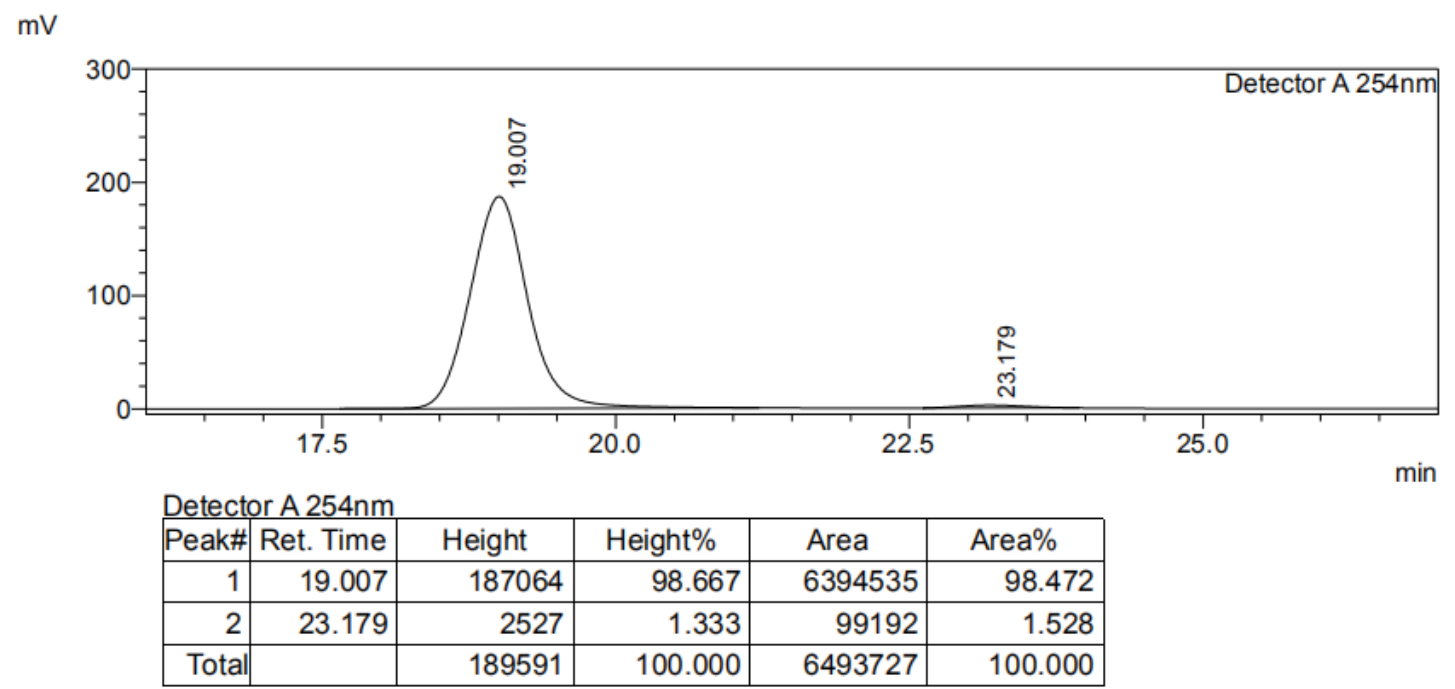

\section{Determination of Absolute Configuration of the Chiral Products}

(1) The absolute configurations of products $\mathbf{3}$ and $\mathbf{4}$, excepting the product $\mathbf{3 o}$, were assigned as $S$ respectively by X-ray crystallographic analysis of a single crystal of enantiopure 3e (Figure S3). CCDC 2086866 contains the supplementary crystallographic data of $\mathbf{3 e}$ for this paper. This data can be obtained free of charge from The Cambridge Crystallographic Data Centre via www.ccdc.cam.ac.uk/data_request/cif.

(2) The absolute configurations of products $\mathbf{6}$ and 7, excepting the product $\mathbf{6 u}$, were assigned as $S$ respectively by $\mathrm{X}$-ray crystallographic analysis of a single crystal of enantiopure 6a (Figure S4). CCDC 2103367 contains the supplementary crystallographic data of $\mathbf{6 a}$ for this paper. This data can be obtained free of charge from The Cambridge Crystallographic Data Centre via www.ccdc.cam.ac.uk/data_request/cif.

(3) A derived compound (S)-6u could be obtained by the transformation of $(S)-\mathbf{6 a}$. Accordingly, the absolute configurations of above desymmetrizing sulfoxide products $6 \mathbf{u}$ and $3 \mathbf{0}$ were determined as $R$ by analogy with this derived $(S)-\mathbf{6 u}$.

(4) The absolute configurations of product $\mathbf{3 j}$ were determined as $S$ by analogy with the CD curve of product $\mathbf{3 j}$ (Figure S5). 

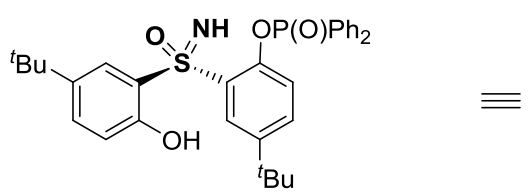

(S)-4-(tert-butyl)-2-(5-(tert-butyl)-2-

hydroxyphenylsulfonimidoyl)phenyl diphenylphosphinate

Figure S3. X-ray structure of 3e.

Table S15. Crystal data and structure refinement for $3 \mathbf{e}$.

\begin{tabular}{|c|c|}
\hline Identification code & $3 e$ \\
\hline Empirical formula & $\mathrm{C}_{32} \mathrm{H}_{36} \mathrm{NO}_{4} \mathrm{PS}$ \\
\hline Formula weight & 681.01 \\
\hline Temperature/K & 150.0 \\
\hline Crystal system & orthorhombic \\
\hline Space group & $\mathrm{P} 2{ }_{1} 2_{1} 2_{1}$ \\
\hline $\mathrm{a} / \AA ̊$ & $11.8935(12)$ \\
\hline $\mathrm{b} / \AA$ & $13.9039(16)$ \\
\hline $\mathrm{c} / \AA ̊ \AA$ & $21.127(3)$ \\
\hline$\alpha /^{\circ}$ & 90 \\
\hline$\beta /{ }^{\circ}$ & 90 \\
\hline$\gamma /{ }^{\circ}$ & 90 \\
\hline Volume $/ \AA^{3}$ & $3493.6(7)$ \\
\hline $\mathrm{Z}$ & 4 \\
\hline$\rho_{\text {calc }} \mathrm{g} / \mathrm{cm}^{3}$ & 1.295 \\
\hline$\mu / \mathrm{mm}^{-1}$ & 0.404 \\
\hline $\mathrm{F}(000)$ & 1424.0 \\
\hline Crystal size $/ \mathrm{mm}^{3}$ & $0.43 \times 0.17 \times 0.07$ \\
\hline Radiation & $\operatorname{MoK} \alpha(\lambda=0.71073)$ \\
\hline $2 \Theta$ range for data collection $/{ }^{\circ}$ & 3.93 to 55.052 \\
\hline Index ranges & $-15 \leq \mathrm{h} \leq 14,-17 \leq \mathrm{k} \leq 18,-27 \leq 1 \leq 26$ \\
\hline Reflections collected & 27119 \\
\hline Independent reflections & $7978\left[\mathrm{R}_{\text {int }}=0.1019, \mathrm{R}_{\text {sigma }}=0.1048\right]$ \\
\hline Data/restraints/parameters & $7978 / 28 / 398$ \\
\hline Goodness-of-fit on $\mathrm{F}^{2}$ & 1.048 \\
\hline Final $\mathrm{R}$ indexes $[\mathrm{I}>=2 \sigma(\mathrm{I})]$ & $\mathrm{R}_{1}=0.0739, \mathrm{wR}_{2}=0.1704$ \\
\hline Final R indexes [all data] & $\mathrm{R}_{1}=0.1240, \mathrm{wR}_{2}=0.2019$ \\
\hline Largest diff. peak/hole / e $\AA^{-3}$ & $0.54 /-0.56$ \\
\hline Flack parameter & $0.01(6)$ \\
\hline
\end{tabular}




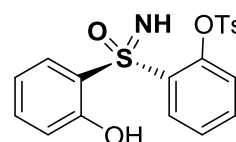

$\equiv$

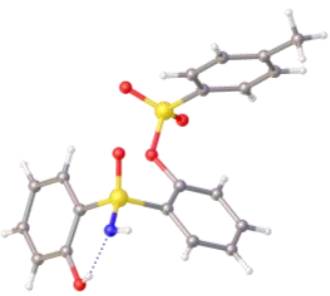

(S)-2-(2-hydroxyphenylsulfonimidoyl)phenyl 4methylbenzenesulfonate

Figure S4. X-ray structure of $\mathbf{6 a}$.

Table S16. Crystal data and structure refinement for $6 \mathbf{a}$.

\begin{tabular}{|c|c|}
\hline Identification code & $6 \mathbf{6 a}$ \\
\hline Empirical formula & $\mathrm{C}_{19} \mathrm{H}_{17} \mathrm{NO}_{5} \mathrm{~S}_{2}$ \\
\hline Formula weight & 403.45 \\
\hline Temperature/K & 304.0 \\
\hline Crystal system & monoclinic \\
\hline Space group & $\mathrm{P} 2_{1}$ \\
\hline $\mathrm{a} / \AA ̊$ & $9.0907(3)$ \\
\hline $\mathrm{b} / \AA$ & $19.9189(8)$ \\
\hline $\mathrm{c} / \AA$ & $10.3884(4)$ \\
\hline$\alpha /^{\circ}$ & 90 \\
\hline$\beta /{ }^{\circ}$ & $94.4070(10)$ \\
\hline$\gamma /{ }^{\circ}$ & 90 \\
\hline Volume $/ \AA^{3}$ & $1875.54(12)$ \\
\hline $\mathrm{Z}$ & 4 \\
\hline$\rho_{\text {calc }} \mathrm{g} / \mathrm{cm}^{3}$ & 1.429 \\
\hline$\mu / \mathrm{mm}^{-1}$ & 0.315 \\
\hline $\mathrm{F}(000)$ & 840.0 \\
\hline Crystal size $/ \mathrm{mm}^{3}$ & $0.35 \times 0.11 \times 0.09$ \\
\hline Radiation & $\operatorname{MoK} \alpha(\lambda=0.71073)$ \\
\hline $2 \Theta$ range for data collection $/{ }^{\circ}$ & 3.932 to 54.994 \\
\hline Index ranges & $-11 \leq \mathrm{h} \leq 10,-25 \leq \mathrm{k} \leq 25,-13 \leq 1 \leq 13$ \\
\hline Reflections collected & 36402 \\
\hline Independent reflections & $8598\left[\mathrm{R}_{\text {int }}=0.0557, \mathrm{R}_{\text {sigma }}=0.0455\right]$ \\
\hline Data/restraints/parameters & $8598 / 9 / 498$ \\
\hline Goodness-of-fit on $\mathrm{F}^{2}$ & 1.018 \\
\hline Final $R$ indexes $[\mathrm{I}>=2 \sigma(\mathrm{I})]$ & $\mathrm{R}_{1}=0.0440, \mathrm{wR}_{2}=0.1000$ \\
\hline Final R indexes [all data] & $\mathrm{R}_{1}=0.0664, \mathrm{wR}_{2}=0.1132$ \\
\hline Largest diff. peak/hole / e $\AA^{-3}$ & $0.68 /-0.37$ \\
\hline Flack parameter & $0.00(3)$ \\
\hline
\end{tabular}


a) CD curve of $\mathbf{3 e}$

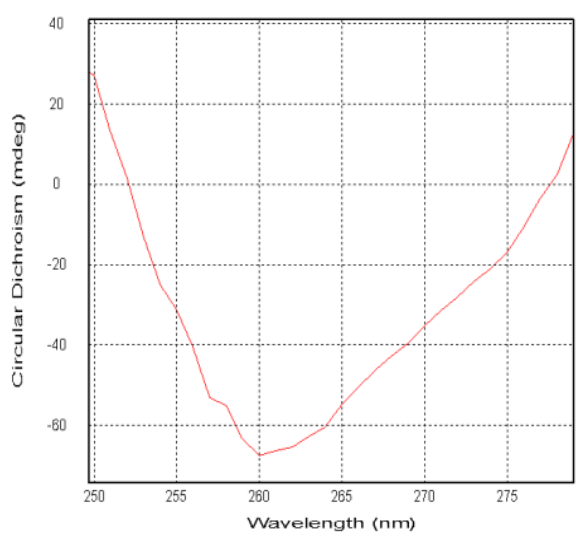

b) CD curve of $\mathbf{3} \mathbf{j}$

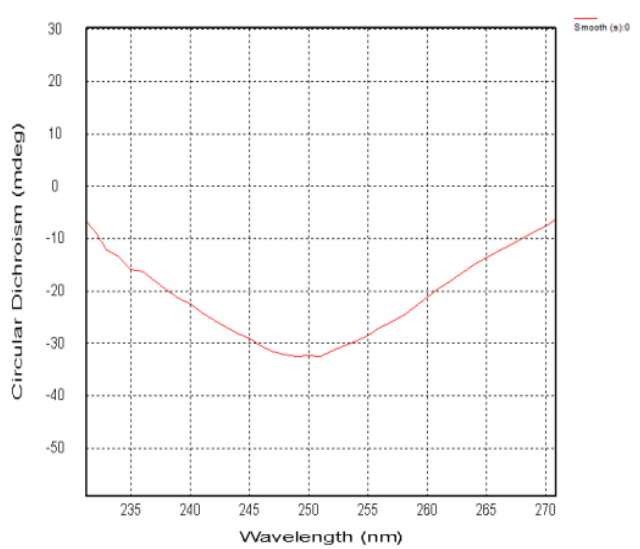

Figure S5. Analysis of CD curve.

\section{Mechanistic Investigations}

A. Control Experiments to investigate whether there exists a KR process in this reaction.

(a) Investigation of Desymmetrizing Asymmetric A-T Reaction Process ${ }^{a}$

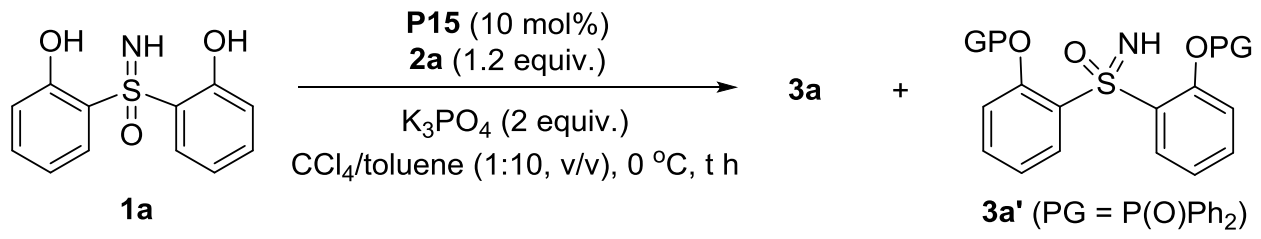

$\begin{array}{cccc}\mathrm{T}(\mathrm{h}) & \text { ee of } \mathbf{3} \mathbf{a}(\%) & \text { yield of } \mathbf{3} \mathbf{a}(\%) & \text { yield of } \mathbf{3} \mathbf{a}^{\prime}(\%) \\ 0 & -- & 0 & \\ 1 & 64 & 13 & 0 \\ 1.5 & 71 & 47 & 5 \\ 2 & 79 & 55 & 6 \\ 2.5 & 83 & 61 & 9 \\ 3.5 & 88 & 79 & 11 \\ 4.5 & 94 & 83 & 17 \\ 5.5 & 96 & 81 & 19 \\ 8.5 & 96 & 81 & 19 \\ 11.5 & 96 & 81 & 19 \\ 16 & 96 & 81 & 19\end{array}$


To a tube with a magnetic stirring bar were added $\mathbf{1 a}(49.8 \mathrm{mg}, 0.2 \mathrm{mmol}), \mathbf{2 a}$ (48.4 mg, $0.24 \mathrm{mmol}$ ), potassium phosphate $(84.8 \mathrm{mg}, 0.4 \mathrm{mmol})$ and $\mathbf{P 1 5}$ (24.0 $\mathrm{mg}$, $10 \mathrm{~mol} \%)$, followed by the addition of $\mathrm{CCl}_{4} /$ toluene $(3.0 \mathrm{~mL}, \mathrm{v} / \mathrm{v}=1 / 10)$. The reaction mixture was stirred at $0{ }^{\circ} \mathrm{C}$, and then both chiral HPLC and ${ }^{31} \mathrm{P}-\mathrm{NMR}$ spectrums were recorded after a period of time. Obviously, the variation of ee of $\mathbf{3 a}$ was closely associated with the formation of byproduct $\mathbf{3} \mathbf{a}^{\prime}$.

\section{(b) Kinetic Resolution of ( \pm -3a/6a}

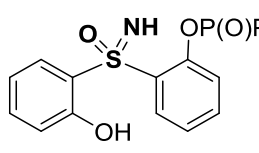

$( \pm)-3 a$

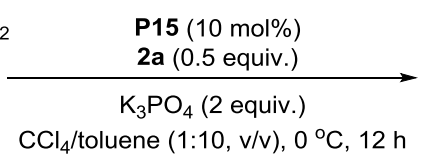

$\mathrm{CCl}_{4} /$ toluene $(1: 10, \mathrm{v} / \mathrm{v}), 0^{\circ} \mathrm{C}, 12 \mathrm{~h}$

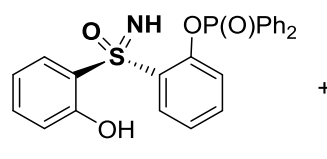

$3 \mathbf{a}$

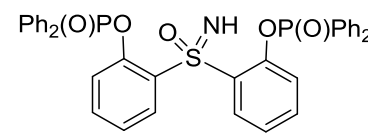

3a'

To a tube with a magnetic stirring bar were added ( \pm )-3a $(44.9 \mathrm{mg}, 0.1 \mathrm{mmol})$, 2a (10.1 mg, $0.05 \mathrm{mmol})$, potassium phosphate (42.4 mg, $0.2 \mathrm{mmol})$ and P15 (12.0 $\mathrm{mg}, 10 \mathrm{~mol} \%)$, followed by the addition of $\mathrm{CCl}_{4} /$ toluene $(2.0 \mathrm{~mL}, \mathrm{v} / \mathrm{v}=1 / 10)$. The reaction mixture was stirred at $0{ }^{\circ} \mathrm{C}$ for $12 \mathrm{~h}$. Then, the reaction mixture was transfered to room temperature, and diluted with dichloromethane. Purification by column chromatography on silica gel (petroleum ether/ethyl acetate $=3: 1$ to $1: 1$ ) afforded the prodcut 3a (23 mg, 51\% yield, 36\% ee) and 3a' (29 mg, 44\% yield).

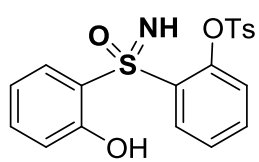

$( \pm)-6 a$

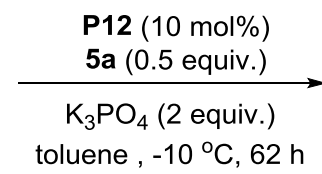

toluene, $-10^{\circ} \mathrm{C}, 62 \mathrm{~h}$

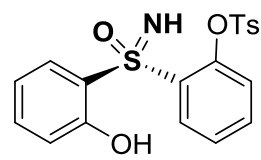

$6 a$

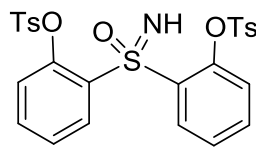

6a'

To a tube with a magnetic stirring bar were added ( \pm )-6a (44.9 mg, $0.1 \mathrm{mmol})$, 5a (9.6 mg, $0.05 \mathrm{mmol}$ ), potassium phosphate (42.4 mg, $0.2 \mathrm{mmol}$ ) and $\mathbf{P 1 2}$ (9.7 $\mathrm{mg}$, $10 \mathrm{~mol} \%)$, followed by the addition of toluene $(2.0 \mathrm{~mL})$. The reaction mixture was stirred at $-10{ }^{\circ} \mathrm{C}$ for $62 \mathrm{~h}$. Then, the reaction mixture was transfered to room temperature, and diluted with dichloromethane. Purification by column chromatography on silica gel (petroleum ether/ethyl acetate $=5: 1$ ) afforded the prodcut 6a (19 mg, 46\% yield, 88\% ee) and 6a' (28 mg, 49\% yield). 


\section{B. a) Chiral Amplification in the Desymmetrizing Reaction of 1a with 2a}

\section{Catalyzed by P15}

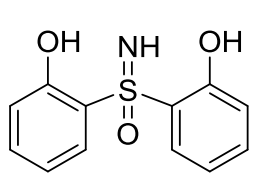

$1 \mathrm{a}$

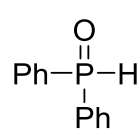

$2 \mathrm{a}$

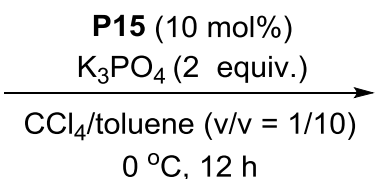

$0{ }^{\circ} \mathrm{C}, 12 \mathrm{~h}$

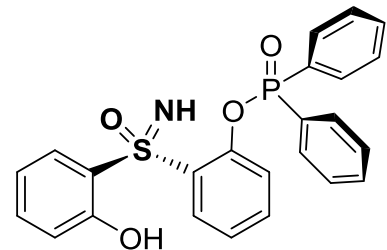

3a ee of P15

20

40

60

80

100 ee of $\mathbf{3 a}(\%)$

21

46

70

81

97

To a tube with a magnetic stirring bar were added $1 \mathbf{a}(24.9 \mathrm{mg}, 0.1 \mathrm{mmol}), 3 \mathbf{a}$ (24.2 mg, $0.12 \mathrm{mmol}$ ), potassium phosphate (42.4 mg, $0.2 \mathrm{mmol}$ ) and P15 (12.0 mg, $10 \mathrm{~mol} \%)$ with various ee values, followed by the addition of $\mathrm{CCl}_{4} /$ toluene $(2.0 \mathrm{~mL}$, $\mathrm{v} / \mathrm{v}=1 / 10)$, respectively. The reaction mixture was stirred at $0{ }^{\circ} \mathrm{C}$ for $12 \mathrm{~h}$. Obviously, a positive nonlinear effect [(+)-NLE] was observed for the desymmetrizing asymmetric A-T reaction.

b) Chiral Amplification in the Desymmetrizing Reaction of 1a with 5a Catalyzed by P12<smiles>N=S(=O)(c1ccccc1O)c1ccccc1O</smiles>

$1 \mathrm{a}$

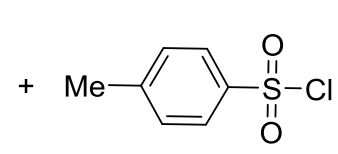

$5 a$

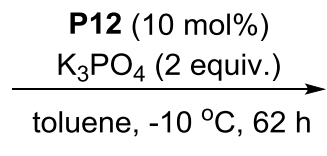<smiles>NS(=O)(=O)c1ccccc1[O-]</smiles>

$6 a$ ee of P12

0

7

25

47 ee of $\mathbf{6 a}(\%)$ 


$\begin{array}{cc}64 & 94 \\ 84 & 96 \\ 100 & >99\end{array}$

To a tube with a magnetic stirring bar were added 1a $(24.9 \mathrm{mg}, 0.1 \mathrm{mmol}), \mathbf{5 a}$ (21.0 mg, $0.11 \mathrm{mmol}$ ), potassium phosphate (42.4 $\mathrm{mg}, 0.2 \mathrm{mmol})$ and $\mathbf{P 1 2}(9.7 \mathrm{mg}$, $10 \mathrm{~mol} \%)$ with various ee values, followed by the addition of toluene $(2.0 \mathrm{~mL})$, respectively. The reaction mixture was stirred at $-10{ }^{\circ} \mathrm{C}$ for $62 \mathrm{~h}$. Obviously, a remarkably positive nonlinear effect [(+)-NLE] was observed for the desymmetrizing asymmetric NAS-type reaction. The addition of $\mathbf{5 a}$ to $\mathbf{1 a}$ in the presence of the catalyst P12 of $64 \%$ ee could even provide the product 6 a with a high ee value $(94 \%)$.

\section{C. ${ }^{1} \mathrm{H}$ NMR Titration Experiments of 1a/5a with Catalyst P12}

Catalyst P12 titration with 1a: ${ }^{1} \mathrm{H}$ NMR titration was conducted in DMSO-d6. The total concentration of the host (P12) and the guest (1a) was $0.02 \mathrm{M}$. The proportion of the concentration of the host vs the total concentration varied from 0.2 equivalent to 1.0. A ${ }^{1} \mathrm{H}$ NMR spectrum was recorded after each addition. ${ }^{1} \mathrm{H}$ NMR spectra were calibrated to the residual DMSO solvent peak.

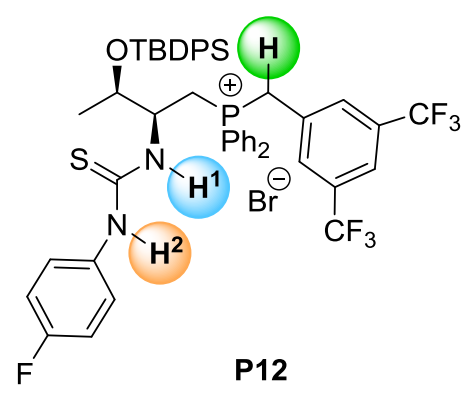



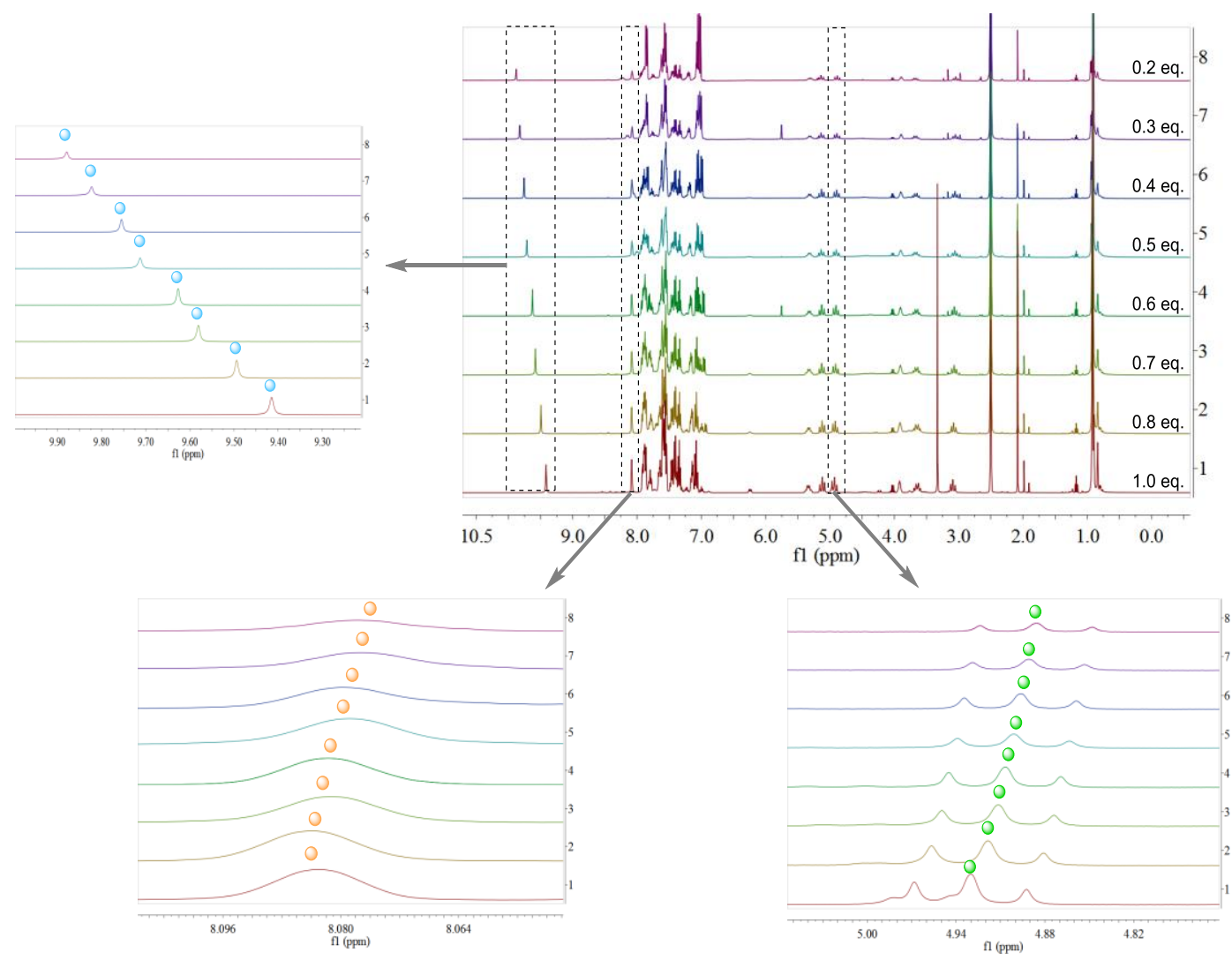

Figure S5. ${ }^{1} \mathrm{H}-\mathrm{NMR}$ spectrum of $\mathbf{P 1 2}$ with 1a

Catalyst P12 titration with 5a: ${ }^{1} \mathrm{H}$ NMR titration was conducted in DMSO-d6. The total concentration of the host $(\mathbf{P 1 2})$ and the guest $(\mathbf{5 a})$ was $0.02 \mathrm{M}$. The proportion of the concentration of the host vs the total concentration varied from 0.2 equivalent to 1.0. A ${ }^{1} \mathrm{H}$ NMR spectrum was recorded after each addition. ${ }^{1} \mathrm{H}$ NMR spectra were calibrated to the residual DMSO solvent peak. 


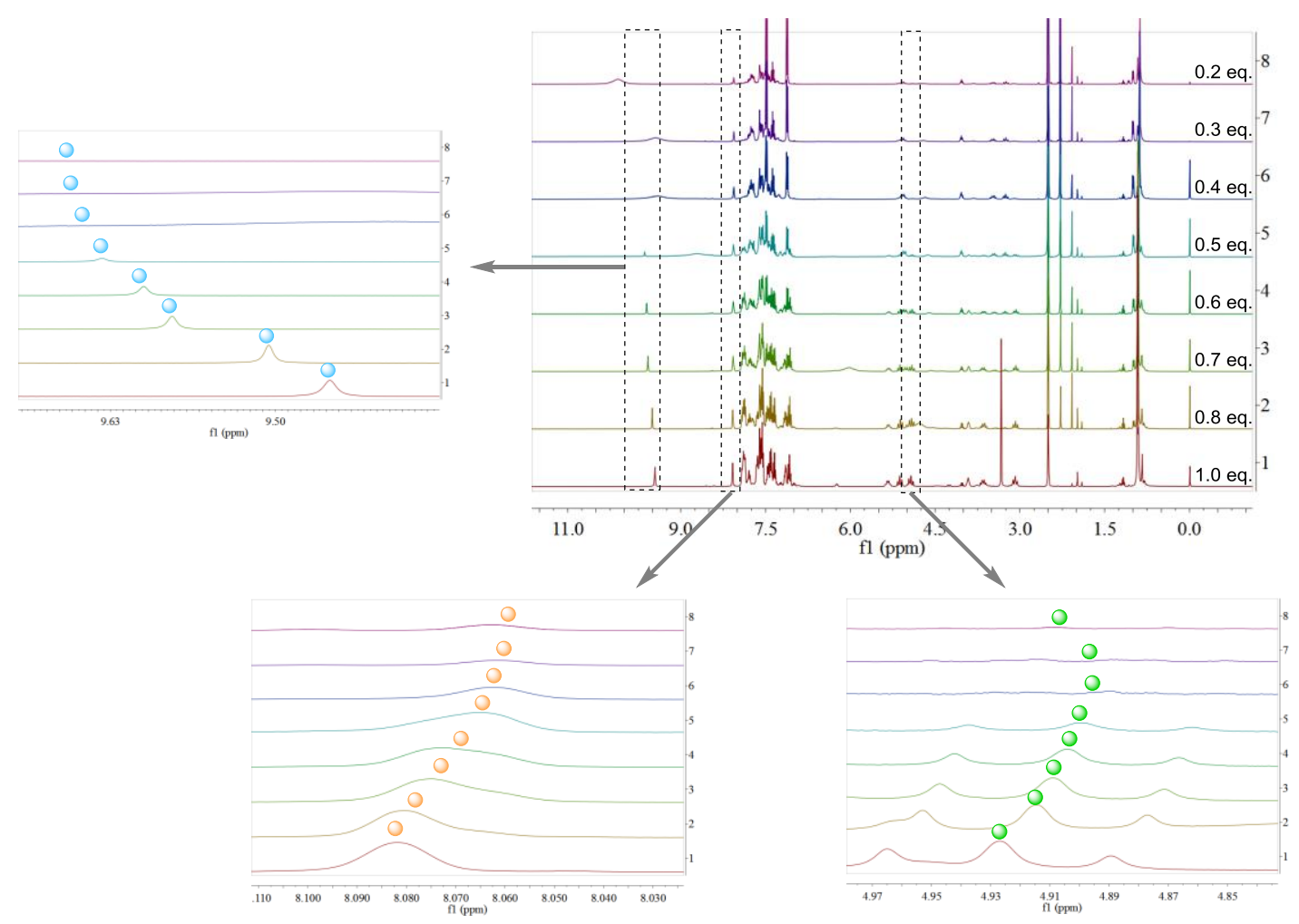

Figure S6. ${ }^{1} \mathrm{H}-\mathrm{NMR}$ spectrum of $\mathbf{P 1 2}$ with $\mathbf{5 a}$

According to the above results, ${ }^{1} \mathrm{H}$ NMR spectral changes of P12 with $\mathbf{1 a}$ and $\mathbf{5 a}$, respectively, were drawn (Figure S7A). Then, we undertook Job plot analyses, which suggest a 1: 1 binding pattern between catalyst P12 and 1a or 5a (Figure S7B). 


\section{A Chemical shift of P12}

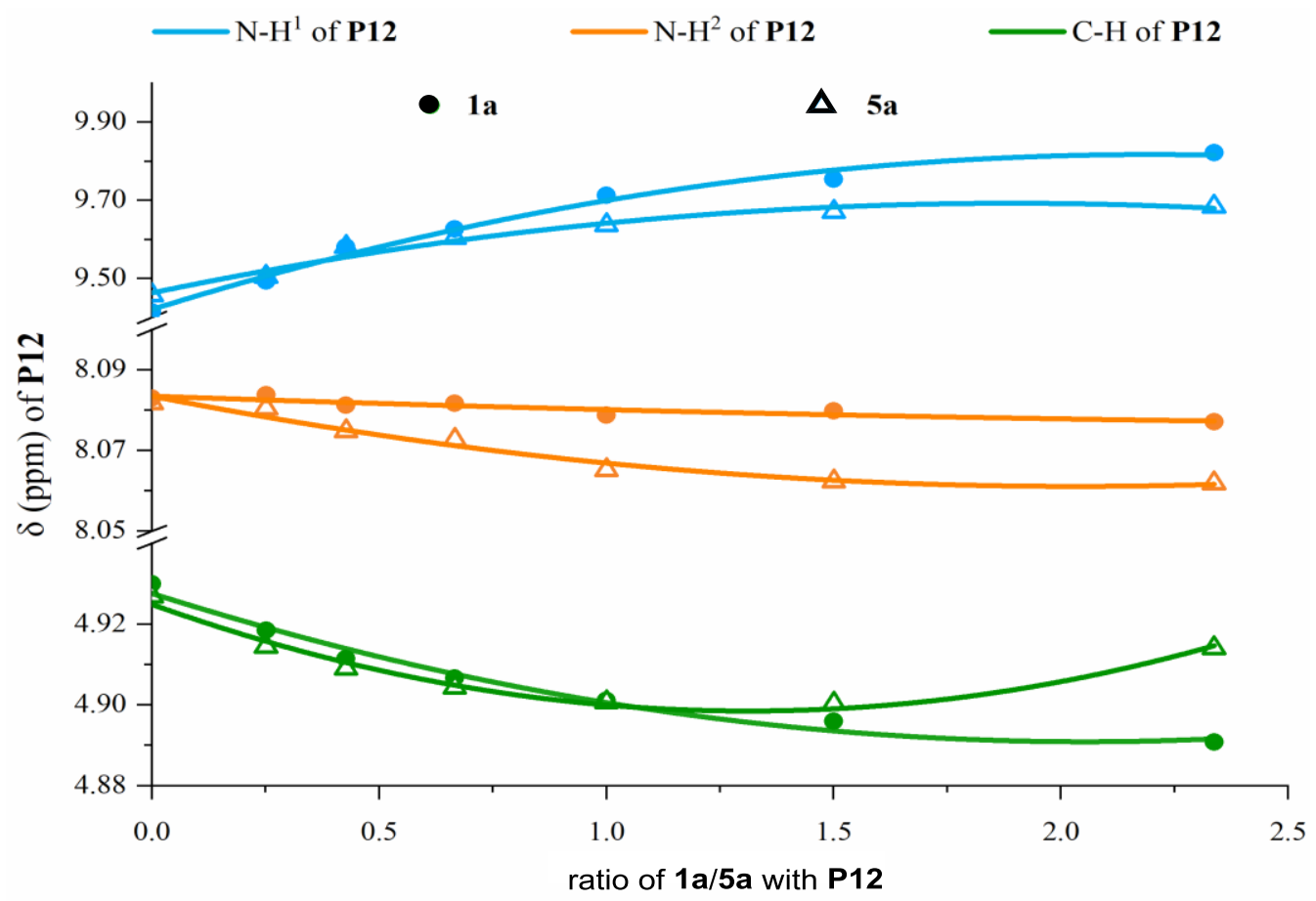

B Job plot analysis

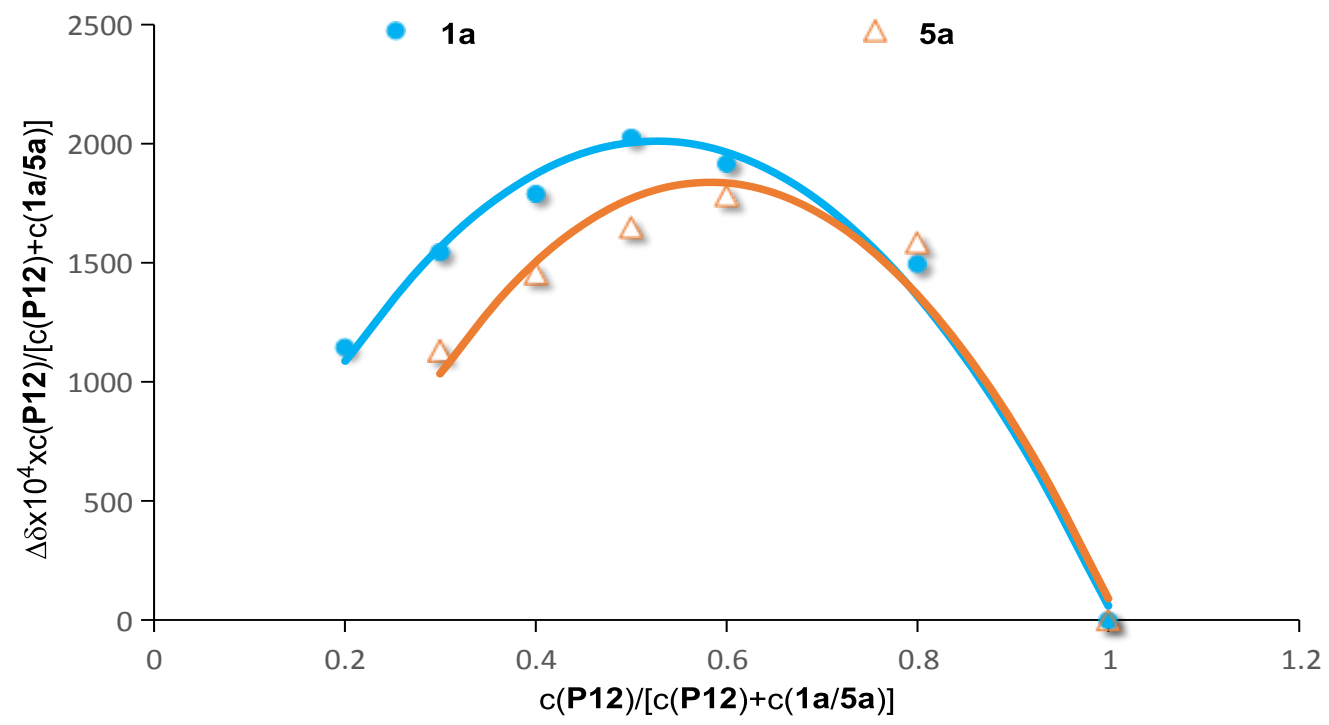

Figure S7. ${ }^{1}$ H NMR titration of $\mathbf{1 a} / \mathbf{5 a}$ with $\mathbf{P 1 2}$

Taking into account the combined results of our mechanistic studies and our previous explorations in phosphonium salt catalysis, a plausible mechanistic cycle is outlined in Figure S8. In general, the base-triggered deprotonation of free sulfoximines 1 that bear pre-existing S-stereocenters initially occurred to generate the 
anionic intermediate In-A, which might be stabilized by phosphonium cation via ion-pair interaction. Then, the nucleophilic substitution of the deprotonated In-A to sulfonyl chlorides such as $\mathbf{5 a}$ proceeded via the transition state TS1-S, thus providing the desymmetrizing $(S)-\mathbf{6}$ as major product with simultaneously releasing the catalyst. In some cases, the unwelcome minority $(R)-6$ would be further transferred into achiral byproduct $\mathbf{6}^{\prime}$ via the second KR cycle, thus facilitating the enantioselectivities for the desymmetrization cycle.

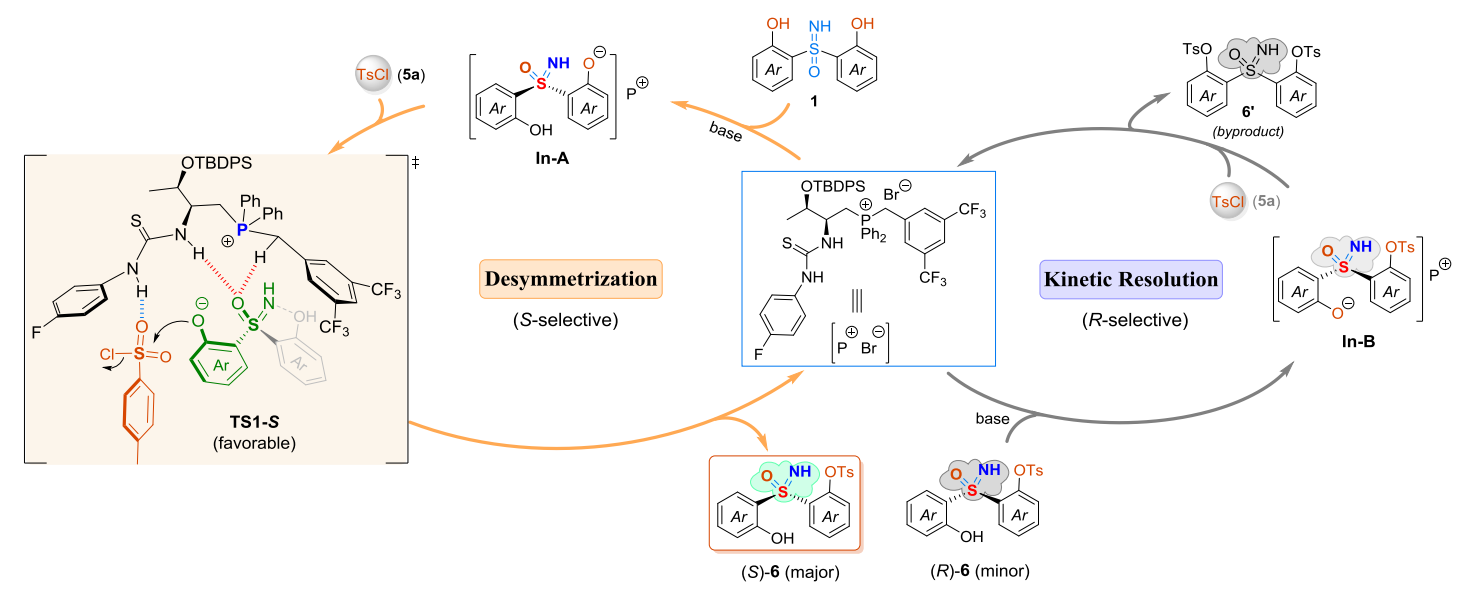

Figure S8. Proposed reaction mechanism 


\section{References}

(1) (a) Han, X.; Wang, Y.; Zhong, F.; Lu, Y. Enantioselective [3+2] Cycloaddition of Allenes to Acrylates Catalyzed by Dipeptide-Derived Phosphines: Facile Creation of Functionalized Cyclopentenes Containing Quaternary Stereogenic Centers. J. Am. Chem. Soc. 2011, 133, 1726-1729. (b) Zhong, F.; Han, X.; Wang, Y.; Lu, Y. Highly Enantioselective [3+2] Annulation of Morita-Baylis-Hillman Adducts Mediated by L-Threonine-Derived Phosphines: Synthesis of 3-Spirocyclopentene-2-oxindoles having Two Contiguous Quaternary Centers. Angew. Chem. Int. Ed. 2011, 50, 7837-7841. (c) Han, X.; Zhong, F.; Wang, Y.; Lu, Y. Versatile Enantioselective [3+2] Cyclization between Imines and Allenoates Catalyzed by Dipeptide-Based Phosphines. Angew. Chem. Int. Ed. 2012, 51, 767-770. (d) Zhong, F.; Han, X.; Wang, Y.; Lu, Y. Highly enantioselective [4+2] annulations catalyzed by amino acid-based phosphines: Synthesis of functionalized cyclohexenes and 3-spirocyclohexene-2-oxindoles. Chem. Sci. 2012, 3, 1231-1234. (e) Zhong, F.; Luo, J.; Chen, G.-Y.; Dou, X.; Lu, Y. J. Am. Chem. Soc. 2012, 134, 10222-10227; (f) Zhong, F.; Dou, X.; Han, X.; Yao, W.; Zhu, Q.; Meng, Y.; Lu, Y. Chiral Phosphine Catalyzed Asymmetric Michael Addition of Oxindoles. Angew. Chem. Int. Ed. 2013, 52, 943-947.

(2) (a) Zhu, L.; Ren, X.; Liao, Z.; Pan, J.; Jiang, C.; Wang. T. Asymmetric Three-Component Cyclizations toward Structurally Spiro Pyrrolidines via Bifunctional Phosphonium Salt Catalysis. Org. Lett. 2019, 21, 8667-8672. (b) Wu, J.-H.; Pan, J.; Du, J.; Wang, X.; Wang, X.; Jiang, C.; Wang. T. Enantioselective Synthesis of Multifunctionalized 4H-Pyrans via Formal [4+2] Annulation Process by Bifunctional Phosphonium Salt Catalysis. Org. Lett. 2020, 22, 395-399. (c) Lu, D.; Wu, J.-H.; Pan, J.; Chen, X.; Ren X.; Wang, T. Asymmetric synthesis of benzothiazolopyrimidines with high catalytic efficiency and stereoselectivity under bifunctional phosphonium salt systems. Chem. Commun. 2020, 56, 11231-11234.

(3) Tsang, K. Y.; Brimble, M. A.; Bremner, J. B. Use of a Sonogashira-Acetylide Coupling Strategy for the Synthesis of the Aromatic Spiroketal Skeleton of $\gamma$-Rubromycin. Org. Lett. 2003, 5, 4425-4427.

(4) Zhao, P.; Yin, H.; Gao, H.; Xi. C. Cu-Catalyzed Synthesis of Diaryl Thioethers and S-Cycles by Reaction of Aryl Iodides with Carbon Disulfide in the Presence of DBU. J. Org. Chem. 2013, 78, 5001-5006.

(5) Tota, A.; Zenzola, M.; Chawner, S. J.; John-Campbell, S. S.; Carlucci, C.; Romanazzi, G.; Degennaro, L.; Bull, J. A.; Luisi, R. Synthesis of NH-sulfoximines from sulfides by chemoselective one-pot N- and O-transfers. Chem. Commun. 2017, 53, 348-351.

(6) Zhu, Y.-C.; Li, Y.; Zhang, B.-C.; Zhang, F.-X.; Yang, Y.-N.; Wang, X.-S. Palladium-Catalyzed Enantioselective C-H Olefination of Diaryl Sulfoxides through Parallel Kinetic Resolution and Desymmetrization. Angew.Chem. Int.Ed. 2018, 57, 5129-5133. 


\section{NMR Spectra of New Compounds}

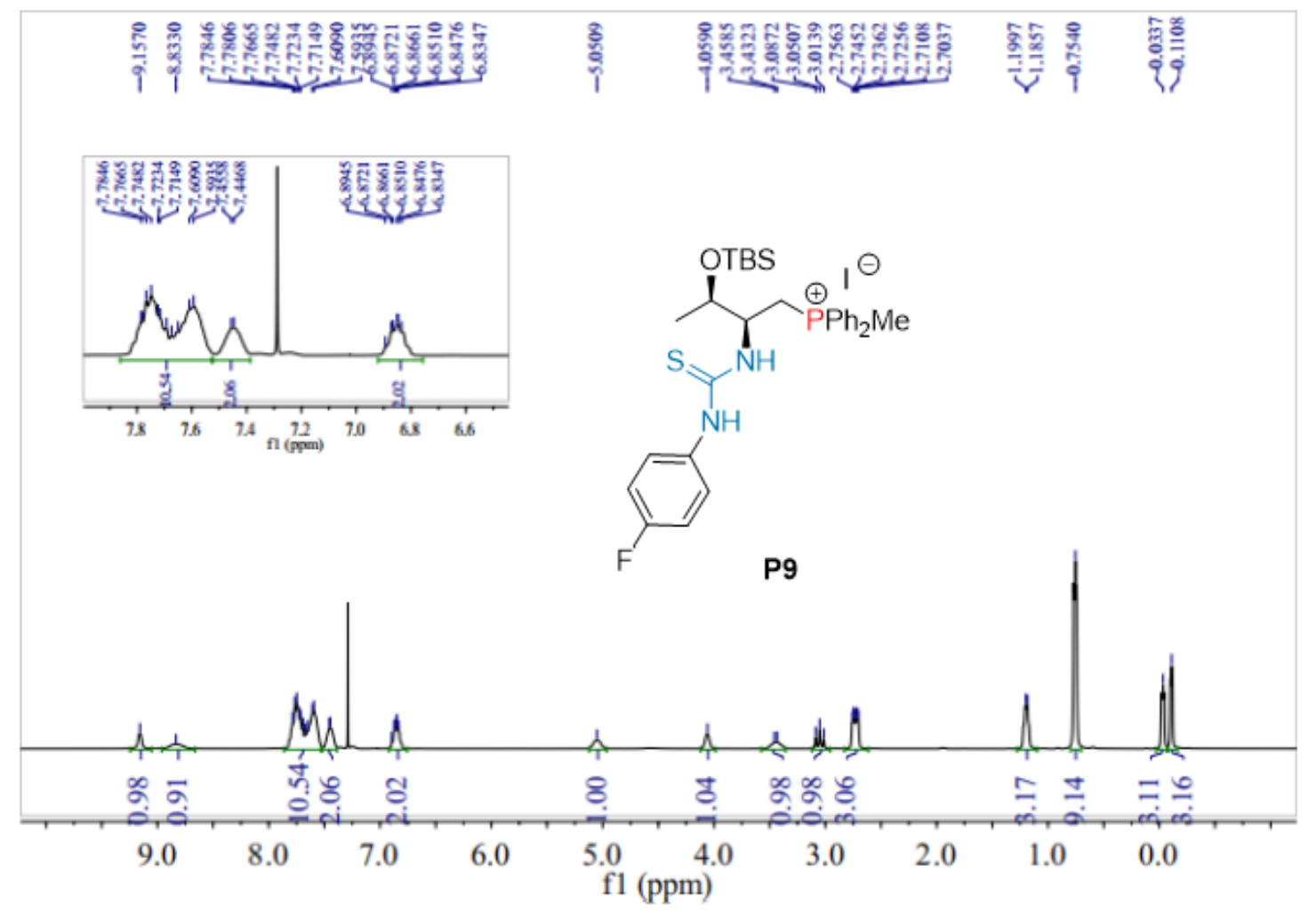

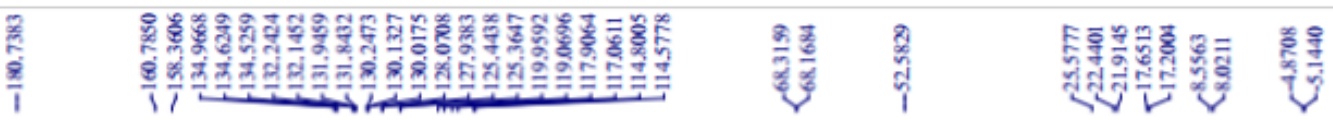
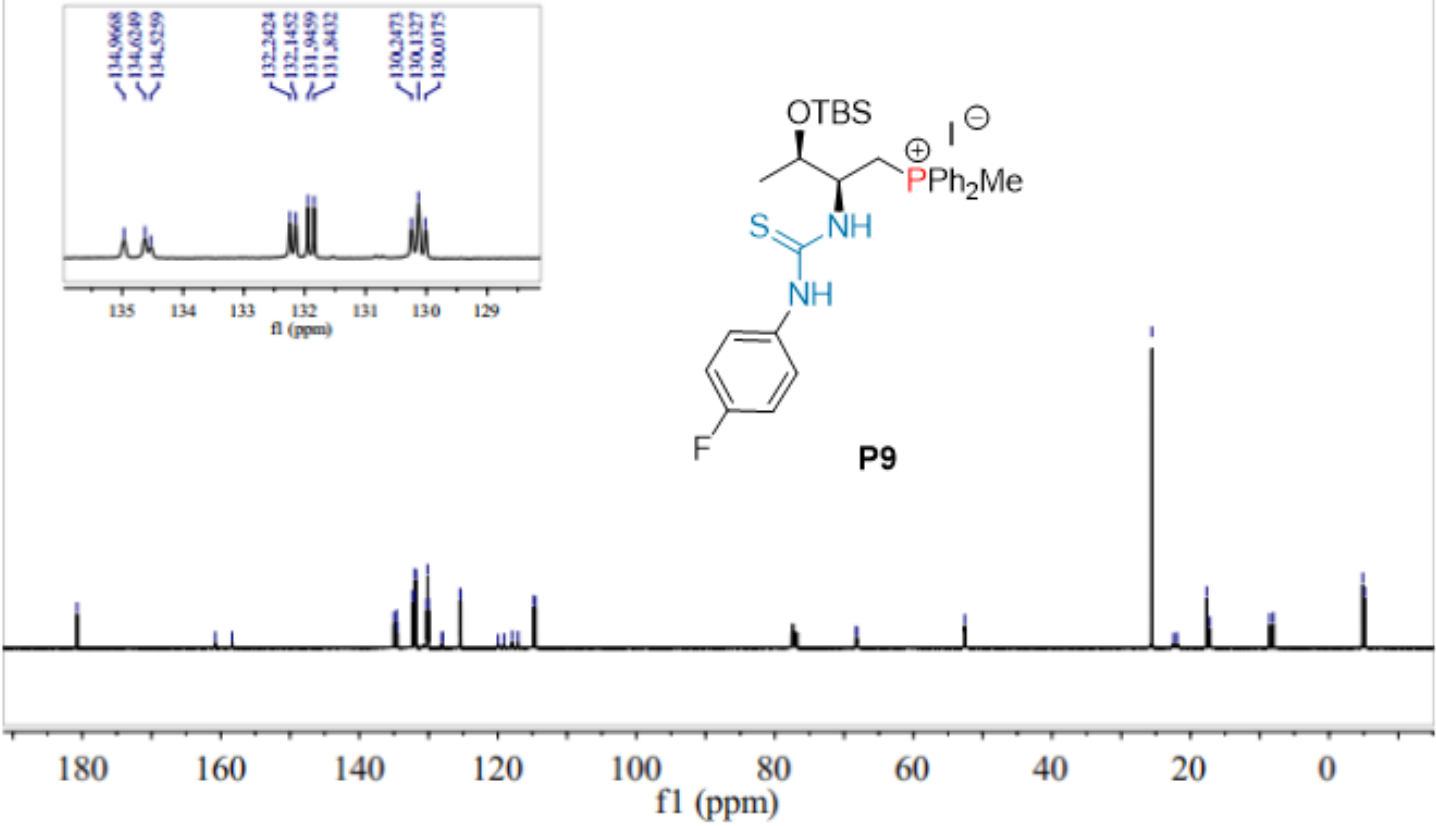

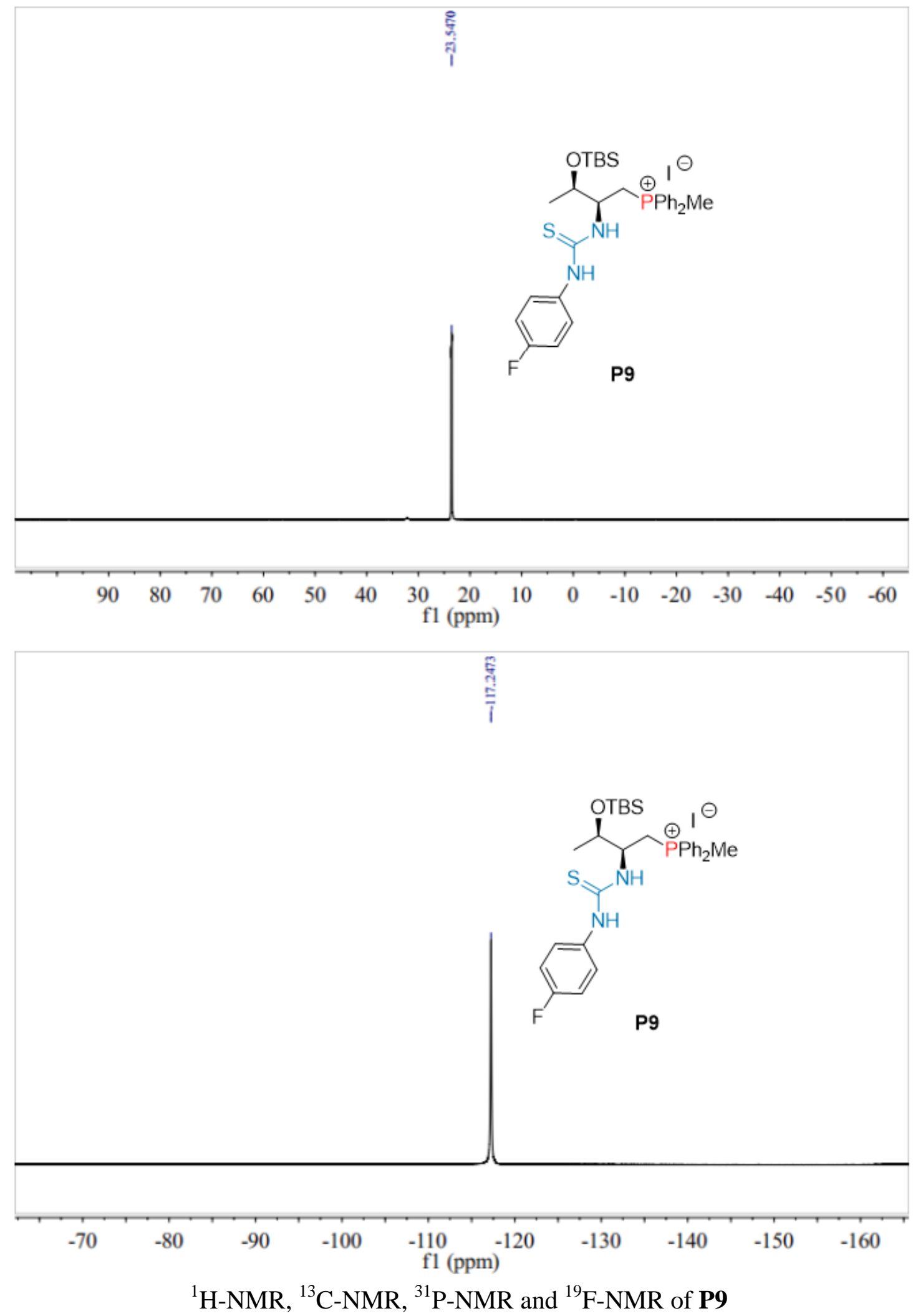


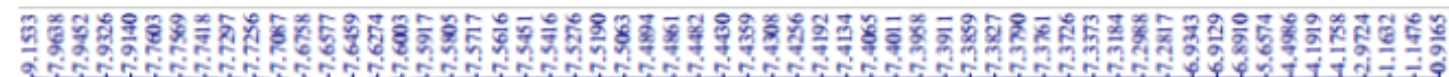
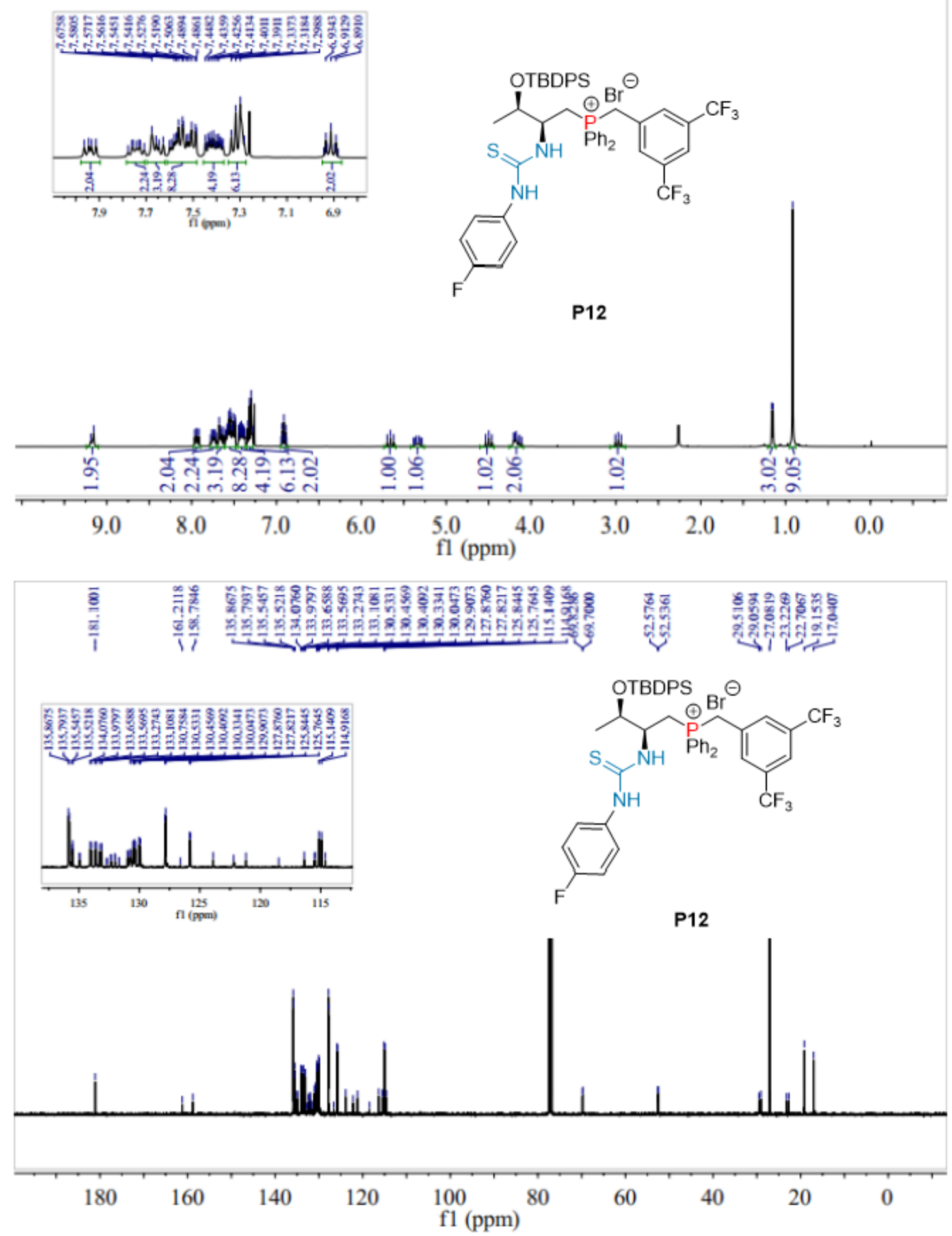

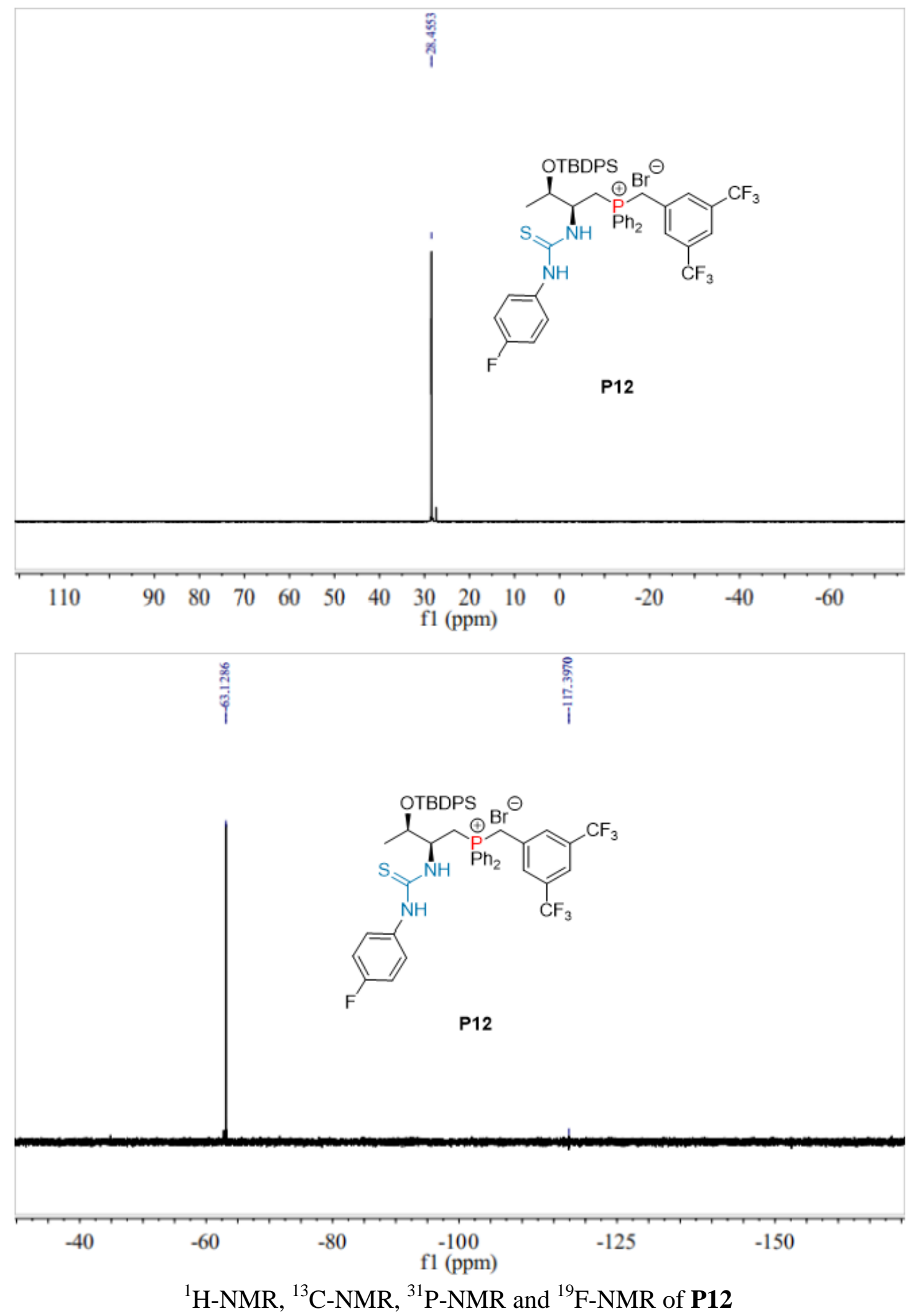


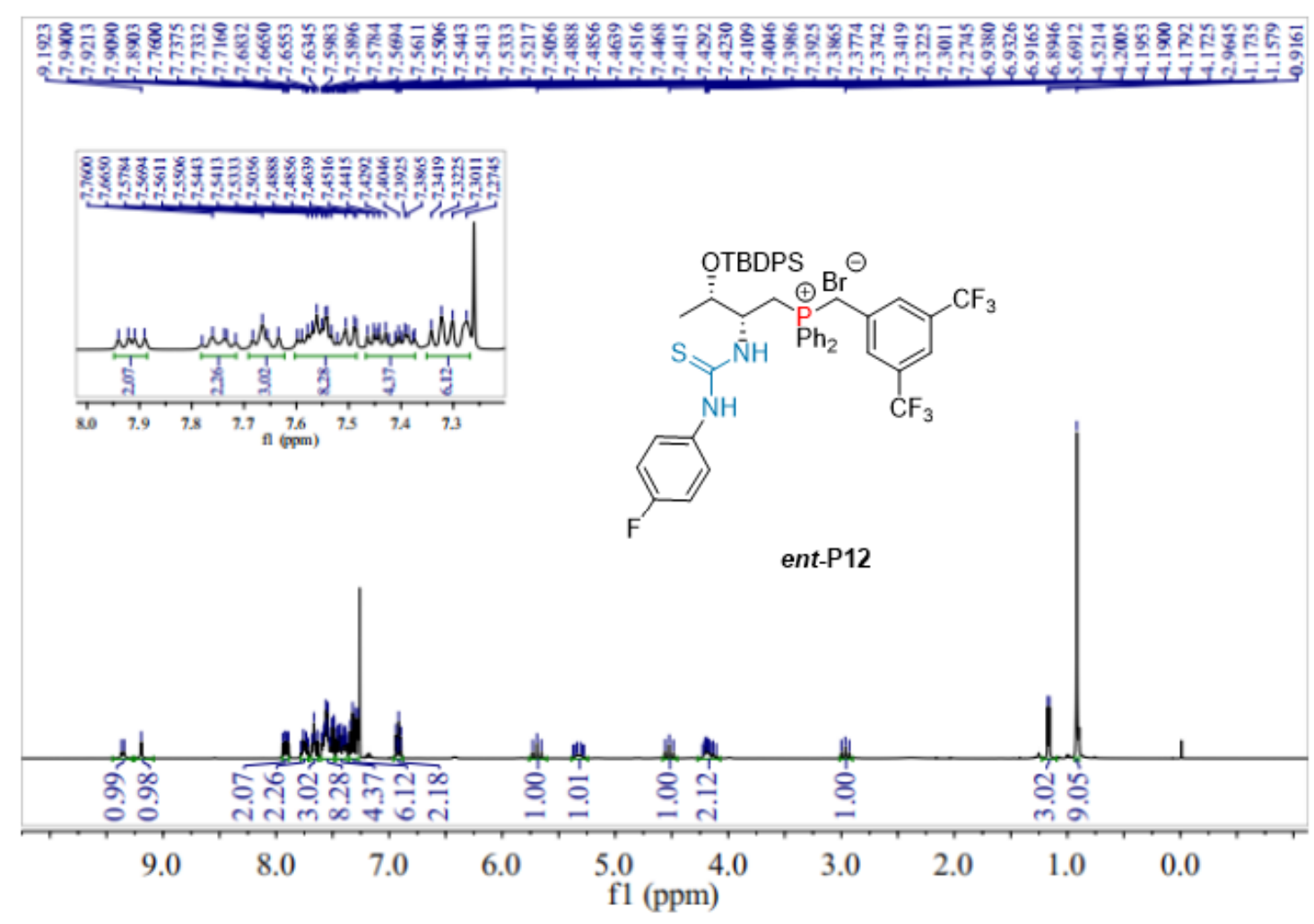

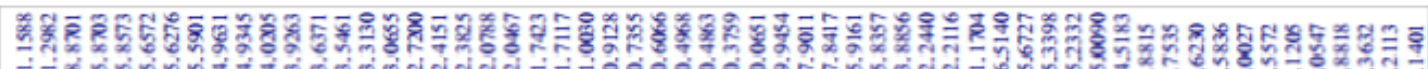

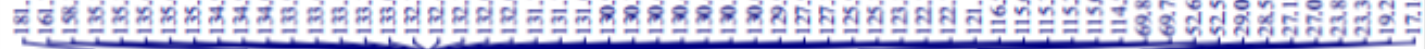

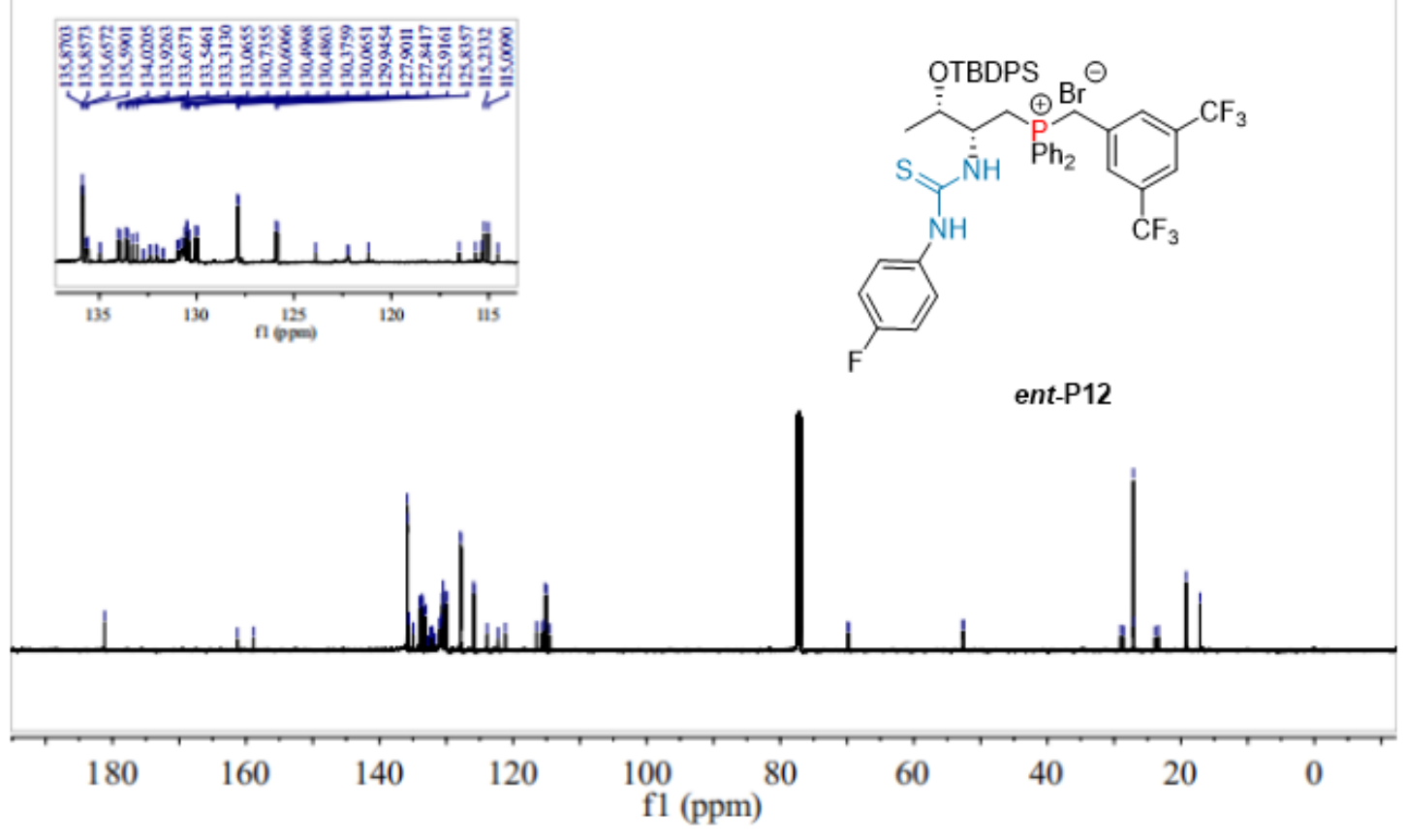



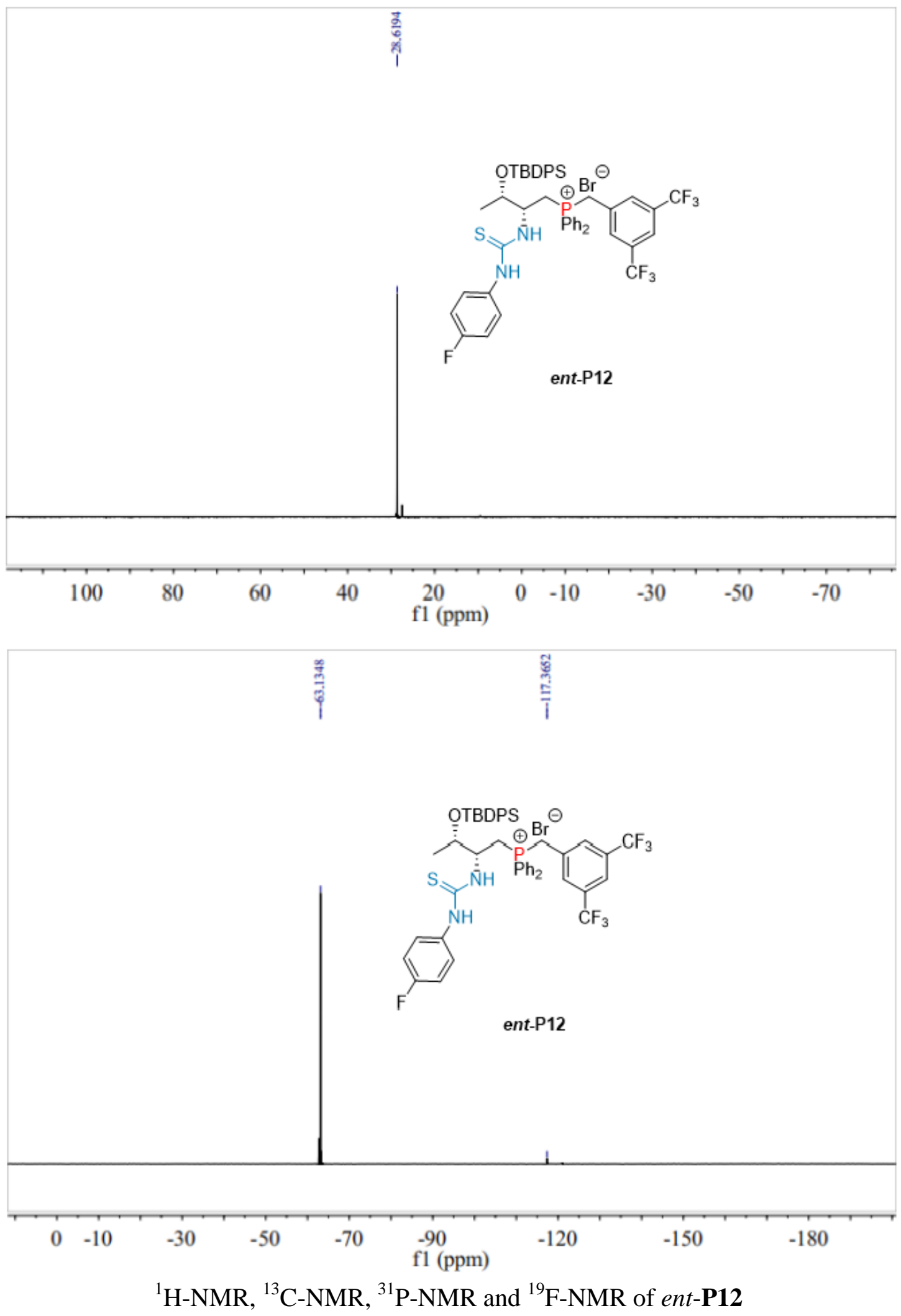

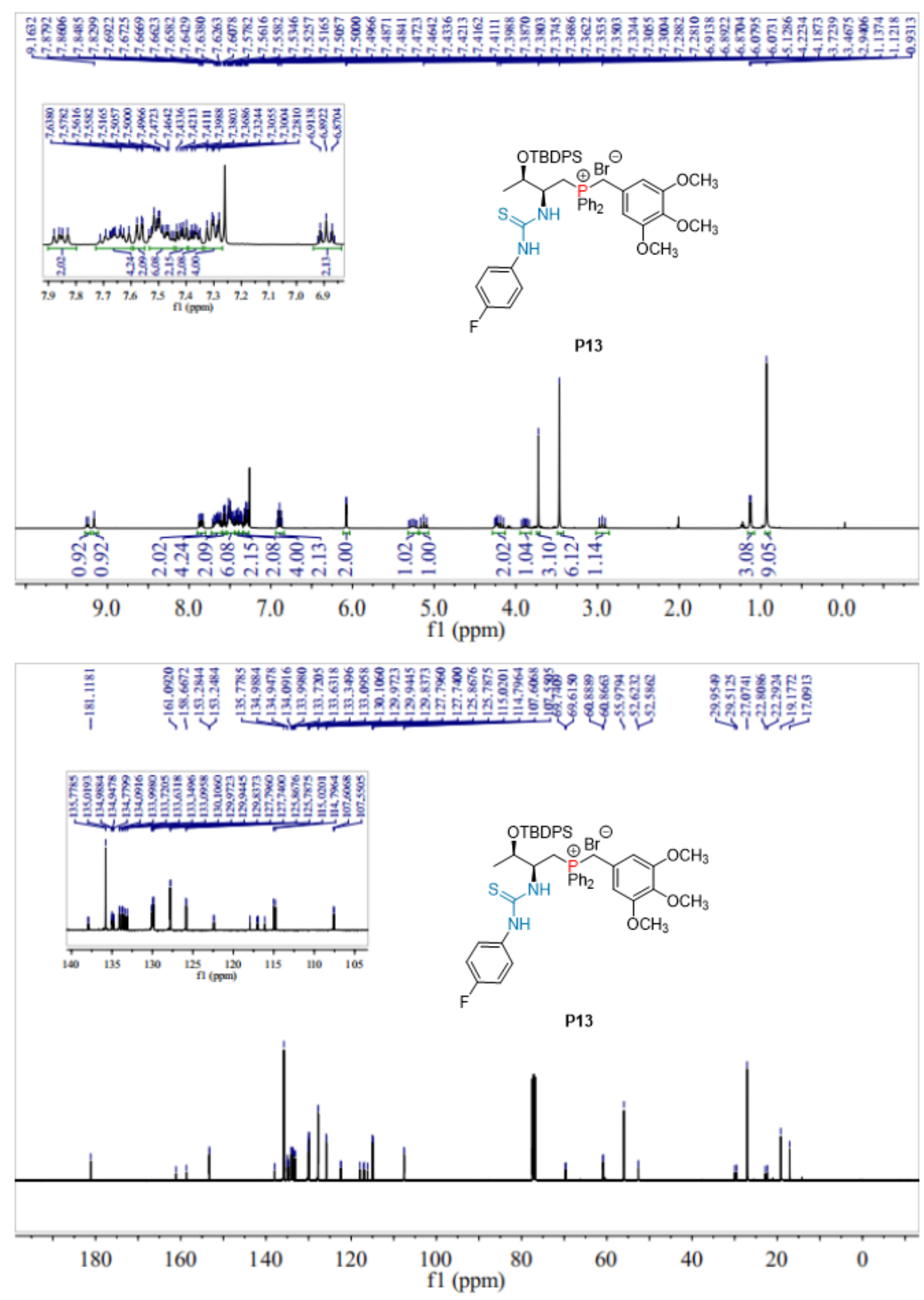

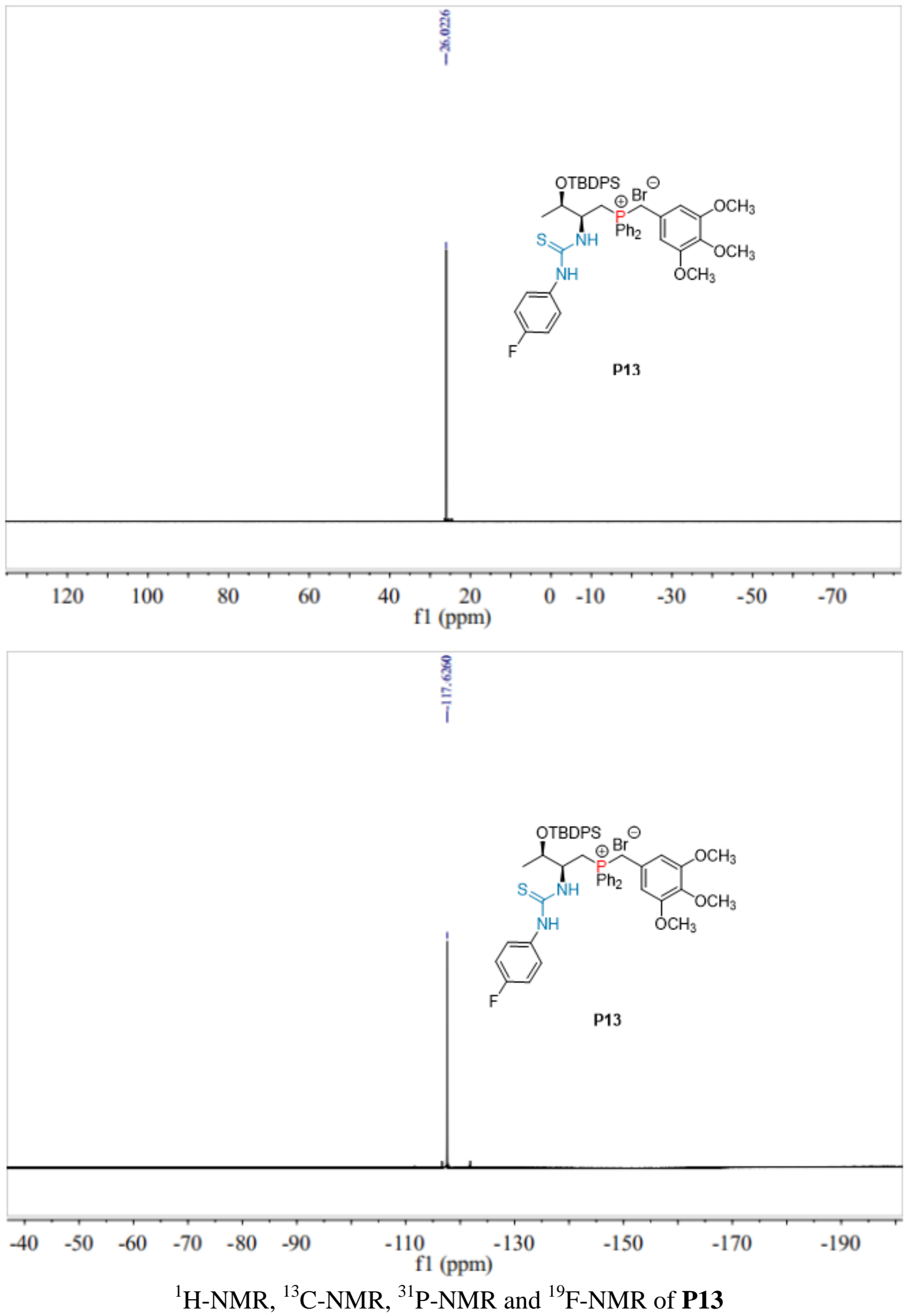

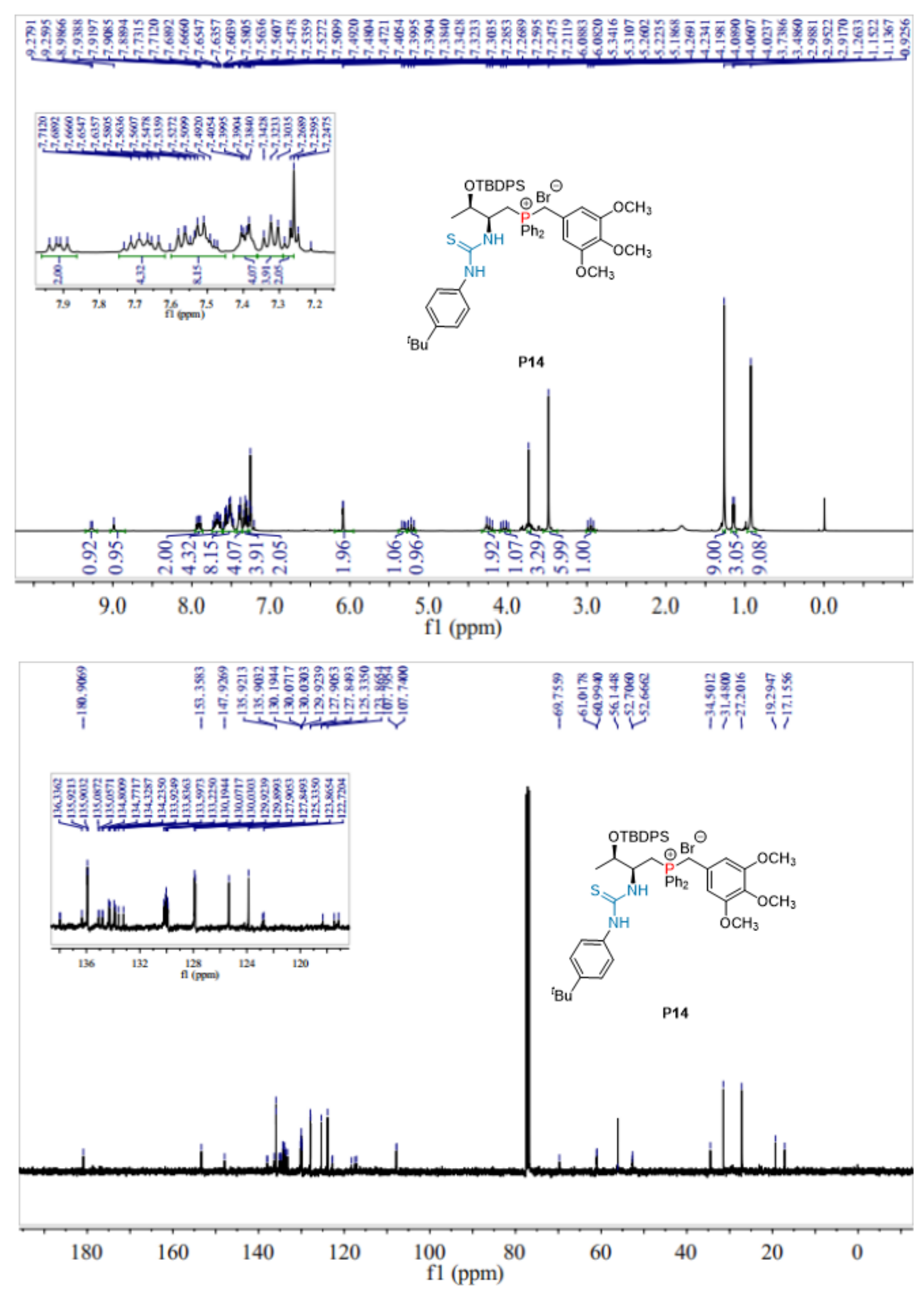


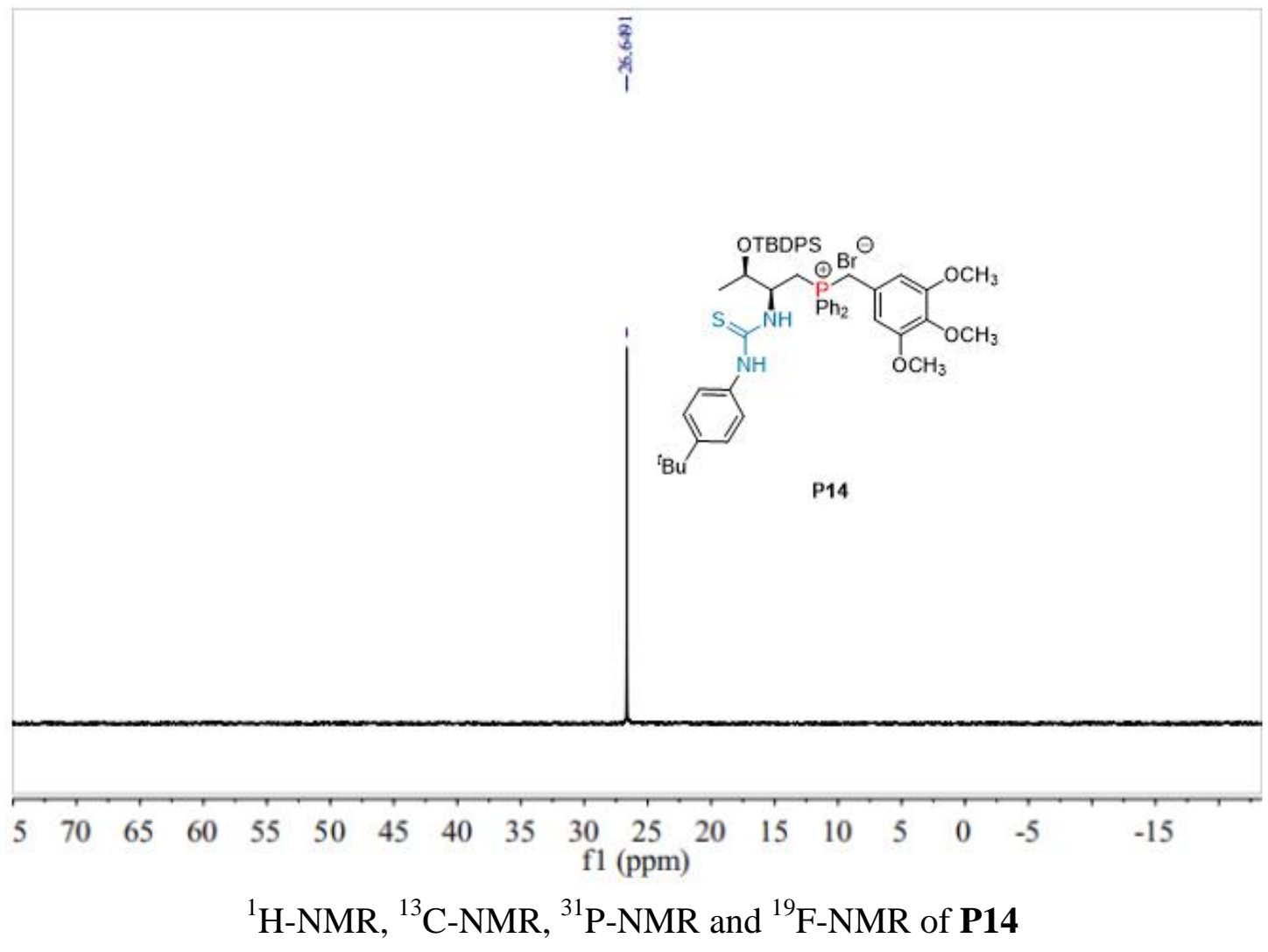



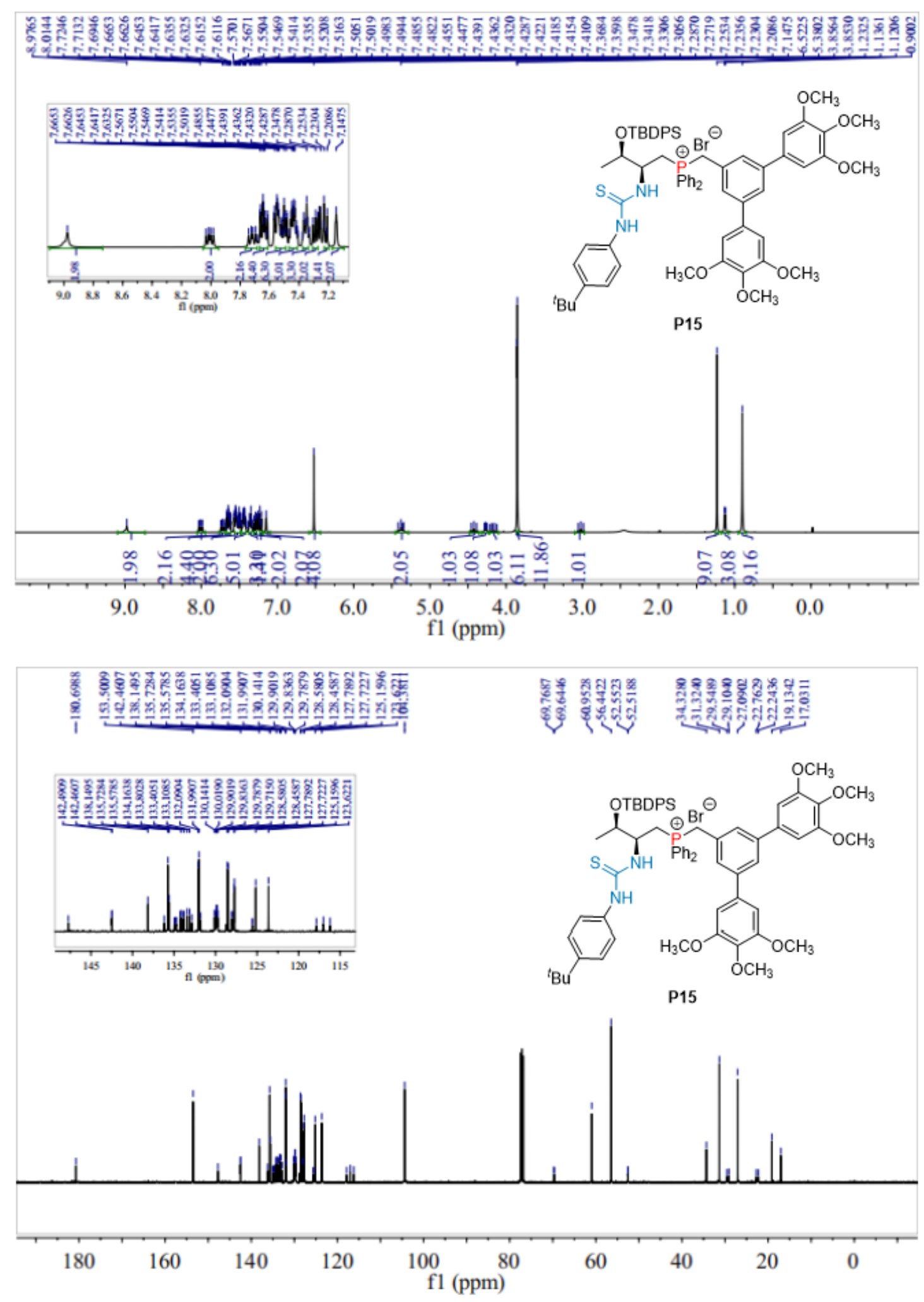


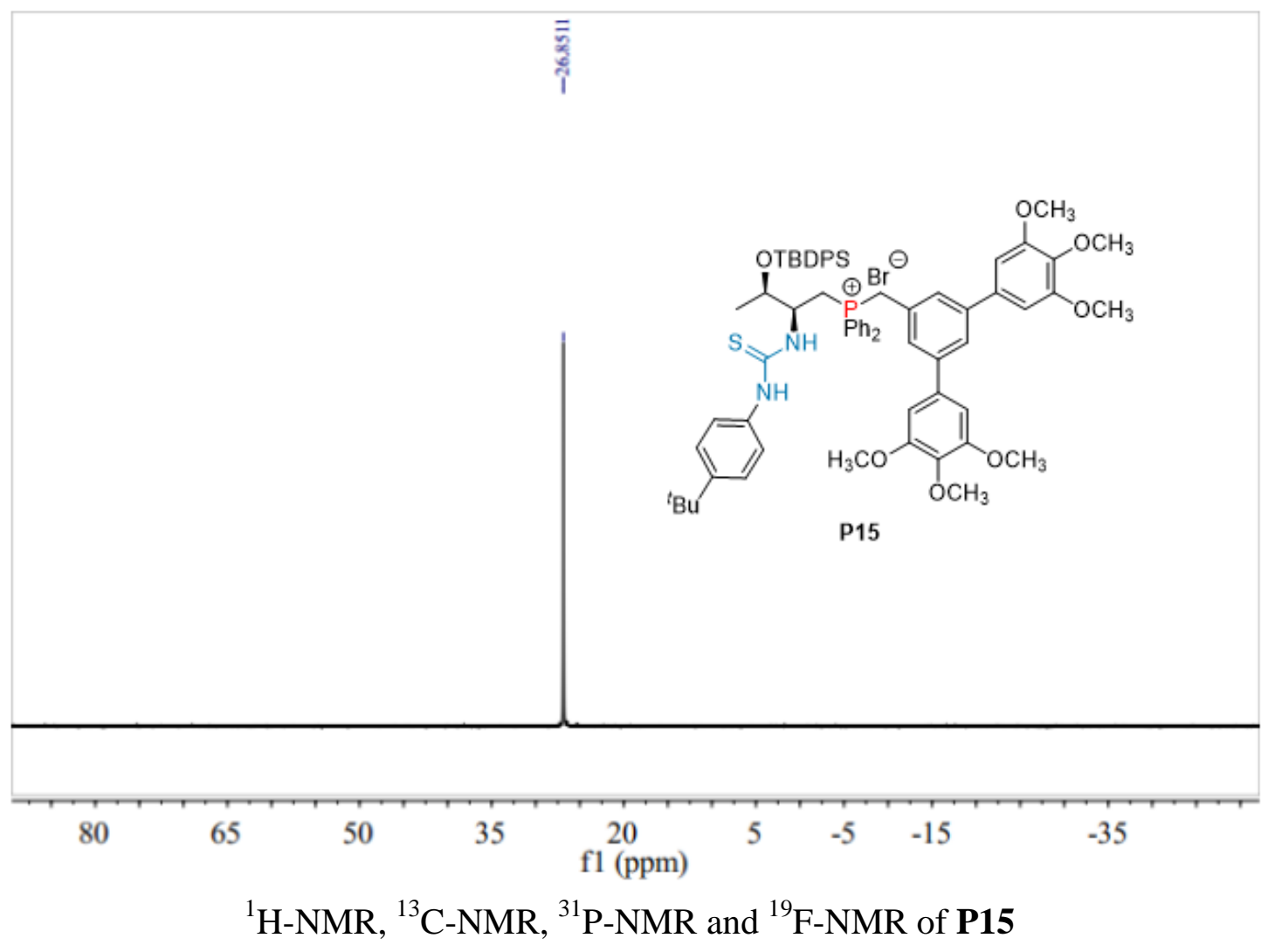




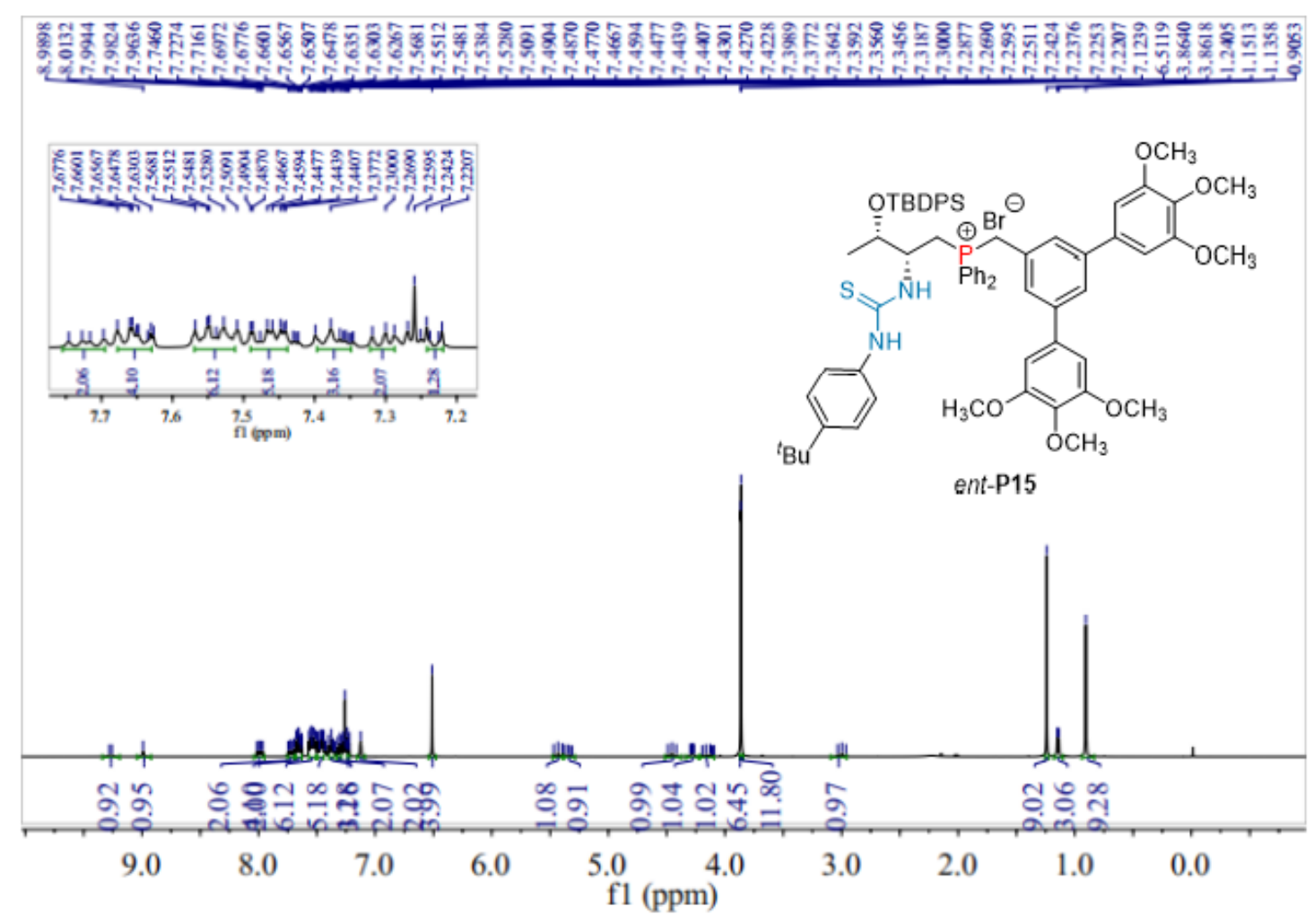

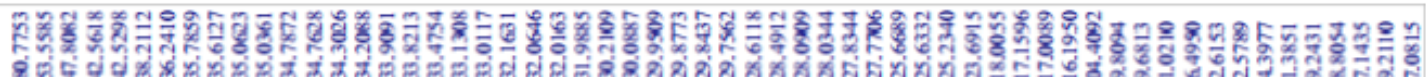

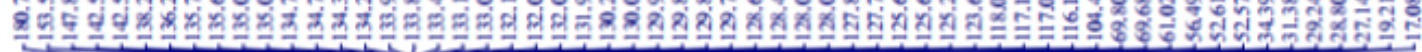

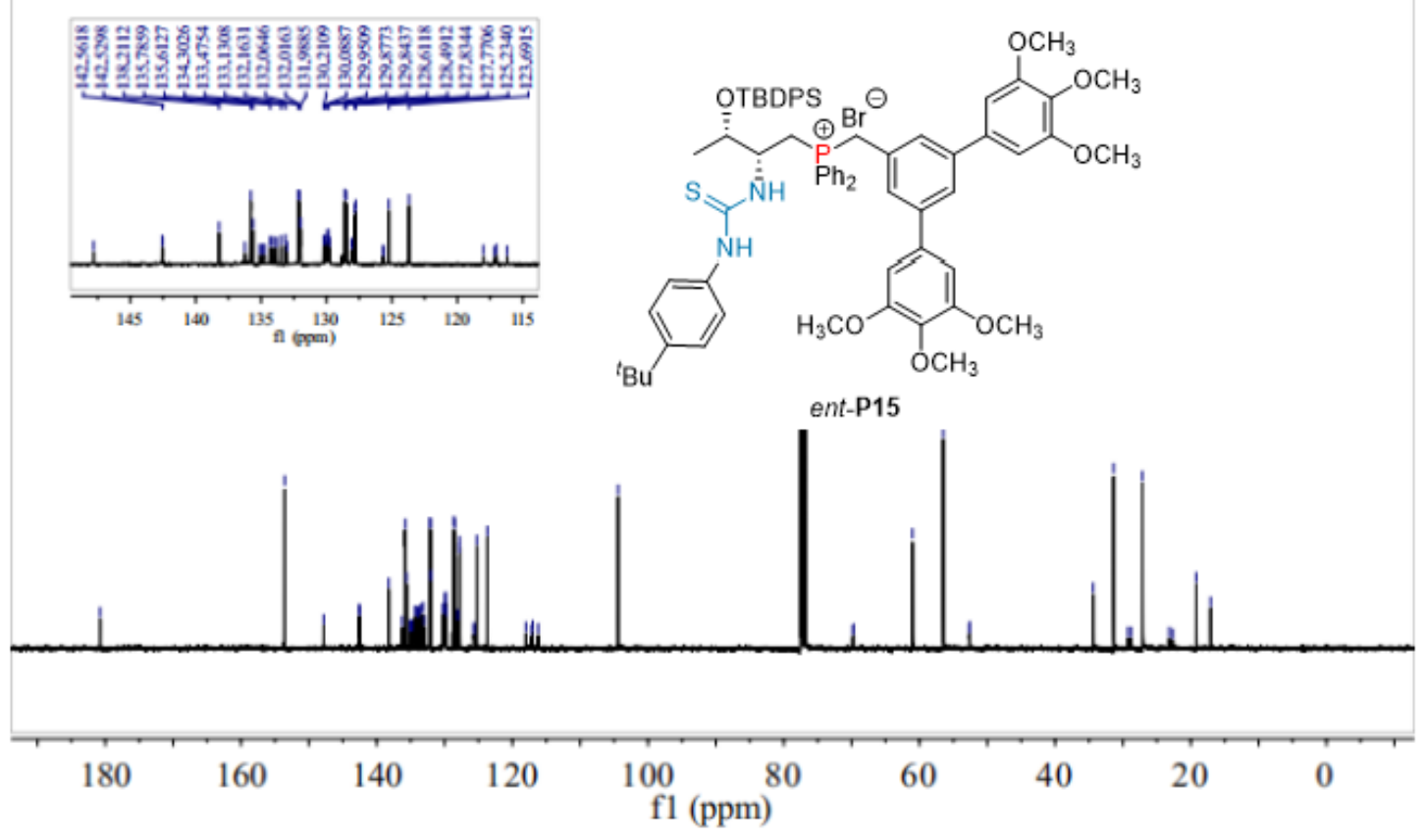




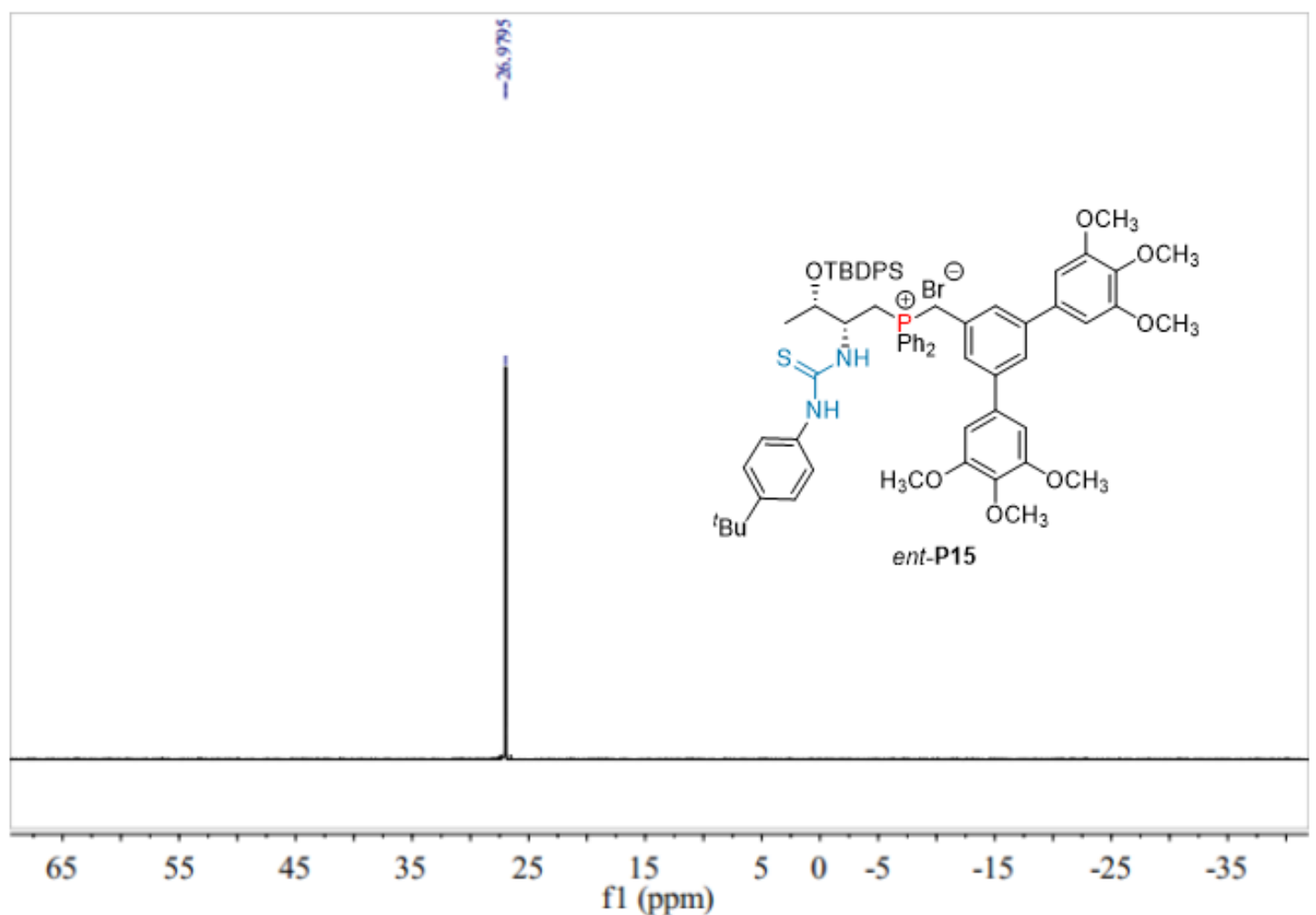

${ }^{1} \mathrm{H}-\mathrm{NMR},{ }^{13} \mathrm{C}-\mathrm{NMR},{ }^{31} \mathrm{P}-\mathrm{NMR}$ and ${ }^{19} \mathrm{~F}-\mathrm{NMR}$ of ent-P15 

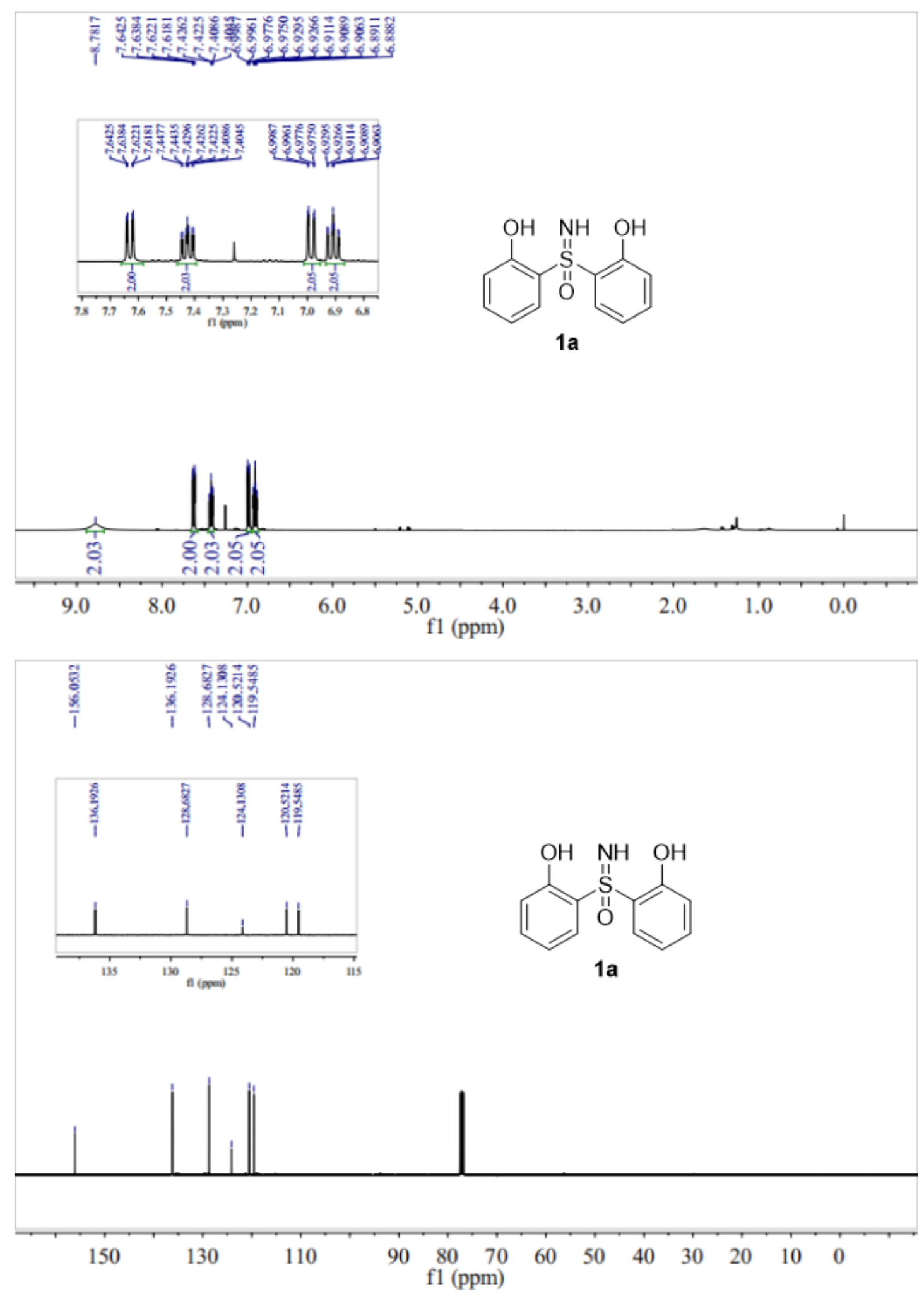

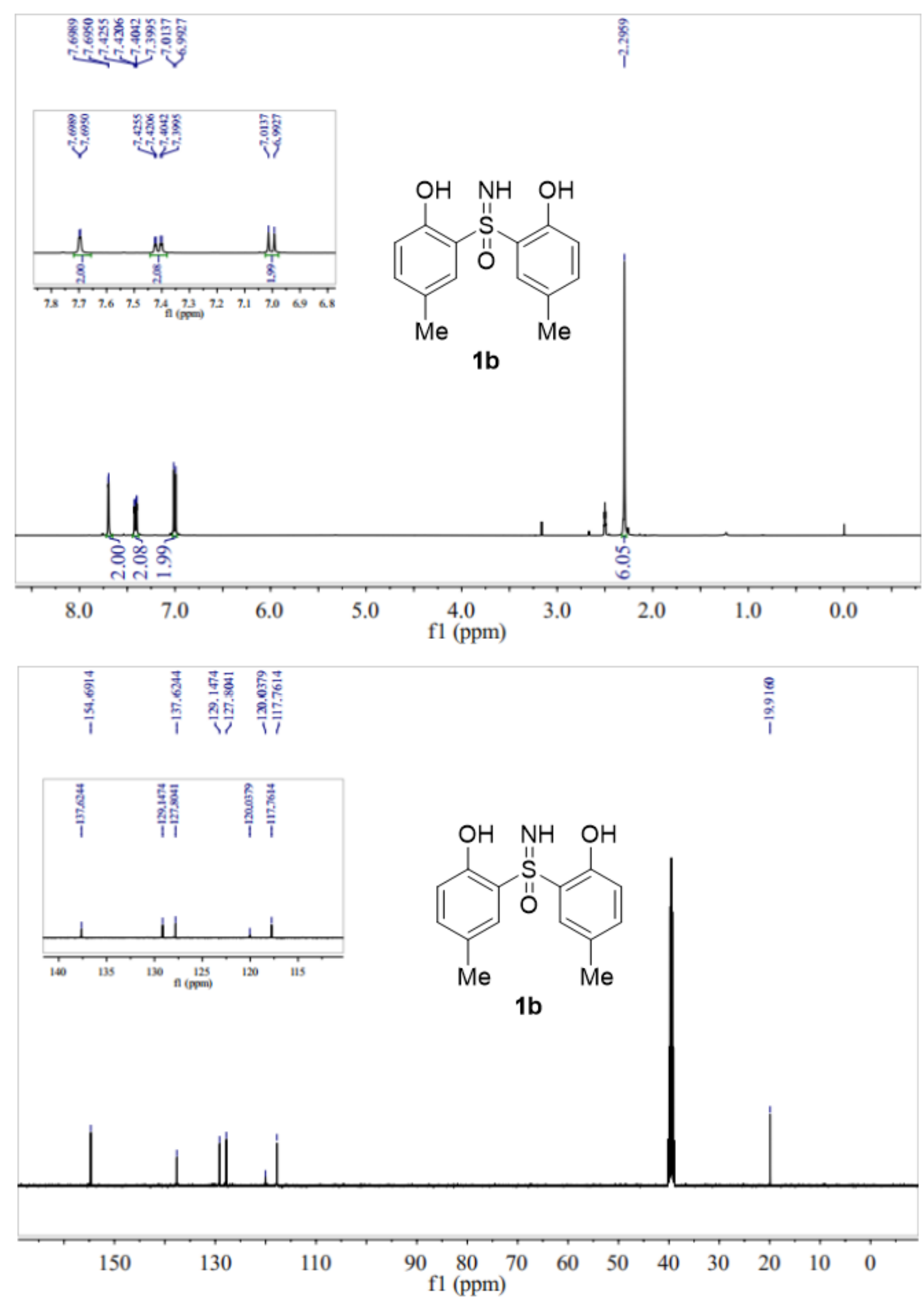

${ }^{1} \mathrm{H}-\mathrm{NMR}$ and ${ }^{13} \mathrm{C}-\mathrm{NMR}$ of $\mathbf{1 b}$ 

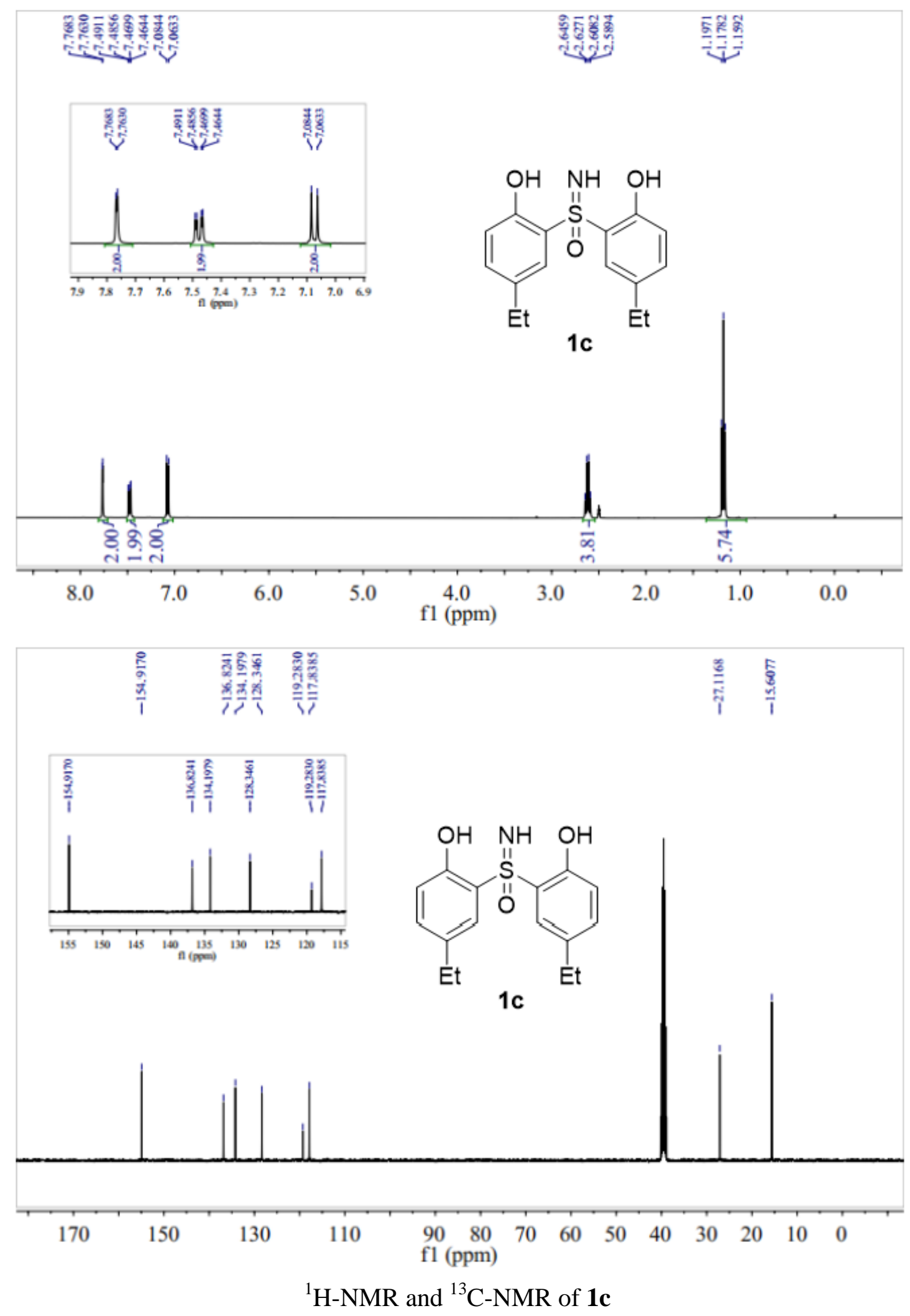

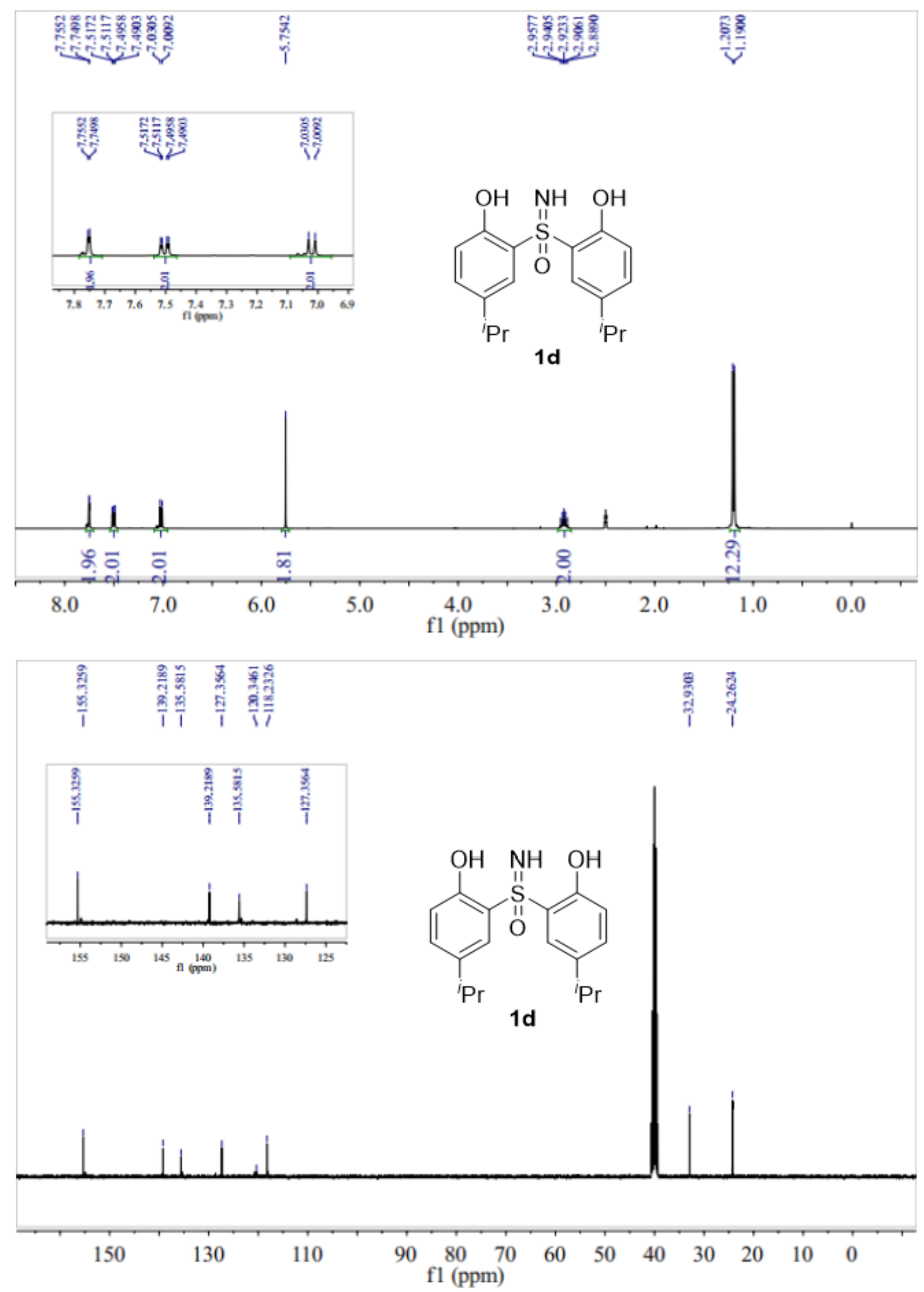

${ }^{1} \mathrm{H}-\mathrm{NMR}$ and ${ }^{13} \mathrm{C}-\mathrm{NMR}$ of $\mathbf{1 d}$ 

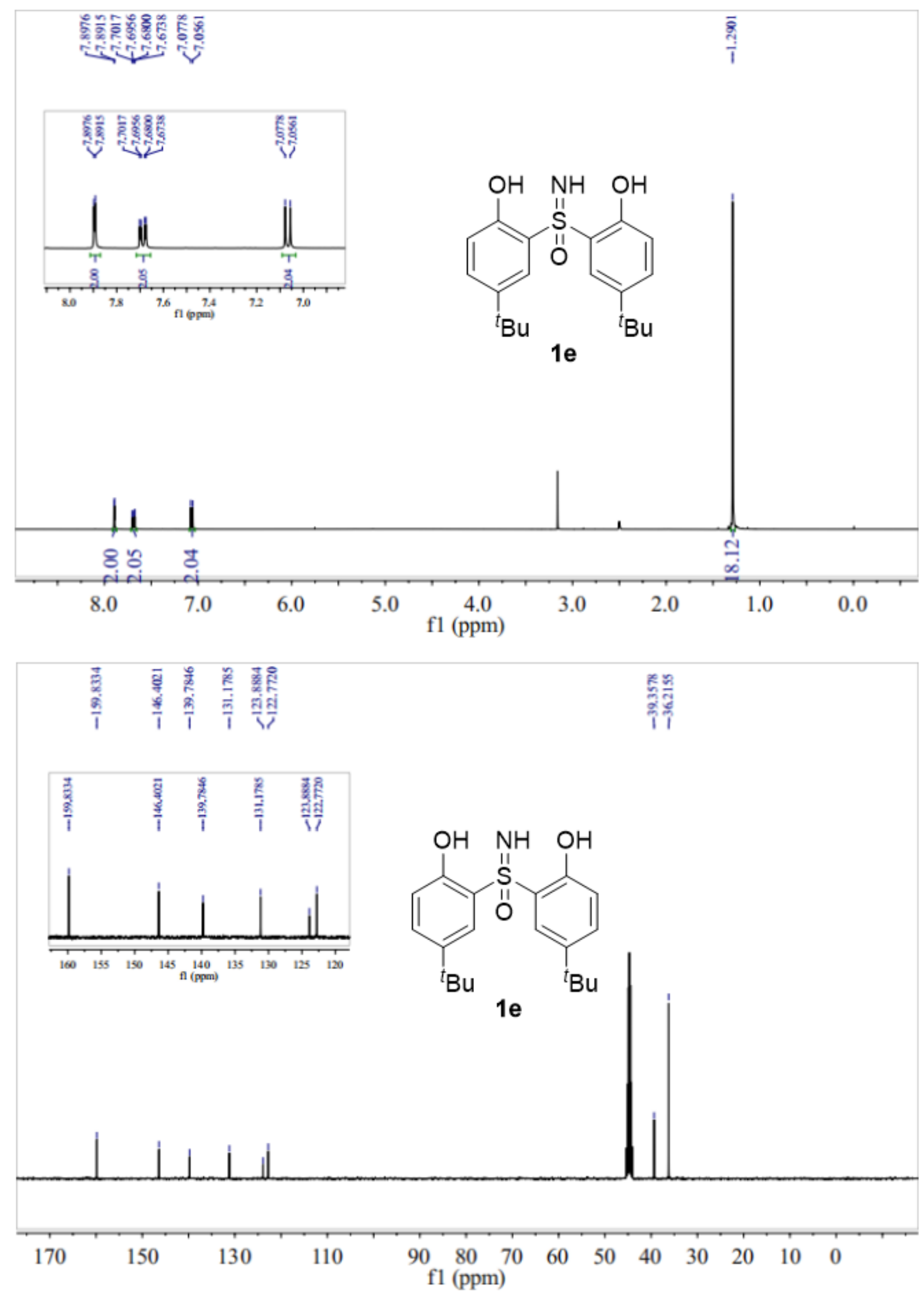

${ }^{1} \mathrm{H}-\mathrm{NMR}$ and ${ }^{13} \mathrm{C}-\mathrm{NMR}$ of $\mathbf{1 e}$ 

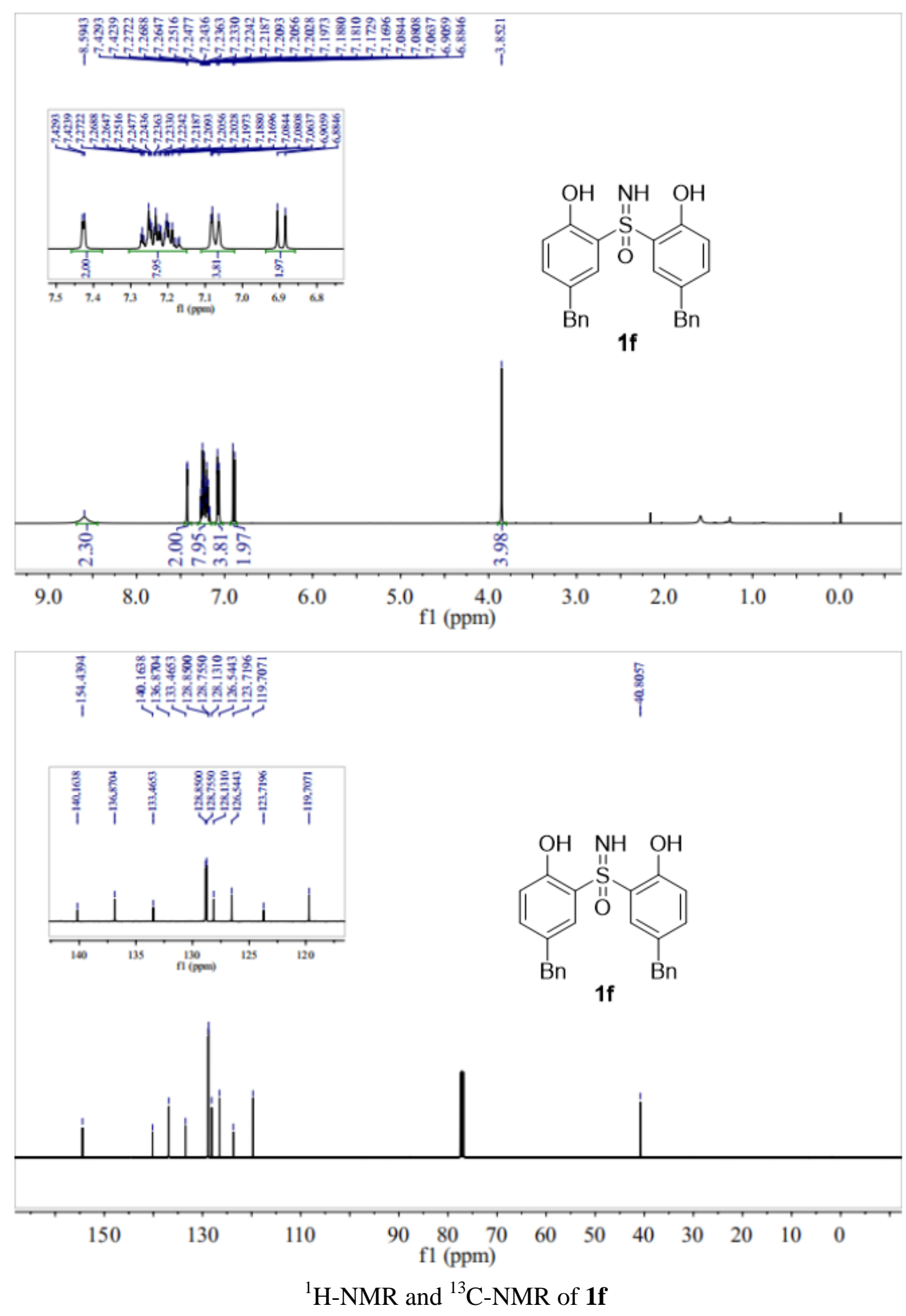


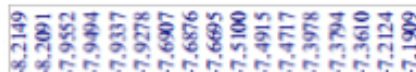

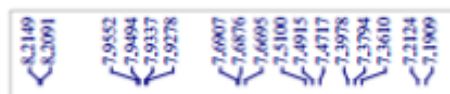
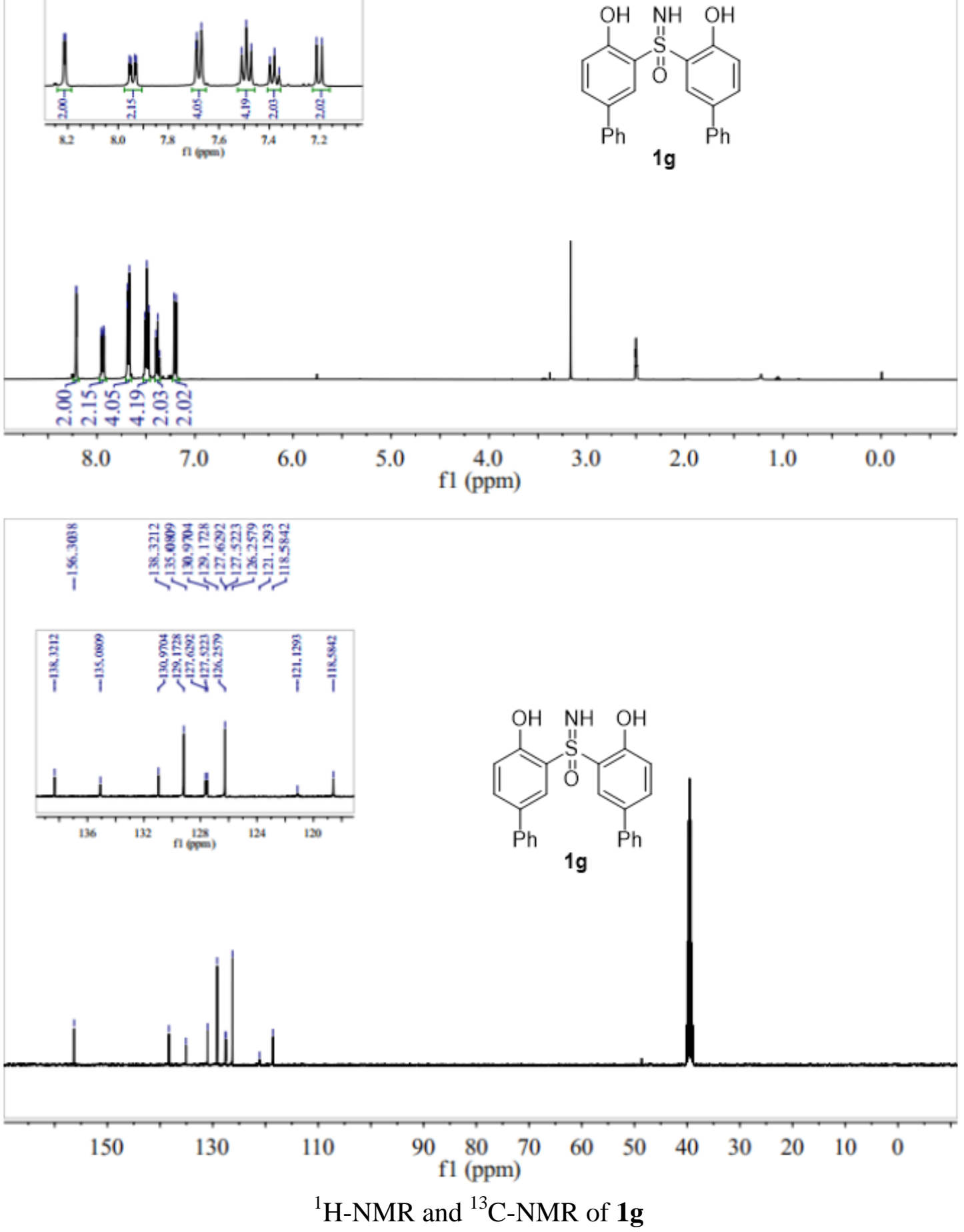

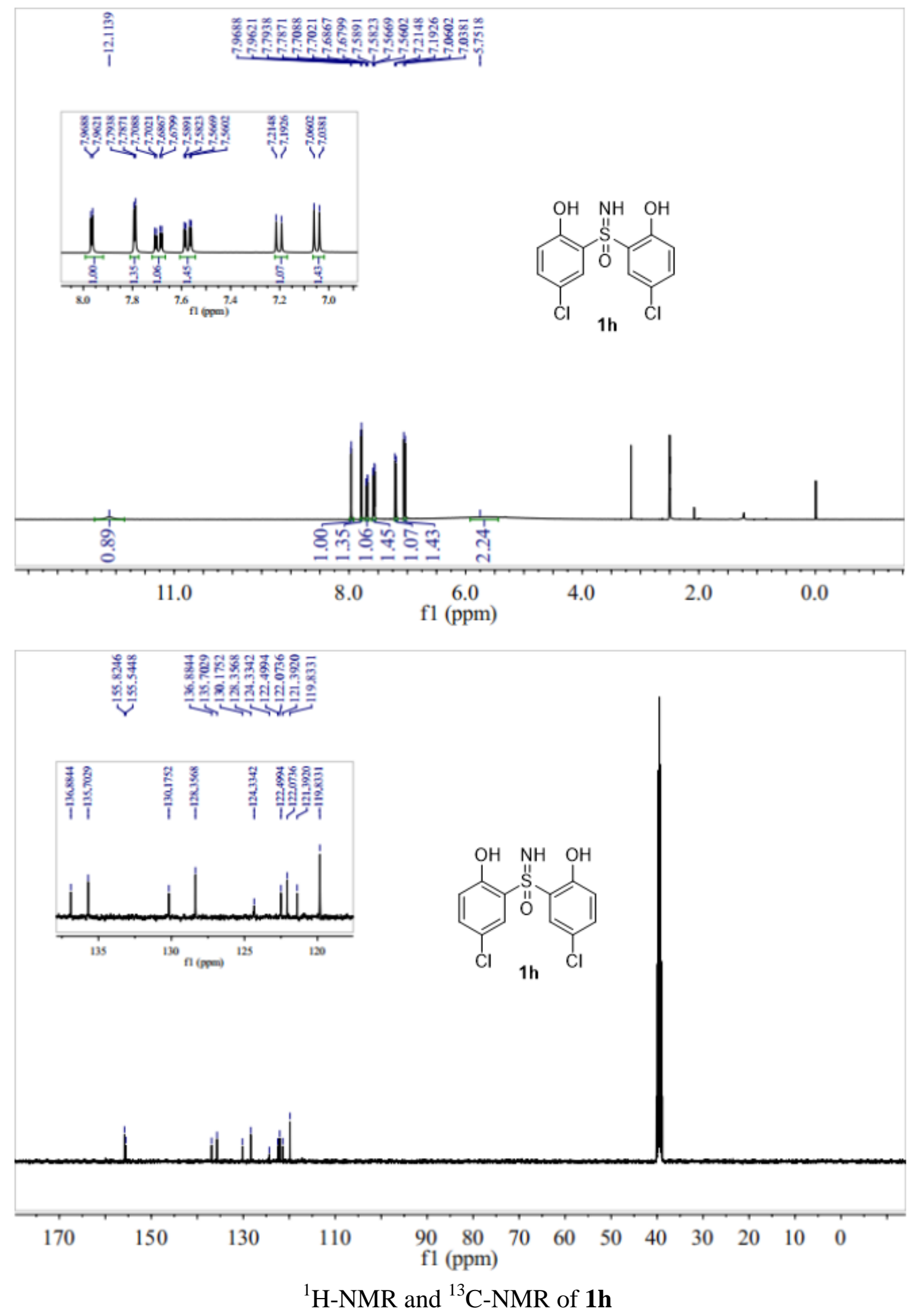

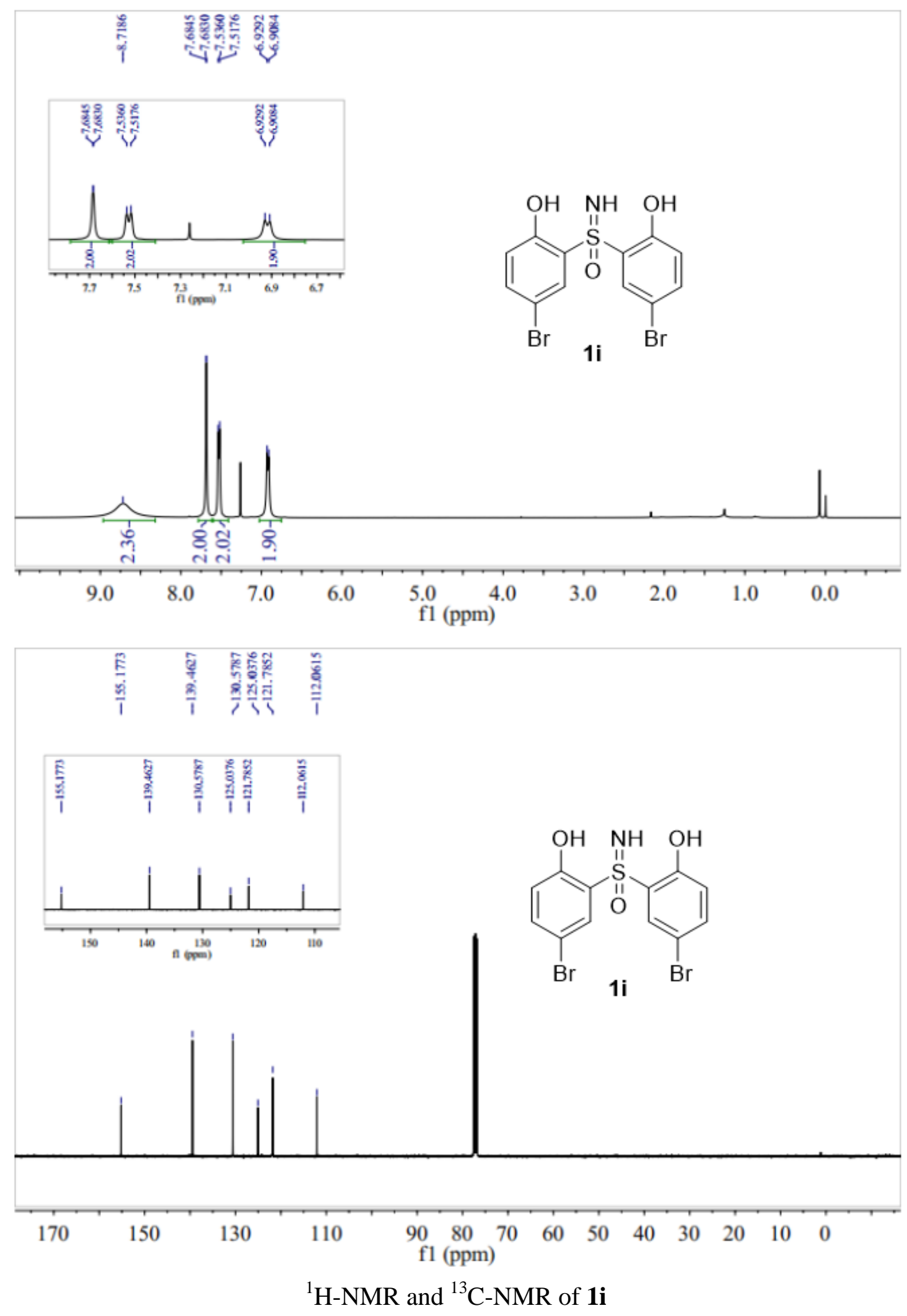

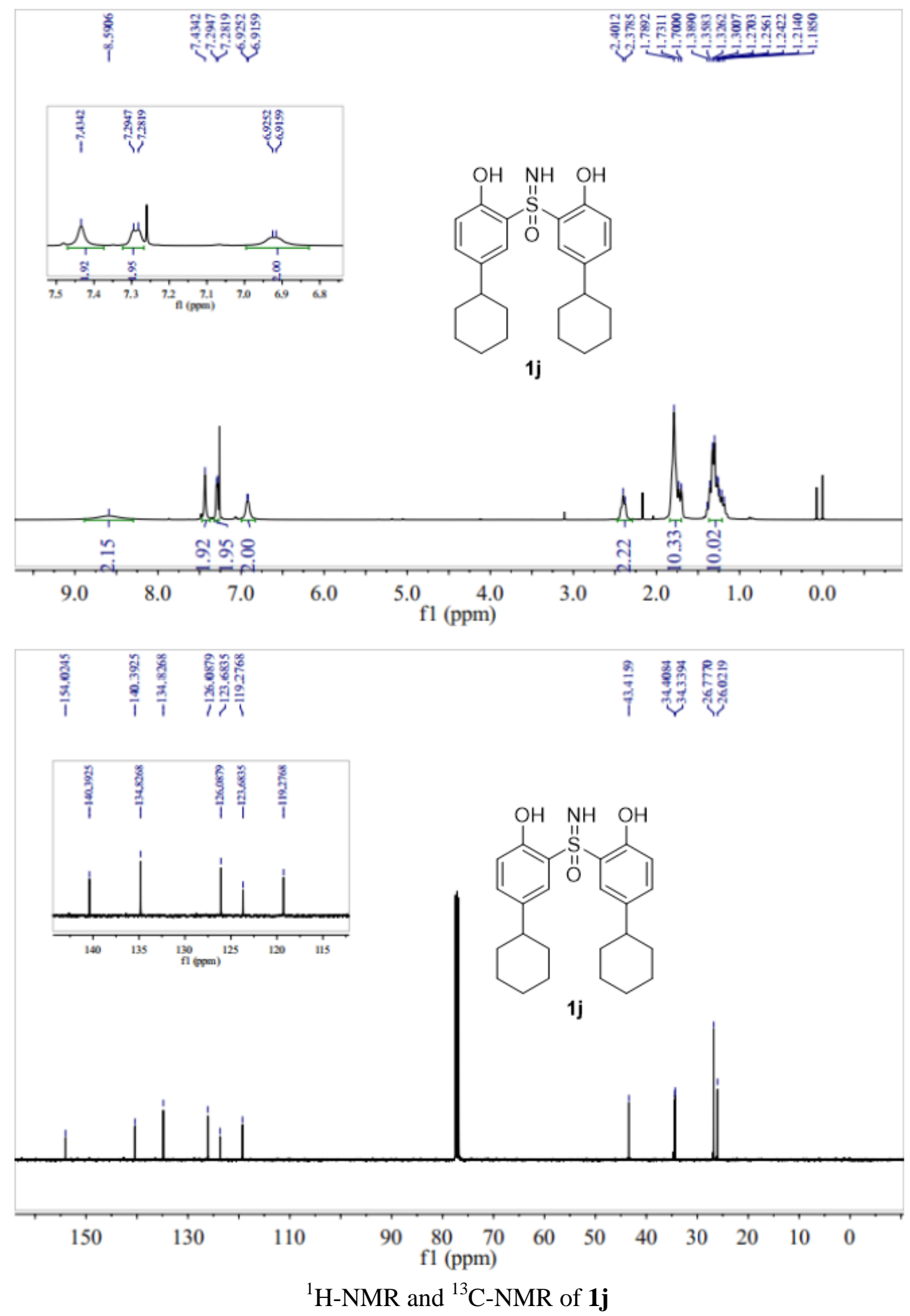

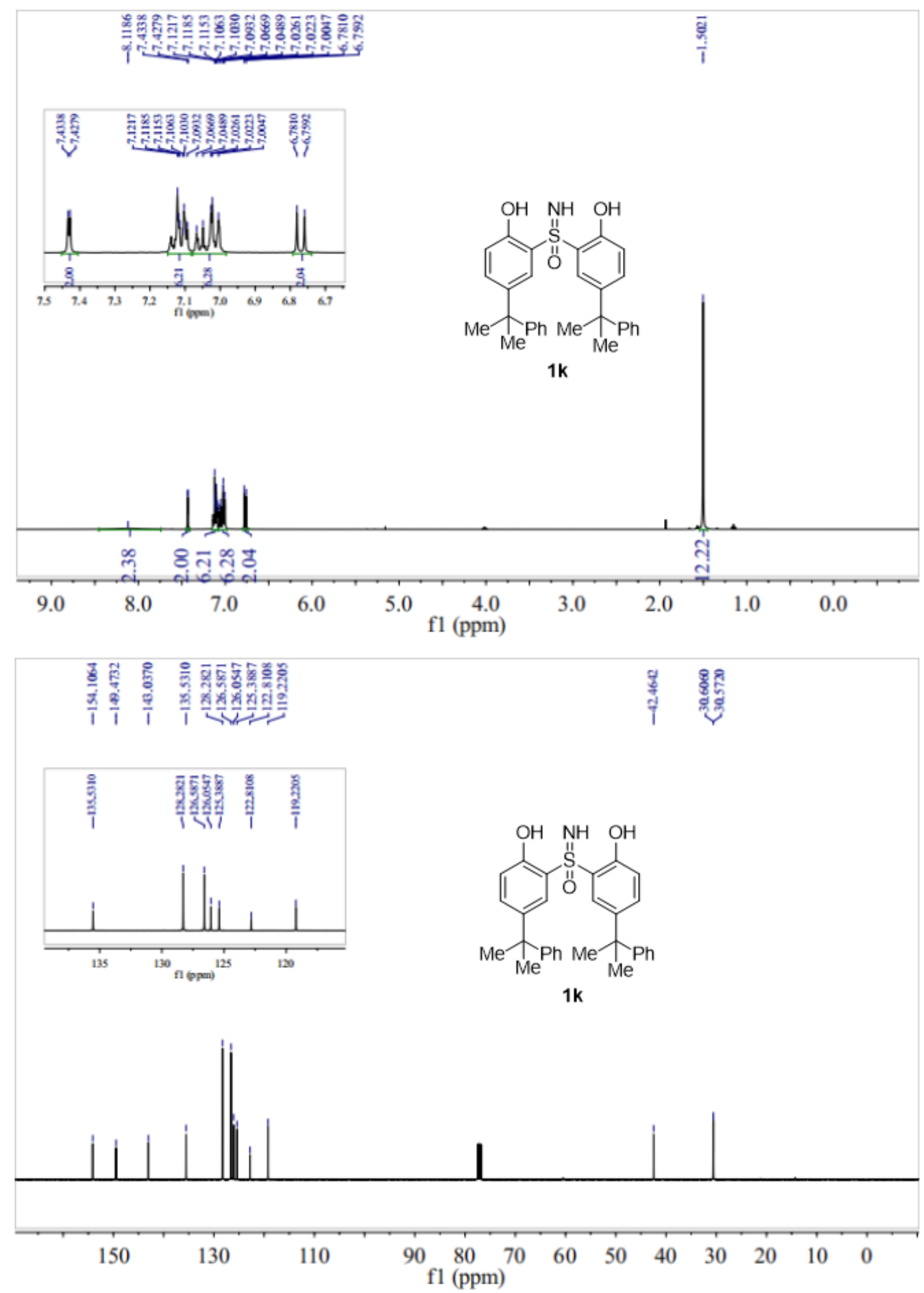

${ }^{1} \mathrm{H}-\mathrm{NMR}$ and ${ }^{13} \mathrm{C}-\mathrm{NMR}$ of $\mathbf{1 k}$ 

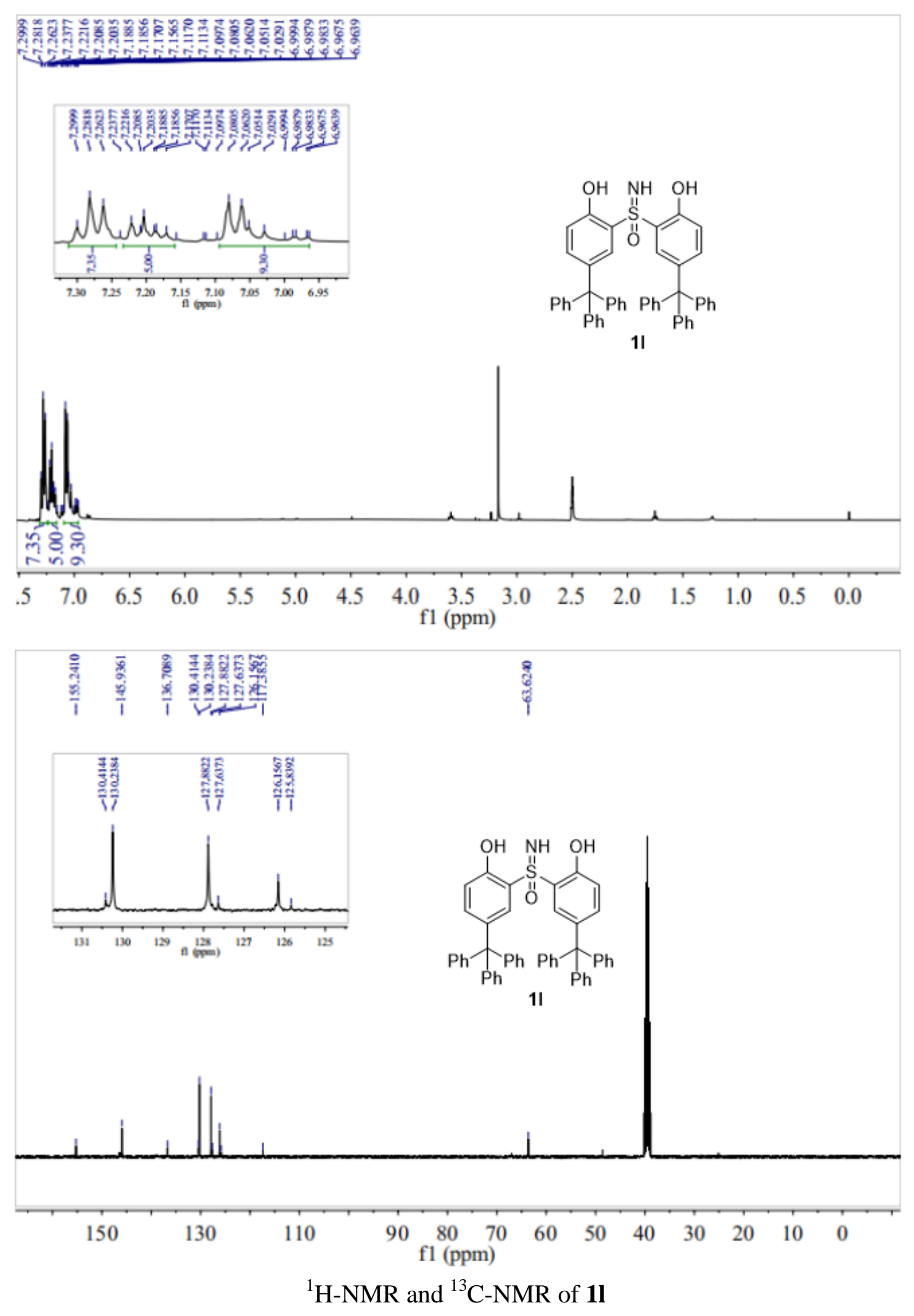

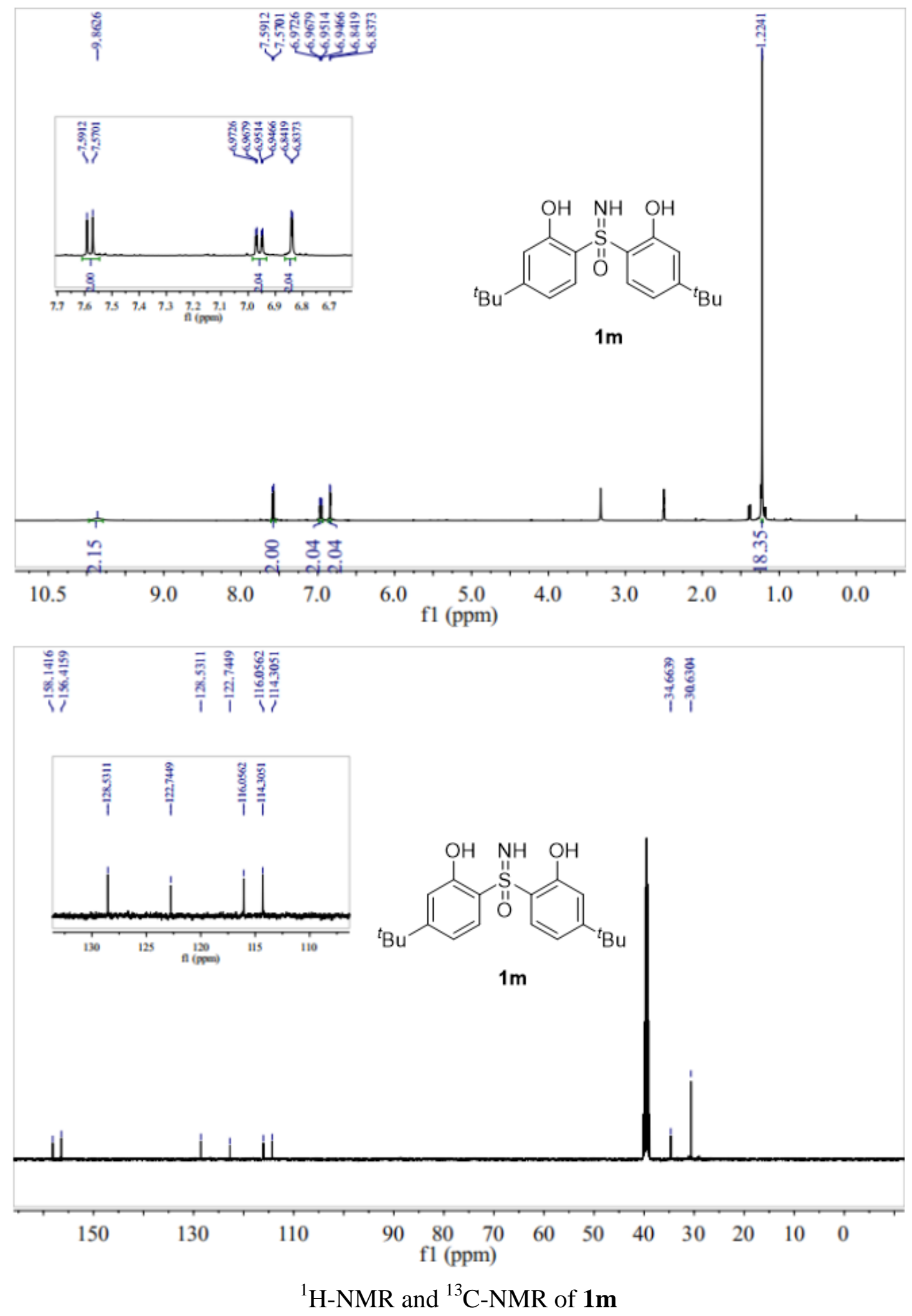

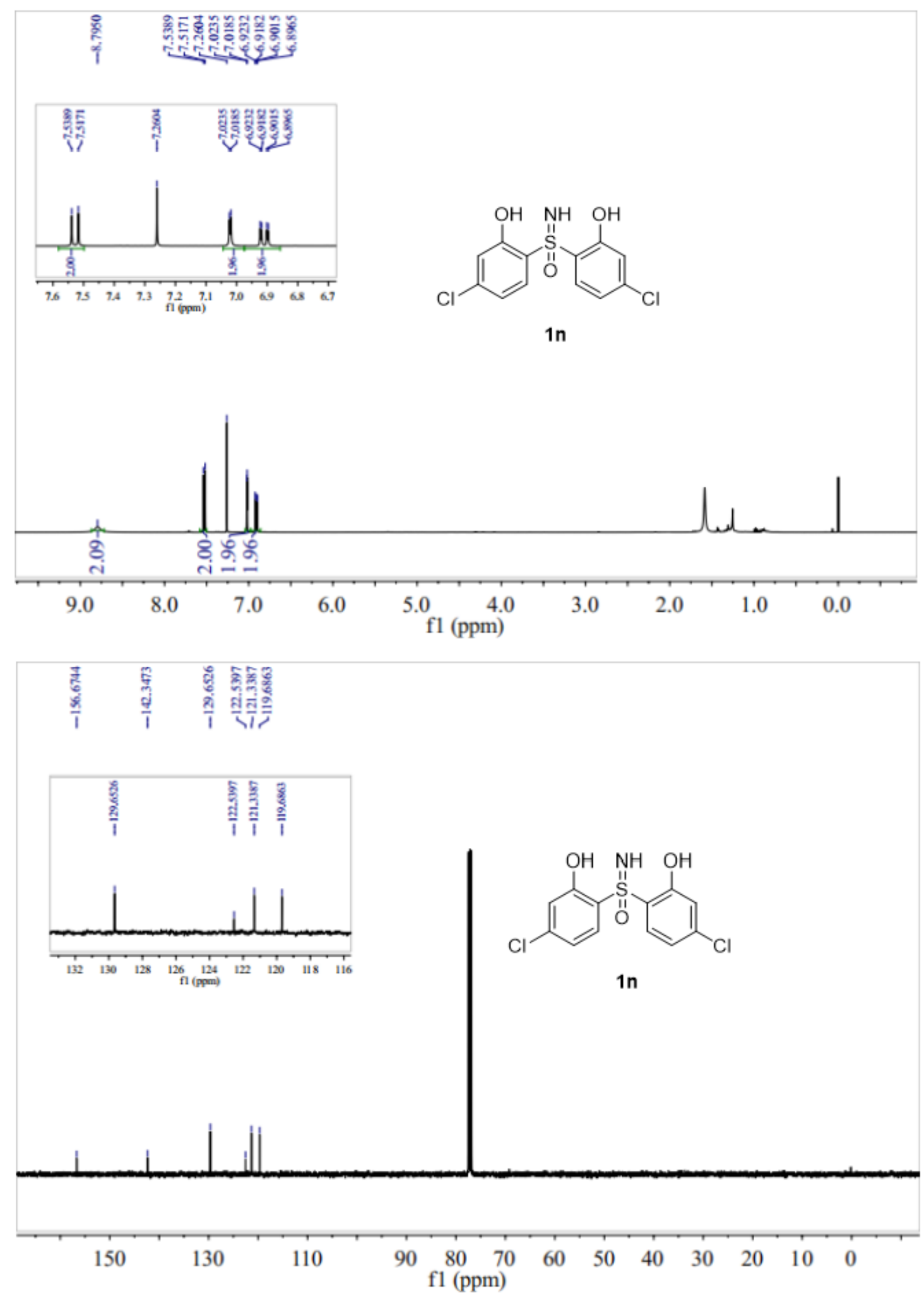

${ }^{1} \mathrm{H}-\mathrm{NMR}$ and ${ }^{13} \mathrm{C}-\mathrm{NMR}$ of $\mathbf{1 n}$ 

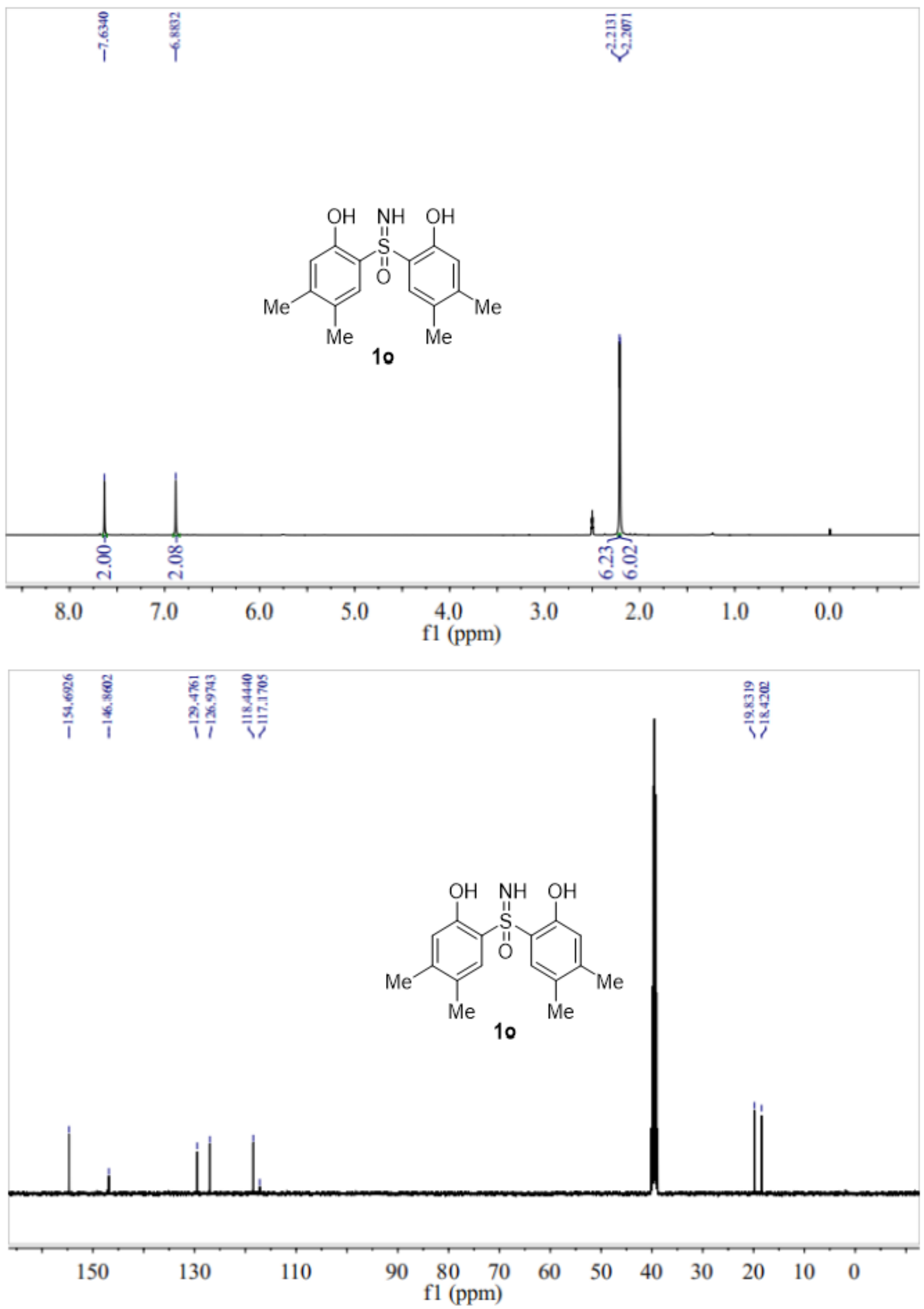

${ }^{1} \mathrm{H}-\mathrm{NMR}$ and ${ }^{13} \mathrm{C}-\mathrm{NMR}$ of $\mathbf{1 o}$ 

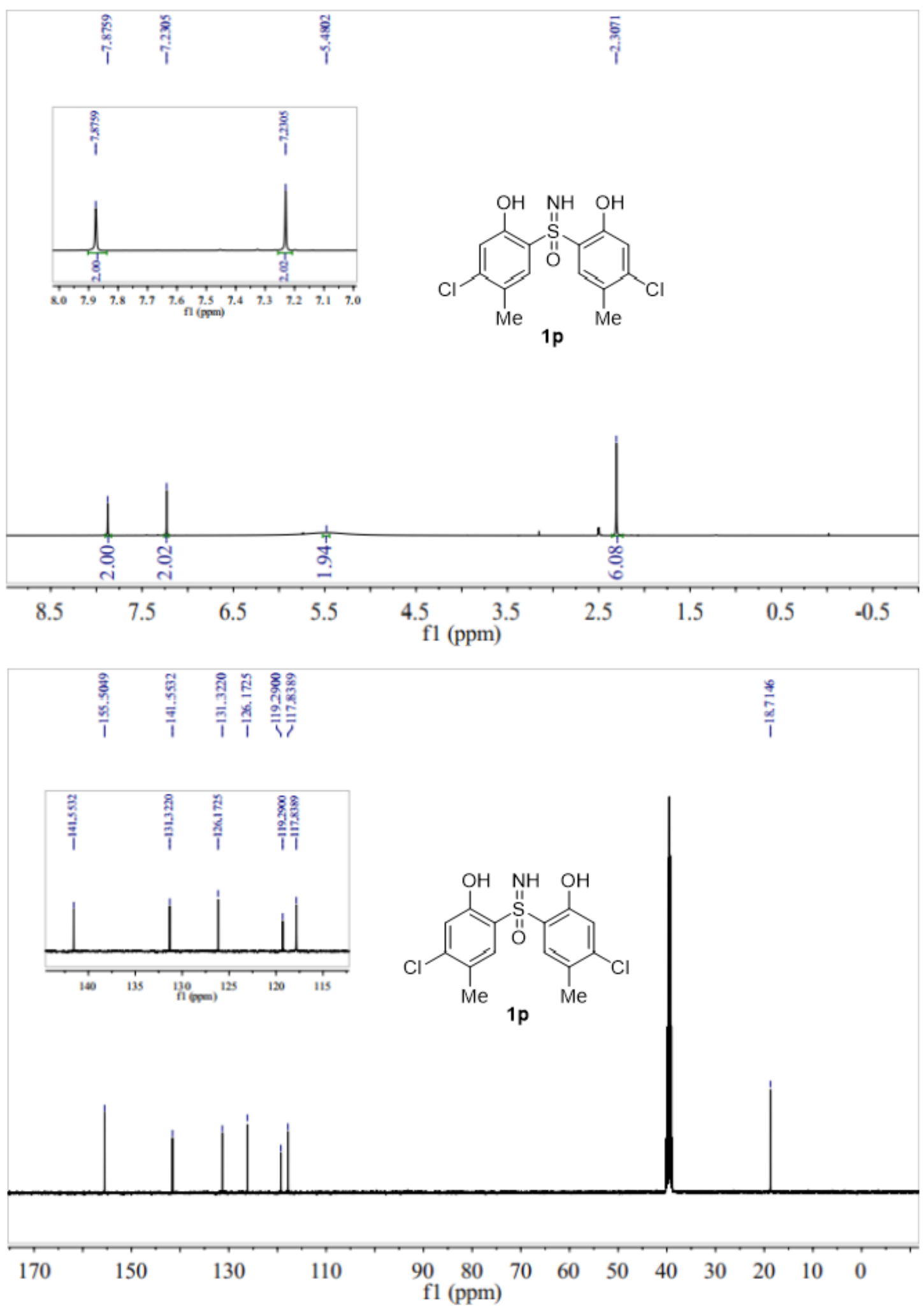

${ }^{1} \mathrm{H}-\mathrm{NMR}$ and ${ }^{13} \mathrm{C}-\mathrm{NMR}$ of $\mathbf{1 p}$ 

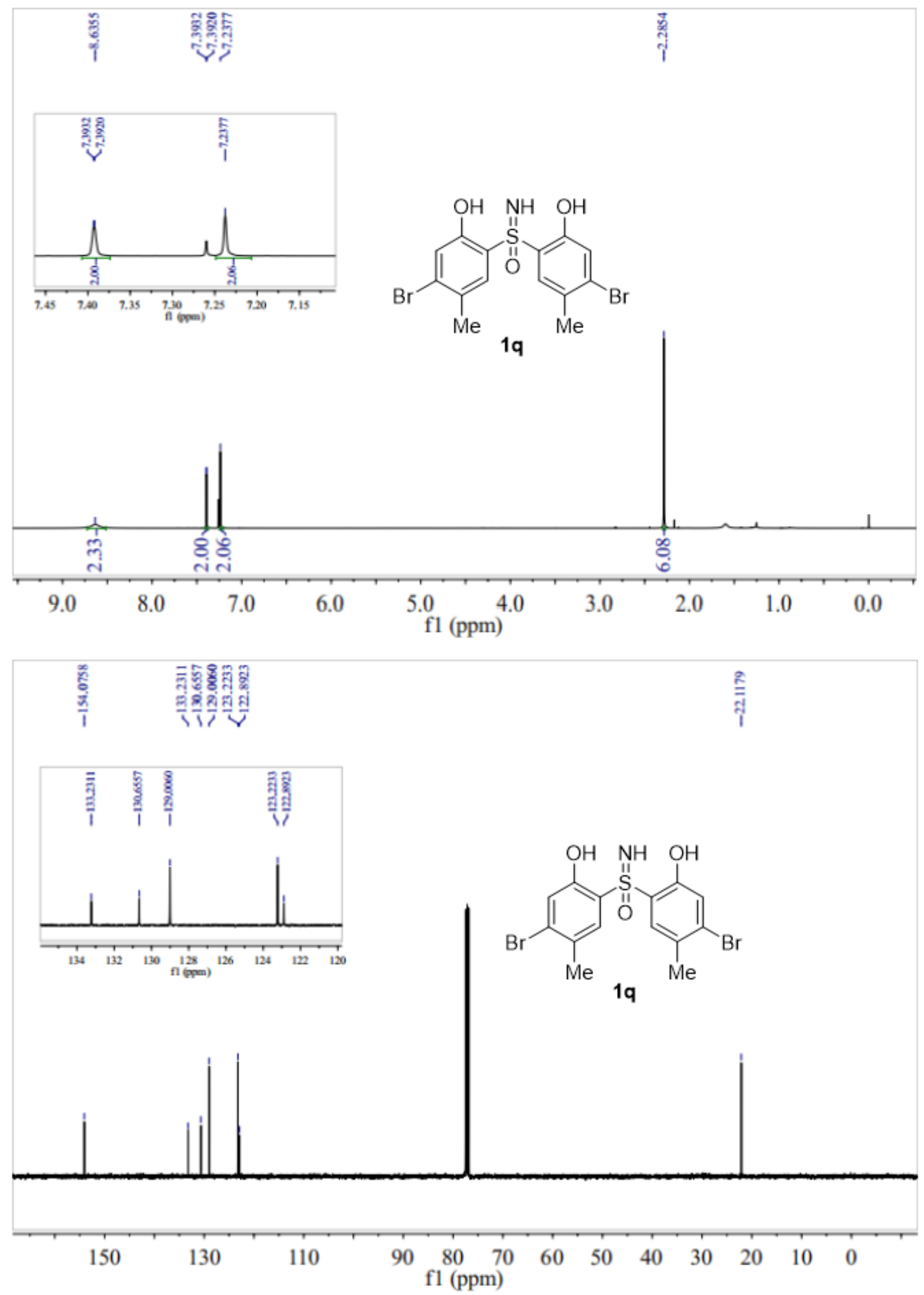

${ }^{1} \mathrm{H}-\mathrm{NMR}$ and ${ }^{13} \mathrm{C}-\mathrm{NMR}$ of $\mathbf{1 q}$ 

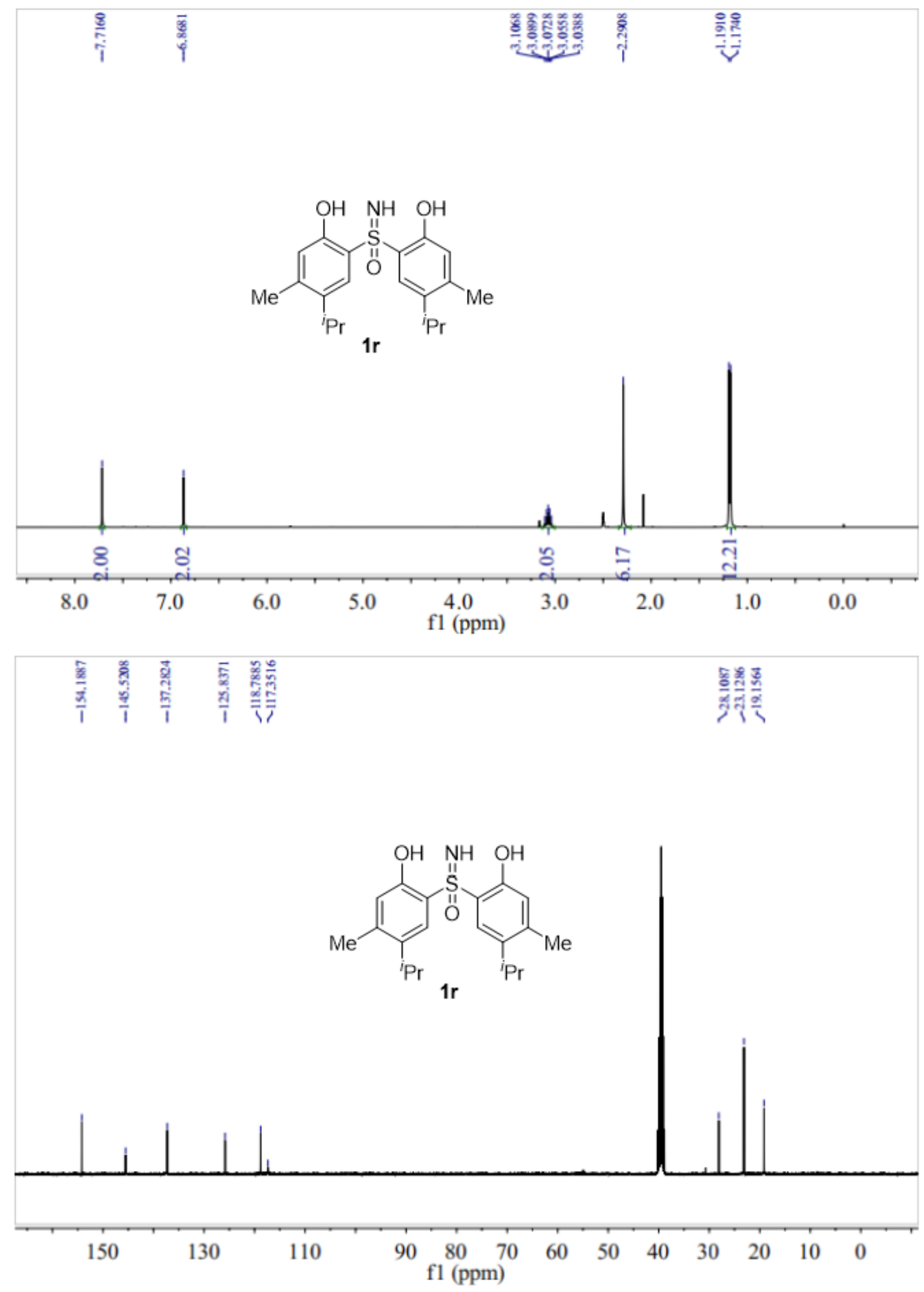

${ }^{1} \mathrm{H}-\mathrm{NMR}$ and ${ }^{13} \mathrm{C}-\mathrm{NMR}$ of $\mathbf{1 r}$ 

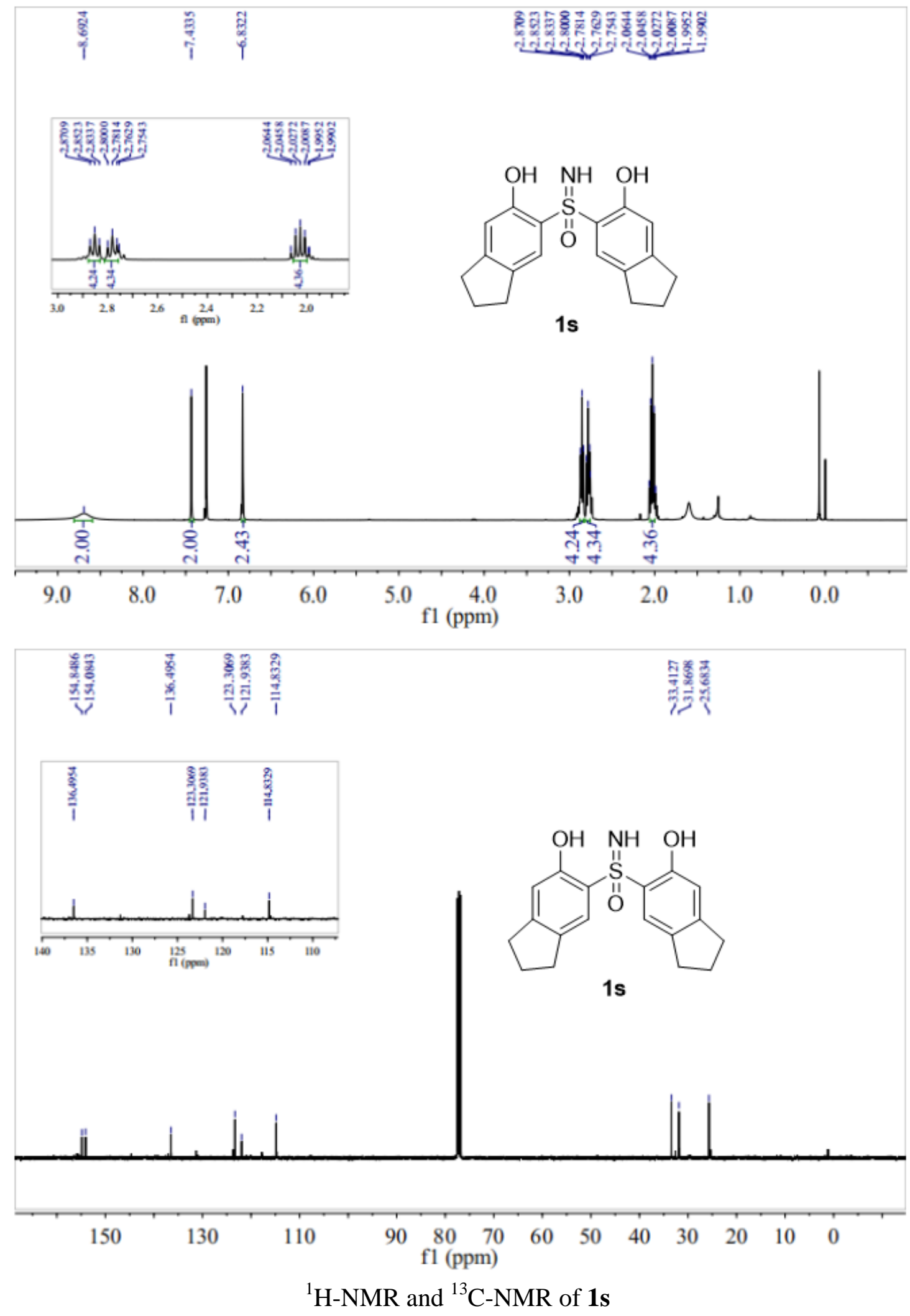

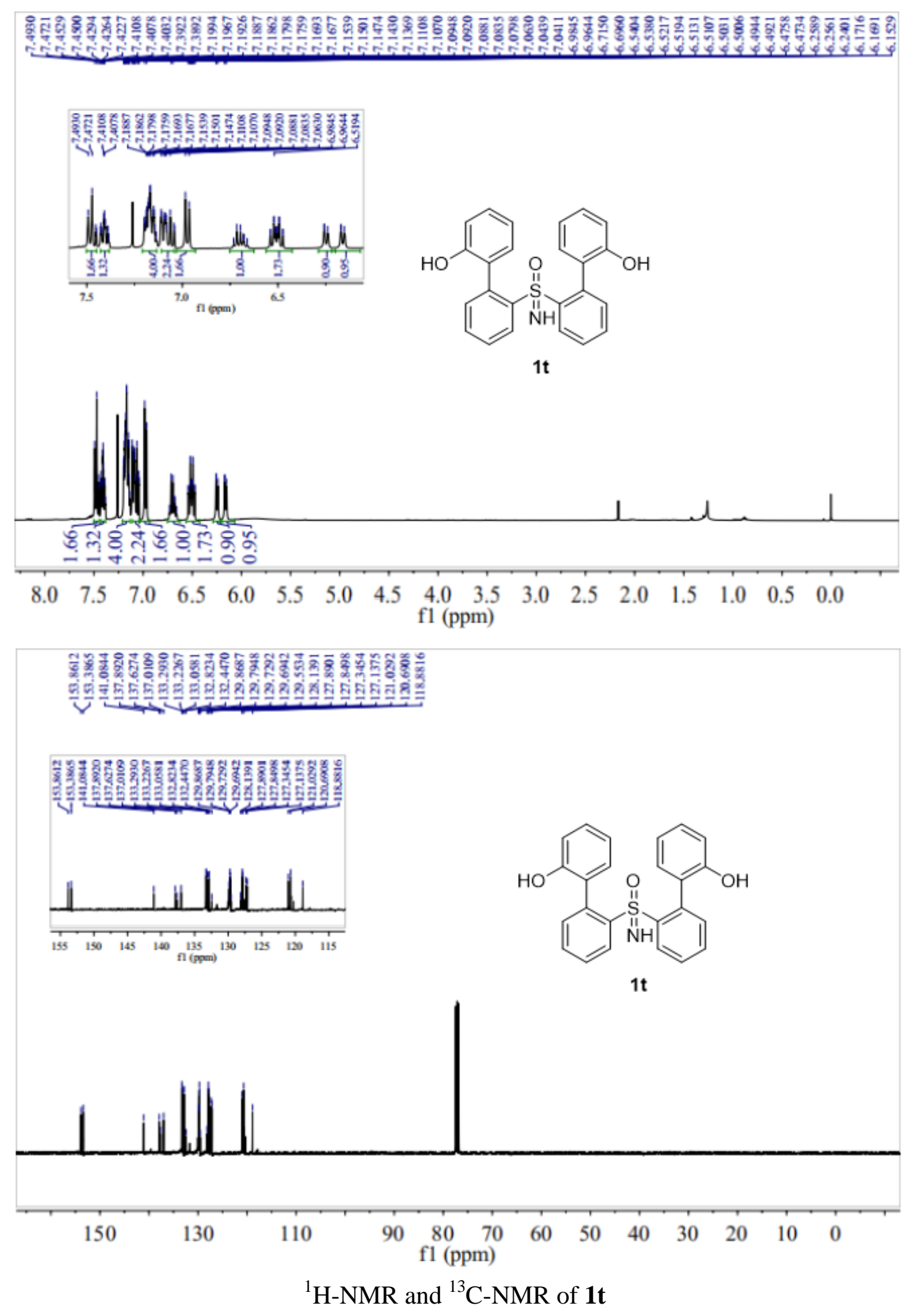

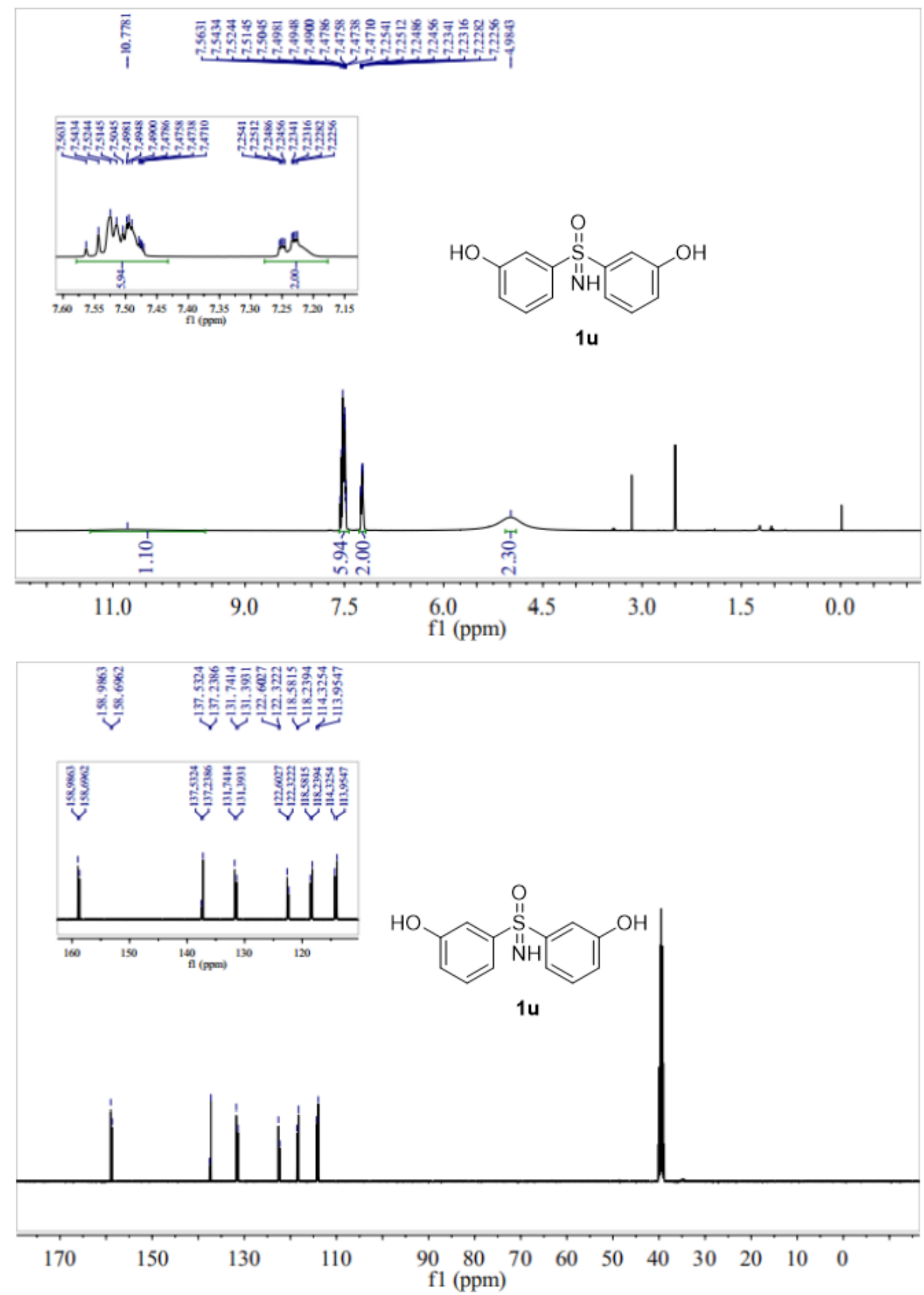

${ }^{1} \mathrm{H}-\mathrm{NMR}$ and ${ }^{13} \mathrm{C}-\mathrm{NMR}$ of $\mathbf{1 u}$ 

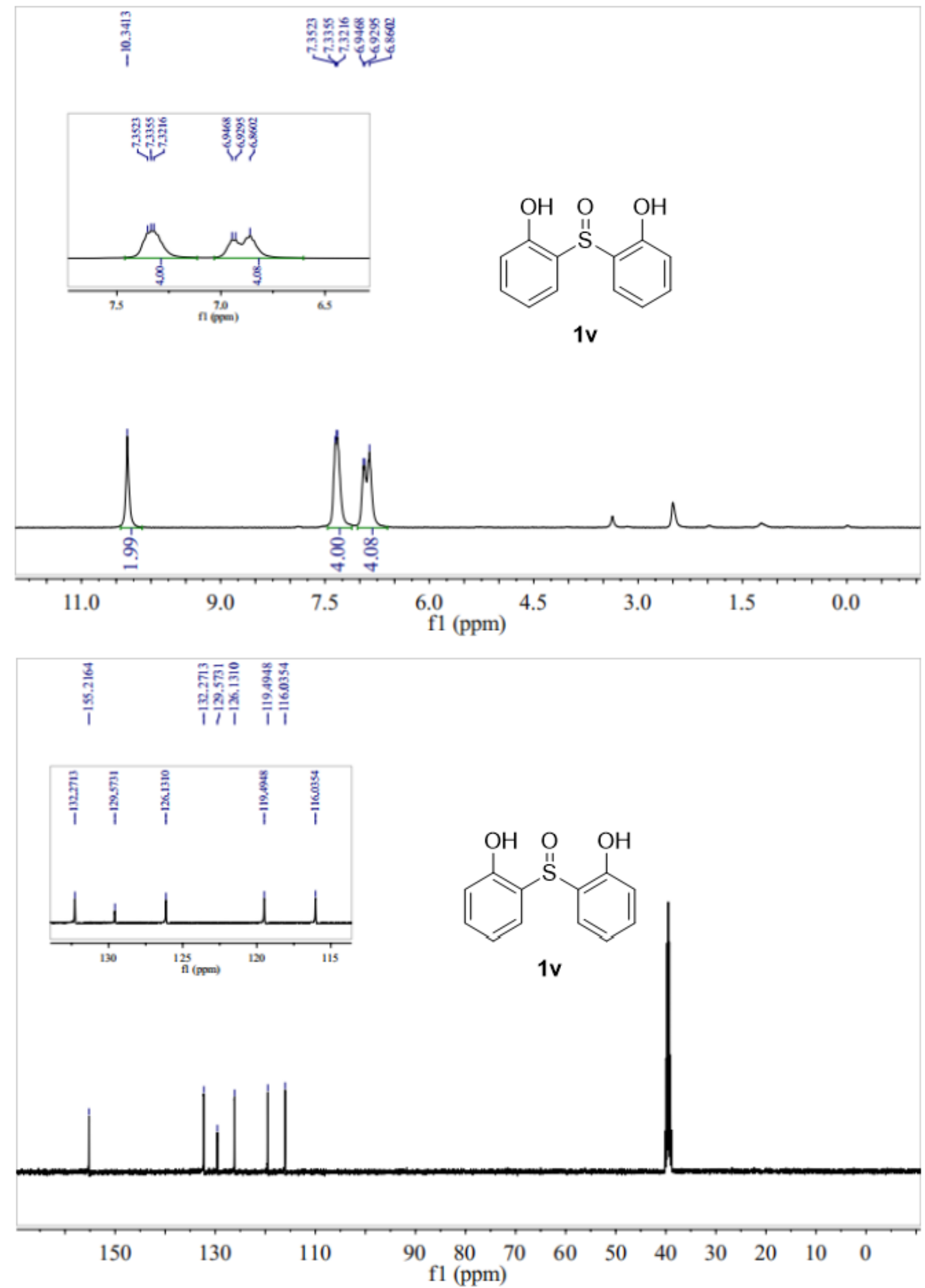

${ }^{1} \mathrm{H}-\mathrm{NMR}$ and ${ }^{13} \mathrm{C}-\mathrm{NMR}$ of $\mathbf{1 v}$ 

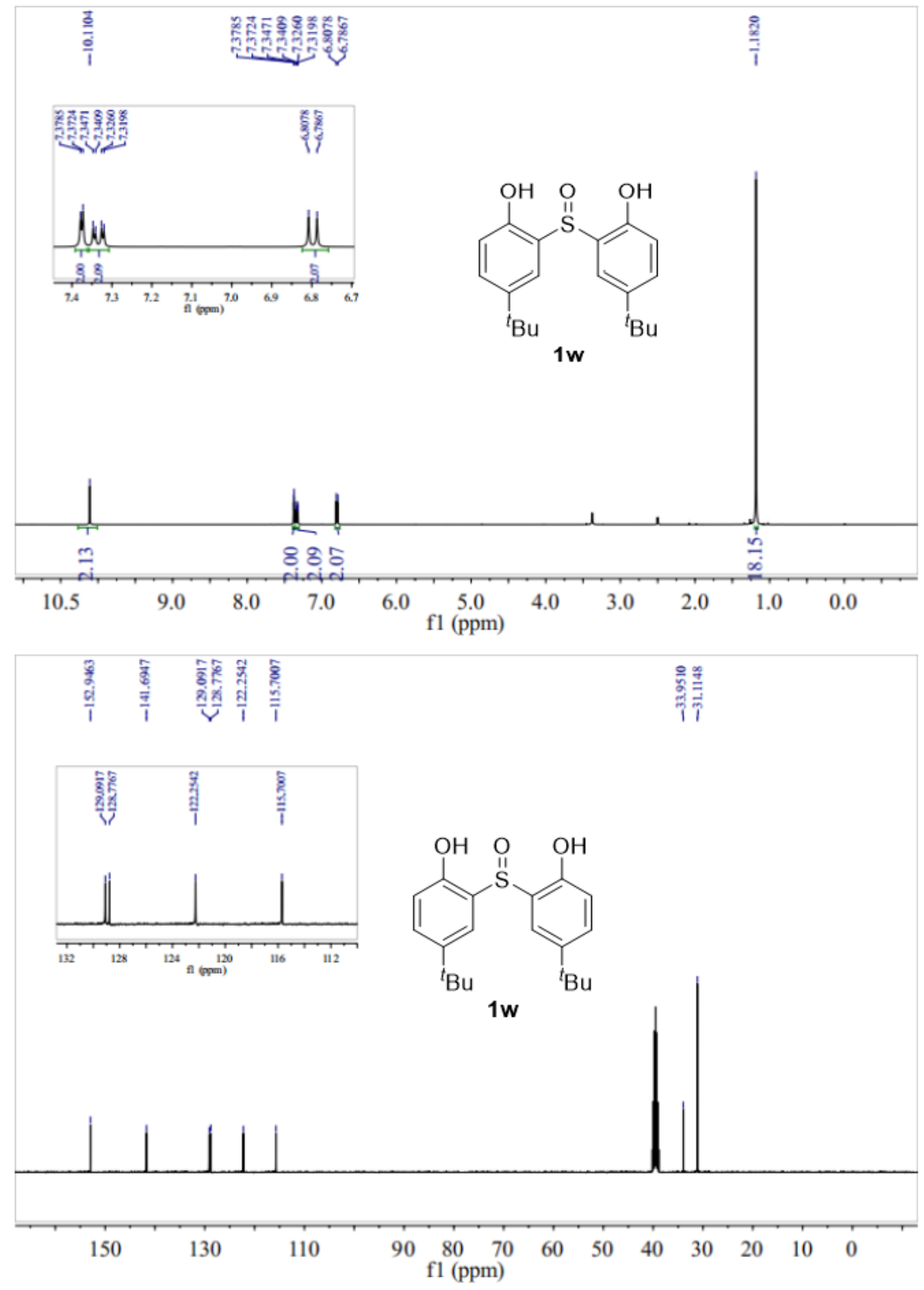

${ }^{1} \mathrm{H}-\mathrm{NMR}$ and ${ }^{13} \mathrm{C}-\mathrm{NMR}$ of $\mathbf{1 w}$ 

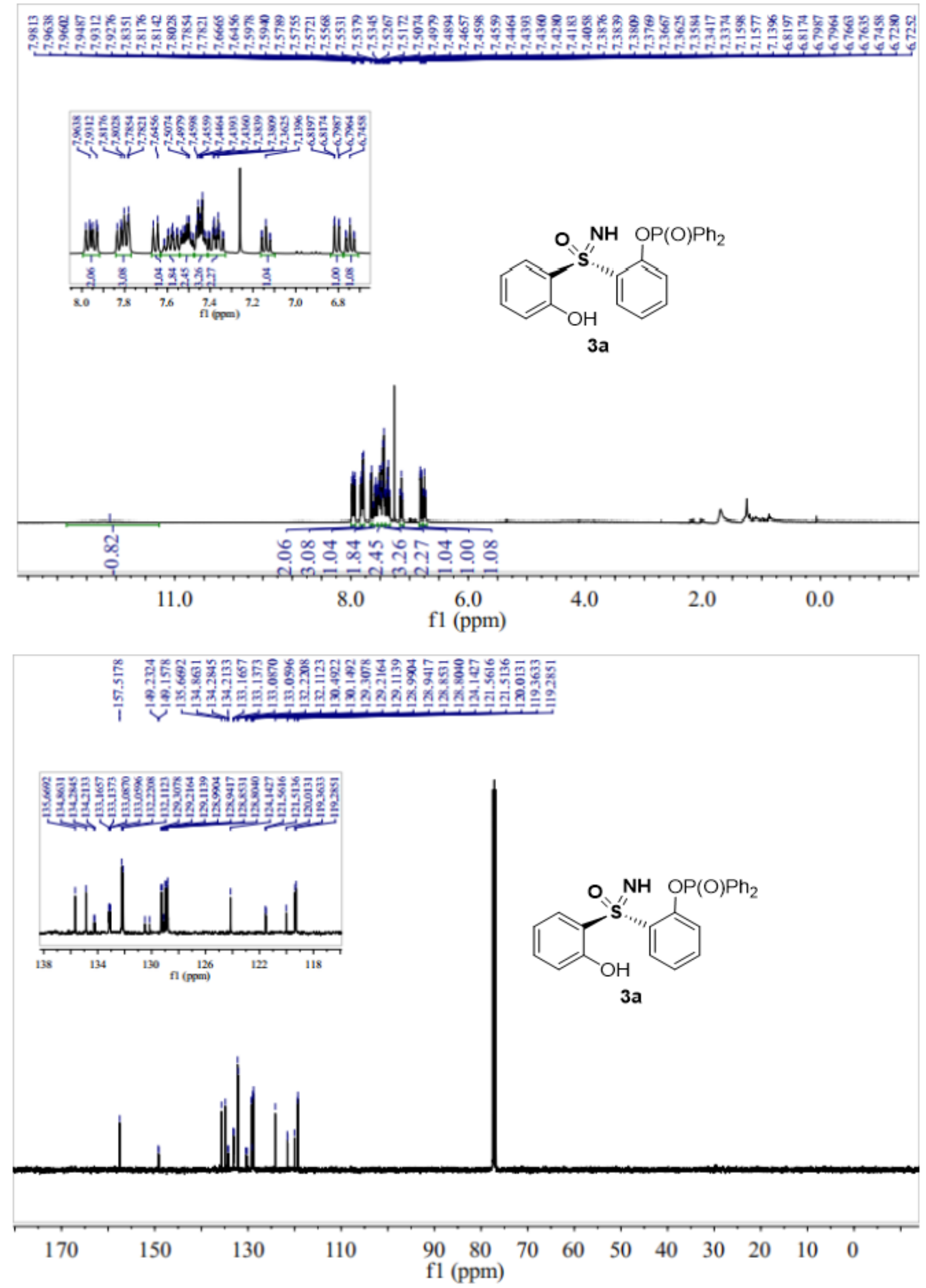


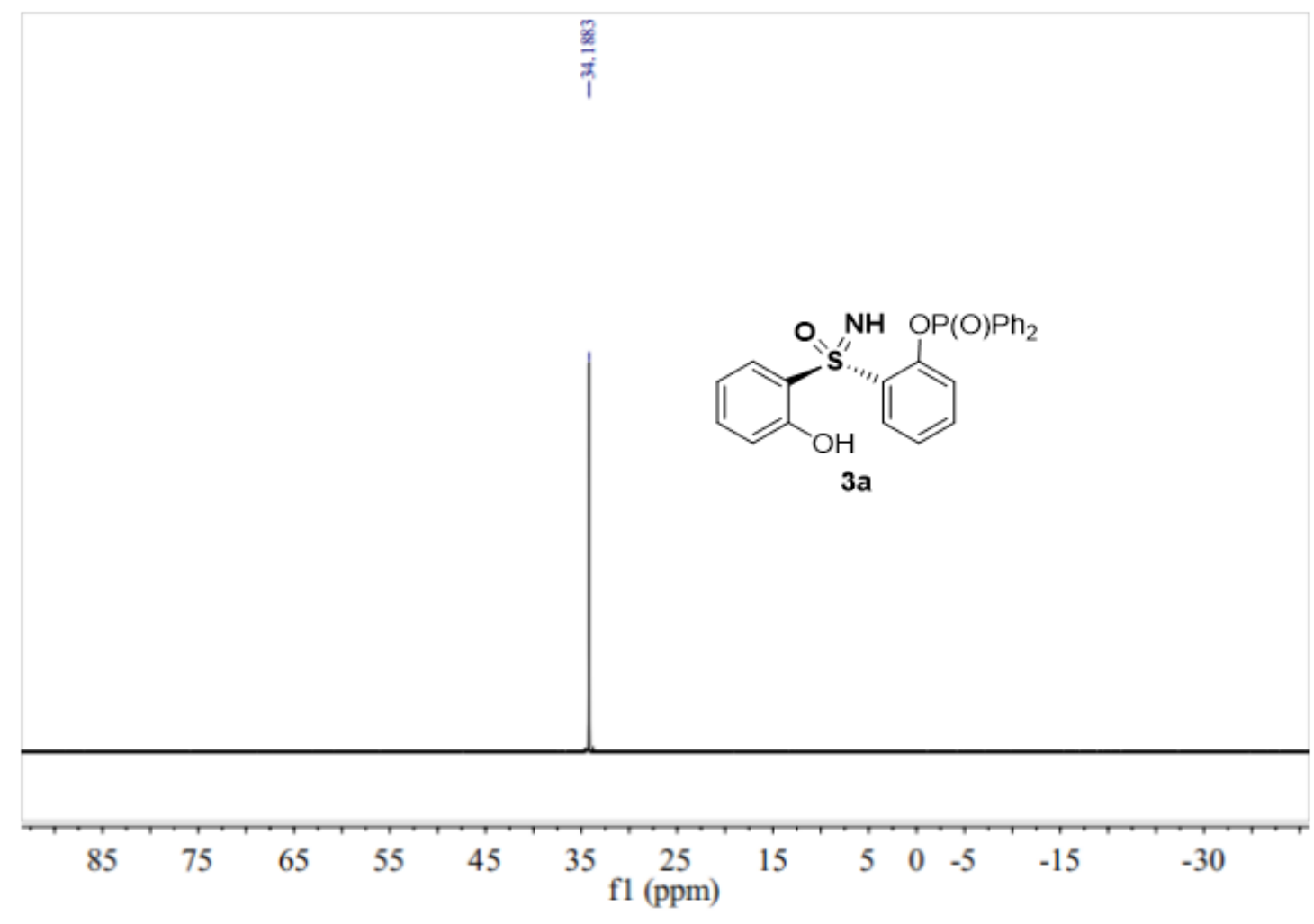

${ }^{1} \mathrm{H}-\mathrm{NMR},{ }^{13} \mathrm{C}-\mathrm{NMR}$ and ${ }^{31} \mathrm{P}-\mathrm{NMR}$ of $\mathbf{3 a}$ 

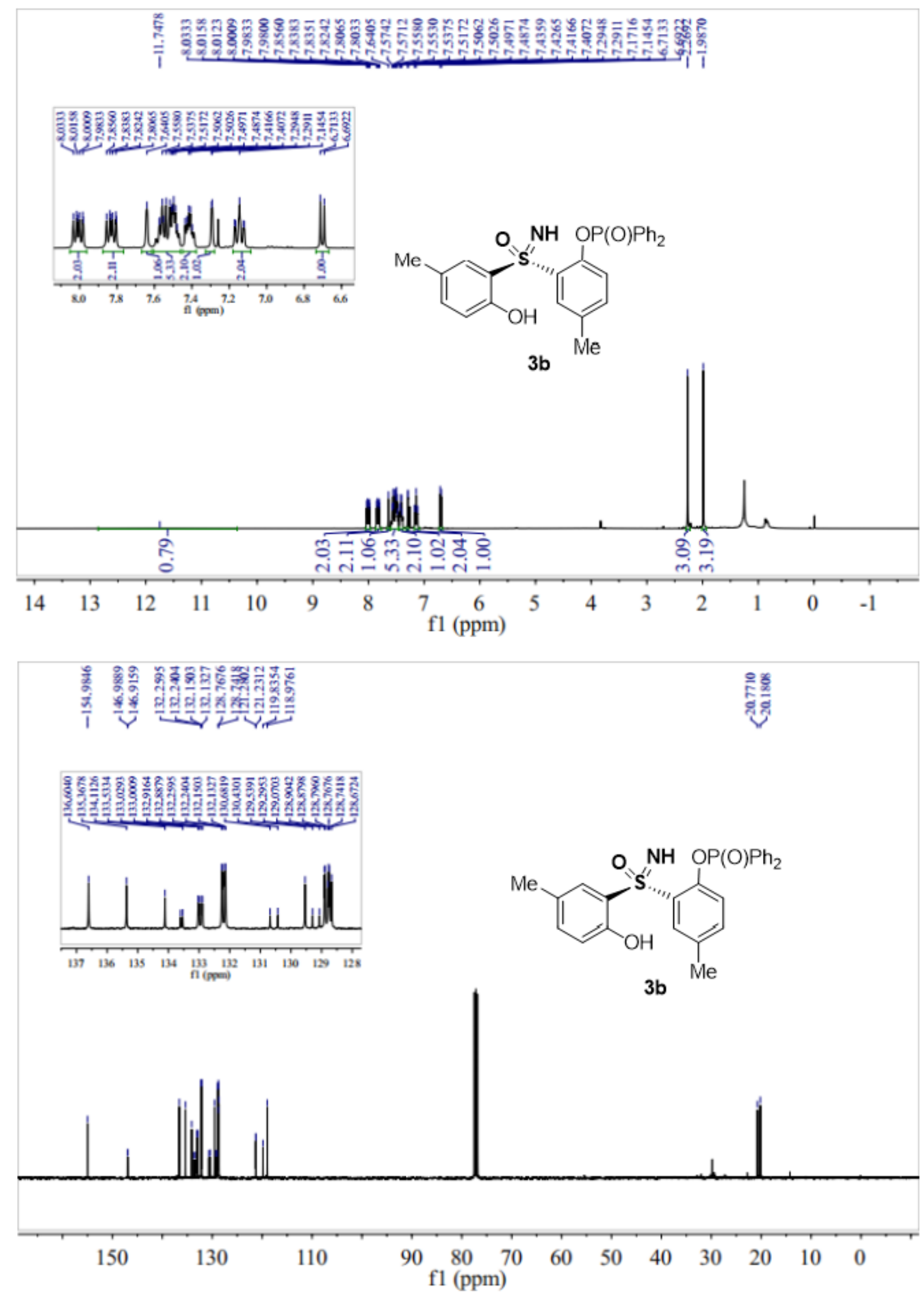


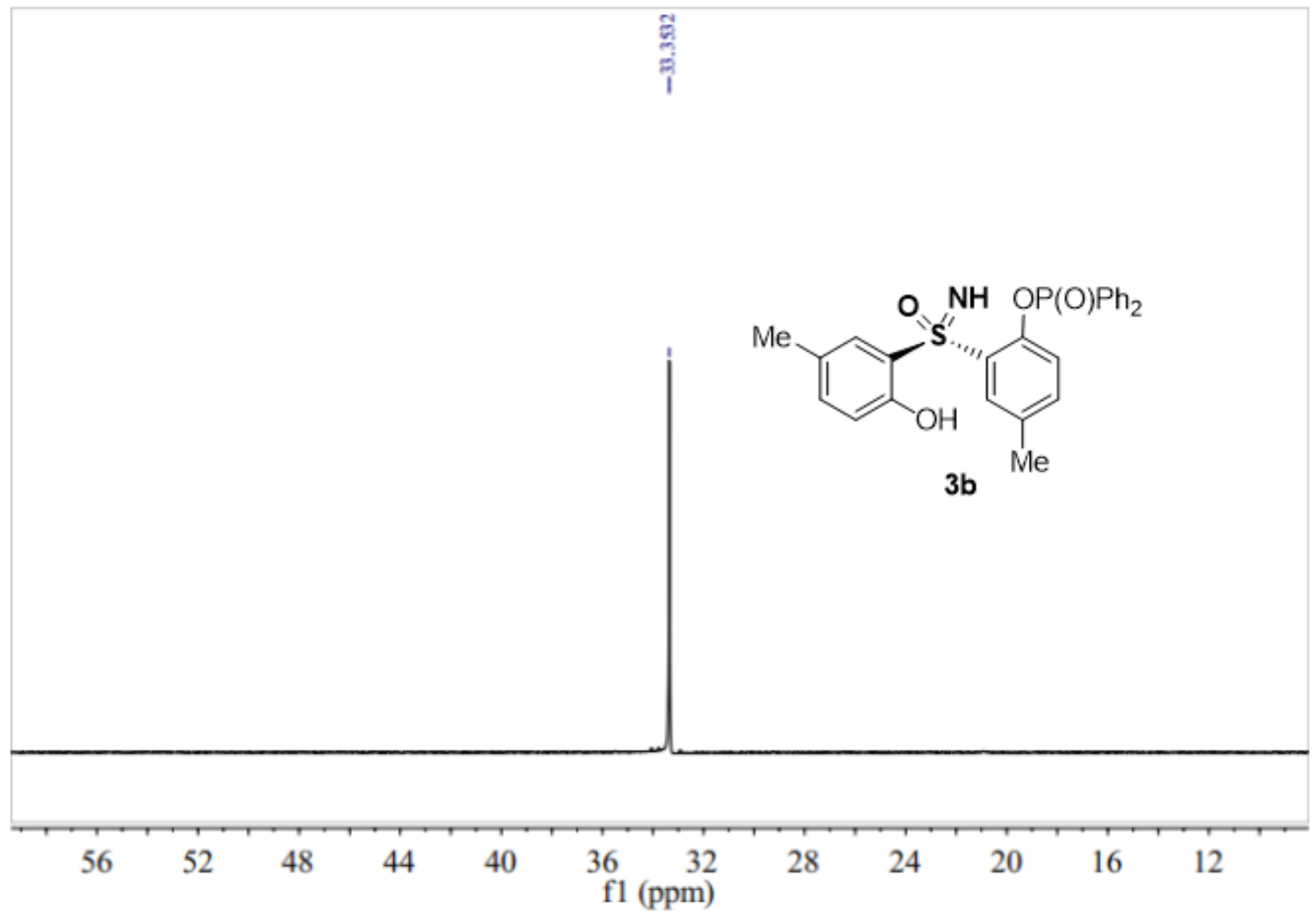

${ }^{1} \mathrm{H}-\mathrm{NMR},{ }^{13} \mathrm{C}-\mathrm{NMR}$ and ${ }^{31} \mathrm{P}-\mathrm{NMR}$ of $\mathbf{3 b}$ 

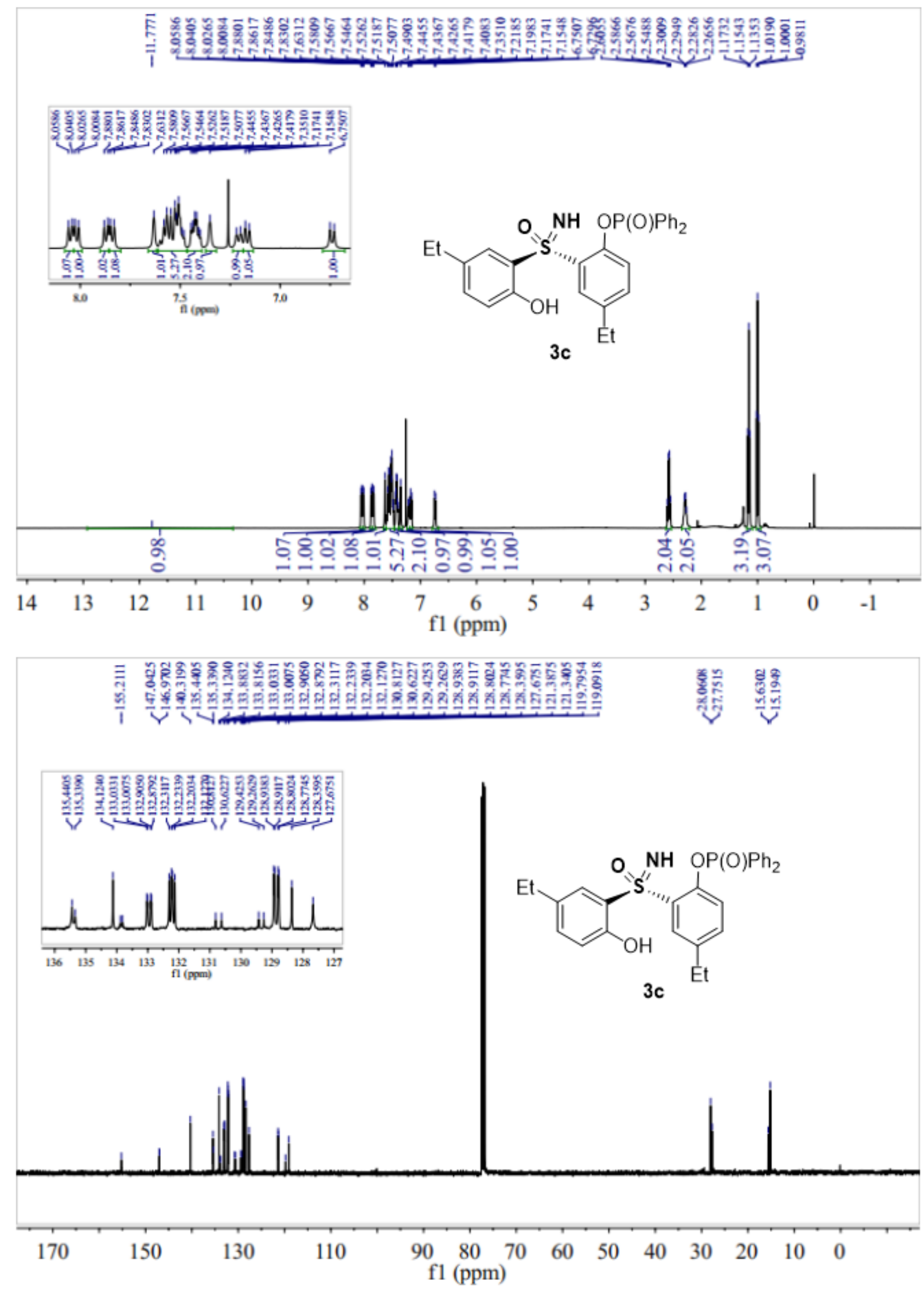


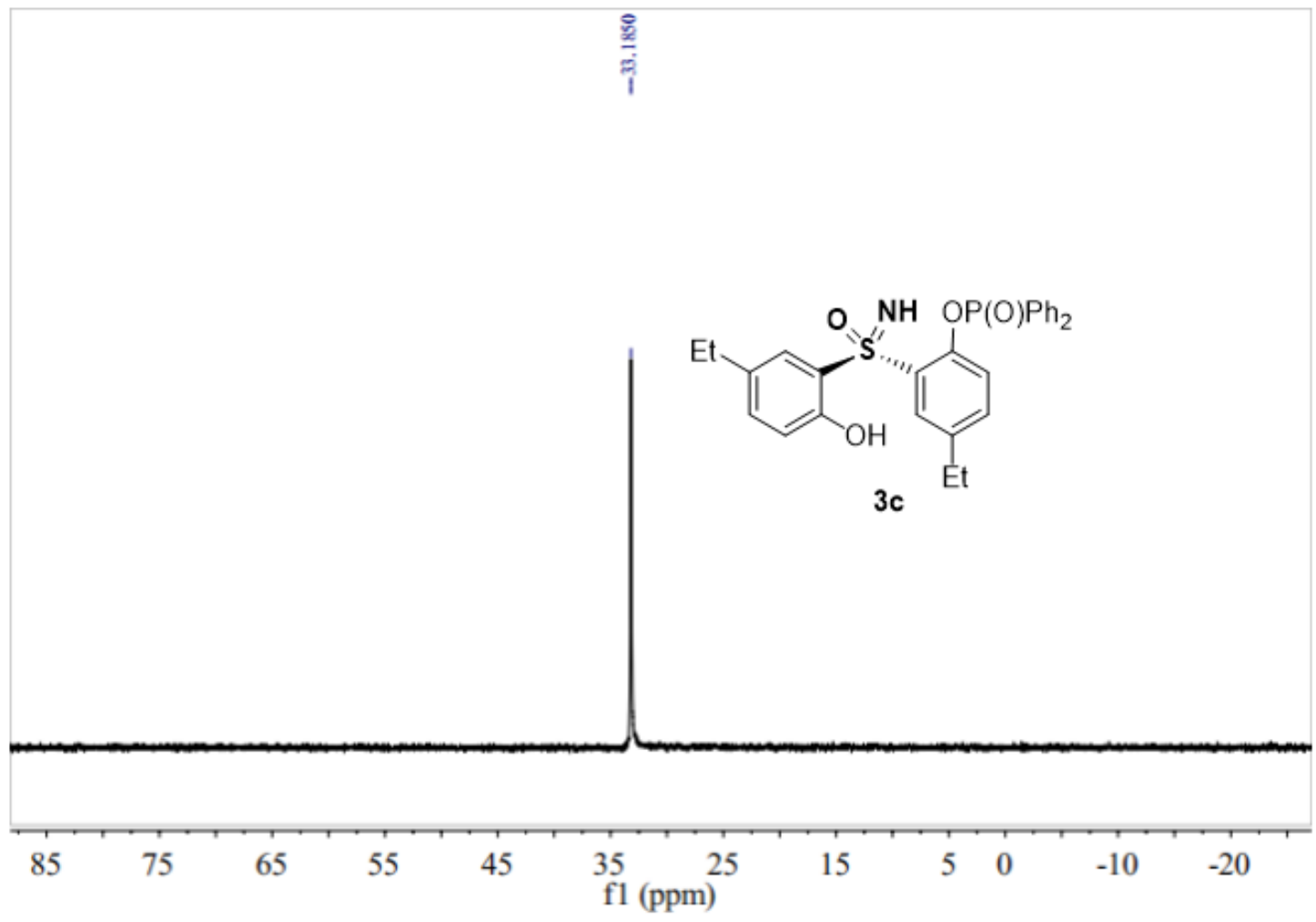

${ }^{1} \mathrm{H}-\mathrm{NMR},{ }^{13} \mathrm{C}-\mathrm{NMR}$ and ${ }^{31} \mathrm{P}-\mathrm{NMR}$ of $\mathbf{3 c}$ 

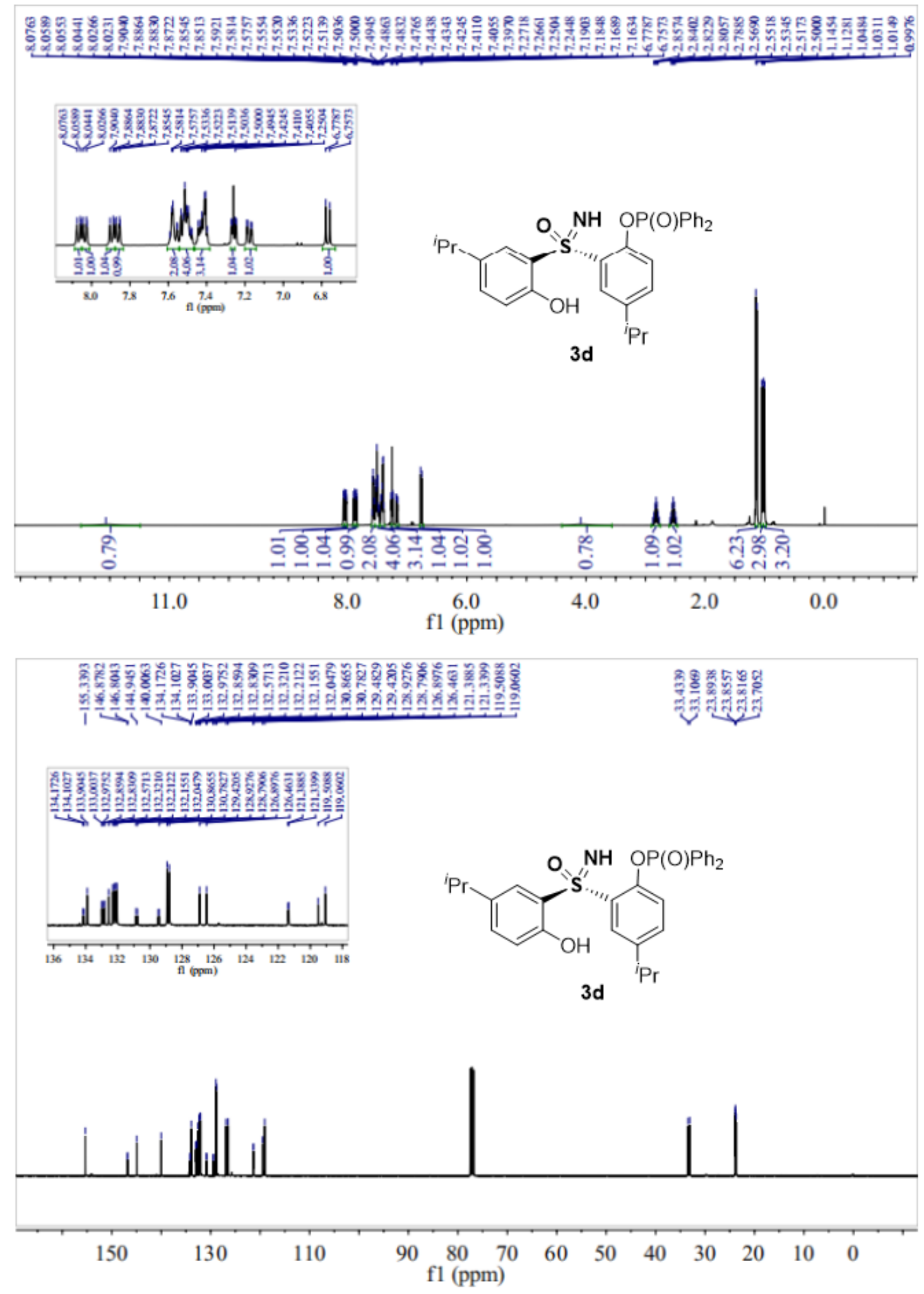


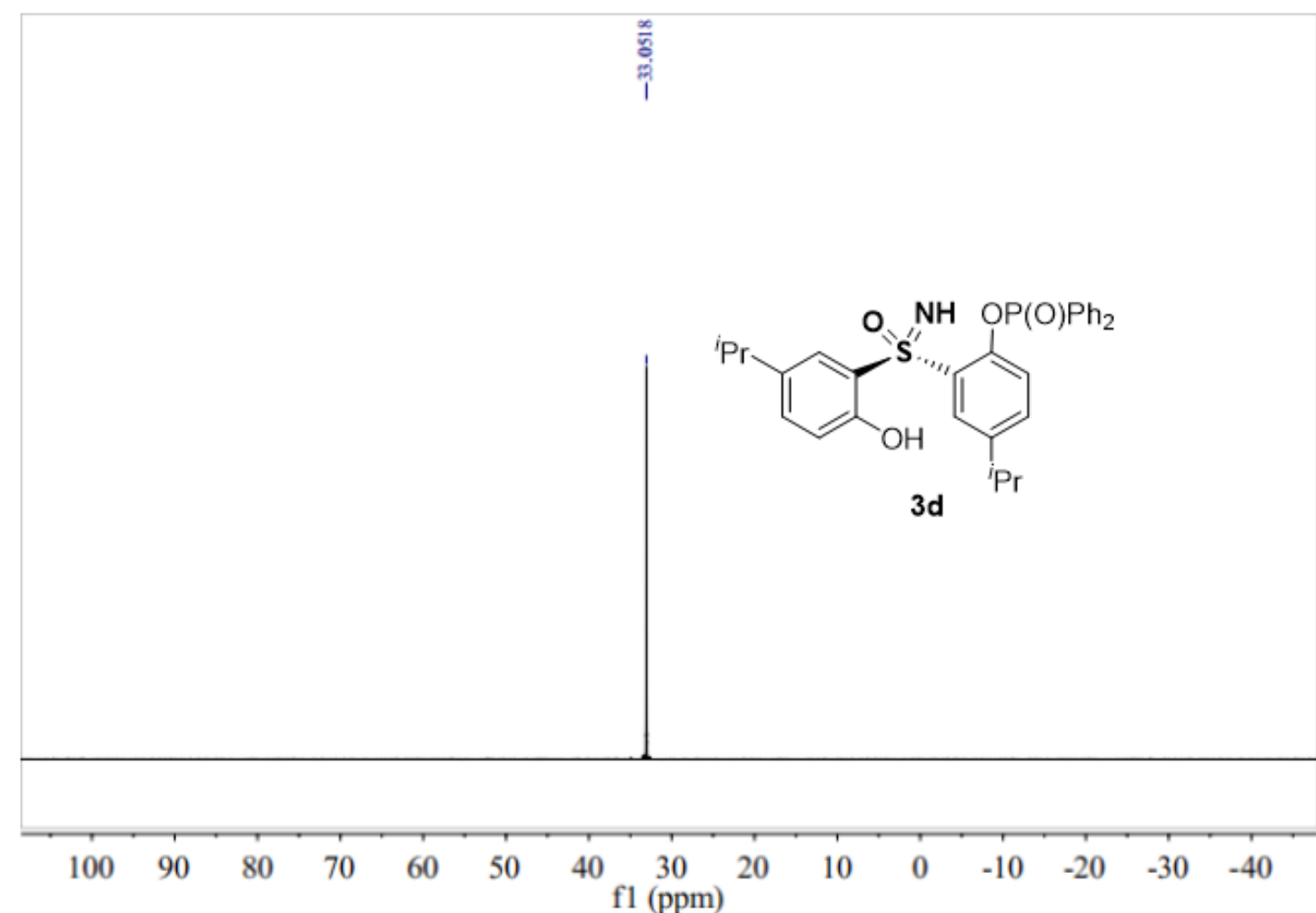

${ }^{1} \mathrm{H}-\mathrm{NMR},{ }^{13} \mathrm{C}-\mathrm{NMR}$ and ${ }^{31} \mathrm{P}-\mathrm{NMR}$ of $\mathbf{3 d}$ 

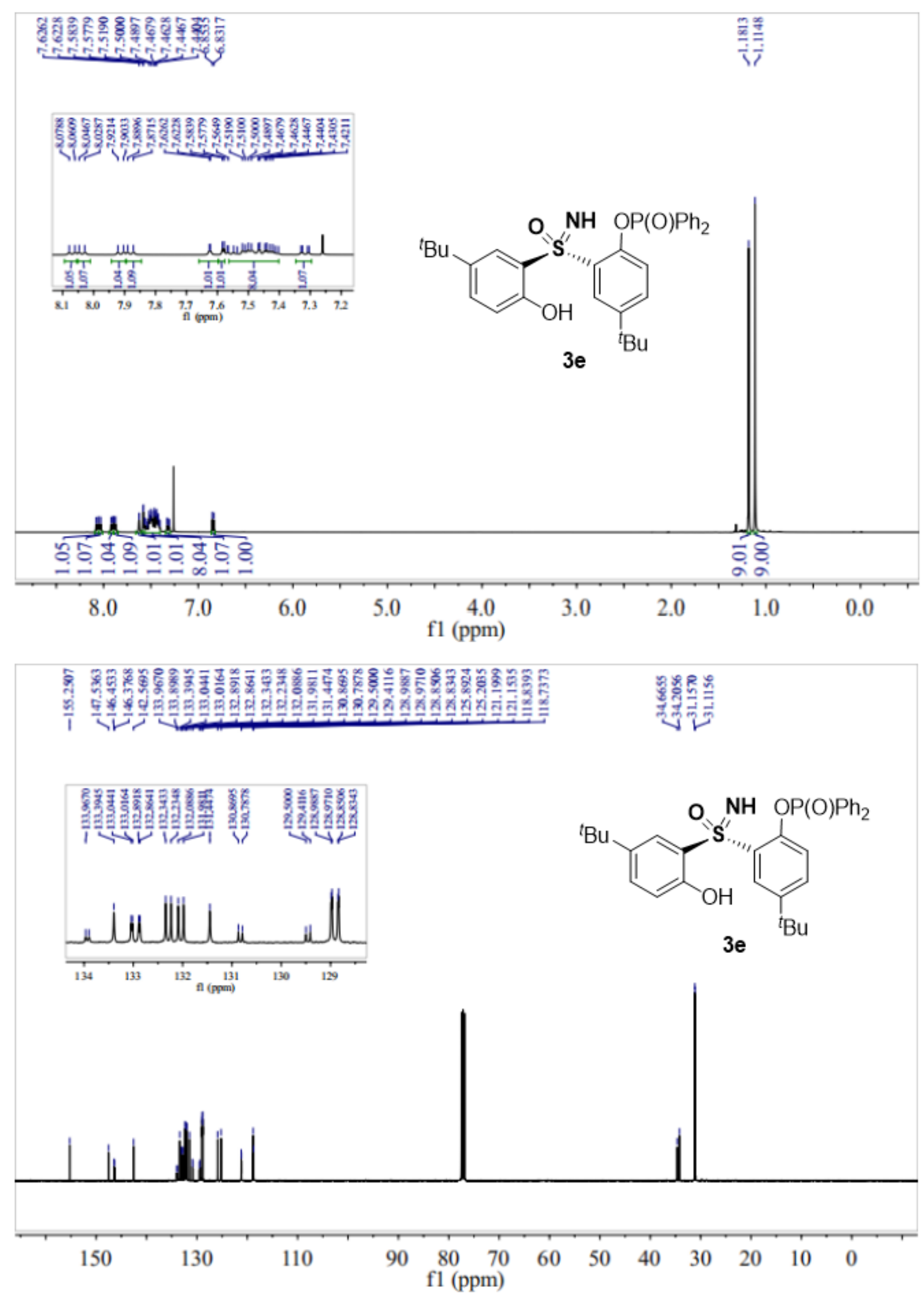


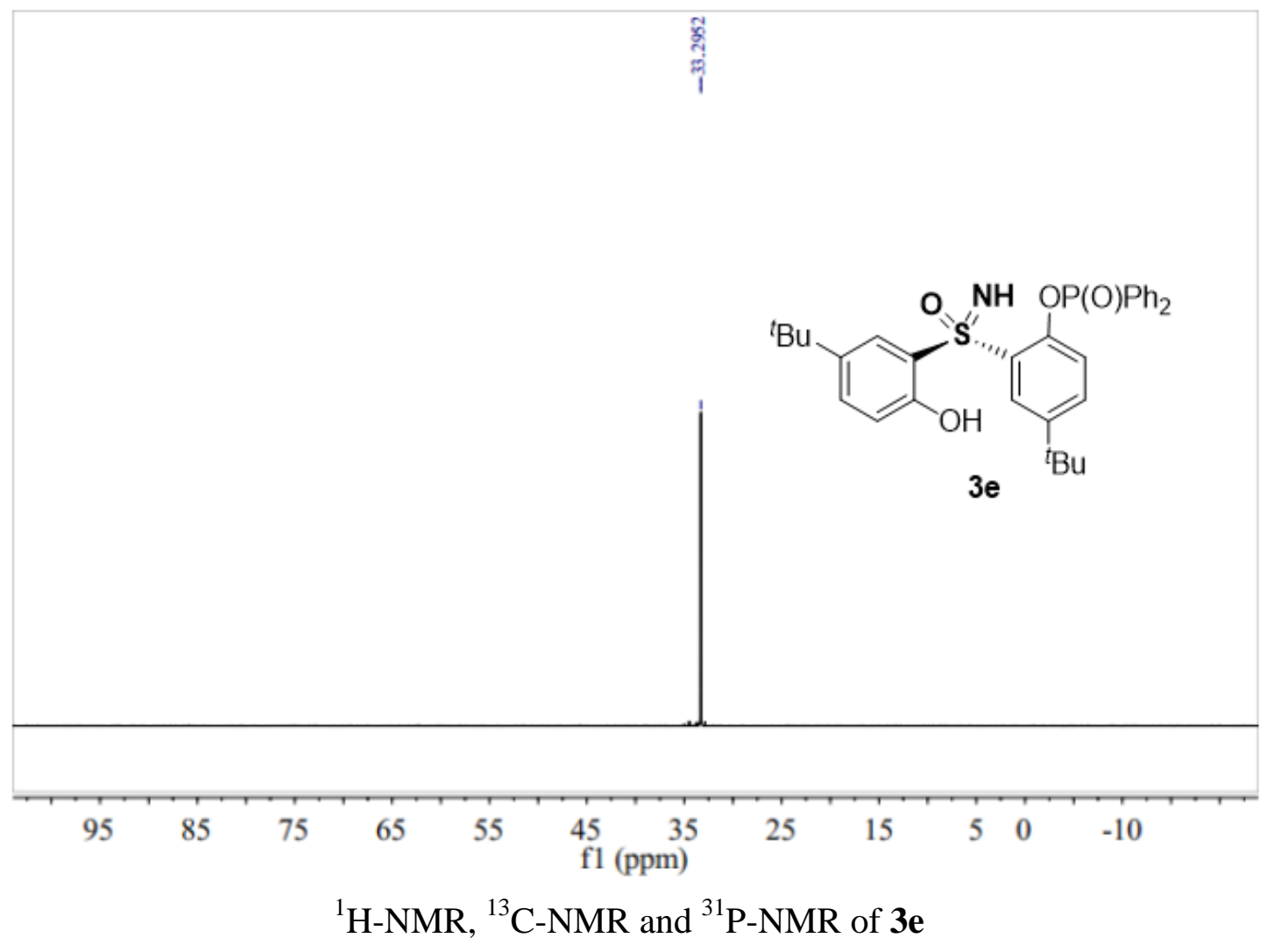




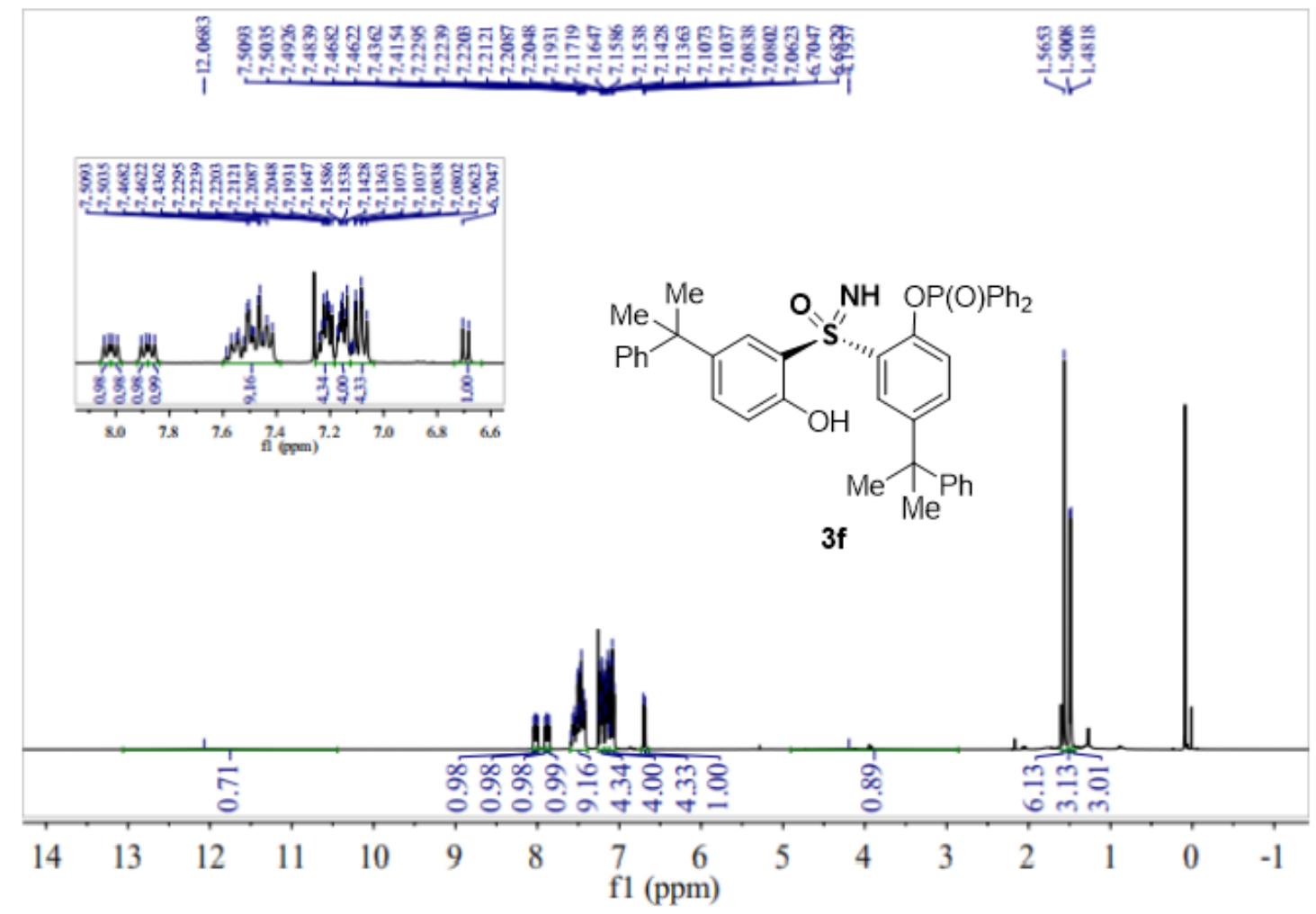

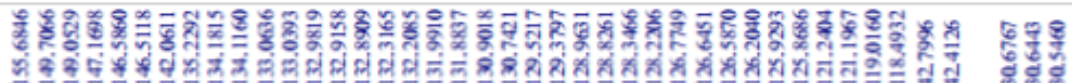

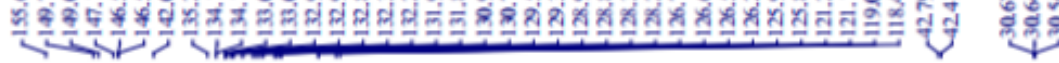

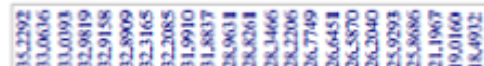

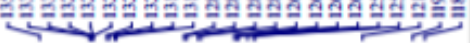
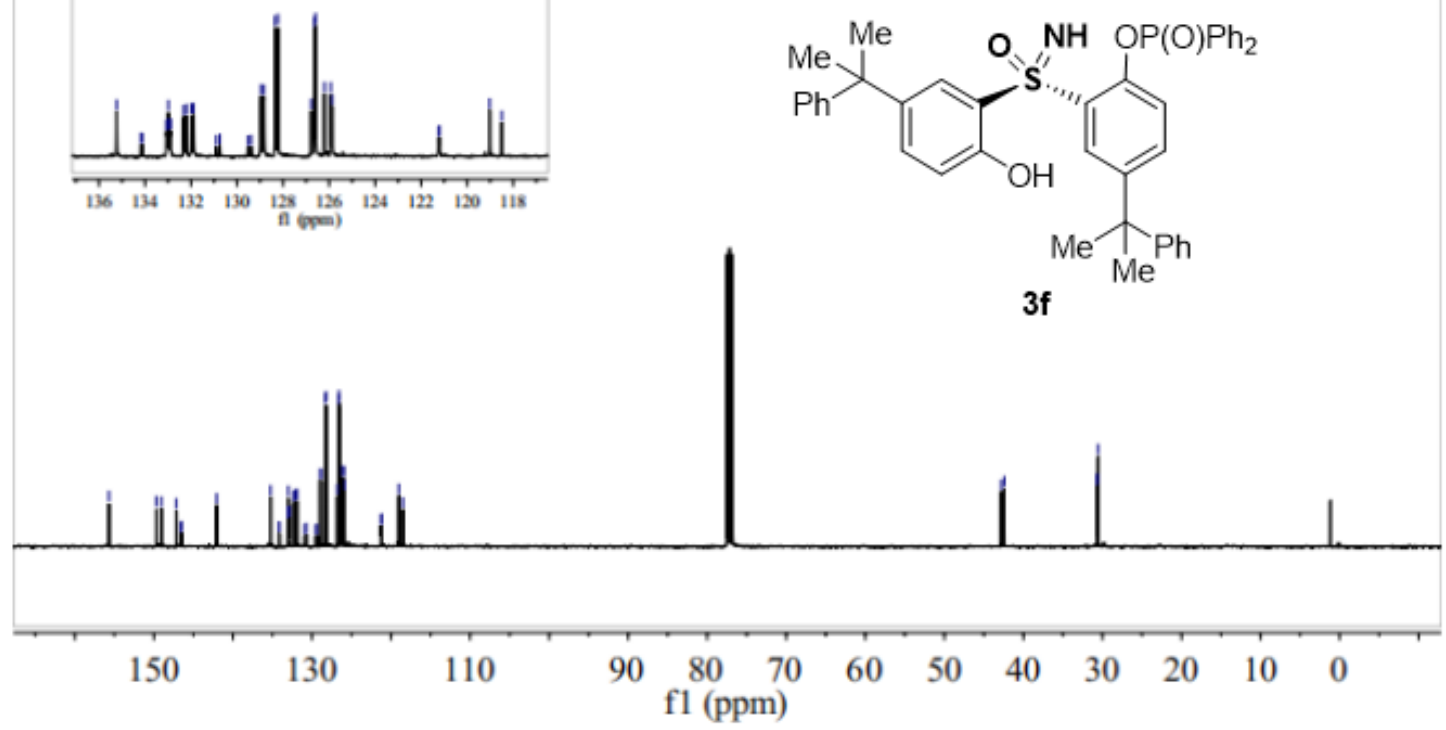


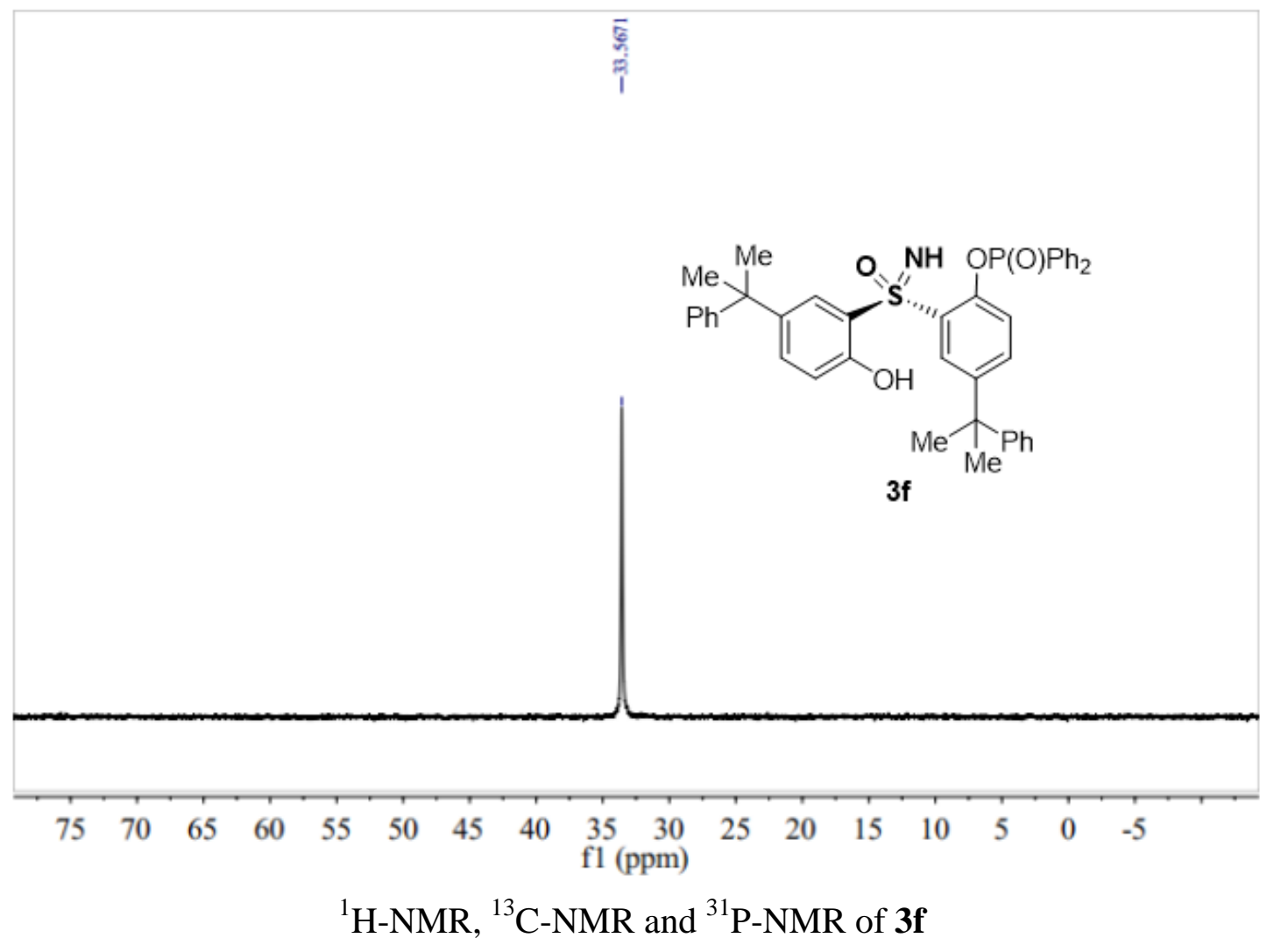



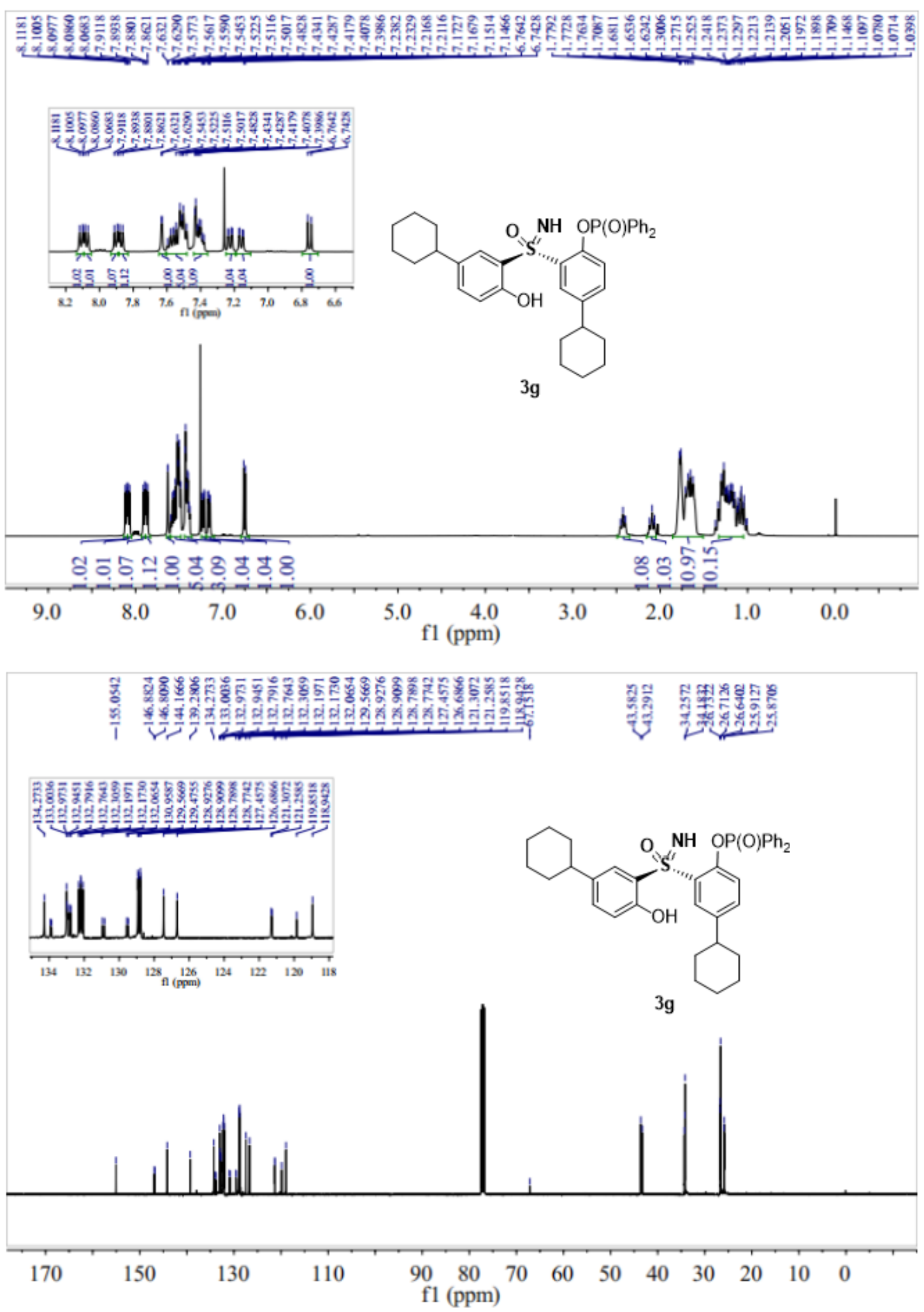


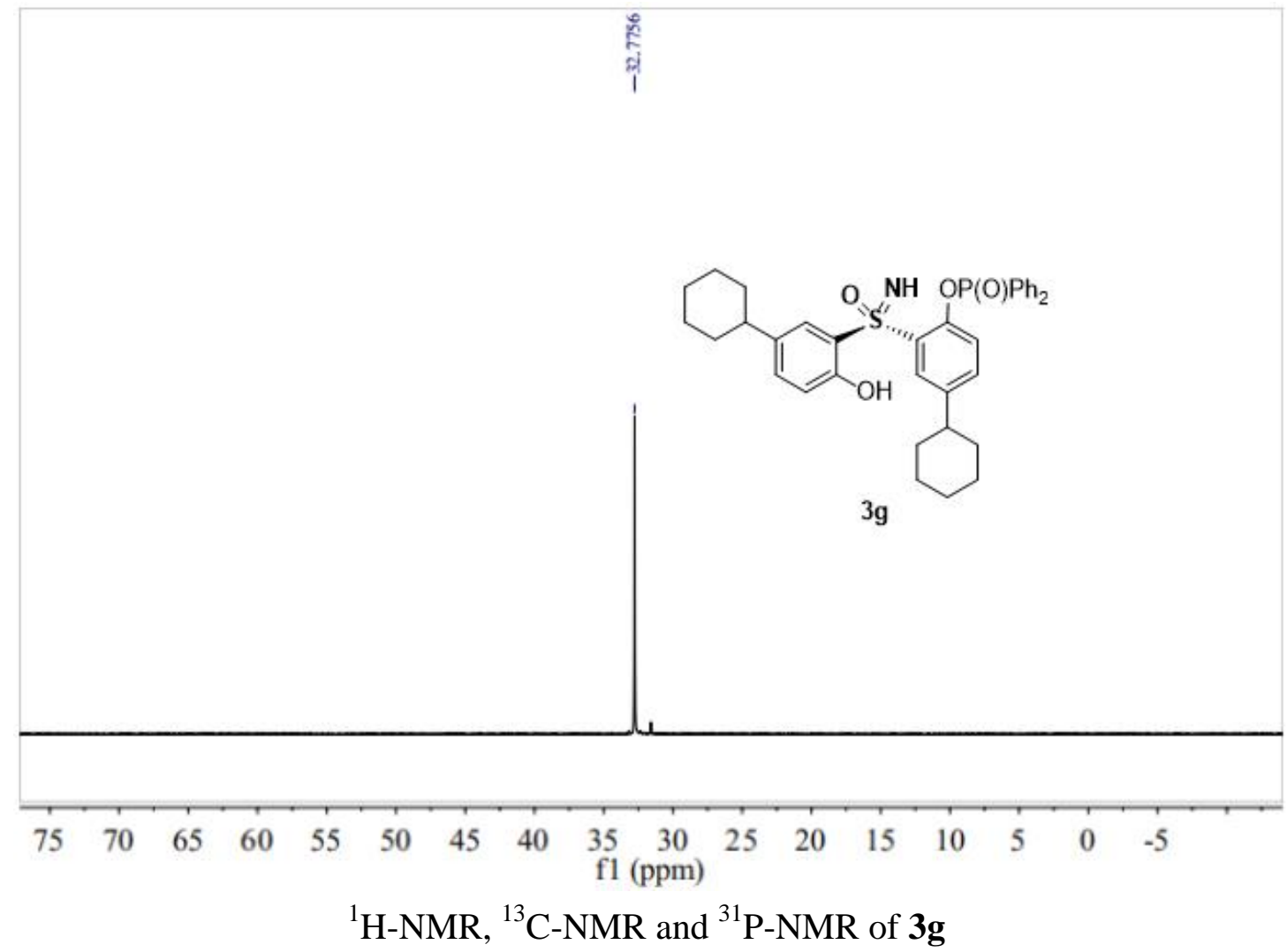



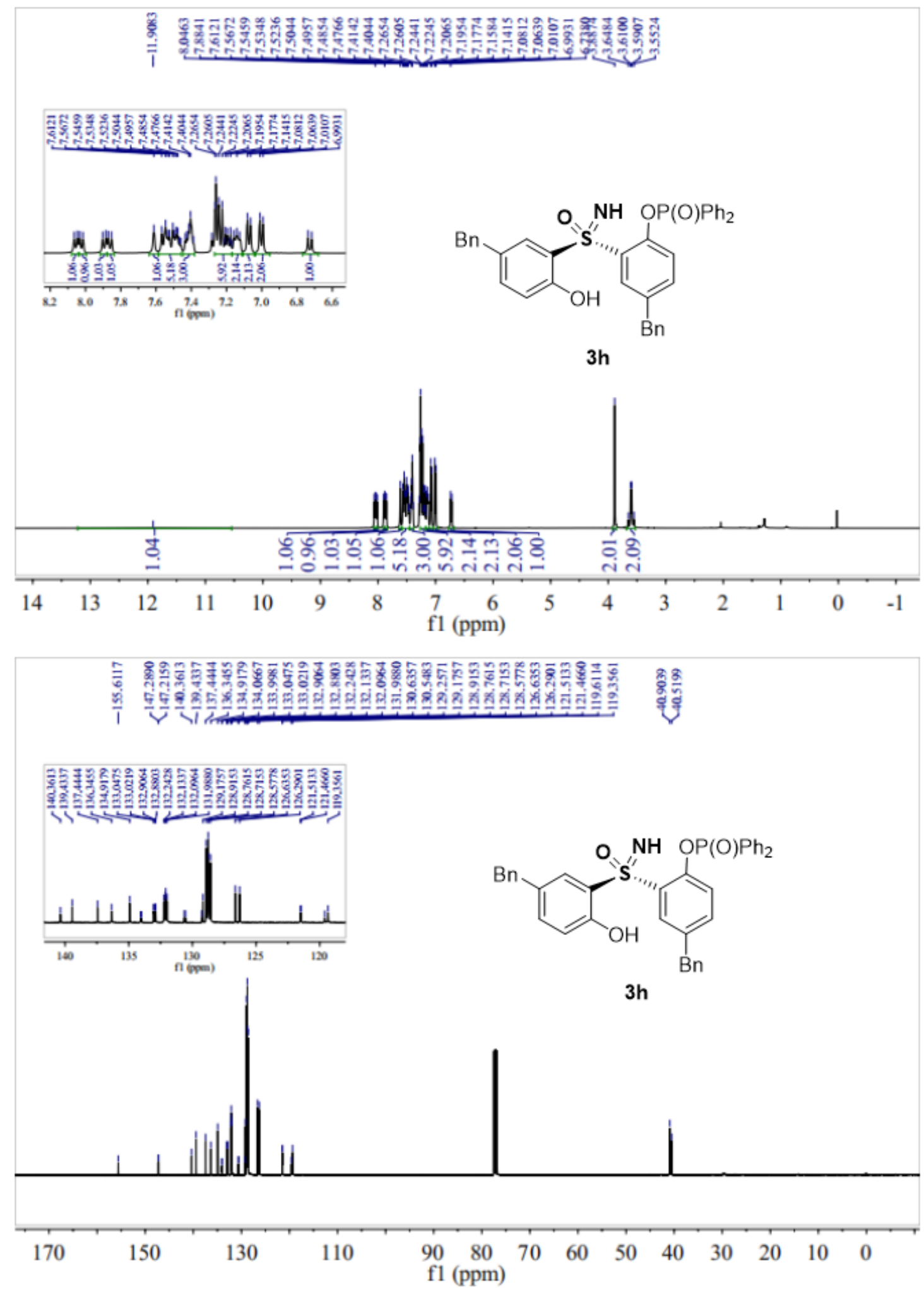


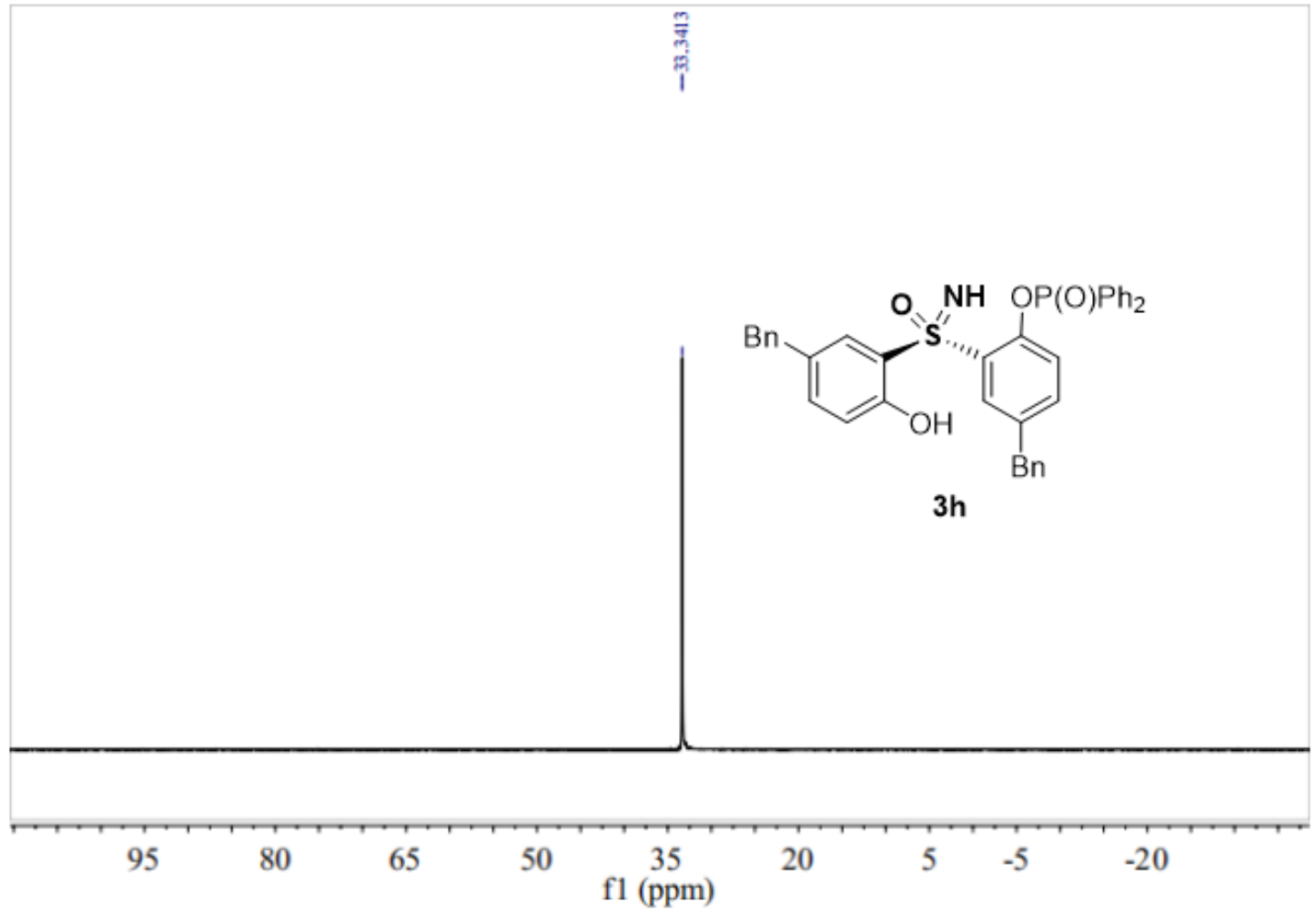

${ }^{1} \mathrm{H}-\mathrm{NMR},{ }^{13} \mathrm{C}-\mathrm{NMR}$ and ${ }^{31} \mathrm{P}-\mathrm{NMR}$ of $\mathbf{3 h}$ 

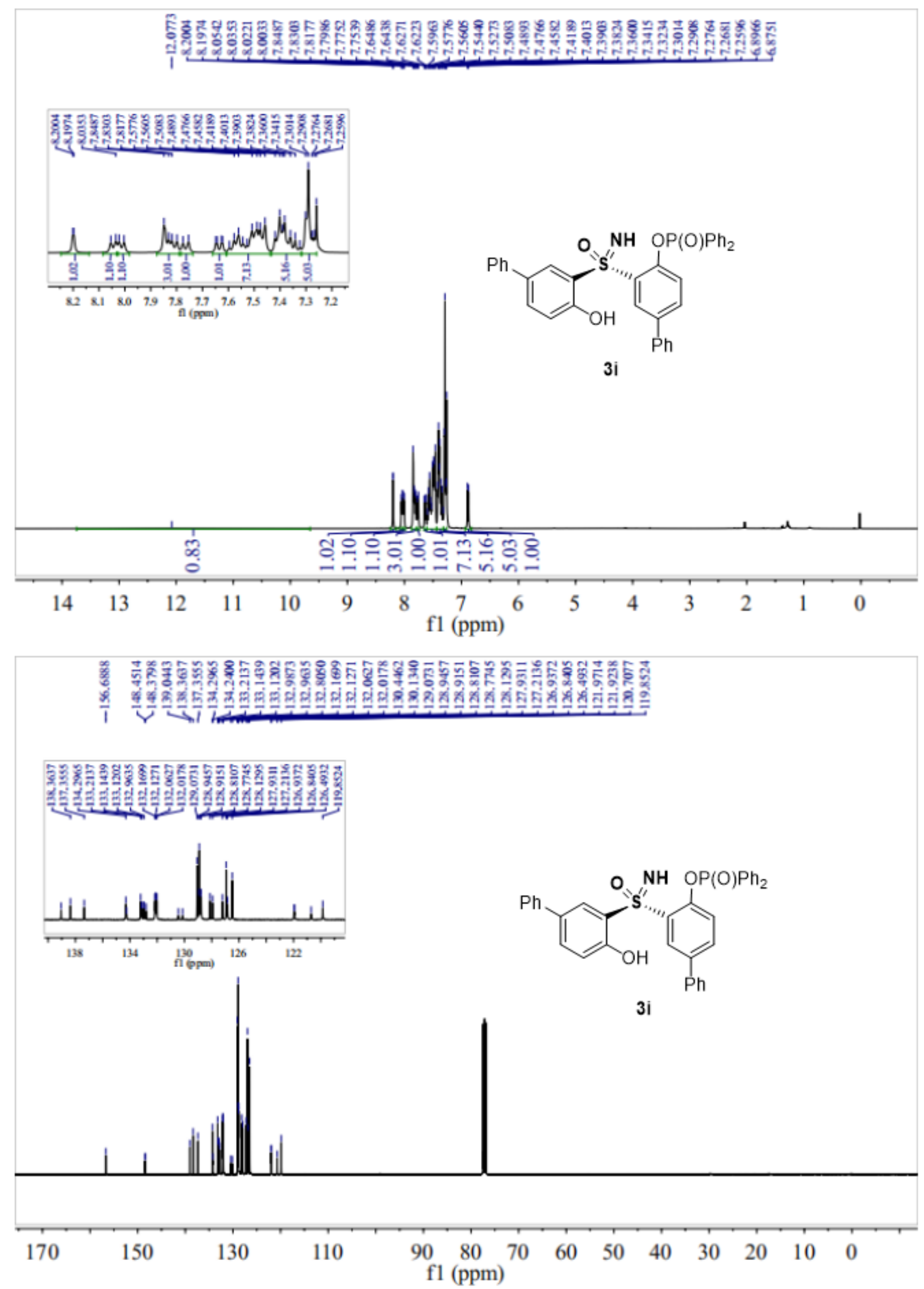


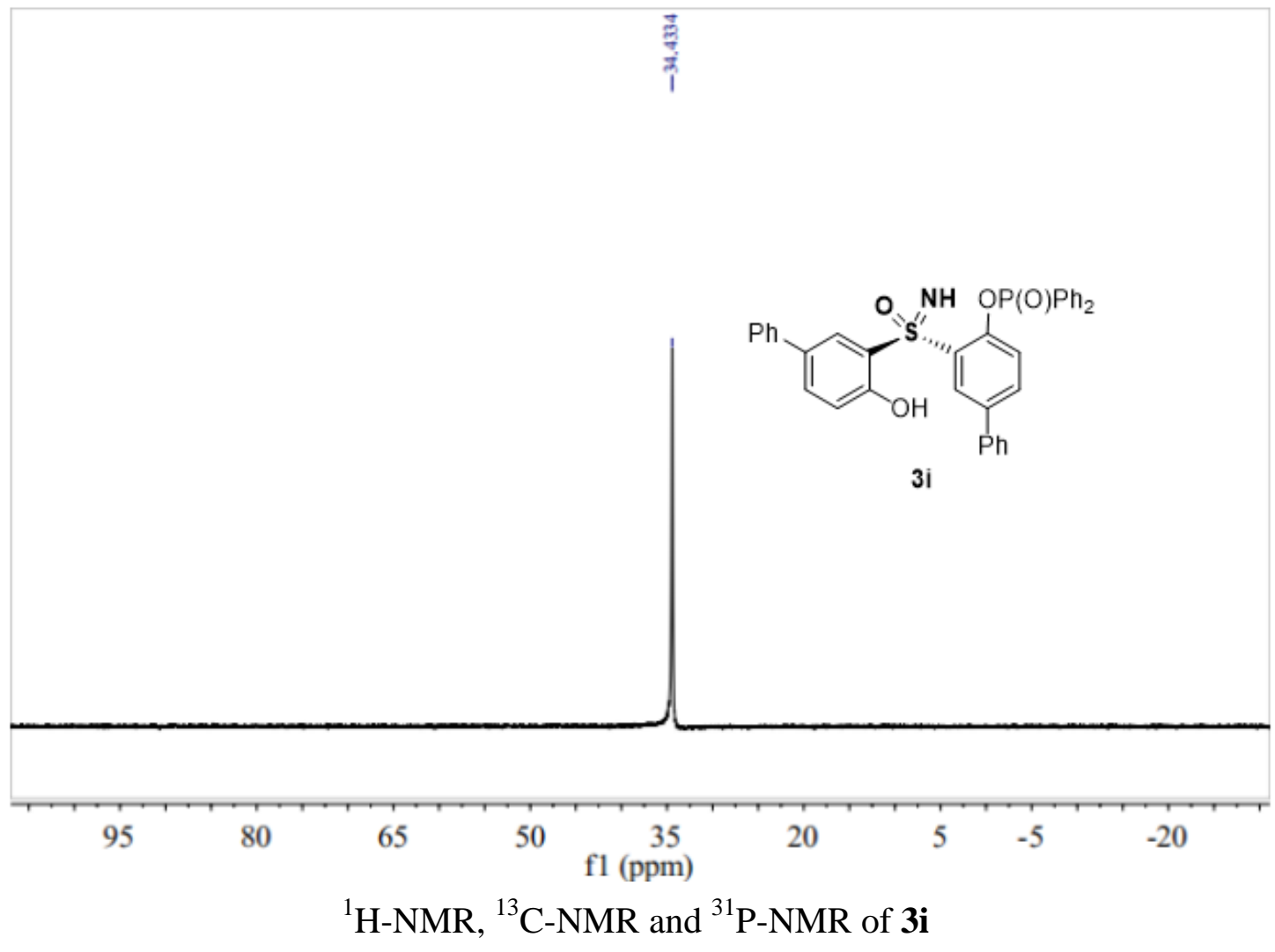



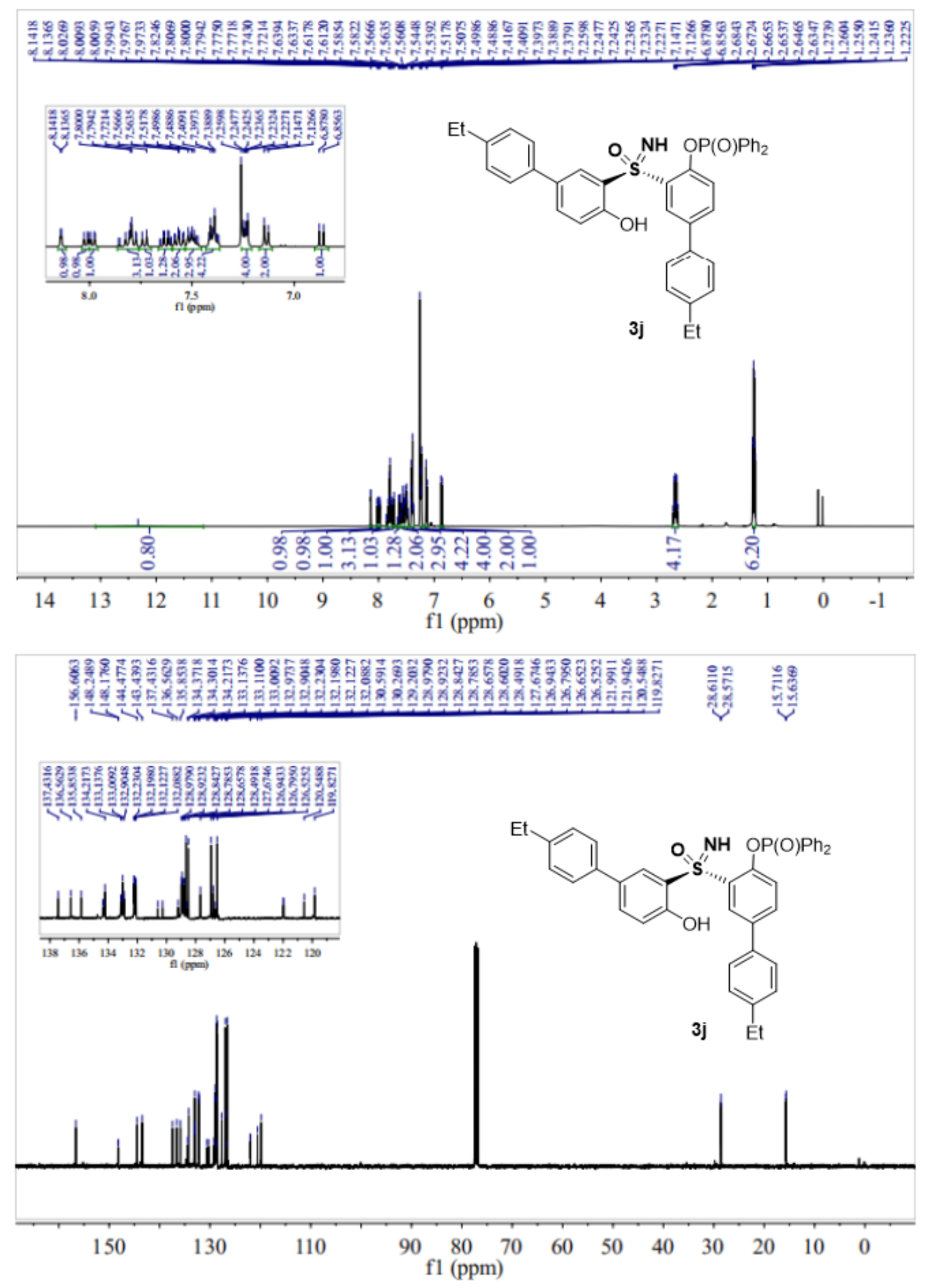


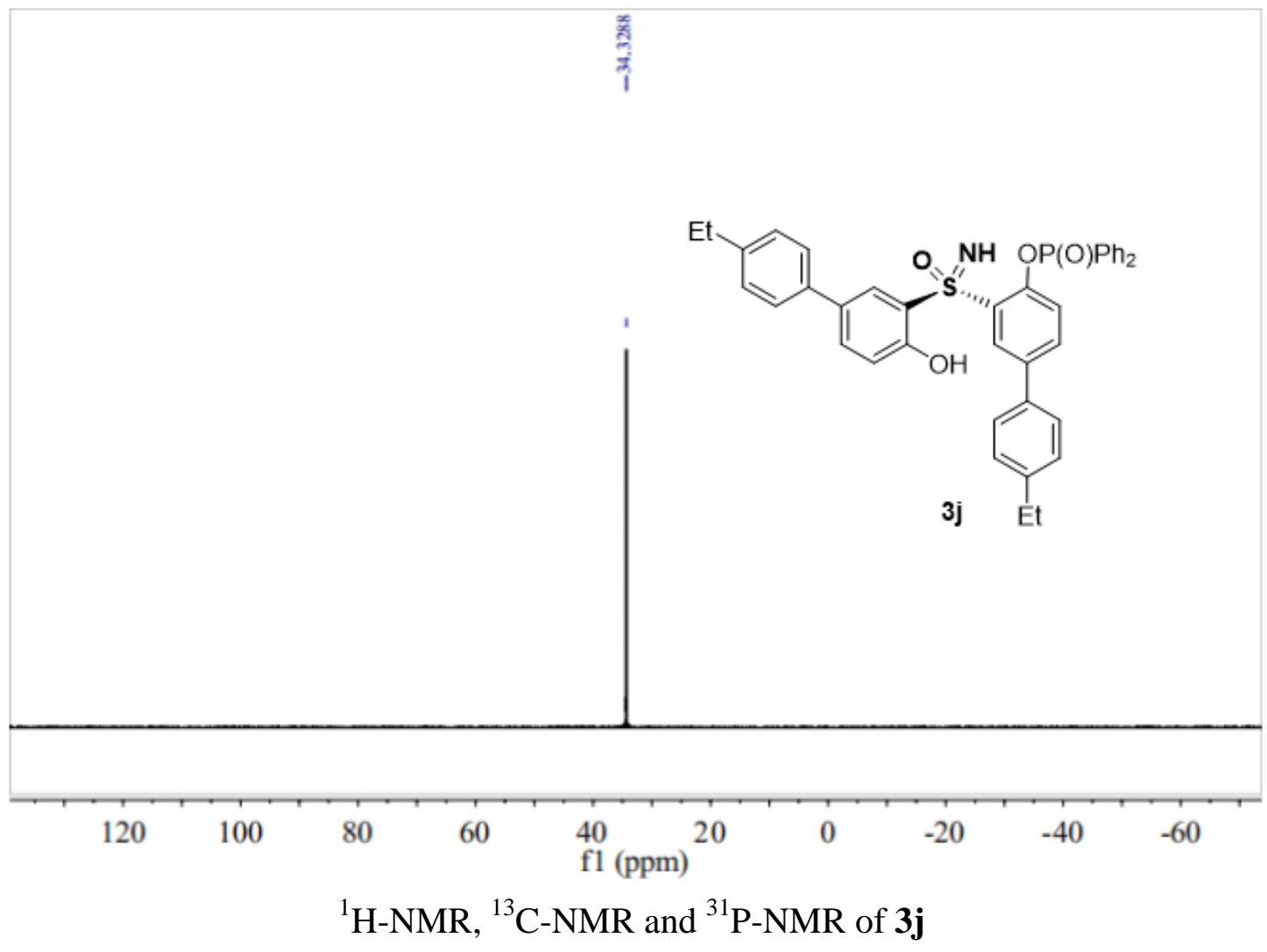



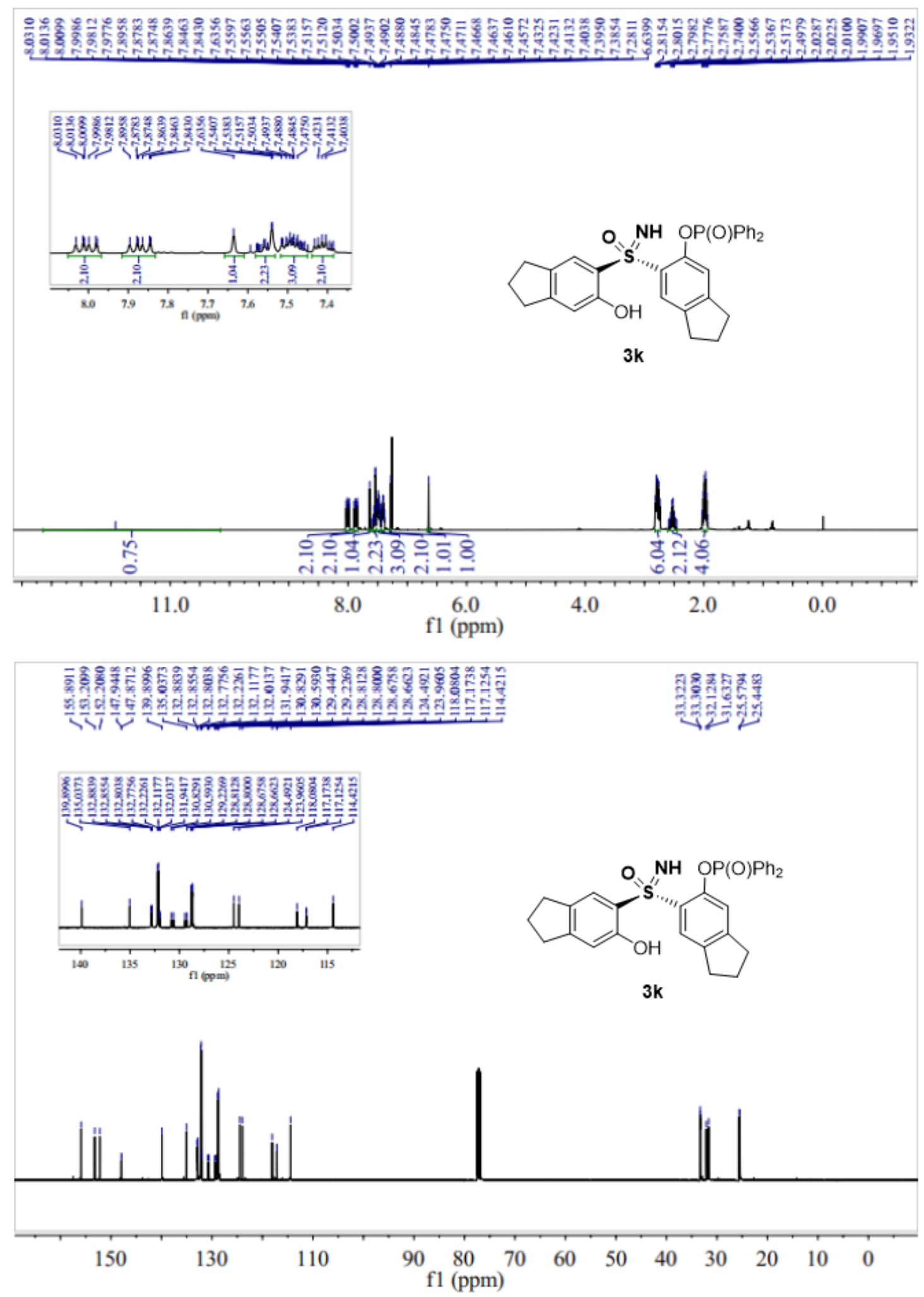


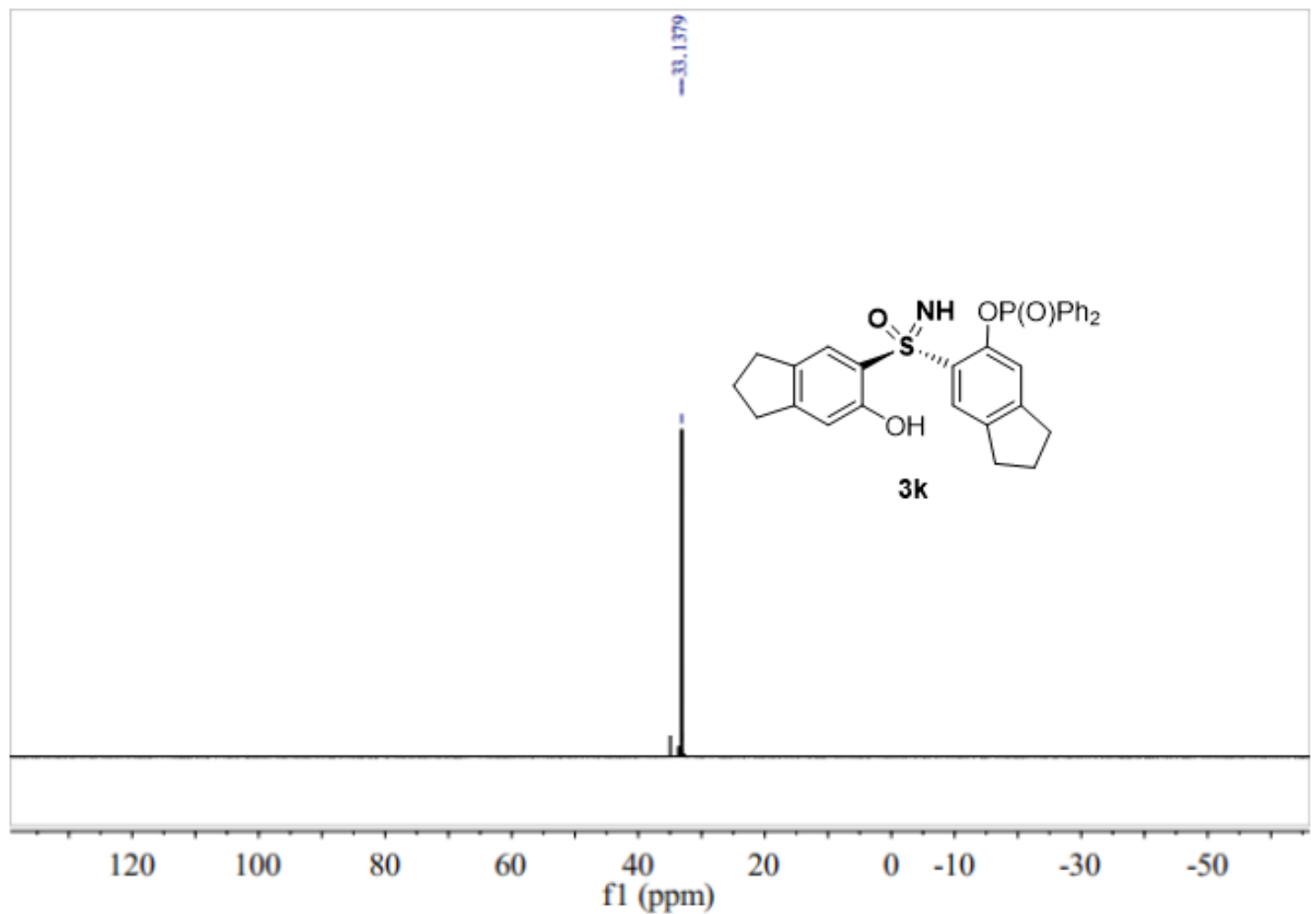

${ }^{1} \mathrm{H}-\mathrm{NMR},{ }^{13} \mathrm{C}-\mathrm{NMR}$ and ${ }^{31} \mathrm{P}-\mathrm{NMR}$ of $\mathbf{3 k}$ 

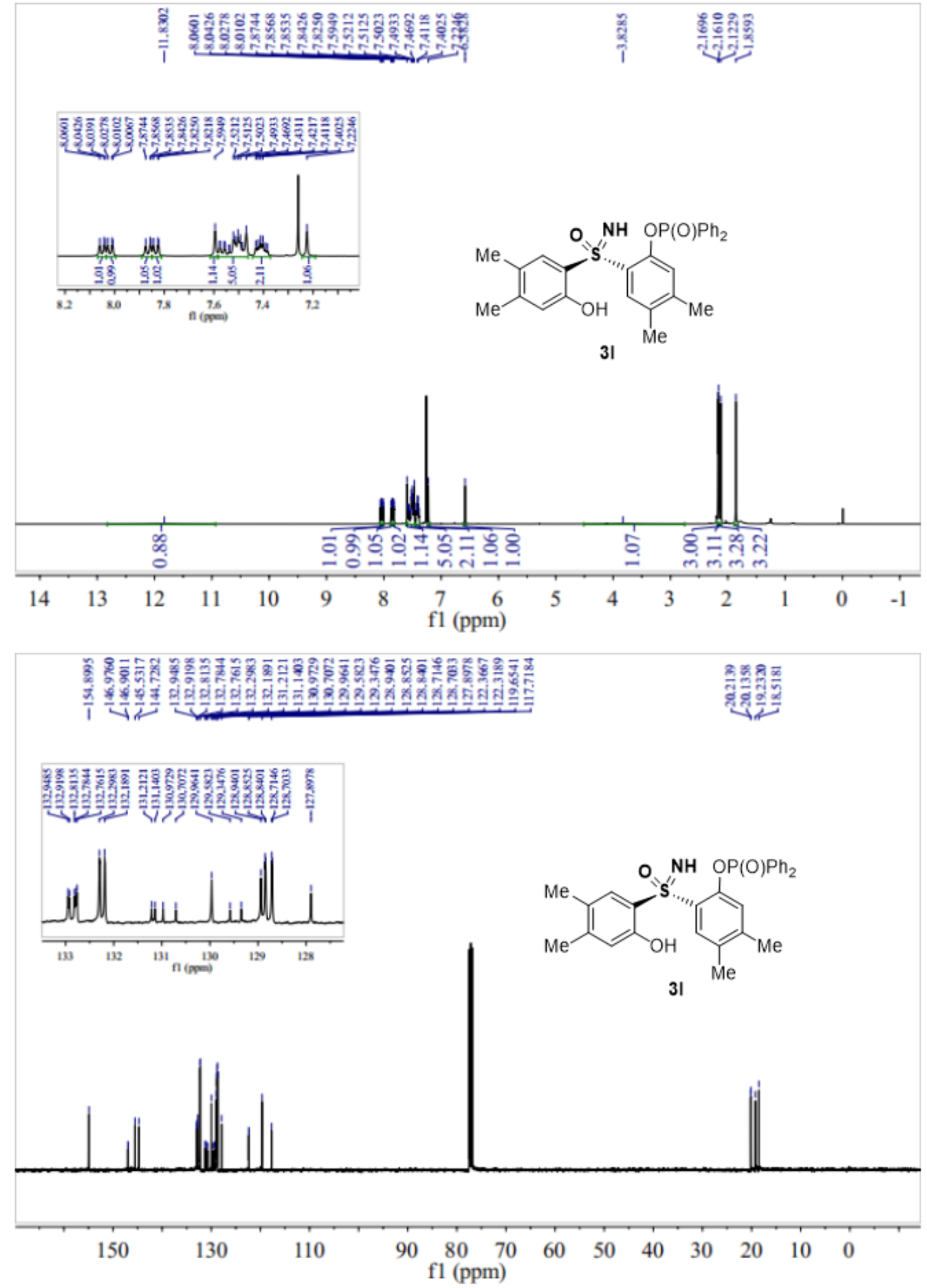


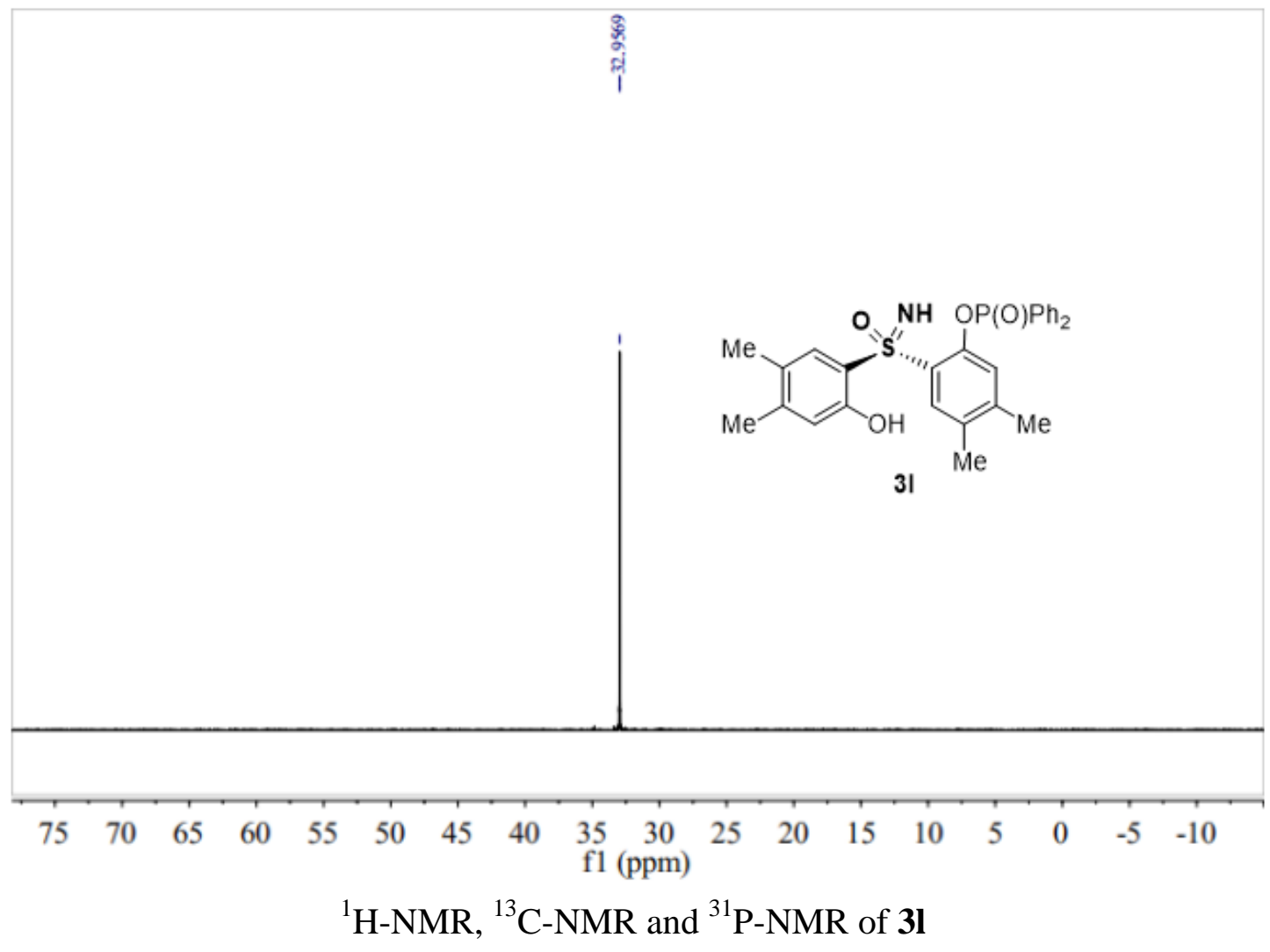



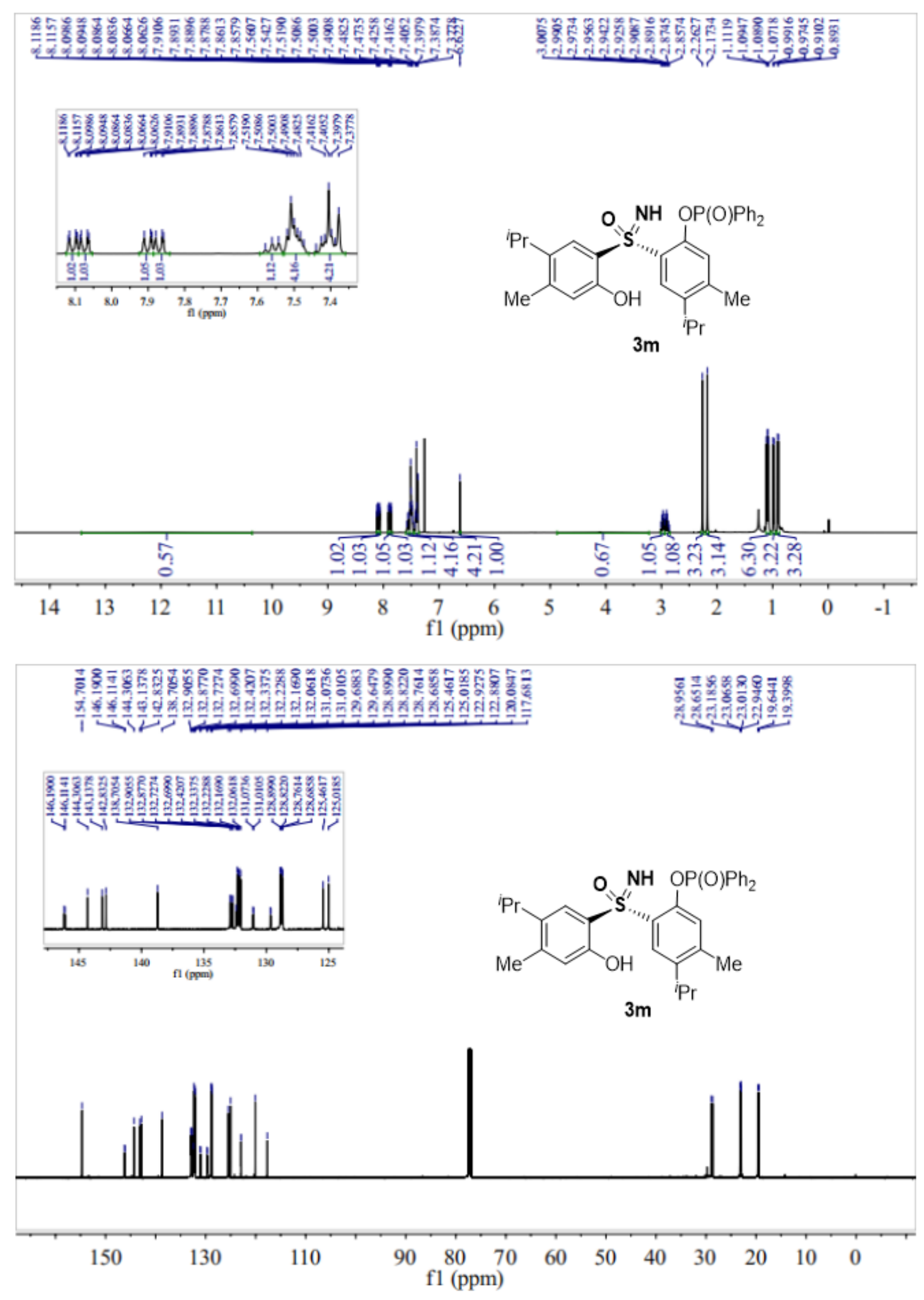


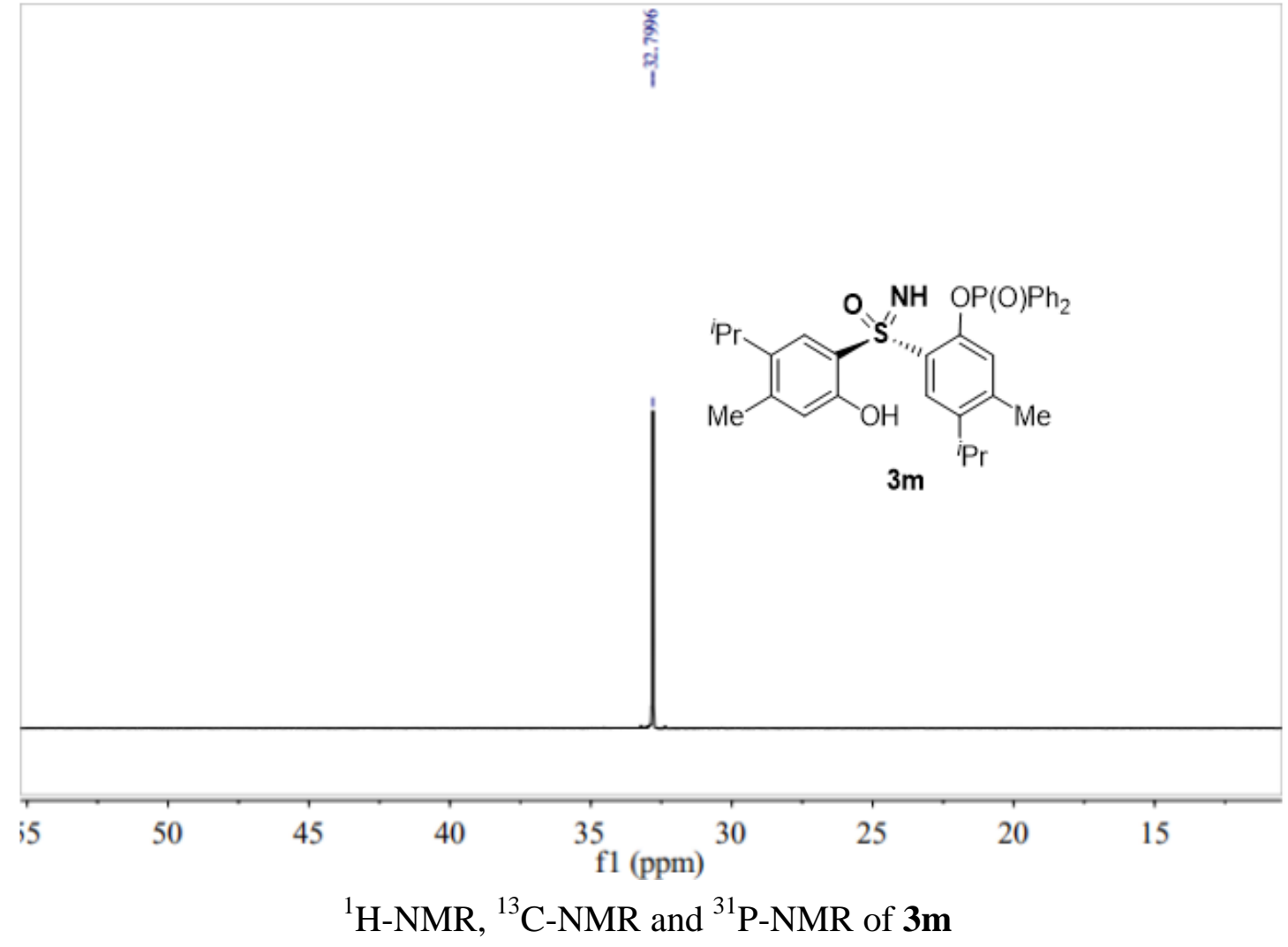



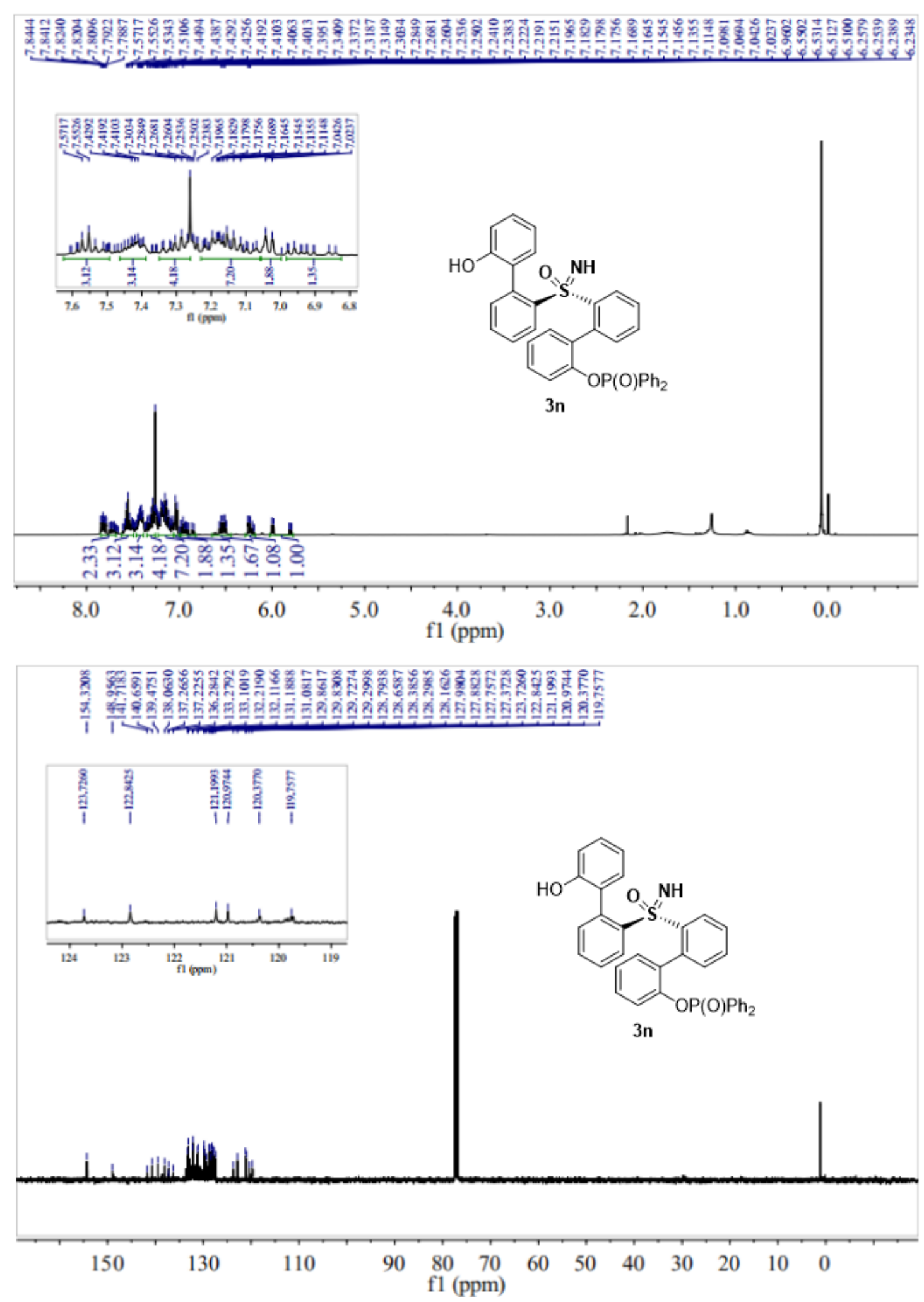


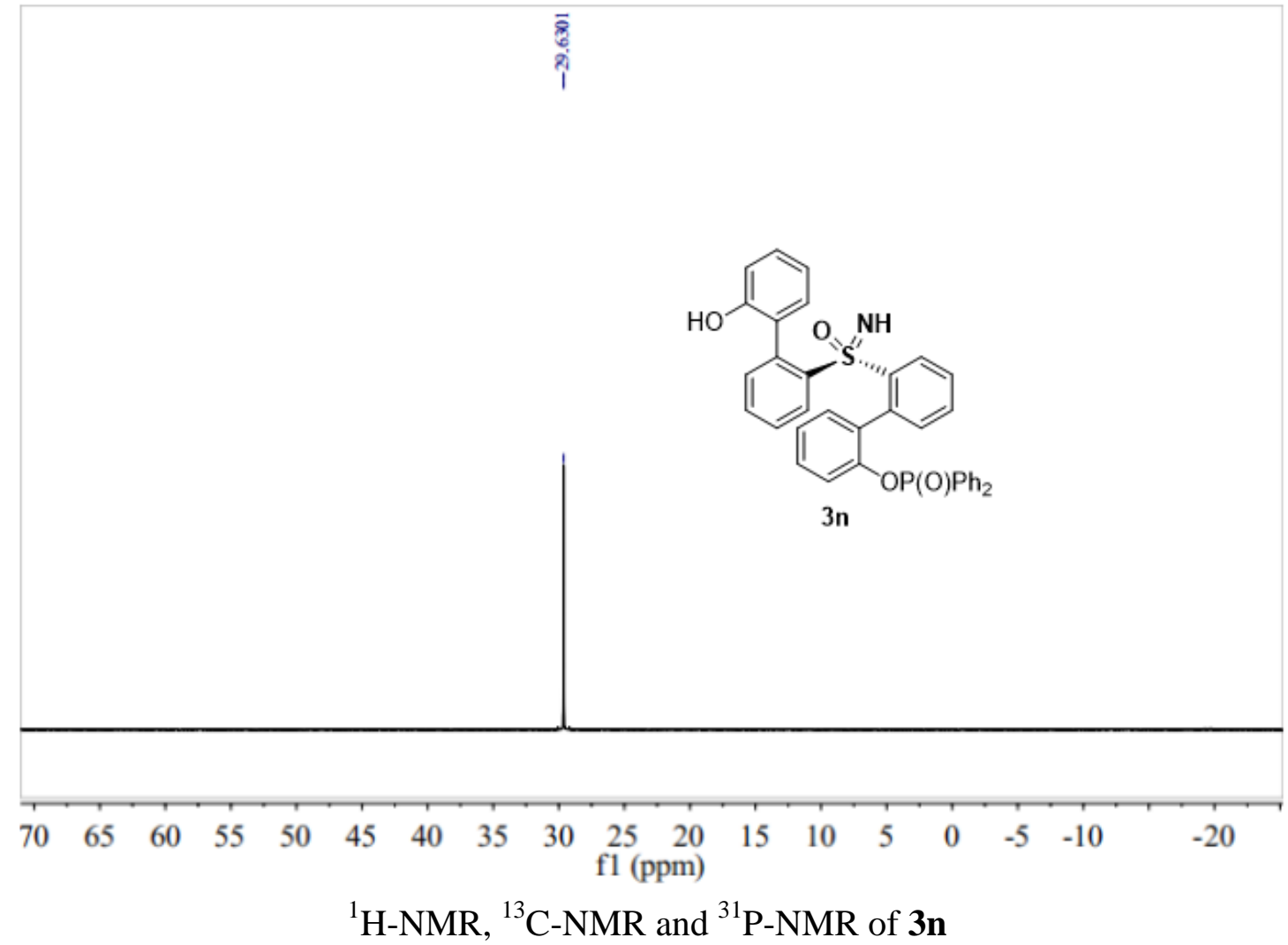



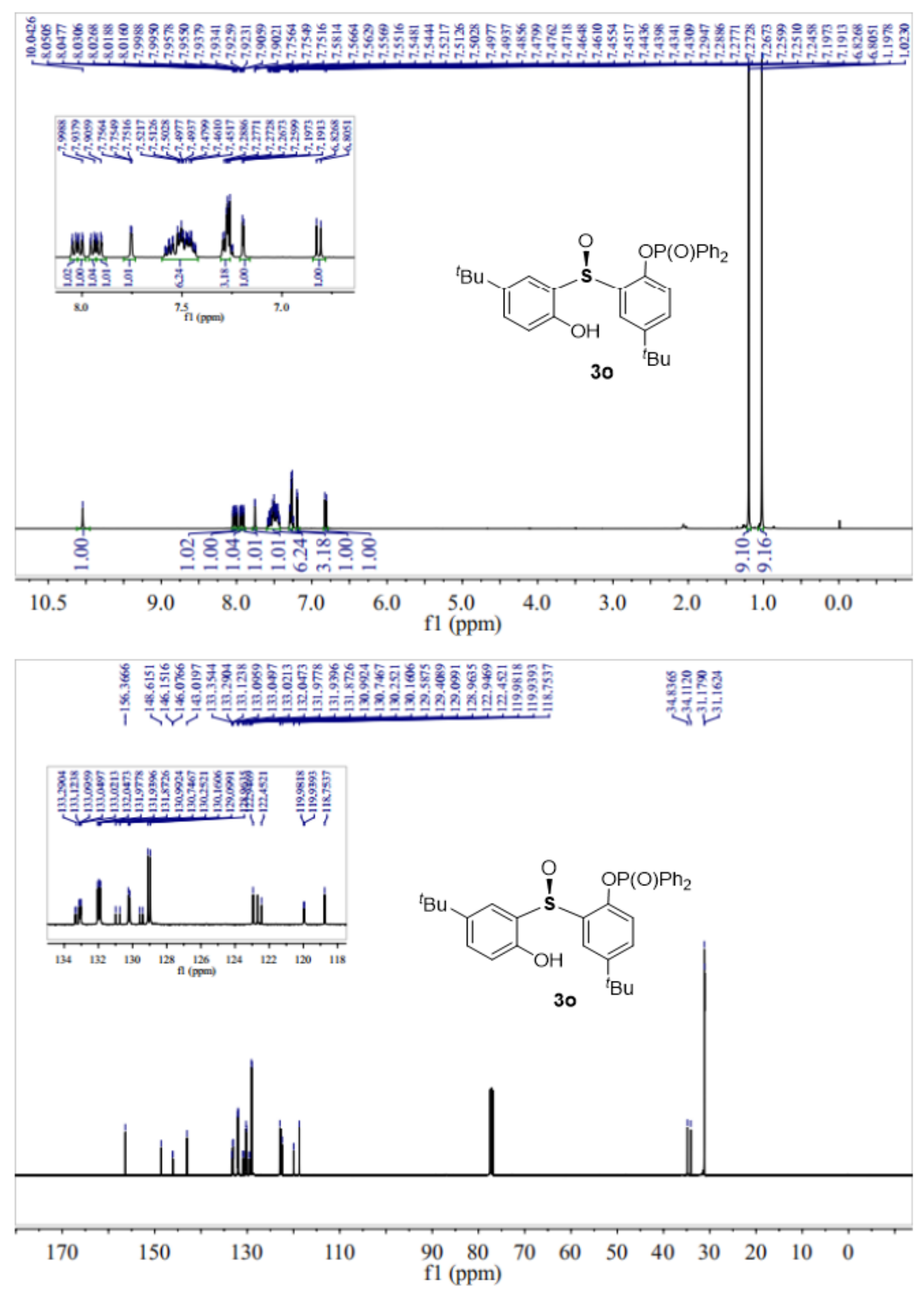


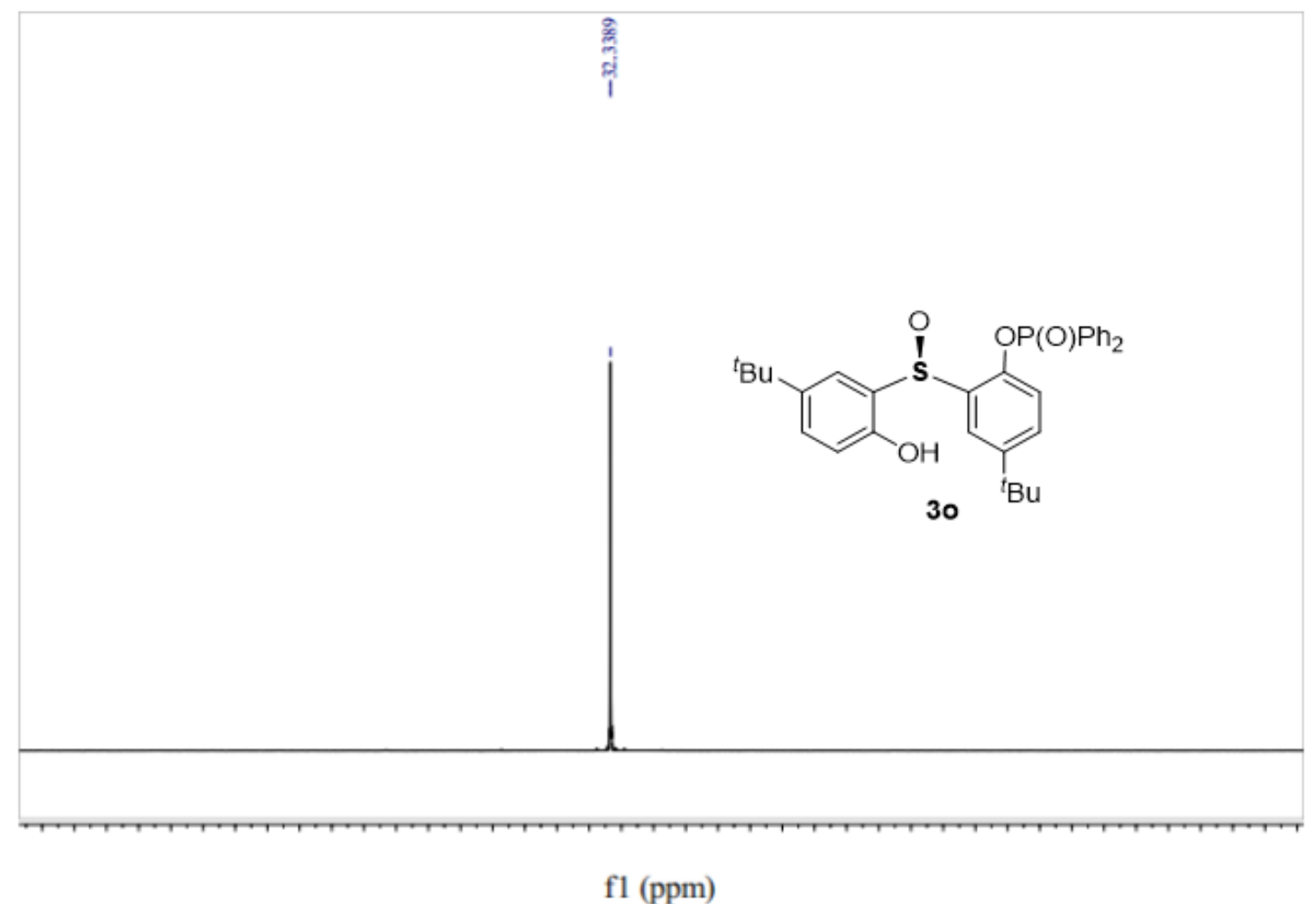

${ }^{1} \mathrm{H}-\mathrm{NMR},{ }^{13} \mathrm{C}-\mathrm{NMR}$ and ${ }^{31} \mathrm{P}-\mathrm{NMR}$ of $\mathbf{3 o}$ 

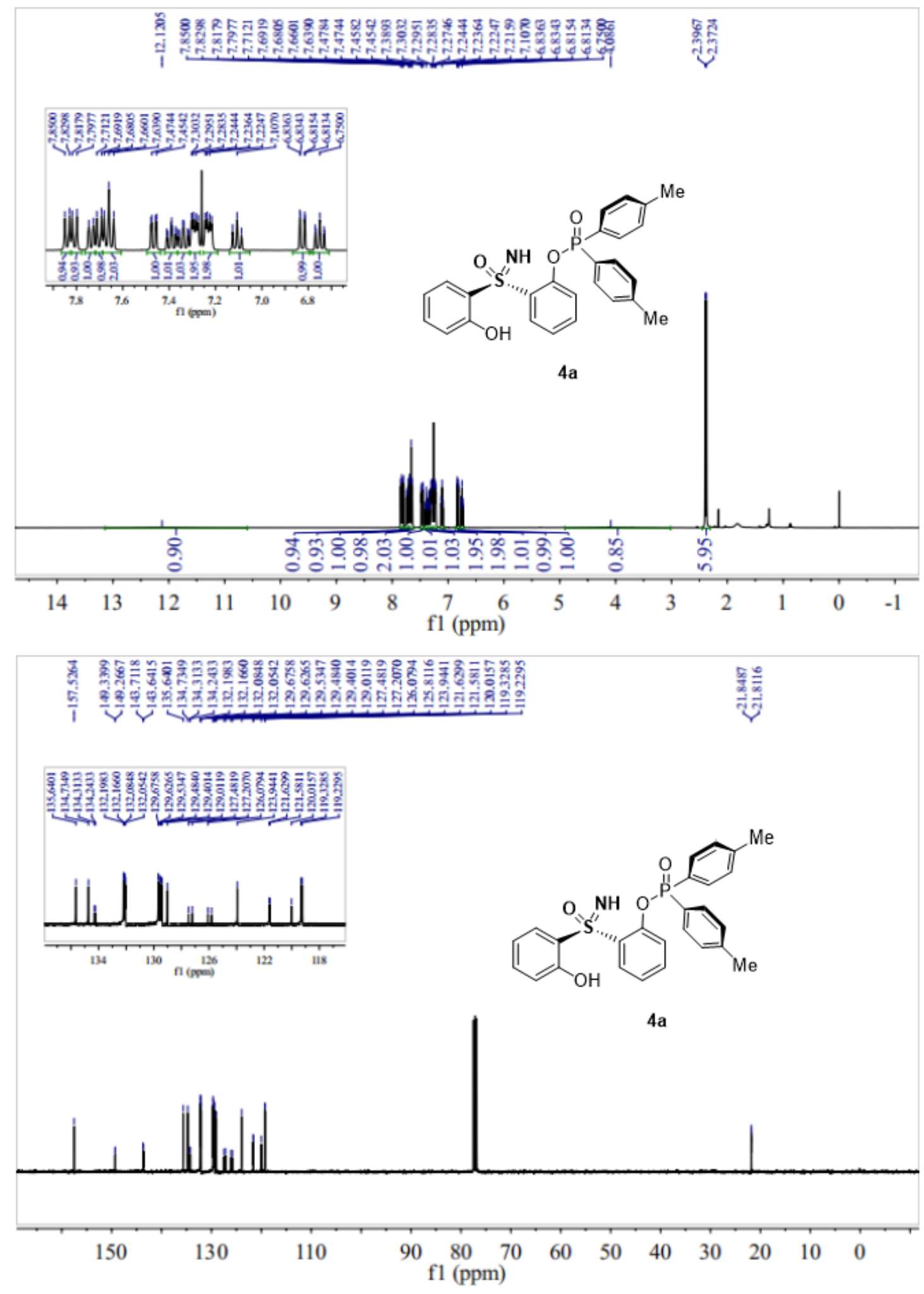


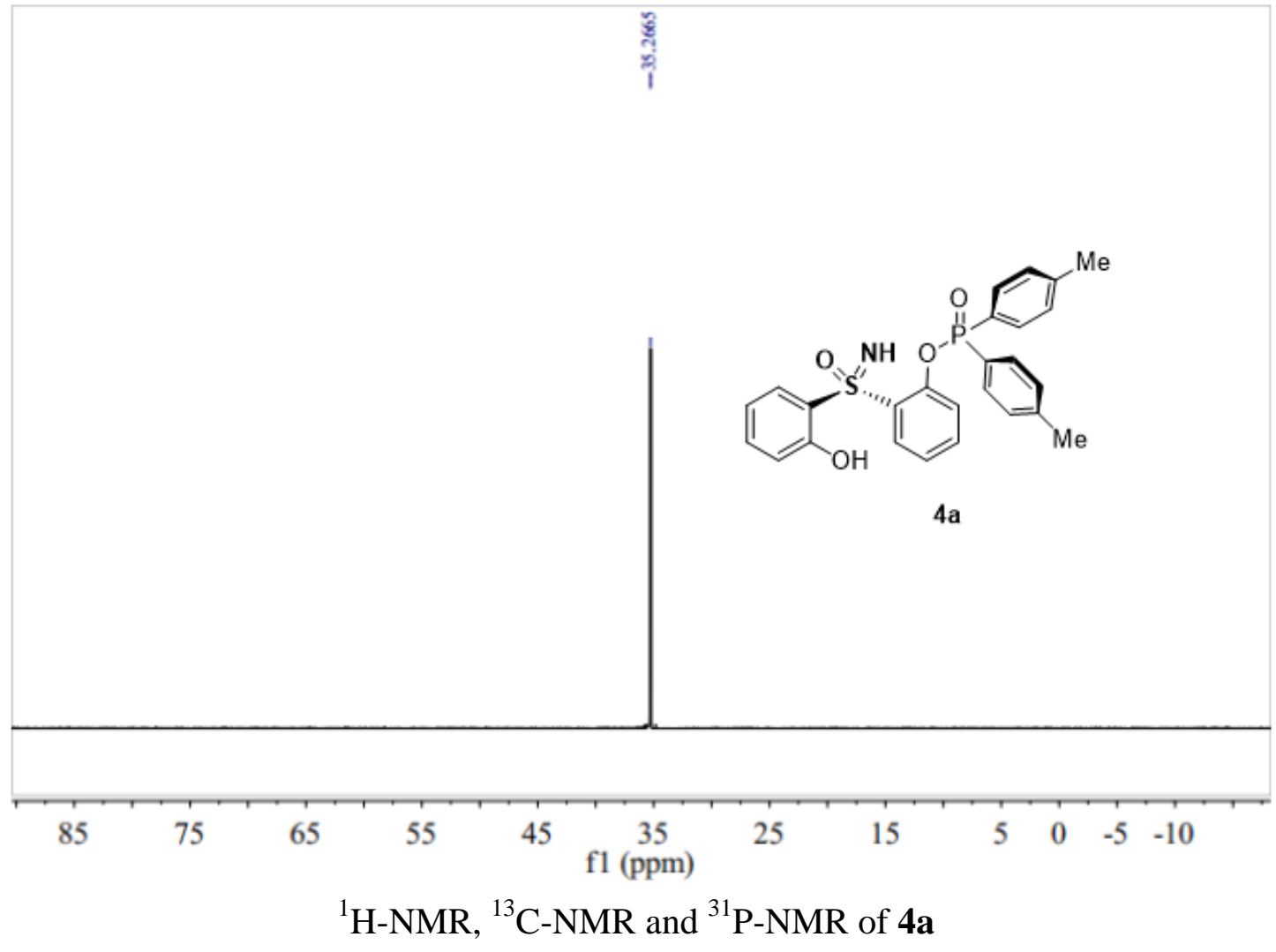



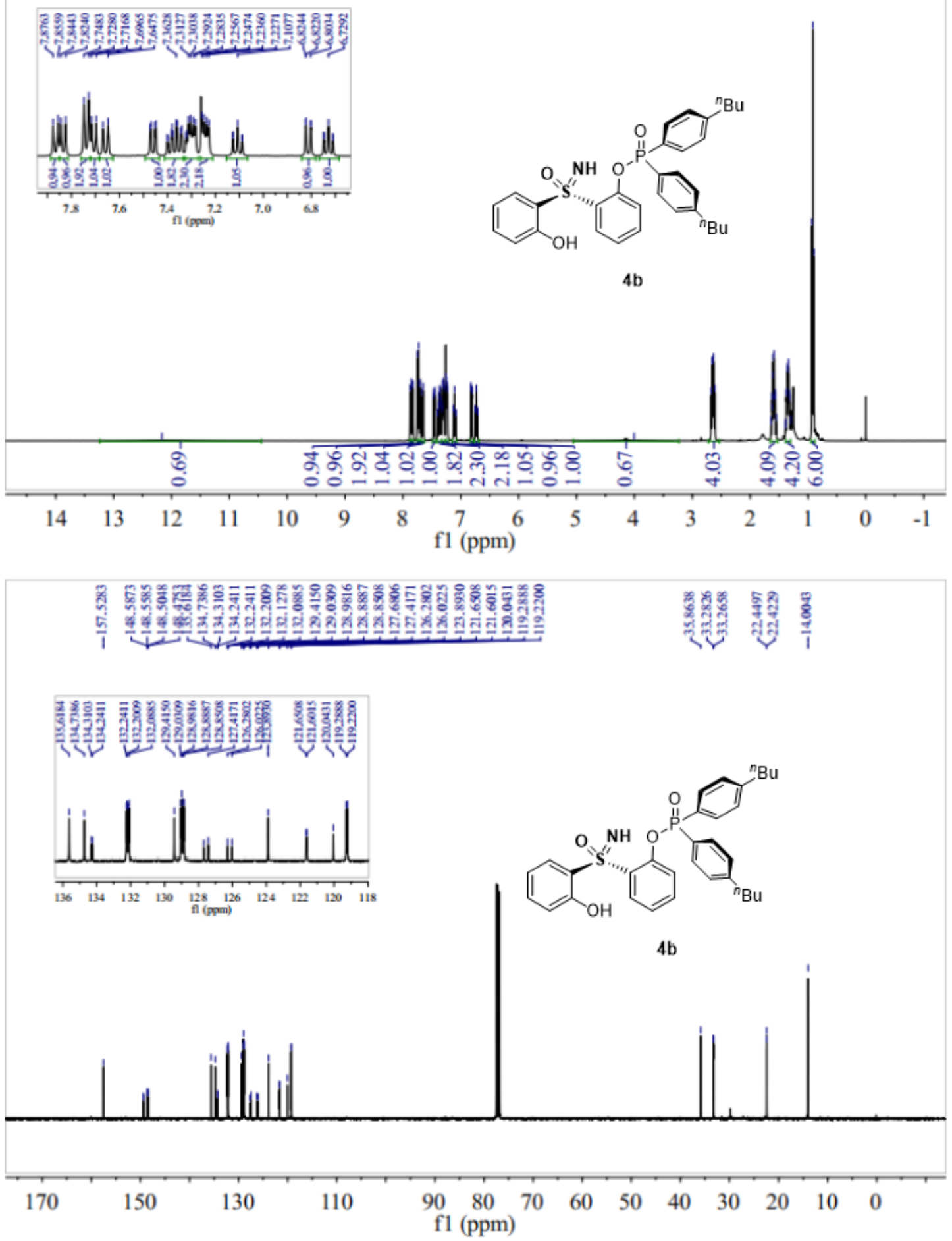


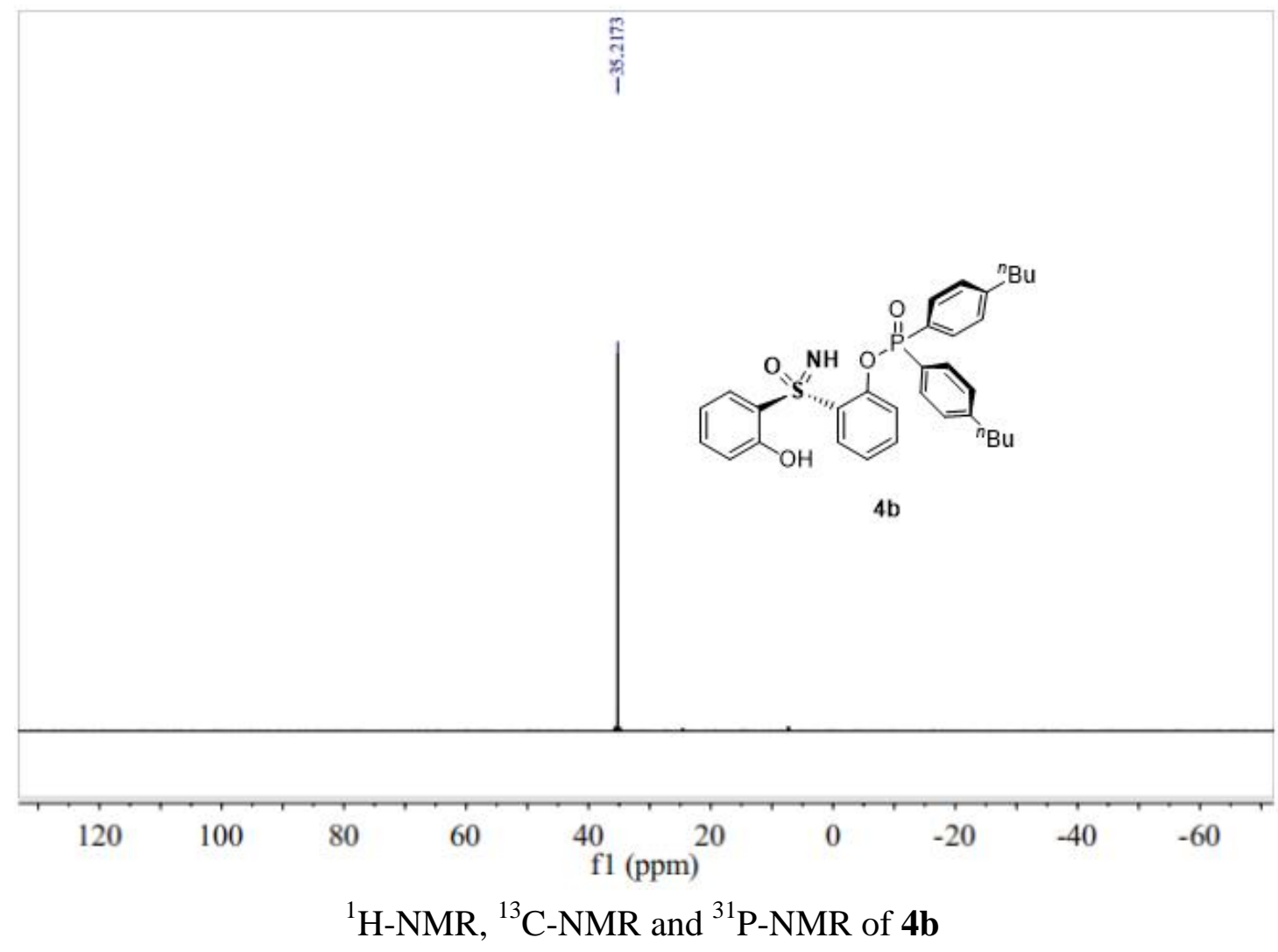




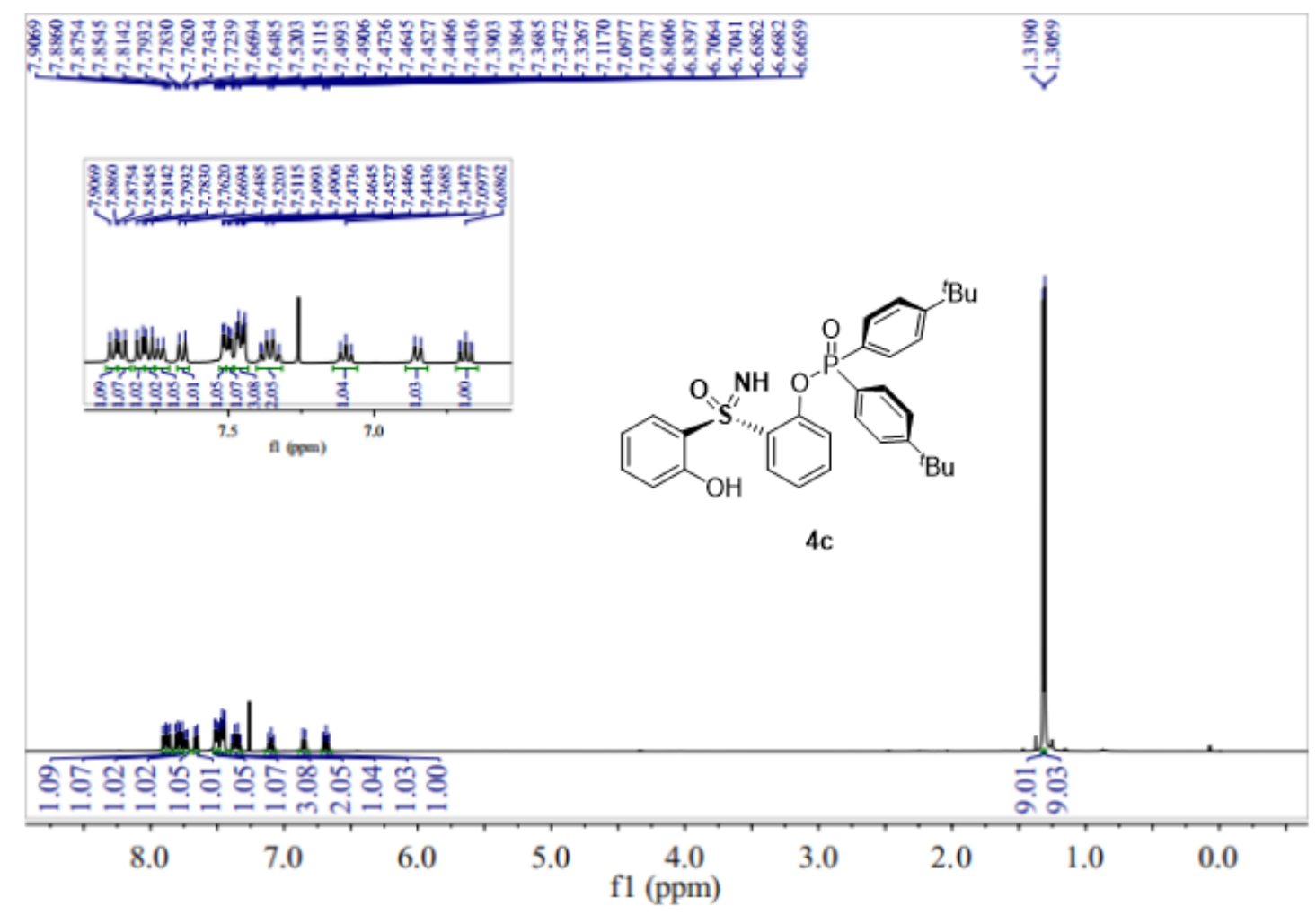

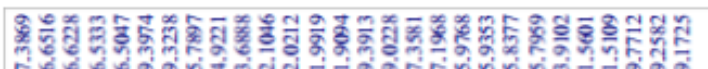

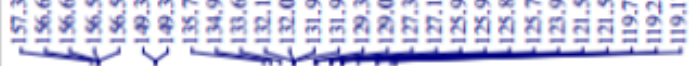
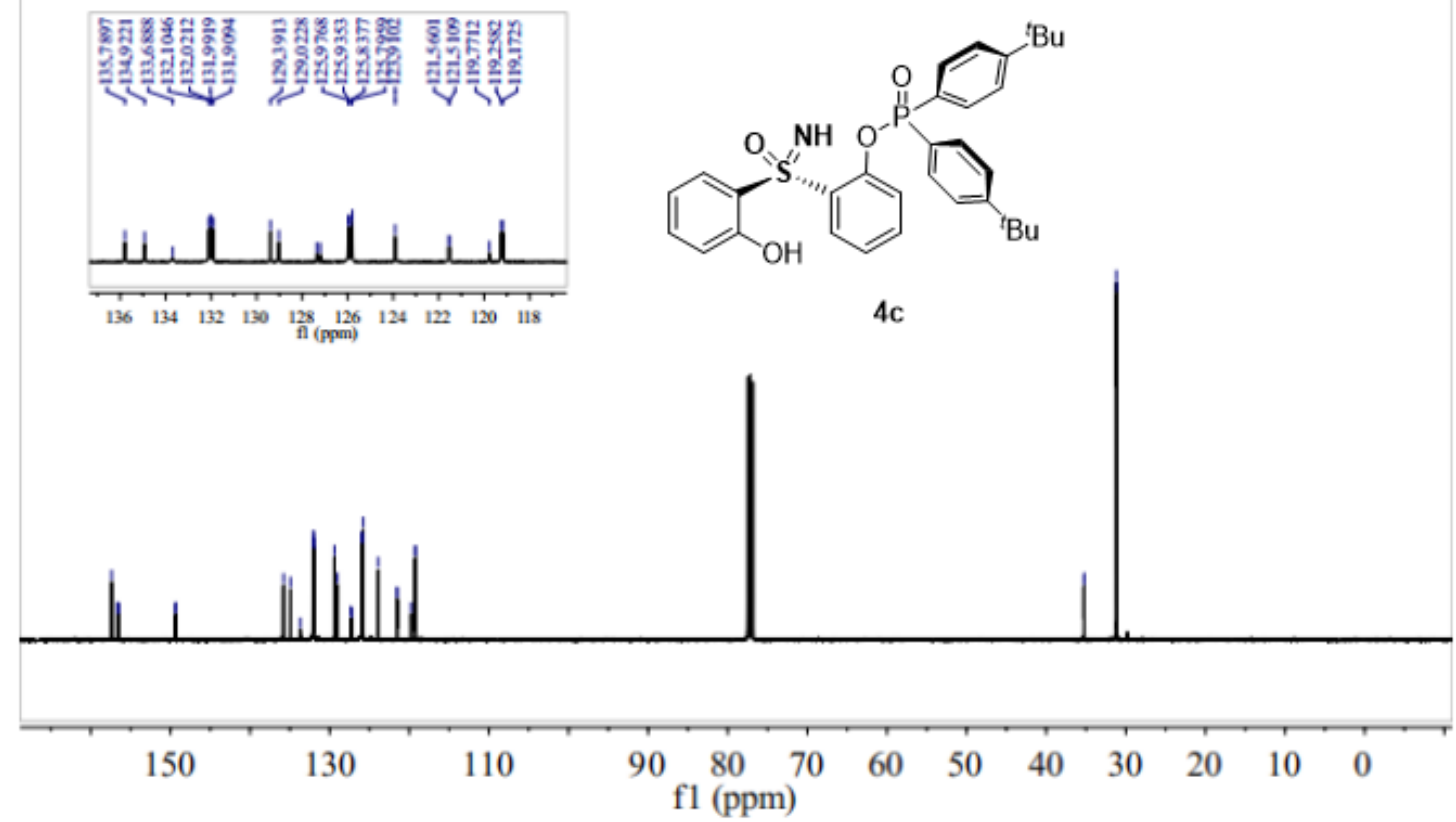


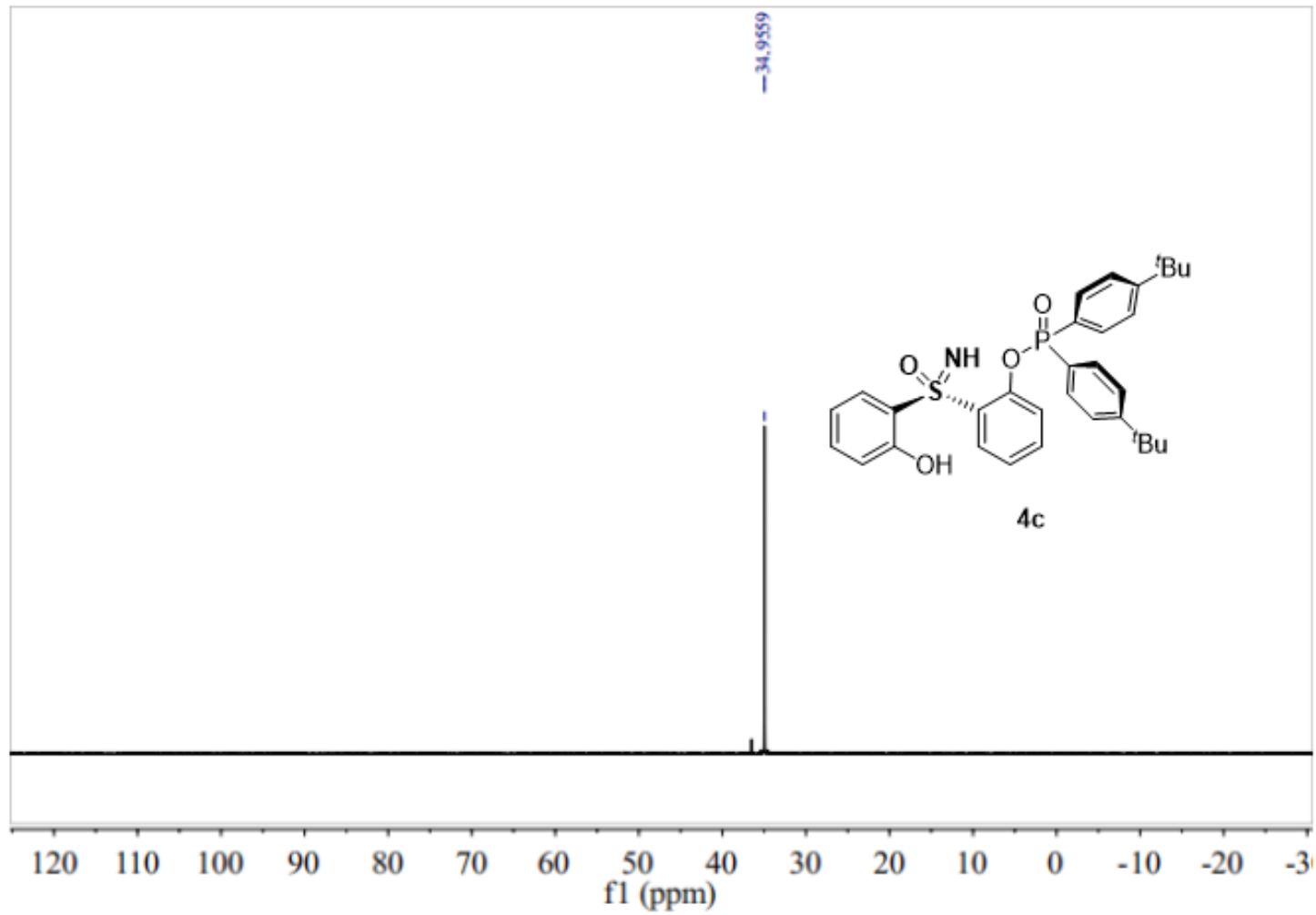

${ }^{1} \mathrm{H}-\mathrm{NMR},{ }^{13} \mathrm{C}-\mathrm{NMR}$ and ${ }^{31} \mathrm{P}-\mathrm{NMR}$ of $\mathbf{4 c}$ 

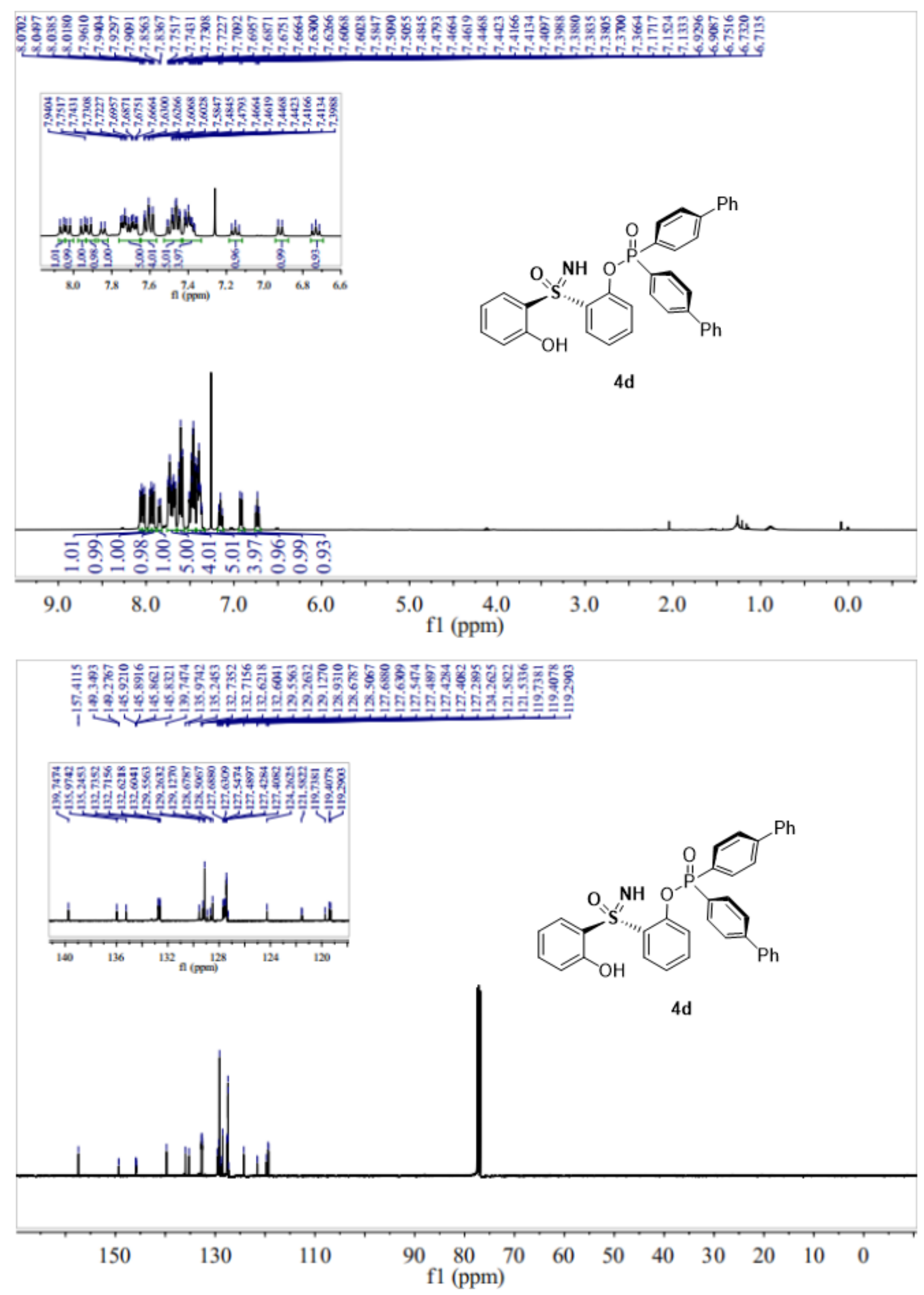


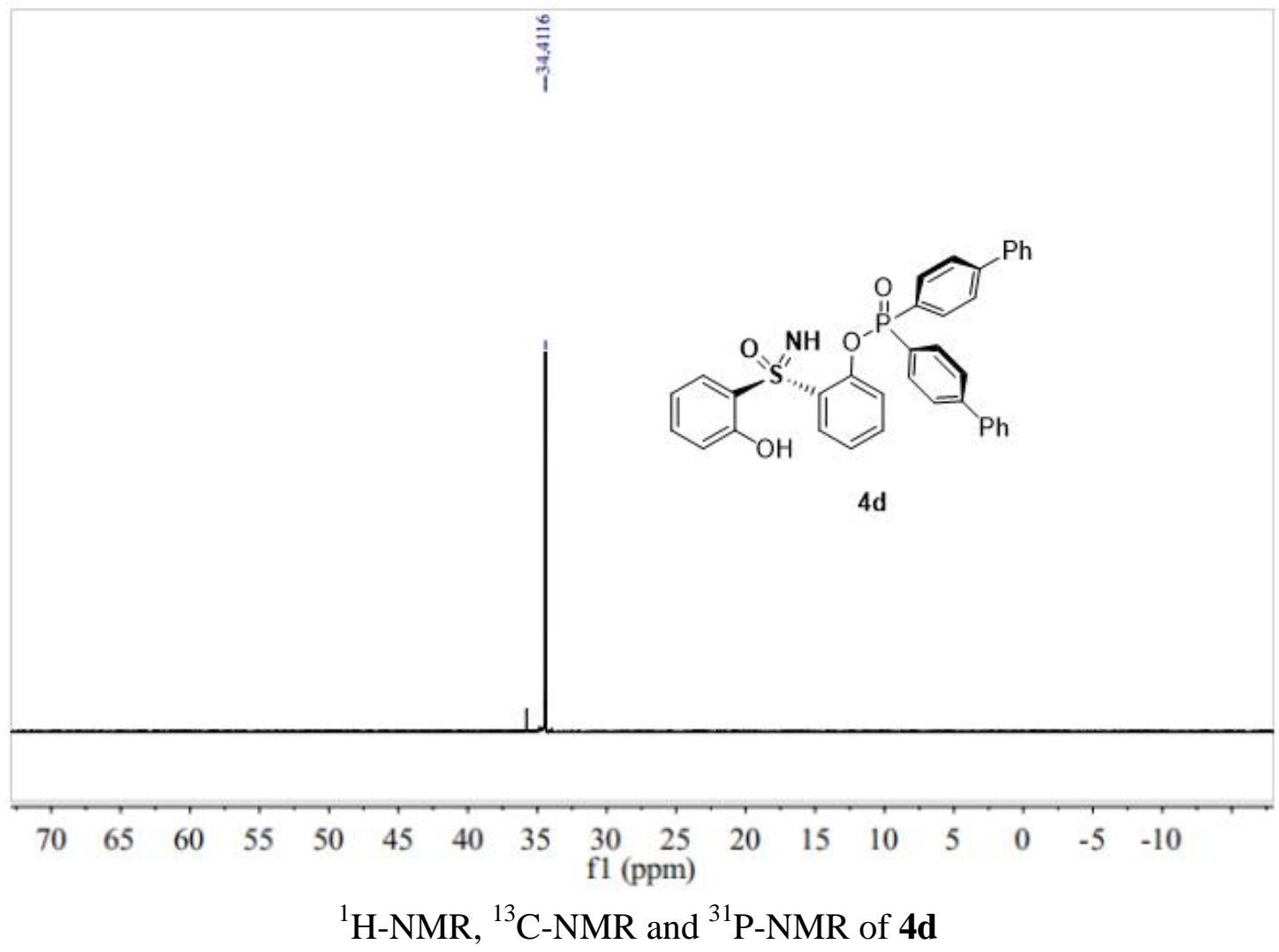



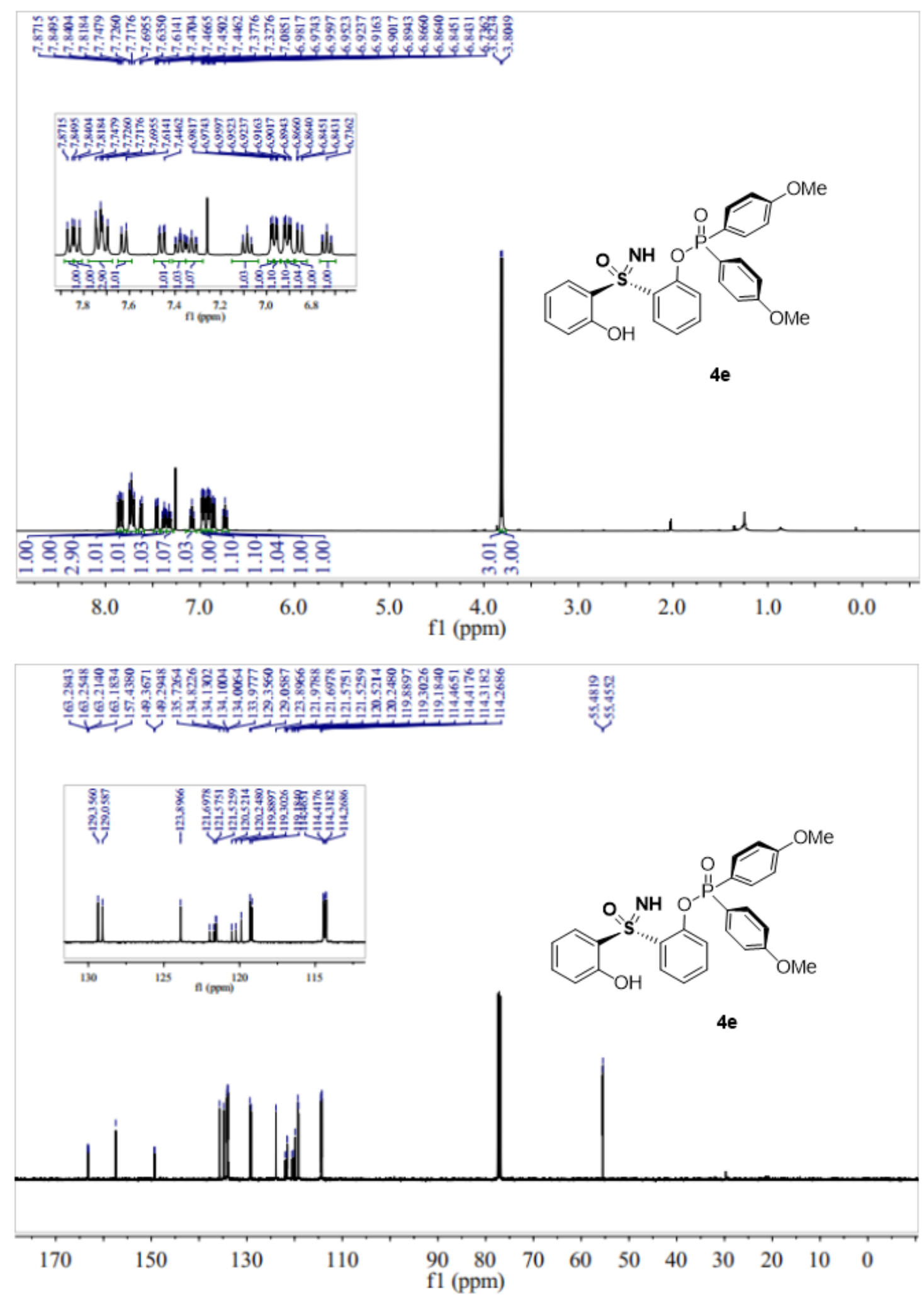


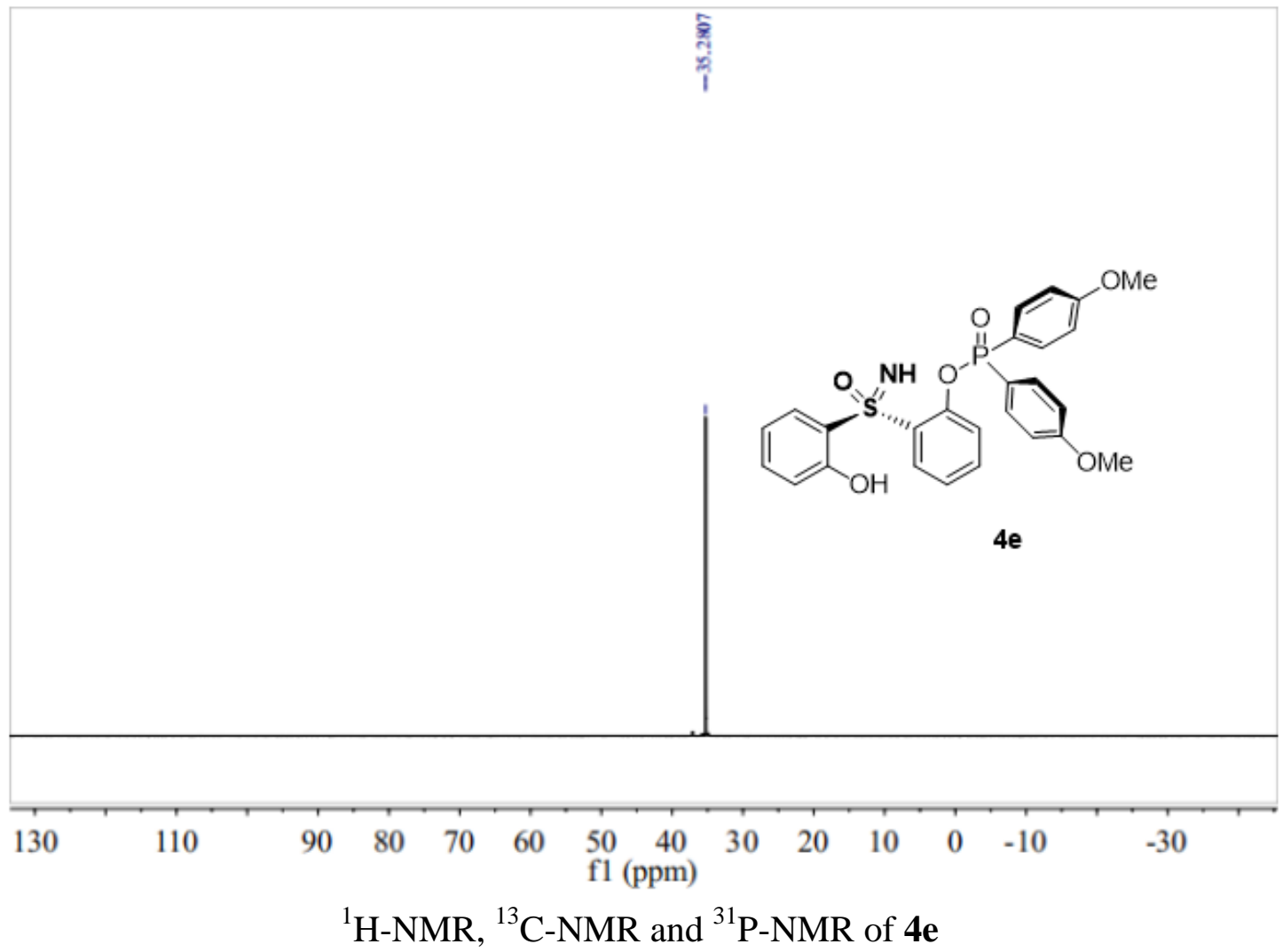



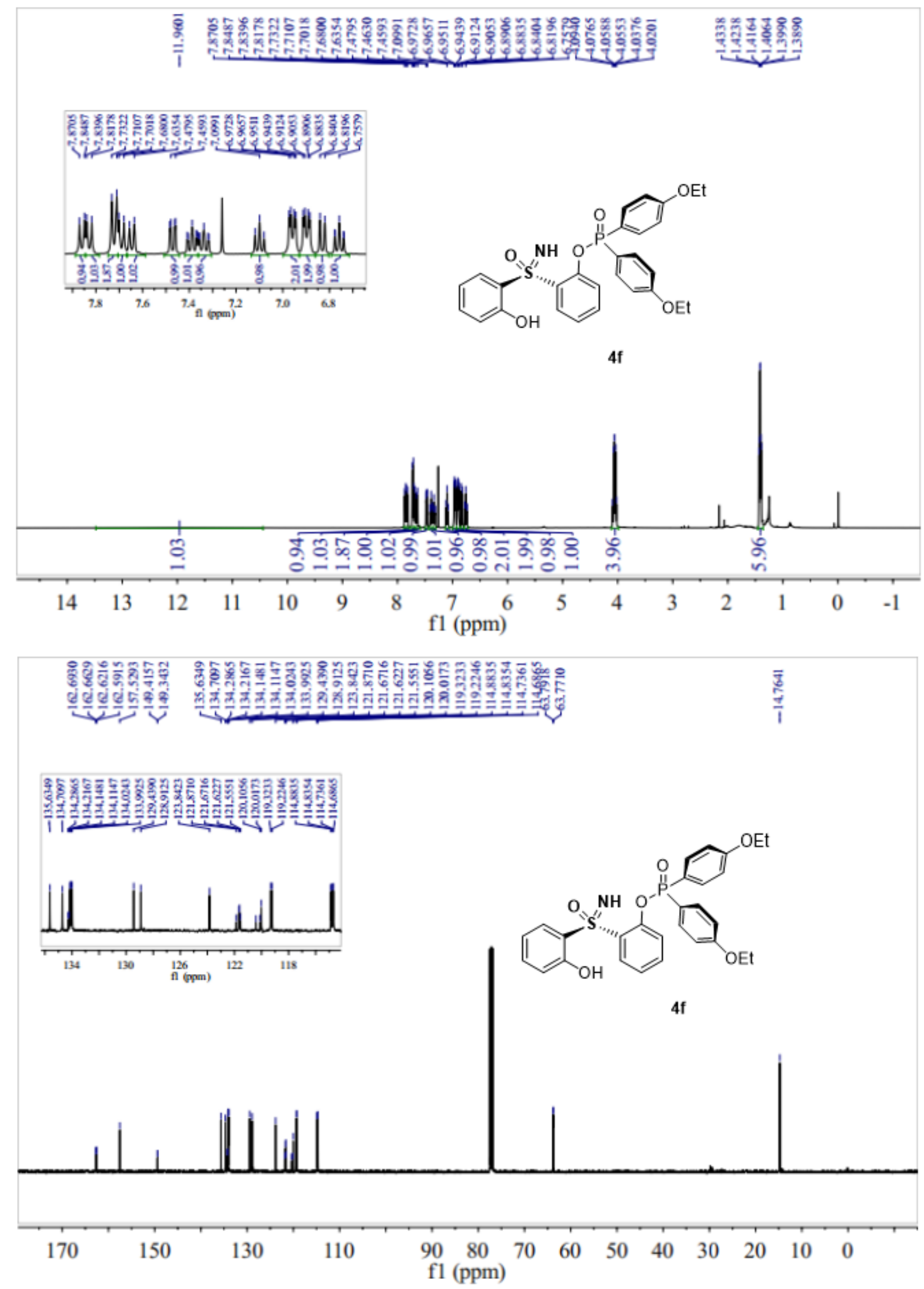


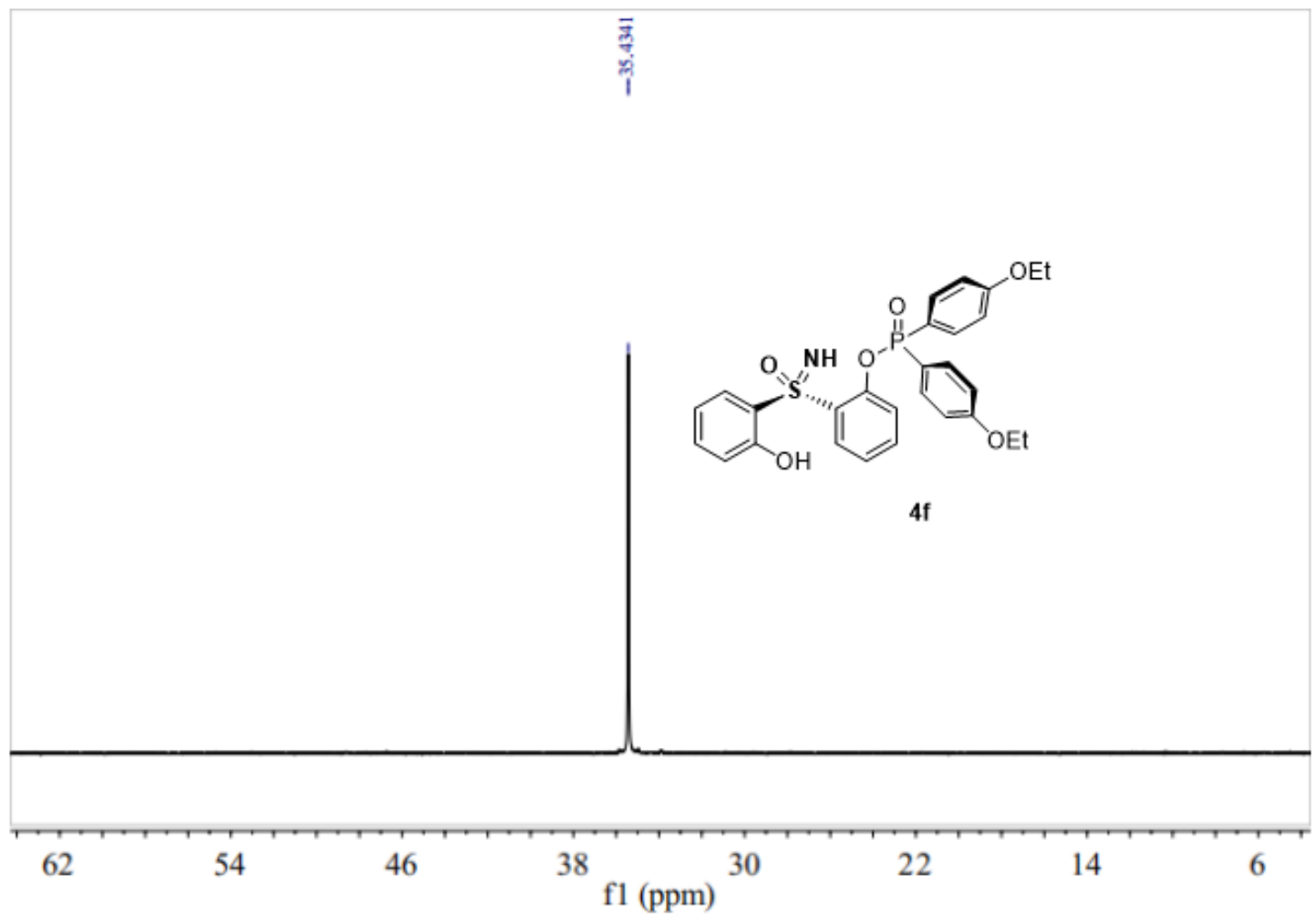

${ }^{1} \mathrm{H}-\mathrm{NMR},{ }^{13} \mathrm{C}-\mathrm{NMR}$ and ${ }^{31} \mathrm{P}-\mathrm{NMR}$ of $\mathbf{4 f}$ 

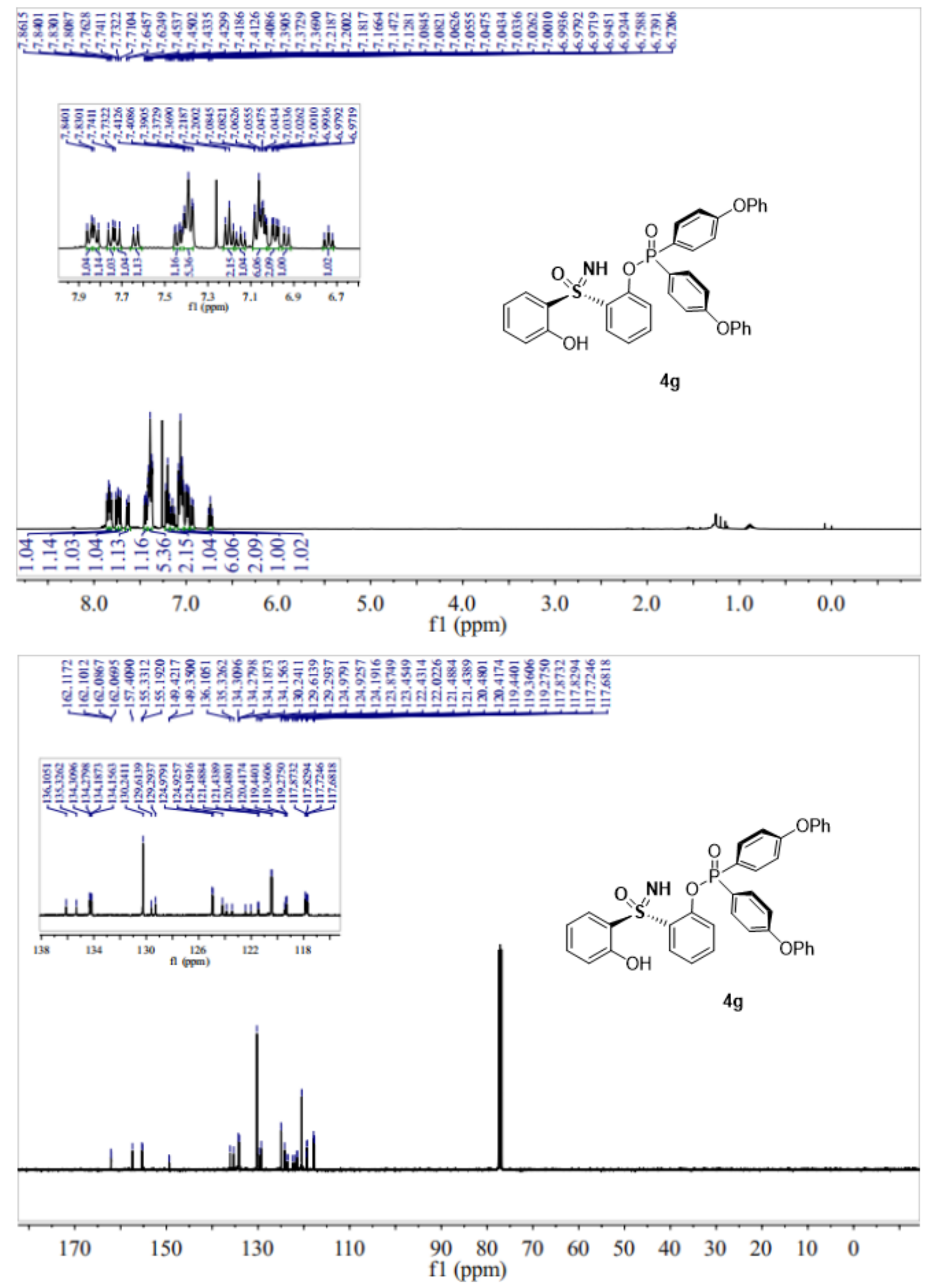


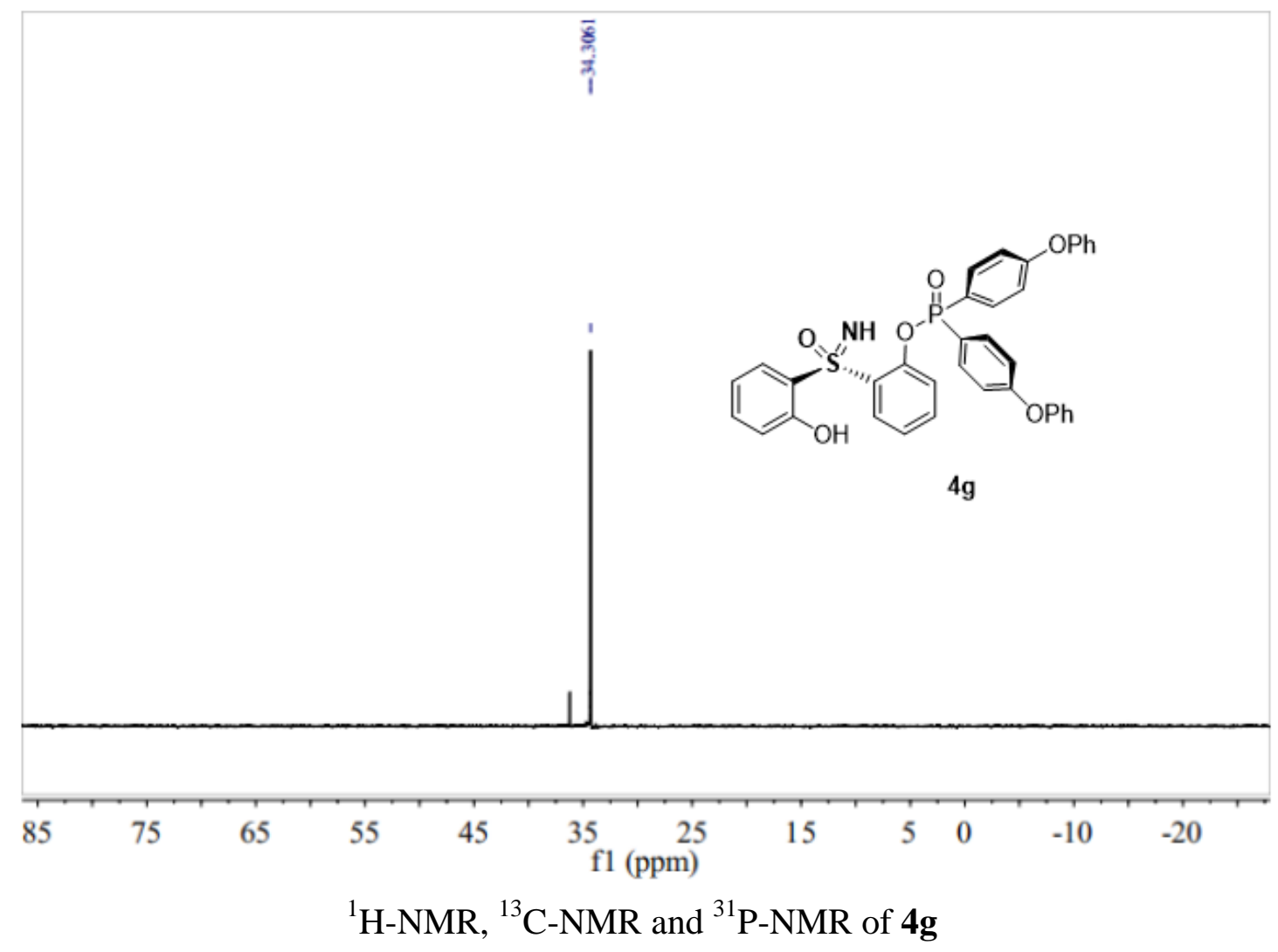



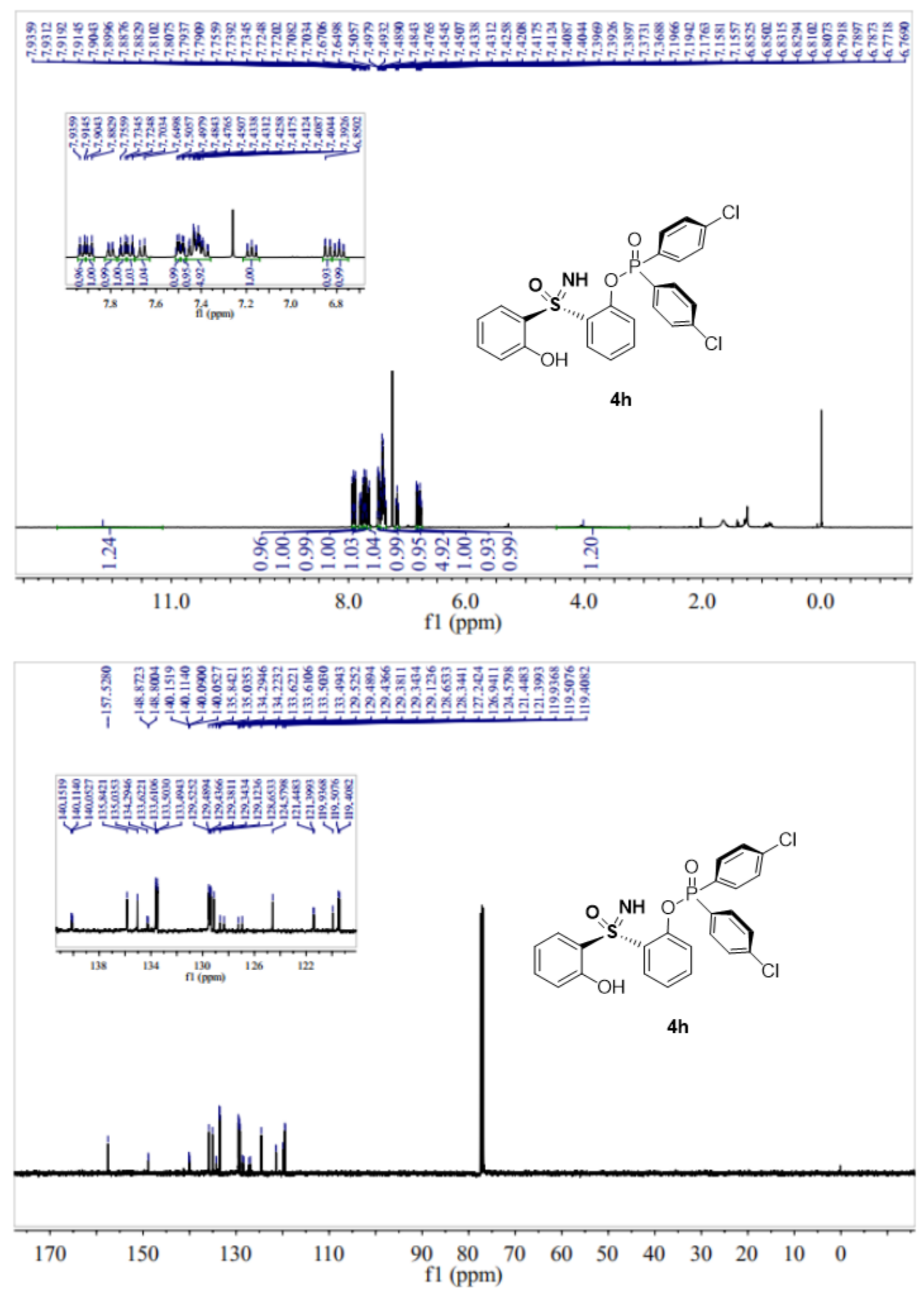


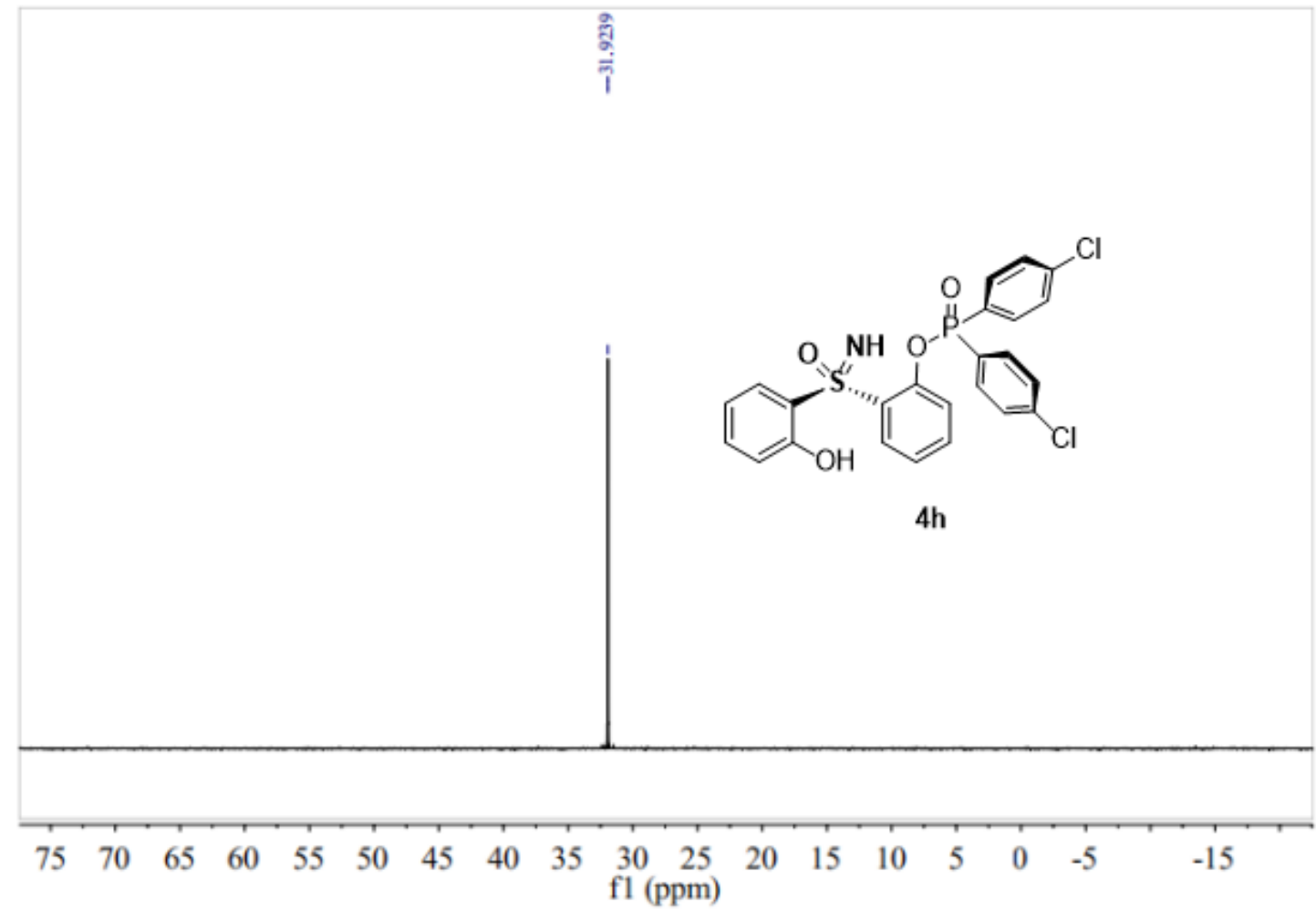

${ }^{1} \mathrm{H}-\mathrm{NMR},{ }^{13} \mathrm{C}-\mathrm{NMR}$ and ${ }^{31} \mathrm{P}-\mathrm{NMR}$ of $\mathbf{4 h}$ 

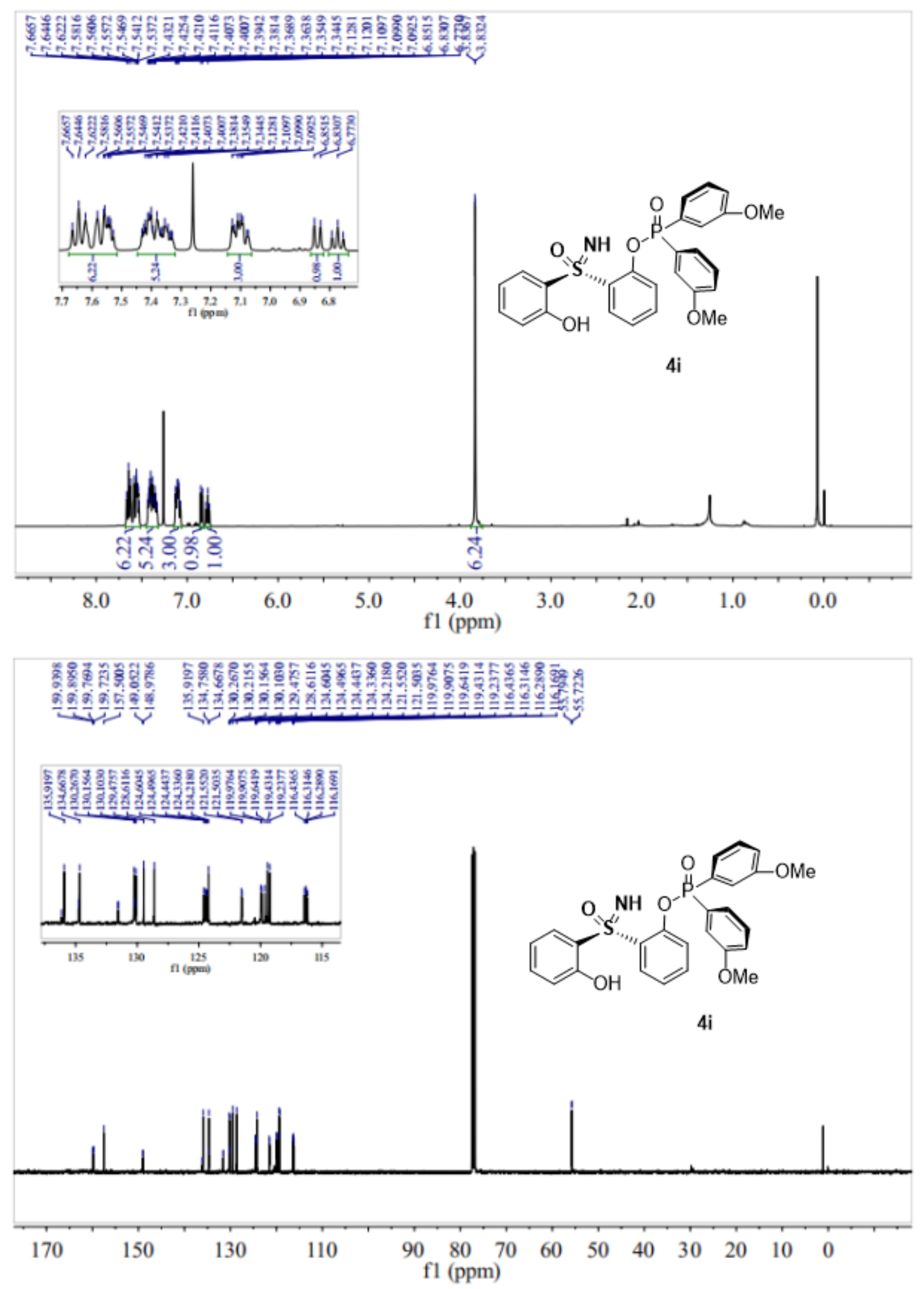


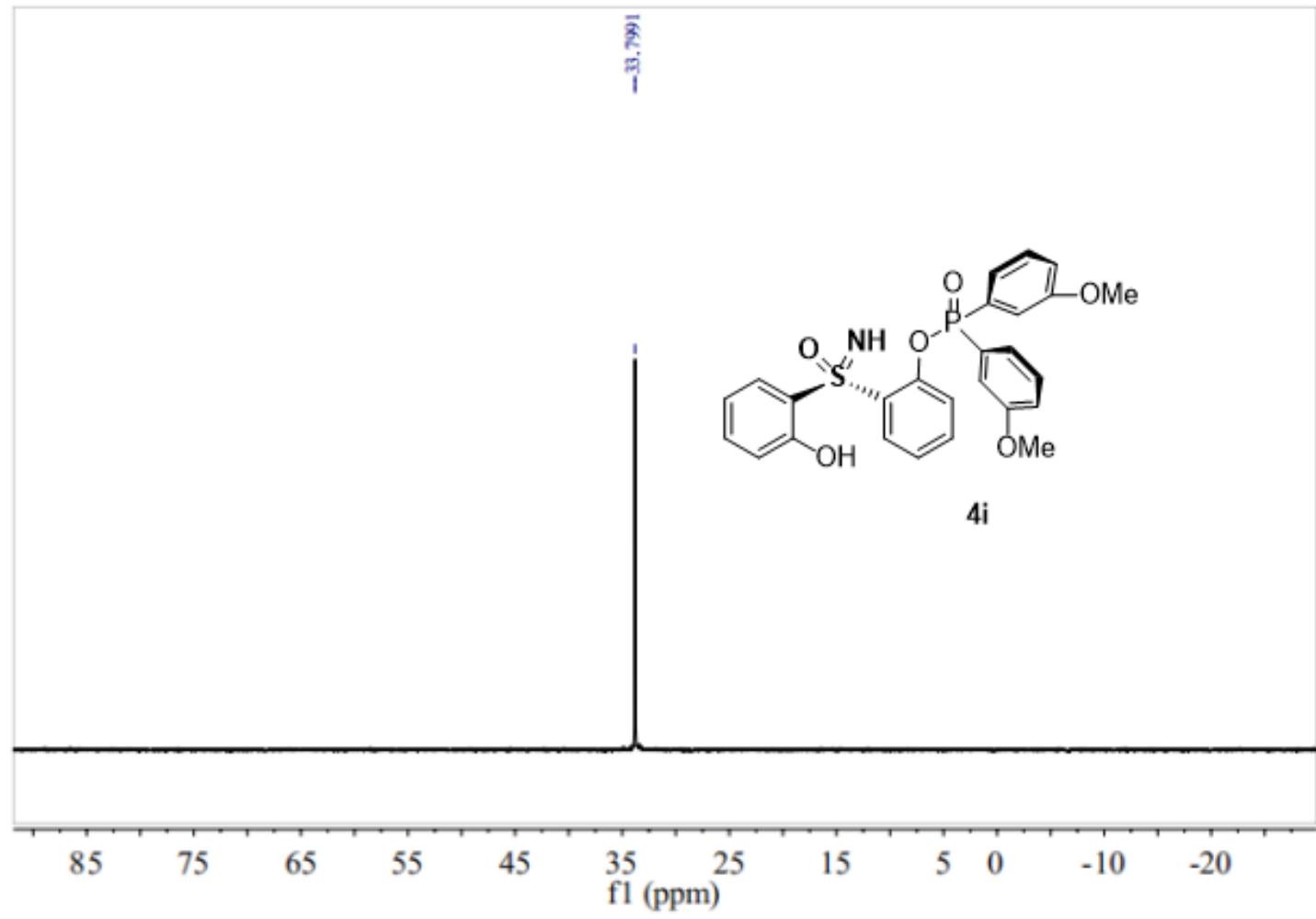

${ }^{1} \mathrm{H}-\mathrm{NMR},{ }^{13} \mathrm{C}-\mathrm{NMR}$ and ${ }^{31} \mathrm{P}-\mathrm{NMR}$ of $\mathbf{4 i}$ 

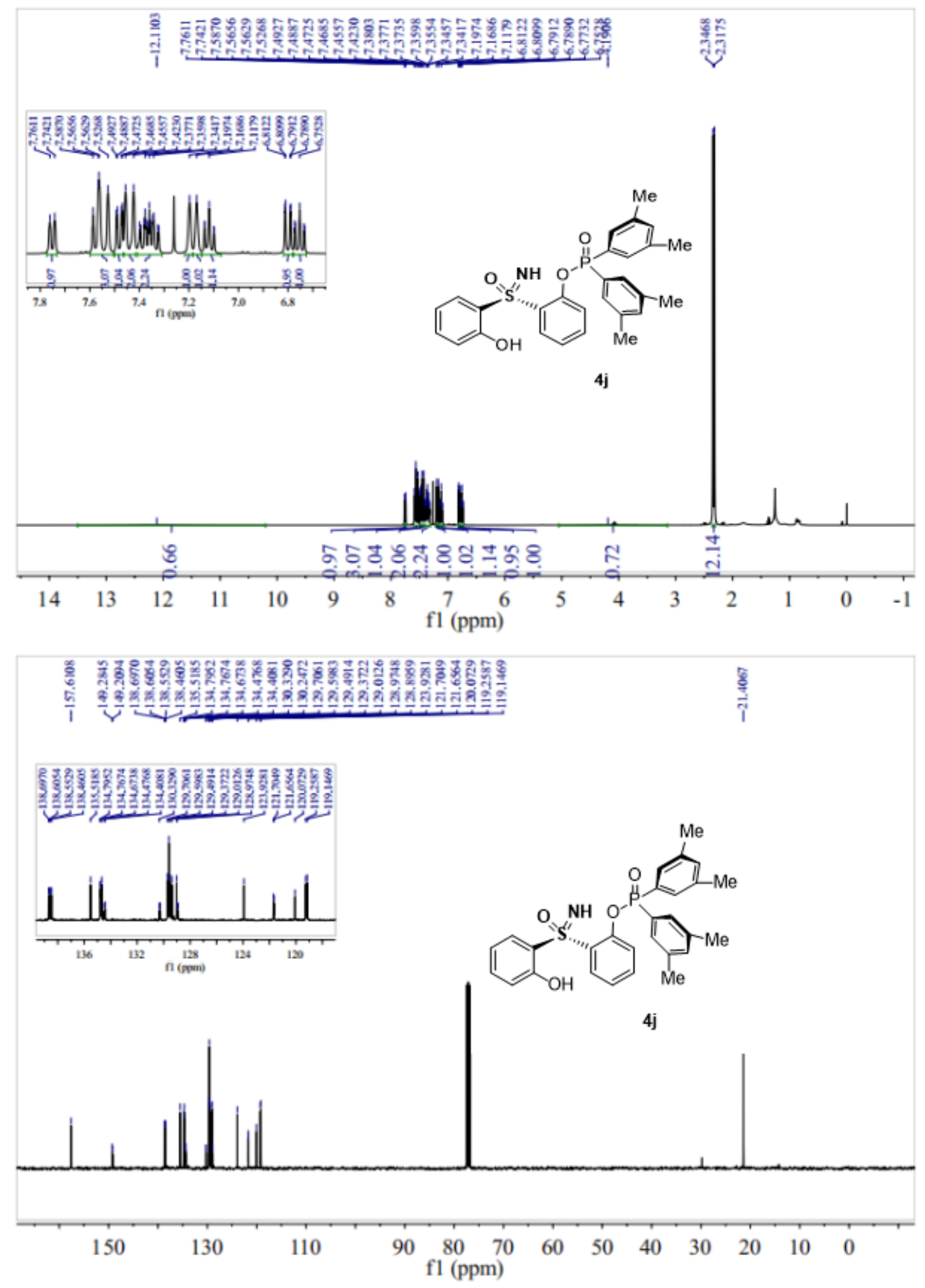


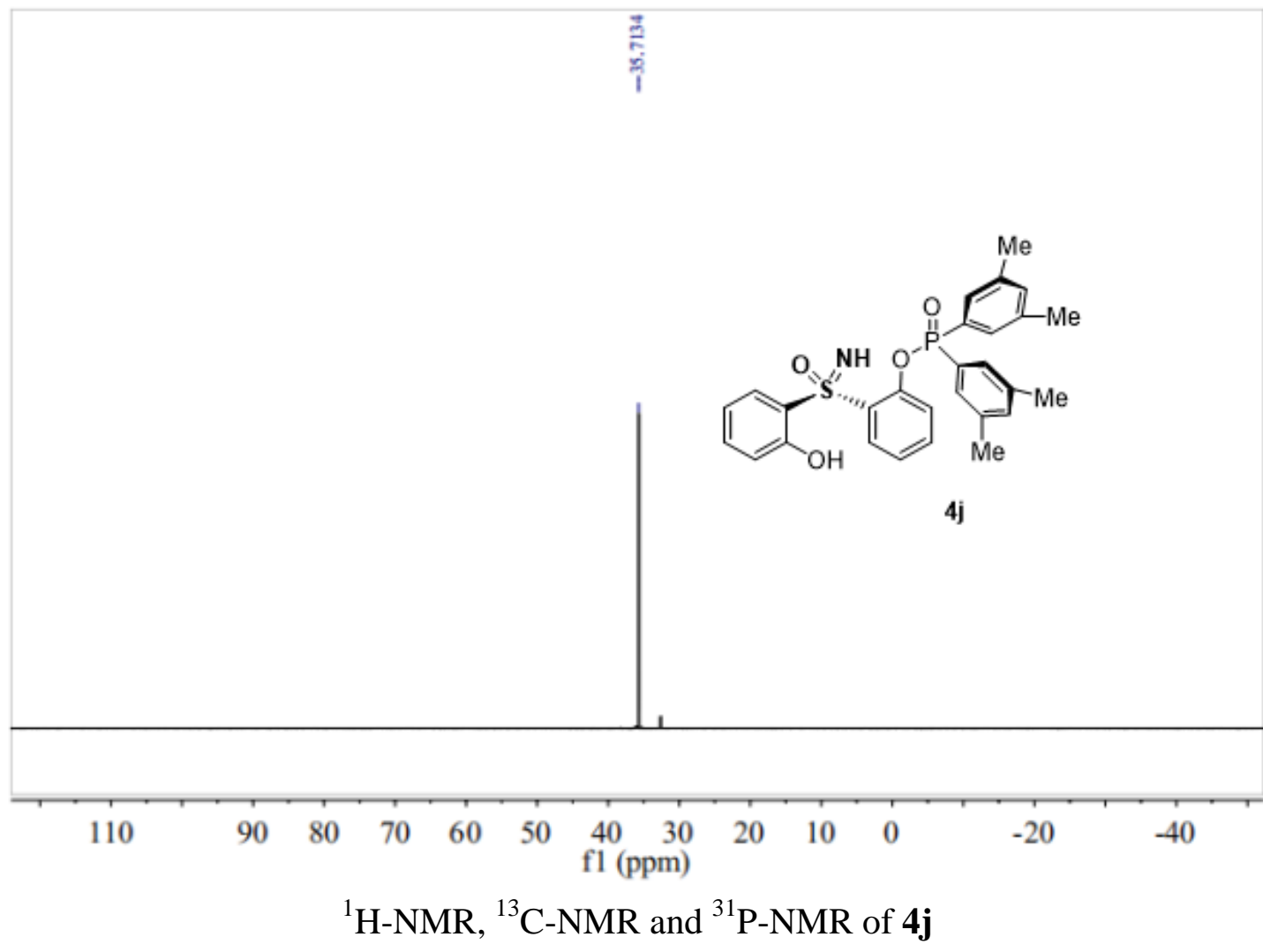



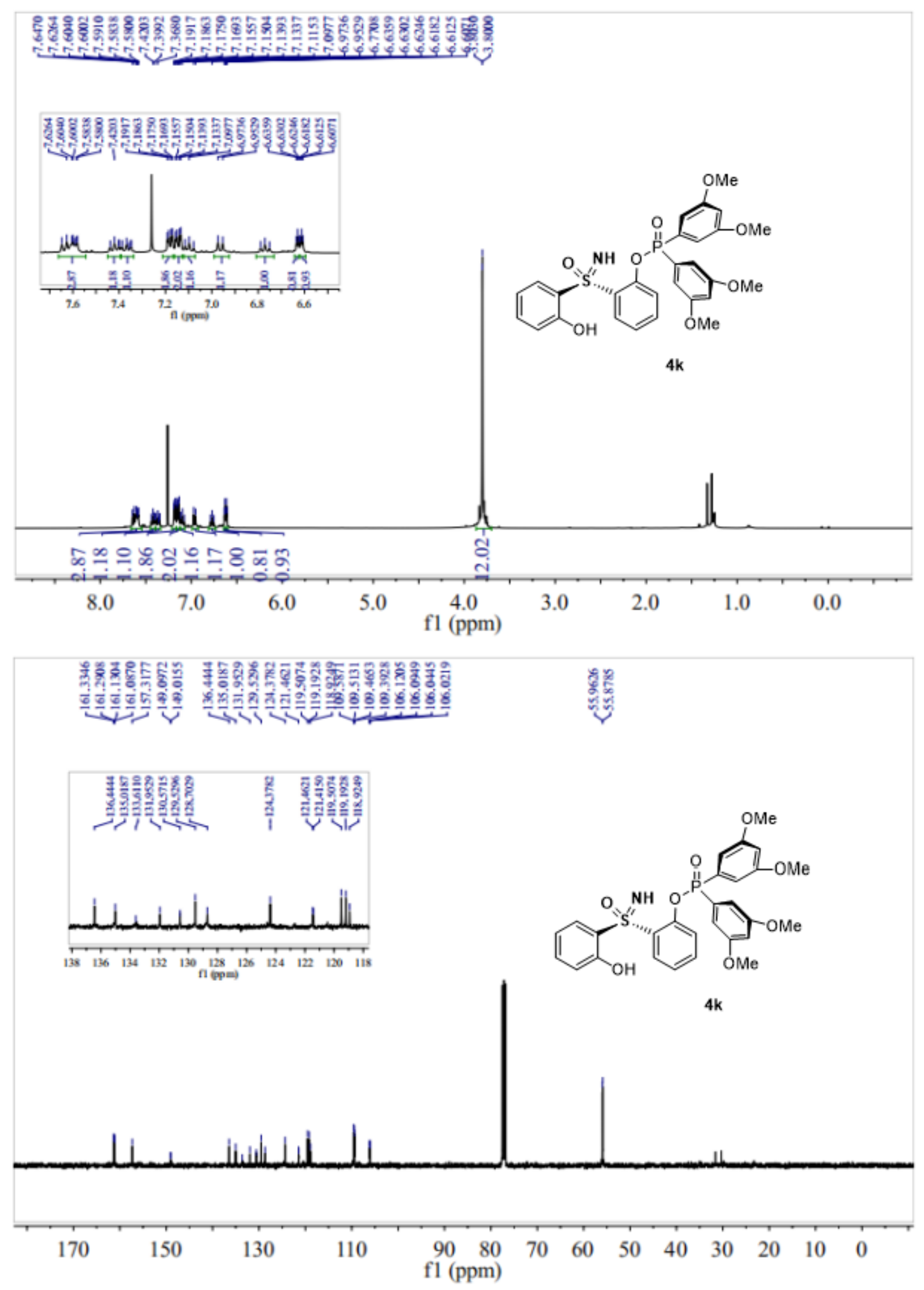


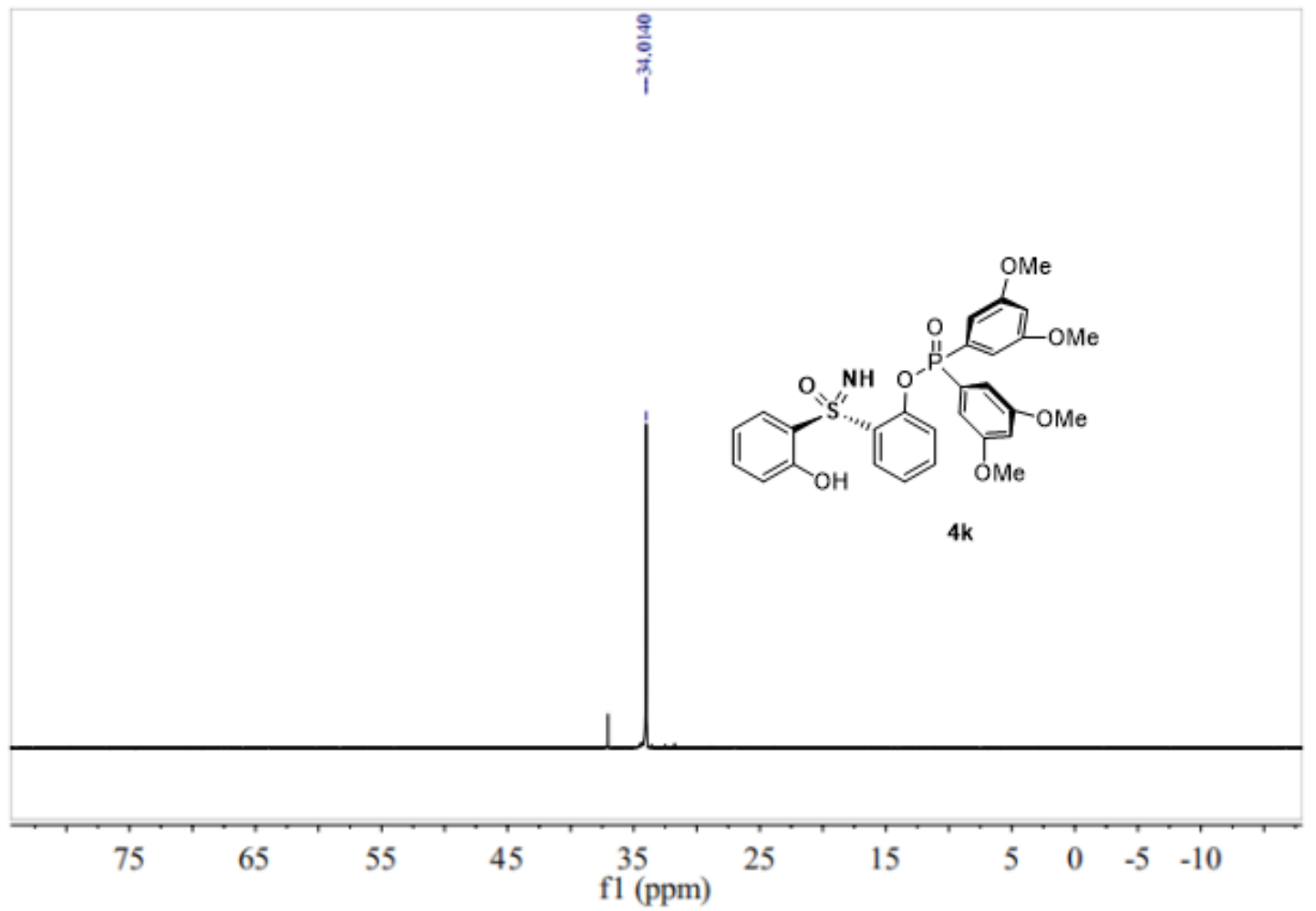

${ }^{1} \mathrm{H}-\mathrm{NMR},{ }^{13} \mathrm{C}-\mathrm{NMR}$ and ${ }^{31} \mathrm{P}-\mathrm{NMR}$ of $\mathbf{4 k}$ 

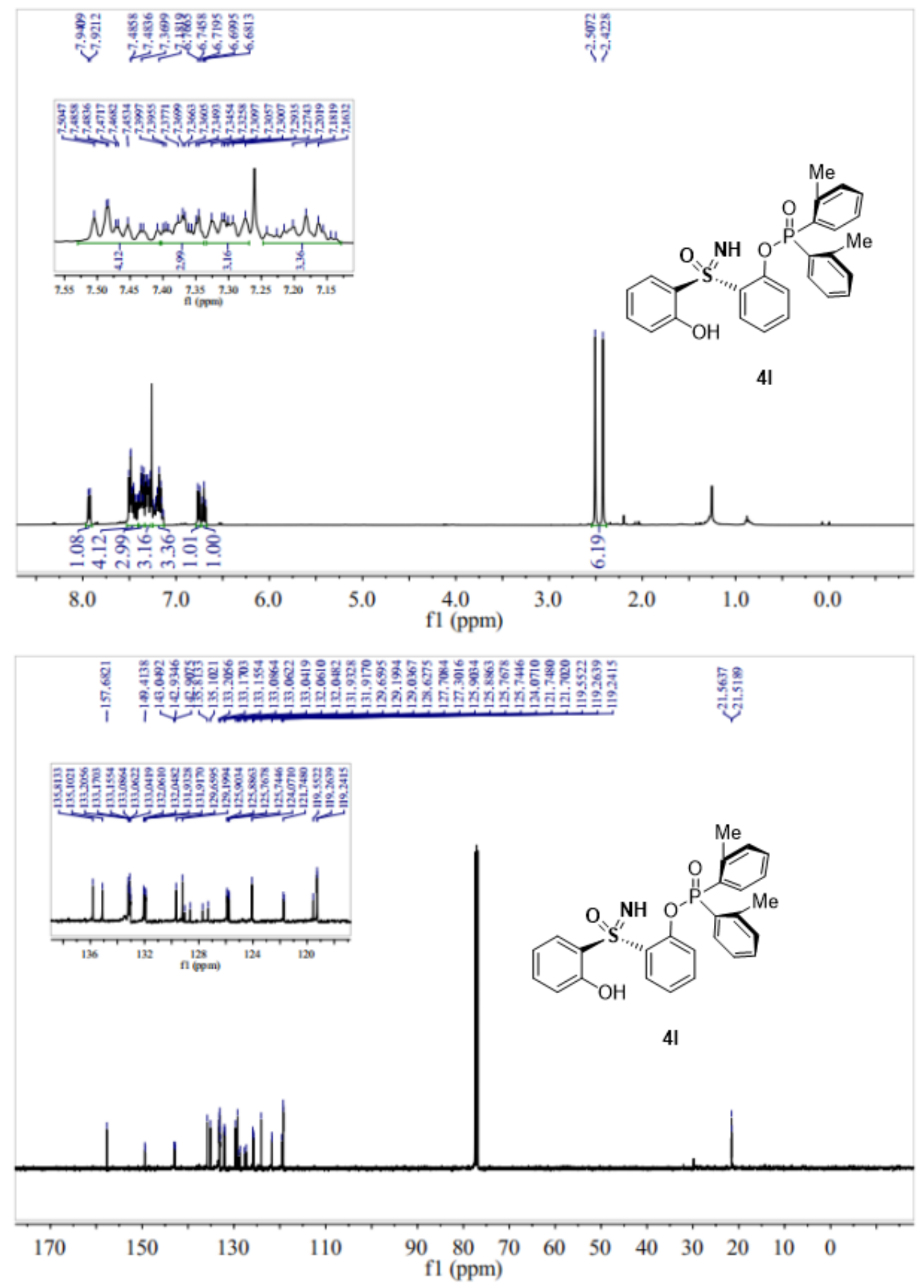


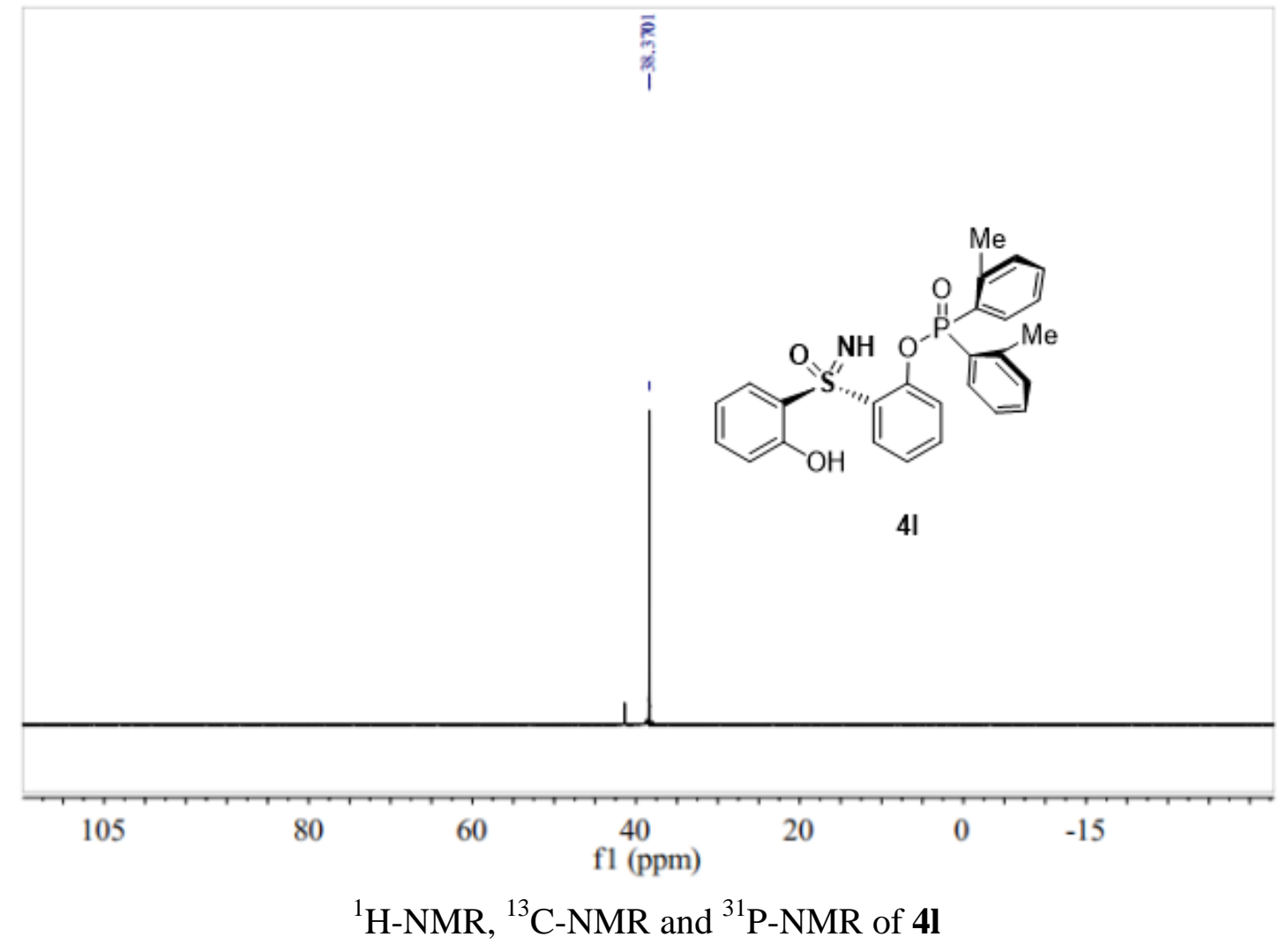



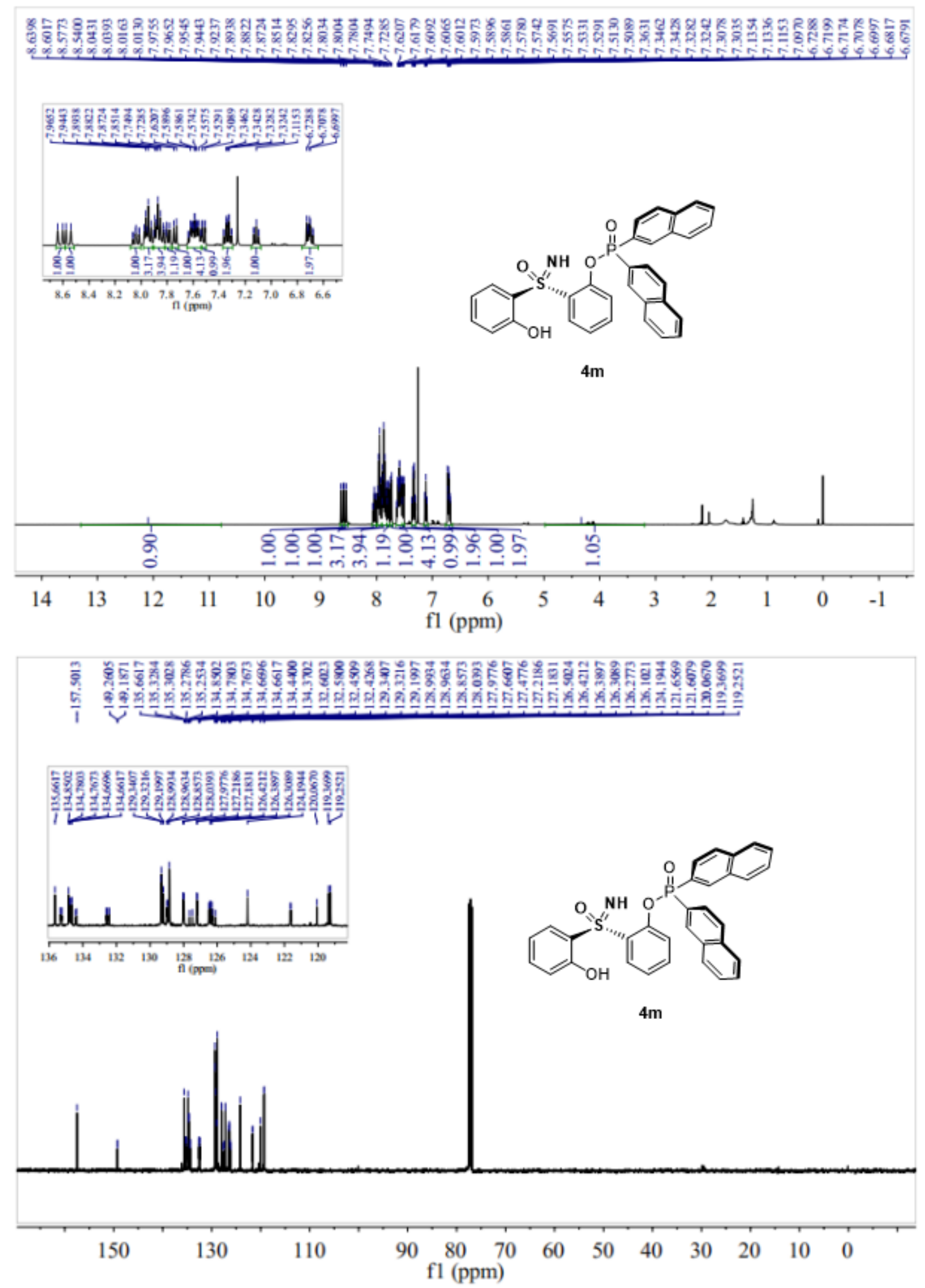


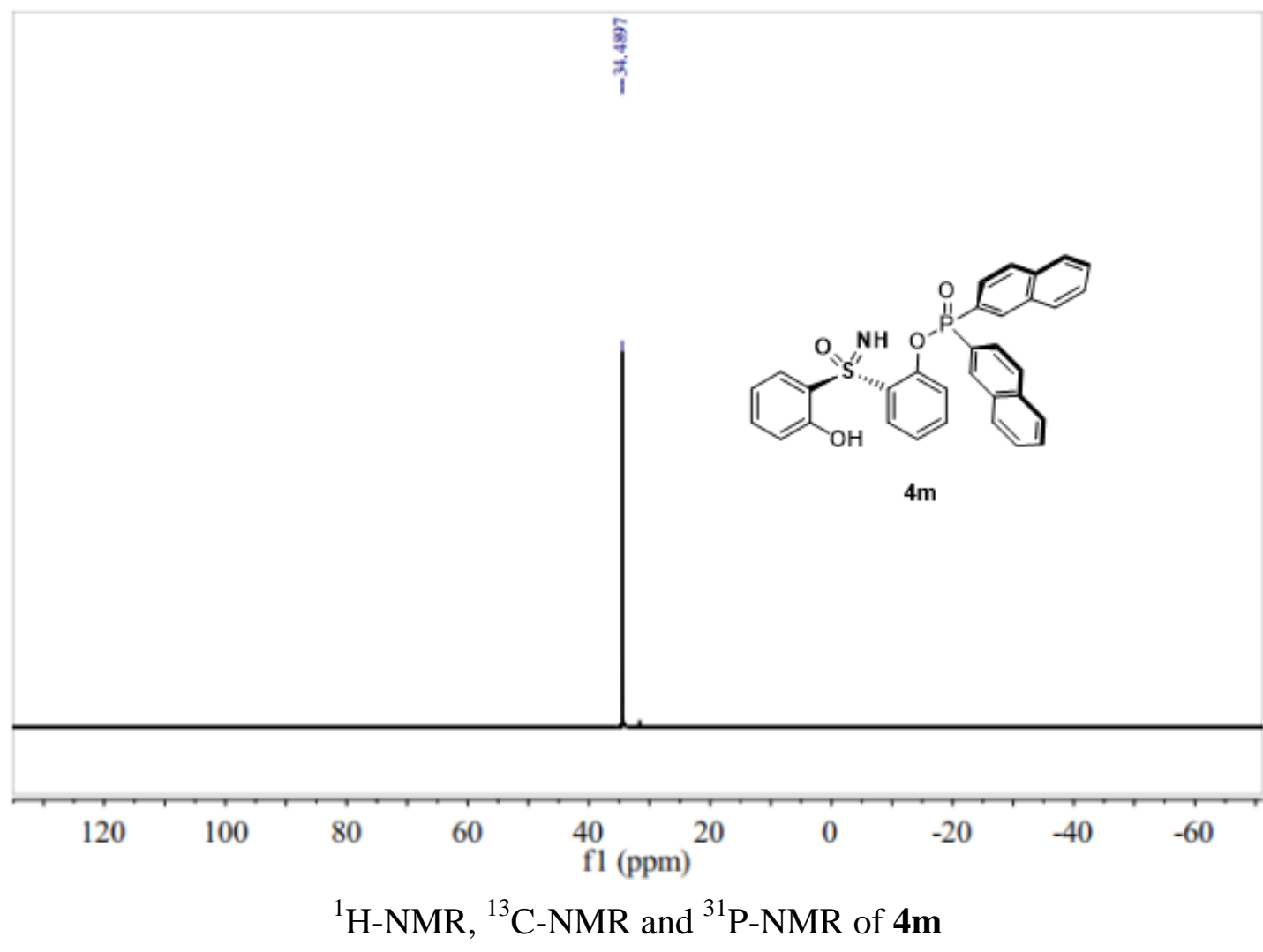



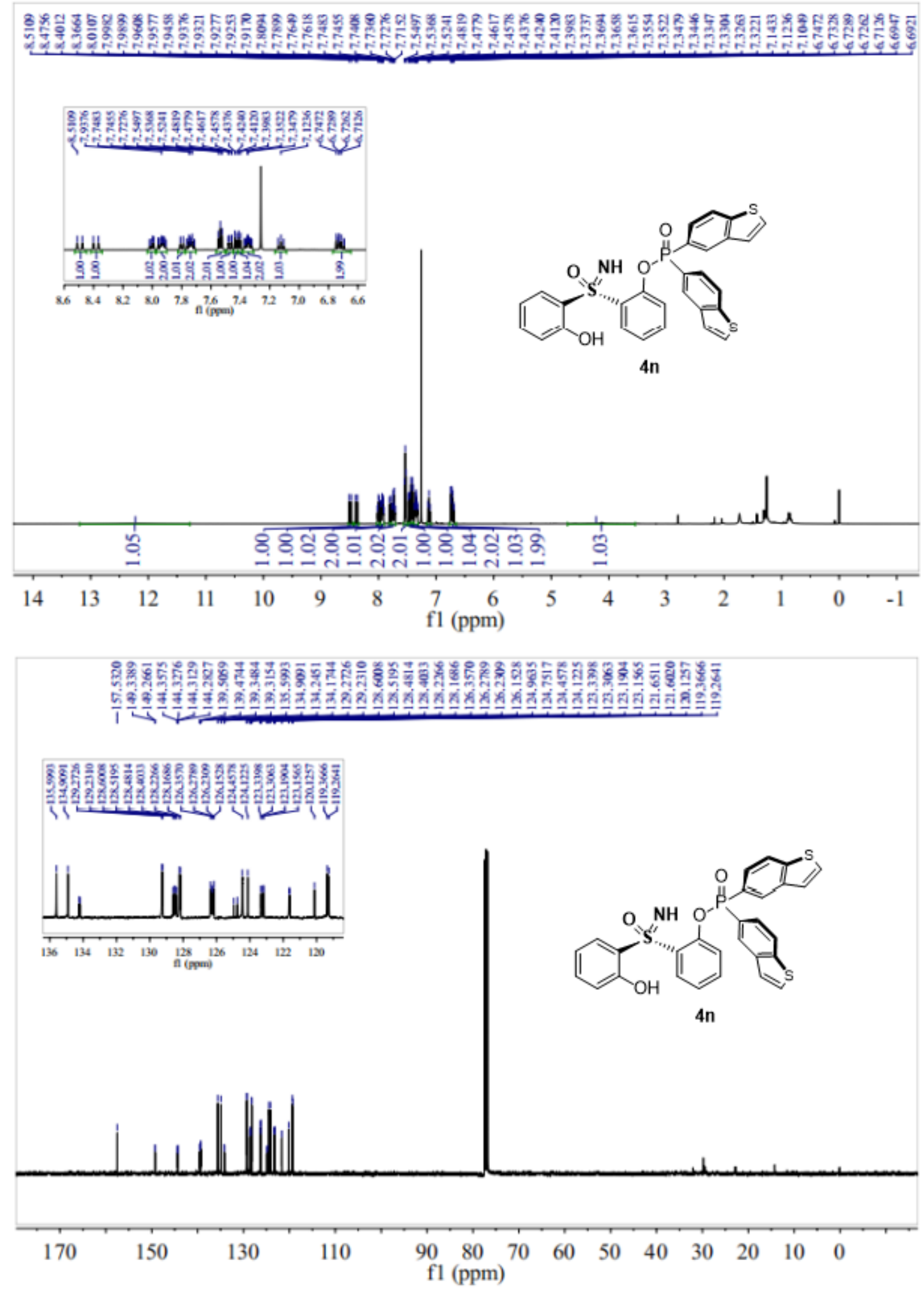


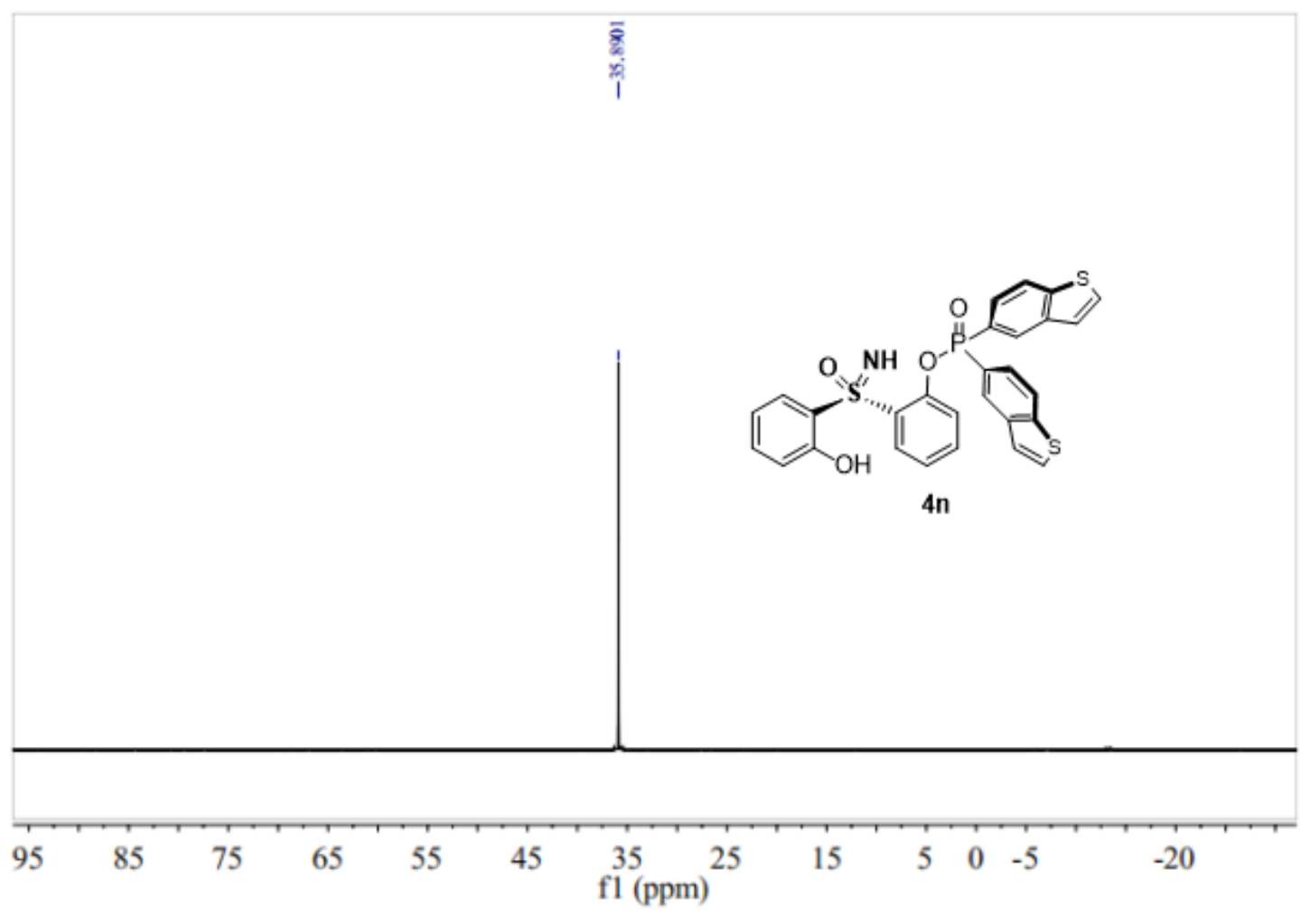

${ }^{1} \mathrm{H}-\mathrm{NMR},{ }^{13} \mathrm{C}-\mathrm{NMR}$ and ${ }^{31} \mathrm{P}-\mathrm{NMR}$ of $\mathbf{4 n}$ 

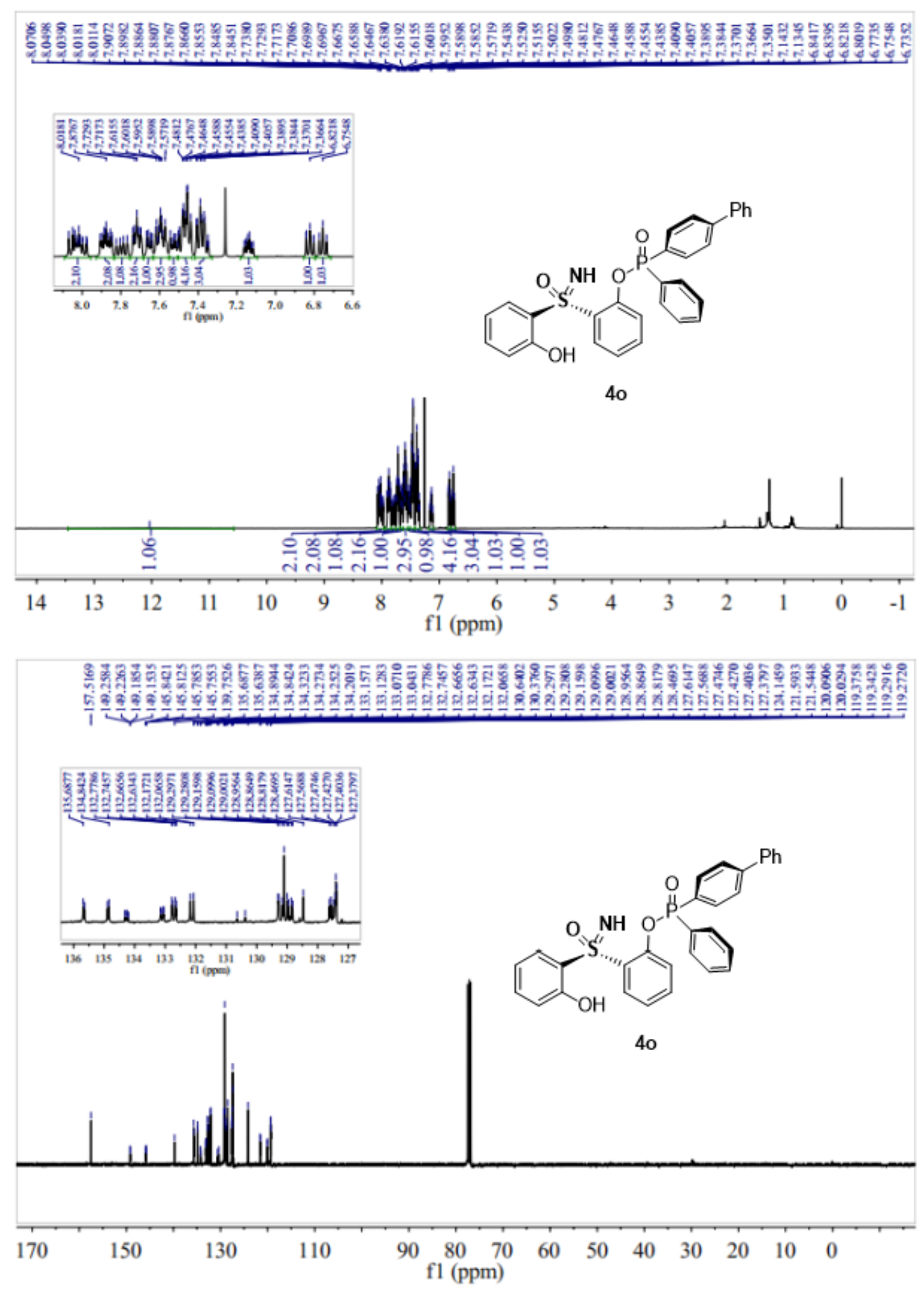


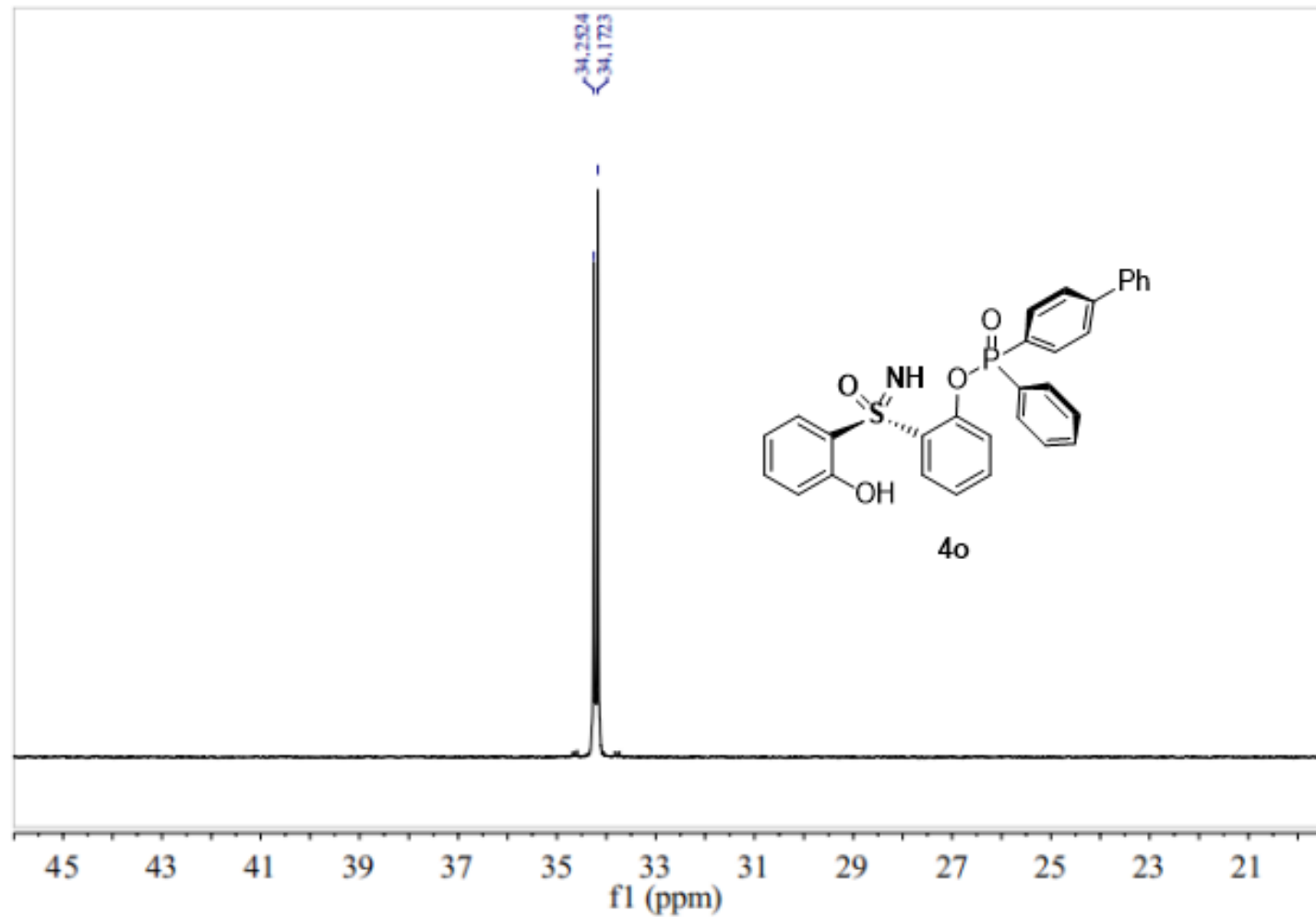

${ }^{1} \mathrm{H}$-NMR, ${ }^{13} \mathrm{C}$-NMR and ${ }^{31} \mathrm{P}$-NMR of 40 

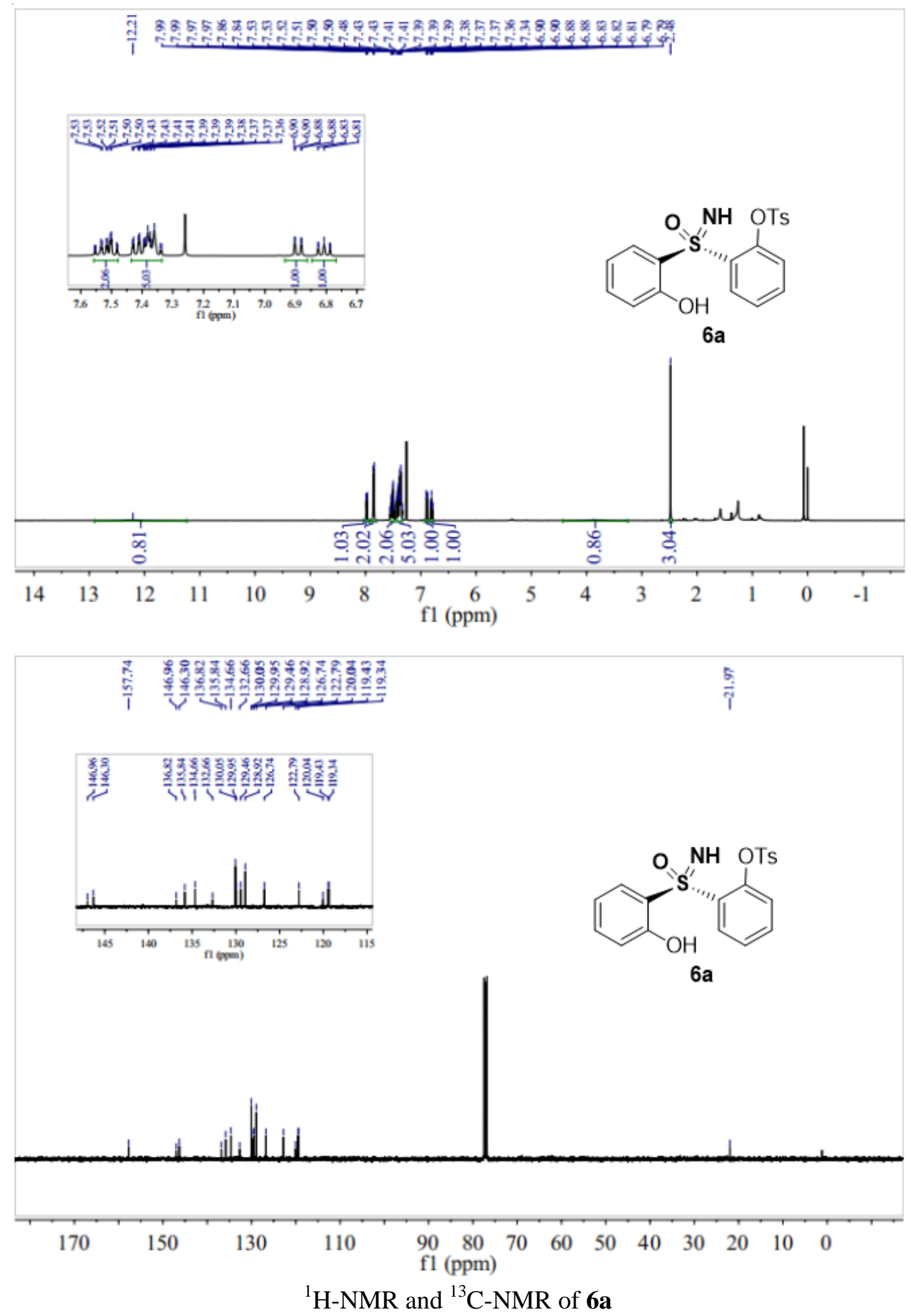

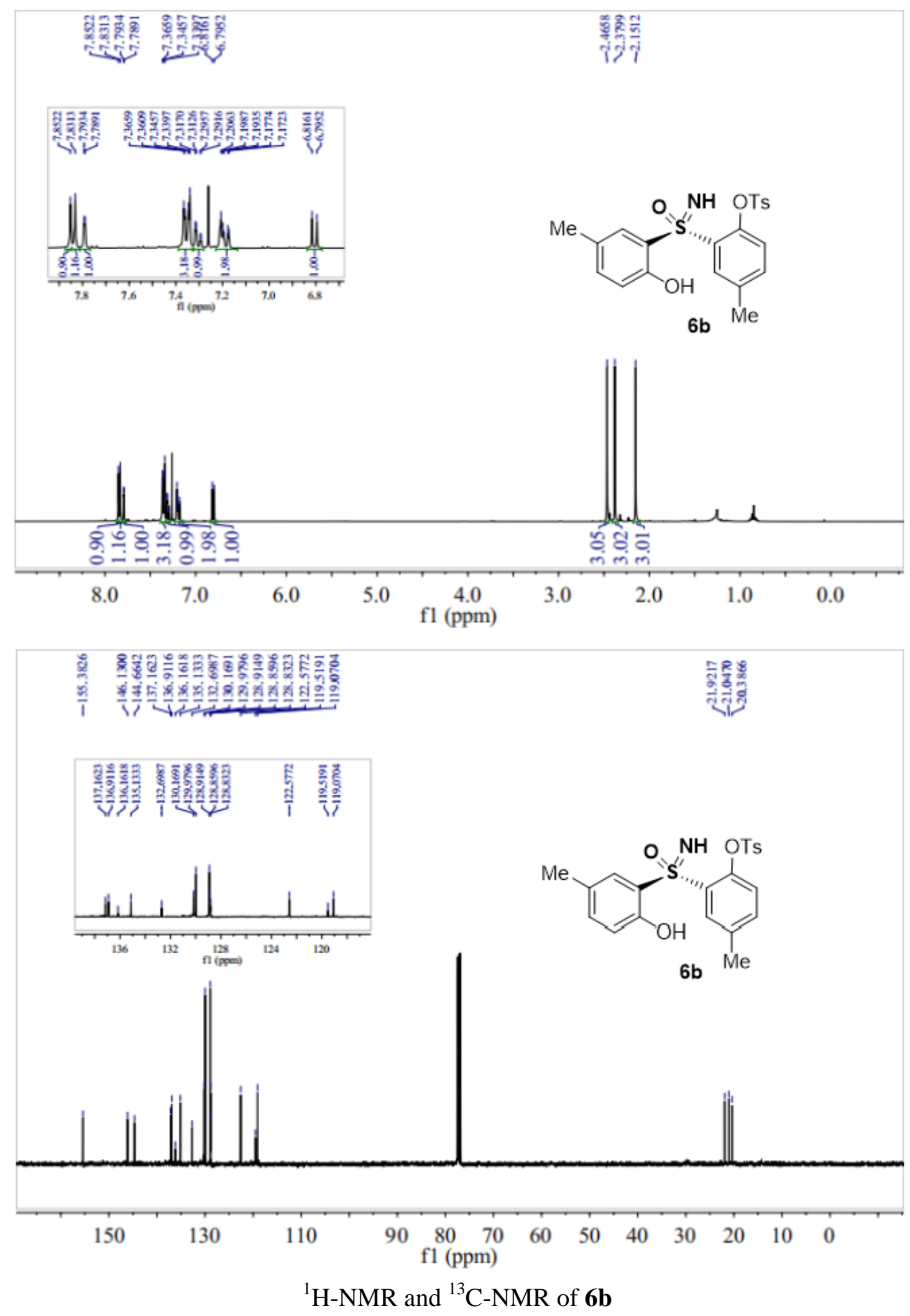

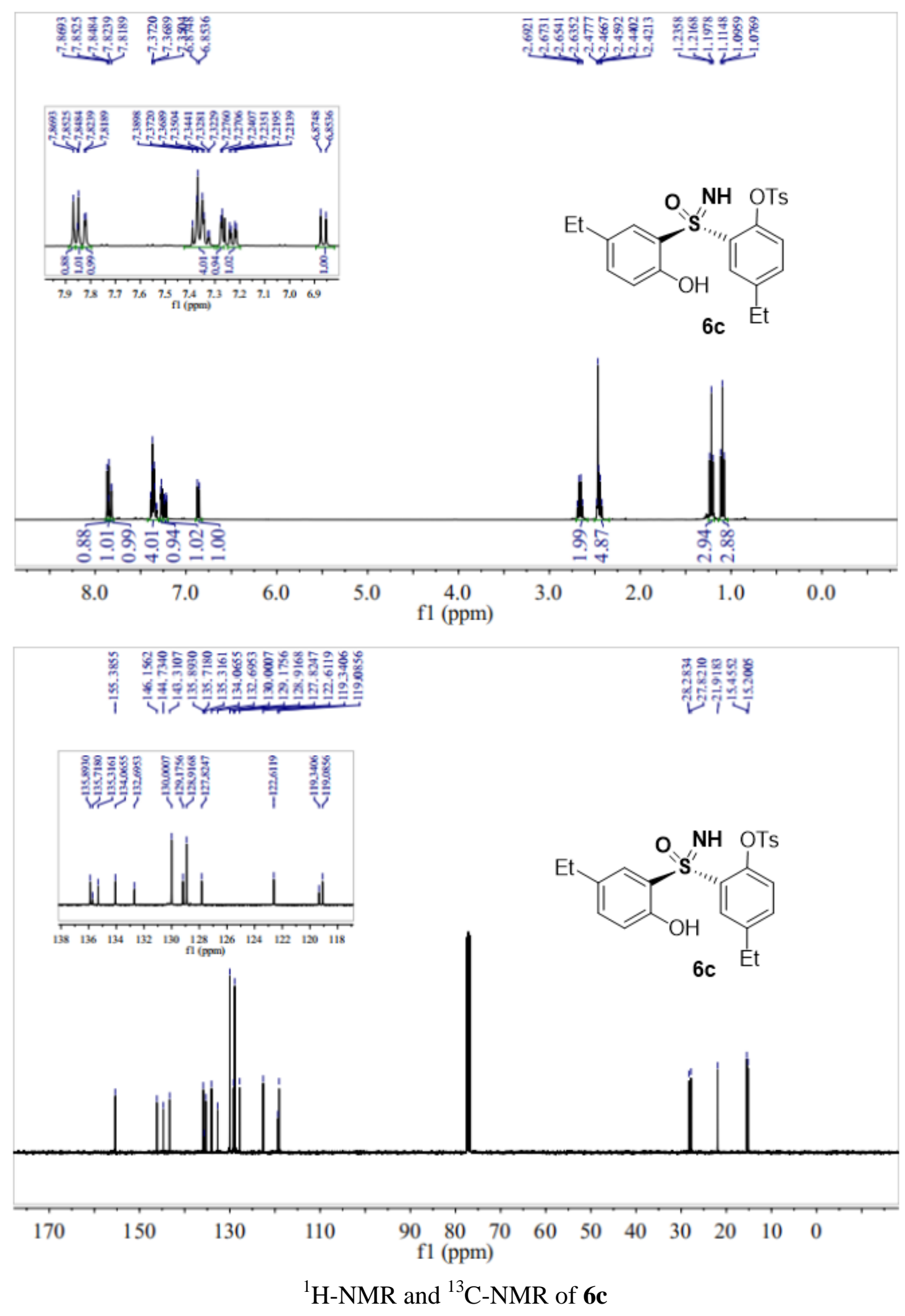

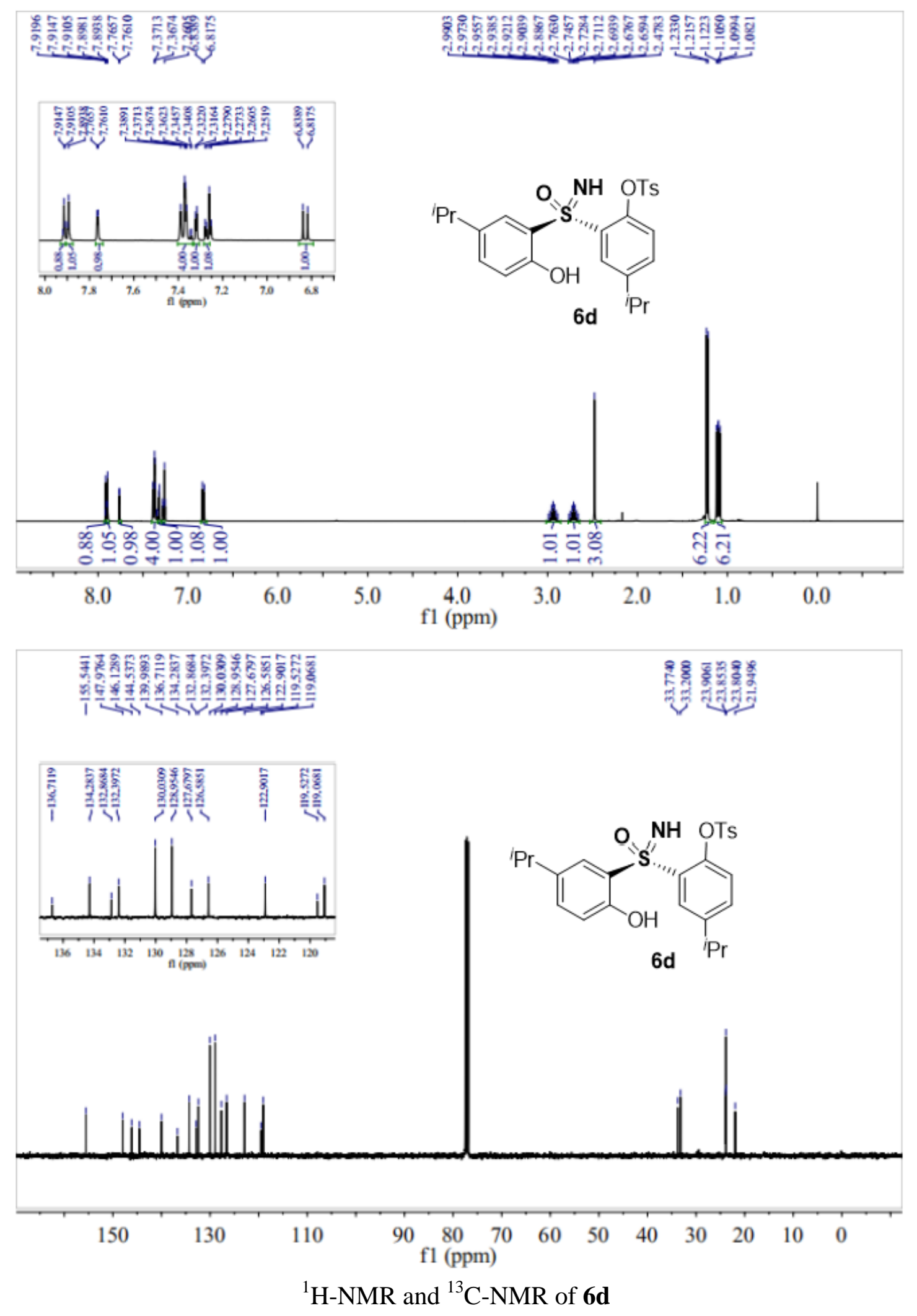

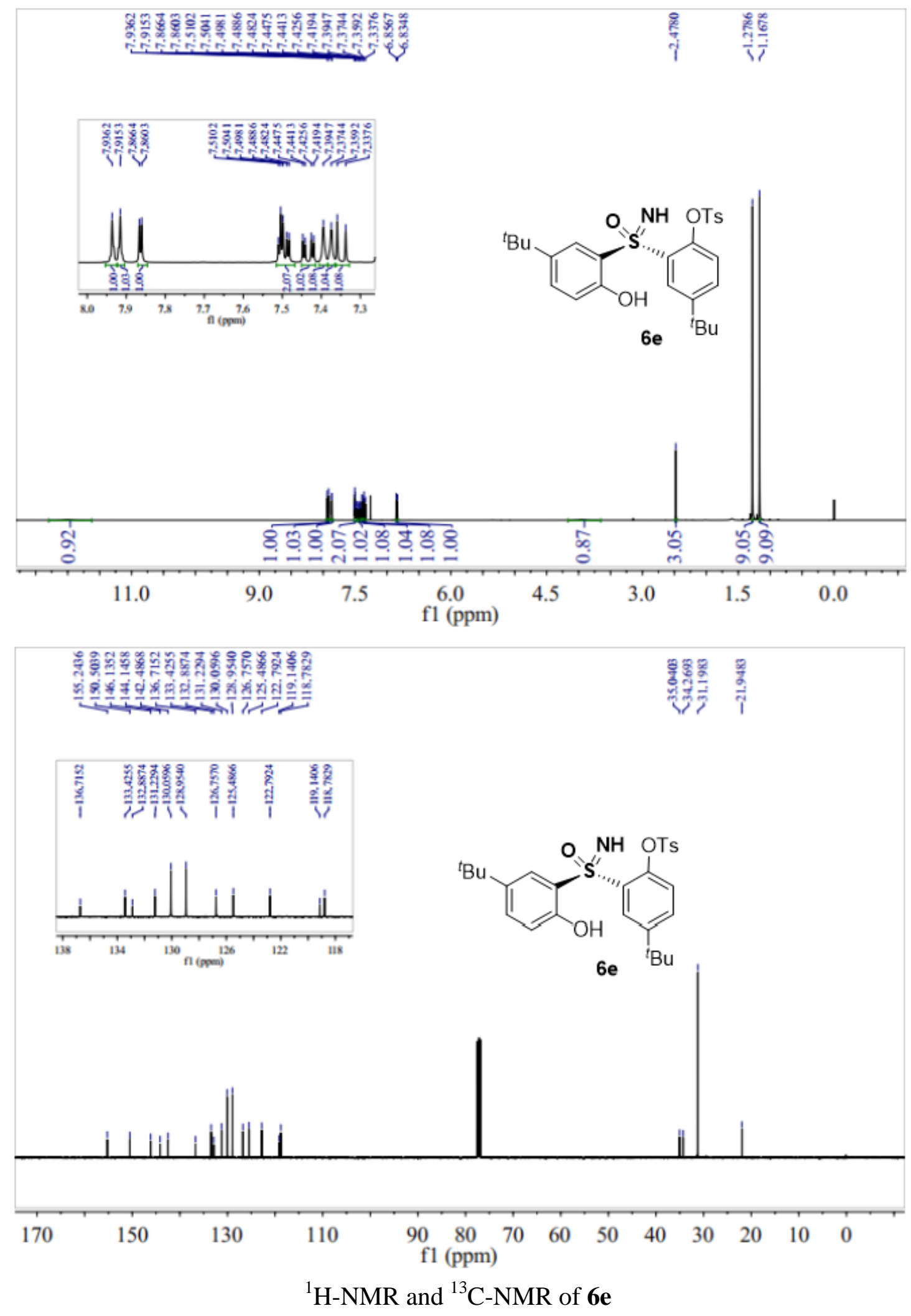

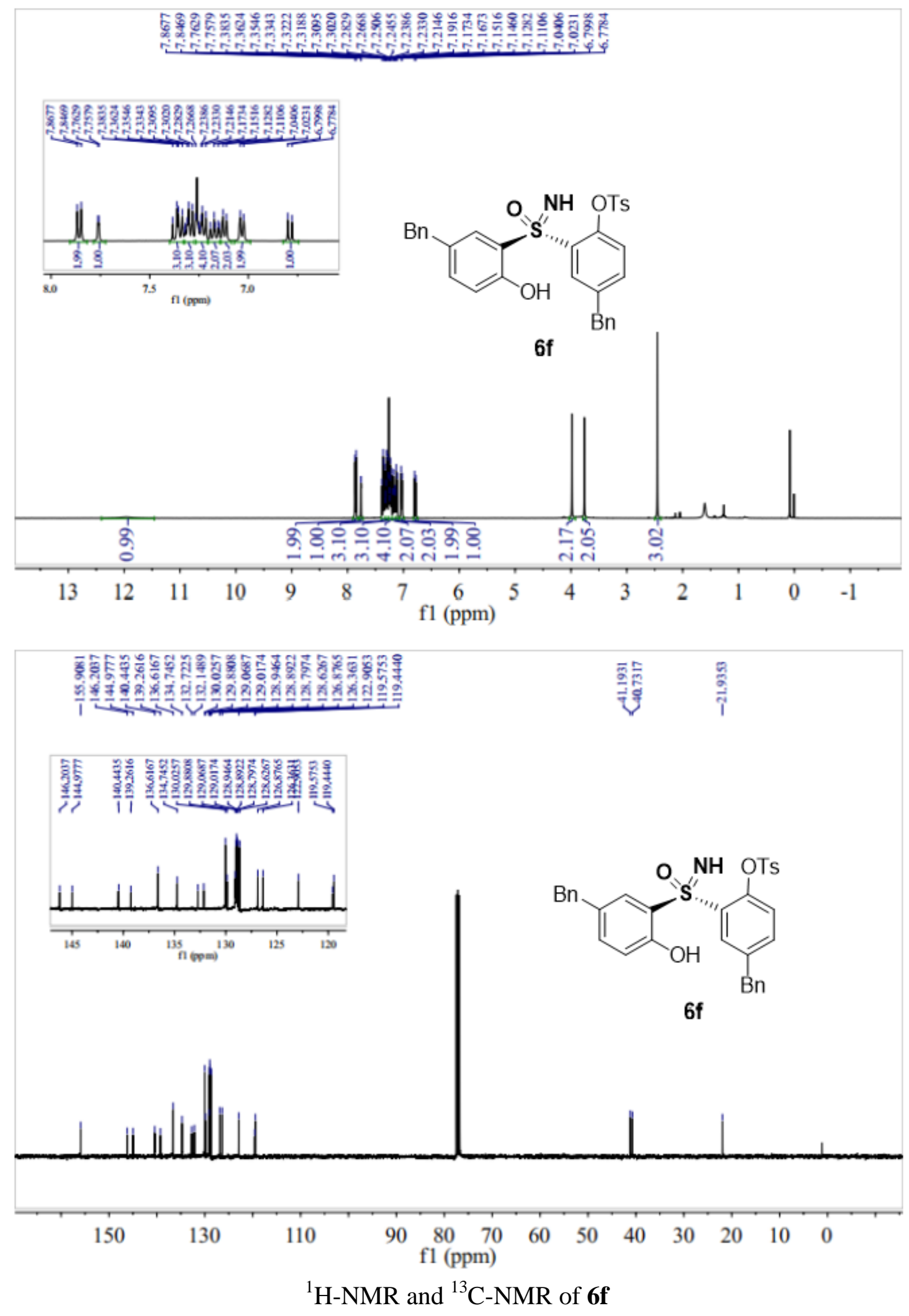

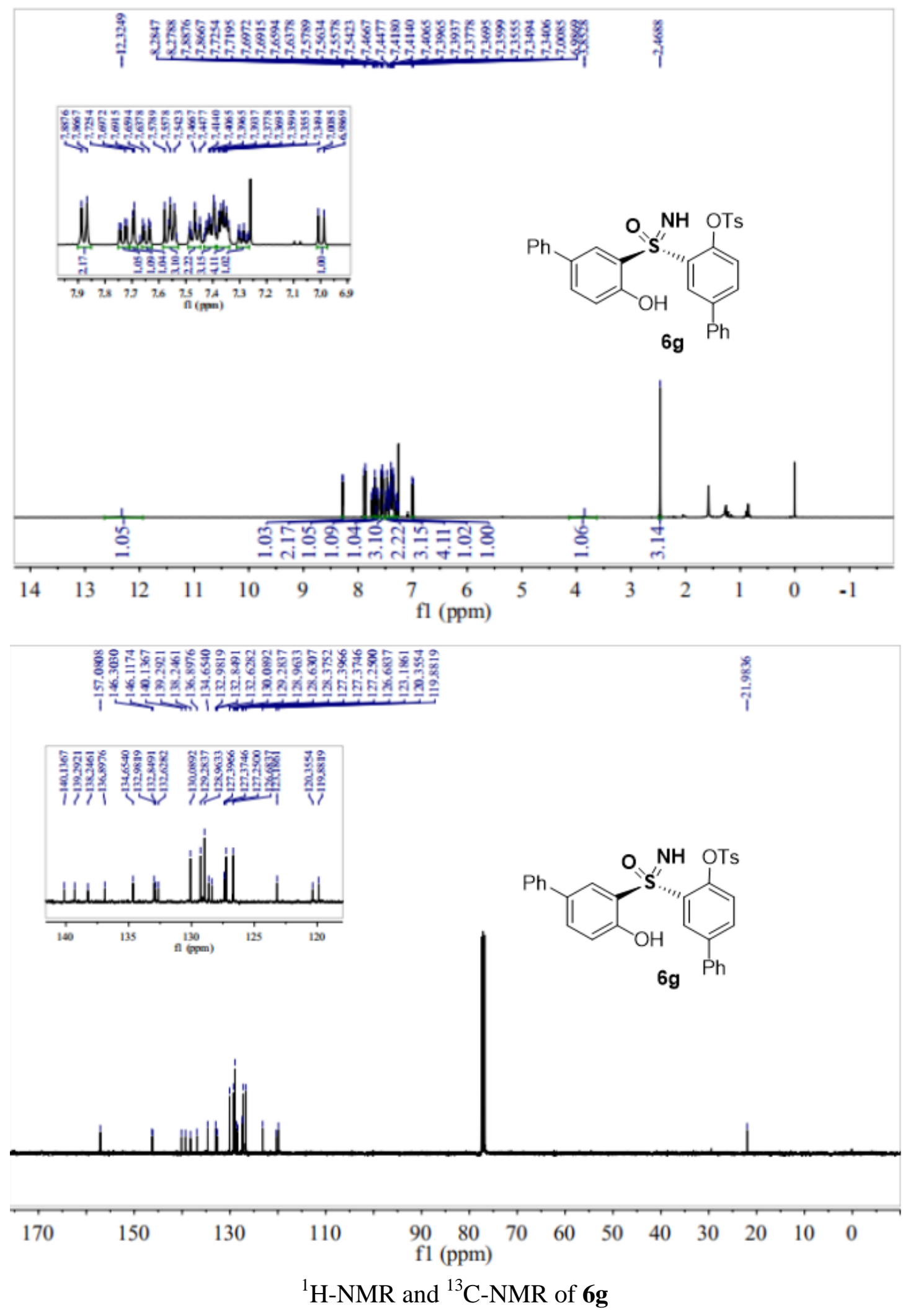

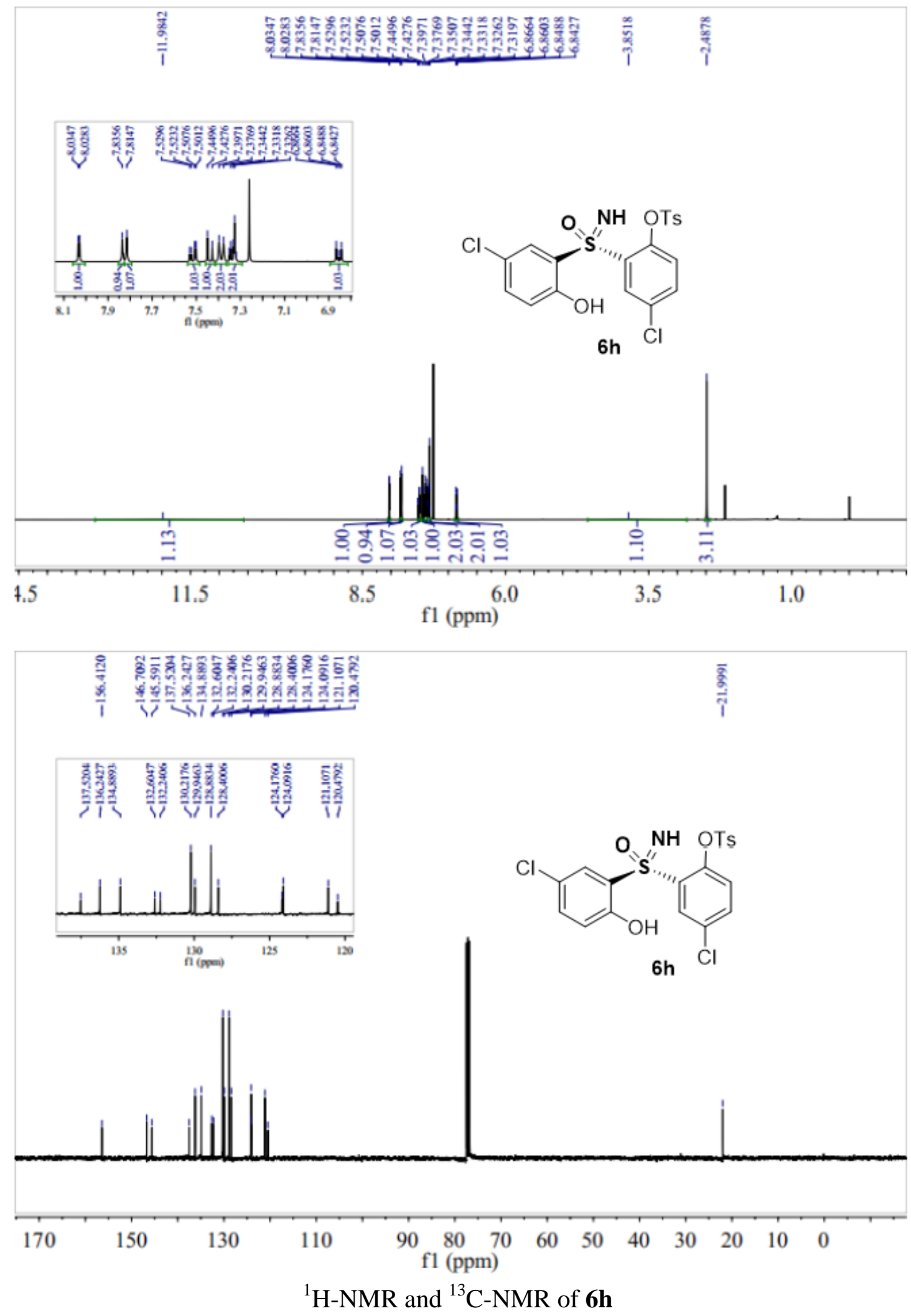

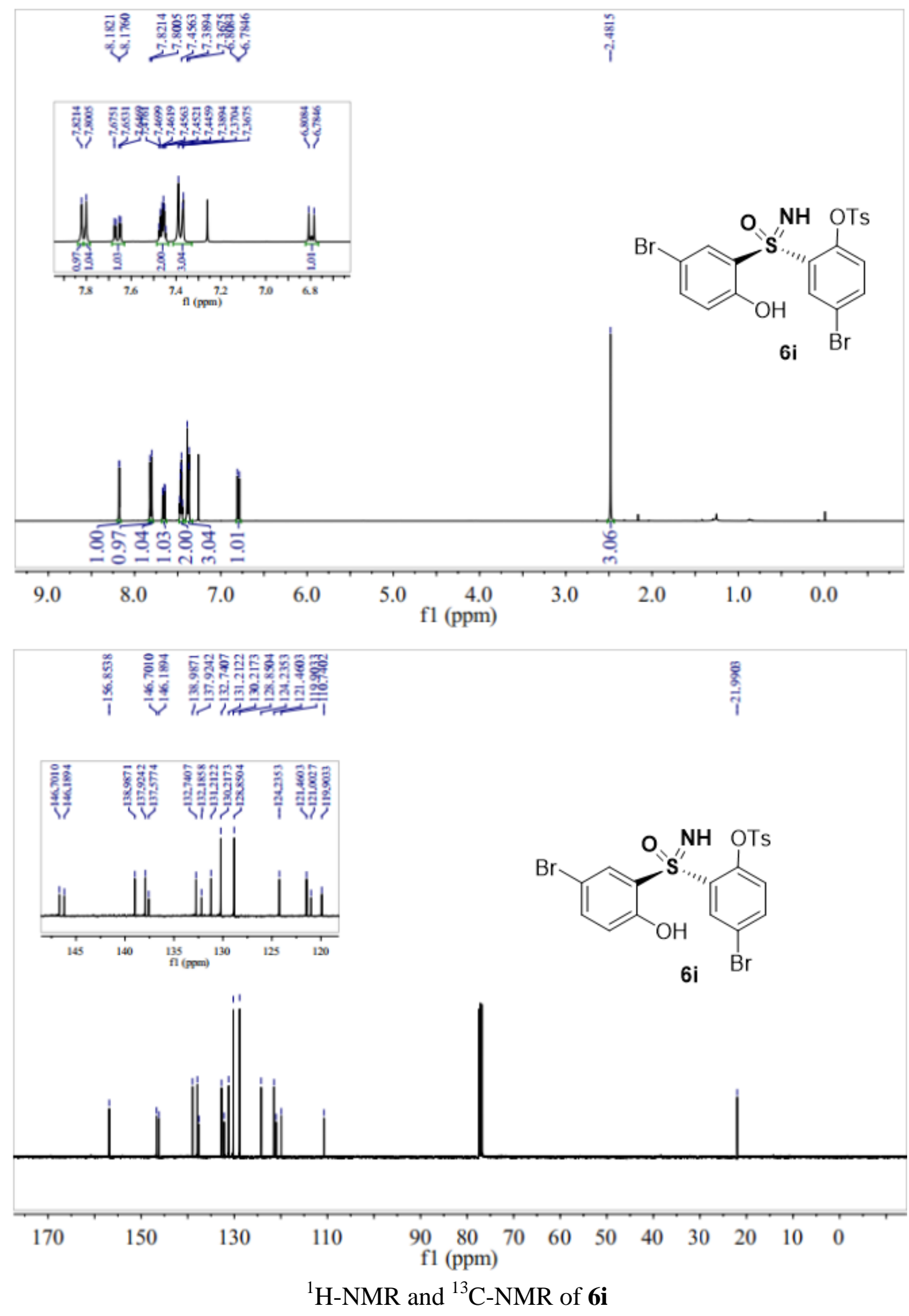

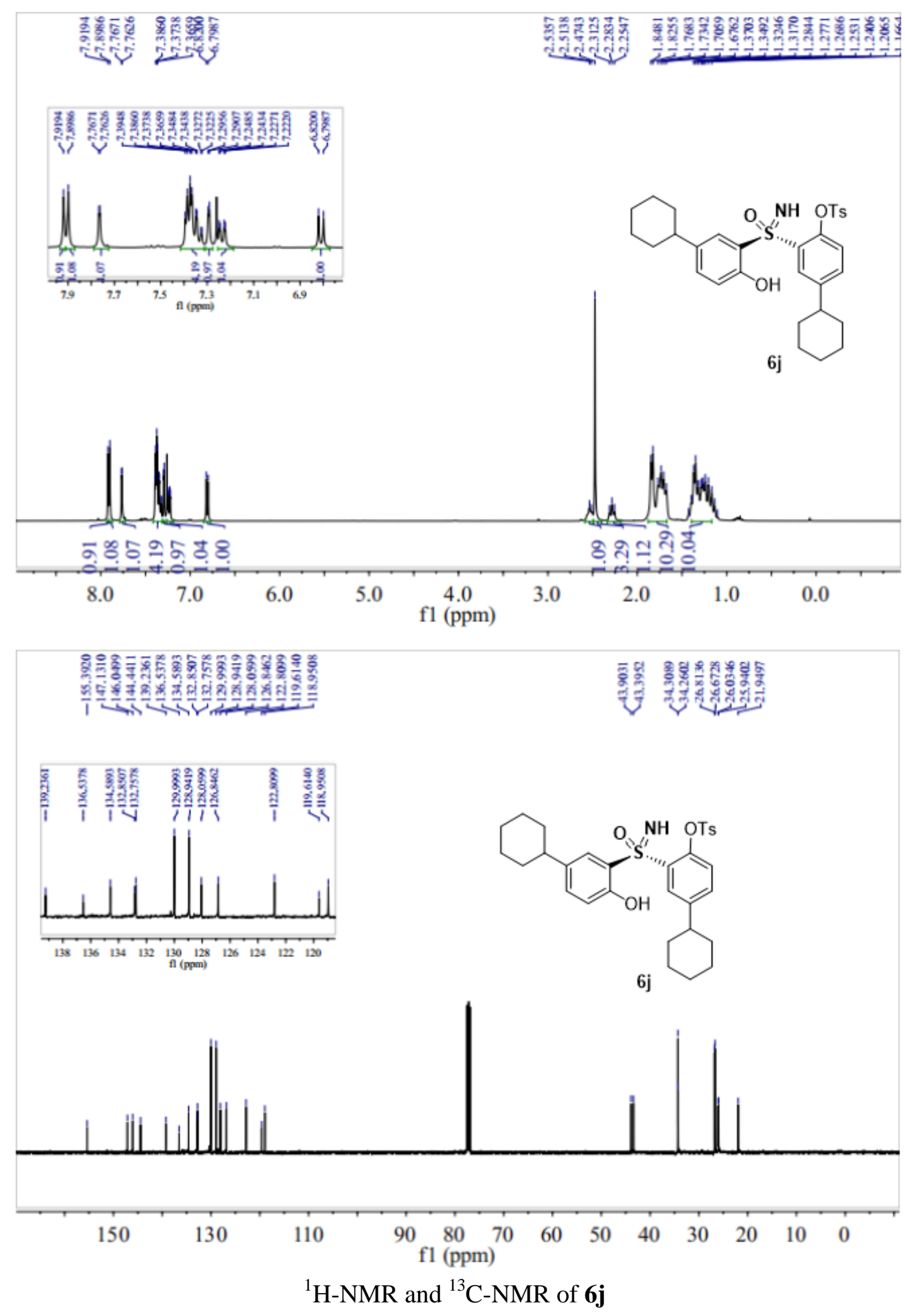


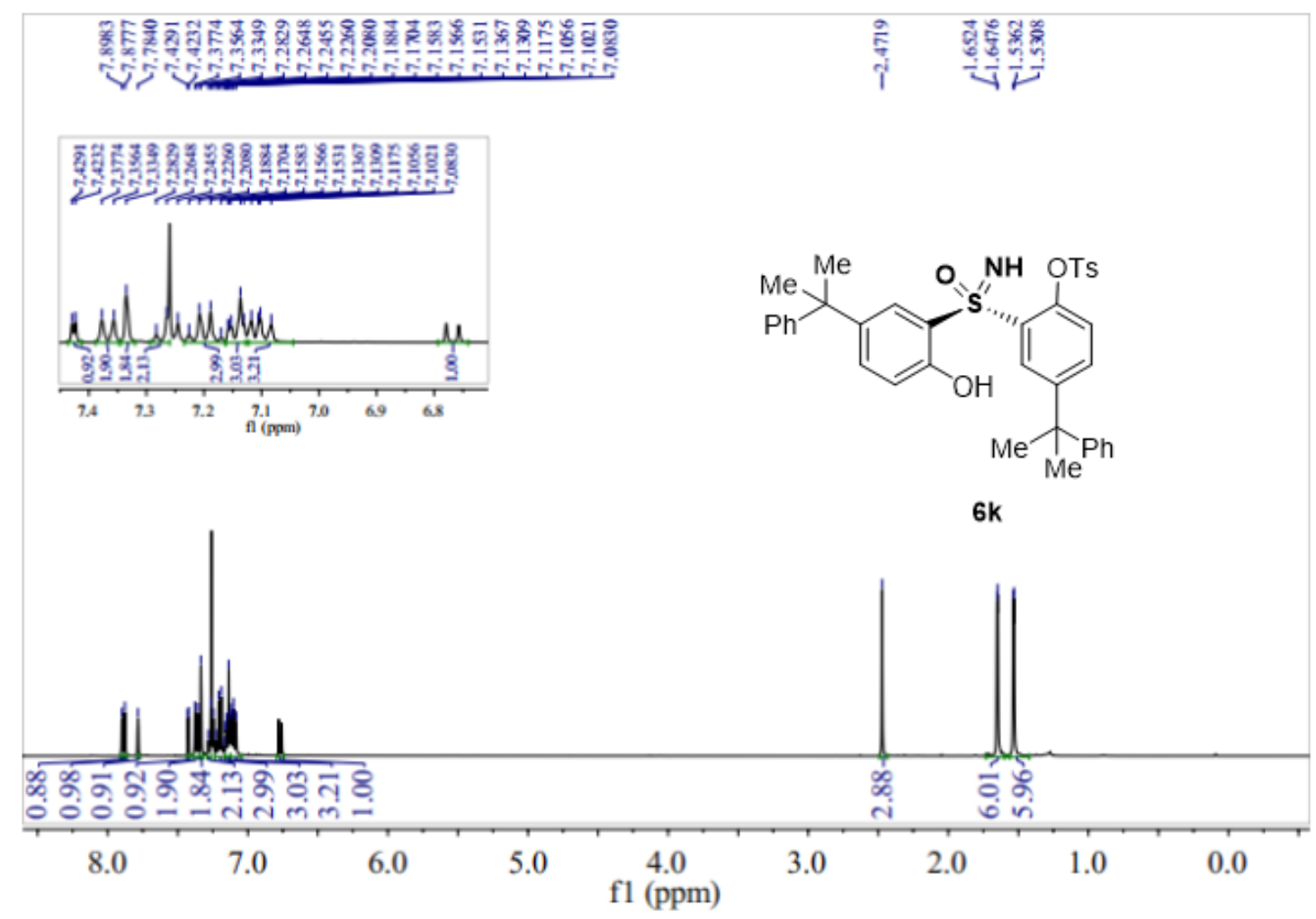

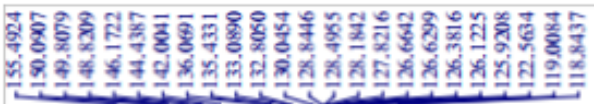

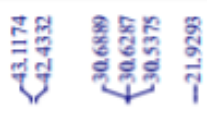

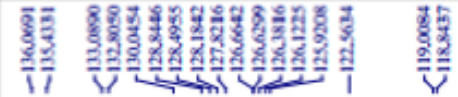
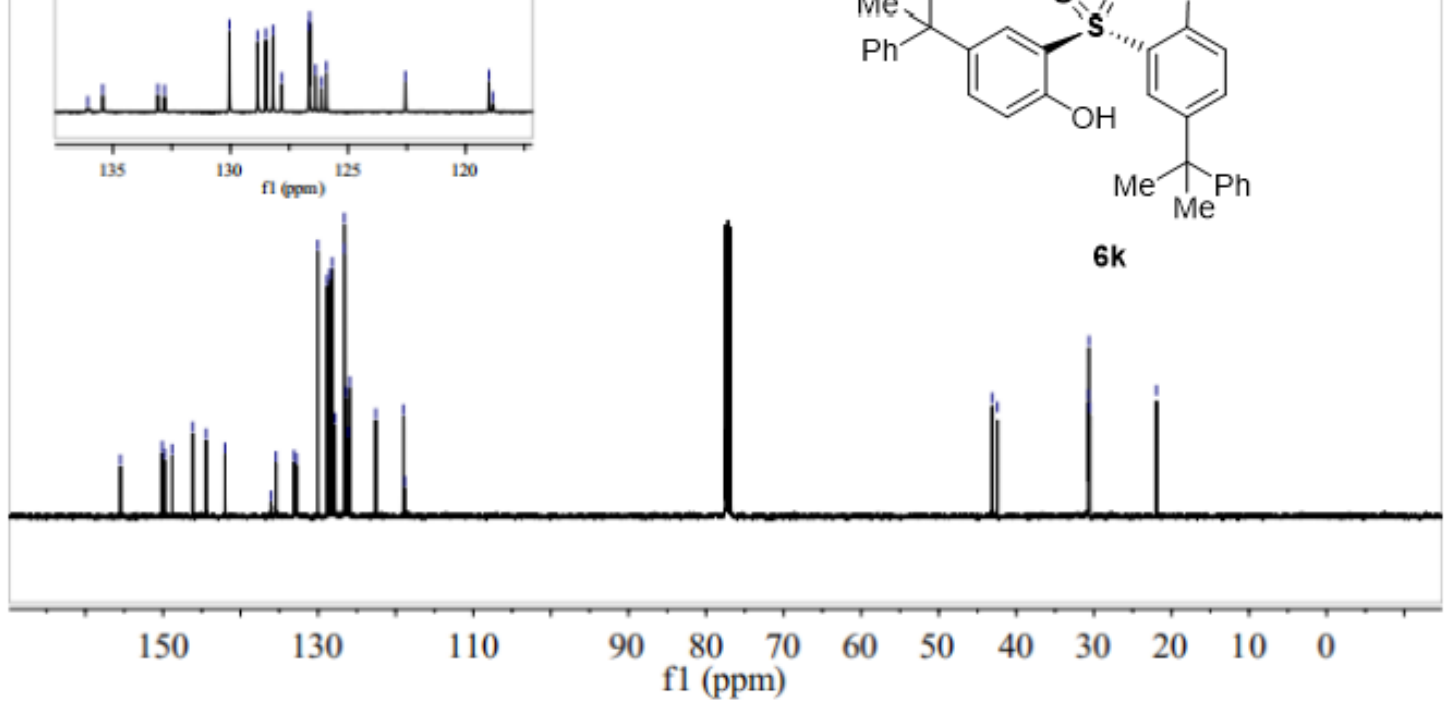

${ }^{1} \mathrm{H}$-NMR and ${ }^{13} \mathrm{C}-\mathrm{NMR}$ of $\mathbf{6 k}$ 

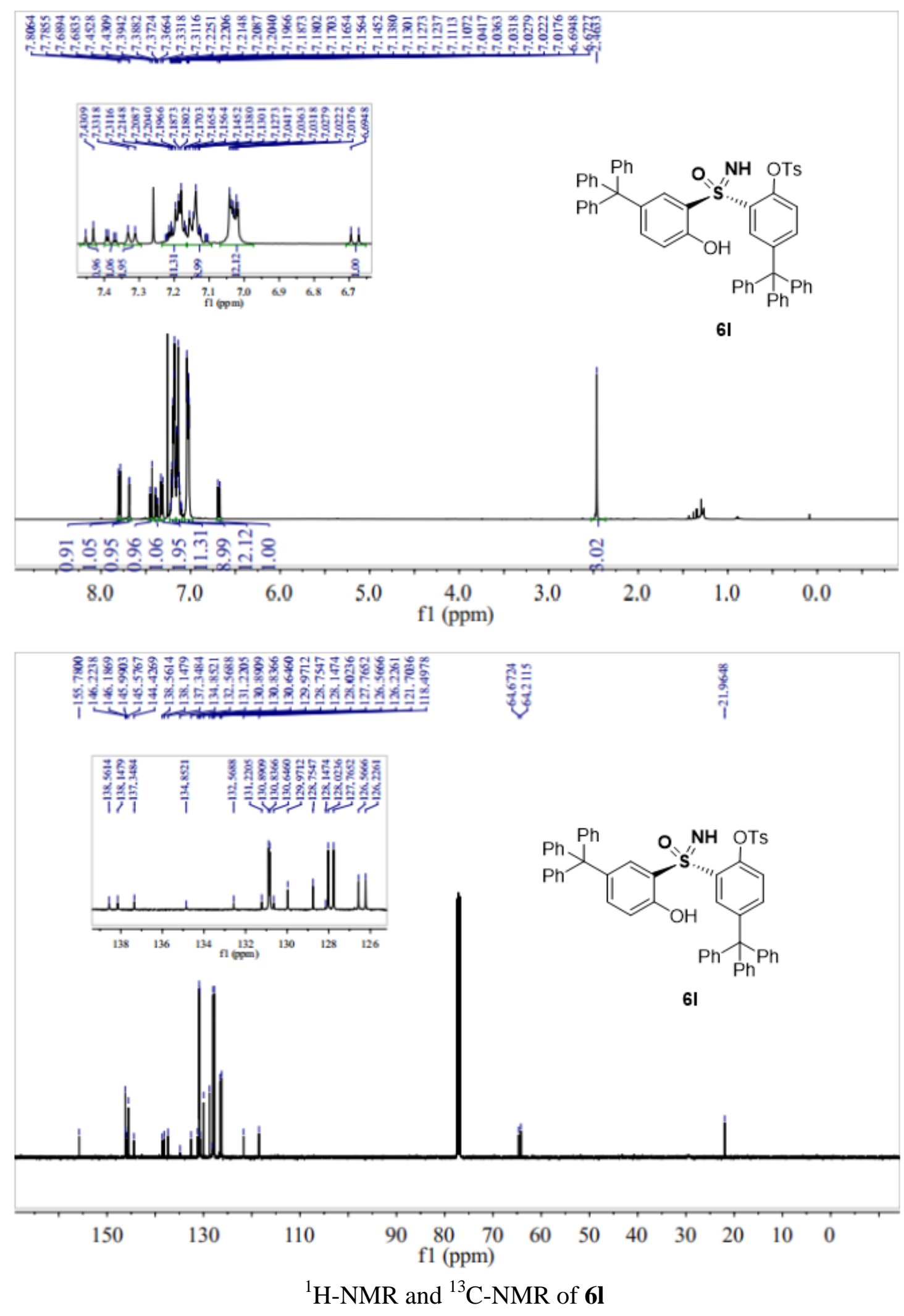

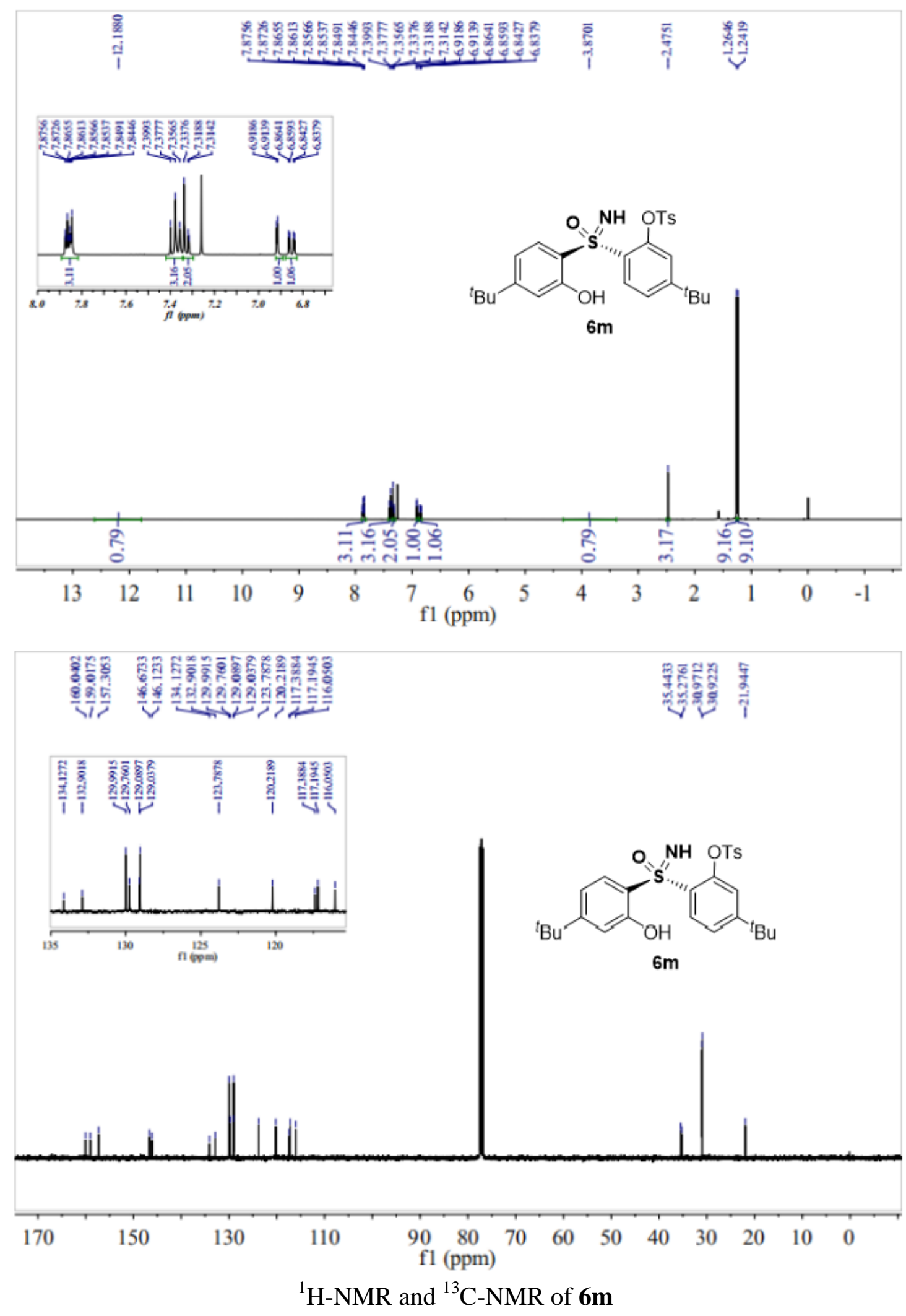

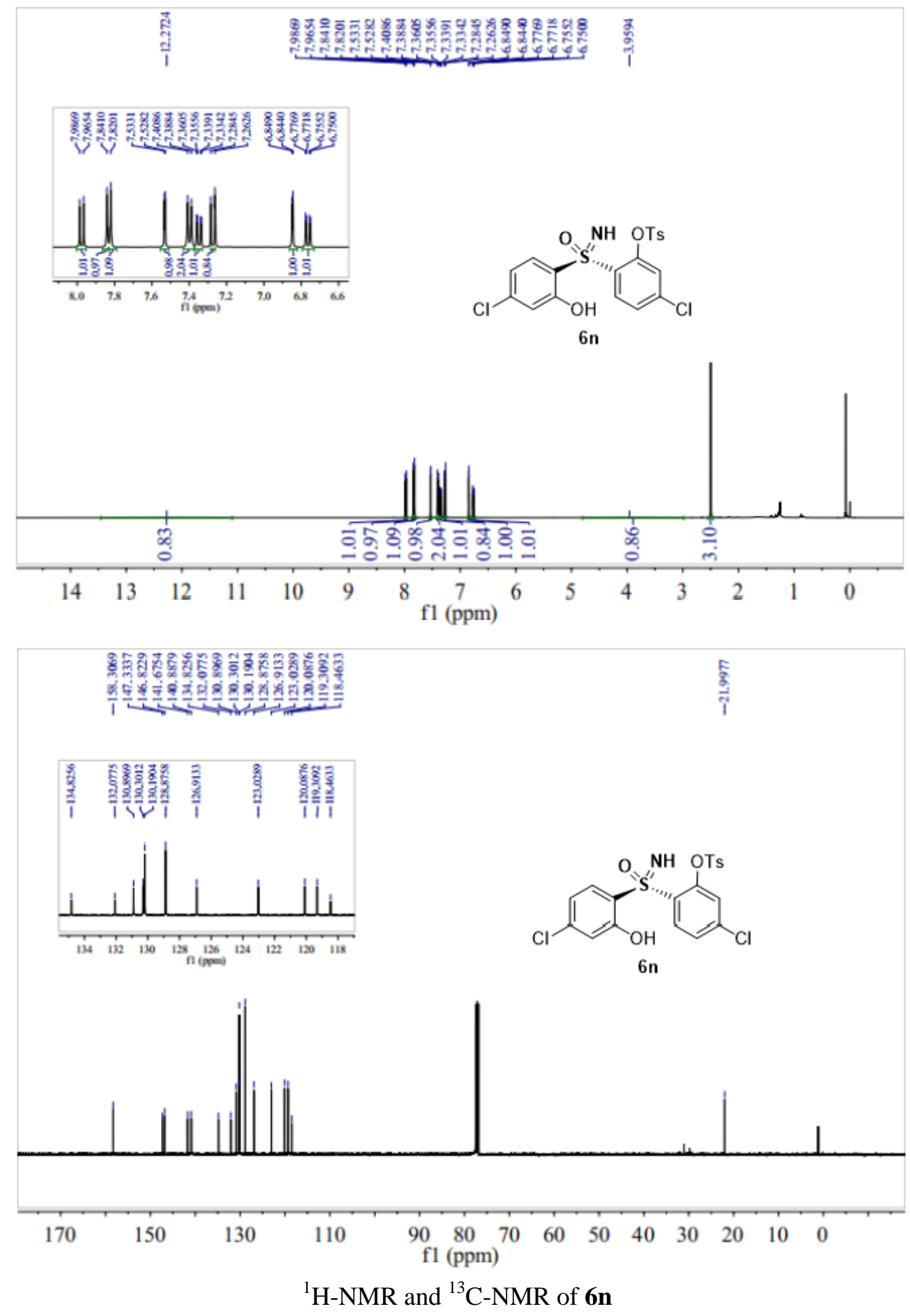

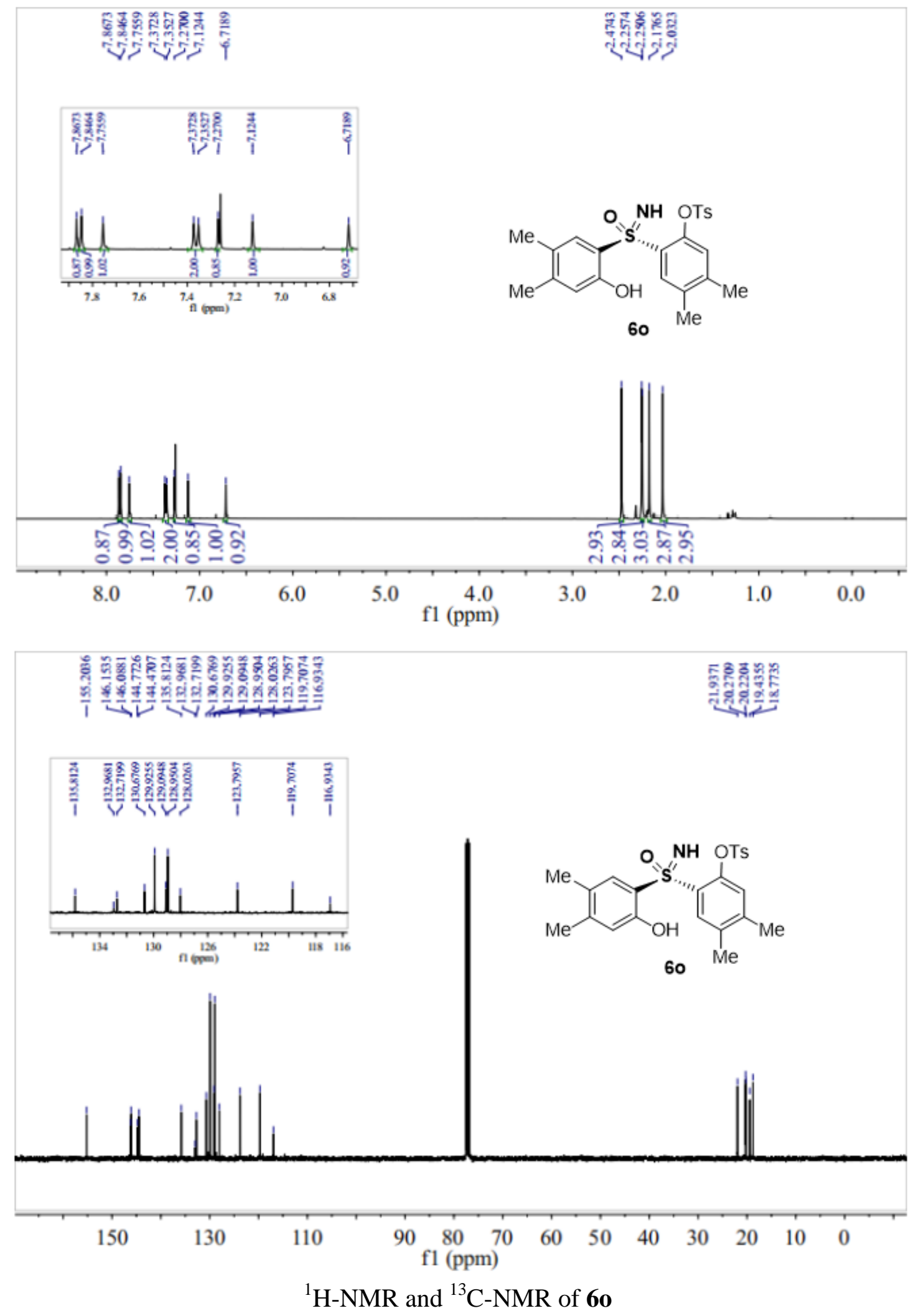

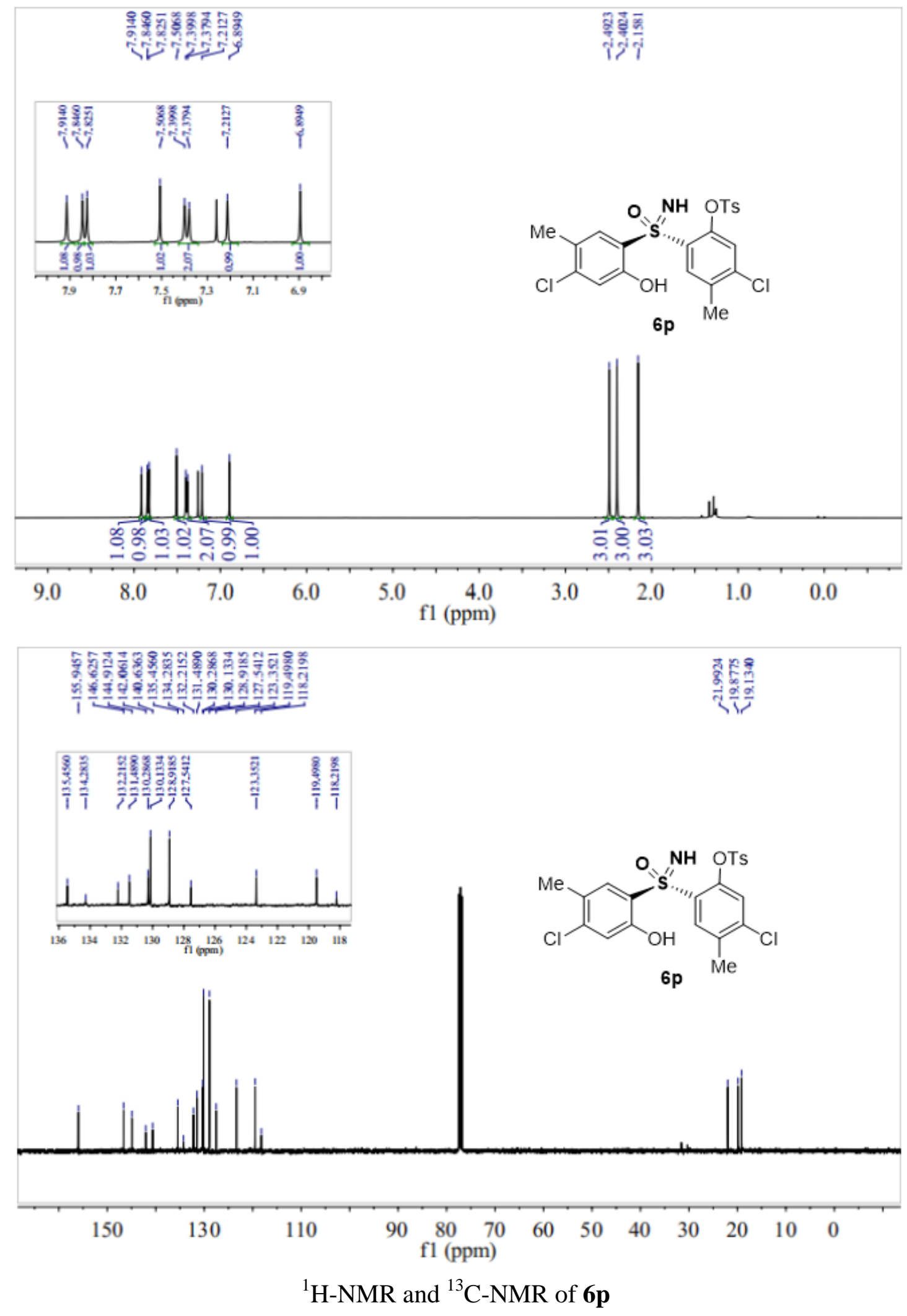

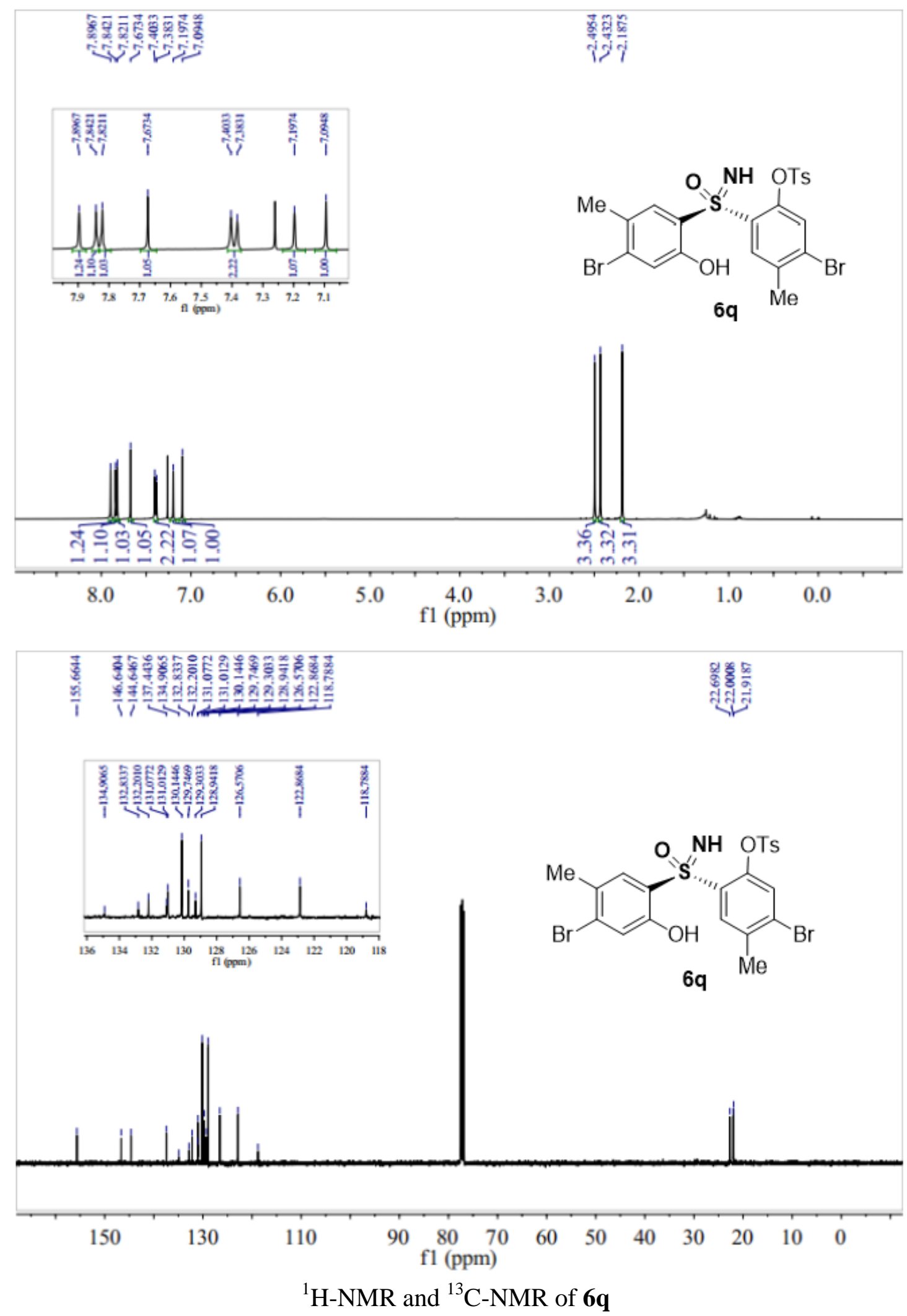

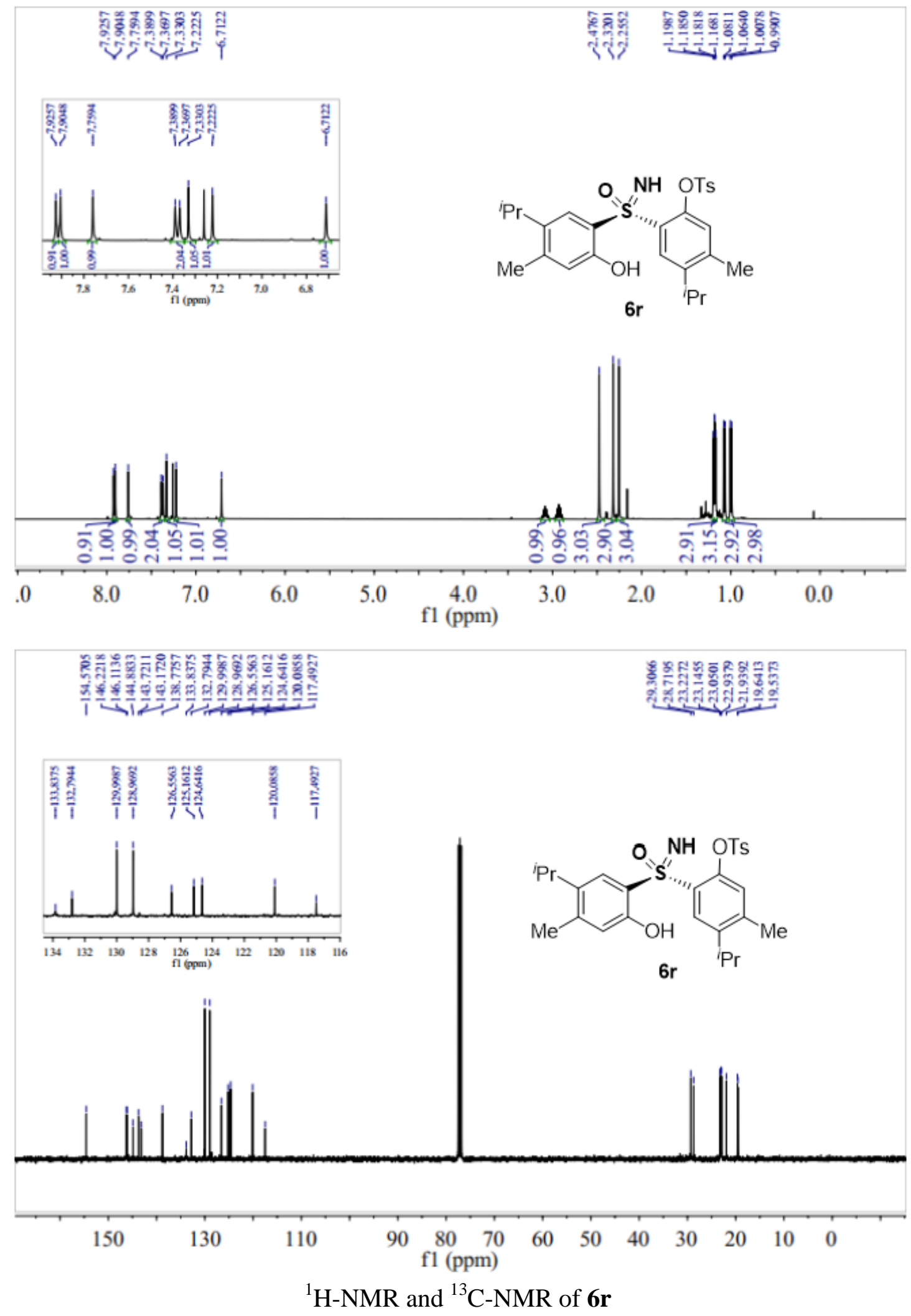

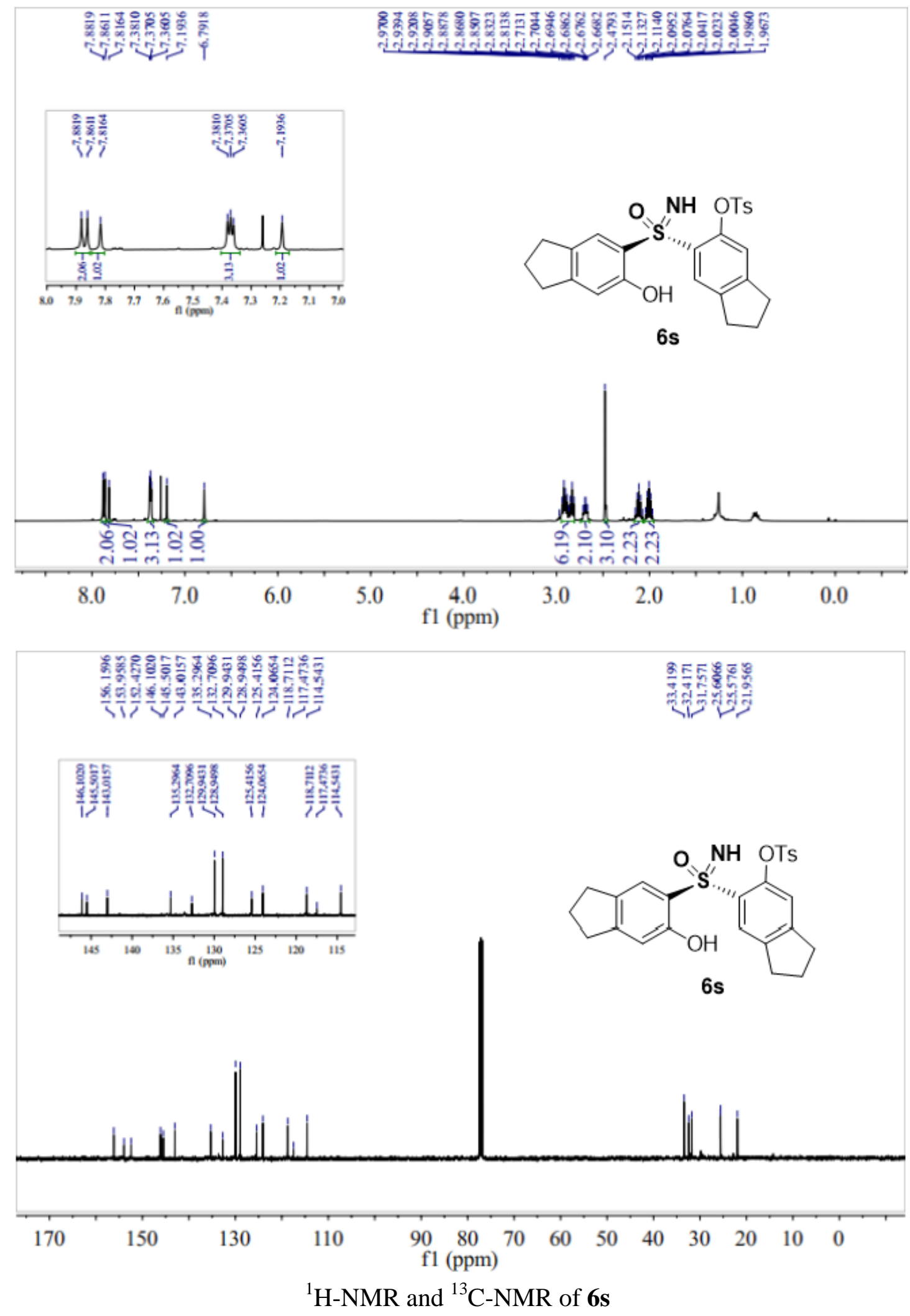

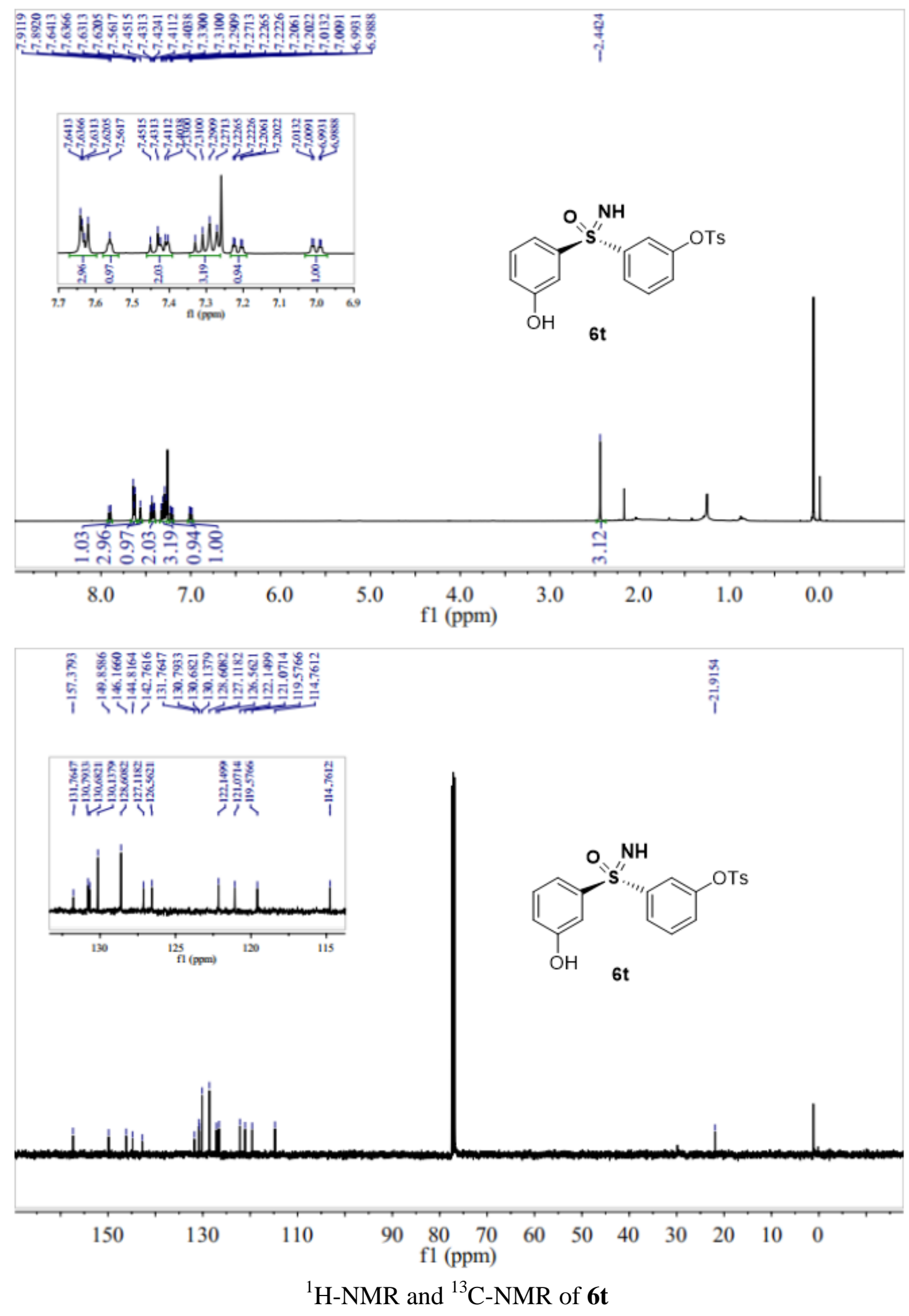

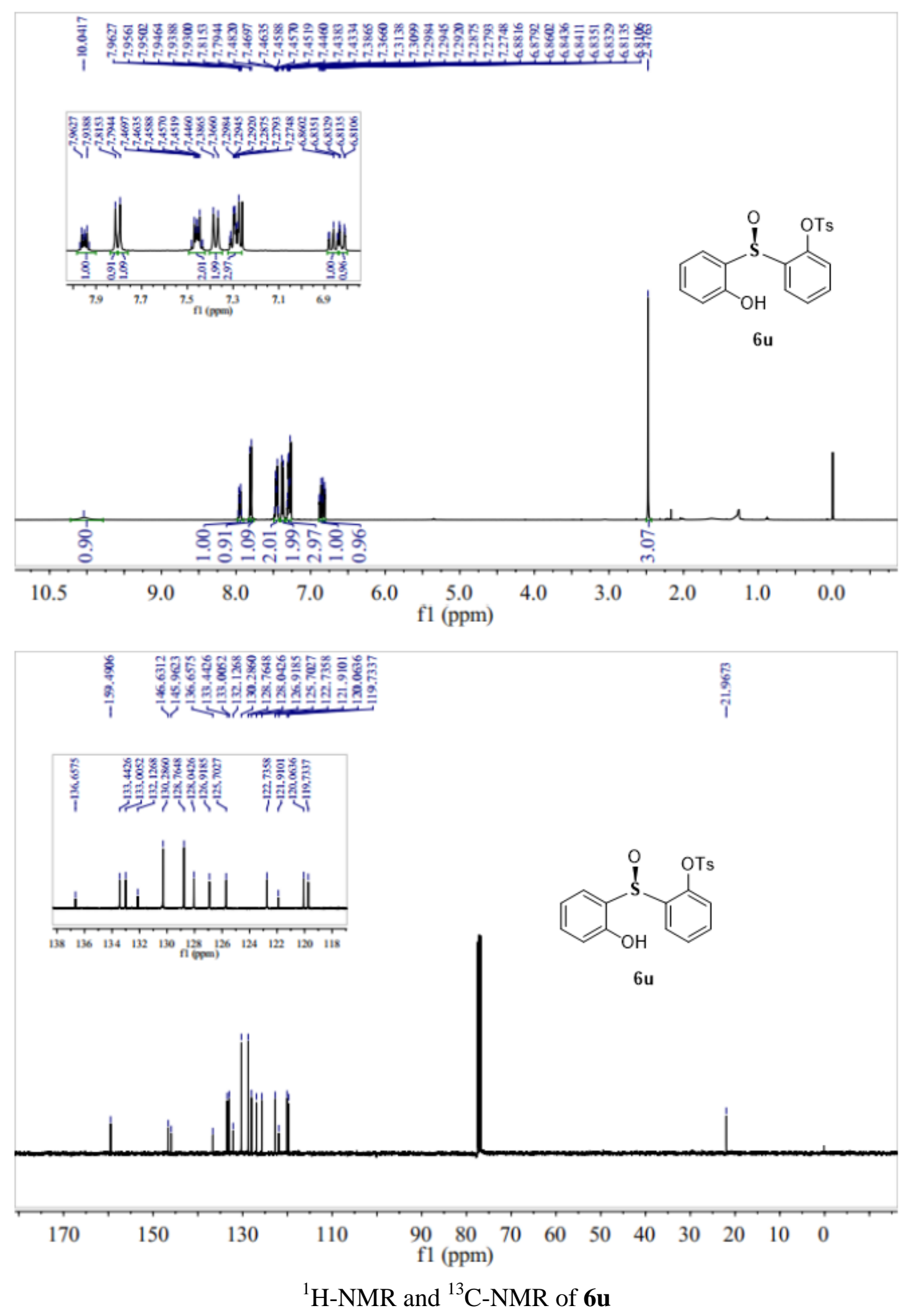

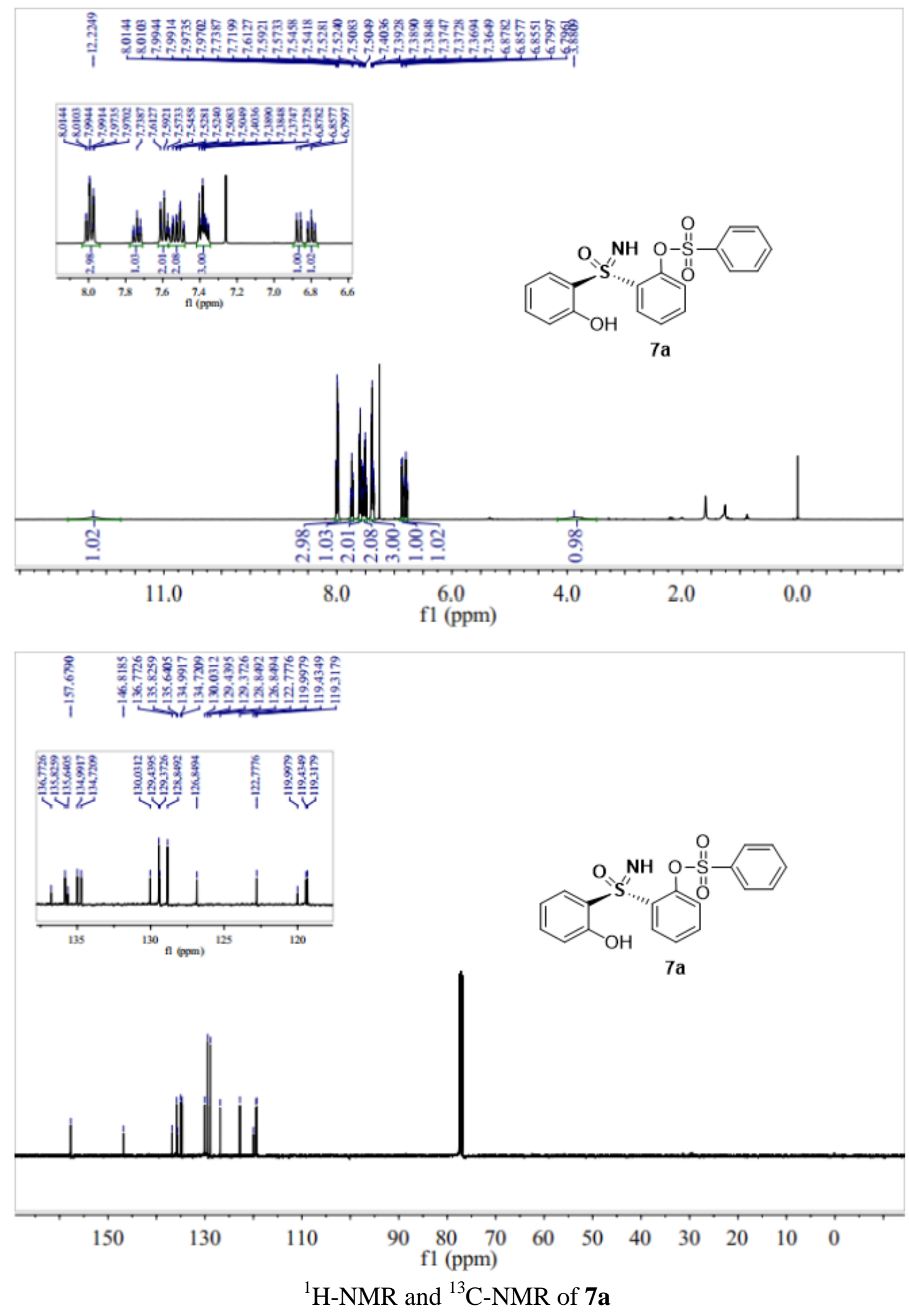

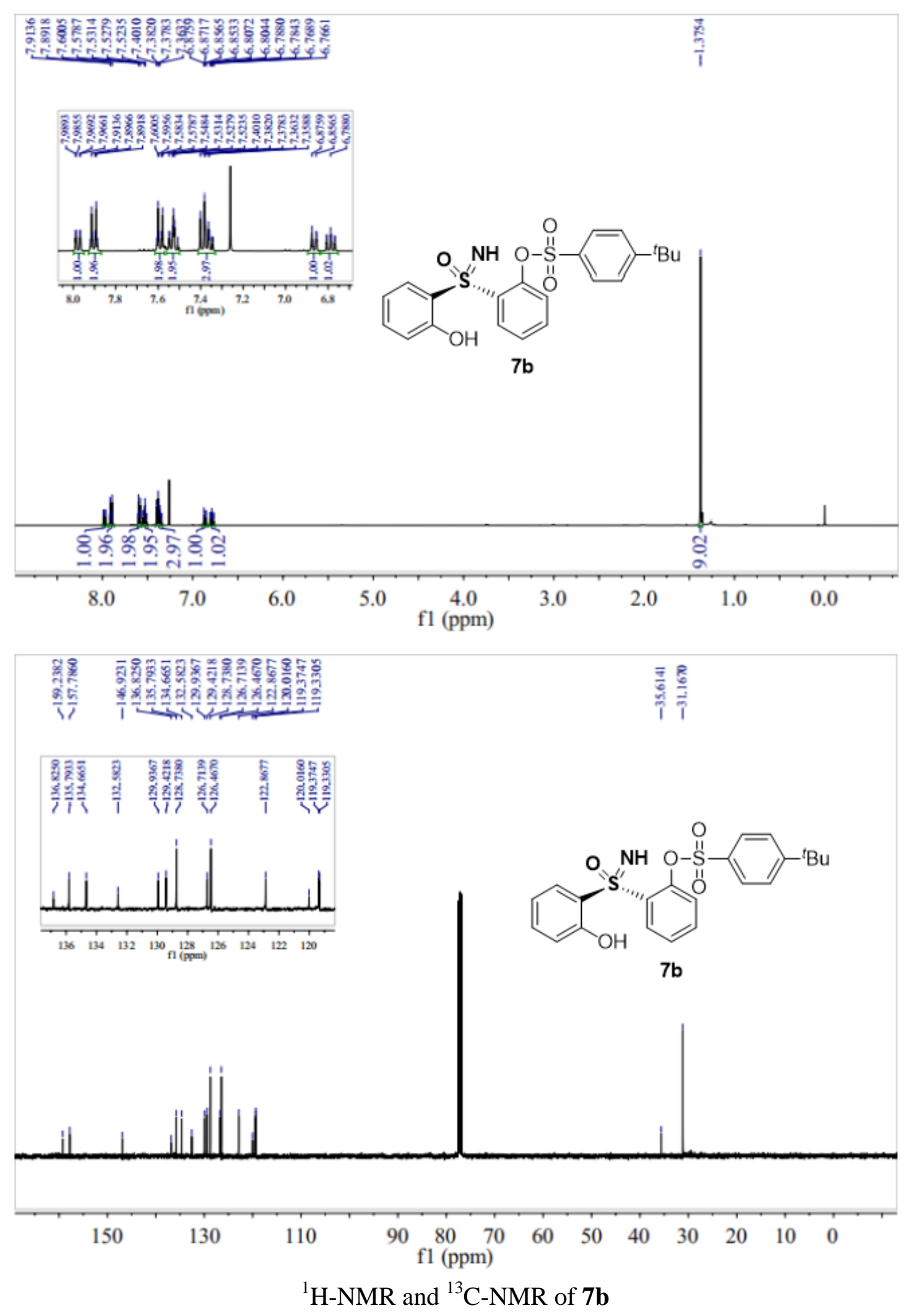

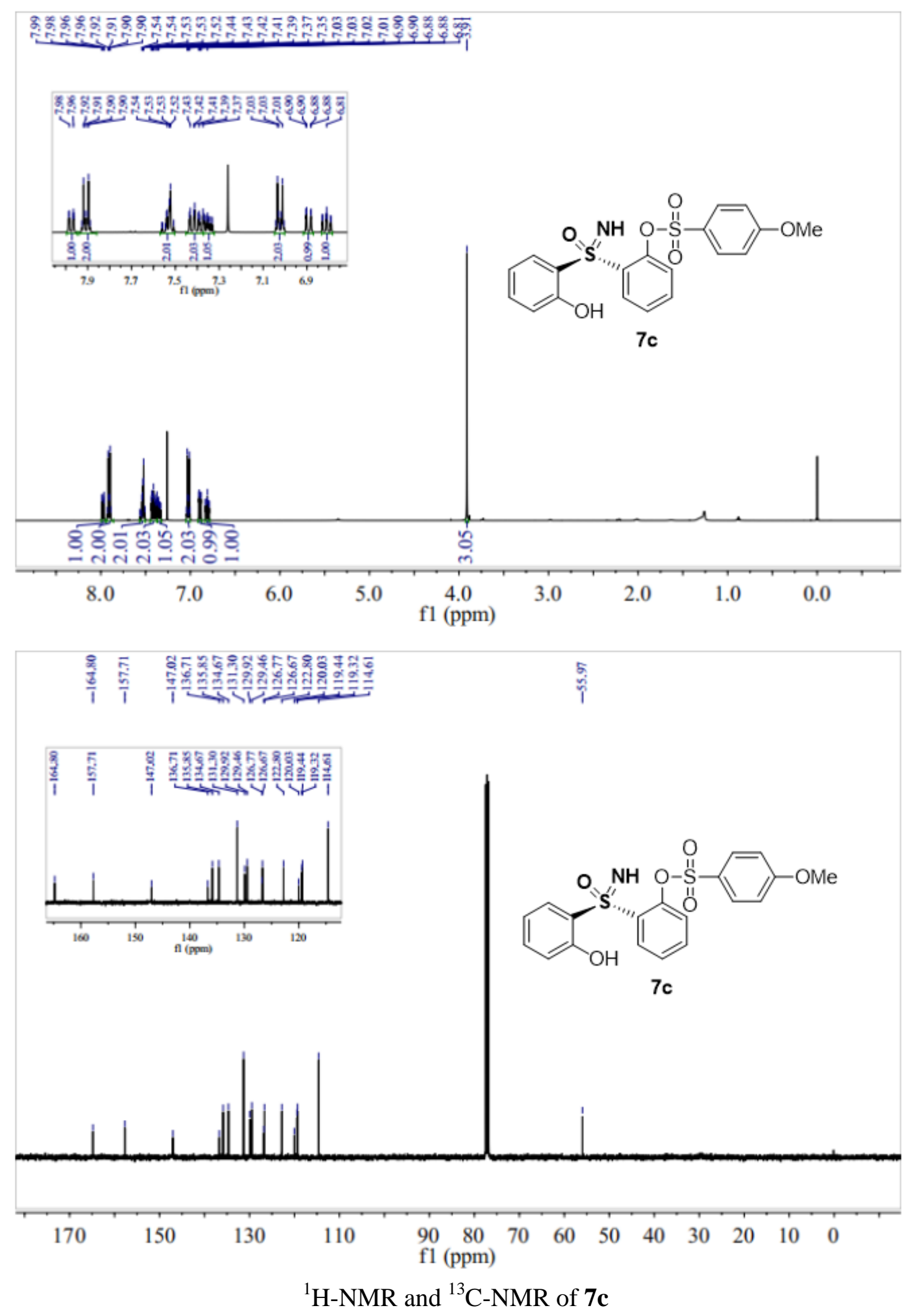

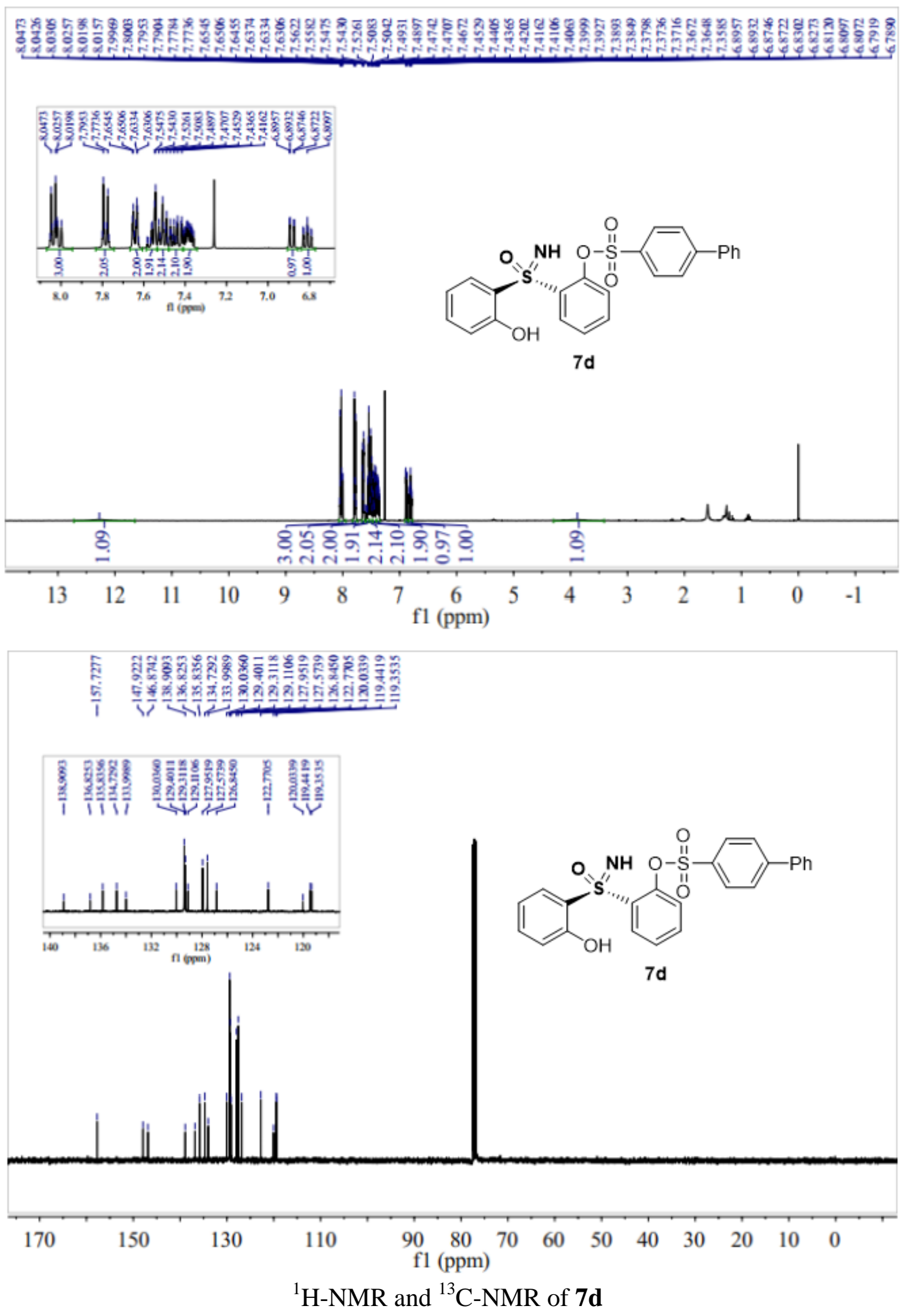

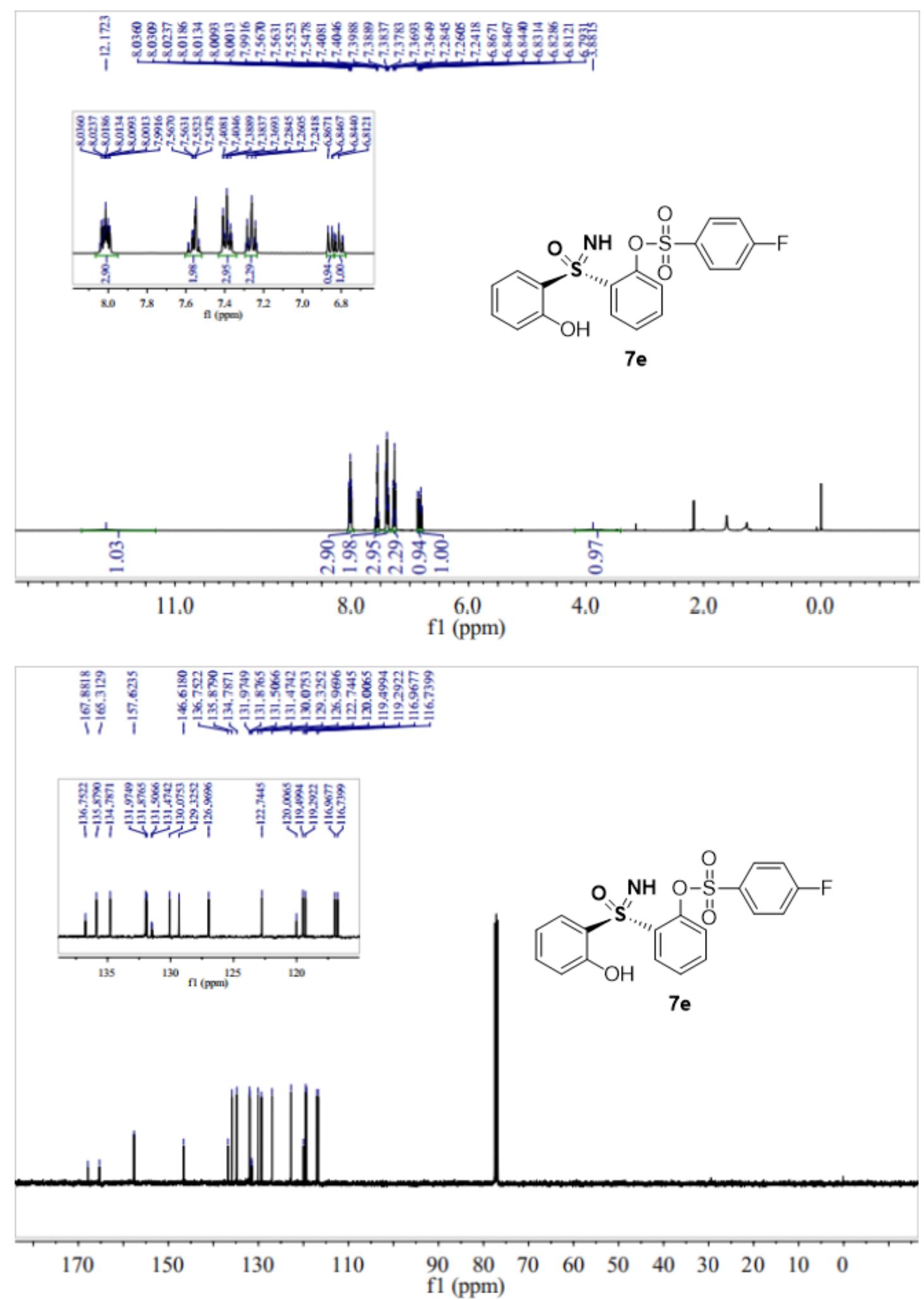


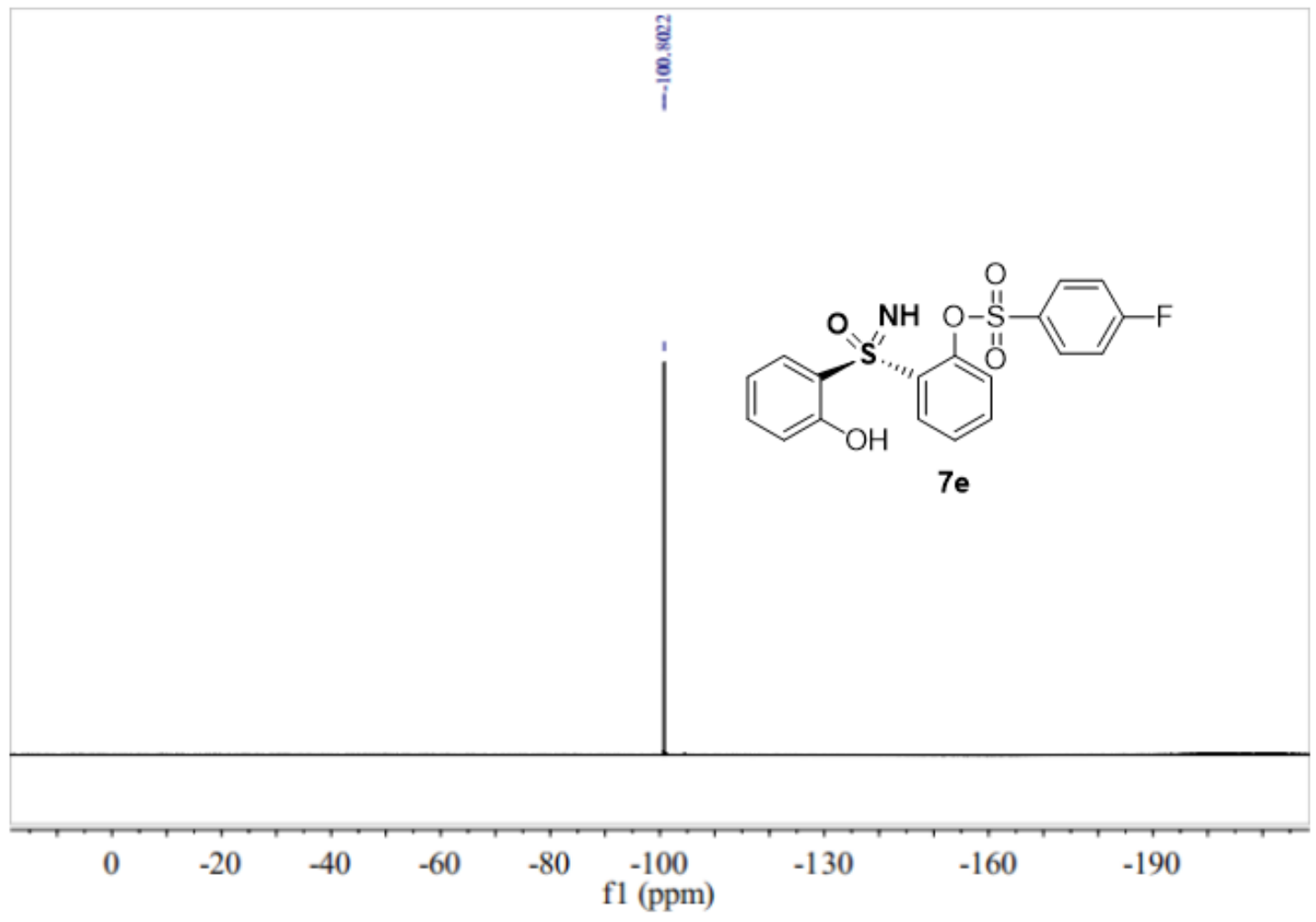

${ }^{1} \mathrm{H}-\mathrm{NMR}$ and ${ }^{13} \mathrm{C}-\mathrm{NMR}$ of $\mathbf{7 e}$ 

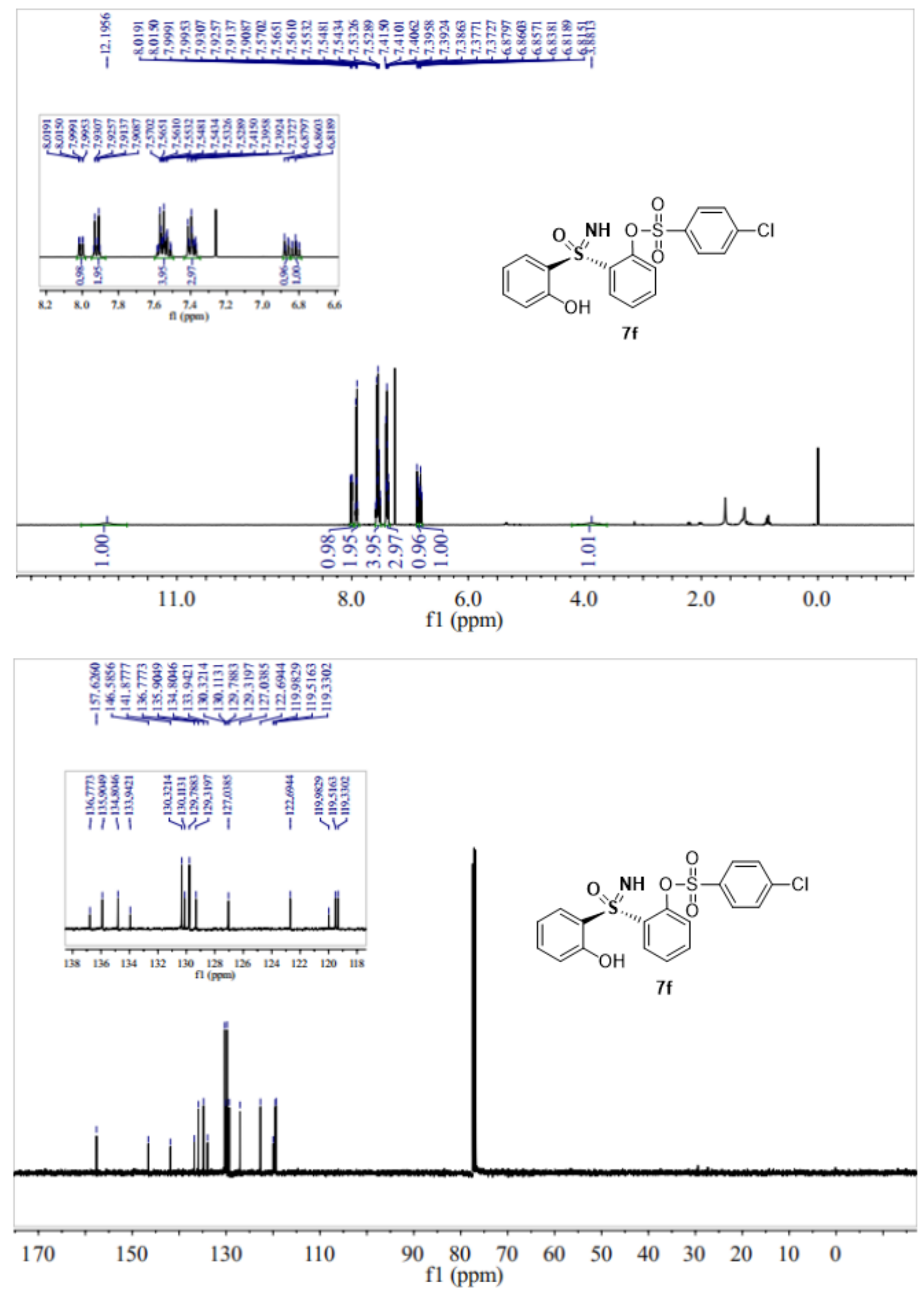

${ }^{1} \mathrm{H}-\mathrm{NMR}$ and ${ }^{13} \mathrm{C}-\mathrm{NMR}$ of $\mathbf{7 f}$ 

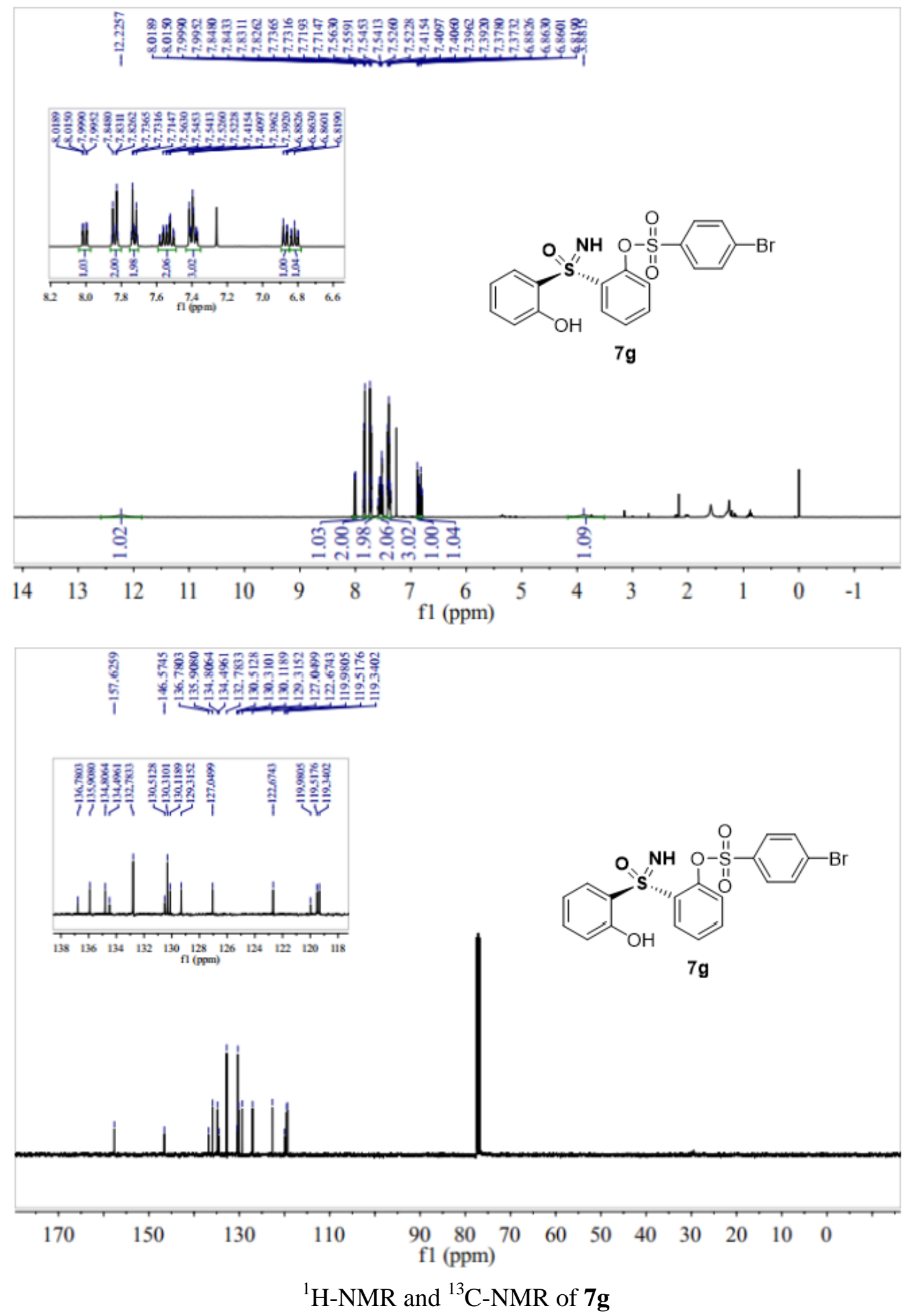

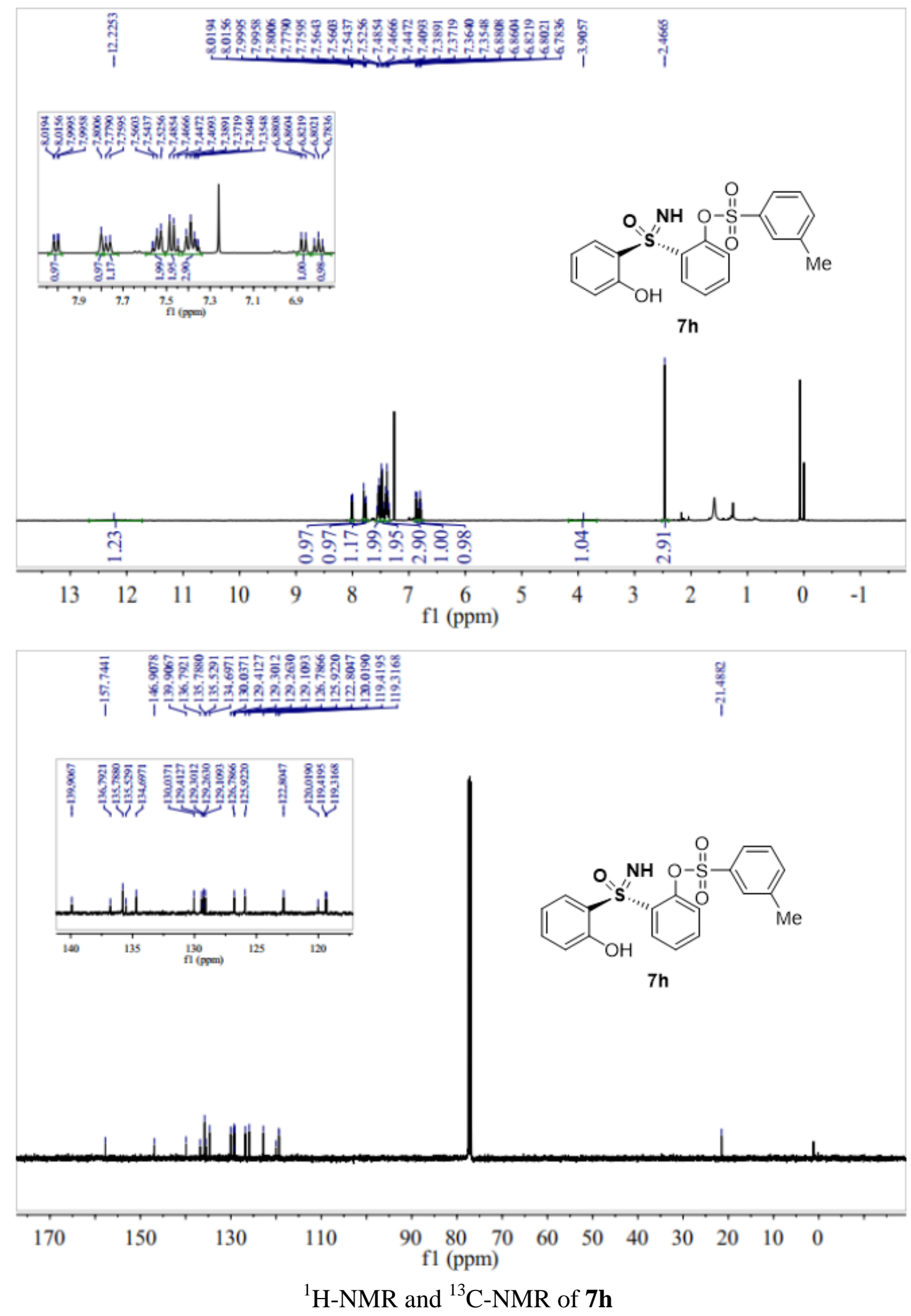

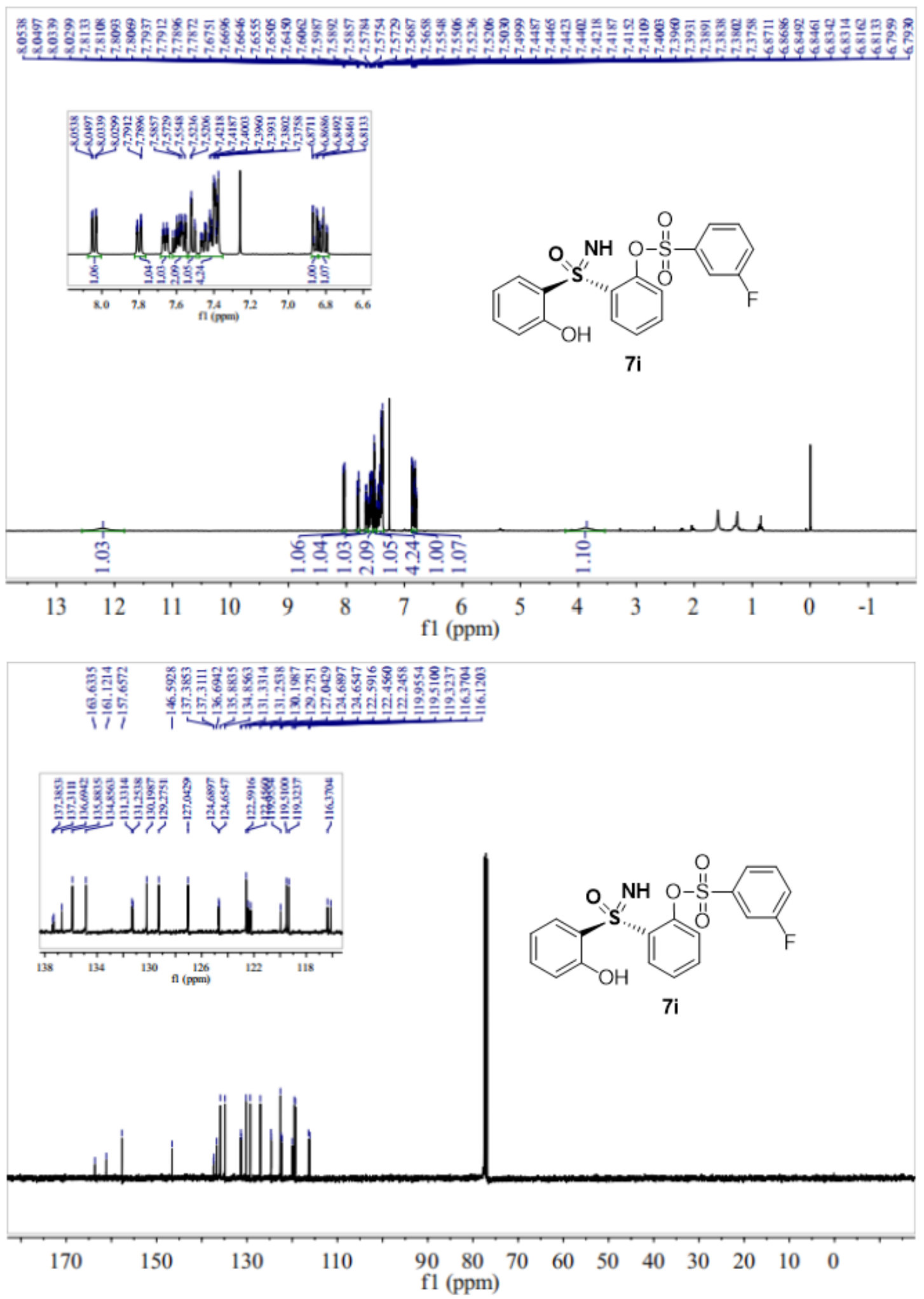


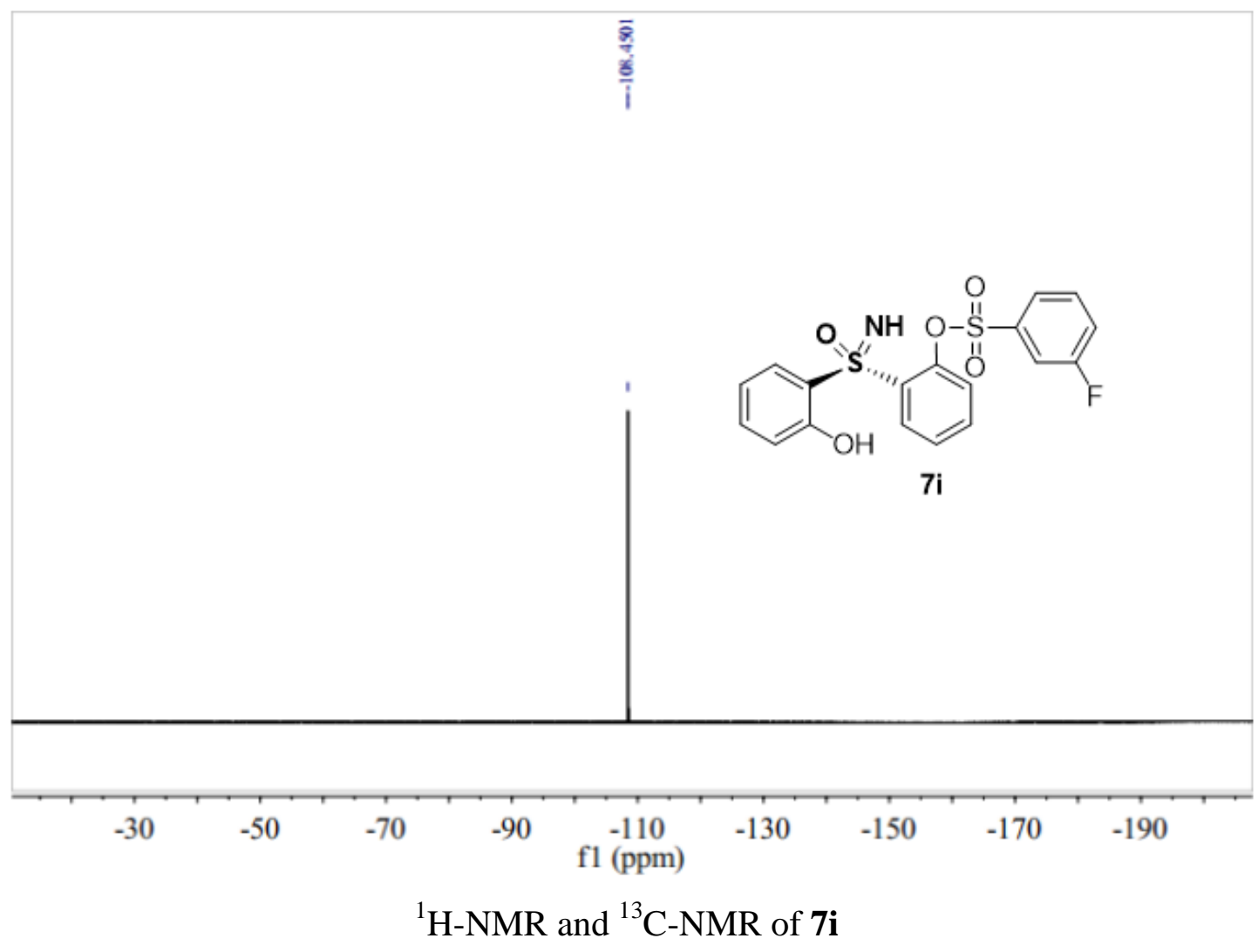



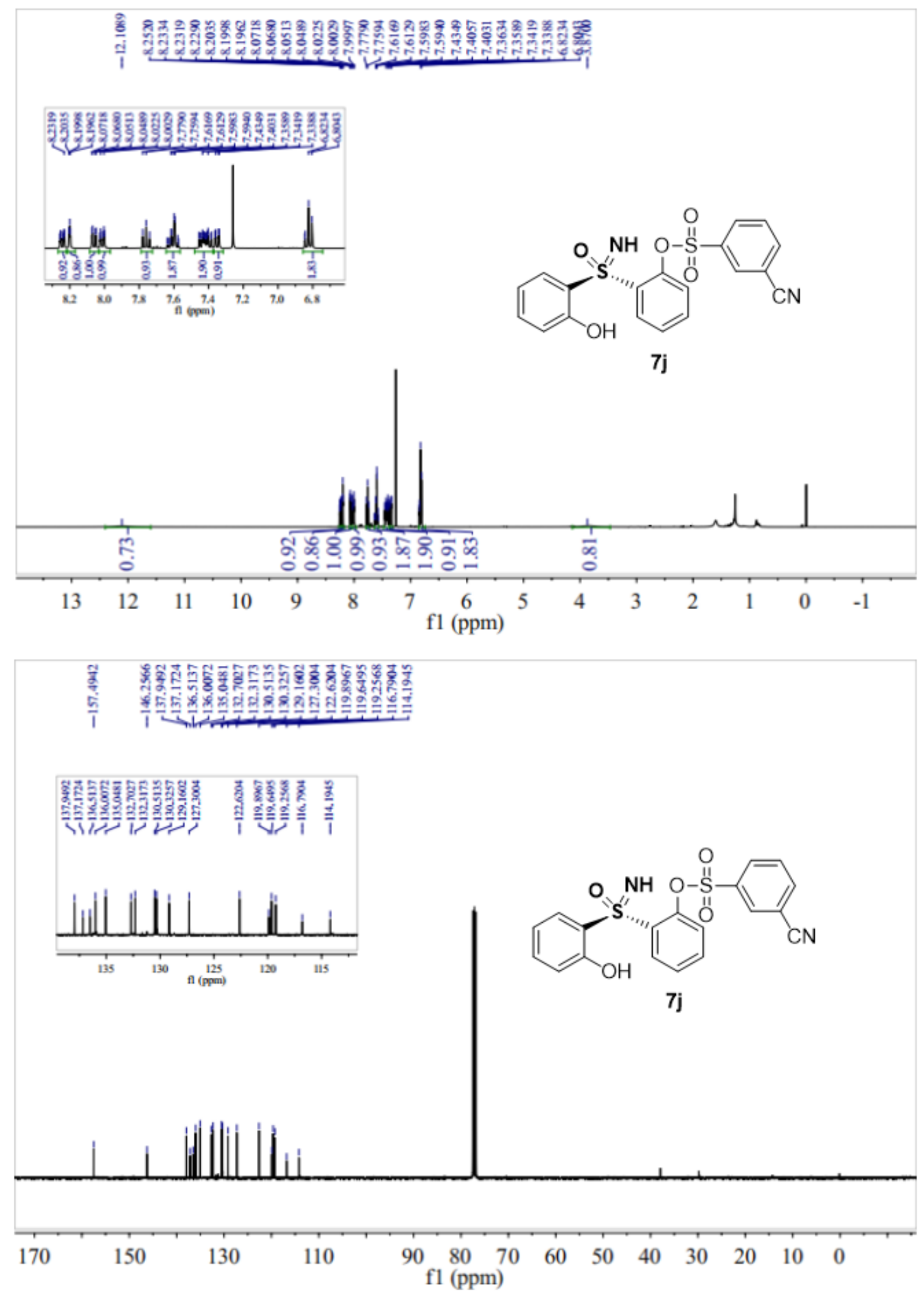

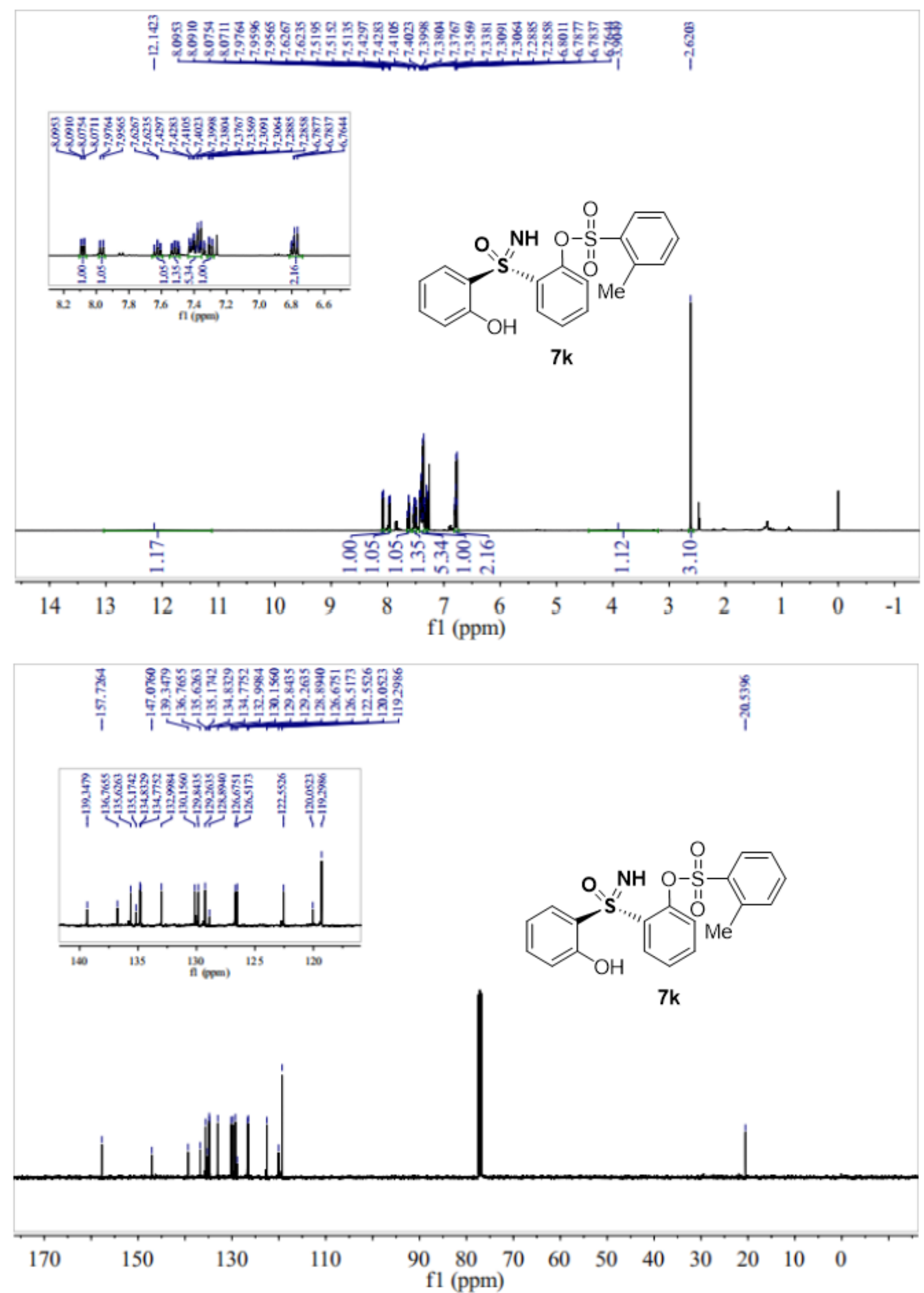

${ }^{1} \mathrm{H}-\mathrm{NMR}$ and ${ }^{13} \mathrm{C}-\mathrm{NMR}$ of $\mathbf{7 k}$ 

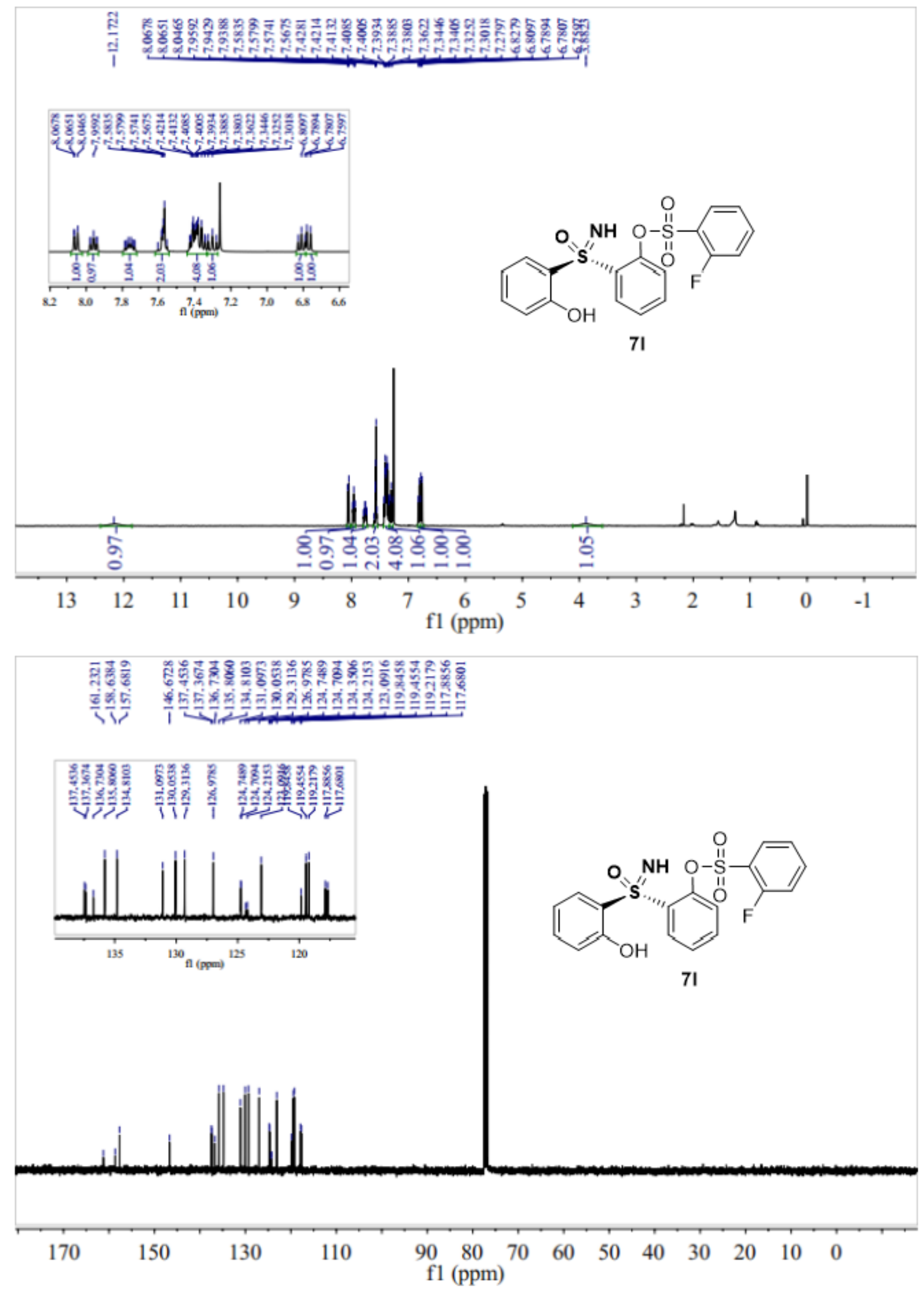


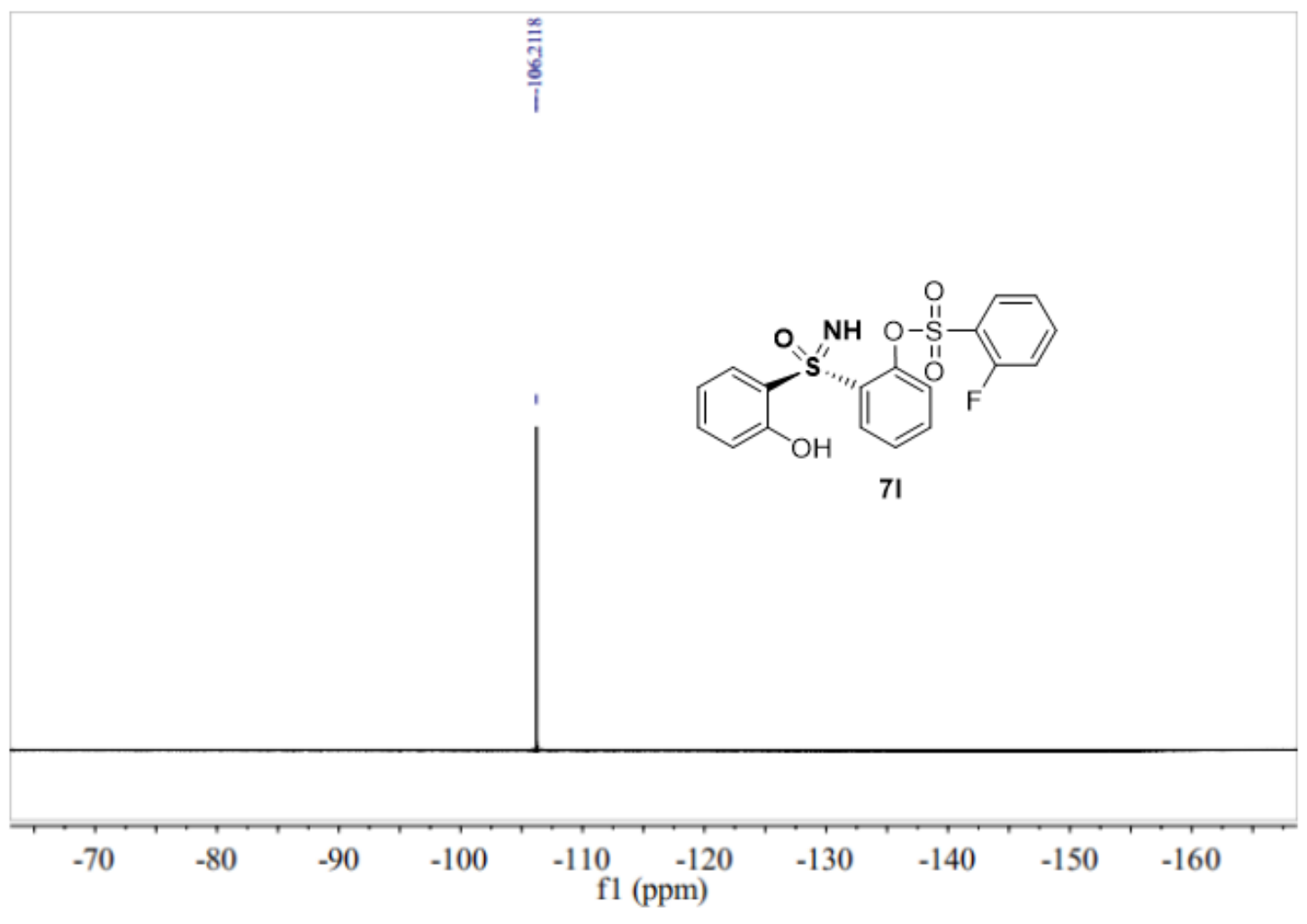

${ }^{1} \mathrm{H}-\mathrm{NMR}$ and ${ }^{13} \mathrm{C}-\mathrm{NMR}$ of $7 \mathbf{l}$ 


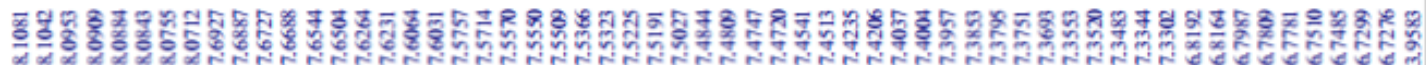
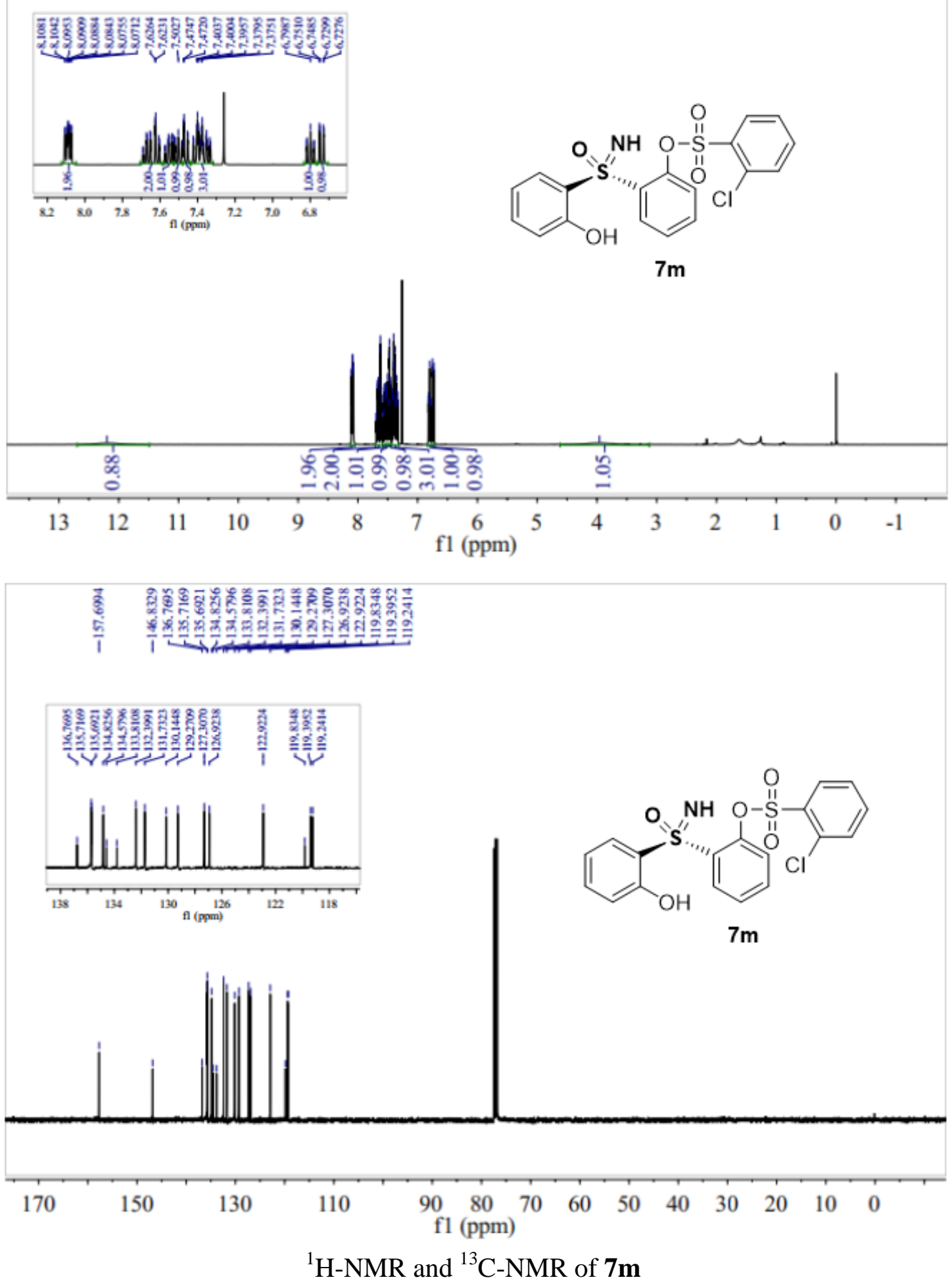

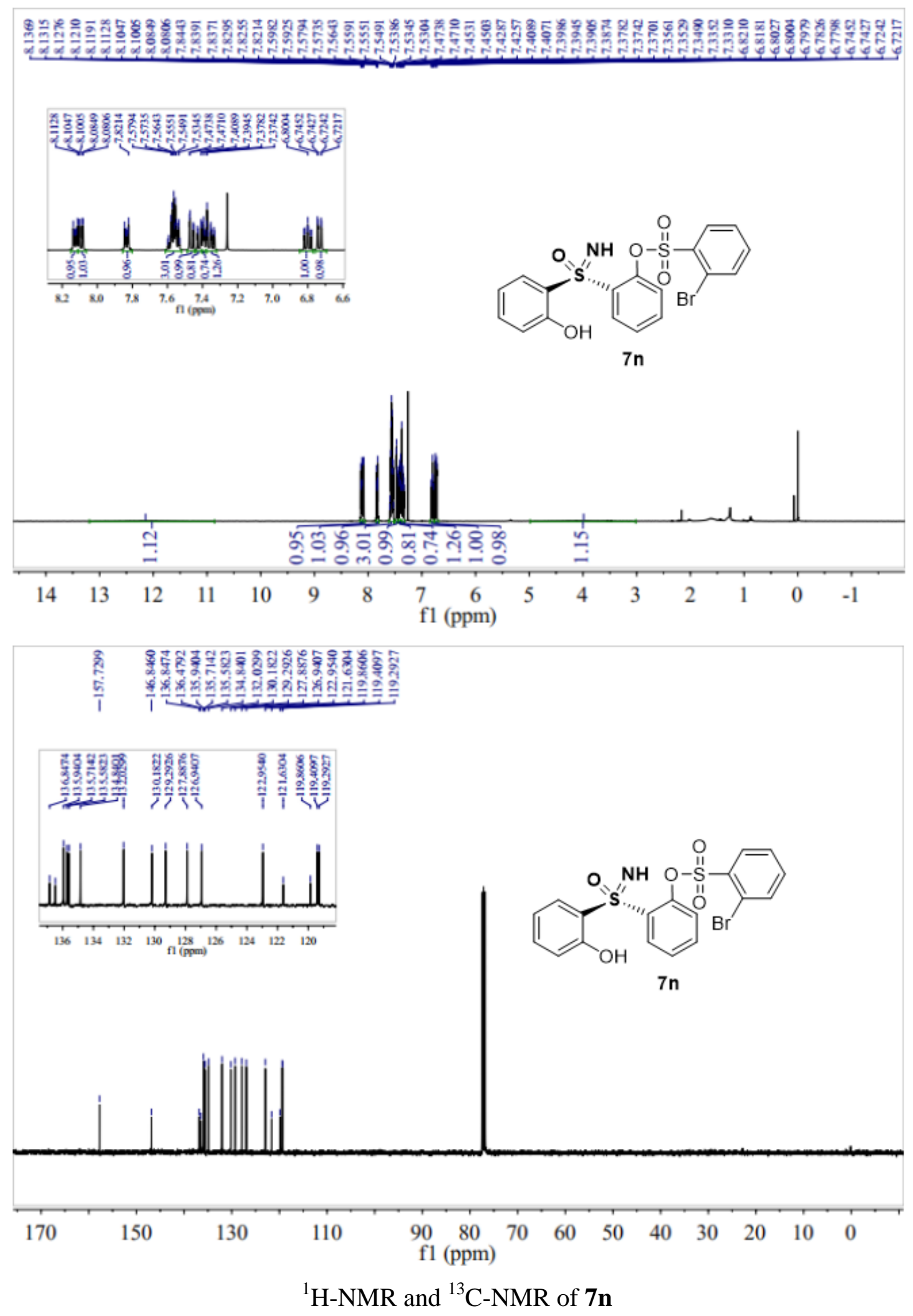

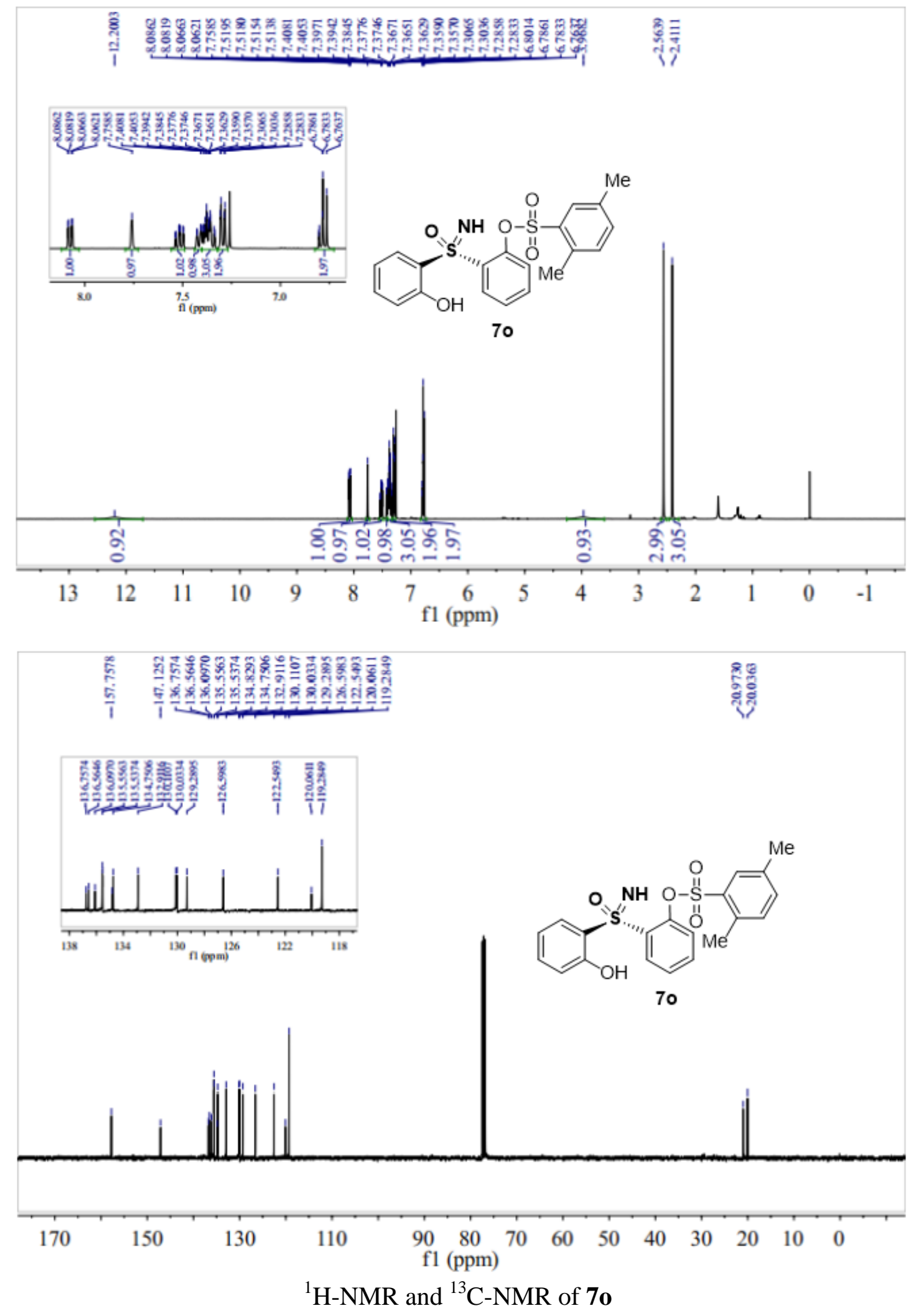

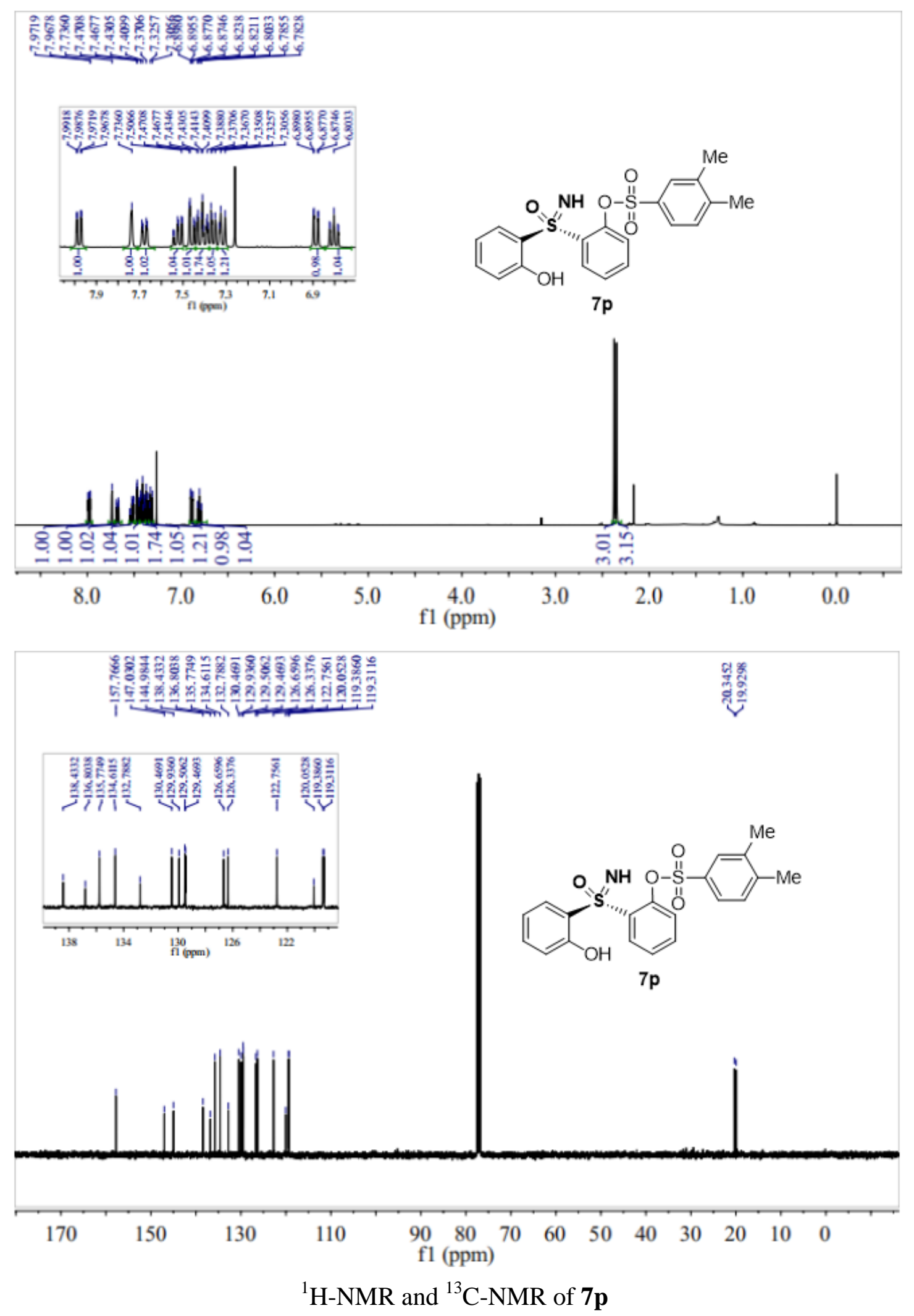

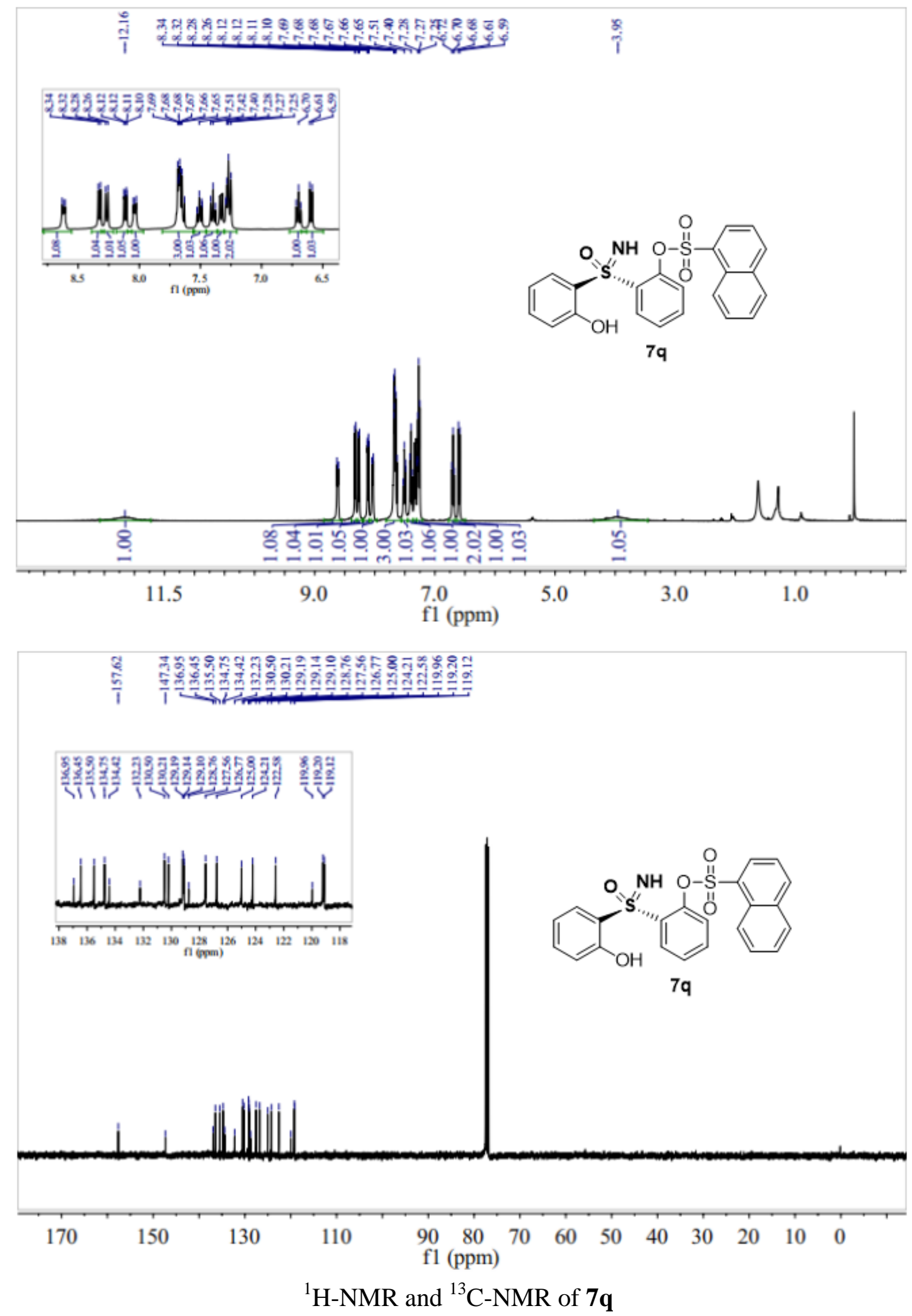

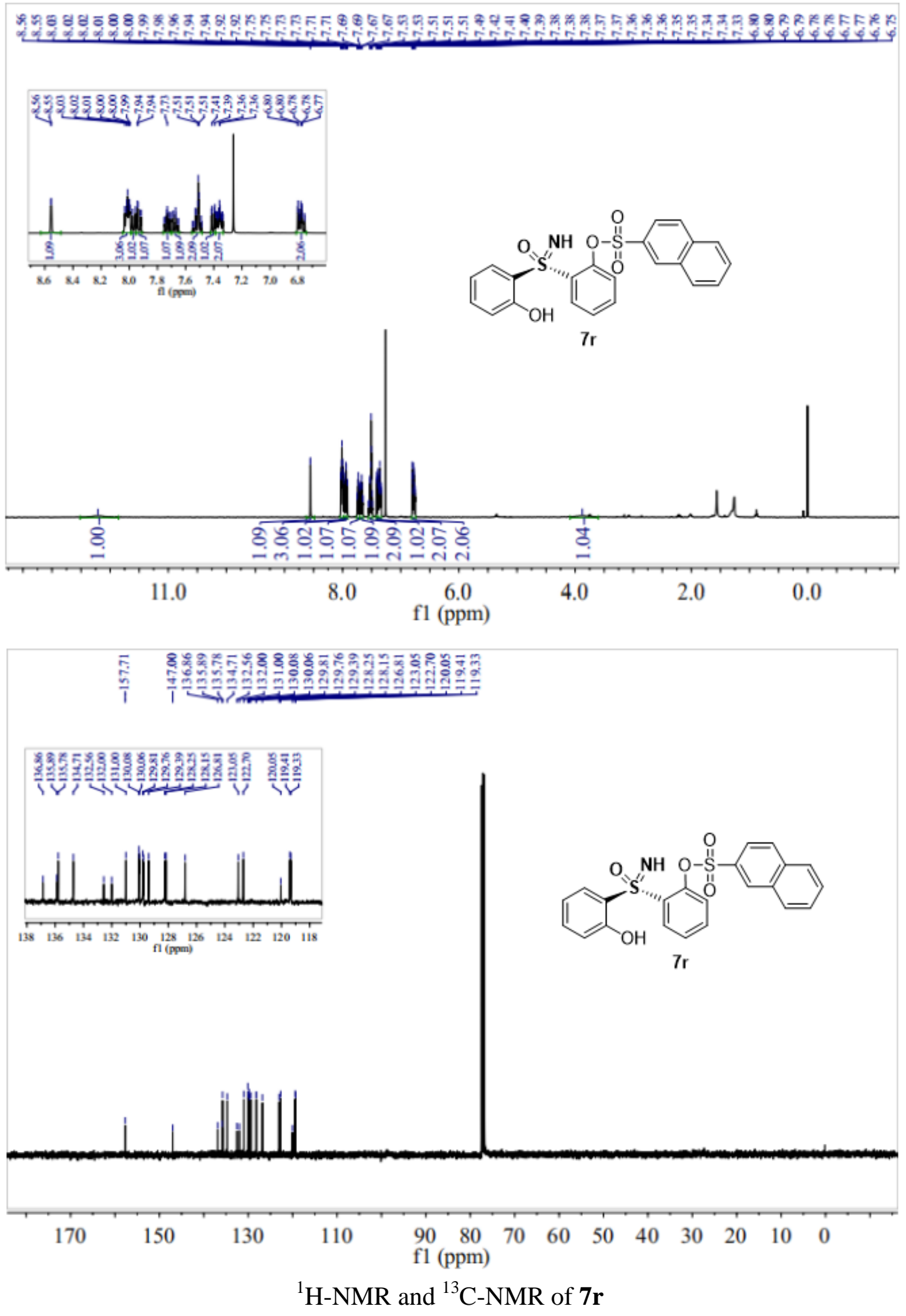

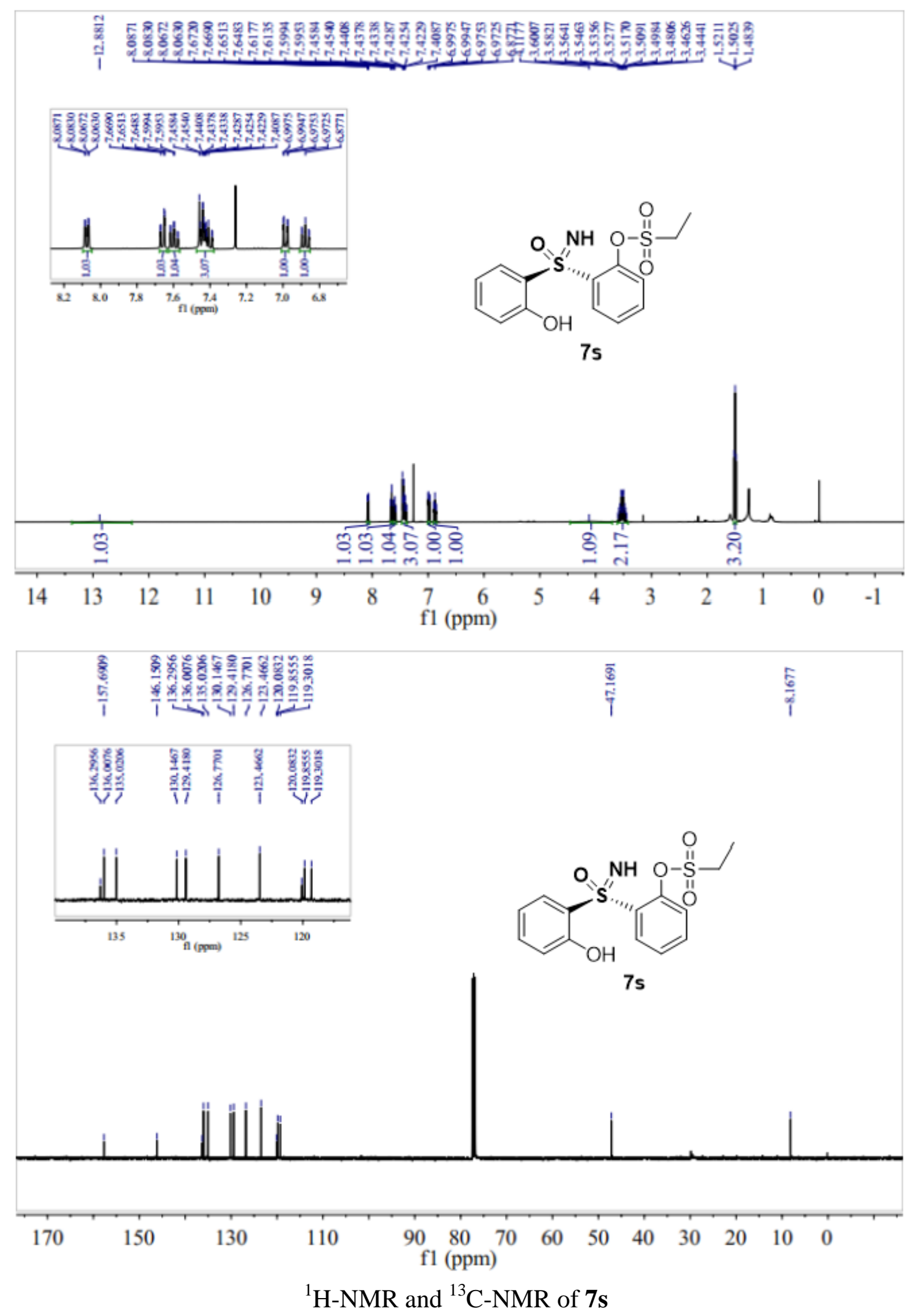

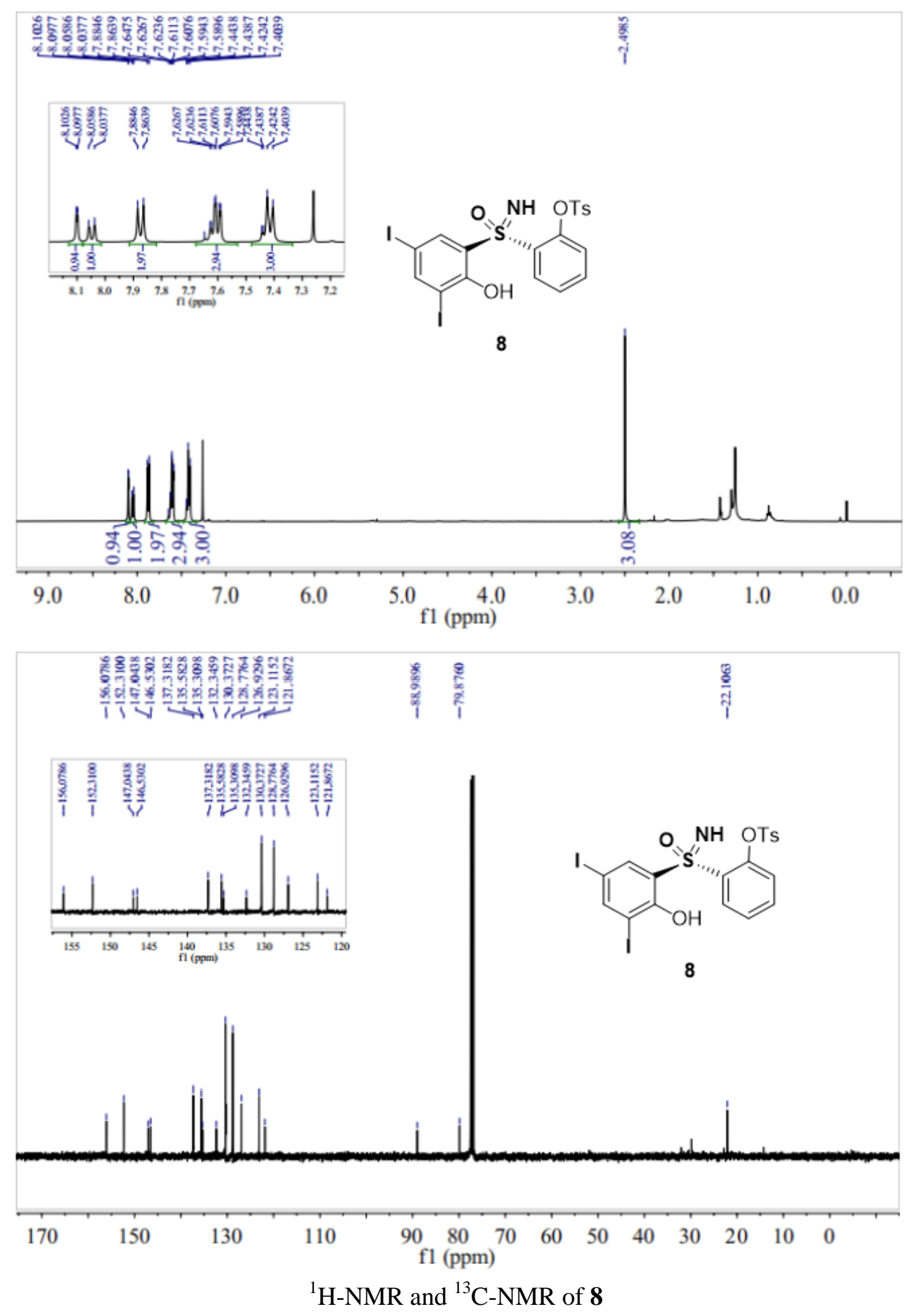

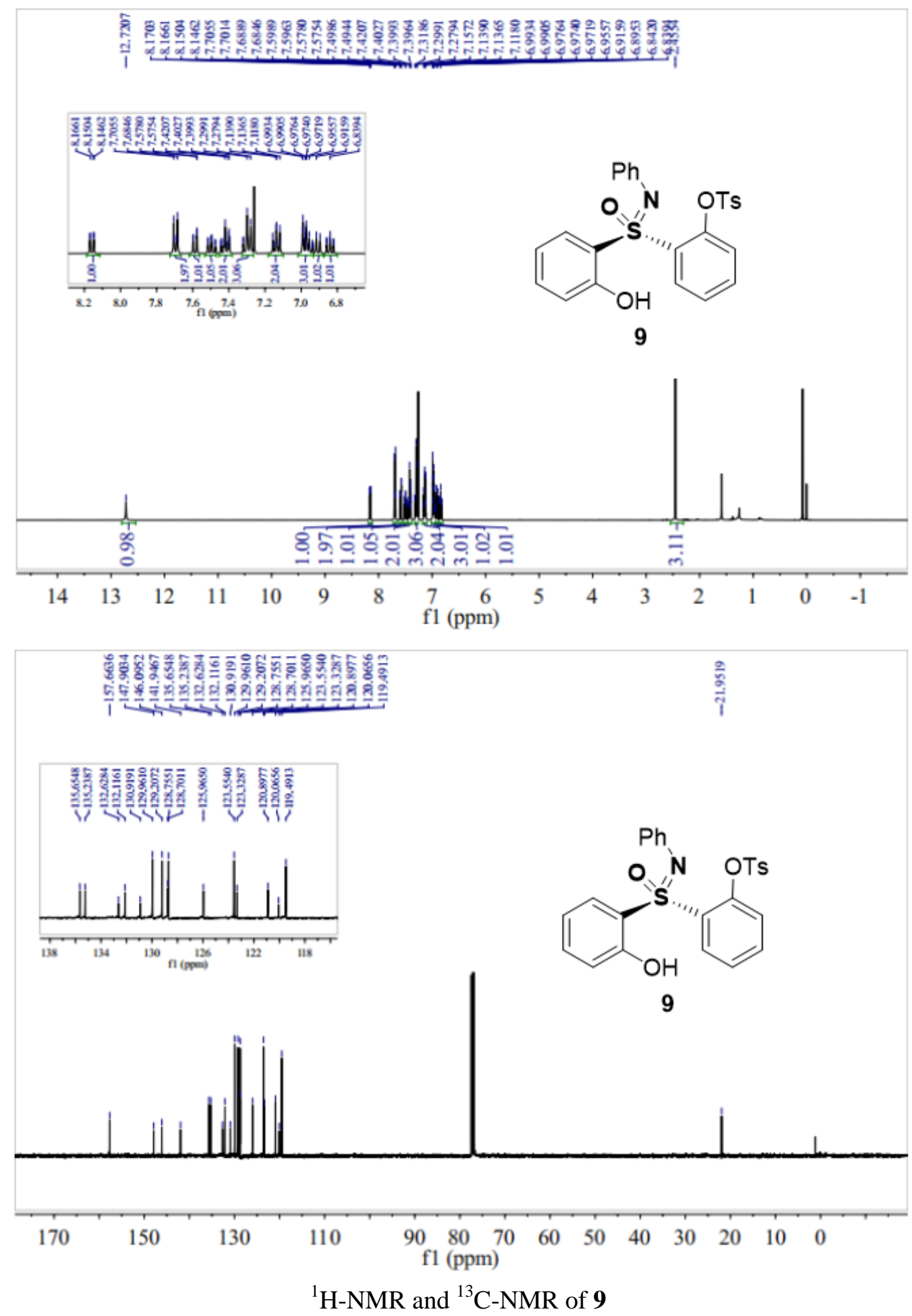

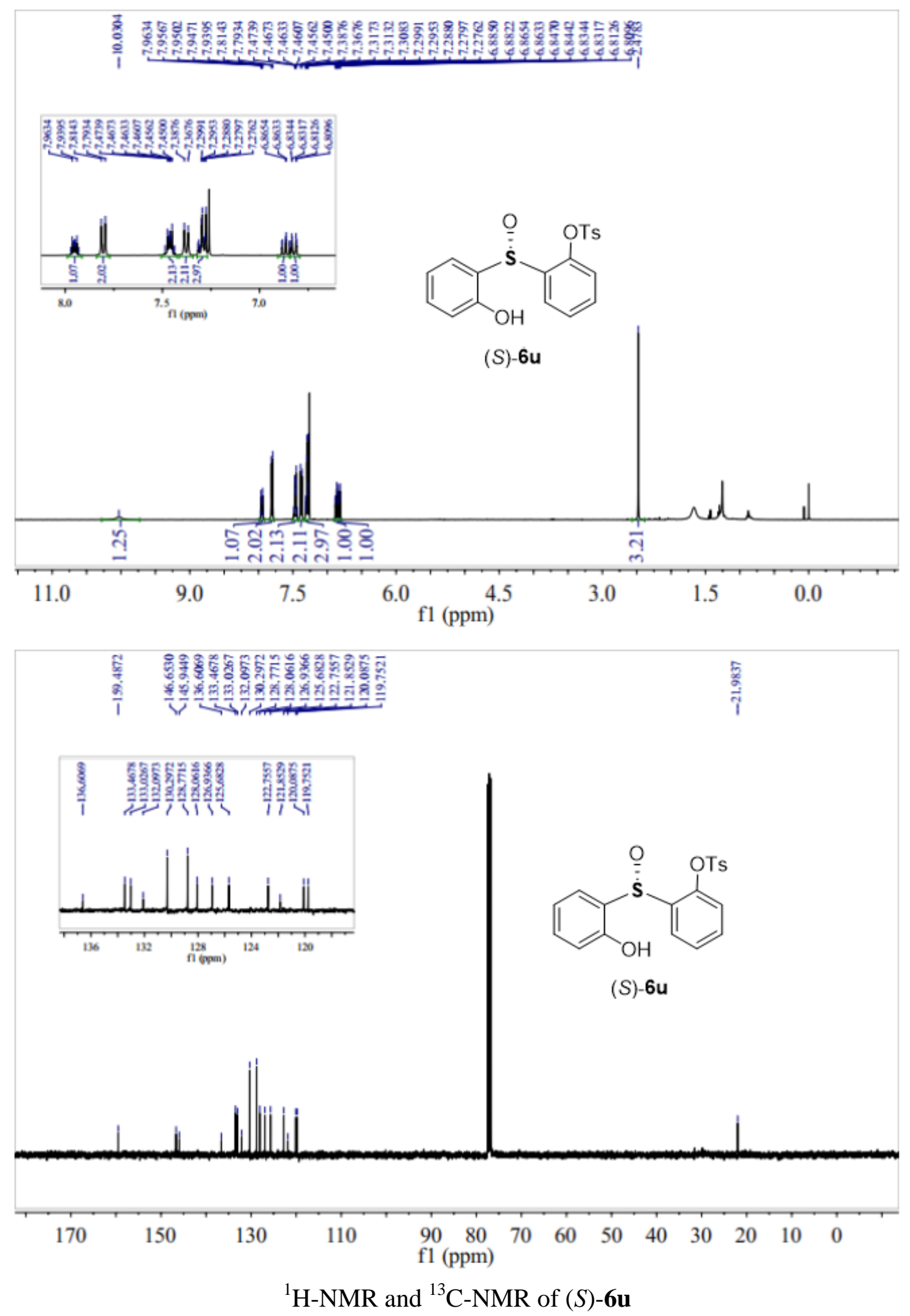

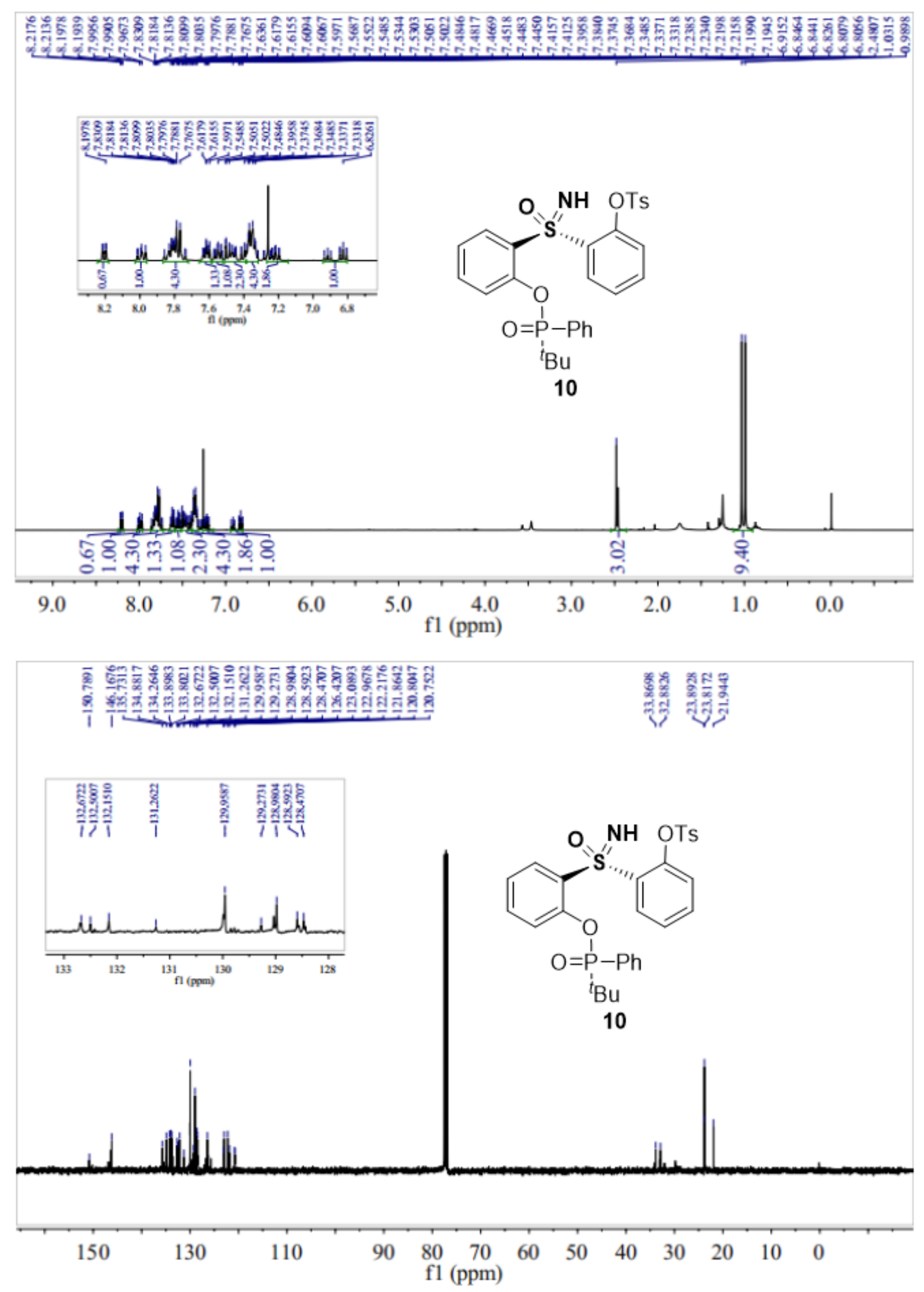


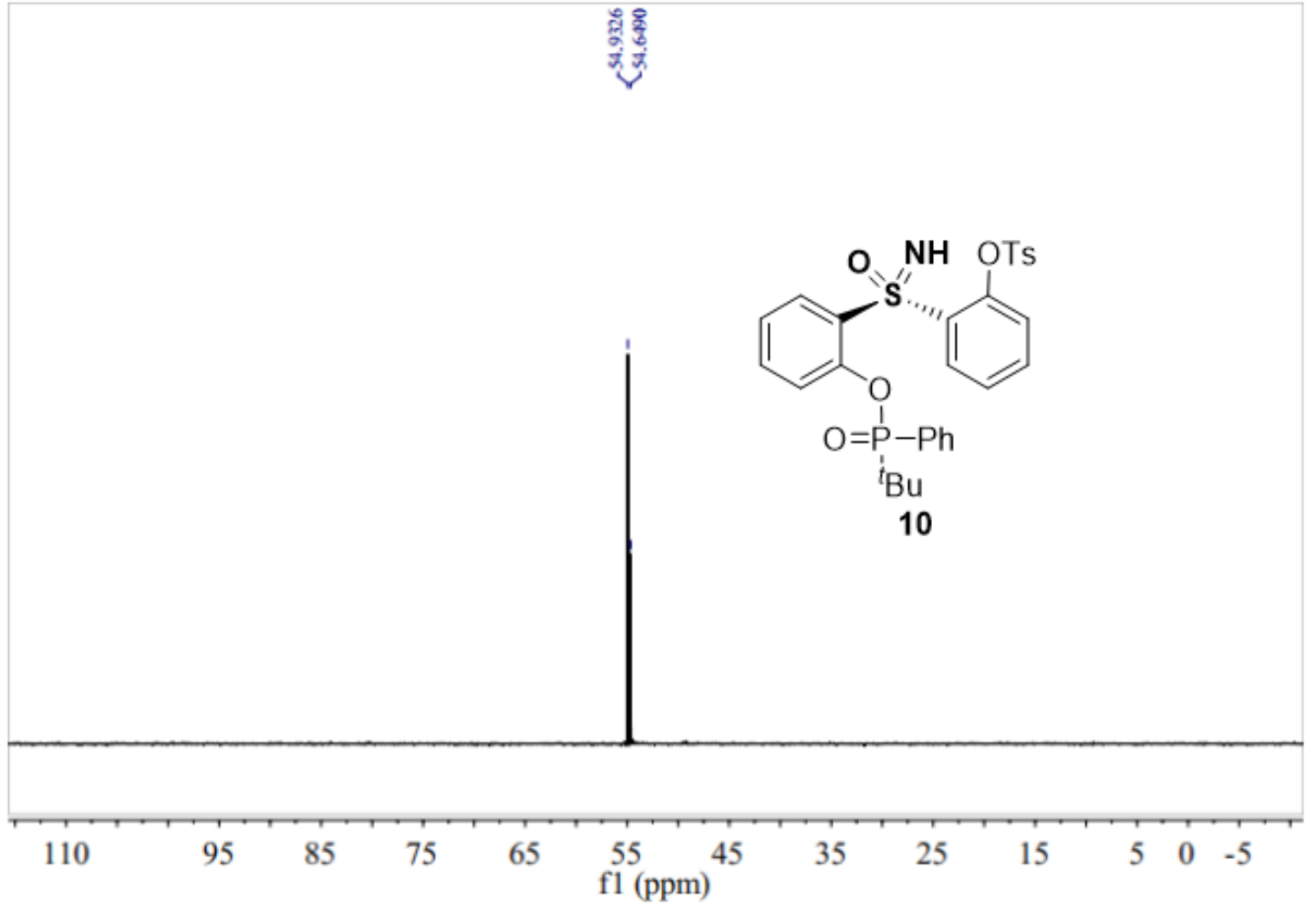

${ }^{1} \mathrm{H}-\mathrm{NMR}$ and ${ }^{13} \mathrm{C}-\mathrm{NMR}$ of $\mathbf{1 0}$ 